\section{BWXT}

Protecting America's Future

Y-12

NATIONAL

SECURITY

COMPLEX

\author{
SUPPLEMENTAL ASSESSMENT \\ OF THE \\ Y-12 GROUNDWATER PROTECTION PROGRAM \\ USING \\ MONITORING AND REMEDIATION \\ OPTIMIZATION SYSTEM SOFTWARE
}

January 2009

Prepared by

Elvado Environmental LLC and GSI Environmental, Inc. Under Subcontract No. 4300063119

for the

Environmental Compliance Department Environmental, Safety, and Health Division Y-12 National Security Complex Oak Ridge, Tennessee 37831

\section{Managed by}

Babcock \& Wilcox Technical Services Y-12, LLC for the U.S. DEPARTMENT OF ENERGY Under Contract No. DE-AC05-00OR22800 


\section{DISCLAIMER}

This report was prepared as an account of work sponsored by an agency of the United States Government. Neither the United States Government nor any agency thereof, nor any of their employees, makes any warranty, express or implied, or assumes any legal liability or responsibility for the accuracy, completeness, or usefulness of any information, apparatus, product, or process disclosed, or represents that its use would not infringe privately owned rights. Reference herein to any specific commercial product, process, or service by trade name, trademark, manufacturer, or otherwise, does not necessarily constitute or imply its endorsement, recommendation, or favoring by the United States Government or any agency thereof. The views and opinions of authors expressed herein do not necessarily state or reflect those of the United States Government or any agency thereof. 


\title{
SUPPLEMENTAL ASSESSMENT OF THE Y-12 GROUNDWATER PROTECTION PROGRAM USING MONITORING AND REMEDIATION OPTIMIZATION SYSTEM SOFTWARE
}

\author{
January 2009 \\ Prepared by \\ Elvado Environmental LLC and GSI Environmental, Inc. \\ Under Subcontract No. 4300063119 \\ for the \\ Environmental Compliance Department \\ Environmental, Safety, and Health Division \\ Y-12 National Security Complex \\ Oak Ridge, Tennessee 37831
}

\section{Managed by}

Babcock \& Wilcox Technical Services Y-12, LLC for the U.S. DEPARTMENT OF ENERGY

Under Contract No. DE-AC05-00OR22800 


\section{CONTENTS}

$\underline{\text { Section }} \quad \underline{\text { Page }}$

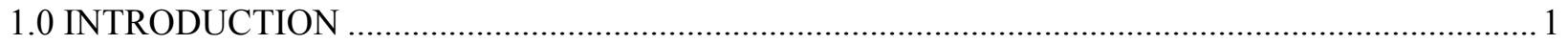

2.0 SUPPLEMENTAL MAROS ASSESSMENT APPROACH...................................................... 1

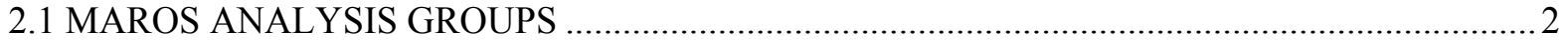

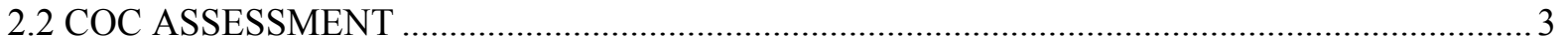

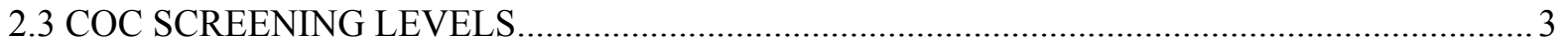

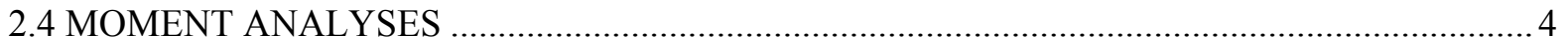

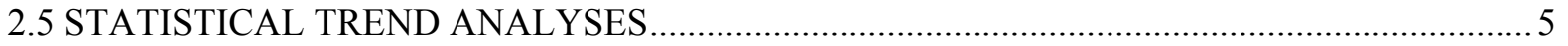

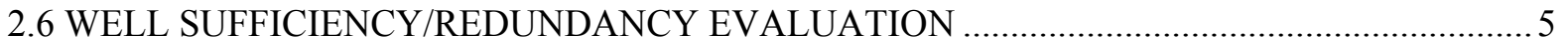

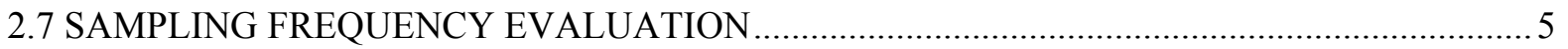

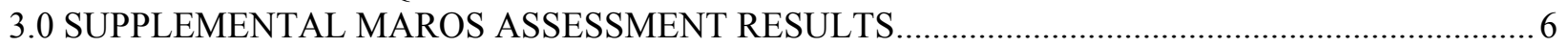

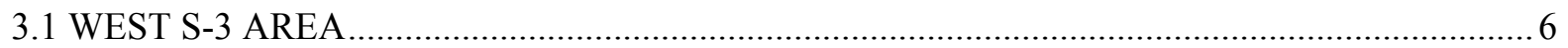

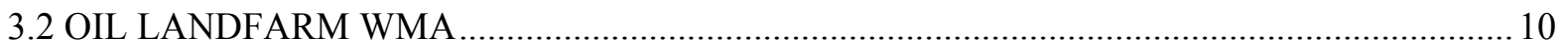

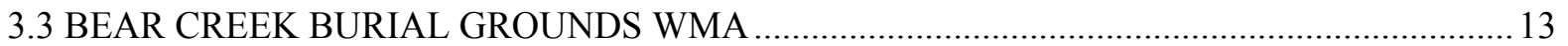

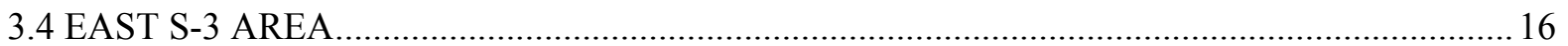

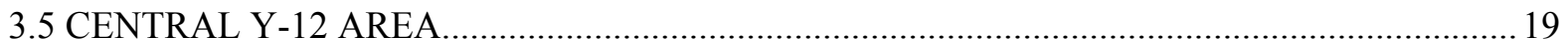

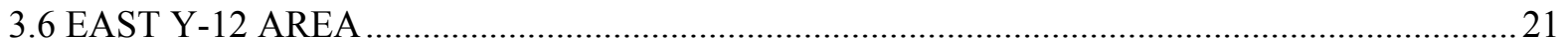

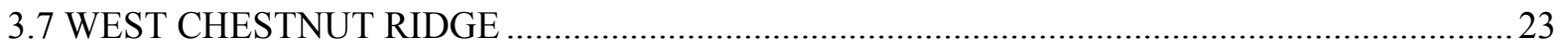

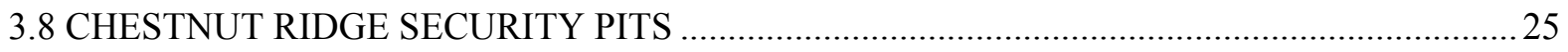

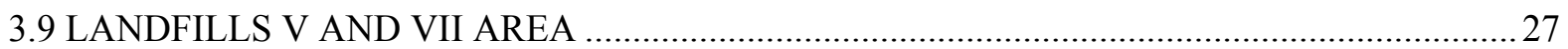

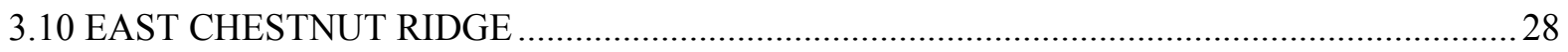

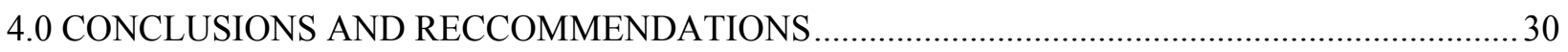

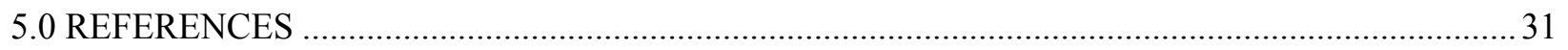

APPENDICES:

A FIGURES

B TABLES

C SUPPLEMENAL MAROS ASSESSMENT SUMMARY REPORTS

C.1 WEST S-3 AREA

C.2 OIL LANDFARM WMA

C.3 BEAR CREEK BURIAL GROUNDS WMA

C.4 EAST S-3 AREA

C.5 CENTRAL Y-12

C.6 EAST Y-12 AREA

C.7 WEST CHESTNUT RIDGE

C.8 CHESTNUT RIDGE SECURITY PITS

C.9 LANDFILLS V AND VII AREA

C.10 EAST CHESTNUT RIDGE

D SUPPLEMENAL MAROS ASSESSMENT SAMPLING RECOMMENDATIONS

D.1 BEAR CREEK HYDROGEOLOGIC REGIME

D.2 UPPER EAST FORK POPLAR CREEK HYDROGEOLOGIC REGIME

D.3 CHESTNUT RIDGE HYDROGEOLOGIC REGIME 


\section{FIGURES \\ (Appendix A)}

A.1 Hydrogeologic regimes at the Y-12 National Security Complex

A.2 Groundwater monitoring wells in the Bear Creek Hydrogeologic Regime

A.3 Groundwater monitoring wells in the Upper East Fork Poplar Creek Hydrogeologic Regime

A.4 Groundwater monitoring wells in the Chestnut Ridge Hydrogeologic Regime

A.5 Components of the Bear Creek Burial Grounds and Oil Landfarm Waste Management Areas

\section{TABLES \\ (Appendix B)}

B.1 Supplemental MAROS assessment results for the West S-3 Area MAROS analysis group

B.2 Supplemental MAROS assessment results for the Oil Landfarm WMA MAROS analysis group

B.3 Supplemental MAROS assessment results for the Bear Creek Burial Grounds WMA MAROS analysis group

B.4 Supplemental MAROS assessment results for the East S-3 Area MAROS analysis group

B.5 Supplemental MAROS assessment results for the Central Y-12 Area MAROS analysis group

B.6 Supplemental MAROS assessment results for the East Y-12 Area MAROS analysis group

B.7 Supplemental MAROS assessment results for the West Chestnut Ridge MAROS analysis group

B.8 Supplemental MAROS assessment results for the Chestnut Ridge Security Pits MAROS analysis group

B.9 Supplemental MAROS assessment results for the Landfills V and VII Area MAROS analysis group

B.10 Supplemental MAROS assessment results for the East Chestnut Ridge MAROS analysis group 


\section{ACRONYMS}

\begin{tabular}{|c|c|}
\hline AFCEE & Air Force Center for Environmental Excellence \\
\hline B4 & Beta 4 Security Pits \\
\hline $\mathrm{B} \& \mathrm{~W} \mathrm{Y}-12$ & Babcock \& Wilcox Technical Services Y-12, LLC \\
\hline $\mathrm{BCV}$ & Bear Creek Valley \\
\hline Bear Creek Regime & Bear Creek Hydrogeologic Regime \\
\hline BCBG & Bear Creek Burial Grounds \\
\hline BWXT & BWXT Y-12, L.L.C. \\
\hline BYBY & Boneyard/Burnyard \\
\hline CDL & Construction/Demolition Landfill \\
\hline CERCLA & Comprehensive Environmental Response, Compensation, and Liability Act \\
\hline Chestnut Ridge Regime & Chestnut Ridge Hydrogeologic Regime \\
\hline $\mathrm{COC}$ & contaminant of concern \\
\hline $\mathrm{CPT}$ & Coal Pile Trench \\
\hline CRBAWP & Chestnut Ridge Borrow Area Waste Pile \\
\hline CRSDB & Chestnut Ridge Sediment Disposal Basin \\
\hline CRSP & Chestnut Ridge Security Pits \\
\hline CTET & carbon tetrachloride \\
\hline $\mathrm{D}$ & decreasing (trend) \\
\hline DNAPL & dense non-aqueous phase liquid \\
\hline DOE & U.S. Department of Energy \\
\hline DQO & data quality objective \\
\hline East Fork Regime & Upper East Fork Poplar Creek Hydrogeologic Regime \\
\hline ECRWP & East Chestnut Ridge Waste Pile \\
\hline Elvado & Elvado Environmental LLC \\
\hline EMWMF & Environmental Management Waste Management Facility \\
\hline $\mathrm{FF}$ & Fuel Facility (Y-12 East End) \\
\hline FTF & Fire Training Facility \\
\hline GSI & GSI Environmental, Inc. (formerly Groundwater Services, Inc.) \\
\hline GWPP & Groundwater Protection Program \\
\hline HWDU & Hazardous Waste Disposal Unit \\
\hline I & increasing (trend) \\
\hline IL & Industrial Landfill \\
\hline LTM & long-term monitoring \\
\hline MAROS & Monitoring and Remediation Optimization System \\
\hline MCES & Modified Cost Effectiveness Sampling (method) \\
\hline MCL & maximum contaminant level \\
\hline MOP & Monitoring Optimization Plan \\
\hline NA & not applicable, insufficient sample results \\
\hline ND & non-detected analytical results \\
\hline NT & no trend \\
\hline ORR & Oak Ridge Reservation \\
\hline PCE & tetrachloroethene \\
\hline PCP & post-closure permit \\
\hline PD & probably decreasing (trend) \\
\hline PI & probably increasing (trend) \\
\hline RCRA & Resource Conservation and Recovery Act \\
\hline RG & Rust Garage Area \\
\hline ROD & record of decision \\
\hline
\end{tabular}




\section{ACRONYMS (continued)}

$\begin{array}{ll}\text { S } & \text { stable (trend) } \\ \text { SCF } & \text { South Campus Facility } \\ \text { SDWA } & \text { Safe Drinking Water Act } \\ \text { SL } & \text { Sanitary Landfill } \\ \text { SWDF } & \text { Solid Waste Disposal Facility } \\ \text { SY } & \text { Salvage Yard (Y-12 Salvage Yard) } \\ \text { Tc-99 } & \text { technetium-99 } \\ \text { TCE } & \text { trichloroethene } \\ \text { TDEC } & \text { Tennessee Department of Environment and Conservation } \\ \text { UEFPC } & \text { Upper East Fork Poplar Creek } \\ \text { UNCS } & \text { United Nuclear Corporation Site } \\ \text { VC } & \text { vinyl chloride } \\ \text { VOC } & \text { volatile organic compound } \\ \text { WCPA } & \text { Waste Coolant Processing Area } \\ \text { WMA } & \text { Waste Management Area } \\ \text { Y-12 } & \text { Y-12 National Security Complex } \\ 11 D C E & 1,1 \text {-dichloroethene } \\ \text { c12DCE } & \text { cis-1,2-dichloroethene }\end{array}$




\subsection{INTRODUCTION}

A supplemental quantitative assessment of the Groundwater Protection Program (GWPP) at the Y-12 National Security Complex (Y-12) in Oak Ridge, TN was performed using the Monitoring and Remediation Optimization System (MAROS) software. This application was previously used as part of a similar quantitative assessment of the GWPP completed in December 2005, hereafter referenced as the "baseline" MAROS assessment (BWXT Y-12 L.L.C. [BWXT] 2005. The MAROS software contains modules that apply statistical analysis techniques to an existing GWPP analytical database in conjunction with hydrogeologic factors, regulatory framework, and the location of potential receptors, to recommend an improved groundwater monitoring network and optimum sampling frequency for individual monitoring locations.

The goal of this supplemental MAROS assessment of the Y-12 GWPP is to review and update monitoring network optimization recommendations resulting from the 2005 baseline report using data collected through December 2007. The supplemental MAROS assessment is based on the findings of the baseline MAROS assessment and includes only the groundwater sampling locations (wells and natural springs) currently granted "Active" status in accordance with the Y-12 GWPP Monitoring Optimization Plan (MOP). The results of the baseline MAROS assessment provided technical rationale regarding the "Active" status designations defined in the MOP (BWXT 2006). One objective of the current report is to provide a quantitative review of data collected from Active but infrequently sampled wells to confirm concentrations at these locations. This supplemental MAROS assessment does not include the extensive qualitative evaluations similar to those presented in the baseline report.

This report was prepared by Elvado Environmental LLC (Elvado) of Knoxville, TN, in collaboration with GSI Environmental, Inc. (GSI), of Houston, TX. Elvado coordinated the performance of the supplemental MAROS assessment and assumed primary responsibility for the preparation of this report. GSI, which performed the baseline MAROS assessment of the Y-12 GWPP (BWXT 2005), provided subject matter expertise, conducted the MAROS analyses, and prepared the MAROS output data tables (Appendix B), MAROS summary reports (Appendix C), and MAROS sampling recommendations (Appendix D).

\subsection{SUPPLEMENTAL MAROS ASSESSMENT APPROACH}

The following subsections provide an overview of the technical approach for the supplemental MAROS assessment, including the MAROS analysis groups, MAROS contaminant of concern (COC) assessment, COC screening levels, MAROS moment analyses, MAROS statistical trend analyses, and the MAROS sampling frequency evaluation. Detailed information regarding the MAROS technical approach and referenced methods used by the MAROS for statistical analysis and data evaluation are deferred to the baseline MAROS assessment report (BWXT 2005) and the MAROS Users Manual (Air Force Center for Environmental Excellence [AFCEE] 2003).

The hydrogeologic system at Y-12 encompasses a complex combination of fracture-dominated anisotropic flow in low permeability non-carbonate bedrock (primarily shale) and conduit-dominated (karst) flow in more highly permeable carbonate bedrock (limestone and dolostone), with a wide range of hydraulic characteristics evident for both types of bedrock. Also, the shallow subsurface in many areas at 
Y-12 was extensively modified by the construction and operation of process facilities and numerous subsurface utilities. Both the baseline and supplemental MAROS assessment assume a simplified hydrogeologic framework for Y-12, including generalized groundwater flow directions in each hydrogeologic regime, a uniform effective porosity (0.01), and an average groundwater flow rate of $200 \mathrm{ft} / \mathrm{yr}\left(2 \times 10^{-4} \mathrm{~cm} / \mathrm{s}\right)$.

Neither the supplemental MAROS assessment nor the baseline MAROS assessment addresses seasonal changes in contaminant concentrations in the groundwater at $\mathrm{Y}-12$, although such seasonal changes are evident for known contaminants at numerous sampling locations. The influence of seasonality in groundwater concentrations may be significant when estimating short-term exposures; however objectives of long-term monitoring (LTM) networks generally involve evaluation of long-term risks and potential for significant change in status of a groundwater contaminant plume. For this reason, MAROS and most other LTM strategies do not address seasonality (BWXT 2005).

\subsection{MAROS ANALYSIS GROUPS}

Based primarily on common source areas and geographic proximity, the network of groundwater sampling locations at Y-12 (wells and springs) included in the supplemental MAROS assessment of the Y-12 GWPP are subdivided into the MAROS analysis groups shown in the following summary. Locations of the wells and springs are shown on Figure A.2 for the MAROS analysis groups in the Bear Creek Hydrogeologic Regime (Bear Creek Regime), Figure A.3 for the MAROS analysis groups in the Upper East Fork Poplar Creek Hydrogeologic Regime (East Fork Regime), and Figure A.4 for the MAROS analysis groups in the Chestnut Ridge Hydrogeologic Regime (Chestnut Ridge Regime).

\section{Hydrogeologic regimes and associated MAROS analysis groups included in the supplemental MAROS assessment of the Y-12 GWPP}

\begin{tabular}{|l|l|l|}
\hline \multicolumn{1}{|c|}{ BEAR CREEK REGIME } & \multicolumn{1}{|c|}{ EAST FORK REGIME } & \multicolumn{1}{c|}{ CHESTNUT RIDGE REGIME } \\
\hline$\bullet$ West S-3 Area & $\bullet$ East S-3 Area & $\bullet$ West Chestnut Ridge \\
$\bullet$ Oil Landfarm Waste Management & $\bullet$ Central Y-12 Area & $\begin{array}{l}\bullet \text { Chestnut Ridge Security Pits (CRSP) } \\
\bullet \text { Landfills V and VII Area } \\
\text { Area (WMA) }\end{array}$ \\
$\begin{array}{l}\text { Bear Creek Burial Grounds } \\
\text { (BCBG) WMA }\end{array}$ & & $\bullet$ East Chestnut Ridge \\
\hline
\end{tabular}

The same MAROS analysis groups were included in the baseline MAROS assessment of the Y-12 GWPP (BWXT 2005), although the respective suite of sampling locations may differ from those included in the baseline MAROS assessment. For example, 15 of the monitoring wells that were in the baseline report are excluded from the supplement report because they are no longer "Active" wells (see Section 1.0); conversely, 18 monitoring wells with recent sampling results (CY 2005 - CY 2007) had insufficient data for the baseline assessment. Also, for the supplemental MAROS assessment, the MAROS analysis group for the Central Y-12 Area includes seven wells located at the Y-12 East End Fuel Facility (FF), which the baseline MAROS assessment addressed as part of a separate MAROS analysis group. The FF wells were added to the MAROS analysis group for the Central Y-12 Area because the MAROS software requires input data from a minimum of six sampling locations with detected COCs per MAROS analysis group (AFCEE 2003) in order to perform spatial computations. Concentrations in the FF area dropped below detection limits at some locations, resulting in less than six wells with detectable levels of constituents for each sample event. 


\subsection{COC ASSESSMENT}

The COCs detected in each MAROS analysis group were prioritized on the basis of: toxicity, prevalence, and mobility in the groundwater; the findings of the baseline MAROS assessment of the Y-12 GWPP; and professional judgment. Prioritization of COCs was necessary because of the number and diversity of constituents analyzed under the Y-12 GWPP. Monitoring networks for each MAROS analysis group were optimized for the priority constituents identified during the COC Assessment. Collectively, the contaminants evaluated for the supplemental MAROS assessment represent a prioritized subset of the contaminants evaluated for the baseline MAROS assessment.

Priority constituents identified for the each analysis group as a whole were used for the moment analyses and other plume-level evaluations. The MAROS statistical trend analyses for individual wells focused on a single priority COC designated for each sampling location per the technical approach established in the baseline MAROS assessment, which involves determining the normalized ratio between the average concentration of the COC and the corresponding screening level (see Sect. 2.3). For each sampling location, the average concentration of each applicable COC is calculated from available sampling results (non-detect results default to a uniform value corresponding to the lowest analytical reporting limit for the COC). Average concentrations were determined from analytical results that meet the data quality objectives (DQOs) of the Y-12 GWPP. Dividing the average concentration of each COC by the applicable screening level determines the normalized ratio, with the COC having the highest normalized ratio being selected as the representative $\mathrm{COC}$ designated for the sampling location.

\subsection{COC SCREENING LEVELS}

The COC screening levels used for the supplemental MAROS assessment of the Y-12 GWPP are: (1) the Safe Drinking Water Act (SDWA) maximum contaminant levels (MCLs) currently adopted by the U.S. Environmental Protection Agency (EPA) and the Tennessee Department of Environment and Conservation (TDEC); (2) the current EPA- and TDEC-approved secondary MCLs for drinking water; and (3) the EPA Region IX preliminary remediation goals for contaminated groundwater. As shown in the following summary, each priority COC for the MAROS analysis groups was selected based on exceeding the applicable MCL. Note that the screening level for gross beta activity is the SDWA threshold value above which the dose equivalent may exceed the drinking water MCL for gross beta activity (4 millirems per year).

Screening levels used for the supplemental MAROS assessment of the Y-12 GWPP

\begin{tabular}{|c|l|c|}
\hline PRIORITY COC & UNITS & MCL \\
\hline Nitrate & milligrams per liter $(\mathrm{mg} / \mathrm{L})$ & 0.01 \\
Cadmium & & 0.03 \\
Uranium & & 15 \\
\hline Gross alpha activity & picoCuries per liter $(\mathrm{pCi} / \mathrm{L})$ & 50 \\
Gross beta activity & & 5 \\
Benzene & micrograms per liter $(\mu \mathrm{g} / \mathrm{L})$ & 5 \\
Carbon tetrachloride (CTET) & & 7 \\
1,1-Dichloroethene (11DCE) & & 70 \\
cis-1,2-Dichloroethene (c12DCE) & & 5 \\
Tetrachloroethene (PCE) & & 5 \\
Trichloroethene (TCE) & & 2 \\
Vinyl chloride (VC) & & \multicolumn{2}{|c|}{} \\
\hline
\end{tabular}


The baseline MAROS assessment of the Y-12 GWPP used these same screening levels for each applicable COC (BWXT 2005).

\subsection{MOMENT ANALYSES}

The MAROS moment analysis module provides a relative estimate of groundwater contaminant plume stability in the context of results from other MAROS modules. The MAROS moment analysis algorithms are simple approximations of complex calculations and are meant to roughly estimate changes in total dissolved plume mass $\left(0^{\text {th }}\right.$ Moment $)$, center of plume mass $\left(1^{\text {st }}\right.$ Moment $)$, and distribution of plume mass about the center ( $2^{\text {nd }}$ Moment). The moment analyses provide a "plume-level" review of constituent mobility. For the supplemental report, the actual MAROS moment analysis results are not reported, but rather the moment trends for the plume mass are indicated for each MAROS analysis group and applicable COC (BWXT 2005).

The MAROS software uses the Mann-Kendall method for statistical analysis of the moment trends. Trend results of the MAROS moment analyses were interpreted as: Decreasing (D), Probably Decreasing (PD), Stable (S), Increasing (I), Probably Increasing (PI), No Trend (NT), no moment analysis because of non-detect (ND) analytical results; and Not Applicable (N/A) due to insufficient sampling results. Details of the statistical algorithm used can be found in the MAROS Users Manual (AFCEE, 2003). Stable to decreasing trends provide evidence that monitoring efforts may be reduced, whereas increasing trends potentially indicate downgradient migration of the plume or generation of daughter products due to biodegradation of parent constituents that may warrant increased monitoring efforts (BWXT 2005). No Trend results indicate the absence of a clear trend in the data and are usually associated with relatively high variability in the data, while Stable (S) usually indicates no definite trend and lower variability in the data.

Moment analysis trends are sensitive to the number and identity of wells used for the moment calculations. Because many sampling locations in the analysis groups are not sampled routinely, the moment analyses are based on annually averaged COC concentrations determined from sampling results that meet the DQOs of the Y-12 GWPP (average values were calculated as described in Section 2.2). Annual moments were calculated for the 1996 to 2007 time period. In order to provide year-to-year comparability in the moment analyses for each analysis group, two datasets were evaluated for certain networks. The first dataset includes data from all of the active wells in the analysis group, while the second dataset includes only data from wells that were sampled frequently at regular intervals during the 1996 to 2007 time interval. The second or "select" dataset includes active wells that were identified as part of the optimized monitoring network in the baseline report, and excludes wells that have not been sampled at least 4 times between 1996 and 2007.

Two datasets were developed for the West S-3 Area, Oil Landfarm WMA, BCBG WMA, and East S-3 Area MAROS analysis groups. By comparing results between the full dataset and the reduced "select" dataset, the statistical impact of intermittently sampled wells can be determined. Significant differences in moments calculated from full and reduced sampling networks may indicate that intermittently sampled wells should be included in the monitoring program on a more frequent basis. 


\subsection{STATISTICAL TREND ANALYSES}

The MAROS software uses the combined results from Mann-Kendall and linear regression methods of statistical trend analysis to evaluate concentration trends for the COC designated for each applicable groundwater sampling location (wells and springs) in each MAROS analytical group. Both trend analysis methods require at least four sampling results for each applicable COC for each sampling location (BWXT 2005).

For each priority COC at each location, the statistical trend analyses addressed the "Recent" trend determined from sampling results obtained between January 1996 and December 2007 and the "Overall" trend determined from sampling results obtained between January 1986 and December 2007. Results of the Recent and Overall trends for each COC were interpreted using the same nomenclature previously described for the MAROS moment trend analyses.

\subsection{WELL SUFFICIENCY/REDUNDANCY EVALUATION}

The MAROS well sufficiency analysis module is intended to identify/recommend locations for new wells in areas where additional plume information is needed because of high uncertainty in groundwater concentrations between existing wells (BWXT 2005). To evaluate well sufficiency, Delaunay triangulation is performed to determine if plume concentrations at each existing well can be predicted from concentrations at its nearest neighbors. Also, the Delaunay method results are derived solely from the two-dimensional spatial configuration of the existing well network and the spatial pattern of the contaminant plume. Therefore, the well sufficiency analysis does not assess potential new well locations needed outside the existing well network (BWXT 2005).

The MAROS well redundancy analysis module is intended to identify existing wells that are redundant within the current well network and can be removed from routine monitoring with minimal impact on the long-term characterization of a contaminant plume (BWXT 2005). To evaluate well redundancy, MAROS uses the Delaunay triangulation to calculate the network area and the average concentration of the plume using data from multiple contaminants and sampling locations. The relative significance of each well within the network is indicated by a slope factor calculated for each well, with the calculated slope factor used to evaluate if excluding data for the well(s) will significantly change the calculated average plume concentration.

\subsection{SAMPLING FREQUENCY EVALUATION}

The MAROS software uses the Modified Cost Effective Sampling (MCES) method to evaluate the optimum sampling frequency for each groundwater sampling location in each MAROS analysis group based on the magnitude, direction, and uncertainty of COC concentration trends from recent and historical analytical data. The MCES method combines quantitative and qualitative evaluations to estimate the lowest-frequency sampling schedule (quarterly, semiannual, annual, biennial, or other) for a given sampling location that will still provide needed information for regulatory and remedial decision-making. Quantitatively, the trend and rate of change are determined by Mann-Kendall and linear regression trend analyses and are used to generate a preliminary sampling frequency recommendation for each sampling location. Qualitative review of the preliminary recommendation, based on considerations such as the 
regulatory requirements or proximity to key monitoring areas, is then performed to designate the final sampling frequency recommendation.

The MCES method requires data from at least four sampling events to evaluate a sampling frequency, and automatically assigns a default sampling frequency (quarterly) to each sampling location with fewer sampling events.

Output for the MCES analysis of the data for each sampling location was considered the "Preliminary" recommended sampling frequency subsequently evaluated in consideration of other factors, including professional judgment, to determine the "Final" recommended sampling frequency. Additionally, for each applicable well and natural spring associated with the following regulatory-driven groundwater monitoring programs, the "Final" recommended sampling frequency was designated as "Regulated" for the purposes of the Y-12 GWPP.

- Post-closure detection and post-closure corrective action groundwater monitoring at hazardous waste treatment, storage, and disposal units addressed under the respective Resource Conservation and Recovery Act (RCRA) post-closure permit (PCP) for the Bear Creek Regime, East Fork Regime, and Chestnut Ridge Regime;

- Baseline monitoring and remedial action effectiveness monitoring in accordance with the Comprehensive Environmental Response, Compensation, and Liability Act (CERCLA) record of decision (ROD) and/or related decision documents for applicable sites in each hydrogeologic regime at $\mathrm{Y}-12$;

- Detection monitoring at the Environmental Management Waste Management Facility (EMWMF), an operating hazardous waste landfill in Bear Creek Valley (BCV) west of Y-12 that receives wastes generated from CERCLA remedial actions on the DOE Oak Ridge Reservation (ORR); and

- Detection monitoring at operating and closed non-hazardous waste landfills on Chestnut Ridge south of Y-12 in accordance with the solid waste disposal facility (SWDF) permit issued by the TDEC and the requirements of the TDEC regulations governing operation, closure, and postclosure care of SWDFs.

\subsection{SUPPLEMENTAL MAROS ASSESSMENT RESULTS}

This section presents the results of the supplemental MAROS assessment of the Y-12 GWPP, with separate subsections for each MAROS analysis group identified in Section 2.1. Each subsection generally follows a standardized format that includes brief discussions (and applicable data summary tables) of the MAROS output/findings regarding the sampling locations, COC assessment, moment analyses, statistical trend analyses, well sufficiency analyses, and sampling frequency evaluation.

\subsection{WEST S-3 AREA}

The MAROS analysis group for the West S-3 Area includes groundwater sampling locations BCV near the western end of Y-12, most of which are located near the former S-3 Ponds (Figure A.2), a closed 
former hazardous waste surface impoundment that is a principal source of groundwater contamination at $\mathrm{Y}-12$.

\section{Sampling Locations}

There are 26 groundwater sampling locations included in the MAROS analysis group for the West S-3 Area (Table B.1): 14 wells completed in the low permeability fractured non-carbonate bedrock (Nolichucky Shale) that underlies the former S-3 Ponds, 11 exit-pathway wells completed in the Maynardville Limestone near the Rust Spoil Area and Spoil Area I, and a natural spring (SS-1) that discharges into Bear Creek approximately $1500 \mathrm{ft}$ west-southwest of the S-3 Ponds (Figure A.2). Twenty-one of the 26 locations were sampled between 2005 and 2007. Several of the wells yield some of the most highly contaminated groundwater in BCV west of Y-12. The baseline MAROS assessment of the Y-12 GWPP included each of these wells and spring SS-1 in the MAROS analysis group for the West S-3 Area (BWXT 2005).

\section{COC Assessment}

On the basis of the supplemental MAROS assessment output (see MAROS COC Assessment report in Appendix C.1), gross beta activity, nitrate, TCE, PCE, and uranium are designated as a principal COC for at least one of the groundwater sampling locations in the West S-3 Area MAROS analysis group. The results are identical to those of the baseline report. Nitrate exceeded screening levels by the highest margin area-wide and was present above screening levels at the greatest number of sampling locations. The COCs listed above were also found to be priority COCs for at least one of the individual groundwater sampling locations included in the MAROS analysis group for the West S-3 Area.

\section{Moment Analysis}

The MAROS moment analyses based on the data for the groundwater sampling locations included in the MAROS analysis group for the West S-3 Area focused on analytical results for gross beta activity, nitrate, PCE, TCE, and uranium (see the MAROS Spatial Moment Analysis Summary report in Appendix C.1). Also, as described in Section 2.4, moment analyses for each COC were performed using one dataset that included analytical results for all the sampling locations included in the MAROS analysis group, and another dataset that excluded analytical results for a selected subset of these sampling locations.

Results of the MAROS moment analyses for each applicable COC and dataset, summarized below, show largely S trends or NT. Gross beta activity, shows D trends for total dissolved mass and distribution of mass perpendicular to groundwater flow $\left(0^{\text {th }}\right.$ Moment and the $2^{\text {nd }}$ Moment (Sigma YY) for the full dataset. Differences between the moment trends for each COC reflect a combination of various potential influences, including inherent analytical variability, the differential transport and anisotropic distribution in the groundwater, and substantial temporal (seasonal and episodic) concentration fluctuations. Similar trends also are indicated by results of the moment analyses performed under the baseline MAROS assessment of the Y-12 GWPP, which focused on sampling results for PCE and gross beta activity (BWXT 2005). These findings do not indicate significant changes in the estimated dissolved mass, relative mass, movement, and spread of the contaminant plumes that may warrant greater monitoring efforts (i.e., increased sampling locations and/or frequencies) for the West S-3 Area. 
Summary of the supplemental MAROS moment trend analysis for the West S-3 Area

\begin{tabular}{|c|c|c|c|c|c|c|c|c|}
\hline \multirow{4}{*}{$\begin{array}{l}\text { PRIORITY } \\
\text { COC }\end{array}$} & \multicolumn{8}{|c|}{ MOMENT TREND $^{1}$} \\
\hline & \multirow{2}{*}{\multicolumn{2}{|c|}{$\begin{array}{l}0^{\text {th }} \text { Moment } \\
\text { (Plume Mass) }\end{array}$}} & \multirow{2}{*}{\multicolumn{2}{|c|}{$\begin{array}{c}1^{\text {st }} \text { Moment } \\
\text { (Plume Center of Mass) }\end{array}$}} & \multicolumn{4}{|c|}{$\begin{array}{c}2^{\text {nd }} \text { Moment } \\
\text { (Plume Spread) }\end{array}$} \\
\hline & & & & & \multicolumn{2}{|c|}{ Sigma XX } & \multicolumn{2}{|c|}{ Sigma YY } \\
\hline & All & Select & All & Select & All & Select & All & Select \\
\hline Gross beta activity & $\mathrm{D}$ & $\mathrm{S}$ & $\mathrm{S}$ & NT & $\mathrm{S}$ & NT & $\mathrm{D}$ & $\mathrm{S}$ \\
\hline Nitrate & $\mathrm{S}$ & $\mathrm{S}$ & $\mathrm{S}$ & $\mathrm{S}$ & $\mathrm{S}$ & NT & $\mathrm{S}$ & $\mathrm{S}$ \\
\hline PCE & $\mathrm{S}$ & $\mathrm{S}$ & NT & $\mathrm{S}$ & NT & $\mathrm{S}$ & NT & $\mathrm{S}$ \\
\hline TCE & $\mathrm{S}$ & $\mathrm{S}$ & NT & $\mathrm{S}$ & NT & $\mathrm{S}$ & $\mathrm{S}$ & $\mathrm{S}$ \\
\hline Uranium & NT & NT & $\mathrm{S}$ & $\mathrm{S}$ & NT & NT & $\mathrm{S}$ & $\mathrm{S}$ \\
\hline
\end{tabular}

No I or PI trends were found for moments calculated for the West S-3 area. The majority of analyses indicate stable or variable no trend results for both the full network and the select (reduced) network. As shown in the preceding data summary, paired results of the separate moment datasets for each COC show the same trends most frequently for nitrate and uranium, the only COC with no disagreement between paired trends for the $0^{\text {th }}$ Moment, $1^{\text {st }}$ Moment, and $2^{\text {nd }}$ Moments (Sigma XX and Sigma YY). The 'select' dataset, with a reduced number of wells, closely represents nitrate and uranium 'plume' trends for the full dataset. Conversely, there is less frequent agreement between the respective paired moment trends for TCE, PCE, and gross beta activity, the only COC for which none of the paired trends are the same. Additionally, the separate analyses of the $0^{\text {th }}$ Moment show agreement between paired trends for each COC except gross beta activity, whereas the separate analytes of the $2^{\text {nd }}$ Moments only show agreement for the paired trends for nitrate (Sigma YY) and uranium (Sigma XX and Sigma YY). These findings suggest that data for intermittently sampled individual or combinations of wells included in the full MAROS analysis group for the West S-3 Area may influence the moment trends for gross beta activity and VOCs, but do not appear to affect the moment trends for nitrate and uranium. Overall, the reduced 'select' network provides adequate data to evaluate the moment trends for the priority COCs in the West S-3 area.

\section{Statistical Trend Analysis}

Statistical trend analysis of data for the groundwater sampling locations included in the MAROS analysis group for the West S-3 Area focused on analytical results for gross alpha activity, gross beta activity, nitrate, PCE, and TCE (see the MAROS Statistical Trend Analysis Summary report in Appendix C.1). For most of the sampling locations, results of the overall statistical trend analyses (1986 - 2007) show a D, $\mathrm{PD}$, or $\mathrm{S}$ concentration trend for the designated COC (Table B.1). Of the 21 wells evaluated for statistical trends, 11 locations show a strongly decreasing concentration trend.

The baseline MAROS assessment of the Y-12 GWPP included sufficient data to determine statistical trends (1996 - 2004) for only five sampling locations (wells GW-276, GW-311, GW-315, GW-829, and spring SS-1). The remaining wells in the network had insufficient recent data to determine a trend. The corresponding results of the statistical analyses also show a D, PD, or S concentration trend for each designated COC (BWXT 2005).

Results of the supplemental MAROS statistical analyses for the overall trends (1986 - 2007) show increasing or probably increasing trends for the priority COC in wells GW-246 (gross beta activity), GW-526 (nitrate), and GW-615 (nitrate). Recent trends (1996 - 2007) for each well are S, S, and NT 
respectively. Each well yields contaminated groundwater from the low permeability fractured noncarbonate bedrock (Nolichucky Shale) that underlies the former S-3 Ponds. The increasing overall trend for gross beta activity in well GW-246, which is located adjacent to the former S-3 Ponds (Figure A.2), may be an artifact of slightly lower concentrations found in 1987 and data variability associated with analytical interferences from the high levels of dissolved solids in the acidic groundwater from the well. Increasing overall concentrations of nitrate in the groundwater from wells GW-526 and GW-615 similarly result from slightly lower concentrations found in 1988 - 1989. Recent data indicate more stable trends for each of these wells.

\section{Well Sufficiency/Redundancy Evaluation}

Results of the supplemental MAROS assessment of well sufficiency for the West S-3 Area MAROS analysis group do not indicate areas of high uncertainty in the concentrations of each $\mathrm{COC}$ in the groundwater that warrant the installation of additional wells. The baseline MAROS assessment of the Y-12 GWPP noted some areas of uncertainty in the concentrations of PCE defined by available data for wells near the former S-3 Ponds, and recommended continued routine sampling of specific wells (GW-243, GW-276, and GW-315) to track PCE concentrations (BWXT 2005).

Results of the supplemental MAROS assessment (Appendix D.1) recommend discontinued sampling (i.e., "Elimination") of the following active wells in the MAROS analysis group for the West S-3 Area that are considered to provide redundant local monitoring coverage in areas with low uncertainty regarding the spatial distribution and concentrations of each applicable COC in the groundwater. Future collection of groundwater samples from these wells will be discontinued if the wells are granted "Inactive" status, as defined and specified in the most current version of the Y-12 GWPP MOP (BWXT 2006).

Summary of supplemental MAROS well sufficiency and well redundancy analysis for the West S-3 Area

\begin{tabular}{|c|l|}
\hline $\begin{array}{c}\text { WELL } \\
\text { NUMBER }\end{array}$ & \multicolumn{1}{|c|}{ BASIS FOR MAROS “ELIMINATION” RECOMMENDATION } \\
\hline GW-122 & Overall decreasing trend, redundant with well GW-616. \\
\hline GW-236 & Decreasing COC concentration trend, redundant with spring SS-1. \\
\hline GW-277 & Stable to decreasing trends, redundant with well GW-276. \\
\hline GW-313 & Redundant with well GW-315. \\
\hline
\end{tabular}

Results of the supplemental MAROS assessment of the sampling locations included in the MAROS analysis group for the West S-3 Area also recommend "Elimination" of wells GW-127, GW-244, GW-245, GW-247, and GW-615 (Appendix D.1). However, these wells are designated as POC wells for RCRA post-closure corrective action monitoring at the former S-3 Ponds and, as noted in Section 2.7, MAROS-based sampling frequency recommendations are not applicable to these wells.

\section{Sampling Frequency Evaluation}

Based on results of the supplemental MAROS assessment of the groundwater sampling frequency appropriate for the sampling locations included in the MAROS analysis group for the West S-3 Area (Appendix D.1), annual sampling is recommended for the 11 sampling locations (10 wells and spring SS-1) that either do not have a regulatory-driven sampling frequency and were not recommended for discontinued sampling ("Elimination”) per the preceding discussion. 


\subsection{OIL LANDFARM WMA}

Located in BCV approximately one mile west of Y-12 (Figure A.2), the Oil Landfarm WMA includes the Oil Landfarm hazardous waste disposal unit (HWDU), the Boneyard/Burnyard (BYBY), the Hazardous Chemical Disposal Area, and Sanitary Landfill I (Figure A.5). Each site is a known or suspected source of groundwater contamination, and the BYBY was the target of CERCLA remedial action, completed in October 2003, that involved the excavation and removal of uranium-related wastes.

\section{Sampling Locations}

There are 32 monitoring wells included in the MAROS analysis group for the Oil Landfarm WMA (Table B.2): 17 wells completed in the low permeability bedrock formations that underlie the Oil Landfarm WMA and the EMWMF, and 15 exit-pathway monitoring wells completed in the Maynardville Limestone south and southwest of the Oil Landfarm HWDU (Figure A.2). Of the 32 wells in the analysis group, 29 had new data collected between 2005 and 2007. The baseline MAROS assessment of the Y-12 GWPP also included these wells in the MAROS analysis group for the Oil Landfarm WMA (BWXT 2005).

\section{COC Assessment}

On the basis of the supplemental MAROS output for the COC assessment (see MAROS COC Assessment report in Appendix C.2), nitrate, TCE, and VC each are designated as a principal COC for one or more of the groundwater sampling locations included in the MAROS analysis group for the Oil Landfarm WMA. These results mirror the findings of the baseline MAROS assessment (BWXT 2005).

\section{Moment Analysis}

The MAROS moment analyses based on the data for the groundwater sampling locations included in the MAROS analysis group for the Oil Landfarm WMA focused on analytical results for gross alpha activity, nitrate, PCE, TCE, and VC (see the MAROS Spatial Moment Analysis Summary report in Appendix C.2). Also, as described in Section 2.4, moment analyses for each COC were performed using one dataset that included analytical results for all the sampling locations included in the MAROS analysis group, and another dataset that excluded analytical results for a selected subset of these sampling locations.

As shown in the following summary, a combination of S trends and NT due to data variability dominate the MAROS moment trends for each COC, with an I trend evident for gross alpha activity ( $1^{\text {st }}$ Moment) and D trends evident for TCE $\left(0^{\text {th }}\right.$ Moment $)$ and gross alpha activity $\left(2^{\text {nd }}\right.$ Moment, Sigma YY $)$. Similar moment trends were evident from the baseline MAROS assessment, which focused on the data for nitrate, PCE, and VC (BWXT 2005). These findings generally do not indicate widespread changes in the relative mass, movement, and spread of the dissolved VOCs released to the groundwater from sources associated with the Oil Landfarm WMA. 
Summary of the MAROS moment trend analysis for the Oil Landfarm WMA

\begin{tabular}{|c|c|c|c|c|c|c|c|c|}
\hline \multirow{4}{*}{ COC } & \multicolumn{8}{|c|}{ MOMENT TREND $^{1}$} \\
\hline & \multirow{2}{*}{\multicolumn{2}{|c|}{$\begin{array}{c}\text { 0 }^{\text {th }} \text { Moment } \\
\text { (Plume Mass) }\end{array}$}} & \multirow{2}{*}{\multicolumn{2}{|c|}{$\begin{array}{c}1^{\text {st }} \text { Moment } \\
\text { (Plume Center of } \\
\text { Mass) }\end{array}$}} & \multicolumn{4}{|c|}{$\begin{array}{c}2^{\text {nd }} \text { Moment } \\
\text { (Plume Spread) }\end{array}$} \\
\hline & & & & & \multicolumn{2}{|c|}{ Sigma XX } & \multicolumn{2}{|c|}{ Sigma YY } \\
\hline & All & Select & All & Select & All & Select & All & Select \\
\hline Gross alpha activity & NT & NT & $\mathrm{I}$ & NT & $\mathrm{S}$ & NT & $\mathrm{S}$ & $\mathrm{D}$ \\
\hline Nitrate & $\mathrm{S}$ & $\mathrm{S}$ & $\mathrm{S}$ & $\mathrm{S}$ & NT & NT & $\mathrm{S}$ & $\mathrm{S}$ \\
\hline PCE & NT & NT & $\mathrm{S}$ & $\mathrm{S}$ & $\mathrm{S}$ & $\mathrm{S}$ & $\mathrm{S}$ & $\mathrm{S}$ \\
\hline TCE & $\mathrm{S}$ & $\mathrm{D}$ & NT & NT & NT & NT & NT & NT \\
\hline $\mathrm{VC}$ & NT & NT & NT & NT & NT & NT & NT & NT \\
\hline
\end{tabular}

Based on the area-wide COC Assessment, TCE is the priority constituent for the Oil Landfarm WMA analysis group. Moment results for TCE indicate NT for first and second moments for both the full and select datasets. The $0^{\text {th }}$ Moments for TCE indicate a stable trend for the full dataset and a decreasing trend for the select well network. The stable trend results from a low confidence factor testing for the decreasing trend. The select network estimates higher average mass in the plume, but a decreasing trend. The select dataset removed well GW-226, a location with relatively high but stable TCE concentration trends.

Moment trends for nitrate indicate a stable plume, and the select network represents the full network accurately. Results for vinyl chloride show variability in data, most likely because only 6 of the sampling locations have detectable concentrations of the COC. The MAROS moment analysis of the gross alpha activity results for all of the wells included in the MAROS analysis group for the Oil Landfarm WMA shows an I trend for the $1^{\text {st }}$ Moment, which potentially indicates an increase in the distance between the source and the center of mass of the COC (BWXT 2005). Uranium isotopes in the groundwater are the source of the gross alpha activity, and uranium-bearing wastes buried at the former BYBY at the Oil Landfarm WMA were a principal source of uranium isotopes in the groundwater and surface water in BCV (DOE 1997). Following excavation and removal of the wastes as part of a CERCLA remedial action completed in March 2003, the center of mass of uranium isotopes released to the groundwater system may now be located at some point downgradient (south-southwest) of the former BYBY, which may by why the data for gross alpha activity show an increasing trend for the $1^{\text {st }}$ Moment.

As shown in the preceding data summary, separate moment analyses for nitrate, PCE, and VC show no disagreement between paired trends for the $0^{\text {th }}$ Moment, $1^{\text {st }}$ Moment, and $2^{\text {nd }}$ Moments (Sigma XX and Sigma YY), whereas paired moment trends for gross alpha activity show the most frequent disagreement. These findings suggest that data for individual or combinations of wells included in the MAROS analysis group for the Oil Landfarm WMA do not appear to disproportionately influence the moment trends for each COC except possibly gross alpha activity.

\section{Statistical Trend Analysis}

Statistical trend analysis of the groundwater sampling data for the wells included in the MAROS analysis group for the Oil Landfarm WMA focused on analytical results for nitrate, PCE, TCE, VC, and uranium (see the MAROS Statistical Trend Analysis Summary report in Appendix C.2). For most of the wells, the results of the MAROS statistical trend analyses show a D trend, PD trend, or no trend because of data variability for the applicable COC (Table B.2). 
An I or PI trend is evident for the COC concentrations in several wells, including well GW-008 (PCE, both recent and overall trend), which is a RCRA POC well that yields VOC-contaminated groundwater from the low-permeability fractured non-carbonate bedrock (Nolichucky Shale) underlying the Oil Landfarm HWDU, and exit-pathway wells GW-225 (TCE, recent), GW-226 (TCE, overall), GW-229 (VC, overall), and GW-365 (VC, overall), each of which yield VOC-contaminated groundwater from the Maynardville Limestone south and southwest of the Oil Landfarm WMA (Figure A.2). Results of the statistical trend analyses performed under the baseline MAROS assessment of the Y-12 GWPP likewise show I trends for the COC concentrations in wells GW-008, GW-225, and GW-226 (BWXT 2005). Increasing concentration trends each of these wells except GW-226 potentially reflect corresponding increases in the relative flux of contaminants (VOCs) via the groundwater flowpaths intercepted by the monitored interval in each well; however, the increasing trend for well GW-226 is probably an artifact of the change in groundwater sampling method (B\&W Y-12 2008). Increasing trends for vinyl chloride may indicate anaerobic biodegradation of parent TCE and PCE.

\section{Well Sufficiency/Redundancy Evaluation}

Results of the supplemental MAROS assessment for well sufficiency for the Oil Landfarm WMA MAROS analysis group do not indicate areas where installation of new wells may be warranted to address high uncertainty regarding the spatial distribution and concentration of each applicable COC in the groundwater. The baseline MAROS assessment reported similar findings, noting some uncertainty in the concentrations of nitrate in area between wells exit-pathways wells GW-225 and GW-795, and did not recommend installation of new wells (BWXT 2005).

Results of the supplemental MAROS assessment recommend discontinued sampling (i.e., "Elimination") of six monitoring wells in the MAROS analysis group for the Oil Landfarm WMA that are considered to provide redundant local monitoring coverage in areas with low uncertainty regarding COC distribution and concentrations in the groundwater (Appendix D.1). All of these wells (GW-226, GW-227, GW-368, GW-369, GW-736, and GW-739) are in the Maynardville Limestone south of the site (Figure A.2). As noted previously, discontinued sampling under the Y-12 GWPP requires these wells to be granted "Inactive" status in accordance with the most current version of the Y-12 GWPP MOP, unless the wells are used for hydrologic monitoring.

Summary of MAROS well sufficiency and well redundancy analysis for the Oil Landfarm WMA

\begin{tabular}{|c|l|}
\hline $\begin{array}{c}\text { WELL } \\
\text { NUMBER }\end{array}$ & \multicolumn{1}{c|}{ BASIS FOR MAROS “ELIMINATION” RECOMMENDATION } \\
\hline GW-226 & Redundant with GW-225. \\
\hline GW-227 & Long-term decreasing trend confirmed, recent reduction in sampling frequency. \\
\hline GW-368 & Redundant with GW-601; decreasing long-term trend for TCE. \\
\hline GW-369 & Redundant with GW-601; decreasing trend long-term for TCE. \\
\hline GW-736 & Redundant with GW-725; decreasing concentrations of nitrate and TCE. \\
\hline GW-739 & $\begin{array}{l}\text { Redundant with GW-740; very stable to decreasing COC concentration trends; low rate of } \\
\text { change supports elimination from routine monitoring. }\end{array}$ \\
\hline
\end{tabular}

\section{Sampling Frequency Evaluation}

Based on results of the supplemental MAROS assessment of the groundwater sampling frequency appropriate for the wells included in the MAROS analysis group for the Oil Landfarm WMA, annual sampling is recommended for most of the wells that do not currently have a regulatory-driven sampling 
frequency (Appendix D.1). Semiannual sampling is recommended for two wells (GW-225 and GW-601) that yield VOC-contaminated groundwater from the Maynardville Limestone south (down dip) of the Oil Landfarm WMA (Figure A.2).

\subsection{BEAR CREEK BURIAL GROUNDS WMA}

The BCBG WMA is in BCV approximately two miles west of Y-12 (Figure A.2) and encompasses several closed waste disposal sites (Figure A.5) designated as Burial Grounds (BG)-A North, BG-A South, BG-B, BG-C East, BG-C West, BG-D, BG-E, and BG-J; the Walk-In Pits; the Uranium Vaults; and the Oil Retention Ponds (No. 1 and No. 2), which were constructed to collect oils seeping from disposal trenches in BG-A South and BG-A North, respectively. These sites generally consist of shallow (i.e., $<25 \mathrm{ft}$ bgs) trenches that a diverse mixture of solid wastes (1955 to 1993) and liquid wastes (1959 to 1979) generated at Y-12, including unknown volumes of waste mop water, all or most of which may have contained varying amounts of radiological contamination.

\section{Sampling Locations}

There are 41 groundwater sampling locations included in the MAROS analysis group for the BCBG WMA (Table B.3): 24 wells completed in the low permeability fractured non-carbonate bedrock underlying the various components of the WMA (e.g., BG-A South), 11 exit-pathway monitoring wells completed in the Maynardville Limestone south (hydraulically downgradient) of the WMA, and six springs that discharge into Bear Creek upstream (east) and downstream (west) of the site (Figure A.2). The baseline MAROS assessment of the Y-12 GWPP also included each of these wells and springs in the MAROS analysis group for the BCBG WMA (BWXT 2005), and all but two of these locations have new monitoring data collected $2005-2007$.

\section{COC Assessment}

On the basis of the MAROS output for the COC assessment (see MAROS COC Assessment report in Appendix C.3), benzene, PCE, TCE, 11DCE, and VC are designated as a principal COC for one or more of the groundwater sampling locations included in the MAROS analysis group for the BCBG WMA.

\section{Moment Analysis}

Moment analyses based on data for the groundwater sampling locations included in the MAROS analysis group for the BCBG WMA focused on the analytical results for gross alpha activity, PCE, TCE, and VC (see the MAROS Spatial Moment Analysis Summary report in Appendix C.3). Also, as described in Section 2.4, moment analyses for each COC were performed using one dataset that included analytical results for all the sampling locations included in the MAROS analysis group, and another dataset that excluded analytical results for a selected subset of these sampling locations.

As shown in the following summary, results of the MAROS moment analyses show primarily S or D moment trends for each COC. These findings are consistent with results of the moment analyses from the baseline MAROS assessment, which focused on sampling data for gross beta activity and PCE and likewise show primarily S or D moment trends for each COC (BWXT 2005). Together, moment analyses from the baseline and supplemental MAROS assessments indicate generally stable or decreasing mass, 
movement, and spread of the intermingled plume of contaminants in the (shallow) groundwater at the BCBG WMA.

Summary of the MAROS moment trend analysis for the BCBG WMA

\begin{tabular}{|c|c|c|c|c|c|c|c|c|}
\hline \multirow{4}{*}{ COC } & \multicolumn{8}{|c|}{ MOMENT TREND $^{1}$} \\
\hline & \multirow{2}{*}{\multicolumn{2}{|c|}{$\begin{array}{c}0^{\text {th }} \text { Moment } \\
\text { (Plume Mass) }\end{array}$}} & \multirow{2}{*}{\multicolumn{2}{|c|}{$\begin{array}{l}\mathbf{1}^{\text {st }} \text { Moment } \\
\text { (Plume Center of Mass) }\end{array}$}} & \multicolumn{4}{|c|}{$\begin{array}{c}2^{\text {nd }} \text { Moment } \\
\text { (Plume Spread) }\end{array}$} \\
\hline & & & & & \multicolumn{2}{|c|}{ Sigma XX } & \multicolumn{2}{|c|}{ Sigma YY } \\
\hline & All & Select & All & Select & All & Select & All & Select \\
\hline Gross alpha activity & $\mathrm{D}$ & $\mathrm{S}$ & $\mathrm{D}$ & $\mathrm{S}$ & $\mathrm{PD}$ & $\mathrm{S}$ & $\mathrm{D}$ & $\mathrm{S}$ \\
\hline PCE & $\mathrm{I}$ & NT & $\mathrm{D}$ & NT & $\mathrm{D}$ & $\mathrm{S}$ & $\mathrm{D}$ & $\mathrm{S}$ \\
\hline TCE & $\mathrm{PI}$ & $\mathrm{S}$ & $\mathrm{D}$ & $\mathrm{S}$ & $\mathrm{D}$ & $\mathrm{S}$ & $\mathrm{D}$ & $\mathrm{S}$ \\
\hline $\mathrm{VC}$ & NT & $\mathrm{S}$ & $\mathrm{D}$ & $\mathrm{S}$ & $\mathrm{D}$ & $\mathrm{S}$ & $\mathrm{S}$ & $\mathrm{S}$ \\
\hline \multicolumn{9}{|c|}{$\begin{array}{l}\text { Note: } \\
\text { 1. Based on Mann-Kendall statistical trend analysis. } \\
\text { Relative direction of trend denoted by D = Decreasing; I = Increasing; NT = No Trend; PD = Probably Decreasing; } \\
\text { PI = Probably Increasing; S = Stable }\end{array}$} \\
\hline
\end{tabular}

As shown in the preceding summary, there is only one instance of agreement (the S trends for VC, $2^{\text {nd }}$ Moment, Sigma YY) between the moment trends determined from data for all the sampling locations and the corresponding moment trends determined after excluding data for a selected subset of sampling locations. The full dataset generally provides a strong indication of overall decreasing dissolved mass, center of mass and distribution of mass around the center (dilution at the edges of the plumes). This suggests that data for individual or combinations of sampling locations included in the MAROS analysis group for the BCBG WMA disproportionately influence the moment trends for each COC. The select network may provide a more conservative interpretation of plume trends in the BCBG WMA.

As shown in the preceding summary, the $0^{\text {th }}$ Moment (total dissolved mass) for PCE and TCE (based on data for all sampling locations) exhibit I and PI trends, respectively. These moment trends may be indicative of an increase in dissolved mass of PCE and TCE that may have occurred in response to a possible re-mobilization or increased release of a mixture of PCE and TCE from the dense non-aqueous phase liquids (DNAPL) encountered more than $250 \mathrm{ft} \mathrm{bgs}$ in a borehole drilled for a well (GW-628) in the low permeability non-carbonate bedrock (Nolichucky Shale) on the south side of BG-A South (Haase and King 1990).

\section{Statistical Trend Analysis}

Statistical trend analysis of the data for the groundwater sampling locations included in the MAROS analysis group for the BCBG WMA focused on analytical results for benzene, gross alpha activity, gross beta activity, nitrate, PCE, TCE, uranium, VC, and 11DCE (see the MAROS Statistical Trend Analysis Summary report in Appendix C.3). Results of these analyses show a D trend, PD trend, or NT due to data variability for the applicable COC concentrations in most of the sampling locations (Table B.3), with I trends evident for the COC concentrations in wells GW-071 (benzene, PCE, and 11DCE), GW-289 (PCE and TCE), GW-626 (11DCE and VC), GW-627 (PCE, TCE, 11DCE, and VC), and GW-629 (TCE). These wells are located hydraulically downgradient (either down-dip or along strike) of BG-A South (Figure A.2), and the increasing concentration trends for each COC suggest a corresponding change in the relative flux of dissolved VOCs via the groundwater flowpaths intercepted by the monitored interval in each well. The increased flux of VOCs may be related to the disturbance and potential remobilization of the DNAPL noted above. 


\section{Well Sufficiency/Redundancy Evaluation}

Results of the MAROS well sufficiency analysis for the sampling locations included in the MAROS analysis group for the BCBG WMA indicate an area near BG-A South as having high uncertainty for VOC concentrations (including increasing concentration trends). Although no new wells are recommended for the area, the results of the well sufficiency analysis highlight the need to monitor the source areas carefully. The baseline MAROS assessment reported similar findings (BWXT 2005).

Results of the supplemental MAROS assessment recommend discontinued sampling (i.e., "Elimination") of the following wells (excluding alternate RCRA monitoring wells) in the MAROS analysis group for the BCBG WMA that are considered to provide redundant local monitoring coverage in areas with low uncertainty regarding the spatial distribution and concentrations of each $\mathrm{COC}$ in the groundwater (Appendix D.1). Most of these wells (GW-052, GW-072, GW-089, GW-624, and GW-626) yield groundwater from various depths in the low-permeability fractured non-carbonate bedrock underlying the BCBG WMA, and one well (GW-694) is an exit-pathway well in the Maynardville Limestone approximately $1000 \mathrm{ft}$ southeast (hydraulically upgradient) of the BCBG WMA (Figure A.2).

\section{Summary of MAROS well sufficiency and well redundancy analysis for the BCBG WMA}

\begin{tabular}{|c|l|}
\hline $\begin{array}{c}\text { WELL } \\
\text { NUMBER }\end{array}$ & \multicolumn{1}{c|}{ BASIS FOR MAROS “ELIMINATION” RECOMMENDATION } \\
\hline GW-052 & Very few COC detections, decreasing trend for gross alpha activity. \\
\hline GW-072 & Very few COC detections; redundant with well GW-071. \\
\hline GW-089 & Redundant with well GW-242. \\
\hline GW-624 & Redundant with GW-629. \\
\hline GW-626 & Redundant with GW-627. \\
\hline GW-694 & Low COC concentrations and decreasing trends; redundant with wells GW-704 and GW-695. \\
\hline
\end{tabular}

\section{Sampling Frequency Evaluation}

Based on results of the supplemental MAROS assessment of the appropriate sampling frequency for each groundwater sampling location included in the MAROS analysis group for the BCBG WMA that do not have a regulatory-driven sampling frequency, final frequency recommendations include annual, semiannual, and biennial monitoring (Appendix D.1). Annual sampling is recommended for two springs (SS-4 and SS-5) and four wells (GW-082, GW-242, GW-289, and GW-703). Three of the wells are in low permeability fractured non-carbonate bedrock along the western (GW-289 and GW-02) or northern (GW-242) boundary of the BCBG WMA, and well GW-703 is completed in the Maynardville Limestone southeast of the site (Figure A.2)

Semiannual sampling is recommended for six wells (GW-014, GW-068, GW-071, GW-627, GW-629, and GW-653), most of which are located along the southern boundary of the BCBG WMA (Figure A.2) and yield VOC-contaminated groundwater distinguished by a the mixture of petroleum hydrocarbons (primarily benzene) and PCE and its degradation products (TCE, VC, and c12DCE). These wells generally monitor the DNAPL encountered on the south side of BG-A South (see Moment Analysis).

Biennial sampling is recommended for wells GW-053 and GW-058, which yield groundwater from shallow depths in the Maynardville Limestone approximately 500 to $800 \mathrm{ft}$ south of the BCBG WMA (Figure A.2). Groundwater from well GW-053 contains a mixture of VOCs dominated by PCE degradation products (c12DCE and VC), with summed concentrations below $50 \mu \mathrm{g} / \mathrm{L}$ and exhibiting a 
decreasing long-term trend, whereas the groundwater from well GW-058 contains elevated uranium concentrations and gross alpha activity that exhibit stable long-term trends (B\&W Y-12 2008).

\subsection{EAST S-3 AREA}

The MAROS analysis group for the East S-3 Area encompasses sampling locations in the East Fork Regime within the westernmost section of Y-12 near several known and suspected sources of groundwater contamination, including the former S-3 Ponds, the S-2 Site, Beta-4 Security Pits (B4), Rust Garage Area (RG), Fire Training Facility (FTF), Y-12 Salvage Yard (SY), Waste Coolant Processing Facility (WCPA).

\section{Sampling Locations}

There are 29 monitoring wells included in the MAROS analysis group for the East S-3 Area (Table B.4). Most (27) of the wells yield groundwater from low-permeability fractured non-carbonate bedrock in the vicinity of the former S-3 Ponds, B4, RG, SY, WCPA, and at several of the Y-12 Comprehensive Monitoring Plan grid coordinates (Y-12 Grid) in the west-central section of Y-12 (Figure A.2). Wells at the S-2 Site, FTF, and downgradient (east) exit pathway wells yield groundwater from the Maynardville Limestone near the former headwaters channel of Upper East Fork Poplar Creek (UEFPC), which was extensively reworked (filled) and replaced with a subsurface drainage network to accommodate construction of Y-12. The baseline MAROS assessment of the Y-12 GWPP included all but two of these wells (55-2A and GW-622) in the MAROS analysis group for East S-3 Area (BWXT 2005).

\section{COC Assessment}

On the basis of the MAROS output for the COC assessment (see MAROS COC Assessment report in Appendix C.4), cadmium, gross alpha activity, nitrate, PCE, and TCE are designated as a principal COC for one or more of the groundwater sampling locations included in the MAROS analysis group for the East S-3 Area. Of the COCs listed, nitrate is present at the highest levels above the screening level areawide, and PCE is the most widely distributed with 17 monitoring locations affected above screening levels.

\section{Moment Analysis}

Moment analyses based on data for the wells included in the MAROS analysis group for the East S-3 Area focused on analytical results for cadmium, gross beta activity, nitrate, PCE, and TCE (see the MAROS Spatial Moment Analysis Summary report in Appendix C.4). Also, as described in Section 2.4, separate moment analyses for each COC were performed using one dataset that included analytical results for all the wells included in the MAROS analysis group, and another dataset that excluded analytical results for a selected subset of these wells.

As shown in the following summary, results of the moment analyses for each COC generally show $\mathrm{S}$ trends, D trends, and NT due to variability of the data. Similar results were obtained from the baseline MAROS assessment, which focused on moment trends for nitrate and PCE (BWXT 2005). These findings do not indicate changes in the relative mass, movement, and spread of the groundwater 
contaminant plumes that may warrant greater monitoring efforts (i.e., increased sampling locations and/or frequencies) in the East S-3 Area.

Summary of the MAROS moment trend analysis for the East S-3 Area

\begin{tabular}{|c|c|c|c|c|c|c|c|c|}
\hline \multirow{4}{*}{ COC } & \multicolumn{8}{|c|}{ MOMENT TREND $^{1}$} \\
\hline & \multirow{2}{*}{\multicolumn{2}{|c|}{$\begin{array}{c}\text { 0 }^{\text {th }} \text { Moment } \\
\text { (Plume Mass) }\end{array}$}} & \multirow{2}{*}{\multicolumn{2}{|c|}{$\begin{array}{l}1^{\text {st }} \text { Moment } \\
\text { (Plume Center of } \\
\text { Mass) }\end{array}$}} & \multicolumn{4}{|c|}{$\begin{array}{c}2^{\text {nd }} \text { Moment } \\
\text { (Plume Spread) }\end{array}$} \\
\hline & & & & & \multicolumn{2}{|c|}{ Sigma XX } & \multicolumn{2}{|c|}{ Sigma YY } \\
\hline & All & Select & All & Select & All & Select & All & Select \\
\hline Cadmium & NT & NT & $\mathrm{S}$ & $\mathrm{S}$ & NT & NT & $\mathrm{S}$ & $\mathrm{S}$ \\
\hline Gross beta activity & NT & NT & $\mathrm{PD}$ & $\mathrm{S}$ & $\mathrm{S}$ & $\mathrm{D}$ & $\mathrm{D}$ & $\mathrm{D}$ \\
\hline Nitrate & NT & NT & $\mathrm{D}$ & PD & PD & $\mathrm{S}$ & NT & $\mathrm{S}$ \\
\hline PCE & NT & $\mathrm{PI}$ & $\mathrm{D}$ & $\mathrm{D}$ & NT & NT & $\mathrm{S}$ & $\mathrm{S}$ \\
\hline TCE & $\mathrm{S}$ & $\mathrm{S}$ & NT & NT & $\mathrm{S}$ & $\mathrm{S}$ & PD & $\mathrm{S}$ \\
\hline \multicolumn{9}{|c|}{$\begin{array}{l}\text { Note: } \\
\text { 1. Based on Mann-Kendall statistical trend analysis. } \\
\text { Relative direction of trend denoted by D = Decreasing; PD = Potentially Decreasing; PI = Potentially Increasing; NT = No Trend; } \\
\mathrm{S}=\text { Stable }\end{array}$} \\
\hline
\end{tabular}

Note that $\mathrm{D}, \mathrm{PD}$, and $\mathrm{S}$ trends dominate the $1^{\text {st }}$ and $2^{\text {nd }}$ Moment trends for two primary components of the groundwater contaminant plume originating from the former S-3 Ponds: nitrate and gross beta activity, which is primarily from a beta particle-emitting radionuclide, technetium-99 (Tc-99), that is considered the "signature" contaminant from the S-3 Ponds (DOE 1997). Nitrate and Tc-99 share similar transport characteristics in the groundwater and these moment trends suggest continuing reductions in the relative movement and spread in the (shallow) groundwater flow system east of the site.

As shown in the preceding data summary, there are no differences between most of the paired moment trends for each COC except nitrate. Moments calculated using the select monitoring network for nitrate provides a similar, but not identical picture of plume stability. The select network indicates a probably decreasing center of mass rather than a strongly decreasing one and indicates that the distribution of mass about the center is stable. These findings suggest that data for individual or combinations of wells included in the MAROS analysis group for the East S-3 Area do not appear to disproportionately influence the moment trends for each COC.

\section{Statistical Trend Analysis}

Statistical trend analysis of the groundwater sampling data for the wells included in the MAROS analysis group for the East S-3 Area focused on analytical results for benzene, cadmium, gross alpha activity, gross beta activity, nitrate, PCE, TCE, c12DCE, 11DCE, and VC (see the MAROS Statistical Trend Analysis Summary report in Appendix C.4). Results of these statistical trend analyses show a stable trend, no trend, and D or PD trends for the COC concentrations in most of the wells (Table B.4).

The MAROS statistical trend analyses show I or PI trends for the applicable COC in several wells (Table B.4), notably GW-274 (PCE, recent and overall trends), GW-275 (nitrate, recent), and GW-633 (benzene recent and overall trends). Each of these wells is completed at a relatively shallow depth in unconsolidated materials (GW-274 and GW-633) or low-permeability fractured non-carbonate bedrock $(\mathrm{GW}-275)$ and yields highly contaminated groundwater impacted by the contaminant plume originating from the former S-3 Ponds, which is the principal source of groundwater contamination in the East S-3 Area. Nitrate concentrations at GW-274, in the shallow zone are decreasing, while nitrate concentrations are increasing at the deeper adjacent well GW-275 and at 55-2B and C. The moment results for center of 
mass indicate an overall decreasing nitrate trend (lower relative concentrations in tail of plume), but specific locations within the fractured bedrock appear to monitor increasing concentrations. Increasing nitrate concentrations may be following the geologic strike of the bedding while concentrations are decreasing at shallow and off-strike locations.

\section{Well Sufficiency/Redundancy Evaluation}

Results of the supplemental MAROS assessment of well sufficiency based on data for the wells included in the MAROS analysis group for the East S-3 Area indicate some areas of uncertainty regarding COC concentrations in the groundwater, but installation of new wells is not recommended. These findings mirror those of the baseline MAROS assessment (BWXT 2005).

Results of the supplemental MAROS assessment recommend discontinued sampling (i.e., "Elimination") of the following wells in the MAROS analysis group for the East S-3 Area that are considered to provide redundant local monitoring coverage in areas with low uncertainty regarding the spatial distribution and concentrations of each COC in the groundwater (Appendix D.2). Three of the wells yield contaminated groundwater from shallow depths $(<50 \mathrm{ft}$ bgs $)$ in areas underlain by low-permeability fractured noncarbonate bedrock (Nolichucky Shale) east of the former S-3 Ponds (GW-105), near the SY (GW-270 and GW-273), and the WCPA (GW-336), and one well (GW-617) is part of a clustered pair of exit-pathway wells in the Maynardville Limestone approximately $800 \mathrm{ft}$ northeast of the S-2 Site (Figure A.3).

Summary of MAROS well sufficiency and well redundancy analysis for the East S-3 Area

\begin{tabular}{|c|l|}
\hline $\begin{array}{c}\text { WELL } \\
\text { NUMBER }\end{array}$ & \multicolumn{1}{|c|}{ BASIS FOR MAROS “ELIMINATION” RECOMMENDATION } \\
\hline GW-105 & Redundant with well GW-106. \\
\hline GW-270 & Redundant with well GW-505. \\
\hline GW-273 & Redundant with well GW-633; recent ND results for major COCs. \\
\hline GW-336 & Redundant with wells GW-332 and GW-337. \\
\hline GW-617 & Redundant with (clustered) well GW-618. \\
\hline
\end{tabular}

\section{Sampling Frequency Evaluation}

Based on results of the supplemental MAROS assessment of the appropriate sampling frequency for each groundwater sampling location included in the MAROS analysis group for the East S-3 Area that do not have a regulatory-driven sampling frequency (Appendix D.2), annual sampling is recommended for most (10) of the wells.

Biennial sampling is recommended for five wells: three wells (GW-106, GW-109, and GW-505) that yield contaminated groundwater from the low permeability fractured non-carbonate bedrock east of the former S-3 Ponds in the vicinity of the RG, one well (55-2A) located farther east, and one well (GW-619) in Maynardville Limestone to the south (Figure A.3).

Sampling once every 5 years is recommended for wells near the B4 (GW-192), the northern portion of the SY (GW-265 and GW-269), and approximately $600 \mathrm{ft}$ northeast of the WCPA (55-1A) (Figure A.3). Each well yields moderately contaminated groundwater, with results of the MAROS statistical trend analyses indicating $\mathrm{S}$ trends for the applicable $\mathrm{COC}$ in wells 55-1A (nitrate) and GW-265 (PCE), a D trend for the COC in well GW-269 (11DCE), and NT (due to data variability) for TCE in well GW-192 (Appendix D.2). 


\subsection{CENTRAL Y-12 AREA}

The MAROS analysis group for the Central Y-12 Area encompasses the primary operations and process facilities that occupy the central section of Y-12 and several known or suspected sources of groundwater contamination, including Bldg. 8110, Bldg. 9201-2, the Coal Pile Trench, and Eastern S-3 Site Plume, which is the eastern extent of the contaminant plume originating from the former S-3 Ponds. The FF MAROS analysis group was included in the Central Y-12 Area for the purpose of the supplemental report because the number of locations with detected concentrations in the FF group fell below 6 , the minimum number of wells for the MAROS analysis.

\section{Sampling Locations}

The MAROS analysis group for the Central Y-12 Area includes one surface water sampling location (UEFPC-SP17) in the exposed channel of UEFPC and 47 monitoring wells (Table B.5). Most of the wells, including two wells (GW-954 and GW-956) equipped with BarCad samplers that provide samples from multiple discrete depth intervals, yield groundwater from the low-permeability fractured noncarbonate bedrock at several Y-12 Grid locations and at various other general (non-grid) locations within the Central Y-12 Area north of UEFPC. Two exit-pathway wells (GW-605 and GW-606) yield groundwater from the Maynardville Limestone north of the main channel of UEFPC approximately 1,300 ft west (upstream) of New Hope Pond (Figure A.3). Also, seven wells at the FF were included in the Central Y-12 analysis group.

Groundwater sampling results for many of the wells in the MAROS analysis group for the Central Y-12 Area, including several of the Y-12 Grid wells (56-6A, GW-762, GW-763, GW-775, and GW-776), were not included in the MAROS analysis group for the Central Y-12 Area that was evaluated under the baseline MAROS assessment of the Y-12 GWPP (BWXT 2005). Additionally, as noted in Section 2.1, the MAROS analysis group for the Central Y-12 Area includes wells at the FF that the baseline MAROS assessment addressed in a separate MAROS analysis group. Thus, the supplemental MAROS assessment for this MAROS analysis group is more comprehensive than the baseline assessment.

\section{COC Assessment}

On the basis of the MAROS output for the COC assessment (see MAROS COC Assessment report in Appendix C.5), CTET, PCE, TCE, VC, and uranium are each designated as a principal COC for the Central Y-12 Area. The primary COC for the Central Y-12 analysis group is PCE, based on results above screening levels and number of affected wells in the network. Of the 53 locations analyzed for the COC Assessment, only six locations had results that exceed screening levels for CTET, however, the exceedences at these locations are significant.

\section{Moment Analysis}

Moment analyses based on data for the groundwater sampling locations included in the MAROS analysis group for the Central Y-12 Area focused on analytical results for benzene, CTET, PCE, TCE, and uranium (see the MAROS Spatial Moment Analysis Summary report in Appendix C.5). Under the baseline MAROS assessment of the Y-12 GWPP, the Central Y-12 Area moment analyses were based on groundwater sampling data for PCE and uranium (BWXT 2005). 
As shown in the following summary, a combination of moment trends are evident for PCE, TCE, and uranium, including I trends, D trends, S trends, and NT (due to data variability). Moment analysis for benzene was performed because this VOC occurs near the FF. However, of greater interest are the I or PI moment trends for CTET, which is a principal contaminant in the majority of wells that yield VOCcontaminated groundwater from the Maynardville Limestone in the vicinity of New Hope Pond. The CTET-dominated plume apparently originates from an unidentified source upgradient (west) of New Hope Pond and extends thousands of feet eastward in the Maynardville Limestone (parallel with geologic strike of bedding) into Union Valley east of the ORR boundary along Scarboro Road. An ongoing CERCLA remedial action that began full-time operation in October 2000 involves on-site treatment (air stripping) to remove dissolved VOCs from groundwater pumped from an extraction well (GW-845) located east of New Hope Pond (Figure A.3). Mobilization of the CTET induced by the long-term operation of the groundwater extraction well potentially accounts for the increasing and probably increasing moment trends determined for CTET.

Summary of the MAROS moment trend analysis for the Central Y-12 Area

\begin{tabular}{|c|c|c|c|c|}
\hline \multirow{3}{*}{ COC } & \multicolumn{4}{|c|}{ MOMENT TREND $^{1}$} \\
\hline & \multirow{2}{*}{$\begin{array}{c}0^{\text {th }} \text { Moment } \\
\text { (Plume Mass) }\end{array}$} & \multirow{2}{*}{$\begin{array}{c}1^{\text {st }} \text { Moment } \\
\text { (Plume Center of } \\
\text { Mass) }\end{array}$} & \multicolumn{2}{|c|}{$\begin{array}{c}2^{\text {nd }} \text { Moment } \\
\text { (Plume Spread) }\end{array}$} \\
\hline & & & Sigma XX & Sigma YY \\
\hline Benzene & I & I & I & NT \\
\hline CTET & $\mathrm{I}$ & $\mathrm{I}$ & $\mathrm{I}$ & PI \\
\hline PCE & NT & $\mathrm{S}$ & $\mathrm{I}$ & NT \\
\hline TCE & I & $\mathrm{S}$ & NT & $\mathrm{D}$ \\
\hline Uranium & I & $\mathrm{S}$ & I & NT \\
\hline
\end{tabular}

\section{Statistical Trend Analysis}

Statistical trend analysis of data for the groundwater sampling locations included in the MAROS analysis group for the Central Y-12 Area focused on analytical results for benzene, CTET, PCE, TCE, c12DCE, 11DCE, VC, uranium, gross alpha activity, and gross beta activity (see the MAROS Statistical Trend Analysis Summary report in Appendix C.5). Results of the statistical trend analyses for the applicable COC in most of the sampling locations show D, PD, and S trends or NT due to data variability (Table B.5). Findings of the baseline MAROS assessment likewise show D, PD, and S trends for the COC in most of the sampling locations included in the MAROS analysis group for the Central Y-12 that had sufficient data for statistical trend analysis (BWXT 2005).

The MAROS statistical analysis show I or PI trends for the applicable COC in several wells included in the MAROS analysis group for the Central Y-12 Area (Table B.5), including wells GW-762 (PCE), GW-769 (CTET), and GW-770 (CTET), which yield groundwater from the low permeability fractured non-carbonate bedrock (Nolichucky Shale) at designated Y-12 Grid locations, and Maynardville Limestone wells GW-691 (PCE) at the Coal Pile Trench, GW-698 (TCE) at Bldg. 8110, and GW-605 (CTET) upgradient (west) of New Hope Pond (Figure A. 3). Increasing concentrations of CTET in wells GW-769 and GW-770 also were evident from the statistical trend analyses performed under the baseline MAROS assessment (BWXT 2005). Increasing concentrations of CTET in the groundwater from well GW-605 potentially reflects a corresponding increase in the eastward flux of CTET toward the extraction well for the groundwater remediation system noted above. 


\section{Well Sufficiency/Redundancy Evaluation}

Results of the supplemental MAROS assessment of well sufficiency based on data for the wells included in the MAROS analysis group for the Central Y-12 Area indicate some areas of uncertainty regarding COC concentrations in the groundwater, but installation of new wells is not recommended. Concentration uncertainty can be explained by heterogeneities in the subsurface geology. Also, none of the wells provide redundant local monitoring coverage in areas having low uncertainty regarding the spatial distribution and concentrations of each COC in the groundwater. Similar findings were obtained from the baseline MAROS assessment (BWXT 2005).

\section{Sampling Frequency Evaluation}

Based on results of the supplemental MAROS assessment of the sampling frequency appropriate for each well included in the MAROS analysis group for the Central Y-12 Area (Table B.5), annual sampling is recommended for most (22) of the wells (including both wells with BarCAD multiport sampling equipment) that do not have a regulatory-driven sampling frequency (Appendix D.2).

Semiannual sampling is recommended for a cluster of wells (55-3A, 55-3B, and 55-3C) that yield contaminated groundwater but have limited sampling histories, and for several wells (GW-691, GW-698, GW-769, and GW-770) that exhibit increasing COC concentration trends.

Biennial sampling is recommended for nine wells (56-6A, 60-1A, GW-204, GW-656, GW-765, GW-775, GW-776, GW-779, and GW-792) that exhibit stable concentration trends for the applicable COC or show no concentration trend because of the variability of the data for the COC.

\subsection{EAST Y-12 AREA}

The MAROS analysis group for the East Y-12 Area encompasses sampling locations within the easternmost sections of Y-12, primarily in the vicinity of New Hope Pond, a closed former surface impoundment used from 1963 to 1988 to regulate the quantity and quality of water exiting Y-12 via UEFPC, and in Union Valley east of the ORR boundary along Scarboro Road (Figure A.3).

\section{Sampling Locations}

There are a total of 27 sampling locations in the MAROS analysis group for the East Y-12 Area (Table B.6), including 25 wells and two natural springs that discharge into unnamed surface drainage features east-southeast of Y-12 (Figure A.3). All but three of the wells yield groundwater from various depths in the Maynardville Limestone; wells GW-744, GW-747, and GW-748 yield groundwater from

Y-12 Grid locations underlain by the low permeability fractured non-carbonate bedrock west-northwest of New Hope Pond. All of these sampling locations were included in the East Y-12 Area MAROS analysis group evaluated under the baseline MAROS assessment of the Y-12 GWPP (BWXT 2005).

\section{COC Assessment}

On the basis of the MAROS output for the COC assessment (see MAROS COC Assessment report in Appendix C.6), CTET, PCE, TCE, and gross alpha activity are designated as a primary COC for one or 
more of the sampling locations included in the MAROS analysis group for the East Y-12 Area. The priority COC for the East Y-12 Area analysis group is CTET, based on area-wide exceedence of screening levels, mobility in the subsurface $(\mathrm{Kd})$ and the number of locations that exceed screening levels.

\section{Moment Analysis}

Moment analyses of data for the groundwater sampling locations included in the MAROS analysis group for the East Y-12 Area focused on analytical results for CTET, gross alpha activity, PCE, and TCE (see the MAROS Spatial Moment Analysis Summary report in Appendix C.6). As shown in the following summary, D or PD moment trends dominate for CTET, gross alpha activity, and gross beta activity (both from uranium isotopes and associated daughter products), whereas the moment trends for PCE show an S trend or NT due to data variability. Somewhat similar results were obtained from the moment analyses performed under the baseline MAROS assessment, which were based on data for CTET and showed a PI trend for $0^{\text {th }}$ Moment, a D trend for the $1^{\text {st }}$ Moment, and D and PD trends for the $2^{\text {nd }}$ Moments (BWXT 2005). Results of the moment analyses from the baseline and supplemental MAROS assessments indicate reductions in the relative mass, movement, and spread of CTET and uranium isotopes (gross alpha and gross beta activity), but minimal impact on PCE. These findings suggest that CTET and uranium isotopes may share a common source area and/or primary migration pathway(s) that differ from that of PCE.

Summary of the MAROS moment trend analysis for the East Y-12 Area

\begin{tabular}{|c|c|c|c|c|}
\hline \multirow{3}{*}{ COC } & \multicolumn{4}{|c|}{ MOMENT TREND $^{1}$} \\
\hline & \multirow{2}{*}{$\begin{array}{c}0^{\text {th }} \text { Moment } \\
\text { (Plume Mass) }\end{array}$} & \multirow{2}{*}{$\begin{array}{c}1^{\text {st }} \text { Moment } \\
\text { (Plume Center of } \\
\text { Mass) }\end{array}$} & \multicolumn{2}{|c|}{$\begin{array}{c}2^{\text {nd }} \text { Moment } \\
\text { (Plume Spread) }\end{array}$} \\
\hline & & & Sigma XX & Sigma YY \\
\hline CTET & $\mathrm{PD}$ & PD & PD & $\mathrm{S}$ \\
\hline Gross alpha activity & $\mathrm{D}$ & $\mathrm{D}$ & $\mathrm{D}$ & $\mathrm{D}$ \\
\hline Gross beta activity & $\mathrm{PD}$ & $\mathrm{D}$ & $\mathrm{PD}$ & $\mathrm{PD}$ \\
\hline PCE & NT & NT & $\mathrm{S}$ & $\mathrm{S}$ \\
\hline $\begin{array}{l}\text { Note: } \\
\text { 1. Based on Mann-Kendall stat } \\
\text { Relative direction of trend de }\end{array}$ & $\begin{array}{l}\text { alysis. } \\
\text { Decreasi }\end{array}$ & atially Decreasing & $\mathrm{nd} ; \mathrm{S}=\mathrm{Stab}$ & \\
\hline
\end{tabular}

\section{Statistical Trend Analysis}

Statistical trend analysis of the data for the groundwater sampling locations included in the MAROS analysis group for the East Y-12 Area focused on analytical results for CTET, gross alpha activity, gross beta activity, PCE, and TCE (see the MAROS Statistical Trend Analysis Summary report in Appendix C.6). Results of the statistical trend analyses show D, PD, or S trends for the applicable COC in most (17 wells and two springs) of the sampling locations included in the MAROS analysis group for the East Y-12 Area, with I or PI trends evident only for wells near New Hope Pond (Table B.6). The remaining sampling locations, including several exit-pathways wells in Union Valley, show NT for the designated COC because of data variability, or the trend analysis is not applicable because of insufficient sampling results (the MAROS trend analysis requires at least four sampling results).

The MAROS statistical analysis show I or PI trends for the applicable COC in wells GW-151 (CTET), GW-154 (gross alpha activity), GW-220 (CTET), and GW-383 (c12DCE) in the East Y-12 Area (Table B.6). Increasing concentrations of CTET in the groundwater from wells GW-151 and GW-220 
may result from a combination of reduced recharge following closure of NHP and the strong local hydrologic influence of the UEFPC distribution channel underdrain (DOE 1998). Biologically mediated degradation of related parent compounds (PCE and TCE) may account for the increasing trend for c12DCE concentrations in groundwater from well GW-383, which have increased almost $400 \%$ since the early 1990s, and increased levels of gross alpha activity indicated by the sampling results for well GW-154 may be an artifact of the groundwater sampling method or reflect a conspicuous "spike" in activity in August 2002 (B\&W Y-12 2008).

\section{Well Sufficiency/Redundancy Evaluation}

Results of the supplemental MAROS assessment of well sufficiency based on data for the sampling locations included in the MAROS analysis group for the East Y-12 Area do not indicate areas of significant uncertainty regarding $\mathrm{COC}$ concentrations in the groundwater that warrant installation of new wells. Also, none of the wells provide redundant local monitoring coverage in areas having low uncertainty regarding the spatial distribution and concentrations of contaminants in the groundwater. Similar findings were obtained from the baseline MAROS assessment (BWXT 2005).

\section{Sampling Frequency Evaluation}

Based on results of the supplemental MAROS assessment of the sampling frequency appropriate for each well included in the MAROS analysis group for the East Y-12 Area that do not have a regulatory-driven sampling frequency (Appendix D.2), annual sampling is recommended for three wells (GW-240, GW-735, and GW-763) and biennial sampling is recommended for three wells (GW-148, GW-748, and GW-750).

\subsection{WEST CHESTNUT RIDGE}

The MAROS analysis group for West Chestnut Ridge includes sampling locations within the westernmost section of the Chestnut Ridge Regime to the south-southwest of Y-12, in the general vicinity of Construction/Demolition Landfill VI (CDL VI), Industrial Landfill IV (IL IV), Sanitary Landfill II (SL II), and the United Nuclear Corporation Site (UNCS). Groundwater monitoring at the landfills is performed in accordance the TDEC regulations governing operation and management of SWDFs and the requirements specified in the operating permit for IL IV, which is an active SWDF, and the respective post-closure care requirements for SL II and CDL VI, which are closed SWDFs. Groundwater sampling at the UNCS is performed as required under a CERCLA ROD for the site.

\section{Sampling Locations}

There are 19 sampling locations in the MAROS analysis group for West Chestnut Ridge (Table B.7): three natural springs on the southern flank of Chestnut Ridge that are monitored as exit-pathway locations, four wells at CDL VI, five wells at IL IV, three wells at SL II, and four wells at the UNCS (Figure A.4). The baseline MAROS assessment of the Y-12 GWPP included these same wells and natural springs in the MAROS analysis group for West Chestnut Ridge (BWXT 2005). 


\section{COC Assessment}

Available groundwater sampling results obtained to date show that all of the sampling locations included in the MAROS analysis group for West Chestnut Ridge yield uncontaminated groundwater except well GW-305 at IL IV, which monitors VOC-contaminated groundwater, and well GW-205 at the UNCS, which monitors groundwater with elevated levels of gross beta activity. Also, historical sampling data show that contaminant concentrations in the groundwater from wells GW-305 and GW-205 rarely exceed applicable screening levels. Thus, the MAROS COC assessment of the sampling data for the West Chestnut Ridge indicated no area-wide priority COCs routinely exceeding screening levels.

\section{Moment Analysis}

The baseline MAROS assessment of the Y-12 GWPP included results of MAROS moment analyses based on the gross alpha activity data for the wells and springs included in the MAROS analysis group for West Chestnut Ridge (BWXT 2005). The MAROS moment analyses performed for the supplemental MAROS assessment addressed other analytes in addition to gross alpha activity (see MAROS Spatial Analysis Summary report in Appendix C.7). However, results of the MAROS moment analyses seem of limited significance considering the overall lack of groundwater contamination at the sites included in the West Chestnut Ridge MAROS analysis group. Also, although groundwater sampling results indicate contaminant releases to the uppermost aquifer at IL IV (GW-305) and UNCS (GW-205), available data for the existing network of sampling locations at each site suggest generally limited contaminant transport/migration via discreet hydrostratigraphic intervals rather than movement as a diffuse "plume" in the groundwater. Thus, analytical results for the bulk of the groundwater sampling locations included in the MAROS analysis group for West Chestnut Ridge do not seem especially suited to MAROS moment analyses.

\section{Statistical Trend Analysis}

Results of the statistical trend analysis of the groundwater sampling data for the sampling locations included in the MAROS analysis group for West Chestnut Ridge are of limited applicability because, as noted previously, only two of the sampling locations (wells GW-205 and GW-305) yield contaminated groundwater (see MAROS Statistical Trend Analysis Summary report in Appendix C.7).

\section{Well Sufficiency/Redundancy Evaluation}

Results of the supplemental MAROS assessment of well sufficiency based on data for the wells included in the MAROS analysis group for West Chestnut Ridge are inconclusive. Areas of significant uncertainty regarding $\mathrm{COC}$ concentrations in the groundwater, which may warrant installation of new wells, are not evident and none of the existing wells provide redundant local monitoring coverage in areas having low uncertainty regarding the spatial distribution and concentrations of contaminants in the groundwater. Similar findings were obtained from the baseline MAROS assessment (BWXT 2005).

\section{Sampling Frequency Evaluation}

As noted above, each groundwater sampling location included in the MAROS analysis group for West Chestnut Ridge has a regulatory-driven sampling frequency (Appendix D.3). Consequently, as noted in Section 2.7, the final MAROS-based sampling frequency recommendations are not applicable. 


\subsection{CHESTNUT RIDGE SECURITY PITS}

The CRSP are located on the crest of Chestnut Ridge directly south of the Central Y-12 Area (Figure A.4). The CRSP consists of a series of east-west oriented waste disposal trenches that received a variety of nonhazardous, hazardous, and classified wastes between 1973 and 1988, and were covered by a multilayer, low permeability cap installed during RCRA closure of the site in 1989. Wastes disposed at the site are the source of an elongated plume of dissolved VOCs in the groundwater extending over $2000 \mathrm{ft}$ to the east-northeast (parallel with geologic strike) and several hundred $\mathrm{ft}$ to the north and south down the flanks of the ridge. The elongated geometry of the VOC plume reflects the preferred, strikeparallel flow of groundwater in the carbonate (karst) aquifer formed by the Knox Group strata that underlie Chestnut Ridge.

\section{Sampling Locations}

There are 13 groundwater sampling locations included in the MAROS analysis group for the CRSP (Table B.8): one natural spring (SCR-3.5SP) that discharges into McCoy Branch on the southern flank of Chestnut Ridge approximately $6200 \mathrm{ft}$ south of the CRSP, ten wells that generally surround the waste disposal trenches at the site, with two wells (GW-514 and GW-831) located near the Filled Coal Ash Pond, south-southwest of the site (Figure A.4). The baseline MAROS assessment of the Y-12 GWPP also included these same sampling locations in the MAROS analysis group for the CRSP (BWXT 2005).

\section{COC Assessment}

On the basis of the MAROS output for the COC assessment (see MAROS COC Assessment report in Appendix C.8), PCE and 11DCE are designated as a principal COC for the CRSP. The baseline MAROS assessment of the Y-12 GWPP also identified each VOC as a COC for the CRSP (BWXT 2005).

\section{Moment Analysis}

Moment analyses based on data for the groundwater sampling locations included in the MAROS analysis group for the CRSP focused on analytical results for gross alpha activity, PCE and 11DCE. Results of the moment analyses, summarized below, show S trends or NT because of data variability (see the MAROS Spatial Moment Analysis Summary report in Appendix C.8). Similar findings were obtained from the moment analyses performed as part of the baseline MAROS assessment of the sampling locations included in the MAROS analysis group for the CRSP, which focused on the analytical results for PCE (BWXT 2005). Together, results of the moment analyses from the baseline and supplemental MAROS assessments do not indicate significant changes in the relative mass, movement, and spread of VOCs (PCE and 11DCE) in the groundwater at the CRSP. 
Summary of the MAROS moment trend analysis for the CRSP MAROS analysis group

\begin{tabular}{|c|c|c|c|c|}
\hline \multirow{3}{*}{ COC } & \multicolumn{4}{|c|}{ MOMENT TREND $^{1}$} \\
\hline & \multirow{2}{*}{$\begin{array}{c}0^{\text {th }} \text { Moment } \\
\text { (Plume Mass) }\end{array}$} & \multirow{2}{*}{$\begin{array}{c}1^{\text {st }} \text { Moment } \\
\text { (Plume Center of } \\
\text { Mass) }\end{array}$} & \multicolumn{2}{|c|}{$\begin{array}{c}2^{\text {nd }} \text { Moment } \\
\text { (Plume Spread) }\end{array}$} \\
\hline & & & Sigma XX & Sigma YY \\
\hline $11 \mathrm{DCE}$ & NT & $\mathrm{S}$ & $\mathrm{S}$ & $\mathrm{S}$ \\
\hline Gross alpha activity & NT & NT & $\mathrm{S}$ & $\mathrm{S}$ \\
\hline PCE & $\mathrm{S}$ & $\mathrm{S}$ & $\mathrm{S}$ & NT \\
\hline \multicolumn{5}{|c|}{$\begin{array}{l}\text { Note: } \\
\text { 1. Based on Mann-Kendall statistical trend analysis. } \quad \text { Relative direction of trend denoted by NT }=\text { No Trend; } S=\text { Stable }\end{array}$} \\
\hline
\end{tabular}

\section{Statistical Trend Analysis}

Statistical trend analysis of the data for the groundwater sampling locations included in the MAROS analysis group for the CRSP focused on analytical results for gross alpha activity, gross beta activity, PCE, and 11DCE (see the MAROS Statistical Trend Analysis Summary report in Appendix C.8). Results of the MAROS statistical trend analyses show a D, PD, and S trends, or NT (due to data variability) for the applicable COC in most (11 wells) of the sampling locations (Table B.8). These findings are consistent with results of the statistical trend analyses performed under the baseline MAROS assessment of the Y-12 GWPP, which were based on analytical results for PCE and other analytes (gross alpha activity and lead), although only eight wells had sufficient data for statistical analysis, including two wells with maximum concentrations below the corresponding screening level for each COC (BWXT 2005).

Results of the MAROS statistical analyses indicate a PI trend for gross alpha activity in groundwater from spring SCR3.5SP (Table B.8). Results of the baseline MAROS assessment noted a PI trend for gross alpha activity in the spring, but qualified the trend as an apparent artifact of analytical detection limits and data variability (BWXT 2005). Concentrations of gross alpha activity do not exceed screening levels at this location.

Results of the MAROS statistical trend analyses of 11DCE concentrations in wells GW-176, GW-177, and GW-178 also show I or PI trends (overall trends) (Table B.8). Increasing concentrations of 11DCE in the groundwater from these wells may be at least partially attributable to the biotic and/or abiotic (spontaneous chemical degradation Vogel and McCarty, 1987) degradation of 111TCA, a parent compound that is a principal component of the VOC plume believed to originate from the western disposal trenches at the CRSP (B\&W Y-12 2008). Results of the baseline MAROS assessment of the CRSP did not indicate increasing trends for 11DCE in these wells; there were insufficient sampling data for well GW-176 and the respective statistical trend analyses for wells GW-177 and GW-178 were based on sampling results for lead (BWXT 2005).

\section{Well Sufficiency/Redundancy Evaluation}

Results of the supplemental MAROS assessment of well sufficiency based on data for the wells included in the MAROS analysis group for the CRSP do not indicate areas of uncertainty regarding COC concentrations in the groundwater that warrant increased monitoring efforts or installation of new wells. Similar findings were obtained from the baseline MAROS assessment (BWXT 2005).

Based on results of the MAROS well redundancy analysis, well GW-173 at the CRSP, which is approximately $200 \mathrm{ft}$ east of the eastern waste disposal trenches at the site (Figure A.4), provides 
redundant local monitoring coverage in an area with low uncertainty regarding the spatial distribution and concentrations of each applicable COC in the groundwater, and is recommended for "Elimination" (i.e., discontinued sampling) (Appendix D.3). As noted previously, the well must be granted "Inactive" status for the purposes of the Y-12 GWPP, as defined and specified in the most current version of the Y-12 GWPP MOP, to formally discontinue future groundwater sampling.

Summary of MAROS well sufficiency and well redundancy analysis for the CRSP

\begin{tabular}{|c|c|}
\hline $\begin{array}{c}\text { WELL } \\
\text { NUMBER }\end{array}$ & BASIS FOR MAROS “ELIMINATION” RECOMMENDATION \\
\hline GW-173 & Probably decreasing trends; redundant with well GW-174 \\
\hline
\end{tabular}

Sampling Frequency Evaluation

Based on results of the supplemental MAROS assessment sampling frequency appropriate for each sampling location included in the MAROS analysis group for the CRSP (Table B.8), biennial sampling is recommended for most (six) of the wells that either do not have a regulatory-driven sampling frequency (Appendix D.3). Annual sampling is recommended for one well at the site and sampling once every five years is recommended for three wells, each of which exhibit decreasing concentration trends for the applicable COC (Appendix D.3).

\subsection{LANDFILLS V AND VII AREA}

The Landfills V and VII Area MAROS analysis group includes sampling locations associated with IL V and CDL VII, both of which are operating facilities used for the disposal of various non-hazardous solid waste and construction debris generated at Y-12 and elsewhere on the ORR (Figure A.4). Groundwater sampling at each landfill is performed as required by the operating permit for each site and the TDEC regulations governing operation and management of nonhazardous SWDFs.

\section{Sampling Locations}

There are 10 sampling locations in the MAROS analysis group for the Landfills V and VII Area (Table B.9), including a natural spring on the southern flank of Chestnut Ridge, four wells located at CDL VII, and five wells at IL V (Figure A.4). The baseline MAROS assessment of the Y-12 GWPP included these same sampling locations in the MAROS analysis group for the Landfills V and VII Area (BWXT 2005).

\section{COC Assessment}

Available groundwater sampling results obtained to date show that all of the sampling locations included in the MAROS analysis group for Landfills V and VII Area yield uncontaminated groundwater except the upgradient/background well (GW-798) at CDL VII, which intercepts groundwater flow/transport pathways for VOCs released from the waste disposal trenches at the CRSP (B\&W Y-12 2008). Results of the MAROS COC assessment show that none of the COCs exceed applicable screening levels (Appendix C.9). 


\section{Moment Analysis}

As noted previously, the sampling locations included in the MAROS analysis group for the Landfills V and VII (except CDL VII upgradient/background well GW-798) yield uncontaminated groundwater. Consequently, results of the MAROS moment analyses are not significant (see MAROS Spatial Moment Analysis Summary report in Appendix C.9).

\section{Statistical Trend Analysis}

All the sampling locations included in the MAROS analysis group for the Landfills V and VII Area yield uncontaminated groundwater except CDL VII upgradient/background well GW-798. Groundwater samples collected from this well since January 2000 have contained several VOCs, including PCE, TCE, and 111TCA, with the concentrations of these compounds steadily increasing through historical peak concentrations in January 2003 and subsequently decreasing more than 50\% through April 2006 (B\&W Y-12 2008). The MAROS statistical trend analysis of the PCE data for well GW-798 show an I trend (see MAROS Statistical Analysis Summary report in Appendix C.9).

\section{Well Sufficiency/Redundancy Evaluation}

The well sufficiency module was not run on the data for the wells included in the MAROS analysis group for the Landfills V and VII Area because of the small number of wells in the group; similar findings were reported in the baseline MAROS assessment of the Y-12 GWPP (BWXT 2005).

\section{Sampling Frequency Evaluation}

As noted above, each groundwater sampling location included in the MAROS analysis group for the Landfills V and VII Area has a regulatory-driven sampling frequency (Appendix D.3). Consequently, as noted in Section 2.7, the final MAROS-based sampling frequency recommendations are not applicable.

\subsection{EAST CHESTNUT RIDGE}

The MAROS analysis group for East Chestnut Ridge includes groundwater sampling locations associated with the following closed waste management sites in the easternmost section of the Chestnut Ridge Regime directly south-southeast of Y-12: the East Chestnut Ridge Waste Pile (ECRWP), Chestnut Ridge Sediment Disposal Basin (CRSDB), Chestnut Ridge Borrow Area Waste Pile (CRBAWP), and Kerr Hollow Quarry (KHQ) (Figure A.4).

\section{Sampling Locations}

There are 17 groundwater sampling locations included in the MAROS analysis group for East Chestnut Ridge (Table B.10): five wells at the ECRWP, four wells at the CRSDB, two wells at the CRBAWP, four wells at KHQ, and two wells at the South Campus Facility (SCF), which is located on the ORR near the southeastern corner of the Chestnut Ridge Regime (Figure A.4). The baseline MAROS assessment of the Y-12 GWPP included all but three of these sampling locations (wells GW-161, GW-294, and GW-296 at the ECRWP) in the MAROS analysis group for East Chestnut Ridge (BWXT 2005). 


\section{COC Assessment}

Historical sampling data show low levels of VOCs (primarily CTET and PCE) in the groundwater from a few wells at KHQ and somewhat higher levels of TCE in wells at the SCF, with more recent data showing either non-detect results for the applicable COC or concentrations that have remained slightly above or decreased below the corresponding screening level for the COC. Data obtained to date show that all the other groundwater sampling locations included in the MAROS analysis group for East Chestnut Ridge currently yield uncontaminated groundwater. Consequently, results of the MAROS COC assessment are not significant (see MAROS Spatial Moment Analysis Summary report in Appendix C.10).

\section{Moment Analysis}

Historical data for almost all of the wells included in the MAROS analysis group for East Chestnut Ridge do not indicate concentrations of any potential COC that exceed the corresponding screening level. Consequently, results of the MAROS moment analyses generally are not significant (see MAROS Spatial Moment Analysis Summary report in Appendix C.10), which also was noted in the baseline MAROS assessment of the Y-12 GWPP (BWXT 2005).

\section{Statistical Trend Analysis}

Historical data for almost all of the wells included in the MAROS analysis group for East Chestnut Ridge do not indicate concentrations of any potential COC that exceed the corresponding screening level. Also, elevated COC concentrations indicated by historical data for wells at KHQ and the SCF have decreased to below detection limits or only slightly above the applicable screening level. Consequently, results of the MAROS statistical trend analyses generally are not significant (see MAROS Statistical Trend Analysis Summary report in Appendix C.10).

\section{Well Sufficiency/Redundancy Evaluation}

Results of the supplemental MAROS assessment of well sufficiency based on data for the wells included in the MAROS analysis group for East Chestnut Ridge are inconclusive and are generally not relevant in light of the contaminant detection-based purpose of the Similar findings were obtained from the baseline MAROS assessment (BWXT 2005).

\section{Sampling Frequency Evaluation}

The groundwater sampling locations included in the MAROS analysis group for East Chestnut Ridge have regulatory-driven sampling frequencies (Appendix D.3). Consequently, as noted in Section 2.7, the final MAROS-based sampling frequency recommendations are not applicable to these wells. 


\subsection{CONCLUSIONS AND RECCOMMENDATIONS}

Results of the supplemental MAROS assessment of the Y-12 GWPP are consistent with, and generally support, the findings of the baseline MAROS assessment. However, the similar and somewhat duplicative findings suggest diminishing returns from future, fully implemented MAROS assessments. Instead, selected MAROS data evaluation modules could be incorporated into the routine management and implementation of the Y-12 GWPP, specifically the MAROS-recommended sampling frequency and the MAROS moment analyses. Moreover, both MAROS assessments included data evaluations that do not seem applicable and/or appropriate for monitoring data from wells and springs that yield uncontaminated groundwater, and should be excluded from any future MAROS-based evaluations.

The MCES-based sampling frequency evaluation method used by MAROS provides the Y-12 GWPP with a fairly non-subjective technical process to establish an appropriate sampling frequency for each well and spring. The overall technical approach could be incorporated into the Y-12 GWPP MOP, which would define the schedule (e.g., biennial) and scope (e.g., wells and springs sampled during biennial periods) of the sampling frequency evaluations and serve as the forum to document the results of the evaluations. Additionally, the Y-12 GWPP should adopt the MAROS-based sampling frequencies recommended for the groundwater sampling locations that serve a regulatory compliance-driven monitoring program, i.e., RCRA post-closure detection monitoring and RCRA post-closure corrective action monitoring, CERCLA baseline monitoring, remedial effectiveness monitoring, and ROD-specified monitoring; and detection monitoring at operating and closed SWDFs. All the wells and springs associated with these monitoring programs maintain "Active" status per the Y-12 GWPP MOP, and the MAROS-recommended sampling frequency would apply only if these wells or springs were to be sampled for non-regulatory compliance purposes.

The MAROS well sufficiency/redundancy evaluations performed for this supplemental assessment involved a slightly different set of locations than the set used for the baseline assessment, although the total number of sampling locations were similar. As described in Section 1.0, the assessment is performed only on "Active" monitoring locations, and the current monitoring well network excludes 15 wells deemed "Inactive", primarily reflecting recommendations of the baseline assessment. Also, monitoring results for 18 wells (mostly located in the Central Y-12 MAROS analysis group) that had insufficient data for the baseline assessment were included in the supplemental assessment. Based primarily on the well sufficiency/redundancy evaluations of the sampling locations in the supplemental assessment, a total of 32 monitoring wells received recommendations to discontinue sampling (i.e., "Elimination"), including 26 wells in the Bear Creek Regime (Appendix D.1), five wells in the East Fork Regime (Appendix D.2), and one well in the Chestnut Ridge Regime (Appendix D.3).

The MAROS moment analyses of selected COC concentrations in the groundwater provide an effective approach for monitoring for significant changes in the hydrochemical dynamics within groundwater contaminant plumes that may warrant appropriate response actions (e.g., increased or decreased sampling frequency). Results of the moment analyses for each COC foster evaluation of concentration trends within the plume as a whole, an approach that dovetails two principal strengths of the Y-12 GWPP: the extensive network of sampling locations in most of the areas with groundwater contamination, and the large database of long-term sampling results for many of these sampling locations. This approach also seems more technically robust than an evaluation focused on plume boundary conditions, particularly considering the widespread intermingling of contaminant plumes in many areas of Y-12 and the relatively limited number of plume boundary sampling locations. 
Results of the baseline and supplemental MAROS assessments indicate that the data evaluations performed by most of the MAROS modules are not particularly applicable to groundwater monitoring results obtained at each waste management facility in the Chestnut Ridge Regime except the CRSP. Some of the MAROS data evaluations (e.g., statistical trend analysis) are inappropriate because all but a few of the wells and springs associated with these sites yield uncontaminated groundwater. Also, the findings and results for other MAROS data evaluations (e.g., well sufficiency/redundancy and sampling frequency) are not actionable because regulatory (and permit) requirements, rather than purely technical considerations, define the site-specific groundwater sampling protocols. In light of these technical and regulatory constraints, only the groundwater sampling locations associated with the CRSP should be included in any future MAROS-based assessments or evaluations.

\subsection{REFERENCES}

Air Force Center for Environmental Excellence (AFCEE). 2003. Monitoring and Remediation Optimization System (MAROS) 2.1 Software Users Guide, MAROS V2.1 Manual.

Babcock \& Wilcox Technical Services Y-12, LLC (B\&W Y-12). 2008. Y-12 Groundwater Protection Program Groundwater Monitoring Data Compendium, Revision 2: Monitoring Wells 1090 through GW-199, Y/TS-1983/R2/V2; Monitoring Wells GW-200 through GW-699, Y/TS1983/R2/V3; and Monitoring Wells GW-700 through GW-959, Y/TS-1983/R2/V4; April 2008.

BWXT Y-12, LLC (BWXT). 2005. Assessment of the Groundwater Protection Program Y-12 National Security Complex, Oak Ridge, Tennessee, Prepared by Groundwater Services, Inc. Y/TS-1984, December 2005.

BWXT 2006. Y-12 Groundwater Protection Program Monitoring Optimization Plan for Groundwater Monitoring Wells at the U.S. Department of Energy Y-12 National Security Complex, Oak Ridge, Tennessee, Prepared by Elvado Environmental LLC. Y/TS-2031, December 2006.

Haase, S. and H.L. King. 1990. Report and Preliminary Assessment of the Occurrence of Dense, Nonaqueous Phase Liquids in the Bear Creek Burial Grounds Hazardous Waste Disposal Unit at the Oak Ridge Y-12 Plant, Y/TS-629, prepared by Martin Marietta Energy Systems, Inc., Oak Ridge, TN.

U.S. Department of Energy (DOE). 1997. Report on the Remedial Investigation of Bear Creek Valley at the Oak Ridge Y-12 Plant, Oak Ridge, Tennessee, DOE/OR/01-1455/V1\&D2.

DOE. 1998. Report on the Remedial Investigation of the Upper East Fork Poplar Creek Characterization Area at the Oak Ridge Y-12 Plant, Oak Ridge, Tennessee, DOE/OR/01-1641/D1\&V1.

DOE. 1999. Action Memorandum for the Oak Ridge Y-12 Plant East End Volatile Organic Compound Plume, Oak Ridge, Tennessee, DOE/OR/01-1819\&D2, U.S. Department of Energy, Office of Environmental Management, Oak Ridge, TN.

Vogel, T.M. and P. L. McCarty. 1987. Abiotic and biotic transformations of 1,1,1-Trichlorethane under Methanogenic Conditions. Environmental Science and Technology 21(12): p. 1208 - 1213. 
APPENDIX A

FIGURES 


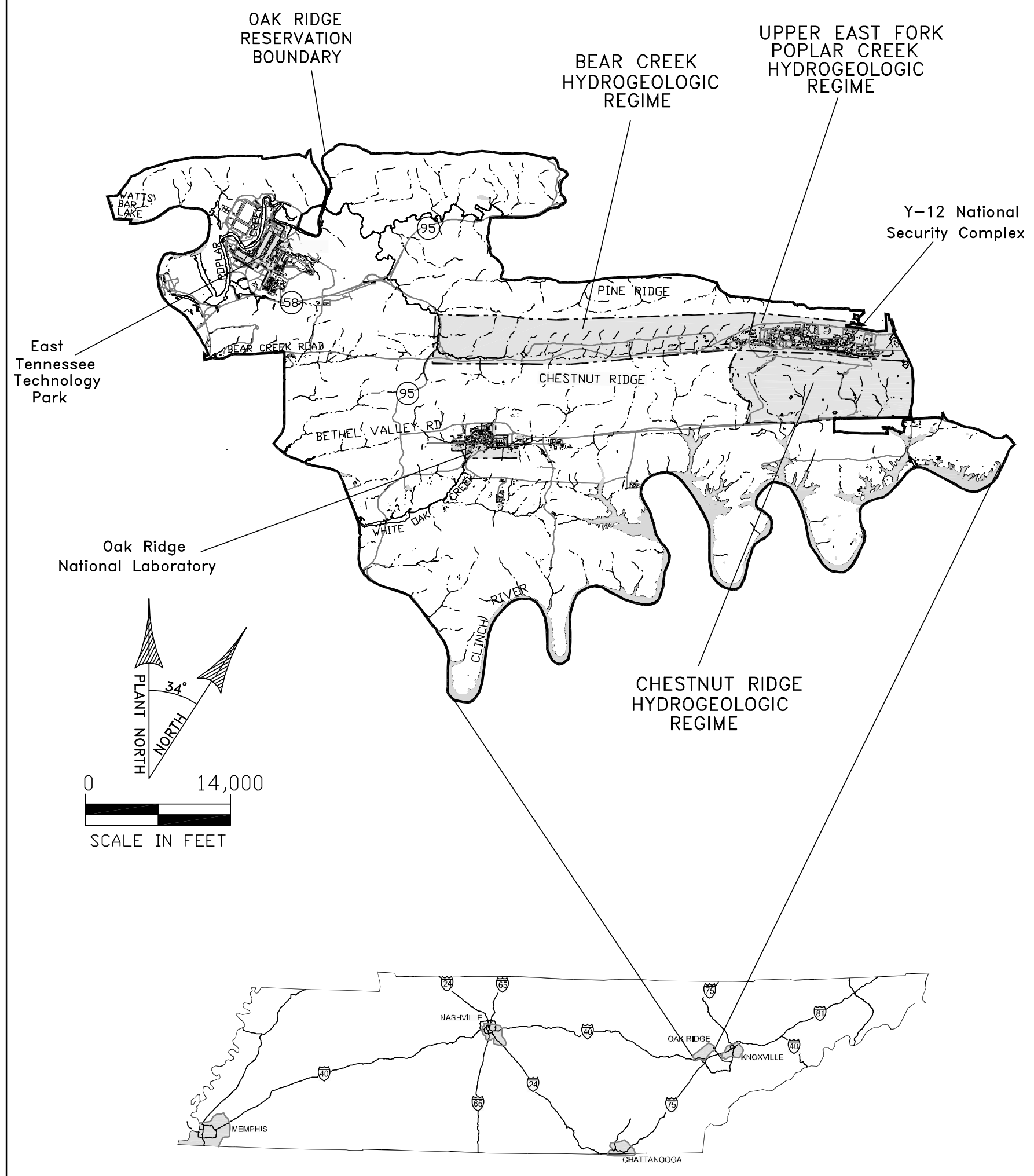

GWPP Fig1

Fig. A.1. Hydrogeologic regimes at the Y-12 National Security Complex. 


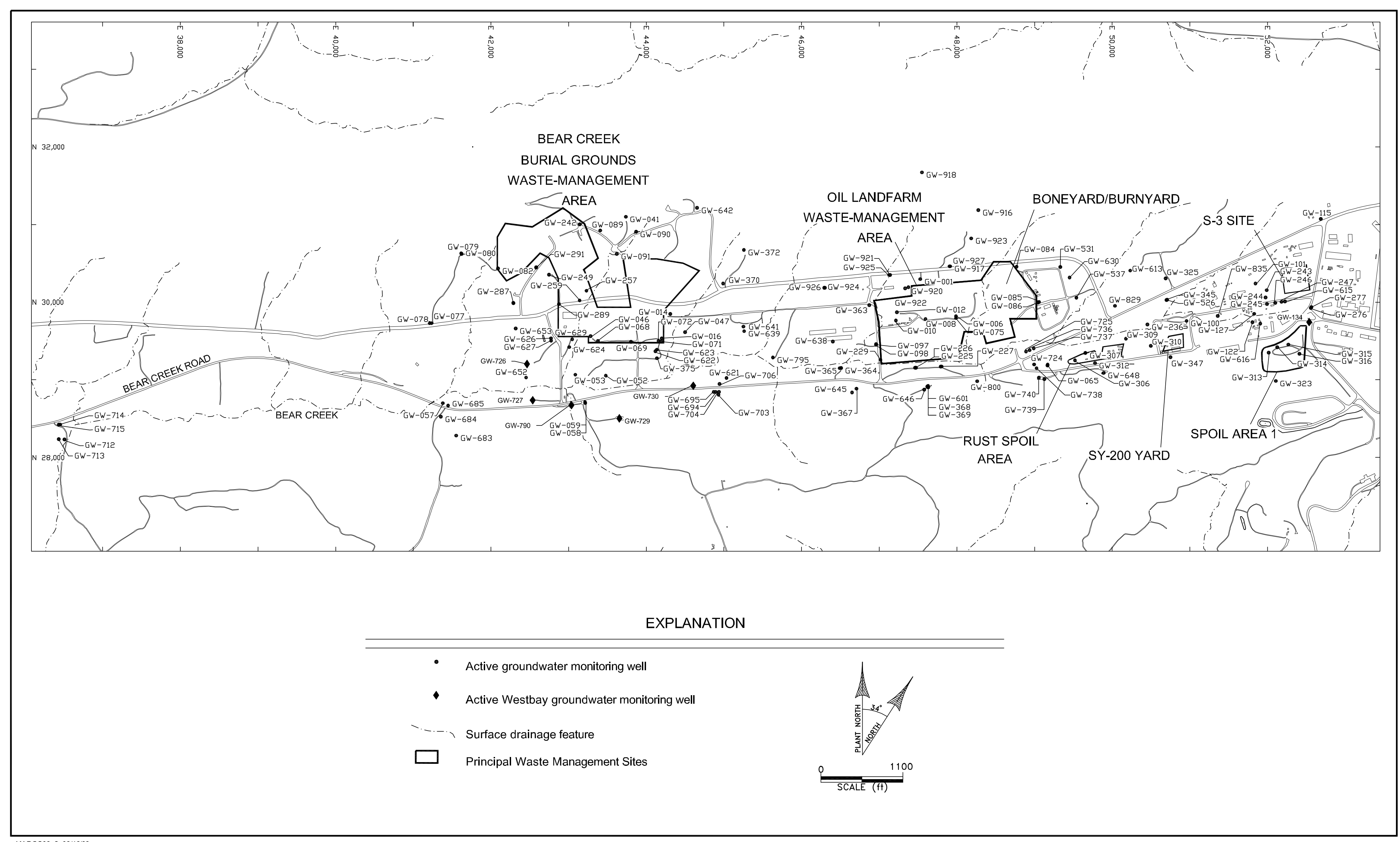




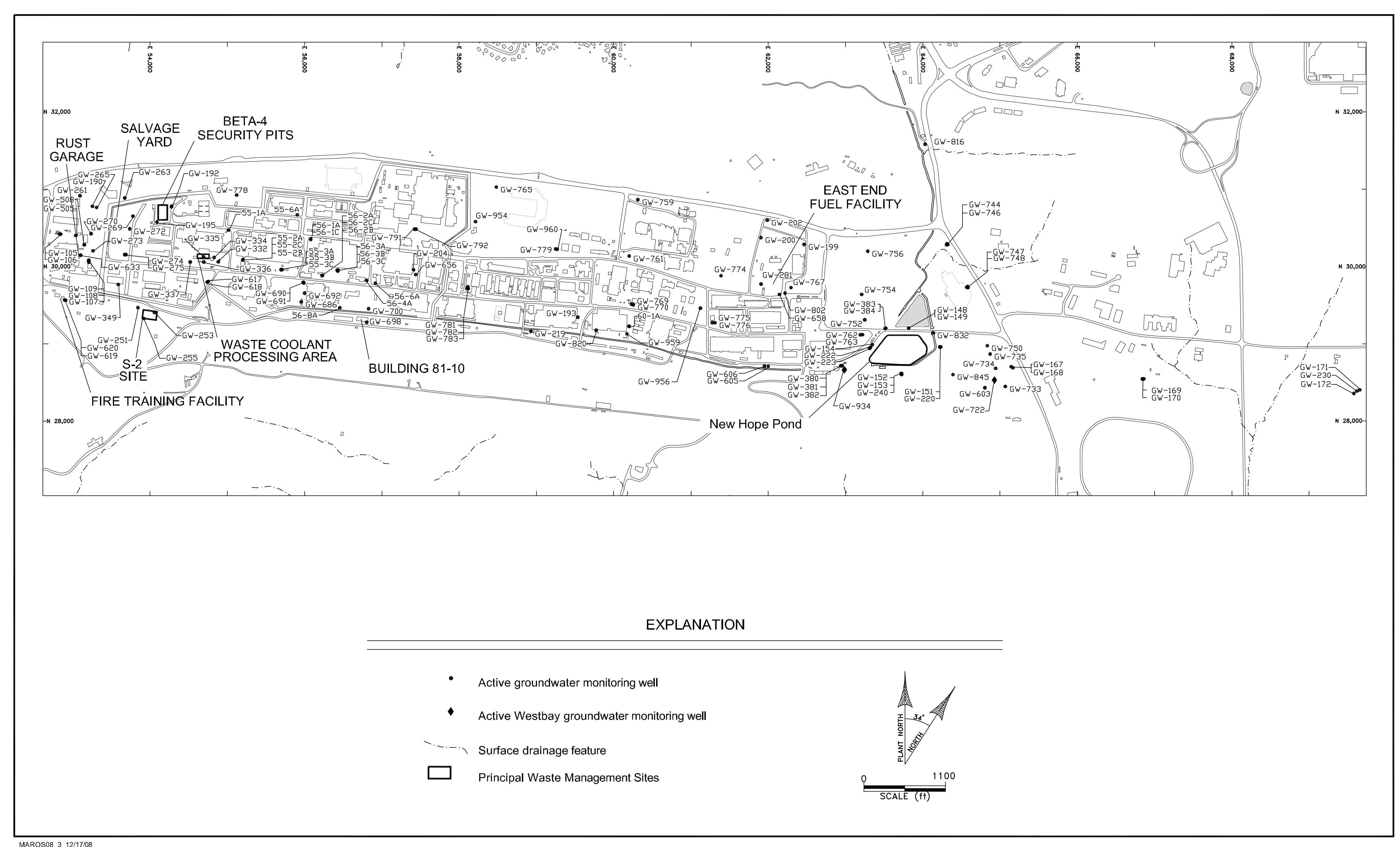




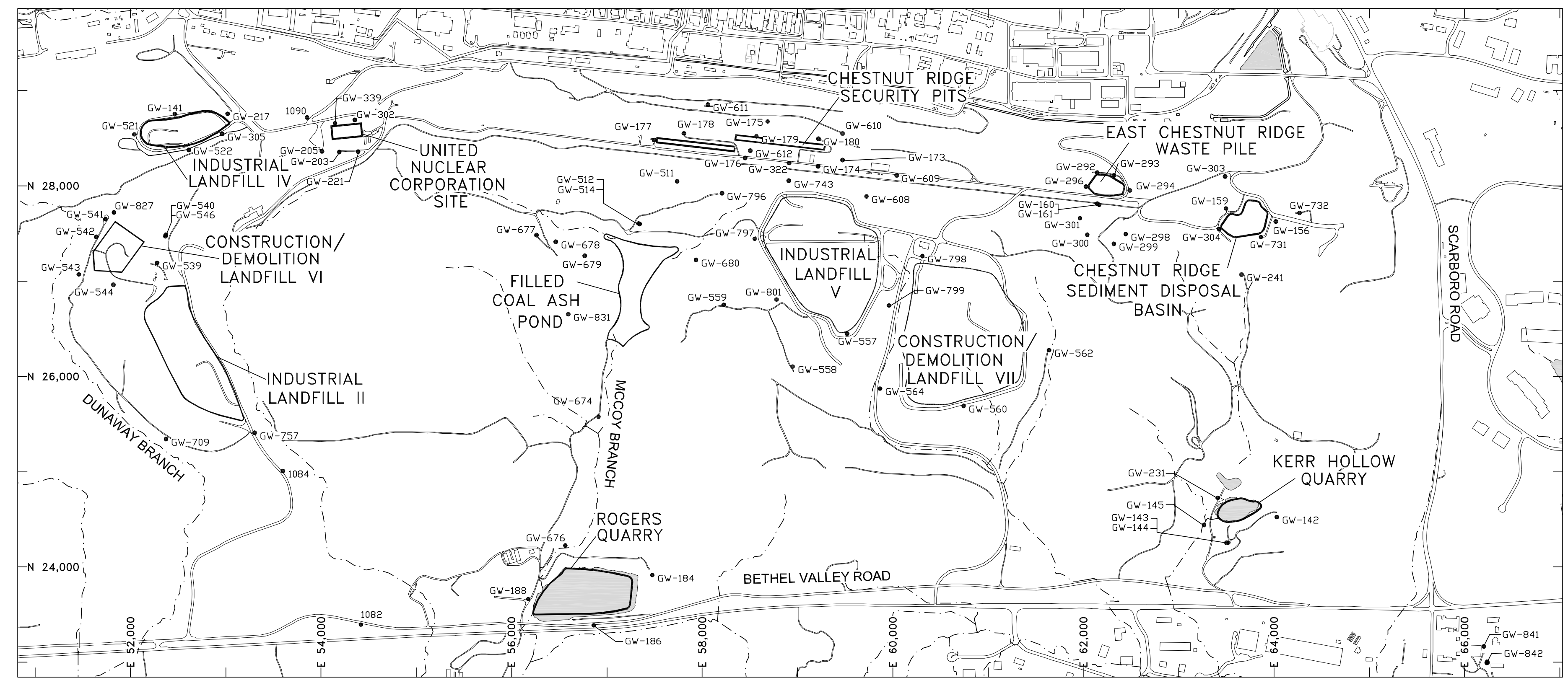

EXPLANATION

- Active groundwater monitoring well

Surface drainage feature

$\square \quad$ Principal Waste Management Sites

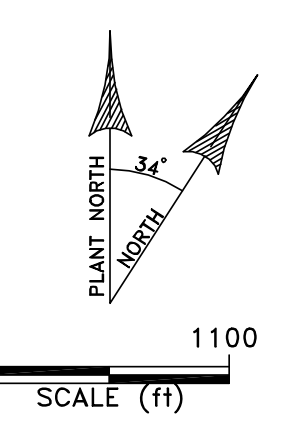




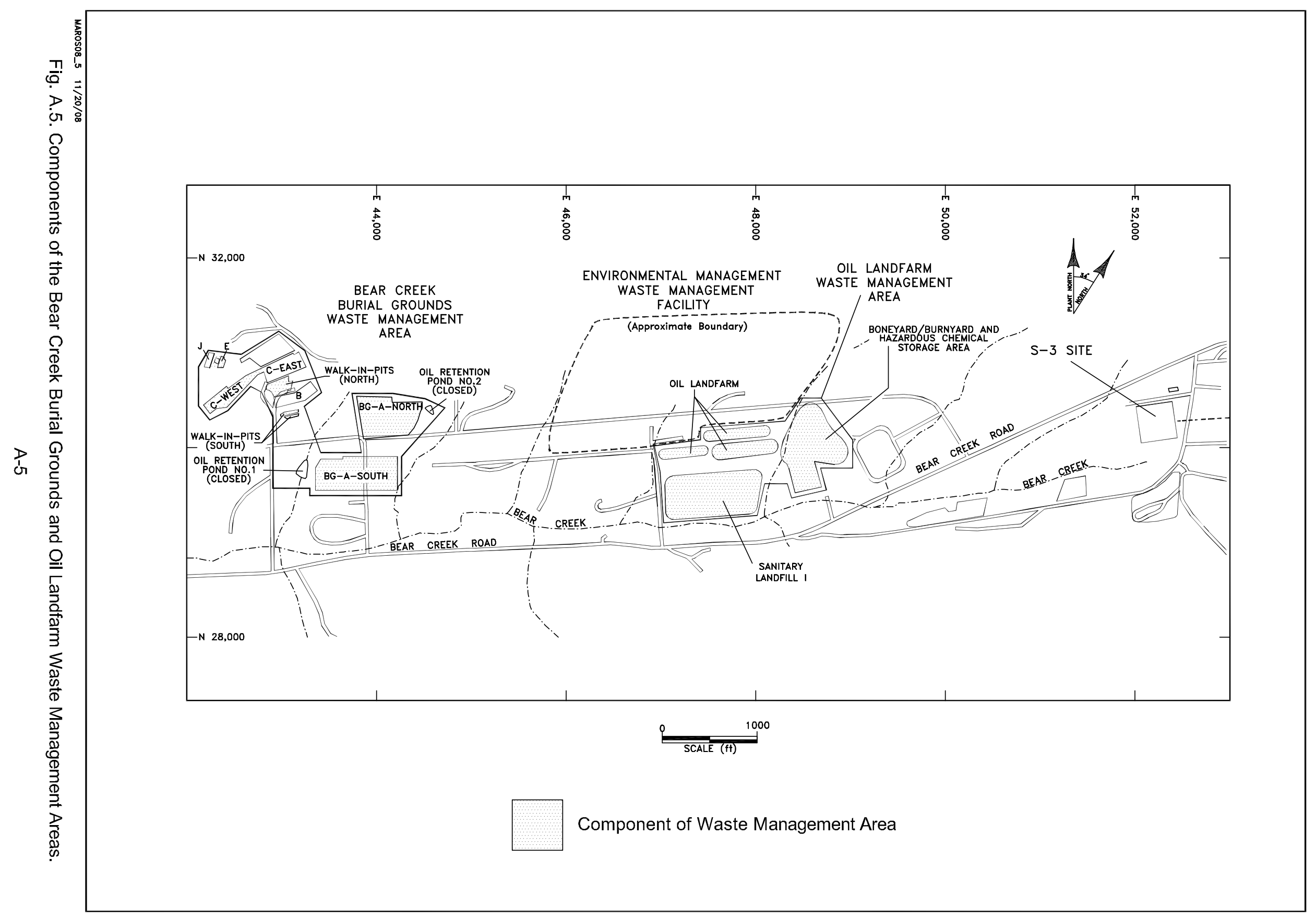


APPENDIX B

TABLES 
TABLE B.1

Supplemental MAROS assessment results for the West S-3 Area MAROS analysis group

\begin{tabular}{|c|c|c|c|c|c|c|c|c|c|}
\hline $\begin{array}{l}\text { Location } \\
\text { Name }\end{array}$ & $\begin{array}{c}\text { Earliest } \\
\text { Sample Date }\end{array}$ & $\begin{array}{l}\text { Most Recent } \\
\text { Sample Date }\end{array}$ & $\begin{array}{l}\text { Location } \\
\text { Type }\end{array}$ & $\begin{array}{c}\text { Number of } \\
\text { Samples } \\
1996-2007\end{array}$ & \begin{tabular}{|c|} 
Average \\
Concentration \\
Exceeds Screening \\
Level
\end{tabular} & Historic Priority Constituent & $\begin{array}{c}\text { Trend for } \\
\text { Priority } \\
\text { Constituent } \\
\text { 1996-2007 } \\
\end{array}$ & $\begin{array}{l}\text { Trend for } \\
\text { Priority } \\
\text { Constituent } \\
1986-2007 \\
\end{array}$ & $\begin{array}{l}\text { Preliminary } \\
\text { Frequency }\end{array}$ \\
\hline GW-100 & $01 / 22 / 86$ & $05 / 07 / 07$ & $\overline{W L}$ & 3 & Yes & NITRATE & $\mathrm{N} / \mathrm{A}$ & $\overline{P D}$ & Quarterly \\
\hline GW-101 & $01 / 23 / 86$ & 05/09/07 & WL & 3 & Yes & NITRATE & N/A & D & Quarterly \\
\hline GW-122 & $12 / 12 / 86$ & 05/09/07 & WL & 4 & Yes & NITRATE & S & D & Annual \\
\hline GW-127 & $12 / 12 / 86$ & 05/07/07 & WL & 3 & Yes & GROSS ALPHA ACTIVITY & N/A & D & Quarterly \\
\hline GW-236 & 04/05/88 & 05/03/07 & WL & 3 & Yes & NITRATE & N/A & D & Quarterly \\
\hline GW-244 & $11 / 17 / 87$ & $10 / 24 / 05$ & WL & 2 & Yes & NITRATE & $\mathrm{N} / \mathrm{A}$ & $\mathrm{D}$ & Quarterly \\
\hline GW-245 & $11 / 23 / 87$ & $10 / 25 / 05$ & WL & 2 & Yes & NITRATE & N/A & $\mathrm{S}$ & Quarterly \\
\hline GW-246 & 03/16/87 & 08/15/07 & WL & 6 & Yes & GROSS BETA ACTIVITY & $\mathrm{s}$ & PI & Annual \\
\hline GW-247 & $11 / 23 / 87$ & 10/25/05 & WL & 2 & Yes & NITRATE & N/A & $\mathrm{s}$ & Quarterly \\
\hline GW-276 & $10 / 29 / 86$ & 07/09/07 & WL & 24 & Yes & URANIUM & D & D & Annual \\
\hline GW-277 & $10 / 29 / 86$ & $05 / 14 / 07$ & $\overline{W L}$ & 3 & Yes & NITRATE & $\mathrm{N} / \mathrm{A}$ & $\mathrm{S}$ & Quarterly \\
\hline GW-307 & $11 / 27 / 89$ & 05/01/07 & WL & 3 & Yes & TRICHLOROETHYLENE (TCE) & N/A & D & Quarterly \\
\hline GW-310 & 05/09/89 & 09/06/06 & WL & 3 & Yes & TRICHLOROETHYLENE (TCE) & N/A & D & Quarterly \\
\hline GW-312 & 05/05/89 & 08/17/05 & WL & 2 & Yes & TRICHLOROETHYLENE (TCE) & N/A & $\mathrm{S}$ & Quarterly \\
\hline GW-313 & $03 / 15 / 90$ & $05 / 01 / 07$ & $\overline{W L}$ & 3 & Yes & $\begin{array}{l}\text { TETRACHLOROETHYLENE(PCE) } \\
\end{array}$ & $\mathrm{N} / \mathrm{A}$ & NT & Quarterly \\
\hline GW-315 & 03/06/90 & 05/01/07 & WL & 22 & Yes & TETRACHLOROETHYLENE(PCE) & D & D & Annual \\
\hline GW-526 & 09/28/88 & 07/25/06 & WL & 5 & Yes & NITRATE & $\mathrm{S}$ & I & Annual \\
\hline GW-615 & 05/16/90 & 05/14/07 & WL & 4 & Yes & NITRATE & NT & I & Quarterly \\
\hline GW-616 & 05/17/90 & 05/10/07 & WL & 6 & Yes & NITRATE & $\mathrm{s}$ & NT & SemiAnnual \\
\hline GW-829 & $06 / 27 / 95$ & $02 / 15 / 06$ & $\mathrm{WL}$ & 12 & Yes & NITRATE & $\bar{D}$ & $\bar{D}$ & Quarterly \\
\hline SS-1 & 08/30/90 & 04/19/06 & SP & 21 & Yes & URANIUM & $\mathrm{D}$ & $\mathrm{D}$ & Annual \\
\hline \multicolumn{4}{|c|}{ Locations with no Data 2005-2007 } & $1986-2007$ & & & $1996-2005$ & $1986-2007$ & \\
\hline GW-306 & 05/09/89 & $04 / 26 / 91$ & WL & 8 & Yes & TRICHLOROETHYLENE (TCE) & $\mathrm{N} / \mathrm{A}$ & NT & Biennial \\
\hline GW-309 & 02/09/88 & 08/30/92 & WL & 16 & Yes & GROSS BETA ACTIVITY & N/A & D & Biennial \\
\hline GW-314 & 03/06/90 & 08/15/92 & WL & 11 & Yes & TETRACHLOROETHYLENE(PCE) & N/A & S & Annual \\
\hline GW-531 & $12 / 15 / 88$ & 06/24/91 & WL & 9 & No & GROSS ALPHA ACTIVITY & N/A & S & Biennial \\
\hline GW-648 & 01/15/91 & 07/23/92 & WL & 6 & No & GROSS ALPHA ACTIVITY & N/A & $\mathrm{S}$ & Biennial \\
\hline
\end{tabular}

Notes:

1. Well sample dates taken from Y-12 Analytical Database 2008.

2. $\mathrm{WL}=$ Groundwater Monitoring Well; $\mathrm{SP}=$ Spring.

3. Trend = Insufficient Data (N/A), Decreasing (D), Probably Decreasing (PD), Stable (S), Probably Increasing (PI), and Increasing (I), No Trend (NT); and non-detect for all sample events (ND).

4. Number of samples between 1996-2007 is the count of analytical samples acquired at the location based on the Analytical Database (2008).

5. Priority Constituent is the $\mathrm{COC}$ with the highest average concentration from the full dataset normalized by the screening level. (e.g. MAX [Ave Conc./Screening Level]).

Priority constituent indicated for wells where average concentrations do not exceed screening levels may be background level.

6. The Preliminary Frequency is the sampling frequency recommended by MAROS for results $1996-2007$. Low number of samples triggers default quarterly sampling. 
TABLE B.2

Supplemental MAROS assessment results for the Oil Landfarm WMA MAROS analysis group

\begin{tabular}{|c|c|c|c|c|c|c|c|c|c|c|}
\hline $\begin{array}{l}\text { Location } \\
\text { Name }\end{array}$ & $\begin{array}{c}\text { Earliest } \\
\text { Sample Date }\end{array}$ & $\begin{array}{l}\text { Most Recent } \\
\text { Sample Date }\end{array}$ & Location Type & $\begin{array}{c}\text { Number of } \\
\text { Samples } \\
1996-2007\end{array}$ & $\begin{array}{c}\text { Percent } \\
\text { Detection } \\
1996-2007\end{array}$ & \begin{tabular}{|c|} 
Average \\
Concentration \\
$\begin{array}{c}\text { Exceeds Screening } \\
\text { Level }\end{array}$ \\
\end{tabular} & Historic Priority Constituent & $\begin{array}{l}\text { Trend for } \\
\text { Priority } \\
\text { Constituent } \\
1996-2007 \\
\end{array}$ & $\begin{array}{c}\text { Trend for } \\
\text { Priority } \\
\text { Constituent } \\
1986-2007 \\
\end{array}$ & $\begin{array}{l}\text { Preliminary } \\
\text { Frequency }\end{array}$ \\
\hline GW-008 & $01 / 26 / 98$ & $07 / 02 / 07$ & WL & 20 & $100 \%$ & Yes & TETRACHLOROETHYLENE(PCE) & $\mathrm{I}$ & $\mathrm{I}$ & Quarterly \\
\hline GW-085 & $11 / 05 / 87$ & $08 / 15 / 07$ & WL & 23 & $100 \%$ & Yes & NITRATE & $\mathrm{s}$ & D & Annual \\
\hline GW-098 & $03 / 17 / 87$ & $04 / 23 / 07$ & WL & 8 & $100 \%$ & Yes & TRICHLOROETHYLENE (TCE) & NT & NT & Annual \\
\hline GW-225 & $01 / 12 / 86$ & $08 / 13 / 07$ & WL & 14 & $100 \%$ & Yes & TRICHLOROETHYLENE (TCE) & I & $\mathrm{D}$ & Quarterly \\
\hline GW-226 & $01 / 12 / 86$ & $08 / 13 / 07$ & WL & 20 & $100 \%$ & Yes & TRICHLOROETHYLENE (TCE) & $\mathrm{S}$ & $\mathrm{T}$ & Annual \\
\hline GW-227 & $01 / 14 / 86$ & 09/08/05 & WL & 2 & $100 \%$ & Yes & TRICHLOROETHYLENE (TCE) & N/A & D & Quarterly \\
\hline GW-229 & $01 / 14 / 86$ & $04 / 23 / 07$ & WL & 10 & $100 \%$ & Yes & VINYL CHLORIDE & NT & I & Annual \\
\hline GW-363 & $09 / 21 / 88$ & $08 / 15 / 07$ & WL & 39 & $2 \%$ & No & BENZENE & NT & NT & Annual \\
\hline GW-365 & $09 / 21 / 88$ & $09 / 01 / 05$ & WL & 4 & $75 \%$ & Yes & VINYL CHLORIDE & NT & $\mathrm{PI}$ & Quarterly \\
\hline GW-368 & $09 / 24 / 88$ & $04 / 26 / 07$ & WL & 3 & $100 \%$ & Yes & TRICHLOROETHYLENE (TCE) & N/A & $\mathrm{D}$ & Quarterly \\
\hline GW-369 & $09 / 22 / 88$ & 04/30/07 & WL & 1 & $100 \%$ & Yes & TRICHLOROETHYLENE (TCE) & N/A & D & Quarterly \\
\hline GW-537 & $12 / 06 / 89$ & 05/03/07 & WL & 21 & $100 \%$ & Yes & NITRATE & $\mathrm{D}$ & $\mathrm{D}$ & Annual \\
\hline GW-601 & $03 / 05 / 90$ & $04 / 24 / 07$ & WL & 4 & $100 \%$ & Yes & TRICHLOROETHYLENE (TCE) & $\mathrm{s}$ & D & Quarterly \\
\hline GW-724 & $05 / 20 / 92$ & $08 / 20 / 07$ & WL & 22 & $100 \%$ & Yes & TRICHLOROETHYLENE (TCE) & $\mathrm{D}$ & NT & Annual \\
\hline GW-725 & 05/21/92 & $08 / 20 / 07$ & WL & 22 & $100 \%$ & Yes & TRICHLOROETHYLENE (TCE) & NT & D & SemiAnnual \\
\hline GW-736 & $05 / 22 / 92$ & $08 / 02 / 05$ & WL & 4 & $100 \%$ & Yes & TRICHLOROETHYLENE (TCE) & $\mathrm{s}$ & $\mathrm{D}$ & Quarterly \\
\hline GW-738 & 05/26/92 & 08/21/07 & WL & 22 & $100 \%$ & Yes & TRICHLOROETHYLENE (TCE) & $\mathrm{D}$ & $\mathrm{D}$ & Annual \\
\hline GW-739 & $05 / 27 / 92$ & $07 / 27 / 05$ & WL & 4 & $100 \%$ & Yes & TRICHLOROETHYLENE (TCE) & $\mathrm{s}$ & $\mathrm{s}$ & Quarterly \\
\hline GW-740 & $06 / 02 / 92$ & $08 / 21 / 07$ & $\mathrm{WL}$ & 22 & $100 \%$ & Yes & TRICHLOROETHYLENE (TCE) & $\mathrm{D}$ & $\mathrm{D}$ & Annual \\
\hline GW-916 & $04 / 18 / 06$ & $08 / 14 / 07$ & WL & 6 & $17 \%$ & No & URANIUM & NT & NT & Biennial \\
\hline GW-917 & $04 / 03 / 01$ & 08/09/07 & WL & 22 & $5 \%$ & No & TRICHLOROETHYLENE (TCE) & NT & NT & Biennial \\
\hline GW-918 & $04 / 02 / 01$ & $08 / 14 / 07$ & WL & 22 & $9 \%$ & No & TRICHLOROETHYLENE (TCE) & NT & NT & Biennial \\
\hline GW-920 & $04 / 18 / 06$ & 08/08/07 & WL & 6 & $33 \%$ & No & URANIUM & NT & NT & Biennial \\
\hline GW-921 & $04 / 24 / 06$ & $08 / 07 / 07$ & WL & 6 & $17 \%$ & No & URANIUM & NT & NT & Biennial \\
\hline GW-922 & $04 / 04 / 01$ & 08/08/07 & WL & 22 & $5 \%$ & No & TETRACHLOROETHYLENE(PCE) & NT & NT & Biennial \\
\hline GW-923 & $04 / 02 / 01$ & $04 / 24 / 07$ & WL & 16 & $6 \%$ & No & TRICHLOROETHYLENE (TCE) & NT & NT & Biennial \\
\hline GW-924 & 03/29/01 & 08/13/07 & WL & 8 & $0 \%$ & No & VINYL CHLORIDE & ND & ND & Annual \\
\hline GW-925 & 04/19/06 & 08/08/07 & WL & 6 & $17 \%$ & No & URANIUM & NT & NT & Biennial \\
\hline GW-926 & $04 / 02 / 01$ & $08 / 13 / 07$ & $\mathrm{WL}$ & 22 & $5 \%$ & No & TRICHLOROETHYLENE (TCE) & NT & NT & Annual \\
\hline GW-927 & 05/30/01 & $05 / 20 / 03$ & WL & 5 & $60 \%$ & No & GROSS ALPHA ACTIVITY & NT & N/A & Annual \\
\hline \multicolumn{7}{|c|}{ Locations with no data collected 2005-2007 } & \multicolumn{4}{|c|}{$1996-2005$} \\
\hline GW-006 & $11 / 11 / 98$ & $08 / 02 / 00$ & $\mathrm{WL}$ & 8 & $100 \%$ & No & TRICHLOROETHYLENE (TCE) & $\mathrm{s}$ & $\mathrm{S}$ & Annual \\
\hline GW-367 & $09 / 19 / 88$ & $05 / 11 / 91$ & WL & 12 & $100 \%$ & Yes & TRICHLOROETHYLENE (TCE) & $\mathrm{N} / \mathrm{A}$ & $\mathrm{D}$ & Biennial \\
\hline
\end{tabular}

Notes:

1. Well sample dates taken from Y-12 Analytical Database 2008.

2. $\mathrm{WL}=$ Groundwater Monitoring Well; $\mathrm{SP}=$ Spring.

3. Trend = Insufficient Data (N/A), Decreasing (D), Probably Decreasing (PD), Stable (S), Probably Increasing (PI),

and Increasing (I), No Trend (NT); and non-detect for all sample events (ND).

4. Number of samples between 1996-2007 is the count of analytical samples acquired at the location based on the Analytical Database (2008).

5. Priority Constituent is the $\mathrm{COC}$ with the highest average concentration from the full dataset normalized by the screening level. (e.g. MAX [Ave Conc./Screening Level]).

Priority constituent indicated for wells where average concentrations do not exceed screening levels may be background level.

6. The Preliminary Frequency is the sampling frequency recommended by MAROS for results $1996-2007$. Low number of samples triggers default quarterly sampling. 
TABLE B.3

Supplemental MAROS assessment results for the Bear Creek Burial Grounds WMA MAROS analysis group

\begin{tabular}{|c|c|c|c|c|c|c|c|c|c|c|}
\hline $\begin{array}{l}\text { Location } \\
\text { Name }\end{array}$ & $\begin{array}{c}\text { Earliest Sample } \\
\text { Date }\end{array}$ & $\begin{array}{l}\text { Most Recent } \\
\text { Sample Date }\end{array}$ & $\begin{array}{l}\text { Location } \\
\text { Type }\end{array}$ & $\begin{array}{c}\text { Number of } \\
\text { Samples } \\
1996-2007\end{array}$ & $\begin{array}{c}\text { Percent } \\
\text { Detection } \\
\text { 1996-2007 }\end{array}$ & \begin{tabular}{|c|} 
Average \\
Concentration \\
$\begin{array}{c}\text { Exceeds Screening } \\
\text { Level }\end{array}$ \\
\end{tabular} & Historic Priority Constituent & $\begin{array}{c}\text { Trend for } \\
\text { Priority } \\
\text { Constituent } \\
1996-2007 \\
\end{array}$ & $\begin{array}{c}\text { Trend for } \\
\text { Priority } \\
\text { Constituent } \\
1986-2007 \\
\end{array}$ & $\begin{array}{l}\text { Preliminary } \\
\text { Frequency }\end{array}$ \\
\hline GW-014 & $03 / 14 / 87$ & $08 / 07 / 07$ & WL & 8 & $100 \%$ & Yes & VINYL CHLORIDE & NT & $\mathrm{D}$ & Quarterly \\
\hline GW-046 & 03/14/87 & 07/02/07 & WL & 20 & $95 \%$ & Yes & TETRACHLOROETHYLENE(PCE) & NT & I & Annual \\
\hline GW-052 & 06/22/90 & $02 / 12 / 07$ & WL & 3 & $100 \%$ & Yes & GROSS ALPHA ACTIVITY & $\mathrm{N} / \mathrm{A}$ & PD & Quarterly \\
\hline GW-053 & $06 / 22 / 90$ & $02 / 13 / 07$ & WL & 14 & $93 \%$ & No & GROSS ALPHA ACTIVITY & NT & NT & SemiAnnual \\
\hline GW-058 & $11 / 24 / 87$ & 06/12/06 & WL & 1 & $100 \%$ & Yes & GROSS ALPHA ACTIVITY & N/A & $\mathrm{s}$ & Quarterly \\
\hline GW-068 & $03 / 18 / 87$ & $06 / 08 / 06$ & WL & 3 & $100 \%$ & Yes & VINYL CHLORIDE & $\mathrm{N} / \mathrm{A}$ & $\mathrm{I}$ & Quarterly \\
\hline GW-071 & 03/17/87 & 08/09/07 & WL & 10 & $100 \%$ & Yes & BENZENE & I & I & Quarterly \\
\hline GW-072 & 03/17/87 & 03/21/07 & WL & 5 & $100 \%$ & No & 1,1-DICHLOROETHENE & NT & $\mathrm{I}$ & Quarterly \\
\hline GW-077 & 01/25/05 & 07/31/07 & WL & 6 & $17 \%$ & No & URANIUM & NT & NT & Biennial \\
\hline GW-078 & $01 / 25 / 05$ & 07/31/07 & WL & 6 & $17 \%$ & No & URANIUM & NT & NT & Biennial \\
\hline GW-079 & $01 / 25 / 05$ & $07 / 31 / 07$ & WL & 6 & $17 \%$ & No & URANIUM & NT & NT & Biennial \\
\hline GW-080 & 01/25/05 & 07/31/07 & WL & 6 & $17 \%$ & No & URANIUM & NT & NT & Biennial \\
\hline GW-082 & 03/23/87 & $02 / 07 / 07$ & WL & 17 & $100 \%$ & Yes & VINYL CHLORIDE & NT & I & Quarterly \\
\hline GW-089 & 06/19/90 & $02 / 12 / 07$ & WL & 1 & $100 \%$ & No & NITRATE & N/A & NT & Annual \\
\hline GW-242 & $11 / 12 / 87$ & $10 / 05 / 05$ & WL & 5 & $100 \%$ & Yes & VINYL CHLORIDE & NT & NT & Quarterly \\
\hline GW-257 & $06 / 23 / 89$ & $02 / 08 / 07$ & WL & 4 & $100 \%$ & Yes & TETRACHLOROETHYLENE(PCE) & NT & NT & SemiAnnual \\
\hline GW-289 & $04 / 11 / 88$ & 02/08/07 & WL & 8 & $100 \%$ & Yes & TETRACHLOROETHYLENE(PCE) & I & $\mathrm{s}$ & Quarterly \\
\hline GW-291 & 05/14/88 & $10 / 10 / 05$ & WL & 6 & $100 \%$ & Yes & TETRACHLOROETHYLENE(PCE) & $\mathrm{s}$ & $\mathrm{s}$ & Quarterly \\
\hline GW-624 & $06 / 28 / 90$ & $10 / 12 / 05$ & WL & 4 & $100 \%$ & Yes & VINYL CHLORIDE & $\mathrm{s}$ & $\mathrm{s}$ & Quarterly \\
\hline GW-626 & $02 / 14 / 90$ & $08 / 02 / 07$ & WL & 10 & $70 \%$ & Yes & VINYL CHLORIDE & $\mathrm{I}$ & $\mathrm{I}$ & Quarterly \\
\hline GW-627 & $02 / 13 / 90$ & 08/06/07 & WL & 25 & $100 \%$ & Yes & TETRACHLOROETHYLENE(PCE) & I & I & Quarterly \\
\hline GW-629 & 06/30/90 & 08/06/07 & WL & 7 & $57 \%$ & Yes & TETRACHLOROETHYLENE(PCE) & $\mathrm{PI}$ & $\mathrm{PI}$ & Quarterly \\
\hline GW-639 & $12 / 06 / 90$ & 08/14/07 & WL & 22 & $5 \%$ & No & TRICHLOROETHYLENE (TCE) & $\mathrm{s}$ & $\mathrm{s}$ & Biennial \\
\hline GW-653 & 03/23/91 & $02 / 07 / 07$ & WL & 22 & $91 \%$ & No & TETRACHLOROETHYLENE(PCE) & NT & I & Annual \\
\hline GW-683 & $05 / 24 / 91$ & $08 / 01 / 07$ & WL & 23 & $100 \%$ & No & GROSS ALPHA ACTIVITY & $\mathrm{D}$ & $\mathrm{D}$ & Annual \\
\hline GW-684 & 05/30/91 & 07/26/07 & WL & 24 & $100 \%$ & No & GROSS ALPHA ACTIVITY & $\mathrm{D}$ & PD & Annual \\
\hline GW-694 & 06/19/91 & 08/16/07 & WL & 10 & $90 \%$ & No & GROSS ALPHA ACTIVITY & NT & D & Annual \\
\hline GW-703 & 06/18/91 & 08/16/07 & WL & 22 & $95 \%$ & Yes & TRICHLOROETHYLENE (TCE) & D & D & Annual \\
\hline GW-704 & $06 / 20 / 91$ & $08 / 01 / 07$ & WL & 24 & $96 \%$ & Yes & TRICHLOROETHYLENE (TCE) & $\mathrm{D}$ & $\mathrm{D}$ & Annual \\
\hline GW-706 & 06/20/91 & 08/01/07 & WL & 24 & $96 \%$ & Yes & TRICHLOROETHYLENE (TCE) & NT & I & Annual \\
\hline GW-712 & $12 / 09 / 91$ & 07/02/07 & WL & 22 & $77 \%$ & No & GROSS BETA ACTIVITY & NT & NT & Biennial \\
\hline GW-713 & 06/13/92 & 07/03/07 & WL & 24 & $4 \%$ & No & TRICHLOROETHYLENE (TCE) & NT & NT & Annual \\
\hline GW-714 & 09/03/92 & 07/02/07 & WL & 23 & $70 \%$ & No & GROSS ALPHA ACTIVITY & $\mathrm{s}$ & PD & Biennial \\
\hline SS-4 & $08 / 30 / 90$ & $08 / 01 / 07$ & SP & 23 & $100 \%$ & Yes & NITRATE & $\mathrm{s}$ & $\mathrm{D}$ & Annual \\
\hline SS-5 & 08/30/90 & 08/01/07 & SP & 25 & $100 \%$ & Yes & NITRATE & NT & $\mathrm{D}$ & Annual \\
\hline SS-6_6 & 03/08/05 & 08/17/06 & $\mathrm{SP}$ & 4 & $0 \%$ & No & URANIUM & ND & ND & Biennial \\
\hline SS-6E & $11 / 01 / 90$ & 07/02/07 & SP & 14 & $86 \%$ & No & GROSS ALPHA ACTIVITY & NT & 1 & Biennial \\
\hline SS-7 & 09/21/05 & 08/17/06 & SP & 3 & $67 \%$ & No & URANIUM & N/A & N/A & Annual \\
\hline SS-8 & 08/30/90 & $08 / 17 / 06$ & SP & 18 & $50 \%$ & No & GROSS BETA ACTIVITY & NT & NT & Biennial \\
\hline \multicolumn{4}{|c|}{ Locations with no data collected 2005-2007 } & $1986-2007$ & & & & $1996-2005$ & $1986-2007$ & \\
\hline GW-259 & $06 / 22 / 89$ & $03 / 05 / 90$ & $\overline{\mathrm{WL}}$ & 4 & $100 \%$ & Yes & TETRACHLOROETHYLENE(PCE) & $\mathrm{N} / \mathrm{A}$ & NT & Annual \\
\hline GW-623 & $06 / 27 / 90$ & 09/27/92 & WL & 8 & $100 \%$ & Yes & TETRACHLOROETHYLENE(PCE) & N/A & I & Annual \\
\hline
\end{tabular}

1. Well sample dates taken from Y-12 Analytical Database 2008 .

2. $\mathrm{WL}=$ Groundwater Monitoring Well; $\mathrm{SP}=$ Spring.

3. Trend = Insufficient Data (N/A), Decreasing (D), Probably Decreasing (PD), Stable (S), Probably Increasing (PI), and Increasing (I), No Trend (NT); and non-detect for all sample events (ND),

4. Number of samples between 1996-2007 is the count of analytical samples acquired at the location based on the Analytical Database (2008).

5. Priority Constituent is the $\mathrm{COC}$ with the highest average concentration from the full dataset normalized by the screening level. (e.g. MAX [Ave Conc./Screening Level]).

Priority constituent indicated for wells where average concentrations do not exceed screening levels may be background level.

6. The Preliminary Frequency is the sampling frequency recommended by MAROS for results $1996-2007$. Low number of samples triggers default quarterly sampling. 
TABLE B.4

Supplemental MAROS assessment results for the East S-3 Area MAROS analysis group

\begin{tabular}{|c|c|c|c|c|c|c|c|c|c|c|}
\hline $\begin{array}{l}\text { Location } \\
\text { Name }\end{array}$ & $\begin{array}{c}\text { Earliest } \\
\text { Sample Date }\end{array}$ & $\begin{array}{l}\text { Most Recent } \\
\text { Sample Date }\end{array}$ & $\begin{array}{l}\text { Location } \\
\text { Type }\end{array}$ & $\begin{array}{c}\text { Number of } \\
\text { Samples } \\
\text { 1996-2007 }\end{array}$ & $\begin{array}{c}\text { Percent } \\
\text { Detection } \\
1996-2007\end{array}$ & \begin{tabular}{|c|} 
Average \\
$\begin{array}{c}\text { Concentration } \\
\text { Exceeds Screening } \\
\text { Level }\end{array}$ \\
\end{tabular} & Historic Priority Constituent & $\begin{array}{c}\text { Trend for } \\
\text { Priority } \\
\text { Constituent } \\
1996-2007 \\
\end{array}$ & $\begin{array}{c}\text { Trend for } \\
\text { Priority } \\
\text { Constituent } \\
1986-2007 \\
\end{array}$ & $\begin{array}{l}\text { Preliminary } \\
\text { Frequency }\end{array}$ \\
\hline $55-1 \mathrm{~A}$ & $06 / 05 / 96$ & $06 / 11 / 07$ & WL & 5 & $100 \%$ & No & NITRATE & $\mathrm{s}$ & $\mathrm{s}$ & Annual \\
\hline $55-2 A$ & $02 / 23 / 06$ & 08/23/07 & WL & 4 & $100 \%$ & Yes & TETRACHLOROETHYLENE(PCE) & D & D & Annual \\
\hline $55-2 B$ & 06/10/96 & 08/23/07 & WL & 9 & $100 \%$ & Yes & TETRACHLOROETHYLENE(PCE) & NT & NT & SemiAnnual \\
\hline $55-2 \mathrm{C}$ & 06/09/96 & $02 / 20 / 07$ & WL & 10 & $100 \%$ & Yes & TETRACHLOROETHYLENE(PCE) & $\mathrm{s}$ & $\mathrm{s}$ & Quarterly \\
\hline GW-105 & $01 / 24 / 86$ & $06 / 14 / 07$ & WL & 4 & $100 \%$ & Yes & NITRATE & NT & $\mathrm{D}$ & Quarterly \\
\hline GW-106 & $01 / 24 / 86$ & $06 / 18 / 07$ & $\mathrm{WL}$ & 4 & $100 \%$ & Yes & NITRATE & $\mathrm{s}$ & $\mathrm{D}$ & Quarterly \\
\hline GW-108 & $01 / 29 / 86$ & 07/10/07 & WL & 11 & $100 \%$ & Yes & NITRATE & D & D & SemiAnnual \\
\hline GW-109 & $01 / 29 / 86$ & 06/20/07 & WL & 6 & $100 \%$ & Yes & NITRATE & NT & NT & Quarterly \\
\hline GW-192 & $03 / 17 / 86$ & 06/11/07 & WL & 15 & $87 \%$ & No & TRICHLOROETHYLENE (TCE) & NT & 1 & SemiAnnual \\
\hline GW-251 & $06 / 10 / 86$ & 06/25/07 & WL & 23 & $100 \%$ & Yes & TETRACHLOROETHYLENE(PCE) & $\mathrm{s}$ & D & Annual \\
\hline GW-253 & $06 / 10 / 86$ & $03 / 08 / 07$ & WL & 17 & $100 \%$ & Yes & CADMIUM & NT & $\mathrm{s}$ & Quarterly \\
\hline GW-265 & $10 / 28 / 86$ & 10/17/07 & WL & 4 & $100 \%$ & Yes & TETRACHLOROETHYLENE(PCE) & $\mathrm{s}$ & D & Annual \\
\hline GW-269 & $10 / 28 / 86$ & 10/17/07 & WL & 6 & $100 \%$ & Yes & 1,1-DICHLOROETHENE & D & $\mathrm{I}$ & Annual \\
\hline GW-270 & $10 / 30 / 86$ & 06/06/07 & WL & 3 & $100 \%$ & Yes & NITRATE & N/A & D & Quarterly \\
\hline GW-272 & $10 / 30 / 86$ & 03/14/07 & WL & 3 & $100 \%$ & Yes & NITRATE & N/A & 1 & Quarterly \\
\hline GW-273 & $10 / 31 / 86$ & $06 / 06 / 07$ & $\overline{W L}$ & 1 & $0 \%$ & No & NITRATE & ND & $\mathrm{D}$ & Annual \\
\hline GW-274 & $11 / 04 / 86$ & 03/13/07 & WL & 6 & $100 \%$ & Yes & TETRACHLOROETHYLENE(PCE) & 1 & 1 & Quarterly \\
\hline GW-275 & $11 / 04 / 86$ & 03/13/07 & WL & 6 & $100 \%$ & Yes & NITRATE & $\mathrm{PI}$ & $\mathrm{s}$ & Quarterly \\
\hline GW-332 & $05 / 24 / 89$ & 03/06/07 & WL & 5 & $100 \%$ & Yes & TETRACHLOROETHYLENE(PCE) & NT & NT & Quarterly \\
\hline GW-336 & $05 / 25 / 89$ & 03/06/07 & WL & 4 & $100 \%$ & Yes & TETRACHLOROETHYLENE(PCE) & $\mathrm{s}$ & NT & Quarterly \\
\hline GW-337 & $05 / 25 / 89$ & $03 / 06 / 07$ & WL & 8 & $100 \%$ & Yes & TETRACHLOROETHYLENE(PCE) & $\mathrm{s}$ & $\mathrm{D}$ & Quarterly \\
\hline GW-505 & $12 / 29 / 88$ & 06/18/07 & WL & 4 & $100 \%$ & Yes & GROSS ALPHA ACTIVITY & $\mathrm{s}$ & NT & SemiAnnual \\
\hline GW-617 & $05 / 14 / 90$ & 05/16/06 & WL & 6 & $100 \%$ & No & NITRATE & $\mathrm{s}$ & NT & Annual \\
\hline GW-618 & 05/14/90 & 03/08/07 & WL & 20 & $100 \%$ & Yes & TETRACHLOROETHYLENE(PCE) & D & $\mathrm{D}$ & Quarterly \\
\hline GW-619 & 05/10/90 & 05/15/06 & WL & 6 & $100 \%$ & Yes & TETRACHLOROETHYLENE(PCE) & PD & $\mathrm{D}$ & Quarterly \\
\hline GW-620 & $05 / 10 / 90$ & $06 / 19 / 07$ & WL & 24 & $96 \%$ & Yes & TETRACHLOROETHYLENE(PCE) & $\mathrm{D}$ & $\mathrm{D}$ & Annual \\
\hline GW-633 & $03 / 11 / 91$ & 05/10/06 & WL & 11 & $100 \%$ & Yes & BENZENE & 1 & I & Quarterly \\
\hline \multicolumn{4}{|c|}{ Locations with no data collected 2005-2007 } & $1986-2007$ & & & & $1996-2005$ & $1986-2007$ & \\
\hline GW-190 & $06 / 20 / 90$ & $10 / 21 / 03$ & $\mathrm{WL}$ & 18 & $89 \%$ & Yes & TETRACHLOROETHYLENE(PCE) & ND & NT & Biennial \\
\hline GW-508 & $12 / 11 / 90$ & $03 / 02 / 94$ & WL & 11 & $100 \%$ & Yes & BENZENE & N/A & $\mathrm{D}$ & Not Evaluated \\
\hline
\end{tabular}

Notes:

1. Well sample dates taken from Y-12 Analytical Database 2008.

2. $\mathrm{WL}=$ Groundwater Monitoring Well; $\mathrm{SP}=$ Spring

3. Trend = Insufficient Data (N/A), Decreasing (D), Probably Decreasing (PD), Stable (S), Probably Increasing (PI),

and Increasing (I), No Trend (NT); and non-detect for all sample events (ND).

4. Number of samples between 1996-2007 is the count of analytical samples acquired at the location based on the Analytical Database (2008).

5. Priority Constituent is the $\mathrm{COC}$ with the highest average concentration from the full dataset normalized by the screening level. (e.g. MAX [Ave Conc./Screening Level]). Priority constituent indicated for wells where average concentrations do not exceed screening levels may be background level.

6. The Preliminary Frequency is the sampling frequency recommended by MAROS for results $1996-2007$. Low number of samples triggers default quarterly sampling. 
TABLE B. 5

Supplemental MAROS assessment results for the Central Y-12 Area MAROS analysis group

\begin{tabular}{|c|c|c|c|c|c|c|c|c|c|c|}
\hline $\begin{array}{l}\text { Location } \\
\text { Name }\end{array}$ & $\begin{array}{c}\text { Earliest } \\
\text { Sample } \\
\text { Date }\end{array}$ & $\begin{array}{l}\text { Most Recent } \\
\text { Sample Date }\end{array}$ & $\begin{array}{l}\text { Location } \\
\text { Type }\end{array}$ & $\begin{array}{c}\text { Number of } \\
\text { Samples } \\
1996-2007\end{array}$ & $\begin{array}{c}\text { Percent } \\
\text { Detection } \\
1996-2007\end{array}$ & $\begin{array}{c}\text { Average } \\
\text { Concentration } \\
\text { Exceeds } \\
\text { Screening } \\
\text { Level } \\
\end{array}$ & Historic Priority Constituent & $\begin{array}{l}\text { Trend for } \\
\text { Priority } \\
\text { Constituent } \\
\text { 1996-2007 }\end{array}$ & $\begin{array}{l}\text { Trend for } \\
\text { Priority } \\
\text { Constituent } \\
1986-2007\end{array}$ & $\begin{array}{l}\text { Preliminary } \\
\text { Frequency }\end{array}$ \\
\hline $55-3 \mathrm{~A}$ & $11 / 17 / 05$ & $08 / 22 / 07$ & WL & 5 & $100 \%$ & Yes & TETRACHLOROETHYLENE(PCE) & NT & NT & Quarterly \\
\hline $55-3 B$ & $11 / 17 / 05$ & 08/22/07 & WL & 5 & $100 \%$ & Yes & TETRACHLOROETHYLENE(PCE) & NT & NT & Quarterly \\
\hline $55-3 C$ & 03/01/06 & 08/27/07 & WL & 4 & $100 \%$ & Yes & TETRACHLOROETHYLENE(PCE) & NT & NT & Quarterly \\
\hline $56-1 A$ & $05 / 22 / 06$ & $10 / 15 / 07$ & WL & 4 & $25 \%$ & No & GROSS BETA ACTIVITY & NT & -- & Biennial \\
\hline $56-2 A$ & $03 / 23 / 98$ & 03/01/07 & WL & 5 & $100 \%$ & Yes & TETRACHLOROETHYLENE(PCE) & $\mathrm{s}$ & $\mathrm{s}$ & Annual \\
\hline $56-2 B$ & $03 / 23 / 98$ & $03 / 05 / 07$ & WL & 5 & $100 \%$ & Yes & TETRACHLOROETHYLENE(PCE) & $\mathrm{s}$ & $\mathrm{s}$ & Annual \\
\hline $56-2 C$ & $03 / 24 / 98$ & 03/05/07 & WL & 7 & $100 \%$ & Yes & TETRACHLOROETHYLENE(PCE) & PD & PD & Quarterly \\
\hline $56-3 A$ & 03/06/06 & 08/30/07 & WL & 4 & $100 \%$ & Yes & TETRACHLOROETHYLENE(PCE) & $\mathrm{s}$ & $\mathrm{S}$ & Annual \\
\hline $56-3 B$ & $03 / 06 / 06$ & 09/04/07 & WL & 4 & $100 \%$ & Yes & TETRACHLOROETHYLENE(PCE) & NT & NT & Quarterly \\
\hline $56-3 C$ & 03/06/06 & 09/05/07 & WL & 4 & $100 \%$ & Yes & TETRACHLOROETHYLENE(PCE) & $\mathrm{S}$ & $\mathrm{s}$ & Annual \\
\hline $56-4 \mathrm{~A}$ & $03 / 02 / 06$ & $09 / 05 / 07$ & WL & 4 & $75 \%$ & No & TETRACHLOROETHYLENE(PCE) & NT & NT & Annual \\
\hline $56-6 \mathrm{~A}$ & $03 / 02 / 06$ & 09/06/07 & WL & 4 & $50 \%$ & No & GROSS BETA ACTIVITY & NT & -- & Biennial \\
\hline $56-8 \mathrm{~A}$ & $05 / 23 / 06$ & $10 / 12 / 06$ & WL & 2 & $50 \%$ & No & GROSS ALPHA ACTIVITY & N/A & N/A & Annual \\
\hline $60-1 \mathrm{~A}$ & 06/15/06 & $11 / 19 / 07$ & WL & 4 & $25 \%$ & No & GROSS BETA ACTIVITY & NT & -- & Biennial \\
\hline GW-204 & 06/19/90 & $11 / 13 / 07$ & WL & 17 & $100 \%$ & Yes & GROSS ALPHA ACTIVITY & $\mathrm{S}$ & 1 & SemiAnnual \\
\hline GW-219 & $09 / 04 / 98$ & $11 / 28 / 07$ & $\overline{W L}$ & 15 & $100 \%$ & Yes & URANIUM & $\mathrm{S}$ & $\mathrm{S}$ & Quarterly \\
\hline GW-605 & 08/26/91 & 07/09/07 & WL & 26 & $96 \%$ & Yes & TRICHLOROETHYLENE (TCE) & I & I & Quarterly \\
\hline GW-606 & $08 / 26 / 91$ & $07 / 09 / 07$ & WL & 26 & $100 \%$ & Yes & CARBON TETRACHLORIDE & D & D & Annual \\
\hline GW-656 & $03 / 07 / 91$ & $11 / 13 / 07$ & WL & 6 & $100 \%$ & Yes & TRICHLOROETHYLENE (TCE) & D & D & Quarterly \\
\hline GW-686 & 06/06/06 & 10/18/07 & WL & 4 & $50 \%$ & Yes & VINYL CHLORIDE & NT & & Annual \\
\hline GW-690 & $06 / 08 / 96$ & $10 / 22 / 07$ & $\overline{\mathrm{WL}}$ & 7 & $100 \%$ & Yes & $\begin{array}{l}\text { TETRACHLOROETHYLENE(PCE) } \\
\end{array}$ & PD & PD & Quarterly \\
\hline GW-691 & 06/08/96 & $10 / 22 / 07$ & WL & 5 & $100 \%$ & Yes & TETRACHLOROETHYLENE(PCE) & $\mathrm{PI}$ & $\mathrm{PI}$ & Quarterly \\
\hline GW-692 & 06/08/96 & $10 / 16 / 07$ & WL & 5 & $80 \%$ & Yes & TETRACHLOROETHYLENE(PCE) & NT & NT & Annual \\
\hline GW-698 & 06/09/96 & 10/16/07 & WL & 17 & $100 \%$ & Yes & TRICHLOROETHYLENE (TCE) & I & 1 & Quarterly \\
\hline GW-700 & $06 / 08 / 96$ & $10 / 25 / 07$ & WL & 7 & $100 \%$ & Yes & TETRACHLOROETHYLENE(PCE) & $\mathrm{D}$ & $\mathrm{D}$ & Quarterly \\
\hline GW-765 & 07/28/92 & $12 / 03 / 07$ & WL & 3 & $67 \%$ & No & GROSS ALPHA ACTIVITY & N/A & D & Annual \\
\hline GW-769 & $01 / 27 / 93$ & $11 / 13 / 07$ & WL & 25 & $96 \%$ & Yes & CARBON TETRACHLORIDE & I & 1 & Quarterly \\
\hline GW-770 & $01 / 27 / 93$ & $11 / 12 / 07$ & WL & 25 & $80 \%$ & Yes & CARBON TETRACHLORIDE & I & 1 & Annual \\
\hline GW-779 & 06/09/94 & 09/10/07 & WL & 3 & $67 \%$ & No & GROSS ALPHA ACTIVITY & $\mathrm{N} / \mathrm{A}$ & NT & Annual \\
\hline GW-781 & $06 / 15 / 94$ & $11 / 27 / 07$ & WL & 11 & $73 \%$ & Yes & TETRACHLOROETHYLENE(PCE) & NT & NT & Annual \\
\hline GW-782 & $06 / 16 / 94$ & $11 / 27 / 07$ & WL & 24 & $100 \%$ & Yes & TETRACHLOROETHYLENE(PCE) & $\mathrm{D}$ & $\mathrm{D}$ & Annual \\
\hline GW-783 & $06 / 16 / 94$ & $11 / 28 / 07$ & WL & 11 & $100 \%$ & Yes & TETRACHLOROETHYLENE(PCE) & S & D & Quarterly \\
\hline GW-791 & $06 / 07 / 94$ & $11 / 12 / 07$ & WL & 23 & $100 \%$ & Yes & TETRACHLOROETHYLENE(PCE) & $\mathrm{s}$ & D & Annual \\
\hline GW-792 & 06/07/94 & $11 / 12 / 07$ & WL & 11 & $100 \%$ & Yes & TETRACHLOROETHYLENE(PCE) & D & D & SemiAnnual \\
\hline GW-820 & $03 / 26 / 98$ & $11 / 20 / 07$ & WL & 10 & $100 \%$ & Yes & TETRACHLOROETHYLENE(PCE) & $\mathrm{S}$ & $\mathrm{S}$ & Annual \\
\hline GW-954-1 & $06 / 13 / 06$ & $10 / 30 / 07$ & WL & 3 & $67 \%$ & No & GROSS ALPHA ACTIVITY & N/A & N/A & -- \\
\hline GW-954-2 & 06/13/06 & $10 / 30 / 07$ & WL & 4 & $50 \%$ & No & GROSS ALPHA ACTIVITY & NT & NT & -- \\
\hline GW-954-3 & 06/13/06 & 10/30/07 & WL & 4 & $100 \%$ & No & 1,1-DICHLOROETHENE & $\mathrm{s}$ & $\mathrm{s}$ & -- \\
\hline GW-956-1 & 06/14/06 & 10/29/07 & WL & 4 & $25 \%$ & No & TETRACHLOROETHYLENE(PCE) & NT & NT & -- \\
\hline GW-956-2 & $06 / 14 / 06$ & 10/29/07 & WL & 4 & $50 \%$ & No & GROSS ALPHA ACTIVITY & NT & NT & -- \\
\hline
\end{tabular}


TABLE B.5

Supplemental MAROS assessment results for the Central Y-12 Area MAROS analysis group

\begin{tabular}{|c|c|c|c|c|c|c|c|c|c|c|}
\hline $\begin{array}{l}\text { Location } \\
\text { Name }\end{array}$ & $\begin{array}{c}\text { Earliest } \\
\text { Sample } \\
\text { Date }\end{array}$ & $\begin{array}{l}\text { Most Recent } \\
\text { Sample Date }\end{array}$ & $\begin{array}{l}\text { Location } \\
\text { Type }\end{array}$ & $\begin{array}{c}\text { Number of } \\
\text { Samples } \\
1996-2007\end{array}$ & $\begin{array}{c}\text { Percent } \\
\text { Detection } \\
1996-2007\end{array}$ & $\begin{array}{c}\text { Average } \\
\text { Concentration } \\
\text { Exceeds } \\
\text { Screening } \\
\text { Level } \\
\end{array}$ & Historic Priority Constituent & $\begin{array}{l}\text { Trend for } \\
\text { Priority } \\
\text { Constituent } \\
\text { 1996-2007 }\end{array}$ & $\begin{array}{l}\text { Trend for } \\
\text { Priority } \\
\text { Constituent } \\
1986 \text { - } 2007\end{array}$ & $\begin{array}{l}\text { Preliminary } \\
\text { Frequency }\end{array}$ \\
\hline GW-956-3 & $06 / 14 / 06$ & $10 / 29 / 07$ & $\mathrm{WL}$ & 4 & $50 \%$ & No & GROSS ALPHA ACTIVITY & NT & NT & -- \\
\hline GW-956-4 & $06 / 14 / 06$ & $10 / 29 / 07$ & WL & 3 & $33 \%$ & No & GROSS ALPHA ACTIVITY & $\mathrm{N} / \mathrm{A}$ & N/A & -- \\
\hline GW-959 & $03 / 30 / 05$ & $11 / 20 / 07$ & WL & 7 & $71 \%$ & Yes & VINYL CHLORIDE & NT & NT & Annual \\
\hline GW-960 & 02/28/07 & 09/10/07 & WL & 2 & $50 \%$ & No & GROSS BETA ACTIVITY & N/A & N/A & Annual \\
\hline UEFPC-SP17 & 03/25/96 & $04 / 24 / 06$ & $\mathrm{SP}$ & 4 & $100 \%$ & Yes & NITRATE & NT & NT & SemiAnnual \\
\hline \multicolumn{11}{|c|}{ Fuel Station Area } \\
\hline GW-281 & $05 / 04 / 89$ & $05 / 01 / 07$ & $\overline{\mathrm{WL}}$ & 5 & $0 \%$ & No & TETRACHLOROETHYLENE(PCE) & ND & NT & Biennial \\
\hline GW-658 & $04 / 21 / 05$ & 05/01/07 & WL & 9 & $89 \%$ & Yes & BENZENE & NT & NT & Quarterly \\
\hline GW-762 & 02/07/05 & 08/06/07 & WL & 18 & $100 \%$ & Yes & TETRACHLOROETHYLENE(PCE) & I & I & Quarterly \\
\hline GW-763 & 06/28/05 & $12 / 03 / 07$ & WL & 24 & $63 \%$ & Yes & TETRACHLOROETHYLENE(PCE) & NT & NT & Annual \\
\hline GW-775 & $11 / 29 / 07$ & $11 / 29 / 07$ & WL & 12 & $100 \%$ & No & TRICHLOROETHYLENE (TCE) & $\mathrm{s}$ & NT & SemiAnnual \\
\hline GW-776 & $11 / 29 / 07$ & $11 / 29 / 07$ & WL & 12 & $92 \%$ & No & TRICHLOROETHYLENE (TCE) & PD & D & Annual \\
\hline GW-802 & $04 / 21 / 05$ & $05 / 01 / 07$ & WL & 7 & $0 \%$ & No & BENZENE & ND & ND & Biennial \\
\hline \multicolumn{8}{|c|}{ Locations with no data collected 2005-2007 } & $1996-2005$ & $1986-2007$ & \\
\hline GW-193 & $06 / 18 / 90$ & $07 / 13 / 04$ & $\overline{W L}$ & 31 & $84 \%$ & Yes & BENZENE & $\mathrm{D}$ & $\mathrm{D}$ & Biennial \\
\hline
\end{tabular}

Notes:

1. Well sample dates taken from Y-12 Analytical Database 2008.

2. $\mathrm{WL}=$ Groundwater Monitoring Well; $\mathrm{SP}=$ Spring.

3. Trend = Insufficient Data (N/A), Decreasing (D), Probably Decreasing (PD), Stable (S), Probably Increasing (PI), and Increasing (I), No Trend (NT); and non-detect for all sample events (ND).

4. Number of samples between 1996-2007 is the count of analytical samples acquired at the location based on the Analytical Database (2008).

5. Priority Constituent is the $\mathrm{COC}$ with the highest average concentration from the full dataset normalized by the screening level. (e.g. MAX [Ave Conc./Screening Level]). Priority constituent indicated for wells where average concentrations do not exceed screening levels may be background level.

6. The Preliminary Frequency is the sampling frequency recommended by MAROS for results $1996-2007$. Low number of samples triggers default quarterly sampling. If insufficient recent data (2005-2007) were available for evaluation, then no Preliminary Frequency was assigned (--). 
TABLE B.6

Supplemental MAROS assessment results for the East Y-12 Area MAROS analysis group

\begin{tabular}{|c|c|c|c|c|c|c|c|c|c|c|}
\hline $\begin{array}{l}\text { Location } \\
\text { Name }\end{array}$ & $\begin{array}{l}\text { Earliest } \\
\text { Sample } \\
\text { Date }\end{array}$ & $\begin{array}{l}\text { Most Recent } \\
\text { Sample Date }\end{array}$ & $\begin{array}{l}\text { Location } \\
\text { Type }\end{array}$ & $\begin{array}{c}\text { Number of } \\
\text { Samples } \\
1996-2007\end{array}$ & $\begin{array}{c}\text { Percent } \\
\text { Detection } \\
1996-2007\end{array}$ & $\begin{array}{c}\text { Average } \\
\text { Concentration } \\
\text { Exceeds } \\
\text { Screening Level }\end{array}$ & Historic Priority Constituent & $\begin{array}{l}\text { Trend for } \\
\text { Priority } \\
\text { Constituent } \\
\text { 1996-2007 }\end{array}$ & $\begin{array}{l}\text { Trend for } \\
\text { Priority } \\
\text { Constituent } \\
1986 \text { - } 2007\end{array}$ & $\begin{array}{l}\text { Preliminary } \\
\text { Frequency }\end{array}$ \\
\hline GW-148 & $01 / 19 / 06$ & $01 / 19 / 06$ & $\mathrm{WL}$ & 1 & $100 \%$ & No & CIS-1,2-DICHLOROETHENE & $\mathrm{N} / \mathrm{A}$ & $\mathrm{N} / \mathrm{A}$ & -- \\
\hline GW-151 & $02 / 20 / 86$ & $08 / 01 / 07$ & WL & 25 & $96 \%$ & Yes & CARBON TETRACHLORIDE & I & 1 & Quarterly \\
\hline GW-153 & $02 / 24 / 86$ & $03 / 08 / 07$ & WL & 24 & $96 \%$ & Yes & CARBON TETRACHLORIDE & D & D & Annual \\
\hline GW-154 & $02 / 23 / 86$ & $08 / 15 / 07$ & WL & 26 & $100 \%$ & Yes & GROSS ALPHA ACTIVITY & 1 & 1 & Quarterly \\
\hline GW-169 & $02 / 08 / 05$ & $08 / 02 / 07$ & WL & 8 & $13 \%$ & No & TRICHLOROETHYLENE (TCE) & $\mathrm{S}$ & $\mathrm{S}$ & Annual \\
\hline GW-170 & $06 / 04 / 90$ & $08 / 02 / 07$ & WL & 36 & $100 \%$ & Yes & CARBON TETRACHLORIDE & NT & $\mathrm{D}$ & Annual \\
\hline GW-171 & $09 / 27 / 94$ & 08/19/03 & WL & 16 & $100 \%$ & No & GROSS BETA ACTIVITY & NT & NT & -- \\
\hline GW-172 & $09 / 26 / 94$ & 08/19/03 & WL & 15 & $67 \%$ & No & GROSS BETA ACTIVITY & NT & NT & -- \\
\hline GW-220 & $02 / 20 / 86$ & $11 / 20 / 07$ & WL & 28 & $100 \%$ & Yes & CARBON TETRACHLORIDE & 1 & 1 & Quarterly \\
\hline GW-223 & $02 / 25 / 86$ & $08 / 06 / 07$ & WL & 21 & $100 \%$ & Yes & TETRACHLOROETHYLENE(PCE) & $\mathrm{D}$ & $\mathrm{D}$ & Annual \\
\hline GW-230 & $04 / 20 / 05$ & $03 / 07 / 07$ & WL & 4 & $25 \%$ & No & VINYL CHLORIDE & $\mathrm{s}$ & $\mathrm{S}$ & Biennial \\
\hline GW-240 & $02 / 25 / 86$ & 03/08/07 & WL & 10 & $100 \%$ & Yes & CARBON TETRACHLORIDE & $S$ & D & Annual \\
\hline GW-380 & $12 / 22 / 88$ & $08 / 15 / 07$ & WL & 21 & $81 \%$ & No & GROSS ALPHA ACTIVITY & $\mathrm{S}$ & $\mathrm{D}$ & Annual \\
\hline GW-381 & $12 / 17 / 88$ & $12 / 04 / 07$ & WL & 14 & $100 \%$ & Yes & CARBON TETRACHLORIDE & D & D & Annual \\
\hline GW-382 & $12 / 10 / 88$ & 08/02/07 & WL & 17 & $100 \%$ & Yes & CARBON TETRACHLORIDE & $\mathrm{D}$ & $\mathrm{D}$ & Annual \\
\hline GW-383 & $08 / 23 / 88$ & 11/19/07 & WL & 27 & $100 \%$ & Yes & TETRACHLOROETHYLENE(PCE) & $\mathrm{S}$ & $\mathrm{I}$ & Annual \\
\hline GW-733 & $04 / 28 / 92$ & 07/09/07 & WL & 27 & $100 \%$ & Yes & CARBON TETRACHLORIDE & $\mathrm{D}$ & $\mathrm{D}$ & Annual \\
\hline GW-735 & $04 / 28 / 92$ & $03 / 08 / 07$ & WL & 18 & $83 \%$ & No & GROSS ALPHA ACTIVITY & NT & NT & Annual \\
\hline GW-744 & 10/25/92 & $03 / 15 / 07$ & WL & 19 & $84 \%$ & No & GROSS ALPHA ACTIVITY & PD & $\mathrm{s}$ & Annual \\
\hline GW-747 & $04 / 13 / 93$ & $03 / 15 / 07$ & WL & 17 & $82 \%$ & No & GROSS ALPHA ACTIVITY & $\mathrm{S}$ & NT & Biennial \\
\hline GW-748 & $08 / 08 / 92$ & $01 / 10 / 06$ & WL & 4 & $75 \%$ & No & GROSS ALPHA ACTIVITY & $\mathrm{S}$ & $\mathrm{D}$ & Biennial \\
\hline GW-750 & 08/09/92 & 03/07/07 & WL & 21 & $90 \%$ & No & GROSS BETA ACTIVITY & $\mathrm{S}$ & $\mathrm{s}$ & Biennial \\
\hline GW-816 & $09 / 21 / 94$ & $03 / 07 / 07$ & WL & 24 & $83 \%$ & No & GROSS BETA ACTIVITY & D & D & Annual \\
\hline GW-832 & $05 / 14 / 96$ & $08 / 15 / 07$ & WL & 23 & $100 \%$ & Yes & CARBON TETRACHLORIDE & $\mathrm{D}$ & $\mathrm{D}$ & Annual \\
\hline SCR7_1SP & $12 / 13 / 95$ & $01 / 18 / 07$ & SP & 23 & $39 \%$ & No & CARBON TETRACHLORIDE & $\mathrm{D}$ & $\mathrm{D}$ & Annual \\
\hline SCR7_8SP & $04 / 09 / 97$ & $01 / 18 / 07$ & SP & 21 & $19 \%$ & No & TETRACHLOROETHYLENE(PCE) & $\mathrm{D}$ & $\mathrm{D}$ & Biennial \\
\hline \multicolumn{8}{|c|}{ Locations with no data collected 2005-2007 } & $1996-2005$ & $1986-2007$ & \\
\hline GW-222 & $02 / 23 / 86$ & $11 / 30 / 04$ & WL & 36 & $94 \%$ & Yes & TETRACHLOROETHYLENE(PCE) & $\mathrm{S}$ & NT & Annual \\
\hline
\end{tabular}

Notes:

1. Well sample dates taken from Y-12 Analytical Database 2008.

2. $\mathrm{WL}=$ Groundwater Monitoring Well; $\mathrm{SP}=$ Spring.

3. Trend = Insufficient Data (N/A), Decreasing (D), Probably Decreasing (PD), Stable (S), Probably Increasing (PI), and Increasing (I), No Trend (NT); and non-detect for all sample events (ND).

4. Number of samples between 1996-2007 is the count of analytical samples acquired at the location based on the Analytical Database (2008).

5. Priority Constituent is the $\mathrm{COC}$ with the highest average concentration from the full dataset normalized by the screening level. (e.g. MAX [Ave Conc./Screening Level]). Priority constituent indicated for wells where average concentrations do not exceed screening levels may be background level.

6. The Preliminary Frequency is the sampling frequency recommended by MAROS for results $1996-2007$. Low number of samples triggers default quarterly sampling. If insufficient recent data (2005-2007) were available for evaluation, then no Preliminary Frequency was assigned (--). 
TABLE B.7

Supplemental MAROS assessment results for the West Chestnut Ridge MAROS analysis group

\begin{tabular}{|c|c|c|c|c|c|c|c|c|c|c|}
\hline $\begin{array}{l}\text { Location } \\
\text { Name }\end{array}$ & $\begin{array}{c}\text { Earliest } \\
\text { Sample } \\
\text { Date }\end{array}$ & $\begin{array}{l}\text { Most Recent } \\
\text { Sample Date }\end{array}$ & $\begin{array}{l}\text { Location } \\
\text { Type }\end{array}$ & $\begin{array}{c}\text { Number of } \\
\text { Samples } \\
1996-2007\end{array}$ & $\begin{array}{c}\text { Percent } \\
\text { Detection } \\
1996-2007\end{array}$ & $\begin{array}{c}\text { Average } \\
\text { Concentration } \\
\text { Exceeds } \\
\text { Screening Level }\end{array}$ & Historic Priority Constituent & $\begin{array}{l}\text { Trend for } \\
\text { Priority } \\
\text { Constituent } \\
\text { 1996-2007 }\end{array}$ & $\begin{array}{l}\text { Trend for } \\
\text { Priority } \\
\text { Constituent } \\
1986-2007\end{array}$ & $\begin{array}{l}\text { Preliminary } \\
\text { Frequency }\end{array}$ \\
\hline 1090 & $02 / 13 / 86$ & $07 / 25 / 07$ & $\overline{\mathrm{WL}}$ & 22 & $64 \%$ & No & GROSS ALPHA ACTIVITY & $\bar{S}$ & $\bar{D}$ & Biennial \\
\hline GW-141 & $02 / 18 / 88$ & $07 / 16 / 07$ & WL & 24 & $21 \%$ & No & CADMIUM & 1 & 1 & Biennial \\
\hline GW-203 & $02 / 06 / 86$ & $07 / 25 / 07$ & WL & 21 & $67 \%$ & No & GROSS ALPHA ACTIVITY & NT & 1 & Biennial \\
\hline GW-205 & $02 / 06 / 86$ & $07 / 26 / 07$ & WL & 22 & $95 \%$ & Yes & GROSS BETA ACTIVITY & 1 & 1 & Annual \\
\hline GW-217 & $02 / 17 / 88$ & $07 / 12 / 07$ & WL & 23 & $61 \%$ & No & GROSS BETA ACTIVITY & NT & $\mathrm{D}$ & Biennial \\
\hline GW-221 & $02 / 07 / 86$ & $07 / 25 / 07$ & $\mathrm{WL}$ & 22 & $41 \%$ & No & GROSS ALPHA ACTIVITY & NT & $\mathrm{D}$ & Annual \\
\hline GW-305 & $01 / 18 / 05$ & $10 / 31 / 07$ & WL & 12 & $100 \%$ & No & 1,1-DICHLOROETHENE & NT & NT & Annual \\
\hline GW-521 & 09/08/89 & $07 / 12 / 07$ & WL & 21 & $29 \%$ & No & GROSS BETA ACTIVITY & D & D & Biennial \\
\hline GW-522 & $01 / 18 / 05$ & $07 / 12 / 07$ & WL & 6 & $83 \%$ & No & NITRATE & $\mathrm{s}$ & $\mathrm{s}$ & Biennial \\
\hline GW-540 & 03/16/91 & 07/18/07 & WL & 20 & $50 \%$ & No & GROSS BETA ACTIVITY & NT & PD & Biennial \\
\hline GW-542 & $03 / 17 / 91$ & $07 / 18 / 07$ & WL & 22 & $77 \%$ & No & GROSS BETA ACTIVITY & $\mathrm{D}$ & $\mathrm{D}$ & Biennial \\
\hline GW-543 & 01/24/05 & $07 / 18 / 07$ & WL & 6 & $83 \%$ & No & NITRATE & NT & NT & Biennial \\
\hline GW-544 & $01 / 24 / 05$ & 07/18/07 & WL & 6 & $100 \%$ & No & NITRATE & NT & NT & Biennial \\
\hline GW-709 & 06/19/91 & 07/16/07 & WL & 21 & $48 \%$ & No & GROSS ALPHA ACTIVITY & NT & D & Biennial \\
\hline GW-757 & $10 / 12 / 92$ & $07 / 16 / 07$ & WL & 24 & $100 \%$ & No & GROSS BETA ACTIVITY & 1 & I & Biennial \\
\hline GW-827 & $11 / 05 / 96$ & $07 / 18 / 07$ & $\mathrm{WL}$ & 22 & $50 \%$ & No & GROSS BETA ACTIVITY & PD & PD & Biennial \\
\hline SCR1_25SP & $02 / 16 / 99$ & $07 / 17 / 07$ & SP & 18 & $78 \%$ & No & GROSS ALPHA ACTIVITY & NT & NT & Biennial \\
\hline SCR2 $1 \mathrm{SP}$ & $04 / 07 / 97$ & $05 / 02 / 07$ & SP & 17 & $82 \%$ & No & GROSS ALPHA ACTIVITY & $\mathrm{D}$ & $\mathrm{D}$ & Annual \\
\hline SCR2 2SP & 03/15/95 & $07 / 19 / 04$ & SP & 14 & $14 \%$ & No & LEAD & NT & NT & Annual \\
\hline
\end{tabular}

Notes:

1. Well sample dates taken from Y-12 Analytical Database 2008

2. $\mathrm{WL}=$ Groundwater Monitoring Well; $\mathrm{SP}=$ Spring.

3. Trend = Insufficient Data (N/A), Decreasing (D), Probably Decreasing (PD), Stable (S), Probably Increasing (PI),

and Increasing (I), No Trend (NT); and non-detect for all sample events (ND).

4. Number of samples between 1996-2007 is the count of analytical samples acquired at the location based on the Analytical Database (2008).

5. Priority Constituent is the $\mathrm{COC}$ with the highest average concentration from the full dataset normalized by the screening level. (e.g. MAX [Ave Conc./Screening Level]). Priority constituent indicated for wells where average concentrations do not exceed screening levels may be background level.

6. The Preliminary Frequency is the sampling frequency recommended by MAROS for results $1996-2007$. Low number of samples triggers default quarterly sampling. 
TABLE B.8

Supplemental MAROS assessment results for the Chestnut Ridge Security Pits MAROS analysis group

\begin{tabular}{|c|c|c|c|c|c|c|c|c|c|c|}
\hline $\begin{array}{l}\text { Location } \\
\text { Name }\end{array}$ & $\begin{array}{c}\text { Earliest } \\
\text { Sample } \\
\text { Date }\end{array}$ & $\begin{array}{l}\text { Most Recent } \\
\text { Sample Date }\end{array}$ & $\begin{array}{l}\text { Location } \\
\text { Type }\end{array}$ & $\begin{array}{c}\text { Number of } \\
\text { Samples } \\
\text { 1996-2007 }\end{array}$ & $\begin{array}{c}\text { Percent } \\
\text { Detection } \\
\text { 1996-2007 }\end{array}$ & $\begin{array}{c}\text { Average } \\
\text { Concentration } \\
\quad \text { Exceeds } \\
\text { Screening Level }\end{array}$ & Historic Priority Constituent & $\begin{array}{l}\text { Trend for } \\
\text { Priority } \\
\text { Constituent } \\
1996-2007\end{array}$ & $\begin{array}{l}\text { Trend for } \\
\text { Priority } \\
\text { Constituent } \\
1986 \text { - } 2007\end{array}$ & $\begin{array}{l}\text { Preliminary } \\
\text { Frequency }\end{array}$ \\
\hline GW-174 & $02 / 04 / 86$ & $05 / 15 / 07$ & $\overline{W L}$ & 3 & $100 \%$ & Yes & TETRACHLOROETHYLENE(PCE) & N/A & $\mathrm{D}$ & Quarterly \\
\hline GW-175 & 08/17/88 & 05/01/06 & WL & 6 & $100 \%$ & Yes & TETRACHLOROETHYLENE(PCE) & $\mathrm{s}$ & D & Annual \\
\hline GW-176 & $02 / 04 / 86$ & $05 / 17 / 07$ & WL & 2 & $100 \%$ & Yes & 1,1-DICHLOROETHENE & N/A & 1 & Quarterly \\
\hline GW-177 & $02 / 04 / 86$ & 07/11/07 & WL & 13 & $92 \%$ & No & 1,1-DICHLOROETHENE & $\mathrm{s}$ & 1 & Annual \\
\hline GW-178 & 03/12/88 & $05 / 02 / 06$ & WL & 3 & $100 \%$ & No & 1,1-DICHLOROETHENE & N/A & $\mathrm{PI}$ & Annual \\
\hline GW-179 & $02 / 10 / 86$ & $05 / 21 / 07$ & WL & 2 & $100 \%$ & Yes & 1,1-DICHLOROETHENE & N/A & $\mathrm{D}$ & Quarterly \\
\hline GW-180 & 03/11/88 & 05/16/07 & WL & 2 & $100 \%$ & Yes & TETRACHLOROETHYLENE(PCE) & N/A & D & Quarterly \\
\hline GW-322 & $03 / 12 / 88$ & 05/08/06 & WL & 4 & $100 \%$ & Yes & 1,1-DICHLOROETHENE & $\mathrm{S}$ & NT & Annual \\
\hline GW-514 & $02 / 11 / 89$ & 05/01/06 & WL & 3 & $67 \%$ & No & GROSS ALPHA ACTIVITY & N/A & $\mathrm{s}$ & Annual \\
\hline GW-609 & $02 / 14 / 90$ & $05 / 02 / 06$ & WL & 15 & $87 \%$ & Yes & TETRACHLOROETHYLENE(PCE) & $\mathrm{D}$ & $\mathrm{D}$ & Annual \\
\hline GW-612 & $02 / 10 / 90$ & $05 / 17 / 07$ & WL & 6 & $100 \%$ & Yes & 1,1-DICHLOROETHENE & $\mathrm{S}$ & $\mathrm{D}$ & Annual \\
\hline GW-831 & $01 / 13 / 05$ & 07/10/06 & WL & 4 & $50 \%$ & Yes & GROSS BETA ACTIVITY & NT & NT & Quarterly \\
\hline SCR3_5SP & $04 / 08 / 97$ & $07 / 17 / 07$ & SP & 19 & $58 \%$ & No & GROSS ALPHA ACTIVITY & $\mathrm{PI}$ & $\mathrm{PI}$ & Annual \\
\hline
\end{tabular}

Notes:

1. Well sample dates taken from Y-12 Analytical Database 2008.

2. $\mathrm{WL}=$ Groundwater Monitoring Well; $\mathrm{SP}=$ Spring.

3. Trend = Insufficient Data (N/A), Decreasing (D), Probably Decreasing (PD), Stable (S), Probably Increasing (PI), and Increasing (I), No Trend (NT); and non-detect for all sample events (ND).

4. Number of samples between 1996-2007 is the count of analytical samples acquired at the location based on the Analytical Database (2008).

5. Priority Constituent is the $\mathrm{COC}$ with the highest average concentration from the full dataset normalized by the screening level. (e.g. MAX [Ave Conc./Screening Level]). Priority constituent indicated for wells where average concentrations do not exceed screening levels may be background level.

6. The Preliminary Frequency is the sampling frequency recommended by MAROS for results $1996-2007$. Low number of samples triggers default quarterly sampling. 
TABLE B.9

Supplemental MAROS assessment results for the Landfills V and VII Area MAROS analysis group

\begin{tabular}{|c|c|c|c|c|c|c|c|c|c|c|}
\hline $\begin{array}{l}\text { Location } \\
\text { Name }\end{array}$ & $\begin{array}{c}\text { Earliest } \\
\text { Sample } \\
\text { Date }\end{array}$ & $\begin{array}{l}\text { Most Recent } \\
\text { Sample Date }\end{array}$ & $\begin{array}{l}\text { Location } \\
\text { Type }\end{array}$ & $\begin{array}{c}\text { Number of } \\
\text { Samples } \\
1996-2007\end{array}$ & $\begin{array}{c}\text { Percent } \\
\text { Detection } \\
\text { 1996-2007 }\end{array}$ & $\begin{array}{c}\text { Average } \\
\text { Concentration } \\
\quad \text { Exceeds } \\
\text { Screening Level }\end{array}$ & Historic Priority Constituent & $\begin{array}{l}\text { Trend for } \\
\text { Priority } \\
\text { Constituent } \\
\text { 1996-2007 }\end{array}$ & $\begin{array}{l}\text { Trend for } \\
\text { Priority } \\
\text { Constituent } \\
1986 \text { - } 2007\end{array}$ & $\begin{array}{l}\text { Preliminary } \\
\text { Frequency }\end{array}$ \\
\hline GW-557 & $01 / 20 / 05$ & $07 / 16 / 07$ & $\mathrm{WL}$ & 6 & $83 \%$ & No & NITRATE & PD & PD & Annual \\
\hline GW-560 & 01/19/05 & 07/17/07 & WL & 6 & $17 \%$ & No & GROSS ALPHA ACTIVITY & NT & NT & Annual \\
\hline GW-562 & 01/19/05 & 07/17/07 & WL & 6 & $17 \%$ & No & GROSS ALPHA ACTIVITY & NT & NT & Annual \\
\hline GW-564 & 01/19/05 & 07/17/07 & WL & 6 & $83 \%$ & No & NITRATE & $\mathrm{s}$ & $\mathrm{s}$ & Annual \\
\hline GW-796 & $01 / 18 / 05$ & 07/16/07 & WL & 6 & $33 \%$ & No & GROSS BETA ACTIVITY & $\mathrm{S}$ & $\mathrm{s}$ & Annual \\
\hline GW-797 & $01 / 20 / 05$ & $07 / 17 / 07$ & $\mathrm{WL}$ & 6 & $100 \%$ & No & NITRATE & $\mathrm{I}$ & $\mathrm{I}$ & Annual \\
\hline GW-798 & $06 / 23 / 93$ & 07/17/07 & WL & 27 & $70 \%$ & No & TETRACHLOROETHYLENE(PCE) & I & I & Annual \\
\hline GW-799 & $01 / 20 / 05$ & 07/17/07 & WL & 6 & $33 \%$ & No & GROSS ALPHA ACTIVITY & $\mathrm{s}$ & $\mathrm{s}$ & Annual \\
\hline GW-801 & 01/19/05 & 07/16/07 & WL & 6 & $83 \%$ & No & NITRATE & $\mathrm{s}$ & $\mathrm{s}$ & Annual \\
\hline SCR4 3SP & $02 / 14 / 94$ & 07/17/07 & SP & 24 & $0 \%$ & No & TETRACHLOROETHYLENE(PCE) & ND & ND & Biennial \\
\hline
\end{tabular}

Notes:

1. Well sample dates taken from Y-12 Analytical Database 2008.

2. $\mathrm{WL}=$ Groundwater Monitoring Well; $\mathrm{SP}=$ Spring.

3. Trend = Insufficient Data (N/A), Decreasing (D), Probably Decreasing (PD), Stable (S), Probably Increasing (PI), and Increasing (I), No Trend (NT); and non-detect for all sample events (ND).

4. Number of samples between 1996-2007 is the count of analytical samples acquired at the location based on the Analytical Database (2008).

5. Priority Constituent is the $\mathrm{COC}$ with the highest average concentration from the full dataset normalized by the screening level. (e.g. MAX [Ave Conc./Screening Level]). Priority constituent indicated for wells where average concentrations do not exceed screening levels may be background level.

6. The Preliminary Frequency is the sampling frequency recommended by MAROS for results $1996-2007$. Low number of samples triggers default quarterly sampling. 
TABLE B.10

Supplemental MAROS assessment results for the East Chestnut Ridge MAROS analysis group

\begin{tabular}{|c|c|c|c|c|c|c|c|c|c|c|}
\hline $\begin{array}{l}\text { Location } \\
\text { Name }\end{array}$ & $\begin{array}{c}\text { Earliest } \\
\text { Sample } \\
\text { Date }\end{array}$ & $\begin{array}{l}\text { Most Recent } \\
\text { Sample Date }\end{array}$ & $\begin{array}{l}\text { Location } \\
\text { Type }\end{array}$ & $\begin{array}{c}\text { Number of } \\
\text { Samples } \\
1996-2007\end{array}$ & $\begin{array}{c}\text { Percent } \\
\text { Detection } \\
\text { 1996-2007 }\end{array}$ & $\begin{array}{c}\text { Average } \\
\text { Concentration } \\
\text { Exceeds } \\
\text { Screening Level }\end{array}$ & Historic Priority Constituent & $\begin{array}{c}\text { Trend for } \\
\text { Priority } \\
\text { Constituent } \\
1996-2007\end{array}$ & $\begin{array}{l}\text { Trend for } \\
\text { Priority } \\
\text { Constituent } \\
1986-2007\end{array}$ & $\begin{array}{l}\text { Preliminary } \\
\text { Frequency }\end{array}$ \\
\hline GW-143 & $04 / 07 / 05$ & $01 / 08 / 07$ & WL & 5 & $100 \%$ & No & GROSS BETA ACTIVITY & $\mathrm{s}$ & $\mathrm{s}$ & Biennial \\
\hline GW-144 & $02 / 28 / 86$ & $01 / 04 / 07$ & WL & 24 & $96 \%$ & No & GROSS ALPHA ACTIVITY & NT & D & Biennial \\
\hline GW-145 & $03 / 05 / 86$ & $01 / 08 / 07$ & WL & 24 & $100 \%$ & No & GROSS ALPHA ACTIVITY & $\mathrm{s}$ & D & Annual \\
\hline GW-156 & 01/18/95 & $01 / 08 / 07$ & WL & 22 & $45 \%$ & No & URANIUM & D & D & Biennial \\
\hline GW-159 & $01 / 15 / 95$ & $01 / 08 / 07$ & WL & 22 & $45 \%$ & No & URANIUM & D & D & Biennial \\
\hline GW-161 & $01 / 25 / 07$ & $07 / 10 / 07$ & $\mathrm{WL}$ & 2 & $50 \%$ & No & CADMIUM & N/A & $\mathrm{N} / \mathrm{A}$ & Quarterly \\
\hline GW-231 & $04 / 07 / 05$ & $01 / 04 / 07$ & WL & 5 & $40 \%$ & No & GROSS BETA ACTIVITY & $\mathrm{S}$ & $\mathrm{S}$ & Biennial \\
\hline GW-292 & $01 / 23 / 07$ & $07 / 10 / 07$ & WL & 2 & $100 \%$ & No & NITRATE & N/A & N/A & Annual \\
\hline GW-293 & $01 / 24 / 07$ & 07/10/07 & WL & 2 & $100 \%$ & No & NITRATE & N/A & N/A & Annual \\
\hline GW-294 & $01 / 22 / 07$ & $07 / 11 / 07$ & WL & 2 & $100 \%$ & No & NITRATE & N/A & $\mathrm{N} / \mathrm{A}$ & Annual \\
\hline GW-296 & $01 / 22 / 07$ & $07 / 10 / 07$ & WL & 2 & $100 \%$ & No & NITRATE & $\mathrm{N} / \mathrm{A}$ & $\mathrm{N} / \mathrm{A}$ & Annual \\
\hline GW-298 & $02 / 03 / 88$ & 07/09/07 & WL & 3 & $67 \%$ & No & GROSS ALPHA ACTIVITY & N/A & NT & Annual \\
\hline GW-301 & 01/13/05 & 07/10/06 & WL & 4 & $50 \%$ & Yes & GROSS BETA ACTIVITY & NT & NT & Quarterly \\
\hline GW-731 & $01 / 17 / 95$ & $01 / 09 / 07$ & WL & 22 & $23 \%$ & No & URANIUM & NT & NT & Biennial \\
\hline GW-732 & 01/18/95 & $01 / 09 / 07$ & WL & 22 & $41 \%$ & No & URANIUM & $\mathrm{PD}$ & $\mathrm{D}$ & Biennial \\
\hline GW-841 & $02 / 06 / 97$ & $03 / 08 / 07$ & $\mathrm{WL}$ & 13 & $100 \%$ & Yes & TRICHLOROETHYLENE (TCE) & PD & PD & Quarterly \\
\hline GW-842 & $02 / 05 / 97$ & 03/08/07 & WL & 16 & $94 \%$ & No & TRICHLOROETHYLENE (TCE) & NT & NT & Annual \\
\hline
\end{tabular}

Notes:

1. Well sample dates taken from Y-12 Analytical Database 2008.

2. $\mathrm{WL}=$ Groundwater Monitoring Well; $\mathrm{SP}=$ Spring.

3. Trend = Insufficient Data (N/A), Decreasing (D), Probably Decreasing (PD), Stable (S), Probably Increasing (PI), and Increasing (I), No Trend (NT); and non-detect for all sample events (ND).

4. Number of samples between 1996-2007 is the count of analytical samples acquired at the location based on the Analytical Database (2008).

5. Priority Constituent is the $\mathrm{COC}$ with the highest average concentration from the full dataset normalized by the screening level. (e.g. MAX [Ave Conc./Screening Level]). Priority constituent indicated for wells where average concentrations do not exceed screening levels may be background level.

6. The Preliminary Frequency is the sampling frequency recommended by MAROS for results $1996-2007$. Low number of samples triggers default quarterly sampling. 
APPENDIX C

SUPPLEMENTAL MAROS ASSESSMENT SUMMARY REPORTS 
APPENDIX C.1

WEST S-3 AREA 


\section{MAROS COC Assessment}

Project: BC West S3

Location: West S-3

Toxicity:

\begin{tabular}{lccc}
\hline Contaminant of Concern & $\begin{array}{c}\text { Representative } \\
\text { Concentration } \\
\text { (mg/L) }\end{array}$ & $\begin{array}{c}\text { PRG } \\
\text { (mg/L) }\end{array}$ & $\begin{array}{c}\text { Percent } \\
\text { Above } \\
\text { PRG }\end{array}$ \\
\hline NITRATE & $1.5 \mathrm{E}+03$ & $1.0 \mathrm{E}+01$ & $14633.8 \%$ \\
GROSS BETA ACTIVITY & $1.5 \mathrm{E}+03$ & $5.0 \mathrm{E}+01$ & $2972.3 \%$ \\
CADMIUM & $7.0 \mathrm{E}-02$ & $5.0 \mathrm{E}-03$ & $1294.4 \%$ \\
GROSS ALPHA ACTIVITY & $1.2 \mathrm{E}+02$ & $1.5 \mathrm{E}+01$ & $722.1 \%$ \\
URANIUM & $2.2 \mathrm{E}-01$ & $3.0 \mathrm{E}-02$ & $635.2 \%$ \\
TETRACHLOROETHYLENE(PCE) & $9.9 \mathrm{E}-03$ & $5.0 \mathrm{E}-03$ & $97.5 \%$ \\
TRICHLOROETHYLENE (TCE) & $9.3 \mathrm{E}-03$ & $5.0 \mathrm{E}-03$ & $86.3 \%$
\end{tabular}

Note: Top COCs by toxicity were determined by examining a representative concentration for each compound over the entire site. The compound representative concentrations are then compared with the chosen PRG for that compound, with the percentage exceedance from the PRG determining the compound's toxicity. All compounds above exceed the PRG.

\section{Prevalence:}

\begin{tabular}{|c|c|c|c|c|c|}
\hline Contaminant of Concern & Class & $\begin{array}{l}\text { Total } \\
\text { Wells }\end{array}$ & $\begin{array}{c}\text { Total } \\
\text { Exceedances }\end{array}$ & $\begin{array}{c}\text { Percent } \\
\text { Exceedances }\end{array}$ & $\begin{array}{l}\text { Total } \\
\text { detects }\end{array}$ \\
\hline NITRATE & INO & 22 & 19 & $86.4 \%$ & 22 \\
\hline GROSS BETA ACTIVITY & INO & 22 & 13 & $59.1 \%$ & 22 \\
\hline GROSS ALPHA ACTIVITY & INO & 22 & 13 & $59.1 \%$ & 22 \\
\hline URANIUM & MET & 22 & 7 & $31.8 \%$ & 22 \\
\hline TETRACHLOROETHYLENE(PCE) & ORG & 22 & 7 & $31.8 \%$ & 18 \\
\hline CADMIUM & MET & 22 & 6 & $27.3 \%$ & 6 \\
\hline TRICHLOROETHYLENE (TCE) & ORG & 22 & 5 & $22.7 \%$ & 13 \\
\hline
\end{tabular}

Note: Top COCs by prevalence were determined by examining a representative concentration for each well location at the site. The total exceedances (values above the chosen PRGs) are compared to the total number of wells to determine the prevalence of the compound.

\section{Mobility:}

Contaminant of Concern

$\mathrm{Kd}$

\begin{tabular}{lr}
\hline NITRATE & \\
GROSS BETA ACTIVITY & \\
GROSS ALPHA ACTIVITY & \\
TRICHLOROETHYLENE (TCE) & 0.297 \\
TETRACHLOROETHYLENE(PCE) & 0.923 \\
CADMIUM & 15 \\
URANIUM & 2960
\end{tabular}

Note: Top COCs by mobility were determined by examining each detected compound in the dataset and comparing their mobilities (Koc's for organics, assume foc $=0.001$, and Kd's for metals).

\section{Contaminants of Concern (COC's)}

NITRATE 
Project: BC West S3

User Name: MV

Location: West S-3

State: Tennessee

TRICHLOROETHYLENE (TCE)

TETRACHLOROETHYLENE(PCE)

URANIUM

GROSS BETA ACTIVITY 


\section{MAROS Spatial Moment Analysis Summary}

Project: BC West S3

Location: West S-3
User Name: MV

State: Tennessee 2nd Moment (Spread)

Sigma XX Sigma YY

$(s q \mathrm{ft}) \quad(s q \mathrm{ft})$
Number of

Wells

\section{GROSS BETA ACTIVITY}

$\begin{array}{rrrrrrrr}7 / 1 / 2004 & 1.1 \mathrm{E}+04 & 51,951 & 29,819 & 338 & 109,137 & 15,266 & 10 \\ 7 / 1 / 2005 & 8.9 \mathrm{E}+03 & 52,204 & 29,904 & 330 & 41,290 & 12,072 & 16 \\ 7 / 1 / 2006 & 7.7 \mathrm{E}+03 & 51,948 & 29,864 & 294 & 185,647 & 10,892 & 11 \\ 7 / 1 / 2007 & 5.1 \mathrm{E}+03 & 52,206 & 29,820 & 399 & 55,340 & 6,534 & 13\end{array}$

NITRATE

$\begin{array}{llllllll}7 / 1 / 2004 & 1.8 \mathrm{E}+04 & 51,802 & 29,899 & 318 & 91,516 & 20,861 & 8 \\ 7 / 1 / 2005 & 2.2 \mathrm{E}+04 & 51,637 & 29,835 & 476 & 307,867 & 17,378 & 16 \\ 7 / 1 / 2006 & 5.2 \mathrm{E}+04 & 51,539 & 29,871 & 533 & 131,684 & 10,834 & 11 \\ 7 / 1 / 2007 & 1.3 \mathrm{E}+04 & 52,037 & 29,874 & 285 & 123,436 & 27,145 & 13\end{array}$

TETRACHLOROETHYLENE(PCE)

$\begin{array}{llllllll}7 / 1 / 2004 & 6.3 \mathrm{E}-01 & 51,693 & 29,751 & 501 & 136,757 & 20,842 & 10 \\ 7 / 1 / 2005 & 4.2 \mathrm{E}-01 & 51,657 & 29,676 & 584 & 444,759 & 34,547 & 16 \\ 7 / 1 / 2006 & 4.3 \mathrm{E}-01 & 51,569 & 29,713 & 610 & 299,393 & 28,376 & 11 \\ 7 / 1 / 2007 & 3.5 \mathrm{E}-01 & 51,699 & 29,709 & 532 & 340,457 & 31,787 & 13\end{array}$

TRICHLOROETHYLENE (TCE)

\begin{tabular}{|c|c|c|c|c|c|c|c|c|}
\hline & $7 / 1 / 2004$ & 2.6E-01 & 51,367 & 29,652 & 801 & 197,781 & 33,101 & 10 \\
\hline & $7 / 1 / 2005$ & 4.2E-01 & 50,854 & 29,511 & 1,306 & 466,970 & 36,365 & 16 \\
\hline & $7 / 1 / 2006$ & 2.2E-01 & 51,122 & 29,640 & 1,010 & 287,888 & 30,200 & 11 \\
\hline & $7 / 1 / 2007$ & 2.5E-01 & 51,148 & 29,589 & 1,015 & 332,238 & 30,110 & 13 \\
\hline \multicolumn{9}{|l|}{ URANIUM } \\
\hline & 7/1/2004 & $2.0 \mathrm{E}+00$ & 52,195 & 29,863 & 357 & 60,560 & 14,928 & 10 \\
\hline & $7 / 1 / 2005$ & $6.8 \mathrm{E}-01$ & 51,905 & 29,748 & 415 & 561,050 & 23,108 & 16 \\
\hline & $7 / 1 / 2006$ & $2.5 \mathrm{E}+00$ & 52,192 & 29,795 & 412 & 120,142 & 5,085 & 11 \\
\hline & $7 / 1 / 2007$ & $3.5 E+00$ & 52,155 & 29,883 & 318 & 88,937 & 15,570 & 13 \\
\hline
\end{tabular}


Project: BC West S3

Location: West S-3
User Name: $\mathrm{MV}$

State: Tennessee

\begin{tabular}{llllll} 
Moment Type & Constituent & $\begin{array}{l}\text { Coefficient } \\
\text { of Variation }\end{array}$ & $\begin{array}{c}\text { Mann-Kendall } \\
\text { S Statistic }\end{array}$ & $\begin{array}{c}\text { Confidence } \\
\text { in Trend }\end{array}$ & $\begin{array}{c}\text { Moment } \\
\text { Trend }\end{array}$ \\
\hline
\end{tabular}

Zeroth Moment: Mass

$\begin{array}{llclc}\text { GROSS BETA ACTIVITY } & 0.30 & -6 & 95.8 \% & \mathrm{D} \\ \text { NITRATE } & 0.67 & 0 & 37.5 \% & \mathrm{~S} \\ \text { TETRACHLOROETHYLENE(PCE) } & 0.26 & -4 & 83.3 \% & \mathrm{~S} \\ \text { TRICHLOROETHYLENE (TCE) } & 0.31 & -2 & 62.5 \% & \mathrm{~S} \\ \text { URANIUM } & 0.54 & 4 & 83.3 \% & \text { NT }\end{array}$

1st Moment: Distance to Source

\begin{tabular}{|c|c|c|c|}
\hline GROSS BETA ACTIVITY & 0.13 & 0 & $37.5 \%$ \\
\hline NITRATE & 0.30 & 0 & $37.5 \%$ \\
\hline TETRACHLOROETHYLENE(PCE) & 0.09 & 2 & $62.5 \%$ \\
\hline TRICHLOROETHYLENE (TCE) & 0.20 & 2 & $62.5 \%$ \\
\hline URANIUM & 0.13 & -2 & $62.5 \%$ \\
\hline
\end{tabular}

2nd Moment: Sigma XX

$\begin{array}{llllc}\text { GROSS BETA ACTIVITY } & 0.67 & 0 & 37.5 \% & \mathrm{~S} \\ \text { NITRATE } & 0.60 & 0 & 37.5 \% & \mathrm{~S} \\ \text { TETRACHLOROETHYLENE(PCE) } & 0.42 & 2 & 62.5 \% & \text { NT } \\ \text { TRICHLOROETHYLENE (TCE) } & 0.35 & 2 & 62.5 \% & \text { NT } \\ \text { URANIUM } & 1.14 & 0 & 37.5 \% & \text { NT }\end{array}$

2nd Moment: Sigma YY

\begin{tabular}{lcccc} 
GROSS BETA ACTIVITY & 0.32 & -6 & $95.8 \%$ & $\mathrm{D}$ \\
NITRATE & 0.36 & 0 & $37.5 \%$ & $\mathrm{~S}$ \\
TETRACHLOROETHYLENE(PCE) & 0.21 & 2 & $62.5 \%$ & $\mathrm{NT}$ \\
TRICHLOROETHYLENE (TCE) & 0.09 & -4 & $83.3 \%$ & $\mathrm{~S}$ \\
URANIUM & 0.50 & 0 & $37.5 \%$ & $\mathrm{~S}$ \\
\hline
\end{tabular}

Note: The following assumptions were applied for the calculation of the Zeroth Moment:

Porosity: $0.10 \quad$ Saturated Thickness: Uniform: $50 \mathrm{ft}$

Mann-Kendall Trend test performed on all sample events for each constituent. Increasing (I); Probably Increasing (PI); Stable (S); Probably Decreasing (PD); Decreasing (D); No Trend (NT); Not Applicable (N/A)-Due to insufficient Data (< 4 sampling events).

Note: The Sigma $X X$ and Sigma $Y Y$ components are estimated using the given field coordinate system and then rotated to align with the estimated groundwater flow direction. Moments are not calculated for sample events with less than 6 wells. 


\section{MAROS Statistical Trend Analysis Summary}

Project: $\quad$ BC West S3

Location: West S-3

Time Period: $1 / 1 / 1996$ to $7 / 15 / 2007$

Consolidation Period: No Time Consolidation

Consolidation Type: Geometric Mean

Duplicate Consolidation: Average

ND Values: Specified Detection Limit

J Flag Values : Actual Value
User Name: MV

State: Tennessee

\begin{tabular}{|c|c|c|c|c|c|c|c|c|}
\hline Well & $\begin{array}{c}\text { Sourcel } \\
\text { Tail }\end{array}$ & $\begin{array}{c}\text { Number } \\
\text { of } \\
\text { Samples }\end{array}$ & $\begin{array}{c}\text { Number } \\
\text { of } \\
\text { Detects }\end{array}$ & $\begin{array}{c}\text { Average } \\
\text { Conc. } \\
\text { (mg/L) }\end{array}$ & $\begin{array}{l}\text { Median } \\
\text { Conc. } \\
\text { (mg/L) }\end{array}$ & $\begin{array}{c}\text { All } \\
\text { Samples } \\
\text { "ND" ? }\end{array}$ & $\begin{array}{l}\text { Mann- } \\
\text { Kendall } \\
\text { Trend }\end{array}$ & $\begin{array}{l}\text { Linear } \\
\text { Regression } \\
\text { Trend }\end{array}$ \\
\hline \multicolumn{9}{|c|}{ GROSS BETA ACTIVITY } \\
\hline GW-100 & $\mathrm{T}$ & 3 & 2 & $1.4 \mathrm{E}+00$ & $1.5 \mathrm{E}+00$ & No & N/A & $N / A$ \\
\hline GW-101 & $\mathrm{T}$ & 3 & 2 & $2.8 \mathrm{E}+00$ & $2.3 E+00$ & No & N/A & N/A \\
\hline GW-122 & $\mathrm{T}$ & 4 & 1 & $4.0 \mathrm{E}+00$ & $1.0 \mathrm{E}-02$ & No & NT & NT \\
\hline GW-127 & $\mathrm{T}$ & 3 & 2 & $1.2 \mathrm{E}+01$ & $1.5 \mathrm{E}+01$ & No & N/A & N/A \\
\hline GW-236 & $\mathrm{T}$ & 4 & 4 & $6.2 \mathrm{E}+01$ & $6.3 E+01$ & No & $\mathrm{D}$ & D \\
\hline GW-244 & $\mathrm{T}$ & 2 & 2 & $4.0 \mathrm{E}+03$ & $4.0 \mathrm{E}+03$ & No & N/A & N/A \\
\hline GW-245 & $\mathrm{T}$ & 2 & 2 & $1.0 \mathrm{E}+03$ & $1.0 \mathrm{E}+03$ & No & N/A & N/A \\
\hline GW-246 & S & 6 & 6 & $1.7 \mathrm{E}+04$ & $1.7 \mathrm{E}+04$ & No & $s$ & NT \\
\hline GW-247 & $\mathrm{T}$ & 2 & 2 & $3.9 \mathrm{E}+03$ & $3.9 \mathrm{E}+03$ & No & N/A & N/A \\
\hline GW-276 & $\mathrm{s}$ & 23 & 23 & $4.3 E+02$ & $3.7 \mathrm{E}+02$ & No & $\mathrm{D}$ & $\mathrm{D}$ \\
\hline GW-277 & $\mathrm{T}$ & 3 & 3 & $9.6 \mathrm{E}+02$ & $1.1 \mathrm{E}+03$ & No & N/A & N/A \\
\hline GW-307 & $\mathrm{T}$ & 3 & 2 & $8.3 E+00$ & $1.1 \mathrm{E}+01$ & No & $\mathrm{N} / \mathrm{A}$ & N/A \\
\hline GW-310 & $\mathrm{T}$ & 3 & 3 & $1.7 \mathrm{E}+01$ & $1.8 \mathrm{E}+01$ & No & N/A & N/A \\
\hline GW-311 & $\mathrm{T}$ & 16 & 13 & $5.7 \mathrm{E}+00$ & $4.3 E+00$ & No & $\mathrm{D}$ & $\mathrm{D}$ \\
\hline GW-312 & $\mathrm{T}$ & 2 & 2 & $8.0 \mathrm{E}+00$ & $8.0 \mathrm{E}+00$ & No & N/A & N/A \\
\hline GW-313 & $\mathrm{T}$ & 3 & 0 & $1.0 \mathrm{E}-02$ & $1.0 \mathrm{E}-02$ & Yes & ND & ND \\
\hline GW-315 & $\mathrm{T}$ & 22 & 22 & $2.6 \mathrm{E}+01$ & $2.6 \mathrm{E}+01$ & No & $\mathrm{s}$ & $\mathrm{D}$ \\
\hline GW-526 & $\mathrm{T}$ & 18 & 10 & $8.3 E+01$ & $2.7 \mathrm{E}+01$ & No & NT & NT \\
\hline GW-615 & S & 4 & 4 & $6.6 \mathrm{E}+02$ & $7.1 \mathrm{E}+02$ & No & $\mathrm{s}$ & NT \\
\hline GW-616 & $\mathrm{T}$ & 5 & 1 & $1.3 \mathrm{E}-01$ & $1.0 \mathrm{E}-02$ & No & NT & $\mathrm{D}$ \\
\hline GW-829 & $\mathrm{T}$ & 13 & 12 & $4.9 \mathrm{E}+01$ & $4.5 \mathrm{E}+00$ & No & NT & $D$ \\
\hline SS-1 & $\mathrm{T}$ & 21 & 21 & $4.0 \mathrm{E}+01$ & $3.7 \mathrm{E}+01$ & No & NT & NT \\
\hline
\end{tabular}

NITRATE

$\begin{array}{lc}\text { GW-100 } & \text { T } \\ \text { GW-101 } & \text { T } \\ \text { GW-122 } & \text { T } \\ \text { GW-127 } & \text { T } \\ \text { GW-236 } & \text { T } \\ \text { GW-244 } & \text { T } \\ \text { GW-245 } & \text { T } \\ \text { GW-246 } & \text { S } \\ \text { GW-247 } & \text { T } \\ \text { GW-276 } & \text { S } \\ \text { GW-277 } & \text { T }\end{array}$

$\begin{array}{lll}4.7 \mathrm{E}+01 & 5.1 \mathrm{E}+01 & \mathrm{~N} \\ 5.7 \mathrm{E}+01 & 6.1 \mathrm{E}+01 & \mathrm{~N} \\ 3.6 \mathrm{E}+02 & 3.4 \mathrm{E}+02 & \mathrm{~N} \\ 1.3 \mathrm{E}-01 & 1.4 \mathrm{E}-01 & \mathrm{~N} \\ 5.3 \mathrm{E}+01 & 5.3 \mathrm{E}+01 & \mathrm{~N} \\ 4.3 \mathrm{E}+03 & 4.3 \mathrm{E}+03 & \mathrm{~N} \\ 2.2 \mathrm{E}+03 & 2.2 \mathrm{E}+03 & \mathrm{~N} \\ 3.0 \mathrm{E}+03 & 2.9 \mathrm{E}+03 & \mathrm{~N} \\ 3.2 \mathrm{E}+03 & 3.2 \mathrm{E}+03 & \mathrm{~N} \\ 7.6 \mathrm{E}+01 & 7.2 \mathrm{E}+01 & \mathrm{~N} \\ 4.1 \mathrm{E}+02 & 4.1 \mathrm{E}+02 & \mathrm{~N}\end{array}$

No
No
No
No
No
No
No
No
No
No
No

N/A
N/A
S
N/A
N/A
N/A
N/A
NT
N/A
D
N/A

\section{N/A}

N/A

$S$

N/A

N/A

N/A

NT

N/A

N/A
N/A

N/A

N/A

N/A

NT

N/A

$D$

N/A 


\section{MAROS Statistical Trend Analysis Summary}

\begin{tabular}{ccccccccc} 
& & Number & Number & Average & Median & All & Mann- & Linear \\
Well & Sourcel & of & of & Conc. & Conc. & Samples & Kendall & Regression \\
Tail & Samples & Detects & (mg/L) & (mg/L) & "ND" ? & Trend & Trend \\
\hline
\end{tabular}

NITRATE

GW-307

GW-310

GW-311

GW-312

GW-313

GW-315

GW-526

GW-615

GW-616

GW-829

SS-1

$\begin{array}{lll}2.0 \mathrm{E}+00 & 2.2 \mathrm{E}+00 & \text { No } \\ 6.9 \mathrm{E}+00 & 7.3 \mathrm{E}+00 & \text { No } \\ 3.5 \mathrm{E}-01 & 3.3 \mathrm{E}-01 & \text { No } \\ 5.7 \mathrm{E}-01 & 5.7 \mathrm{E}-01 & \text { No } \\ 1.3 \mathrm{E}+00 & 1.5 \mathrm{E}+00 & \text { No } \\ 7.6 \mathrm{E}+00 & 7.8 \mathrm{E}+00 & \text { No } \\ 1.2 \mathrm{E}+03 & 1.2 \mathrm{E}+03 & \text { No } \\ 1.1 \mathrm{E}+04 & 1.1 \mathrm{E}+04 & \text { No } \\ 2.6 \mathrm{E}+02 & 2.7 \mathrm{E}+02 & \text { No } \\ 2.6 \mathrm{E}+01 & 2.4 \mathrm{E}+01 & \text { No } \\ 1.4 \mathrm{E}+01 & 1.4 \mathrm{E}+01 & \text { No }\end{array}$

$\begin{array}{cc}\text { N/A } & \text { N/A } \\ \text { N/A } & \text { N/A } \\ \text { S } & \text { S } \\ \text { N/A } & \text { N/A } \\ \text { N/A } & \text { N/A } \\ \text { D } & \text { S } \\ \text { S } & \text { S } \\ \text { NT } & \text { NT } \\ \text { S } & \text { S } \\ \text { D } & \text { PD } \\ \text { PD } & \text { PD }\end{array}$

TETRACHLOROETHYLENE(PCE)

\begin{tabular}{|c|c|c|c|c|c|c|c|c|}
\hline GW-100 & $T$ & 3 & 0 & 5.0E-04 & $5.0 \mathrm{E}-04$ & Yes & ND & ND \\
\hline GW-101 & $\mathrm{T}$ & 3 & 0 & 5.0E-04 & $5.0 \mathrm{E}-04$ & Yes & ND & ND \\
\hline GW-122 & $\mathrm{T}$ & 4 & 0 & 5.0E-04 & $5.0 \mathrm{E}-04$ & Yes & ND & ND \\
\hline GW-127 & $\mathrm{T}$ & 3 & 0 & 5.0E-04 & $5.0 \mathrm{E}-04$ & Yes & ND & ND \\
\hline GW-236 & $\mathrm{T}$ & 4 & 2 & 1.0E-03 & 7.5E-04 & No & NT & NT \\
\hline GW-244 & $\mathrm{T}$ & 2 & 2 & $5.5 \mathrm{E}-03$ & $5.5 \mathrm{E}-03$ & No & N/A & N/A \\
\hline GW-245 & $\mathrm{T}$ & 2 & 2 & 2.5E-03 & $2.5 \mathrm{E}-03$ & No & N/A & N/A \\
\hline GW-246 & $\mathrm{S}$ & 6 & 6 & 1.1E-01 & $1.1 \mathrm{E}-01$ & No & S & $\mathrm{D}$ \\
\hline GW-247 & $\mathrm{T}$ & 2 & 2 & 8.0E-03 & $8.0 \mathrm{E}-03$ & No & N/A & N/A \\
\hline GW-276 & $\mathrm{S}$ & 24 & 24 & 1.1E-02 & $1.0 \mathrm{E}-02$ & No & D & D \\
\hline GW-277 & $\mathrm{T}$ & 3 & 3 & 2.1E-02 & $1.8 \mathrm{E}-02$ & No & N/A & N/A \\
\hline GW-307 & $\mathrm{T}$ & 3 & 1 & 1.0E-03 & $5.0 \mathrm{E}-04$ & No & N/A & N/A \\
\hline GW-310 & $\mathrm{T}$ & 3 & 3 & 2.3E-03 & $2.0 \mathrm{E}-03$ & No & N/A & N/A \\
\hline GW-311 & $\mathrm{T}$ & 21 & 1 & $5.2 \mathrm{E}-04$ & $5.0 \mathrm{E}-04$ & No & NT & NT \\
\hline GW-312 & $\mathrm{T}$ & 2 & 0 & 5.0E-04 & $5.0 \mathrm{E}-04$ & Yes & ND & ND \\
\hline GW-313 & $\mathrm{T}$ & 3 & 3 & 5.7E-03 & $6.0 \mathrm{E}-03$ & No & N/A & N/A \\
\hline GW-315 & $\mathrm{T}$ & 22 & 22 & 1.1E-02 & $1.0 \mathrm{E}-02$ & No & D & D \\
\hline GW-526 & $\mathrm{T}$ & 15 & 0 & 5.0E-04 & $5.0 \mathrm{E}-04$ & Yes & ND & ND \\
\hline GW-615 & $S$ & 8 & 0 & 5.0E-04 & $5.0 \mathrm{E}-04$ & Yes & ND & ND \\
\hline GW-616 & $\mathrm{T}$ & 6 & 0 & 5.0E-04 & $5.0 \mathrm{E}-04$ & Yes & ND & ND \\
\hline GW-829 & $\mathrm{T}$ & 13 & 0 & 5.0E-04 & $5.0 \mathrm{E}-04$ & Yes & ND & ND \\
\hline SS-1 & $\mathrm{T}$ & 21 & 4 & $6.9 \mathrm{E}-04$ & $5.0 \mathrm{E}-04$ & No & D & D \\
\hline \multicolumn{9}{|c|}{ TRICHLOROETHYLENE (TCE) } \\
\hline GW-100 & $\mathrm{T}$ & 3 & 0 & 5.0E-04 & $5.0 \mathrm{E}-04$ & Yes & ND & ND \\
\hline GW-101 & $\mathrm{T}$ & 3 & 0 & $5.0 \mathrm{E}-04$ & $5.0 \mathrm{E}-04$ & Yes & ND & ND \\
\hline GW-122 & $\mathrm{T}$ & 4 & 0 & 5.0E-04 & $5.0 \mathrm{E}-04$ & Yes & ND & ND \\
\hline GW-127 & $\mathrm{T}$ & 3 & 0 & 5.0E-04 & $5.0 \mathrm{E}-04$ & Yes & ND & ND \\
\hline GW-236 & $\mathrm{T}$ & 4 & 0 & 5.0E-04 & $5.0 \mathrm{E}-04$ & Yes & ND & ND \\
\hline GW-244 & $\mathrm{T}$ & 2 & 0 & $5.0 \mathrm{E}-04$ & $5.0 \mathrm{E}-04$ & Yes & ND & ND \\
\hline GW-245 & $\mathrm{T}$ & 2 & 0 & $5.0 \mathrm{E}-04$ & $5.0 \mathrm{E}-04$ & Yes & ND & ND \\
\hline GW-246 & $\mathrm{S}$ & 6 & 6 & 1.7E-03 & $2.0 \mathrm{E}-03$ & No & $\mathrm{S}$ & S \\
\hline GW-247 & $\mathrm{T}$ & 2 & 0 & 5.0E-04 & $5.0 \mathrm{E}-04$ & Yes & ND & ND \\
\hline
\end{tabular}




\section{MAROS Statistical Trend Analysis Summary}

\begin{tabular}{ccccccccc} 
& & Number & Number & Average & Median & All & Mann- & Linear \\
Well & Sourcel & of & of & Conc. & Conc. & Samples & Kendall & Regression \\
Tail & Samples & Detects & (mg/L) & (mg/L) & "ND" ? & Trend & Trend \\
\hline
\end{tabular}

TRICHLOROETHYLENE (TCE)

\begin{tabular}{|c|c|c|c|c|c|c|c|c|}
\hline GW-276 & $\mathrm{S}$ & 24 & 0 & $5.0 \mathrm{E}-04$ & $5.0 \mathrm{E}-04$ & Yes & ND & ND \\
\hline GW-277 & $\mathrm{T}$ & 3 & 0 & $5.0 \mathrm{E}-04$ & $5.0 \mathrm{E}-04$ & Yes & ND & ND \\
\hline GW-307 & $\mathrm{T}$ & 3 & 3 & $1.8 \mathrm{E}-02$ & $2.1 \mathrm{E}-02$ & No & N/A & N/A \\
\hline GW-310 & $\mathrm{T}$ & 3 & 3 & $5.7 \mathrm{E}-03$ & $5.0 \mathrm{E}-03$ & No & $\mathrm{N} / \mathrm{A}$ & $\mathrm{N} / \mathrm{A}$ \\
\hline GW-311 & $\mathrm{T}$ & 21 & 21 & $5.5 \mathrm{E}-03$ & $4.0 \mathrm{E}-03$ & No & D & $\mathrm{D}$ \\
\hline GW-312 & $\mathrm{T}$ & 2 & 2 & $3.8 \mathrm{E}-02$ & $3.8 \mathrm{E}-02$ & No & $\mathrm{N} / \mathrm{A}$ & N/A \\
\hline GW-313 & $\mathrm{T}$ & 3 & 3 & $2.0 \mathrm{E}-03$ & $2.0 \mathrm{E}-03$ & No & N/A & $\mathrm{N} / \mathrm{A}$ \\
\hline GW-315 & $\mathrm{T}$ & 22 & 20 & 4.5E-03 & $5.0 \mathrm{E}-03$ & No & D & S \\
\hline GW-526 & $\mathrm{T}$ & 15 & 0 & $5.0 \mathrm{E}-04$ & $5.0 \mathrm{E}-04$ & Yes & ND & ND \\
\hline GW-615 & $S$ & 8 & 0 & $5.0 \mathrm{E}-04$ & $5.0 \mathrm{E}-04$ & Yes & ND & ND \\
\hline GW-616 & $\mathrm{T}$ & 6 & 0 & $5.0 \mathrm{E}-04$ & $5.0 \mathrm{E}-04$ & Yes & ND & ND \\
\hline GW-829 & $\mathrm{T}$ & 13 & 0 & 5.0E-04 & $5.0 \mathrm{E}-04$ & Yes & ND & ND \\
\hline SS-1 & $\mathrm{T}$ & 21 & 2 & $6.4 \mathrm{E}-04$ & $5.0 \mathrm{E}-04$ & No & $\mathrm{s}$ & D \\
\hline \multicolumn{9}{|l|}{ URANIUM } \\
\hline GW-100 & $\mathrm{T}$ & 3 & 2 & 7.2E-04 & $6.9 \mathrm{E}-04$ & No & N/A & N/A \\
\hline GW-101 & $\mathrm{T}$ & 3 & 3 & $2.5 \mathrm{E}-03$ & $1.8 \mathrm{E}-03$ & No & $N / A$ & N/A \\
\hline GW-122 & $\mathrm{T}$ & 4 & 4 & $1.6 \mathrm{E}-03$ & 1.6E-03 & No & 1 & 1 \\
\hline GW-127 & $\mathrm{T}$ & 3 & 3 & 2.6E-02 & $2.8 \mathrm{E}-02$ & No & $N / A$ & N/A \\
\hline GW-236 & $\mathrm{T}$ & 4 & 4 & 8.0E-03 & $6.4 \mathrm{E}-03$ & No & NT & NT \\
\hline GW-244 & $\mathrm{T}$ & 2 & 2 & $2.3 \mathrm{E}-03$ & $2.3 \mathrm{E}-03$ & No & $N / A$ & $\mathrm{~N} / \mathrm{A}$ \\
\hline GW-245 & $\mathrm{T}$ & 2 & 2 & $3.7 \mathrm{E}-02$ & 3.7E-02 & No & $N / A$ & $\mathrm{~N} / \mathrm{A}$ \\
\hline GW-246 & $\mathrm{S}$ & 6 & 6 & $5.3 \mathrm{E}-01$ & $4.5 \mathrm{E}-01$ & No & NT & NT \\
\hline GW-247 & $\mathrm{T}$ & 2 & 2 & $2.4 \mathrm{E}-02$ & $2.4 \mathrm{E}-02$ & No & $N / A$ & $\mathrm{~N} / \mathrm{A}$ \\
\hline GW-276 & $\mathrm{S}$ & 24 & 24 & $7.9 \mathrm{E}-01$ & 7.5E-01 & No & D & $\mathrm{D}$ \\
\hline GW-277 & $\mathrm{T}$ & 3 & 3 & $2.8 \mathrm{E}-02$ & $6.1 \mathrm{E}-03$ & No & $N / A$ & $\mathrm{~N} / \mathrm{A}$ \\
\hline GW-307 & $\mathrm{T}$ & 3 & 3 & 1.6E-03 & 1.6E-03 & No & $N / A$ & $\mathrm{~N} / \mathrm{A}$ \\
\hline GW-310 & $\mathrm{T}$ & 3 & 3 & $3.8 \mathrm{E}-03$ & $3.8 \mathrm{E}-03$ & No & $N / A$ & $\mathrm{~N} / \mathrm{A}$ \\
\hline GW-311 & $\mathrm{T}$ & 21 & 9 & 2.7E-04 & $1.0 \mathrm{E}-05$ & No & NT & NT \\
\hline GW-312 & $\mathrm{T}$ & 2 & 1 & 4.3E-04 & 4.3E-04 & No & N/A & $\mathrm{N} / \mathrm{A}$ \\
\hline GW-313 & $\mathrm{T}$ & 3 & 1 & 2.6E-04 & $1.0 \mathrm{E}-05$ & No & N/A & $\mathrm{N} / \mathrm{A}$ \\
\hline GW-315 & $\mathrm{T}$ & 22 & 21 & 2.1E-03 & 2.3E-03 & No & NT & NT \\
\hline GW-526 & $\mathrm{T}$ & 15 & 2 & $6.0 \mathrm{E}-05$ & $1.0 \mathrm{E}-05$ & No & NT & D \\
\hline GW-615 & $\mathrm{s}$ & 8 & 8 & 8.4E-01 & 7.9E-01 & No & NT & NT \\
\hline GW-616 & $\mathrm{T}$ & 6 & 0 & $1.0 \mathrm{E}-05$ & $1.0 \mathrm{E}-05$ & Yes & ND & ND \\
\hline GW-829 & $\mathrm{T}$ & 13 & 13 & $6.4 \mathrm{E}-03$ & $1.3 \mathrm{E}-03$ & No & 1 & NT \\
\hline SS-1 & $\mathrm{T}$ & 21 & 21 & 3.5E-02 & 4.1E-02 & No & $\mathrm{D}$ & $\mathrm{D}$ \\
\hline
\end{tabular}

Note: Increasing (I); Probably Increasing (PI); Stable (S); Probably Decreasing (PD); Decreasing (D); No Trend (NT); Not Applicable (N/A); Not Applicable (N/A) - Due to insufficient Data (< 4 sampling events); No Detectable Concentration (NDC)

The Number of Samples and Number of Detects shown above are post-consolidation values. 


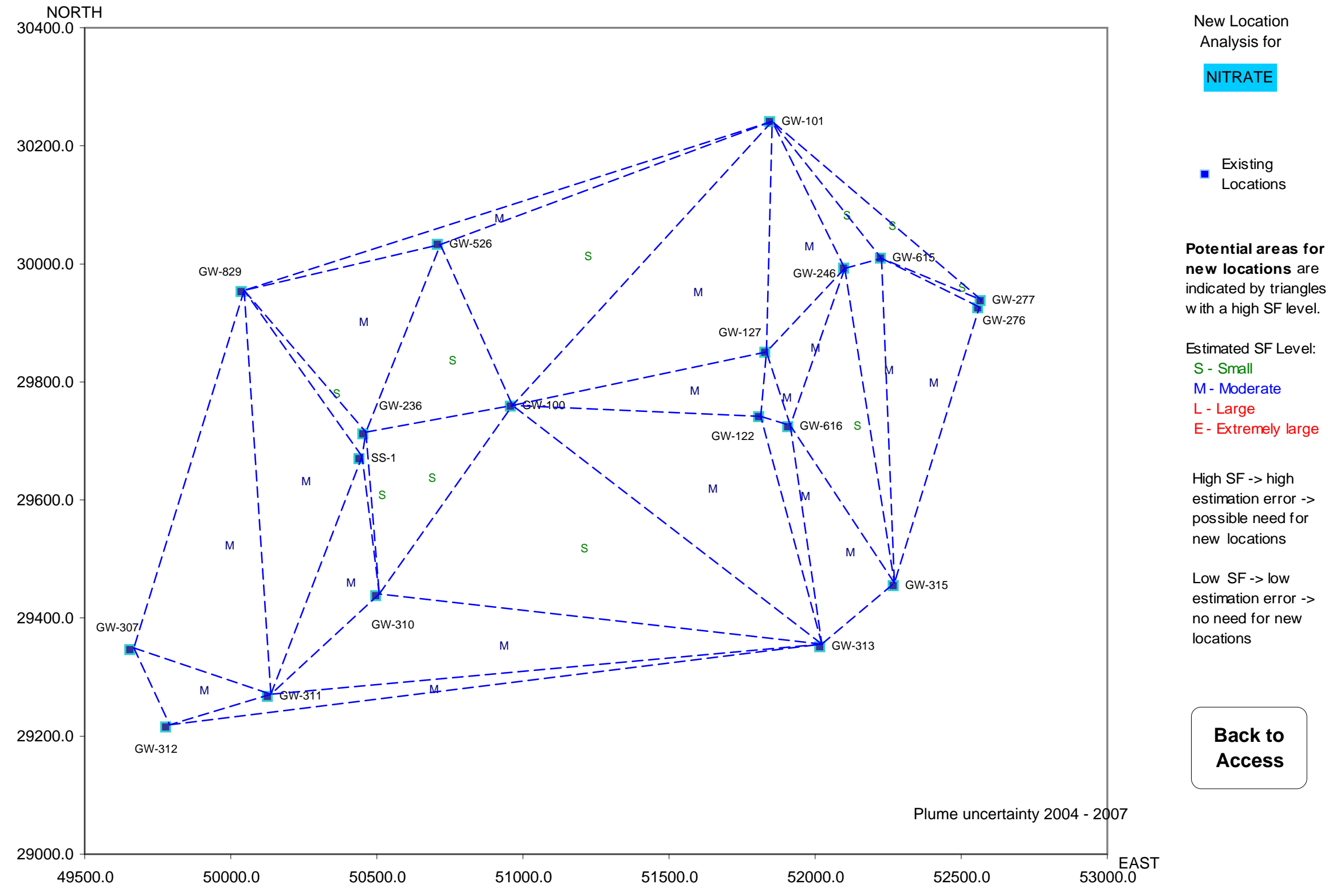


APPENDIX C.2

\section{OIL LANDFARM WMA}




\section{MAROS COC Assessment}

\author{
Project: BC OLF WMA \\ Location: OLFA
}

\section{Toxicity:}

\section{Contaminant of Concern}

TRICHLOROETHYLENE (TCE)

NITRATE

VINYL CHLORIDE
User Name: $\quad$ MV

State Tennessee

Note: Top COCs by toxicity were determined by examining a representative concentration for each compound over the entire site. The compound representative concentrations are then compared with the chosen PRG for that compound, with the percentage exceedance from the PRG determining the compound's toxicity. All compounds above exceed the PRG.

\section{Prevalence:}

\begin{tabular}{|c|c|c|c|c|c|}
\hline Contaminant of Concern & Class & $\begin{array}{l}\text { Total } \\
\text { Wells }\end{array}$ & $\begin{array}{c}\text { Total } \\
\text { Exceedances }\end{array}$ & $\begin{array}{c}\text { Percent } \\
\text { Exceedances }\end{array}$ & $\begin{array}{l}\text { Total } \\
\text { detects }\end{array}$ \\
\hline TRICHLOROETHYLENE (TCE) & ORG & 32 & 17 & $53.1 \%$ & 26 \\
\hline NITRATE & INO & 21 & 10 & $47.6 \%$ & 20 \\
\hline VINYL CHLORIDE & ORG & 32 & 4 & $12.5 \%$ & 4 \\
\hline
\end{tabular}

Note: Top COCs by prevalence were determined by examining a representative concentration for each well location at the site. The total exceedances (values above the chosen PRGs) are compared to the total number of wells to determine the prevalence of the compound.

\section{Mobility:}

Contaminant of Concern

NITRATE

VINYL CHLORIDE

TRICHLOROETHYLENE (TCE)

\begin{tabular}{ccc}
$\begin{array}{c}\text { Representative } \\
\text { Concentration } \\
\text { (mg/L) }\end{array}$ & $\begin{array}{c}\text { PRG } \\
\text { (mg/L) }\end{array}$ & $\begin{array}{c}\text { Percent } \\
\text { Above } \\
\text { PRG }\end{array}$ \\
\hline $3.6 \mathrm{E}-02$ & $5.0 \mathrm{E}-03$ & $611.2 \%$ \\
$5.6 \mathrm{E}+01$ & $1.0 \mathrm{E}+01$ & $462.4 \%$ \\
$3.6 \mathrm{E}-03$ & $2.0 \mathrm{E}-03$ & $81.7 \%$
\end{tabular}

Note: Top COCs by mobility were determined by examining each detected compound in the dataset and comparing their mobilities (Koc's for organics, assume foc $=0.001$, and Kd's for metals).

\section{Contaminants of Concern (COC's)}

TRICHLOROETHYLENE (TCE)

NITRATE

VINYL CHLORIDE 


\section{MAROS Spatial Moment Analysis Summary}

Project: $\quad$ BC EMWMF

Location: OLFA
User Name: MV

State: Tennessee

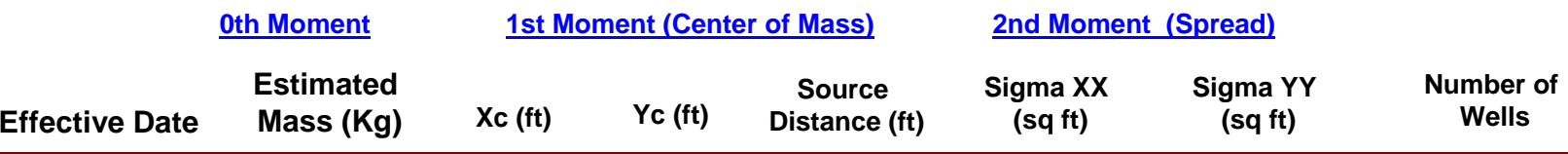

2nd Moment (Spread)

GROSS ALPHA ACTIVITY

$\begin{array}{lrrrrrrr}7 / 1 / 2004 & 2.1 \mathrm{E}+02 & 48,474 & 29,397 & 724 & 242,112 & 55,816 & 10 \\ 7 / 1 / 2005 & 5.9 \mathrm{E}+02 & 48,055 & 29,423 & 1,137 & 107,373 & 16,164 & 19 \\ 7 / 1 / 2006 & 2.1 \mathrm{E}+01 & 47,596 & 29,371 & 1,583 & 380,264 & 20,395 & 10 \\ 7 / 1 / 2007 & 1.4 \mathrm{E}+02 & 47,429 & 29,150 & 1,741 & 77,235 & 10,080 & 12\end{array}$

NITRATE

$\begin{array}{llllllll}7 / 1 / 2004 & 6.5 \mathrm{E}+03 & 48,795 & 29,590 & 544 & 150,060 & 37,737 & 10 \\ 7 / 1 / 2005 & 3.4 \mathrm{E}+03 & 48,928 & 29,613 & 483 & 174,313 & 54,798 & 18 \\ 7 / 1 / 2006 & 4.1 \mathrm{E}+03 & 48,855 & 29,605 & 516 & 156,133 & 35,210 & 10 \\ 7 / 1 / 2007 & 4.6 \mathrm{E}+03 & 48,769 & 29,548 & 533 & 196,118 & 54,482 & 13\end{array}$

TETRACHLOROETHYLENE(PCE)

$\begin{array}{rrrrrrrr}7 / 1 / 2004 & 2.6 \mathrm{E}-01 & 48,033 & 29,633 & 1,217 & 212,173 & 145,823 & 23 \\ 7 / 1 / 2005 & 4.6 \mathrm{E}-01 & 48,109 & 29,598 & 1,134 & 198,793 & 119,252 & 31 \\ 7 / 1 / 2006 & 6.5 \mathrm{E}-01 & 48,141 & 29,594 & 1,103 & 163,154 & 81,238 & 23 \\ 7 / 1 / 2007 & 4.5 \mathrm{E}-01 & 48,100 & 29,612 & 1,147 & 209,031 & 112,088 & 26\end{array}$

TRICHLOROETHYLENE (TCE)

\begin{tabular}{rrrrrrrr}
\hline $7 / 1 / 2004$ & $7.7 \mathrm{E}+00$ & 48,183 & 29,341 & 997 & 167,152 & 29,903 & 23 \\
$7 / 1 / 2005$ & $4.7 \mathrm{E}+00$ & 48,025 & 29,227 & 1,145 & 320,796 & 50,988 & 31 \\
$7 / 1 / 2006$ & $5.2 \mathrm{E}+00$ & 48,128 & 29,337 & 1,051 & 220,187 & 34,817 & 23 \\
$7 / 1 / 2007$ & $4.9 \mathrm{E}+00$ & 48,060 & 29,260 & 1,110 & 260,854 & 43,244 & 26
\end{tabular}

VINYL CHLORIDE

$\begin{array}{lrrrrrrr}7 / 1 / 2004 & 1.3 \mathrm{E}-01 & 48,257 & 29,494 & 960 & 369,526 & 41,859 & 11 \\ 7 / 1 / 2005 & 2.1 \mathrm{E}-01 & 47,884 & 29,353 & 1,295 & 576,684 & 59,199 & 19 \\ 7 / 1 / 2006 & 1.3 \mathrm{E}-01 & 48,239 & 29,491 & 976 & 383,533 & 41,862 & 11 \\ 7 / 1 / 2007 & 4.1 \mathrm{E}-01 & 47,864 & 30,057 & 1,564 & 479,831 & 368,081 & 26\end{array}$


Project: BC EMWMF

Location: OLFA
User Name: MV

State: Tennessee

\begin{tabular}{llllll} 
Moment Type & Constituent & $\begin{array}{l}\text { Coefficient } \\
\text { of Variation }\end{array}$ & $\begin{array}{c}\text { Mann-Kendall } \\
\text { S Statistic }\end{array}$ & $\begin{array}{c}\text { Confidence } \\
\text { in Trend }\end{array}$ & $\begin{array}{c}\text { Moment } \\
\text { Trend }\end{array}$ \\
\hline
\end{tabular}

\section{Zeroth Moment: Mass}

GROSS ALPHA ACTIVITY
NITRATE
TETRACHLOROETHYLENE(PCE)
TRICHLOROETHYLENE (TCE)
VINYL CHLORIDE

1.02
0.29
0.35
0.24
0.61

$-2$

$62.5 \%$

NT

$0 \quad 37.5 \%$

$262.5 \%$

$62.5 \%$

$\mathrm{S}$

$-2$

$62.5 \%$

$83.3 \%$

NT

4

$\mathrm{S}$

1st Moment: Distance to Source

GROSS ALPHA ACTIVITY
NITRATE
TETRACHLOROETHYLENE(PCE)
TRICHLOROETHYLENE (TCE)
VINYL CHLORIDE

0.35
0.05
0.04
0.06
0.24

$95.8 \%$

$37.5 \% \quad \mathrm{~S}$

$62.5 \% \quad \mathrm{~S}$

$62.5 \% \quad$ NT

83.3\% NT

2nd Moment: Sigma XX

GROSS ALPHA ACTIVITY
NITRATE
TETRACHLOROETHYLENE(PCE)
TRICHLOROETHYLENE (TCE)
VINYL CHLORIDE

$\begin{array}{cccc}0.69 & -2 & 62.5 \% & \mathrm{~S} \\ 0.12 & 4 & 83.3 \% & \mathrm{NT} \\ 0.11 & -2 & 62.5 \% & \mathrm{~S} \\ 0.27 & 2 & 62.5 \% & \mathrm{NT} \\ 0.21 & 2 & 62.5 \% & \mathrm{NT}\end{array}$

2nd Moment: Sigma YY

GROSS ALPHA ACTIVITY
NITRATE
TETRACHLOROETHYLENE(PCE)
TRICHLOROETHYLENE (TCE)
VINYL CHLORIDE

$\begin{array}{lccc}0.80 & -4 & 83.3 \% & \mathrm{~S} \\ 0.23 & 0 & 37.5 \% & \mathrm{~S} \\ 0.23 & -4 & 83.3 \% & \mathrm{~S} \\ 0.23 & 2 & 62.5 \% & \mathrm{NT} \\ 1.26 & 4 & 83.3 \% & \mathrm{NT}\end{array}$

Note: The following assumptions were applied for the calculation of the Zeroth Moment:

Porosity: 0.10

Saturated Thickness: Uniform: $50 \mathrm{ft}$

Mann-Kendall Trend test performed on all sample events for each constituent. Increasing (I); Probably Increasing (PI); Stable (S); Probably Decreasing (PD); Decreasing (D); No Trend (NT); Not Applicable (N/A)-Due to insufficient Data (< 4 sampling events).

Note: The Sigma $X X$ and Sigma $Y Y$ components are estimated using the given field coordinate system and then rotated to align with the estimated groundwater flow direction. Moments are not calculated for sample events with less than 6 wells. 


\section{MAROS Statistical Trend Analysis Summary}

Project: $\quad$ BC EMWMF

Location: OLFA

Time Period: $1 / 1 / 1996$ to $7 / 15 / 2007$

Consolidation Period: No Time Consolidation

Consolidation Type: Geometric Mean

Duplicate Consolidation: Average

ND Values: Specified Detection Limit

J Flag Values : Actual Value
User Name: MV

State: Tennessee

\begin{tabular}{|c|c|c|c|c|c|c|c|c|}
\hline Well & $\begin{array}{c}\text { Sourcel } \\
\text { Tail }\end{array}$ & $\begin{array}{l}\text { Number } \\
\text { of } \\
\text { Samples }\end{array}$ & $\begin{array}{c}\text { Number } \\
\text { of } \\
\text { Detects }\end{array}$ & $\begin{array}{l}\text { Average } \\
\text { Conc. } \\
\text { (mg/L) }\end{array}$ & $\begin{array}{l}\text { Median } \\
\text { Conc. } \\
\text { (mg/L) }\end{array}$ & $\begin{array}{c}\text { All } \\
\text { Samples } \\
\text { "ND" ? }\end{array}$ & $\begin{array}{l}\text { Mann- } \\
\text { Kendall } \\
\text { Trend }\end{array}$ & $\begin{array}{l}\text { Linear } \\
\text { Regression } \\
\text { Trend }\end{array}$ \\
\hline \multicolumn{9}{|l|}{ NITRATE } \\
\hline GW-008 & $\mathrm{T}$ & 2 & 0 & $2.0 \mathrm{E}-02$ & $2.0 \mathrm{E}-02$ & Yes & ND & ND \\
\hline GW-085 & $\mathrm{T}$ & 23 & 23 & $1.3 \mathrm{E}+02$ & $1.0 \mathrm{E}+02$ & No & S & $\mathrm{s}$ \\
\hline GW-097 & $\mathrm{T}$ & 2 & 1 & $5.0 \mathrm{E}-02$ & $5.0 \mathrm{E}-02$ & No & N/A & N/A \\
\hline GW-098 & $\mathrm{T}$ & 8 & 0 & $2.0 \mathrm{E}-02$ & 2.0E-02 & Yes & ND & ND \\
\hline GW-225 & S & 14 & 14 & $3.8 \mathrm{E}+01$ & $3.8 \mathrm{E}+01$ & No & I & $\mathrm{PI}$ \\
\hline GW-226 & $\mathrm{T}$ & 19 & 19 & $1.6 \mathrm{E}+01$ & 1.4E+01 & No & NT & S \\
\hline GW-227 & $\mathrm{T}$ & 2 & 2 & $3.4 \mathrm{E}+00$ & $3.4 \mathrm{E}+00$ & No & N/A & N/A \\
\hline GW-229 & $S$ & 10 & 0 & 2.0E-02 & 2.0E-02 & Yes & ND & ND \\
\hline GW-363 & $\mathrm{T}$ & 4 & 1 & 2.2E-02 & 2.0E-02 & No & NT & NT \\
\hline GW-364 & $\mathrm{T}$ & 4 & 3 & 2. $8 \mathrm{E}-01$ & $1.5 \mathrm{E}-01$ & No & NT & D \\
\hline GW-365 & $\mathrm{T}$ & 4 & 0 & 2.0E-02 & 2.0E-02 & Yes & ND & ND \\
\hline GW-368 & $\mathrm{T}$ & 3 & 3 & $8.8 \mathrm{E}+00$ & $8.9 \mathrm{E}+00$ & No & N/A & N/A \\
\hline GW-369 & $\mathrm{T}$ & 1 & 1 & $9.3 E+00$ & $9.3 E+00$ & No & N/A & $N / A$ \\
\hline GW-537 & $\mathrm{T}$ & 21 & 21 & $6.3 E+02$ & $6.3 E+02$ & No & D & D \\
\hline GW-601 & $\mathrm{T}$ & 4 & 4 & $1.8 \mathrm{E}+01$ & $1.9 \mathrm{E}+01$ & No & NT & 1 \\
\hline GW-724 & $\mathrm{T}$ & 21 & 21 & $2.3 \mathrm{E}+01$ & $2.3 E+01$ & No & $\mathrm{D}$ & D \\
\hline GW-725 & S & 21 & 21 & $3.1 \mathrm{E}+01$ & $2.4 \mathrm{E}+01$ & No & $\mathrm{D}$ & $\mathrm{D}$ \\
\hline GW-736 & $\mathrm{T}$ & 4 & 4 & $1.4 \mathrm{E}+01$ & $1.3 \mathrm{E}+01$ & No & $\mathrm{D}$ & $\mathrm{D}$ \\
\hline GW-738 & $\mathrm{T}$ & 21 & 21 & $1.4 \mathrm{E}+01$ & $1.4 \mathrm{E}+01$ & No & s & $\mathrm{s}$ \\
\hline GW-739 & $\mathrm{T}$ & 4 & 4 & $1.5 \mathrm{E}+00$ & $1.5 \mathrm{E}+00$ & No & NT & NT \\
\hline GW-740 & $\mathrm{T}$ & 21 & 21 & $2.5 \mathrm{E}+00$ & $2.3 \mathrm{E}+00$ & No & D & $\mathrm{D}$ \\
\hline
\end{tabular}

TETRACHLOROETHYLENE(PCE)

\begin{tabular}{|c|c|c|c|c|c|c|c|c|}
\hline GW-008 & $\mathrm{T}$ & 20 & 20 & 4.0E-02 & 3.7E-02 & No & I & 1 \\
\hline GW-085 & $\mathrm{T}$ & 24 & 0 & 5.0E-05 & 5.0E-05 & Yes & ND & ND \\
\hline GW-097 & $\mathrm{T}$ & 4 & 1 & 7.9E-04 & 5.0E-05 & No & NT & NT \\
\hline GW-098 & $\mathrm{T}$ & 8 & 0 & 5.0E-05 & 5.0E-05 & Yes & ND & ND \\
\hline GW-225 & $\mathrm{s}$ & 14 & 10 & 1.0E-03 & 1.0E-03 & No & 1 & 1 \\
\hline GW-226 & $\mathrm{T}$ & 20 & 4 & $2.9 \mathrm{E}-04$ & 5.0E-05 & No & $\mathrm{PI}$ & 1 \\
\hline GW-227 & $\mathrm{T}$ & 2 & 2 & 1.0E-03 & 1.0E-03 & No & N/A & $\mathrm{N} / \mathrm{A}$ \\
\hline GW-229 & $\mathrm{s}$ & 10 & 2 & 2.4E-04 & 5.0E-05 & No & NT & NT \\
\hline GW-363 & $\mathrm{T}$ & 26 & 0 & 5.0E-05 & 5.0E-05 & Yes & ND & ND \\
\hline GW-364 & $\mathrm{T}$ & 4 & 0 & 5.0E-05 & 5.0E-05 & Yes & ND & ND \\
\hline GW-365 & $\mathrm{T}$ & 4 & 0 & 5.0E-05 & 5.0E-05 & Yes & ND & ND \\
\hline GW-368 & $\mathrm{T}$ & 3 & 0 & $5.0 \mathrm{E}-05$ & $5.0 \mathrm{E}-05$ & Yes & ND & ND \\
\hline
\end{tabular}




\section{MAROS Statistical Trend Analysis Summary}

\begin{tabular}{ccccccccc} 
& & Number & Number & Average & Median & All & Mann- & Linear \\
Well & Sourcel & of & of & Conc. & Conc. & Samples & Kendall & Regression \\
Tail & Samples & Detects & (mg/L) & (mg/L) & "ND" ? & Trend & Trend \\
\hline
\end{tabular}

TETRACHLOROETHYLENE(PCE)

GW-537

GW-601

GW-724

GW-725

GW-736

GW-738

GW-739

GW-740

GW-916

GW-917

GW-918

GW-920

GW-921

GW-922

GW-923

GW-924

GW-925

GW-926

GW-927

\section{E)}

T

$\mathrm{T}$

1
22
4
22
22
4
22
4
22
22
22
22
22
22
22
16
23
22
22
22

TRICHLOROETHYLENE (TCE)

\begin{tabular}{|c|c|c|c|c|c|c|c|c|}
\hline GW-008 & $T$ & 20 & 20 & $9.5 \mathrm{E}-03$ & $9.5 \mathrm{E}-03$ & No & 1 & 1 \\
\hline GW-085 & $\mathrm{T}$ & 24 & 0 & 5.0E-05 & 5.0E-05 & Yes & ND & ND \\
\hline GW-097 & $T$ & 4 & 0 & 5.0E-05 & 5.0E-05 & Yes & ND & ND \\
\hline GW-098 & $\mathrm{T}$ & 8 & 8 & $7.9 \mathrm{E}-03$ & 7.0E-03 & No & NT & NT \\
\hline GW-225 & s & 14 & 14 & 2.3E-01 & 2.3E-01 & No & 1 & 1 \\
\hline GW-226 & $\mathrm{T}$ & 20 & 20 & 1.3E-01 & 1.3E-01 & No & S & $\mathrm{s}$ \\
\hline GW-227 & $\mathrm{T}$ & 2 & 2 & 1.7E-02 & 1.7E-02 & No & N/A & $\mathrm{N} / \mathrm{A}$ \\
\hline GW-229 & $\mathrm{S}$ & 10 & 8 & 8.6E-03 & 8.5E-03 & No & NT & I \\
\hline GW-363 & $\mathrm{T}$ & 26 & 0 & 5.0E-05 & 5.0E-05 & Yes & ND & ND \\
\hline GW-364 & $\mathrm{T}$ & 4 & 4 & $6.5 \mathrm{E}-03$ & $6.0 \mathrm{E}-03$ & No & S & $\mathrm{s}$ \\
\hline GW-365 & $\mathrm{T}$ & 4 & 4 & $2.8 \mathrm{E}-02$ & 2.7E-02 & No & S & $\mathrm{D}$ \\
\hline GW-368 & $\mathrm{T}$ & 3 & 3 & $5.2 \mathrm{E}-02$ & $5.0 \mathrm{E}-02$ & No & N/A & N/A \\
\hline GW-369 & $\mathrm{T}$ & 1 & 1 & 4.6E-02 & 4.6E-02 & No & N/A & $\mathrm{N} / \mathrm{A}$ \\
\hline GW-537 & $\mathrm{T}$ & 22 & 0 & $5.0 \mathrm{E}-05$ & 5.0E-05 & Yes & ND & ND \\
\hline GW-601 & $\mathrm{T}$ & 4 & 4 & 8.5E-02 & 8.5E-02 & No & $\mathrm{s}$ & $\mathrm{s}$ \\
\hline GW-724 & $\mathrm{T}$ & 22 & 22 & $9.9 \mathrm{E}-02$ & 1.0E-01 & No & $\mathrm{D}$ & NT \\
\hline GW-725 & $\mathrm{S}$ & 22 & 22 & 4.7E-02 & 1.8E-02 & No & NT & NT \\
\hline GW-736 & $\mathrm{T}$ & 4 & 4 & 8.0E-03 & 8.0E-03 & No & S & $S$ \\
\hline GW-738 & $\mathrm{T}$ & 22 & 22 & 3.1E-02 & 3.0E-02 & No & $\mathrm{D}$ & $\mathrm{D}$ \\
\hline GW-739 & $\mathrm{T}$ & 4 & 4 & 3.4E-02 & 3.5E-02 & No & S & $\mathrm{D}$ \\
\hline GW-740 & $\mathrm{T}$ & 22 & 22 & 5.7E-02 & 5.7E-02 & No & $\mathrm{D}$ & $\mathrm{D}$ \\
\hline GW-916 & $\mathrm{T}$ & 22 & 2 & 5.1E-04 & 5.0E-05 & No & NT & NT \\
\hline GW-917 & $\mathrm{T}$ & 22 & 1 & $6.6 \mathrm{E}-05$ & $5.0 \mathrm{E}-05$ & No & NT & NT \\
\hline GW-918 & $\mathrm{T}$ & 22 & 2 & $1.5 \mathrm{E}-04$ & $5.0 \mathrm{E}-05$ & No & NT & NT \\
\hline
\end{tabular}




\section{MAROS Statistical Trend Analysis Summary}

\begin{tabular}{ccccccccc} 
& & Number & Number & Average & Median & All & Mann- & Linear \\
Well & Sourcel & of & of & Conc. & Conc. & Samples & Kendall & Regression \\
Tail & Samples & Detects & (mg/L) & (mg/L) & "ND" ? & Trend & Trend \\
\hline
\end{tabular}

TRICHLOROETHYLENE (TCE)

$\begin{array}{llllllllc}\text { GW-920 } & \text { T } & 22 & 0 & 5.0 E-05 & 5.0 E-05 & \text { Yes } & \text { ND } & \text { ND } \\ \text { GW-921 } & \text { T } & 22 & 1 & 5.2 E-05 & 5.0 E-05 & \text { No } & \text { S } & \text { ND } \\ \text { GW-922 } & \text { T } & 22 & 0 & 5.0 E-05 & 5.0 E-05 & \text { Yes } & \text { ND } \\ \text { GW-923 } & \text { T } & 16 & 1 & 1.7 E-04 & 5.0 E-05 & \text { No } & \text { ND } & \text { NT } \\ \text { GW-924 } & \text { T } & 23 & 0 & 5.0 E-05 & 5.0 E-05 & \text { Yes } & \text { ND } \\ \text { GW-925 } & \text { T } & 22 & 2 & 6.4 E-05 & 5.0 E-05 & \text { No } & \text { NT } & \text { NT } \\ \text { GW-926 } & \text { T } & 22 & 1 & 2.3 E-04 & 5.0 E-05 & \text { No } & \text { NT } & \text { NT } \\ \text { GW-927 } & \text { T } & 22 & 0 & 5.0 E-05 & 5.0 E-05 & \text { Yes } & \text { ND } & \text { ND }\end{array}$

URANIUM

\begin{tabular}{|c|c|c|c|c|c|c|c|c|}
\hline GW-008 & $\mathrm{T}$ & 6 & 2 & $2.0 \mathrm{E}-05$ & 4.6E-06 & No & NT & 1 \\
\hline GW-085 & $\mathrm{T}$ & 6 & 0 & 4.6E-06 & 4.6E-06 & Yes & ND & ND \\
\hline GW-097 & $\mathrm{T}$ & 2 & 2 & $6.4 \mathrm{E}-04$ & $6.4 \mathrm{E}-04$ & No & $\mathrm{N} / \mathrm{A}$ & N/A \\
\hline GW-098 & $\mathrm{T}$ & 4 & 4 & $1.5 \mathrm{E}-03$ & $1.5 \mathrm{E}-03$ & No & s & PD \\
\hline GW-225 & $\mathrm{S}$ & 6 & 6 & $2.9 \mathrm{E}-03$ & $2.9 \mathrm{E}-03$ & No & NT & $\mathrm{s}$ \\
\hline GW-226 & $\mathrm{T}$ & 6 & 6 & 7.6E-03 & $6.8 \mathrm{E}-03$ & No & s & $\mathrm{s}$ \\
\hline GW-227 & $\mathrm{T}$ & 2 & 2 & $5.3 \mathrm{E}-02$ & 5.3E-02 & No & $\mathrm{N} / \mathrm{A}$ & $\mathrm{N} / \mathrm{A}$ \\
\hline GW-229 & $\mathrm{S}$ & 4 & 4 & $1.7 \mathrm{E}-01$ & 1.7E-01 & No & NT & NT \\
\hline GW-363 & $\mathrm{T}$ & 6 & 0 & 4.6E-06 & 4.6E-06 & Yes & ND & ND \\
\hline GW-364 & $\mathrm{T}$ & 2 & 2 & $1.9 \mathrm{E}-03$ & $1.9 \mathrm{E}-03$ & No & N/A & $N / A$ \\
\hline GW-365 & $\mathrm{T}$ & 2 & 2 & $8.1 \mathrm{E}-04$ & 8.1E-04 & No & N/A & $\mathrm{N} / \mathrm{A}$ \\
\hline GW-368 & $\mathrm{T}$ & 3 & 3 & $1.7 \mathrm{E}-03$ & 1.6E-03 & No & $\mathrm{N} / \mathrm{A}$ & N/A \\
\hline GW-369 & $\mathrm{T}$ & 1 & 0 & 4.6E-06 & 4.6E-06 & Yes & ND & $N D$ \\
\hline GW-537 & $\mathrm{T}$ & 4 & 4 & $1.2 \mathrm{E}-03$ & $1.2 \mathrm{E}-03$ & No & s & $\mathrm{s}$ \\
\hline GW-601 & $\mathrm{T}$ & 3 & 0 & 4.6E-06 & 4.6E-06 & Yes & ND & $N D$ \\
\hline GW-724 & $\mathrm{T}$ & 4 & 3 & $5.5 \mathrm{E}-04$ & $6.8 \mathrm{E}-04$ & No & s & $\mathrm{s}$ \\
\hline GW-725 & $\mathrm{S}$ & 4 & 4 & $1.0 \mathrm{E}-02$ & 1.1E-02 & No & NT & NT \\
\hline GW-736 & $\mathrm{T}$ & 2 & 2 & $3.1 \mathrm{E}-02$ & 3.1E-02 & No & N/A & $N / A$ \\
\hline GW-738 & $\mathrm{T}$ & 4 & 4 & $2.6 \mathrm{E}-03$ & 2.6E-03 & No & NT & NT \\
\hline GW-739 & $\mathrm{T}$ & 2 & 2 & $6.2 \mathrm{E}-04$ & $6.2 \mathrm{E}-04$ & No & $\mathrm{N} / \mathrm{A}$ & $N / A$ \\
\hline GW-740 & $\mathrm{T}$ & 4 & 0 & 4.6E-06 & 4.6E-06 & Yes & ND & ND \\
\hline GW-916 & $\mathrm{T}$ & 6 & 1 & $6.5 \mathrm{E}-04$ & 4.6E-06 & No & NT & PD \\
\hline GW-917 & $\mathrm{T}$ & 6 & 0 & 4.6E-06 & 4.6E-06 & Yes & ND & ND \\
\hline GW-918 & $\mathrm{T}$ & 6 & 0 & 4.6E-06 & 4.6E-06 & Yes & ND & $N D$ \\
\hline GW-920 & $\mathrm{T}$ & 6 & 2 & $1.1 \mathrm{E}-03$ & 4.6E-06 & No & NT & $\mathrm{PD}$ \\
\hline GW-921 & $\mathrm{T}$ & 6 & 1 & $1.2 \mathrm{E}-03$ & 4.6E-06 & No & NT & PD \\
\hline GW-922 & $\mathrm{T}$ & 6 & 1 & $7.2 \mathrm{E}-04$ & 4.6E-06 & No & NT & PD \\
\hline GW-923 & $\mathrm{T}$ & 4 & 1 & $1.6 \mathrm{E}-03$ & 4.6E-06 & No & NT & PD \\
\hline GW-924 & $\mathrm{T}$ & 6 & 0 & 4.6E-06 & 4.6E-06 & Yes & ND & $N D$ \\
\hline GW-925 & $\mathrm{T}$ & 6 & 1 & $9.0 \mathrm{E}-04$ & 4.6E-06 & No & NT & NT \\
\hline GW-926 & $\mathrm{T}$ & 6 & 3 & $1.7 \mathrm{E}-03$ & 1.1E-03 & No & NT & $\mathrm{D}$ \\
\hline GW-927 & $\mathrm{T}$ & 6 & 0 & 4.6E-06 & 4.6E-06 & Yes & ND & ND \\
\hline \multicolumn{9}{|c|}{ VINYL CHLORIDE } \\
\hline GW-008 & $\mathrm{T}$ & 20 & 0 & $5.0 \mathrm{E}-04$ & $5.0 \mathrm{E}-04$ & Yes & ND & $N D$ \\
\hline GW-085 & $\mathrm{T}$ & 24 & 0 & $5.0 \mathrm{E}-04$ & $5.0 \mathrm{E}-04$ & Yes & ND & ND \\
\hline
\end{tabular}




\section{MAROS Statistical Trend Analysis Summary}

\begin{tabular}{|c|c|c|c|c|c|c|c|c|}
\hline Well & $\begin{array}{c}\text { Sourcel } \\
\text { Tail }\end{array}$ & $\begin{array}{c}\text { Number } \\
\text { of } \\
\text { Samples }\end{array}$ & $\begin{array}{l}\text { Number } \\
\text { of } \\
\text { Detects }\end{array}$ & $\begin{array}{l}\text { Average } \\
\text { Conc. } \\
\text { (mg/L) }\end{array}$ & $\begin{array}{l}\text { Median } \\
\text { Conc. } \\
\text { (mg/L) }\end{array}$ & $\begin{array}{c}\text { All } \\
\text { Samples } \\
\text { "ND" ? }\end{array}$ & $\begin{array}{c}\text { Mann- } \\
\text { Kendall } \\
\text { Trend }\end{array}$ & $\begin{array}{c}\text { Linear } \\
\text { Regression } \\
\text { Trend }\end{array}$ \\
\hline \multicolumn{9}{|c|}{ VINYL CHLORIDE } \\
\hline GW-097 & $\mathrm{T}$ & 4 & 0 & $5.0 \mathrm{E}-04$ & $5.0 \mathrm{E}-04$ & Yes & ND & ND \\
\hline GW-098 & $\mathrm{T}$ & 8 & 0 & $5.0 \mathrm{E}-04$ & $5.0 \mathrm{E}-04$ & Yes & ND & ND \\
\hline GW-225 & s & 14 & 0 & $5.0 \mathrm{E}-04$ & $5.0 \mathrm{E}-04$ & Yes & ND & ND \\
\hline GW-226 & $T$ & 20 & 0 & $5.0 \mathrm{E}-04$ & $5.0 \mathrm{E}-04$ & Yes & ND & ND \\
\hline GW-227 & $\mathrm{T}$ & 2 & 0 & $5.0 \mathrm{E}-04$ & $5.0 \mathrm{E}-04$ & Yes & ND & ND \\
\hline GW-229 & s & 10 & 10 & 3.3E-02 & $3.1 \mathrm{E}-02$ & No & NT & $\mathrm{s}$ \\
\hline GW-363 & $\mathrm{T}$ & 11 & 0 & $5.0 \mathrm{E}-04$ & $5.0 \mathrm{E}-04$ & Yes & ND & ND \\
\hline GW-364 & $\mathrm{T}$ & 4 & 0 & $5.0 \mathrm{E}-04$ & $5.0 \mathrm{E}-04$ & Yes & ND & ND \\
\hline GW-365 & $\mathrm{T}$ & 4 & 3 & $3.1 \mathrm{E}-03$ & $3.5 \mathrm{E}-03$ & No & NT & $\mathrm{PI}$ \\
\hline GW-368 & $\mathrm{T}$ & 3 & 0 & $5.0 \mathrm{E}-04$ & $5.0 \mathrm{E}-04$ & Yes & ND & ND \\
\hline GW-369 & $\mathrm{T}$ & 1 & 0 & 5.0E-04 & $5.0 \mathrm{E}-04$ & Yes & ND & ND \\
\hline GW-537 & $\mathrm{T}$ & 22 & 0 & $5.0 \mathrm{E}-04$ & $5.0 \mathrm{E}-04$ & Yes & ND & ND \\
\hline GW-601 & $\mathrm{T}$ & 4 & 0 & $5.0 \mathrm{E}-04$ & $5.0 \mathrm{E}-04$ & Yes & ND & ND \\
\hline GW-724 & $\mathrm{T}$ & 22 & 0 & $5.0 \mathrm{E}-04$ & $5.0 \mathrm{E}-04$ & Yes & ND & ND \\
\hline GW-725 & S & 22 & 0 & 5.0E-04 & $5.0 \mathrm{E}-04$ & Yes & ND & ND \\
\hline GW-736 & $\mathrm{T}$ & 4 & 0 & $5.0 \mathrm{E}-04$ & $5.0 \mathrm{E}-04$ & Yes & ND & ND \\
\hline GW-738 & $T$ & 22 & 0 & 5.0E-04 & $5.0 \mathrm{E}-04$ & Yes & ND & ND \\
\hline GW-739 & $\mathrm{T}$ & 4 & 0 & $5.0 \mathrm{E}-04$ & $5.0 \mathrm{E}-04$ & Yes & ND & ND \\
\hline GW-740 & $\mathrm{T}$ & 22 & 0 & $5.0 \mathrm{E}-04$ & $5.0 \mathrm{E}-04$ & Yes & ND & ND \\
\hline GW-916 & $\mathrm{T}$ & 7 & 0 & $5.0 \mathrm{E}-04$ & $5.0 \mathrm{E}-04$ & Yes & ND & ND \\
\hline GW-917 & $\mathrm{T}$ & 7 & 0 & $5.0 \mathrm{E}-04$ & $5.0 \mathrm{E}-04$ & Yes & ND & ND \\
\hline GW-918 & $\mathrm{T}$ & 7 & 0 & $5.0 \mathrm{E}-04$ & $5.0 \mathrm{E}-04$ & Yes & ND & ND \\
\hline GW-920 & $\mathrm{T}$ & 7 & 0 & $5.0 \mathrm{E}-04$ & $5.0 \mathrm{E}-04$ & Yes & ND & ND \\
\hline GW-921 & $\mathrm{T}$ & 7 & 0 & $5.0 \mathrm{E}-04$ & $5.0 \mathrm{E}-04$ & Yes & ND & ND \\
\hline GW-922 & $\mathrm{T}$ & 7 & 0 & $5.0 \mathrm{E}-04$ & $5.0 \mathrm{E}-04$ & Yes & ND & ND \\
\hline GW-923 & $\mathrm{T}$ & 6 & 0 & $5.0 \mathrm{E}-04$ & $5.0 \mathrm{E}-04$ & Yes & ND & ND \\
\hline GW-924 & $T$ & 8 & 0 & $5.0 \mathrm{E}-04$ & $5.0 \mathrm{E}-04$ & Yes & ND & ND \\
\hline GW-925 & $T$ & 7 & 0 & $5.0 \mathrm{E}-04$ & $5.0 \mathrm{E}-04$ & Yes & ND & ND \\
\hline GW-926 & $\mathrm{T}$ & 7 & 0 & $5.0 \mathrm{E}-04$ & $5.0 \mathrm{E}-04$ & Yes & ND & ND \\
\hline GW-927 & $\mathrm{T}$ & 7 & 0 & $5.0 \mathrm{E}-04$ & $5.0 \mathrm{E}-04$ & Yes & ND & ND \\
\hline
\end{tabular}

Note: Increasing (I); Probably Increasing (PI); Stable (S); Probably Decreasing (PD); Decreasing (D); No Trend (NT); Not Applicable (N/A); Not Applicable (N/A) - Due to insufficient Data (< 4 sampling events); No Detectable Concentration (NDC)

The Number of Samples and Number of Detects shown above are post-consolidation values. 


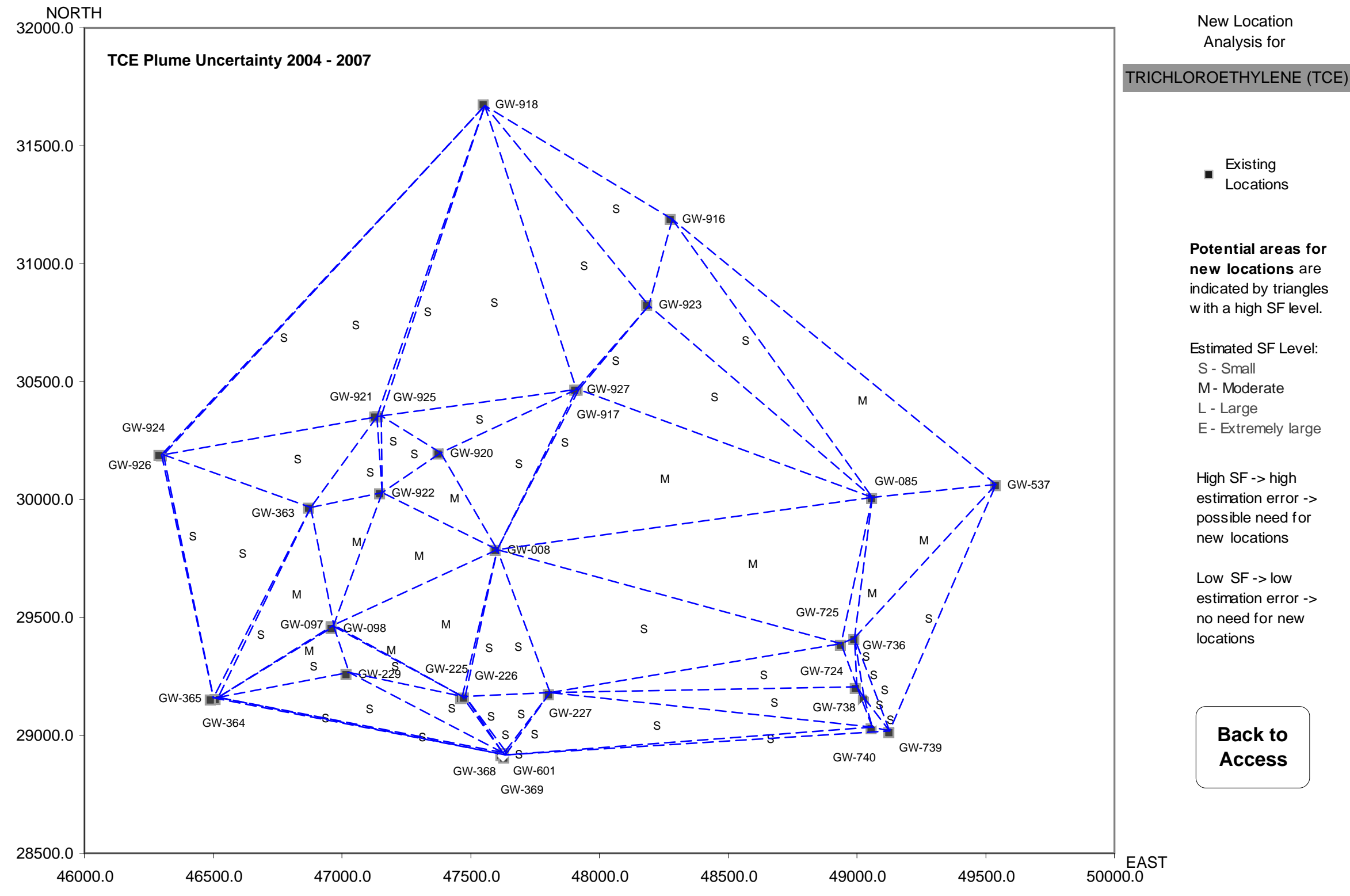


APPENDIX C.3

BEAR CREEK BURIAL GROUNDS WMA 


\section{MAROS COC Assessment}

Project: $\quad$ BC Burial Grounds

Location: Bear Creek Burial Grounds

Toxicity:

\begin{tabular}{lccc}
\hline Contaminant of Concern & $\begin{array}{c}\text { Representative } \\
\text { Concentration } \\
\mathbf{( m g / L )}\end{array}$ & $\begin{array}{c}\text { PRG } \\
\mathbf{( m g / L )}\end{array}$ & $\begin{array}{c}\text { Percent } \\
\text { Above } \\
\text { PRG }\end{array}$ \\
\hline TETRACHLOROETHYLENE(PCE) & $7.1 \mathrm{E}-01$ & $5.0 \mathrm{E}-03$ & $14080.5 \%$ \\
TRICHLOROETHYLENE (TCE) & $3.9 \mathrm{E}-01$ & $5.0 \mathrm{E}-03$ & $7765.4 \%$ \\
VINYL CHLORIDE & $6.7 \mathrm{E}-02$ & $2.0 \mathrm{E}-03$ & $3232.9 \%$ \\
BENZENE & $3.2 \mathrm{E}-02$ & $5.0 \mathrm{E}-03$ & $532.7 \%$ \\
cis-1,2-DICHLOROETHYLENE & $2.6 \mathrm{E}-01$ & $7.0 \mathrm{E}-02$ & $265.6 \%$ \\
1,1-DICHLOROETHENE & $2.4 \mathrm{E}-02$ & $7.0 \mathrm{E}-03$ & $249.7 \%$ \\
CARBON TETRACHLORIDE & $1.2 \mathrm{E}-02$ & $5.0 \mathrm{E}-03$ & $144.1 \%$ \\
1,2-DICHLOROETHANE & $1.2 \mathrm{E}-02$ & $5.0 \mathrm{E}-03$ & $143.4 \%$ \\
1,1-DICHLOROETHANE & $1.1 \mathrm{E}-01$ & $8.1 \mathrm{E}-02$ & $34.5 \%$
\end{tabular}

Note: Top COCs by toxicity were determined by examining a representative concentration for each compound over the entire site. The compound representative concentrations are then compared with the chosen PRG for that compound, with the percentage exceedance from the PRG determining the compound's toxicity. All compounds above exceed the PRG.

\section{Prevalence:}

\begin{tabular}{|c|c|c|c|c|c|}
\hline Contaminant of Concern & Class & $\begin{array}{l}\text { Total } \\
\text { Wells }\end{array}$ & $\begin{array}{c}\text { Total } \\
\text { Exceedances }\end{array}$ & $\begin{array}{c}\text { Percent } \\
\text { Exceedances }\end{array}$ & $\begin{array}{c}\text { Total } \\
\text { detects }\end{array}$ \\
\hline TRICHLOROETHYLENE (TCE) & ORG & 41 & 17 & $41.5 \%$ & 29 \\
\hline VINYL CHLORIDE & ORG & 41 & 16 & $39.0 \%$ & 16 \\
\hline TETRACHLOROETHYLENE(PCE) & ORG & 41 & 13 & $31.7 \%$ & 25 \\
\hline 1,1-DICHLOROETHENE & ORG & 41 & 11 & $26.8 \%$ & 19 \\
\hline BENZENE & ORG & 41 & 9 & $22.0 \%$ & 22 \\
\hline cis-1,2-DICHLOROETHYLENE & ORG & 41 & 8 & $19.5 \%$ & 26 \\
\hline 1,1-DICHLOROETHANE & ORG & 41 & 7 & $17.1 \%$ & 19 \\
\hline 1,2-DICHLOROETHANE & ORG & 41 & 6 & $14.6 \%$ & 10 \\
\hline CARBON TETRACHLORIDE & ORG & 41 & 5 & $12.2 \%$ & 11 \\
\hline
\end{tabular}

Note: Top COCs by prevalence were determined by examining a representative concentration for each well location at the site. The total exceedances (values above the chosen PRGs) are compared to the total number of wells to determine the prevalence of the compound.

\section{Mobility:}

Contaminant of Concern

VINYL CHLORIDE

1,1-DICHLOROETHANE

1,2-DICHLOROETHANE

cis-1,2-DICHLOROETHYLENE

BENZENE

1,1-DICHLOROETHENE

CARBON TETRACHLORIDE

TRICHLOROETHYLENE (TCE)

TETRACHLOROETHYLENE(PCE)
Kd
0.042
0.0573
0.0679
0.0724
0.0984
0.13
0.277
0.297
0.923 
Project: BC Burial Grounds

Location: Bear Creek Burial Grounds
User Name: MV

State: Tennessee

Note: Top COCs by mobility were determined by examining each detected compound in the dataset and comparing their mobilities (Koc's for organics, assume foc $=0.001$, and Kd's for metals).

\section{Contaminants of Concern (COC's)}

\section{TETRACHLOROETHYLENE(PCE)}

TRICHLOROETHYLENE (TCE)

VINYL CHLORIDE

BENZENE

1,1-DICHLOROETHENE 


\section{MAROS Spatial Moment Analysis Summary}

Project: $\quad$ BC Burial Ground

Location: Bear Creek Burial Grounds
User Name: MV

State: Tennessee 2nd Moment (Spread)

0th Moment

1st Moment (Center of Mass)
Estimated

Mass $(\mathrm{Kg})$

Source

Effective Date

GROSS ALPHA ACTIVITY

\begin{tabular}{|c|c|c|c|c|c|c|c|}
\hline 7/1/1996 & $1.1 \mathrm{E}+04$ & 41,134 & 29,089 & 2,201 & $3,049,075$ & 115,977 & 17 \\
\hline 7/1/1997 & $4.6 \mathrm{E}+03$ & 41,259 & 28,829 & 2,153 & $6,117,309$ & 129,101 & 24 \\
\hline 7/1/1998 & $7.9 \mathrm{E}+03$ & 39,613 & 29,132 & 3,696 & $14,187,110$ & 256,925 & 32 \\
\hline 7/1/1999 & $1.1 \mathrm{E}+04$ & 39,168 & 29,107 & 4,141 & $12,757,027$ & 98,789 & 21 \\
\hline $7 / 1 / 2000$ & $4.8 \mathrm{E}+03$ & 41,654 & 28,808 & 1,796 & $11,663,396$ & 84,297 & 24 \\
\hline 7/1/2001 & $4.1 \mathrm{E}+03$ & 42,121 & 28,845 & 1,367 & $5,170,597$ & 98,504 & 22 \\
\hline $7 / 1 / 2002$ & $9.1 \mathrm{E}+03$ & 42,879 & 28,930 & 751 & $1,526,762$ & 79,578 & 26 \\
\hline $7 / 1 / 2003$ & $4.8 \mathrm{E}+03$ & 42,300 & 28,900 & 1,186 & $2,315,758$ & 97,143 & 19 \\
\hline $7 / 1 / 2004$ & $5.8 \mathrm{E}+03$ & 41,451 & 28,994 & 1,920 & $2,753,573$ & 87,936 & 15 \\
\hline $7 / 1 / 2005$ & $9.3 \mathrm{E}+02$ & 42,549 & 28,884 & 1,001 & $3,992,575$ & 99,284 & 28 \\
\hline $7 / 1 / 2006$ & $9.8 \mathrm{E}+02$ & 41,865 & 28,893 & 1,569 & $4,811,866$ & 80,278 & 24 \\
\hline $7 / 1 / 2007$ & $1.8 \mathrm{E}+03$ & 42,673 & 28,781 & 992 & $1,585,966$ & 45,727 & 23 \\
\hline
\end{tabular}

TETRACHLOROETHYLENE(PCE)

\begin{tabular}{|c|c|c|c|c|c|c|c|}
\hline 7/1/1996 & $1.2 \mathrm{E}+00$ & 41,670 & 29,202 & 1,654 & $2,830,090$ & 134,905 & 18 \\
\hline $7 / 1 / 1997$ & $1.9 \mathrm{E}+00$ & 38,378 & 29,235 & 4,917 & $13,306,682$ & 172,560 & 25 \\
\hline 7/1/1998 & $8.2 \mathrm{E}+00$ & 41,755 & 29,866 & 1,559 & $7,075,371$ & 279,412 & 32 \\
\hline 7/1/1999 & $8.2 \mathrm{E}+00$ & 41,965 & 29,585 & 1,319 & $7,652,905$ & 160,136 & 26 \\
\hline $7 / 1 / 2000$ & $6.8 \mathrm{E}+00$ & 41,699 & 29,445 & 1,589 & $8,336,983$ & 113,558 & 24 \\
\hline $7 / 1 / 2001$ & $7.2 \mathrm{E}+00$ & 41,835 & 29,528 & 1,449 & $8,172,749$ & 121,125 & 24 \\
\hline $7 / 1 / 2002$ & $3.9 \mathrm{E}+01$ & 42,885 & 29,752 & 442 & $1,750,761$ & 90,430 & 28 \\
\hline $7 / 1 / 2003$ & $1.2 \mathrm{E}+01$ & 42,313 & 29,461 & 976 & $5,540,474$ & 130,122 & 23 \\
\hline $7 / 1 / 2004$ & $4.5 \mathrm{E}+01$ & 43,245 & 29,671 & 115 & 920,297 & 38,165 & 24 \\
\hline $7 / 1 / 2005$ & $4.0 \mathrm{E}+01$ & 43,127 & 29,796 & 282 & $1,027,640$ & 80,603 & 34 \\
\hline $7 / 1 / 2006$ & $6.1 \mathrm{E}+01$ & 42,987 & 29,613 & 302 & 655,905 & 64,956 & 31 \\
\hline $7 / 1 / 2007$ & $5.7 \mathrm{E}+01$ & 43,062 & 29,730 & 278 & 295,286 & 46,091 & 31 \\
\hline
\end{tabular}

TRICHLOROETHYLENE (TCE)

\begin{tabular}{rrrrrrrr}
\hline $7 / 1 / 1996$ & $1.8 \mathrm{E}+00$ & 42,356 & 29,125 & 1,026 & $2,887,021$ & 125,444 \\
$7 / 1 / 1997$ & $2.3 \mathrm{E}+00$ & 39,169 & 29,134 & 4,137 & $14,557,612$ & 153,735 \\
$7 / 1 / 1998$ & $1.3 \mathrm{E}+01$ & 42,788 & 29,492 & 501 & $5,776,002$ & 187,810 \\
$7 / 1 / 1999$ & $1.8 \mathrm{E}+01$ & 42,989 & 29,559 & 295 & $4,566,656$ & 162,435 & 32 \\
$7 / 1 / 2000$ & $1.1 \mathrm{E}+01$ & 42,632 & 29,297 & 704 & $6,167,351$ & 97,077 & 26 \\
$7 / 1 / 2001$ & $1.1 \mathrm{E}+01$ & 42,603 & 29,386 & 703 & $6,245,095$ & 108,856 \\
$7 / 1 / 2002$ & $2.3 \mathrm{E}+01$ & 42,985 & 29,510 & 303 & $3,139,120$ & 133,477 \\
$7 / 1 / 2003$ & $1.3 \mathrm{E}+01$ & 42,602 & 29,367 & 710 & $5,322,219$ & 115,353 \\
$7 / 1 / 2004$ & $8.1 \mathrm{E}+00$ & 42,733 & 29,408 & 572 & $4,317,234$ & 103,898 & 24 \\
$7 / 1 / 2005$ & $2.2 \mathrm{E}+01$ & 43,197 & 29,706 & 168 & $1,882,923$ & 118,790 \\
$7 / 1 / 2006$ & $1.8 \mathrm{E}+01$ & 43,077 & 29,494 & 218 & $2,454,621$ & 80,661 \\
\hline
\end{tabular}


Project: BC Burrial Ground

Location: Bear Creek Burial Grounds

\begin{tabular}{|c|c|c|c|c|c|c|c|}
\hline \multirow[b]{2}{*}{ Effective Date } & \multirow{2}{*}{$\begin{array}{l}\text { th Moment } \\
\text { Estimated } \\
\text { Mass (kg) }\end{array}$} & \multicolumn{3}{|c|}{ 1st Moment (Center of Mass) } & \multicolumn{2}{|c|}{ 2nd Moment (Spread) } & \multirow[b]{2}{*}{$\begin{array}{c}\text { Number of } \\
\text { Wells }\end{array}$} \\
\hline & & $\mathrm{Xc}(\mathrm{ft})$ & Yc (ft) & $\begin{array}{c}\text { Source } \\
\text { Distance (ft) }\end{array}$ & $\begin{array}{l}\text { Sigma } X X \\
(s q \mathrm{ft})\end{array}$ & $\begin{array}{l}\text { Sigma } Y Y \\
(s q \mathrm{ft})\end{array}$ & \\
\hline \multicolumn{8}{|c|}{ LOROETHYLENE (TCE) } \\
\hline $7 / 1 / 2007$ & $1.6 \mathrm{E}+01$ & 43,160 & 29,587 & 126 & 947,769 & 86,251 & 31 \\
\hline \multicolumn{8}{|l|}{ IUM } \\
\hline $7 / 1 / 2005$ & $2.3 E+00$ & 43,050 & 28,776 & 820 & $2,224,008$ & 47,465 & 35 \\
\hline $7 / 1 / 2006$ & $1.9 \mathrm{E}+00$ & 42,636 & 28,764 & 1,029 & $5,589,823$ & 59,898 & 33 \\
\hline $7 / 1 / 2007$ & $2.8 \mathrm{E}+00$ & 42,554 & 28,887 & 995 & $3,144,909$ & 38,522 & 29 \\
\hline
\end{tabular}

VINYL CHLORIDE

\begin{tabular}{|c|c|c|c|c|c|c|c|}
\hline 7/1/1996 & 9.0E-01 & 41,210 & 29,099 & 2,125 & $2,643,358$ & 141,754 & 18 \\
\hline 7/1/1997 & $2.1 \mathrm{E}+00$ & 38,747 & 29,262 & 4,546 & $13,886,210$ & 208,505 & 25 \\
\hline $7 / 1 / 1998$ & 8.7E+00 & 42,085 & 29,686 & 1,205 & $8,532,893$ & 220,956 & 32 \\
\hline 7/1/1999 & $1.1 \mathrm{E}+01$ & 42,216 & 29,825 & 1,100 & $6,842,224$ & 224,829 & 26 \\
\hline $7 / 1 / 2000$ & $3.5 \mathrm{E}+00$ & 40,647 & 29,298 & 2,650 & $13,502,526$ & 139,278 & 24 \\
\hline $7 / 1 / 2001$ & $1.2 \mathrm{E}+01$ & 42,392 & 29,706 & 903 & $5,549,706$ & 99,593 & 24 \\
\hline $7 / 1 / 2002$ & $5.9 \mathrm{E}+00$ & 41,719 & 29,540 & 1,565 & $9,214,245$ & 150,058 & 28 \\
\hline $7 / 1 / 2003$ & $1.6 \mathrm{E}+01$ & 42,551 & 29,733 & 752 & $4,308,663$ & 85,671 & 23 \\
\hline $7 / 1 / 2004$ & $3.4 \mathrm{E}+00$ & 41,418 & 29,430 & 1,870 & $7,130,354$ & 179,406 & 23 \\
\hline $7 / 1 / 2005$ & $1.2 \mathrm{E}+01$ & 42,887 & 29,739 & 435 & $3,324,239$ & 175,896 & 33 \\
\hline $7 / 1 / 2006$ & $6.6 \mathrm{E}+00$ & 42,592 & 29,495 & 695 & $5,261,212$ & 135,274 & 30 \\
\hline $7 / 1 / 2007$ & $5.0 \mathrm{E}+00$ & 42,879 & 29,727 & 437 & $2,389,302$ & 187,983 & 31 \\
\hline
\end{tabular}


Project: BC Burrial Ground

Location: Bear Creek Burial Grounds
User Name: $\mathrm{MV}$

State: Tennessee

\begin{tabular}{|c|c|c|c|c|c|}
\hline Moment Type & Constituent & $\begin{array}{l}\text { Coefficient } \\
\text { of Variation }\end{array}$ & $\begin{array}{l}\text { Mann-Kendall } \\
\text { S Statistic }\end{array}$ & $\begin{array}{l}\text { Confidence } \\
\text { in Trend }\end{array}$ & $\begin{array}{c}\text { Moment } \\
\text { Trend }\end{array}$ \\
\hline \multicolumn{6}{|c|}{ Zeroth Moment: Mass } \\
\hline & GROSS ALPHA ACTIVITY & 0.64 & -32 & $98.4 \%$ & $\mathrm{D}$ \\
\hline & TETRACHLOROETHYLENE(PCE) & 0.95 & 50 & $100.0 \%$ & 1 \\
\hline & TRICHLOROETHYLENE (TCE) & 0.52 & 24 & $94.2 \%$ & $\mathrm{PI}$ \\
\hline & URANIUM & 0.00 & 0 & $0.0 \%$ & $\mathrm{~N} / \mathrm{A}$ \\
\hline & VINYL CHLORIDE & 0.64 & 18 & $87.5 \%$ & NT \\
\hline \multicolumn{6}{|c|}{ 1st Moment: Distance to Source } \\
\hline & GROSS ALPHA ACTIVITY & 0.56 & -34 & $99.0 \%$ & $\mathrm{D}$ \\
\hline & TETRACHLOROETHYLENE(PCE) & 1.05 & -48 & $100.0 \%$ & $\mathrm{D}$ \\
\hline & TRICHLOROETHYLENE (TCE) & 1.38 & -36 & $99.3 \%$ & $\mathrm{D}$ \\
\hline & URANIUM & 0.00 & 0 & $0.0 \%$ & $\mathrm{~N} / \mathrm{A}$ \\
\hline & VINYL CHLORIDE & 0.77 & -38 & $99.6 \%$ & $\mathrm{D}$ \\
\hline \multicolumn{6}{|c|}{ 2nd Moment: Sigma XX } \\
\hline & GROSS ALPHA ACTIVITY & 0.77 & -24 & $94.2 \%$ & PD \\
\hline & TETRACHLOROETHYLENE(PCE) & 0.87 & -40 & $99.7 \%$ & $\mathrm{D}$ \\
\hline & TRICHLOROETHYLENE (TCE) & 0.72 & -32 & $98.4 \%$ & $\mathrm{D}$ \\
\hline & URANIUM & 0.00 & 0 & $0.0 \%$ & $\mathrm{~N} / \mathrm{A}$ \\
\hline & VINYL CHLORIDE & 0.56 & -26 & $95.7 \%$ & $\mathrm{D}$ \\
\hline \multicolumn{6}{|c|}{ 2nd Moment: Sigma YY } \\
\hline & GROSS ALPHA ACTIVITY & 0.49 & -36 & $99.3 \%$ & $\mathrm{D}$ \\
\hline & TETRACHLOROETHYLENE(PCE) & 0.55 & -44 & $99.9 \%$ & $\mathrm{D}$ \\
\hline & TRICHLOROETHYLENE (TCE) & 0.26 & -32 & $98.4 \%$ & $\mathrm{D}$ \\
\hline & URANIUM & 0.00 & 0 & $0.0 \%$ & $\mathrm{~N} / \mathrm{A}$ \\
\hline & VINYL CHLORIDE & 0.28 & -8 & $68.1 \%$ & $\mathrm{~S}$ \\
\hline
\end{tabular}

Note: The following assumptions were applied for the calculation of the Zeroth Moment:

Porosity: $0.10 \quad$ Saturated Thickness: Uniform: $50 \mathrm{ft}$

Mann-Kendall Trend test performed on all sample events for each constituent. Increasing (I); Probably Increasing (PI); Stable (S); Probably Decreasing (PD); Decreasing (D); No Trend (NT); Not Applicable (N/A)-Due to insufficient Data (< 4 sampling events).

Note: The Sigma $X X$ and Sigma $Y Y$ components are estimated using the given field coordinate system and then rotated to align with the estimated groundwater flow direction. Moments are not calculated for sample events with less than 6 wells. 


\section{MAROS Statistical Trend Analysis Summary}

Project: $\quad$ BC Burial Grounds

Location: Bear Creek Burial Grounds

Time Period: $1 / 1 / 1996$ to $7 / 15 / 2007$

Consolidation Period: No Time Consolidation

Consolidation Type: Median

Duplicate Consolidation: Average

ND Values: Specified Detection Limit

J Flag Values : Actual Value
User Name: MV

State: Tennessee

\begin{tabular}{|c|c|c|c|c|c|c|c|c|}
\hline lell & $\begin{array}{c}\text { Sourcel } \\
\text { Tail }\end{array}$ & $\begin{array}{c}\text { Number } \\
\text { of } \\
\text { Samples }\end{array}$ & $\begin{array}{c}\text { Number } \\
\text { of } \\
\text { Detects }\end{array}$ & $\begin{array}{c}\text { Average } \\
\text { Conc. } \\
\text { (mg/L) }\end{array}$ & $\begin{array}{l}\text { Median } \\
\text { Conc. } \\
\text { (mg/L) }\end{array}$ & $\begin{array}{c}\text { All } \\
\text { Samples } \\
\text { "ND" ? }\end{array}$ & $\begin{array}{l}\text { Mann- } \\
\text { Kendall } \\
\text { Trend }\end{array}$ & $\begin{array}{l}\text { Linear } \\
\text { Regression } \\
\text { Trend }\end{array}$ \\
\hline
\end{tabular}

1,1-DICHLOROETHENE

\begin{tabular}{|c|c|c|c|c|c|c|c|c|}
\hline GW-014 & $\mathrm{s}$ & 8 & 8 & $7.2 \mathrm{E}-02$ & $7.7 \mathrm{E}-02$ & No & NT & 1 \\
\hline GW-046 & $\mathrm{S}$ & 20 & 20 & $9.1 \mathrm{E}-02$ & 8.3E-02 & No & S & PD \\
\hline GW-052 & $\mathrm{T}$ & 3 & 0 & $5.0 \mathrm{E}-04$ & $5.0 \mathrm{E}-04$ & Yes & ND & ND \\
\hline GW-053 & $\mathrm{T}$ & 15 & 0 & $5.0 \mathrm{E}-04$ & $5.0 \mathrm{E}-04$ & Yes & ND & ND \\
\hline GW-058 & $\mathrm{T}$ & 1 & 0 & $5.0 \mathrm{E}-04$ & $5.0 \mathrm{E}-04$ & Yes & ND & ND \\
\hline GW-068 & $\mathrm{T}$ & 3 & 3 & $1.2 \mathrm{E}-01$ & $1.1 \mathrm{E}-01$ & No & $N / A$ & N/A \\
\hline GW-071 & $\mathrm{s}$ & 10 & 10 & $1.2 \mathrm{E}-01$ & 1.3E-01 & No & 1 & 1 \\
\hline GW-072 & $\mathrm{T}$ & 5 & 5 & $6.8 \mathrm{E}-03$ & $6.0 \mathrm{E}-03$ & No & NT & NT \\
\hline GW-077 & $\mathrm{T}$ & 21 & 0 & $5.0 \mathrm{E}-04$ & $5.0 \mathrm{E}-04$ & Yes & ND & ND \\
\hline GW-078 & $\mathrm{T}$ & 21 & 0 & $5.0 \mathrm{E}-04$ & $5.0 \mathrm{E}-04$ & Yes & ND & ND \\
\hline GW-079 & $T$ & 24 & 0 & $5.0 \mathrm{E}-04$ & $5.0 \mathrm{E}-04$ & Yes & ND & ND \\
\hline GW-080 & $\mathrm{T}$ & 23 & 0 & $5.0 \mathrm{E}-04$ & $5.0 \mathrm{E}-04$ & Yes & ND & ND \\
\hline GW-082 & $\mathrm{s}$ & 17 & 10 & $1.4 \mathrm{E}-02$ & $1.0 \mathrm{E}-03$ & No & NT & NT \\
\hline GW-089 & $\mathrm{T}$ & 1 & 0 & $5.0 \mathrm{E}-04$ & $5.0 \mathrm{E}-04$ & Yes & ND & ND \\
\hline GW-242 & $\mathrm{T}$ & 5 & 0 & $5.0 \mathrm{E}-04$ & $5.0 \mathrm{E}-04$ & Yes & ND & ND \\
\hline GW-257 & $\mathrm{T}$ & 4 & 0 & $5.0 \mathrm{E}-04$ & 5.0E-04 & Yes & ND & ND \\
\hline GW-289 & $T$ & 8 & 1 & $5.6 \mathrm{E}-04$ & $5.0 \mathrm{E}-04$ & No & NT & NT \\
\hline GW-291 & $\mathrm{T}$ & 6 & 0 & $5.0 \mathrm{E}-04$ & $5.0 \mathrm{E}-04$ & Yes & ND & ND \\
\hline GW-624 & $\mathrm{s}$ & 4 & 4 & $1.1 \mathrm{E}-01$ & $1.1 \mathrm{E}-01$ & No & NT & NT \\
\hline GW-626 & $\mathrm{s}$ & 10 & 10 & 4.1E-02 & 2.6E-02 & No & NT & $\mathrm{PI}$ \\
\hline GW-627 & $T$ & 25 & 25 & $1.8 \mathrm{E}-02$ & $1.5 \mathrm{E}-02$ & No & 1 & 1 \\
\hline GW-629 & $\mathrm{T}$ & 7 & 3 & $1.3 \mathrm{E}-01$ & $5.0 \mathrm{E}-04$ & No & 1 & 1 \\
\hline GW-639 & $\mathrm{T}$ & 4 & 0 & $5.0 \mathrm{E}-04$ & $5.0 \mathrm{E}-04$ & Yes & ND & ND \\
\hline GW-653 & $\mathrm{T}$ & 22 & 10 & $1.5 \mathrm{E}-03$ & $5.0 \mathrm{E}-04$ & No & NT & NT \\
\hline GW-683 & $\mathrm{T}$ & 23 & 0 & $5.0 \mathrm{E}-04$ & $5.0 \mathrm{E}-04$ & Yes & ND & ND \\
\hline GW-684 & $\mathrm{T}$ & 24 & 0 & $5.0 \mathrm{E}-04$ & $5.0 \mathrm{E}-04$ & Yes & ND & ND \\
\hline GW-694 & $\mathrm{T}$ & 10 & 0 & $5.0 \mathrm{E}-04$ & $5.0 \mathrm{E}-04$ & Yes & ND & ND \\
\hline GW-703 & $\mathrm{T}$ & 22 & 3 & 7.0E-04 & $5.0 \mathrm{E}-04$ & No & S & D \\
\hline GW-704 & $\mathrm{T}$ & 24 & 21 & $3.9 \mathrm{E}-03$ & $4.0 \mathrm{E}-03$ & No & D & D \\
\hline GW-706 & $\mathrm{T}$ & 24 & 3 & $6.9 \mathrm{E}-04$ & $5.0 \mathrm{E}-04$ & No & $\mathrm{Pl}$ & 1 \\
\hline GW-712 & $\mathrm{T}$ & 24 & 0 & $5.0 \mathrm{E}-04$ & $5.0 \mathrm{E}-04$ & Yes & ND & ND \\
\hline GW-713 & $\mathrm{T}$ & 24 & 0 & $5.0 \mathrm{E}-04$ & $5.0 \mathrm{E}-04$ & Yes & ND & ND \\
\hline GW-714 & $\mathrm{T}$ & 24 & 0 & $5.0 \mathrm{E}-04$ & $5.0 \mathrm{E}-04$ & Yes & ND & ND \\
\hline SS-4 & $T$ & 23 & 3 & $6.5 \mathrm{E}-04$ & $5.0 \mathrm{E}-04$ & No & NT & NT \\
\hline SS-5 & $\mathrm{T}$ & 26 & 0 & $5.0 \mathrm{E}-04$ & $5.0 \mathrm{E}-04$ & Yes & ND & ND \\
\hline
\end{tabular}


MAROS Statistical Trend Analysis Summary

\begin{tabular}{ccccccccc} 
& & Number & Number & Average & Median & All & Mann- & Linear \\
Well & Sourcel & of & of & Conc. & Conc. & Samples & Kendall & Regression \\
Tail & Samples & Detects & (mg/L) & (mg/L) & "ND" ? & Trend & Trend \\
\hline
\end{tabular}

1,1-DICHLOROETHENE

$\begin{array}{lllllllll}\text { SS-6_6 } & \text { T } & 16 & 0 & 5.0 \mathrm{E}-04 & 5.0 \mathrm{E}-04 & \text { Yes } & \text { ND } & \text { ND } \\ \text { SS-6E } & \text { T } & 14 & 0 & 5.0 \mathrm{E}-04 & 5.0 \mathrm{E}-04 & \text { Yes } & \text { ND } & \text { ND } \\ \text { SS-7 } & \text { T } & 13 & 0 & 5.0 \mathrm{E}-04 & 5.0 \mathrm{E}-04 & \text { Yes } & \text { ND } & \text { ND } \\ \text { SS-8 } & \text { T } & 14 & 0 & 5.0 \mathrm{E}-04 & 5.0 \mathrm{E}-04 & \text { Yes } & \text { ND } & \text { ND }\end{array}$

BENZENE

\begin{tabular}{|c|c|c|c|c|c|c|c|c|}
\hline GW-014 & $\mathrm{s}$ & 8 & 7 & 2.6E-03 & $2.5 \mathrm{E}-03$ & No & NT & 1 \\
\hline GW-046 & $\mathrm{S}$ & 20 & 19 & 4.2E-02 & 2.3E-02 & No & NT & NT \\
\hline GW-052 & $T$ & 3 & 0 & $5.0 \mathrm{E}-05$ & 5.0E-05 & Yes & ND & ND \\
\hline GW-053 & $T$ & 15 & 0 & $5.0 \mathrm{E}-05$ & 5.0E-05 & Yes & ND & ND \\
\hline GW-058 & $T$ & 1 & 0 & $5.0 \mathrm{E}-05$ & 5.0E-05 & Yes & ND & ND \\
\hline GW-068 & $T$ & 3 & 3 & 4.5E-02 & 4.7E-02 & No & N/A & $\mathrm{N} / \mathrm{A}$ \\
\hline GW-071 & $\mathrm{S}$ & 10 & 10 & $1.5 \mathrm{E}+00$ & $1.3 \mathrm{E}+00$ & No & I & 1 \\
\hline GW-072 & $T$ & 5 & 2 & 3.4E-04 & 5.0E-05 & No & NT & NT \\
\hline GW-077 & $T$ & 21 & 0 & $5.0 \mathrm{E}-05$ & $5.0 \mathrm{E}-05$ & Yes & ND & ND \\
\hline GW-078 & $T$ & 21 & 0 & $5.0 \mathrm{E}-05$ & 5.0E-05 & Yes & ND & ND \\
\hline GW-079 & $\mathrm{T}$ & 24 & 0 & $5.0 \mathrm{E}-05$ & $5.0 \mathrm{E}-05$ & Yes & ND & ND \\
\hline GW-080 & $\mathrm{T}$ & 23 & 0 & $5.0 \mathrm{E}-05$ & 5.0E-05 & Yes & ND & ND \\
\hline GW-082 & $\mathrm{S}$ & 17 & 11 & $1.4 \mathrm{E}-02$ & 1.0E-03 & No & NT & NT \\
\hline GW-089 & $\mathrm{T}$ & 1 & 0 & $5.0 \mathrm{E}-05$ & 5.0E-05 & Yes & ND & ND \\
\hline GW-242 & $\mathrm{T}$ & 5 & 0 & $5.0 \mathrm{E}-05$ & 5.0E-05 & Yes & ND & ND \\
\hline GW-257 & $\mathrm{T}$ & 4 & 0 & $5.0 \mathrm{E}-05$ & 5.0E-05 & Yes & ND & ND \\
\hline GW-289 & $\mathrm{T}$ & 8 & 0 & $5.0 \mathrm{E}-05$ & 5.0E-05 & Yes & ND & ND \\
\hline GW-291 & $\mathrm{T}$ & 6 & 0 & $5.0 \mathrm{E}-05$ & 5.0E-05 & Yes & ND & ND \\
\hline GW-624 & $\mathrm{S}$ & 4 & 3 & $1.9 \mathrm{E}-02$ & 2.2E-02 & No & NT & NT \\
\hline GW-626 & $\mathrm{S}$ & 10 & 6 & $5.8 \mathrm{E}-02$ & $9.0 \mathrm{E}-03$ & No & NT & $\mathrm{PI}$ \\
\hline GW-627 & $\mathrm{T}$ & 25 & 0 & $5.0 \mathrm{E}-05$ & 5.0E-05 & Yes & ND & ND \\
\hline GW-629 & $\mathrm{T}$ & 7 & 3 & $2.6 \mathrm{E}-03$ & 5.0E-05 & No & $\mathrm{PI}$ & 1 \\
\hline GW-639 & $\mathrm{T}$ & 19 & 1 & $3.1 \mathrm{E}-04$ & 5.0E-05 & No & NT & D \\
\hline GW-653 & $\mathrm{T}$ & 22 & 0 & $5.0 \mathrm{E}-05$ & 5.0E-05 & Yes & ND & ND \\
\hline GW-683 & $\mathrm{T}$ & 23 & 0 & $5.0 \mathrm{E}-05$ & 5.0E-05 & Yes & ND & ND \\
\hline GW-684 & $\mathrm{T}$ & 24 & 0 & $5.0 \mathrm{E}-05$ & 5.0E-05 & Yes & ND & ND \\
\hline GW-694 & $\mathrm{T}$ & 10 & 0 & $5.0 \mathrm{E}-05$ & 5.0E-05 & Yes & ND & ND \\
\hline GW-703 & $\mathrm{T}$ & 22 & 1 & $9.3 \mathrm{E}-05$ & 5.0E-05 & No & NT & NT \\
\hline GW-704 & $\mathrm{T}$ & 24 & 0 & $5.0 \mathrm{E}-05$ & 5.0E-05 & Yes & ND & ND \\
\hline GW-706 & $\mathrm{T}$ & 24 & 0 & $5.0 \mathrm{E}-05$ & 5.0E-05 & Yes & ND & ND \\
\hline GW-712 & $\mathrm{T}$ & 24 & 0 & 5.0E-05 & 5.0E-05 & Yes & ND & ND \\
\hline GW-713 & $\mathrm{T}$ & 24 & 0 & 5.0E-05 & 5.0E-05 & Yes & ND & ND \\
\hline GW-714 & $\mathrm{T}$ & 24 & 0 & 5.0E-05 & 5.0E-05 & Yes & ND & ND \\
\hline SS-4 & $\mathrm{T}$ & 23 & 0 & 5.0E-05 & 5.0E-05 & Yes & ND & ND \\
\hline SS-5 & $\mathrm{T}$ & 26 & 0 & 5.0E-05 & 5.0E-05 & Yes & ND & ND \\
\hline SS-6_6 & $\mathrm{T}$ & 16 & 1 & $1.1 \mathrm{E}-04$ & 5.0E-05 & No & NT & NT \\
\hline SS-6E & $\mathrm{T}$ & 14 & 0 & 5.0E-05 & 5.0E-05 & Yes & ND & ND \\
\hline SS-7 & $\mathrm{T}$ & 13 & 0 & $5.0 \mathrm{E}-05$ & 5.0E-05 & Yes & ND & ND \\
\hline SS-8 & $\mathrm{T}$ & 14 & 0 & $5.0 \mathrm{E}-05$ & 5.0E-05 & Yes & ND & ND \\
\hline
\end{tabular}




\section{MAROS Statistical Trend Analysis Summary}

\begin{tabular}{ccccccccc} 
Well & $\begin{array}{c}\text { Sourcel } \\
\text { Tail }\end{array}$ & $\begin{array}{c}\text { Number } \\
\text { of } \\
\text { Samples }\end{array}$ & $\begin{array}{c}\text { Number } \\
\text { of } \\
\text { Detects }\end{array}$ & $\begin{array}{c}\text { Average } \\
\text { Conc. } \\
\text { (mg/L) }\end{array}$ & $\begin{array}{c}\text { Median } \\
\text { Conc. } \\
(\mathbf{m g} / \mathrm{L})\end{array}$ & $\begin{array}{c}\text { All } \\
\text { Samples } \\
\text { "ND" ? }\end{array}$ & $\begin{array}{c}\text { Mann- } \\
\text { Kendall } \\
\text { Trend }\end{array}$ & $\begin{array}{c}\text { Linear } \\
\text { Regression } \\
\text { Trend }\end{array}$ \\
\hline TETRACHLOROETHYLENE(PCE) \\
\hline
\end{tabular}

\section{TETRACHLOROETHYLENE(PCE)}

\begin{tabular}{|c|c|c|c|c|c|c|c|c|}
\hline GW-014 & $\mathrm{S}$ & 8 & 8 & 3.2E-02 & 2.9E-02 & No & NT & 1 \\
\hline GW-046 & $\mathrm{S}$ & 20 & 19 & $2.0 \mathrm{E}+00$ & $1.2 \mathrm{E}+00$ & No & NT & NT \\
\hline GW-052 & $T$ & 3 & 0 & $5.0 \mathrm{E}-04$ & $5.0 \mathrm{E}-04$ & Yes & ND & ND \\
\hline GW-053 & $\mathrm{T}$ & 15 & 4 & 7.7E-04 & 5.0E-04 & No & $\mathrm{s}$ & $\mathrm{s}$ \\
\hline GW-058 & $\mathrm{T}$ & 1 & 1 & 4.0E-03 & 4.0E-03 & No & $\mathrm{N} / \mathrm{A}$ & N/A \\
\hline GW-068 & $\mathrm{T}$ & 3 & 3 & 4.3E-03 & 2.0E-03 & No & N/A & N/A \\
\hline GW-071 & $\mathrm{S}$ & 10 & 10 & 8.6E-01 & 9.2E-01 & No & 1 & 1 \\
\hline GW-072 & $T$ & 5 & 1 & $6.5 \mathrm{E}-04$ & 5.0E-04 & No & $S$ & NT \\
\hline GW-077 & $\mathrm{T}$ & 21 & 1 & 5.2E-04 & 5.0E-04 & No & $\mathrm{s}$ & $P D$ \\
\hline GW-078 & $T$ & 21 & 0 & 5.0E-04 & 5.0E-04 & Yes & ND & ND \\
\hline GW-079 & $\mathrm{T}$ & 24 & 0 & 5.0E-04 & 5.0E-04 & Yes & ND & ND \\
\hline GW-080 & $T$ & 23 & 0 & $5.0 \mathrm{E}-04$ & $5.0 \mathrm{E}-04$ & Yes & ND & ND \\
\hline GW-082 & $\mathrm{S}$ & 17 & 2 & 7.4E-04 & $5.0 \mathrm{E}-04$ & No & NT & NT \\
\hline GW-089 & $T$ & 1 & 0 & $5.0 \mathrm{E}-04$ & $5.0 \mathrm{E}-04$ & Yes & ND & ND \\
\hline GW-242 & $\mathrm{T}$ & 5 & 1 & $6.0 \mathrm{E}-04$ & $5.0 \mathrm{E}-04$ & No & NT & NT \\
\hline GW-257 & $\mathrm{T}$ & 4 & 4 & 2.1E-01 & 2.1E-01 & No & NT & NT \\
\hline GW-289 & $\mathrm{T}$ & 8 & 8 & $6.5 \mathrm{E}-01$ & 6.4E-01 & No & 1 & 1 \\
\hline GW-291 & $\mathrm{T}$ & 6 & 6 & 4.3E-01 & 4.1E-01 & No & $\mathrm{S}$ & $P D$ \\
\hline GW-624 & $\mathrm{S}$ & 4 & 4 & 3.8E-01 & 3.8E-01 & No & $\mathrm{S}$ & NT \\
\hline GW-626 & $\mathrm{S}$ & 10 & 10 & 3.4E-01 & 2.5E-01 & No & $\mathrm{PI}$ & 1 \\
\hline GW-627 & $\mathrm{T}$ & 25 & 25 & 5.1E-01 & 4.7E-01 & No & 1 & 1 \\
\hline GW-629 & $\mathrm{T}$ & 7 & 4 & $7.0 \mathrm{E}+00$ & 8.0E-04 & No & $\mathrm{PI}$ & 1 \\
\hline GW-639 & $\mathrm{T}$ & 22 & 1 & $4.9 \mathrm{E}-04$ & $5.0 \mathrm{E}-04$ & No & $\mathrm{S}$ & S \\
\hline GW-653 & $\mathrm{T}$ & 22 & 20 & 4.5E-03 & $3.5 \mathrm{E}-03$ & No & NT & NT \\
\hline GW-683 & $\mathrm{T}$ & 23 & 0 & 5.0E-04 & 5.0E-04 & Yes & ND & ND \\
\hline GW-684 & $\mathrm{T}$ & 24 & 0 & 5.0E-04 & 5.0E-04 & Yes & ND & ND \\
\hline GW-694 & $\mathrm{T}$ & 10 & 1 & $6.5 \mathrm{E}-04$ & 5.0E-04 & No & $\mathrm{s}$ & $\mathrm{s}$ \\
\hline GW-703 & $\mathrm{T}$ & 22 & 0 & 5.0E-04 & 5.0E-04 & Yes & ND & ND \\
\hline GW-704 & $\mathrm{T}$ & 24 & 0 & 5.0E-04 & 5.0E-04 & Yes & ND & ND \\
\hline GW-706 & $\mathrm{T}$ & 24 & 0 & 5.0E-04 & 5.0E-04 & Yes & ND & ND \\
\hline GW-712 & $\mathrm{T}$ & 24 & 0 & $5.0 \mathrm{E}-04$ & $5.0 \mathrm{E}-04$ & Yes & ND & ND \\
\hline GW-713 & $\mathrm{T}$ & 23 & 0 & 5.0E-04 & 5.0E-04 & Yes & ND & ND \\
\hline GW-714 & $\mathrm{T}$ & 24 & 0 & $5.0 \mathrm{E}-04$ & $5.0 \mathrm{E}-04$ & Yes & ND & ND \\
\hline SS-4 & $\mathrm{T}$ & 23 & 0 & $5.0 \mathrm{E}-04$ & $5.0 \mathrm{E}-04$ & Yes & ND & ND \\
\hline SS-5 & $\mathrm{T}$ & 26 & 0 & $5.0 \mathrm{E}-04$ & $5.0 \mathrm{E}-04$ & Yes & ND & ND \\
\hline SS-6_6 & $\mathrm{T}$ & 16 & 0 & $5.0 \mathrm{E}-04$ & $5.0 \mathrm{E}-04$ & Yes & ND & ND \\
\hline SS-6E & $\mathrm{T}$ & 14 & 0 & 5.0E-04 & 5.0E-04 & Yes & ND & ND \\
\hline SS-7 & $\mathrm{T}$ & 13 & 0 & $5.0 \mathrm{E}-04$ & $5.0 \mathrm{E}-04$ & Yes & ND & ND \\
\hline SS-8 & $\mathrm{T}$ & 14 & 0 & $5.0 \mathrm{E}-04$ & $5.0 \mathrm{E}-04$ & Yes & ND & ND \\
\hline \multicolumn{9}{|c|}{ TRICHLOROETHYLENE (TCE) } \\
\hline GW-014 & $\mathrm{S}$ & 8 & 8 & 2.2E-01 & 2.2E-01 & No & NT & 1 \\
\hline GW-046 & $\mathrm{S}$ & 20 & 20 & $1.8 \mathrm{E}+00$ & $1.5 \mathrm{E}+00$ & No & $\mathrm{D}$ & $\mathrm{D}$ \\
\hline GW-052 & $\mathrm{T}$ & 3 & 0 & 5.0E-04 & 5.0E-04 & Yes & ND & ND \\
\hline
\end{tabular}


MAROS Statistical Trend Analysis Summary

\begin{tabular}{|c|c|c|c|c|c|c|c|c|}
\hline Well & $\begin{array}{c}\text { Sourcel } \\
\text { Tail }\end{array}$ & $\begin{array}{l}\text { Number } \\
\text { of } \\
\text { Samples }\end{array}$ & $\begin{array}{c}\text { Number } \\
\text { of } \\
\text { Detects }\end{array}$ & $\begin{array}{l}\text { Average } \\
\text { Conc. } \\
\text { (mg/L) }\end{array}$ & $\begin{array}{l}\text { Median } \\
\text { Conc. } \\
\text { (mg/L) }\end{array}$ & $\begin{array}{c}\text { All } \\
\text { Samples } \\
\text { "ND" ? }\end{array}$ & $\begin{array}{l}\text { Mann- } \\
\text { Kendall } \\
\text { Trend }\end{array}$ & $\begin{array}{c}\text { Linear } \\
\text { Regression } \\
\text { Trend }\end{array}$ \\
\hline
\end{tabular}

TRICHLOROETHYLENE (TCE)

\begin{tabular}{|c|c|c|c|c|c|c|c|c|}
\hline GW-053 & $\mathrm{T}$ & 15 & 15 & $2.7 \mathrm{E}-03$ & 3.0E-03 & No & NT & 1 \\
\hline GW-058 & $\mathrm{T}$ & 1 & 0 & $5.0 \mathrm{E}-04$ & $5.0 \mathrm{E}-04$ & Yes & ND & ND \\
\hline GW-068 & $\mathrm{T}$ & 3 & 3 & 7.4E-02 & 7.0E-02 & No & $\mathrm{N} / \mathrm{A}$ & $\mathrm{N} / \mathrm{A}$ \\
\hline GW-071 & $\mathrm{S}$ & 10 & 10 & $1.2 \mathrm{E}-01$ & $1.1 \mathrm{E}-01$ & No & NT & $\mathrm{PI}$ \\
\hline GW-072 & $\mathrm{T}$ & 5 & 0 & $5.0 \mathrm{E}-04$ & $5.0 \mathrm{E}-04$ & Yes & ND & ND \\
\hline GW-077 & $\mathrm{T}$ & 21 & 0 & $5.0 \mathrm{E}-04$ & $5.0 \mathrm{E}-04$ & Yes & ND & ND \\
\hline GW-078 & $\mathrm{T}$ & 21 & 0 & $5.0 \mathrm{E}-04$ & $5.0 \mathrm{E}-04$ & Yes & ND & ND \\
\hline GW-079 & $\mathrm{T}$ & 24 & 0 & $5.0 \mathrm{E}-04$ & $5.0 \mathrm{E}-04$ & Yes & ND & ND \\
\hline GW-080 & $\mathrm{T}$ & 23 & 0 & $5.0 \mathrm{E}-04$ & $5.0 \mathrm{E}-04$ & Yes & ND & ND \\
\hline GW-082 & $\mathrm{S}$ & 17 & 0 & $5.0 \mathrm{E}-04$ & $5.0 \mathrm{E}-04$ & Yes & ND & ND \\
\hline GW-089 & $\mathrm{T}$ & 1 & 0 & $5.0 \mathrm{E}-04$ & $5.0 \mathrm{E}-04$ & Yes & ND & ND \\
\hline GW-242 & $\mathrm{T}$ & 5 & 4 & $1.7 \mathrm{E}-03$ & $2.0 \mathrm{E}-03$ & No & NT & NT \\
\hline GW-257 & $\mathrm{T}$ & 4 & 0 & $5.0 \mathrm{E}-04$ & $5.0 \mathrm{E}-04$ & Yes & ND & ND \\
\hline GW-289 & $\mathrm{T}$ & 8 & 8 & $1.4 \mathrm{E}-02$ & $1.4 \mathrm{E}-02$ & No & I & 1 \\
\hline GW-291 & $\mathrm{T}$ & 6 & 6 & 4.8E-02 & 4.4E-02 & No & $\mathrm{S}$ & PD \\
\hline GW-624 & $\mathrm{s}$ & 4 & 4 & $5.6 \mathrm{E}-01$ & $4.5 \mathrm{E}-01$ & No & $S$ & $S$ \\
\hline GW-626 & $\mathrm{S}$ & 10 & 10 & $2.2 \mathrm{E}-01$ & $1.6 \mathrm{E}-01$ & No & $\mathrm{PI}$ & 1 \\
\hline GW-627 & $\mathrm{T}$ & 25 & 24 & $1.6 \mathrm{E}-01$ & $1.5 \mathrm{E}-01$ & No & I & 1 \\
\hline GW-629 & $\mathrm{T}$ & 7 & 6 & $1.8 \mathrm{E}+00$ & $5.0 \mathrm{E}-03$ & No & 1 & I \\
\hline GW-639 & $\mathrm{T}$ & 22 & 1 & $5.0 \mathrm{E}-04$ & $5.0 \mathrm{E}-04$ & No & $S$ & $\mathrm{~S}$ \\
\hline GW-653 & $\mathrm{T}$ & 22 & 15 & $2.7 \mathrm{E}-03$ & $2.0 \mathrm{E}-03$ & No & NT & NT \\
\hline GW-683 & $\mathrm{T}$ & 23 & 0 & $5.0 \mathrm{E}-04$ & $5.0 \mathrm{E}-04$ & Yes & ND & ND \\
\hline GW-684 & $\mathrm{T}$ & 24 & 1 & $4.8 \mathrm{E}-04$ & $5.0 \mathrm{E}-04$ & No & $\mathrm{S}$ & D \\
\hline GW-694 & $\mathrm{T}$ & 10 & 9 & $3.0 \mathrm{E}-03$ & 3.0E-03 & No & $\mathrm{D}$ & PD \\
\hline GW-703 & $\mathrm{T}$ & 22 & 21 & $1.4 \mathrm{E}-02$ & $1.5 \mathrm{E}-02$ & No & $\mathrm{D}$ & NT \\
\hline GW-704 & $\mathrm{T}$ & 24 & 23 & 4.8E-02 & 4.3E-02 & No & $\mathrm{D}$ & $S$ \\
\hline GW-706 & $\mathrm{T}$ & 24 & 23 & $1.1 \mathrm{E}-02$ & $1.1 \mathrm{E}-02$ & No & NT & NT \\
\hline GW-712 & $\mathrm{T}$ & 24 & 0 & $5.0 \mathrm{E}-04$ & $5.0 \mathrm{E}-04$ & Yes & ND & ND \\
\hline GW-713 & $\mathrm{T}$ & 24 & 1 & $6.0 \mathrm{E}-04$ & $5.0 \mathrm{E}-04$ & No & NT & $\mathrm{PI}$ \\
\hline GW-714 & $\mathrm{T}$ & 24 & 0 & $5.0 \mathrm{E}-04$ & $5.0 \mathrm{E}-04$ & Yes & ND & ND \\
\hline SS-4 & $\mathrm{T}$ & 23 & 22 & 7.6E-03 & 7.0E-03 & No & PD & $D$ \\
\hline SS-5 & $\mathrm{T}$ & 26 & 5 & 7.5E-04 & $5.0 \mathrm{E}-04$ & No & $\mathrm{S}$ & $\mathrm{S}$ \\
\hline SS-6_6 & $\mathrm{T}$ & 16 & 0 & 5.0E-04 & $5.0 \mathrm{E}-04$ & Yes & ND & ND \\
\hline SS-6E & $\mathrm{T}$ & 14 & 0 & $5.0 \mathrm{E}-04$ & $5.0 \mathrm{E}-04$ & Yes & ND & ND \\
\hline SS-7 & $\mathrm{T}$ & 13 & 0 & $5.0 \mathrm{E}-04$ & $5.0 \mathrm{E}-04$ & Yes & ND & ND \\
\hline SS-8 & $\mathrm{T}$ & 14 & 0 & $5.0 \mathrm{E}-04$ & $5.0 \mathrm{E}-04$ & Yes & ND & ND \\
\hline \multicolumn{9}{|c|}{ VINYL CHLORIDE } \\
\hline GW-014 & $\mathrm{S}$ & 8 & 8 & $1.8 \mathrm{E}-01$ & $1.5 \mathrm{E}-01$ & No & NT & $\mathrm{PI}$ \\
\hline GW-046 & $\mathrm{S}$ & 20 & 20 & $5.1 \mathrm{E}-01$ & 4.7E-01 & No & $\mathrm{D}$ & $\mathrm{D}$ \\
\hline GW-052 & $\mathrm{T}$ & 3 & 0 & $5.0 \mathrm{E}-04$ & $5.0 \mathrm{E}-04$ & Yes & ND & ND \\
\hline GW-053 & $\mathrm{T}$ & 15 & 12 & $3.8 \mathrm{E}-03$ & $5.0 \mathrm{E}-03$ & No & $\mathrm{D}$ & $\mathrm{D}$ \\
\hline GW-058 & $\mathrm{T}$ & 1 & 0 & $5.0 \mathrm{E}-04$ & $5.0 \mathrm{E}-04$ & Yes & ND & ND \\
\hline GW-068 & $\mathrm{T}$ & 3 & 3 & $2.8 \mathrm{E}-01$ & $2.7 \mathrm{E}-01$ & No & $\mathrm{N} / \mathrm{A}$ & $\mathrm{N} / \mathrm{A}$ \\
\hline GW-071 & $\mathrm{S}$ & 10 & 9 & $6.0 \mathrm{E}-03$ & $4.0 \mathrm{E}-03$ & No & $\mathrm{PI}$ & 1 \\
\hline GW-072 & $\mathrm{T}$ & 5 & 2 & $1.1 \mathrm{E}-03$ & $5.0 \mathrm{E}-04$ & No & NT & NT \\
\hline
\end{tabular}




\section{MAROS Statistical Trend Analysis Summary}

\begin{tabular}{|c|c|c|c|c|c|c|c|c|}
\hline Well & $\begin{array}{c}\text { Sourcel } \\
\text { Tail }\end{array}$ & $\begin{array}{c}\text { Number } \\
\text { of } \\
\text { Samples }\end{array}$ & $\begin{array}{c}\text { Number } \\
\text { of } \\
\text { Detects }\end{array}$ & $\begin{array}{c}\text { Average } \\
\text { Conc. } \\
\text { (mg/L) }\end{array}$ & $\begin{array}{l}\text { Median } \\
\text { Conc. } \\
\text { (mg/L) }\end{array}$ & $\begin{array}{c}\text { All } \\
\text { Samples } \\
\text { "ND" ? }\end{array}$ & $\begin{array}{l}\text { Mann- } \\
\text { Kendall } \\
\text { Trend }\end{array}$ & $\begin{array}{c}\text { Linear } \\
\text { Regression } \\
\text { Trend }\end{array}$ \\
\hline \multicolumn{9}{|c|}{ VINYL CHLORIDE } \\
\hline GW-077 & $\mathrm{T}$ & 21 & 0 & $5.0 \mathrm{E}-04$ & $5.0 \mathrm{E}-04$ & Yes & ND & ND \\
\hline GW-078 & $T$ & 21 & 0 & 5.0E-04 & $5.0 \mathrm{E}-04$ & Yes & ND & ND \\
\hline GW-079 & $T$ & 24 & 0 & $5.0 \mathrm{E}-04$ & $5.0 \mathrm{E}-04$ & Yes & ND & ND \\
\hline GW-080 & $T$ & 23 & 0 & $5.0 \mathrm{E}-04$ & $5.0 \mathrm{E}-04$ & Yes & ND & ND \\
\hline GW-082 & S & 17 & 17 & 1.4E-01 & $1.2 \mathrm{E}-01$ & No & NT & $\mathrm{PI}$ \\
\hline GW-089 & $T$ & 1 & 0 & $5.0 \mathrm{E}-04$ & $5.0 \mathrm{E}-04$ & Yes & ND & ND \\
\hline GW-242 & $T$ & 5 & 5 & 3.3E-02 & $3.2 \mathrm{E}-02$ & No & NT & $\mathrm{s}$ \\
\hline GW-257 & $T$ & 4 & 0 & 5.0E-04 & $5.0 \mathrm{E}-04$ & Yes & ND & ND \\
\hline GW-289 & $T$ & 8 & 3 & $9.4 \mathrm{E}-04$ & $5.0 \mathrm{E}-04$ & No & $\mathrm{s}$ & $\mathrm{s}$ \\
\hline GW-291 & $T$ & 6 & 0 & 5.0E-04 & $5.0 \mathrm{E}-04$ & Yes & ND & ND \\
\hline GW-624 & S & 4 & 4 & 3.6E-01 & $3.1 \mathrm{E}-01$ & No & $\mathrm{s}$ & $\mathrm{s}$ \\
\hline GW-626 & S & 10 & 7 & 4.5E-01 & $9.6 \mathrm{E}-02$ & No & 1 & $\mathrm{PI}$ \\
\hline GW-627 & $\mathrm{T}$ & 25 & 22 & $1.8 \mathrm{E}-02$ & 1.3E-02 & No & 1 & 1 \\
\hline GW-629 & $T$ & 7 & 3 & 1.3E-02 & $5.0 \mathrm{E}-04$ & No & $\mathrm{PI}$ & I \\
\hline GW-639 & $T$ & 7 & 0 & $5.0 \mathrm{E}-04$ & $5.0 \mathrm{E}-04$ & Yes & ND & ND \\
\hline GW-653 & $T$ & 22 & 0 & 5.0E-04 & $5.0 \mathrm{E}-04$ & Yes & ND & ND \\
\hline GW-683 & $T$ & 23 & 0 & $5.0 \mathrm{E}-04$ & $5.0 \mathrm{E}-04$ & Yes & ND & ND \\
\hline GW-684 & $T$ & 24 & 0 & $5.0 \mathrm{E}-04$ & $5.0 \mathrm{E}-04$ & Yes & ND & ND \\
\hline GW-694 & $\mathrm{T}$ & 10 & 0 & $5.0 \mathrm{E}-04$ & $5.0 \mathrm{E}-04$ & Yes & ND & ND \\
\hline GW-703 & $\mathrm{T}$ & 22 & 0 & $5.0 \mathrm{E}-04$ & $5.0 \mathrm{E}-04$ & Yes & ND & ND \\
\hline GW-704 & $T$ & 24 & 0 & $5.0 \mathrm{E}-04$ & $5.0 \mathrm{E}-04$ & Yes & ND & ND \\
\hline GW-706 & $\mathrm{T}$ & 24 & 1 & 4.8E-04 & $5.0 \mathrm{E}-04$ & No & $\mathrm{s}$ & $\mathrm{D}$ \\
\hline GW-712 & $T$ & 24 & 0 & 5.0E-04 & $5.0 \mathrm{E}-04$ & Yes & ND & ND \\
\hline GW-713 & $T$ & 24 & 0 & 5.0E-04 & $5.0 \mathrm{E}-04$ & Yes & ND & ND \\
\hline GW-714 & $T$ & 24 & 0 & 5.0E-04 & $5.0 \mathrm{E}-04$ & Yes & ND & ND \\
\hline SS-4 & $T$ & 23 & 0 & 5.0E-04 & $5.0 \mathrm{E}-04$ & Yes & ND & ND \\
\hline SS-5 & $T$ & 26 & 0 & 5.0E-04 & $5.0 \mathrm{E}-04$ & Yes & ND & ND \\
\hline SS-6_6 & $T$ & 16 & 0 & $5.0 \mathrm{E}-04$ & $5.0 \mathrm{E}-04$ & Yes & ND & ND \\
\hline SS-6E & $\mathrm{T}$ & 14 & 0 & $5.0 \mathrm{E}-04$ & $5.0 \mathrm{E}-04$ & Yes & ND & ND \\
\hline SS-7 & $\mathrm{T}$ & 13 & 0 & $5.0 \mathrm{E}-04$ & $5.0 \mathrm{E}-04$ & Yes & ND & ND \\
\hline SS-8 & $\mathrm{T}$ & 14 & 0 & $5.0 \mathrm{E}-04$ & $5.0 \mathrm{E}-04$ & Yes & ND & ND \\
\hline
\end{tabular}

Note: Increasing (I); Probably Increasing (PI); Stable (S); Probably Decreasing (PD); Decreasing (D); No Trend (NT); Not Applicable (N/A); Not Applicable (N/A) - Due to insufficient Data (< 4 sampling events); No Detectable Concentration (NDC)

The Number of Samples and Number of Detects shown above are post-consolidation values. 


\section{MAROS Statistical Trend Analysis Summary}

Project: $\quad$ BC Burial Grounds

Location: Bear Creek Burial Grounds

Time Period: $1 / 1 / 1996$ to $7 / 15 / 2007$

Consolidation Period: No Time Consolidation

Consolidation Type: Median

Duplicate Consolidation: Average

ND Values: Specified Detection Limit

J Flag Values : Actual Value
User Name: MV

State: Tennessee

\begin{tabular}{|c|c|c|c|c|c|c|c|c|}
\hline Well & $\begin{array}{c}\text { Sourcel } \\
\text { Tail }\end{array}$ & $\begin{array}{l}\text { Number } \\
\text { of } \\
\text { Samples }\end{array}$ & $\begin{array}{c}\text { Number } \\
\text { of } \\
\text { Detects }\end{array}$ & $\begin{array}{l}\text { Average } \\
\text { Conc. } \\
\text { (mg/L) }\end{array}$ & $\begin{array}{l}\text { Median } \\
\text { Conc. } \\
\text { (mg/L) }\end{array}$ & $\begin{array}{c}\text { All } \\
\text { Samples } \\
\text { "ND" ? }\end{array}$ & $\begin{array}{l}\text { Mann- } \\
\text { Kendall } \\
\text { Trend }\end{array}$ & $\begin{array}{l}\text { Linear } \\
\text { Regression } \\
\text { Trend }\end{array}$ \\
\hline \multicolumn{9}{|c|}{ GROSS ALPHA ACTIVITY } \\
\hline GW-014 & $\mathrm{s}$ & 7 & 4 & $3.4 \mathrm{E}+00$ & 9.3E-01 & No & NT & NT \\
\hline GW-046 & s & 20 & 15 & $1.5 \mathrm{E}+00$ & $1.1 \mathrm{E}+00$ & No & NT & $\mathrm{D}$ \\
\hline GW-052 & $\mathrm{T}$ & 3 & 3 & $2.0 \mathrm{E}+01$ & $1.7 \mathrm{E}+01$ & No & N/A & N/A \\
\hline GW-053 & $\mathrm{T}$ & 14 & 13 & $2.8 \mathrm{E}+00$ & $2.0 \mathrm{E}+00$ & No & NT & NT \\
\hline GW-058 & $\mathrm{T}$ & 1 & 1 & $1.7 \mathrm{E}+01$ & $1.7 \mathrm{E}+01$ & No & N/A & N/A \\
\hline GW-068 & $T$ & 3 & 0 & 1.0E-02 & $1.0 \mathrm{E}-02$ & Yes & ND & ND \\
\hline GW-071 & s & 8 & 3 & $3.3 E+00$ & 1.0E-02 & No & PD & $\mathrm{D}$ \\
\hline GW-072 & $\mathrm{T}$ & 4 & 1 & 8.5E-02 & $1.0 \mathrm{E}-02$ & No & NT & D \\
\hline GW-077 & $\mathrm{T}$ & 11 & 2 & 3.2E-01 & $1.0 \mathrm{E}-02$ & No & NT & NT \\
\hline GW-078 & $\mathrm{T}$ & 11 & 4 & 5.9E-01 & $1.0 \mathrm{E}-02$ & No & NT & NT \\
\hline GW-079 & $\mathrm{T}$ & 12 & 5 & $1.1 \mathrm{E}+00$ & $1.0 \mathrm{E}-02$ & No & PD & $\mathrm{D}$ \\
\hline GW-080 & $\mathrm{T}$ & 14 & 9 & $1.6 \mathrm{E}+00$ & $7.8 \mathrm{E}-01$ & No & $\mathrm{D}$ & D \\
\hline GW-082 & s & 14 & 9 & $1.2 \mathrm{E}+00$ & 4.3E-01 & No & NT & NT \\
\hline GW-242 & $\mathrm{T}$ & 4 & 2 & $6.6 \mathrm{E}+00$ & $1.6 \mathrm{E}+00$ & No & NT & $\mathrm{D}$ \\
\hline GW-257 & $\mathrm{T}$ & 1 & 0 & 1.0E-02 & $1.0 \mathrm{E}-02$ & Yes & ND & ND \\
\hline GW-289 & $\mathrm{T}$ & 8 & 4 & 7.7E-01 & $2.8 \mathrm{E}-01$ & No & NT & NT \\
\hline GW-291 & $\mathrm{T}$ & 6 & 3 & $6.9 \mathrm{E}-01$ & $3.0 \mathrm{E}-01$ & No & NT & NT \\
\hline GW-624 & $\mathrm{s}$ & 4 & 1 & 9.1E-01 & $1.0 \mathrm{E}-02$ & No & NT & NT \\
\hline GW-626 & $\mathrm{s}$ & 6 & 2 & 3.2E-01 & $1.0 \mathrm{E}-02$ & No & NT & NT \\
\hline GW-627 & $\mathrm{T}$ & 16 & 10 & $2.5 \mathrm{E}+00$ & $1.3 \mathrm{E}+00$ & No & $\mathrm{D}$ & $\mathrm{D}$ \\
\hline GW-629 & $\mathrm{T}$ & 7 & 2 & $2.9 \mathrm{E}+00$ & $1.0 \mathrm{E}-02$ & No & NT & NT \\
\hline GW-639 & $\mathrm{T}$ & 5 & 1 & 6.3E-01 & $1.0 \mathrm{E}-02$ & No & NT & NT \\
\hline GW-653 & $\mathrm{T}$ & 13 & 9 & 7.1E-01 & 4.0E-01 & No & $\mathrm{D}$ & $\mathrm{D}$ \\
\hline GW-683 & $\mathrm{T}$ & 23 & 23 & $1.4 \mathrm{E}+01$ & $1.1 \mathrm{E}+01$ & No & D & D \\
\hline GW-684 & $\mathrm{T}$ & 24 & 24 & $1.4 \mathrm{E}+01$ & $1.3 \mathrm{E}+01$ & No & $\mathrm{D}$ & $\mathrm{D}$ \\
\hline GW-694 & $\mathrm{T}$ & 10 & 9 & $1.4 \mathrm{E}+01$ & $1.3 \mathrm{E}+01$ & No & NT & $\mathrm{PI}$ \\
\hline GW-703 & $\mathrm{T}$ & 22 & 19 & $4.5 \mathrm{E}+00$ & $3.9 \mathrm{E}+00$ & No & NT & $\mathrm{D}$ \\
\hline GW-704 & $\mathrm{T}$ & 22 & 20 & $5.2 \mathrm{E}+00$ & $4.2 \mathrm{E}+00$ & No & NT & PD \\
\hline GW-706 & $\mathrm{T}$ & 25 & 25 & $4.3 \mathrm{E}+01$ & $4.2 \mathrm{E}+01$ & No & $\mathrm{D}$ & $\mathrm{D}$ \\
\hline GW-712 & $\mathrm{T}$ & 23 & 10 & $1.2 \mathrm{E}+00$ & $1.0 \mathrm{E}-02$ & No & D & D \\
\hline GW-713 & $\mathrm{T}$ & 21 & 12 & $2.7 \mathrm{E}+00$ & $1.2 \mathrm{E}+00$ & No & PD & $\mathrm{D}$ \\
\hline GW-714 & $\mathrm{T}$ & 23 & 16 & $2.0 \mathrm{E}+00$ & $1.9 \mathrm{E}+00$ & No & $\mathrm{s}$ & PD \\
\hline SS-4 & $\mathrm{T}$ & 23 & 23 & $4.7 \mathrm{E}+01$ & $4.2 \mathrm{E}+01$ & No & $\mathrm{D}$ & $\mathrm{D}$ \\
\hline SS-5 & $T$ & 22 & 21 & $2.3 \mathrm{E}+01$ & $2.0 \mathrm{E}+01$ & No & PD & PD \\
\hline SS-6_6 & $\mathrm{T}$ & 7 & 6 & $4.8 \mathrm{E}+00$ & $4.1 \mathrm{E}+00$ & No & NT & NT \\
\hline
\end{tabular}




\section{MAROS Statistical Trend Analysis Summary}

\begin{tabular}{ccccccccc} 
& & Number & Number & Average & Median & All & Mann- & Linear \\
Well & Sourcel & of & of & Conc. & Conc. & Samples & Kendall & Regression \\
Tail & Samples & Detects & (mg/L) & (mg/L) & "ND" ? & Trend & Trend \\
\hline
\end{tabular}

GROSS ALPHA ACTIVITY

\begin{tabular}{|c|c|c|c|c|c|c|c|c|}
\hline SS-6E & $\mathrm{T}$ & 14 & 12 & $4.5 \mathrm{E}+00$ & $4.8 \mathrm{E}+00$ & No & NT & s \\
\hline SS-7 & $\mathrm{T}$ & 7 & 5 & $2.9 \mathrm{E}+00$ & $1.5 \mathrm{E}+00$ & No & NT & NT \\
\hline SS-8 & $\mathrm{T}$ & 8 & 3 & 4.7E-01 & $1.0 \mathrm{E}-02$ & No & NT & NT \\
\hline
\end{tabular}

GROSS BETA ACTIVITY

\begin{tabular}{|c|c|c|c|c|c|c|c|c|}
\hline GW-014 & $\mathrm{S}$ & 7 & 2 & $3.1 \mathrm{E}+00$ & 1.0E-02 & No & NT & NT \\
\hline GW-046 & $\mathrm{S}$ & 20 & 17 & $3.8 \mathrm{E}+00$ & $4.1 \mathrm{E}+00$ & No & NT & D \\
\hline GW-052 & $T$ & 3 & 3 & 1.7E+01 & 1.7E+01 & No & $\mathrm{N} / \mathrm{A}$ & N/A \\
\hline GW-053 & $T$ & 14 & 11 & $2.2 \mathrm{E}+00$ & $2.0 \mathrm{E}+00$ & No & $D$ & D \\
\hline GW-058 & $T$ & 1 & 1 & $1.7 \mathrm{E}+01$ & 1.7E+01 & No & N/A & N/A \\
\hline GW-068 & $\mathrm{T}$ & 3 & 3 & $1.2 \mathrm{E}+01$ & $1.1 \mathrm{E}+01$ & No & $\mathrm{N} / \mathrm{A}$ & N/A \\
\hline GW-071 & $\mathrm{S}$ & 7 & 1 & 4.4E-01 & 1.0E-02 & No & NT & D \\
\hline GW-072 & $T$ & 5 & 3 & $2.8 \mathrm{E}+00$ & 4.1E-01 & No & NT & NT \\
\hline GW-077 & $T$ & 11 & 11 & $6.3 \mathrm{E}+00$ & $3.2 \mathrm{E}+00$ & No & NT & PD \\
\hline GW-078 & $\mathrm{T}$ & 11 & 7 & $2.6 \mathrm{E}+00$ & $1.4 \mathrm{E}+00$ & No & NT & NT \\
\hline GW-079 & $\mathrm{T}$ & 13 & 12 & $3.3 \mathrm{E}+00$ & $2.4 \mathrm{E}+00$ & No & $\mathrm{s}$ & S \\
\hline GW-080 & $\mathrm{T}$ & 13 & 10 & $6.4 \mathrm{E}+00$ & $2.6 \mathrm{E}+00$ & No & NT & NT \\
\hline GW-082 & $\mathrm{S}$ & 15 & 10 & $3.9 \mathrm{E}+00$ & $4.3 \mathrm{E}+00$ & No & $\mathrm{s}$ & D \\
\hline GW-089 & $\mathrm{T}$ & 1 & 0 & 1.0E-02 & 1.0E-02 & Yes & ND & ND \\
\hline GW-242 & $T$ & 5 & 3 & $1.1 \mathrm{E}+01$ & $3.3 \mathrm{E}+00$ & No & PD & D \\
\hline GW-257 & $\mathrm{T}$ & 4 & 2 & $1.2 \mathrm{E}+00$ & $1.2 \mathrm{E}+00$ & No & NT & D \\
\hline GW-289 & $T$ & 7 & 3 & $1.9 \mathrm{E}+00$ & 1.0E-02 & No & $\mathrm{D}$ & D \\
\hline GW-291 & $\mathrm{T}$ & 6 & 3 & $1.2 \mathrm{E}+00$ & 8.5E-01 & No & NT & NT \\
\hline GW-624 & $S$ & 4 & 2 & $2.0 \mathrm{E}+00$ & $1.4 \mathrm{E}+00$ & No & NT & D \\
\hline GW-626 & $\mathrm{S}$ & 8 & 3 & $2.2 \mathrm{E}+00$ & 1.0E-02 & No & NT & D \\
\hline GW-627 & $\mathrm{T}$ & 16 & 10 & $5.1 \mathrm{E}+00$ & $3.9 \mathrm{E}+00$ & No & $D$ & $\mathrm{D}$ \\
\hline GW-629 & $\mathrm{T}$ & 7 & 0 & 1.0E-02 & 1.0E-02 & Yes & ND & ND \\
\hline GW-639 & $T$ & 5 & 1 & $9.5 \mathrm{E}-01$ & 1.0E-02 & No & NT & NT \\
\hline GW-653 & $\mathrm{T}$ & 17 & 13 & $2.1 \mathrm{E}+00$ & $2.7 \mathrm{E}+00$ & No & $D$ & $\mathrm{D}$ \\
\hline GW-683 & $T$ & 23 & 23 & $2.1 \mathrm{E}+01$ & $1.9 \mathrm{E}+01$ & No & PD & $\mathrm{S}$ \\
\hline GW-684 & $T$ & 24 & 24 & $2.5 \mathrm{E}+01$ & $2.3 \mathrm{E}+01$ & No & $S$ & $\mathrm{~s}$ \\
\hline GW-694 & $T$ & 9 & 9 & $2.2 \mathrm{E}+01$ & $2.0 \mathrm{E}+01$ & No & NT & NT \\
\hline GW-703 & $\mathrm{T}$ & 22 & 22 & $4.5 \mathrm{E}+01$ & $4.6 \mathrm{E}+01$ & No & 1 & I \\
\hline GW-704 & $\mathrm{T}$ & 24 & 24 & $2.1 \mathrm{E}+01$ & $1.6 \mathrm{E}+01$ & No & $\mathrm{PI}$ & I \\
\hline GW-706 & $\mathrm{T}$ & 25 & 25 & $9.9 \mathrm{E}+01$ & $8.6 \mathrm{E}+01$ & No & $\mathrm{s}$ & $\mathrm{s}$ \\
\hline GW-712 & $\mathrm{T}$ & 22 & 17 & $3.9 \mathrm{E}+00$ & $3.5 \mathrm{E}+00$ & No & NT & $D$ \\
\hline GW-713 & $\mathrm{T}$ & 23 & 18 & $6.3 \mathrm{E}+00$ & $2.9 \mathrm{E}+00$ & No & NT & $D$ \\
\hline GW-714 & $\mathrm{T}$ & 24 & 22 & $4.2 \mathrm{E}+00$ & $4.1 \mathrm{E}+00$ & No & $\mathrm{s}$ & $D$ \\
\hline SS-4 & $\mathrm{T}$ & 23 & 23 & $9.3 \mathrm{E}+01$ & $6.5 \mathrm{E}+01$ & No & NT & 1 \\
\hline SS-5 & $T$ & 26 & 26 & $3.5 \mathrm{E}+01$ & $2.9 \mathrm{E}+01$ & No & NT & NT \\
\hline SS-6_6 & $\mathrm{T}$ & 16 & 15 & $8.8 \mathrm{E}+00$ & $5.5 \mathrm{E}+00$ & No & NT & NT \\
\hline SS-6E & $T$ & 13 & 13 & $1.1 \mathrm{E}+01$ & $9.2 \mathrm{E}+00$ & No & NT & NT \\
\hline SS-7 & $\mathrm{T}$ & 16 & 12 & $5.5 E+00$ & $5.3 \mathrm{E}+00$ & No & 1 & $\mathrm{PI}$ \\
\hline SS-8 & $\mathrm{T}$ & 18 & 9 & $1.3 \mathrm{E}+00$ & 4.7E-01 & No & NT & NT \\
\hline
\end{tabular}


MAROS Statistical Trend Analysis Summary

\begin{tabular}{|c|c|c|c|c|c|c|c|c|}
\hline Well & $\begin{array}{c}\text { Sourcel } \\
\text { Tail }\end{array}$ & $\begin{array}{l}\text { Number } \\
\text { of } \\
\text { Samples }\end{array}$ & $\begin{array}{c}\text { Number } \\
\text { of } \\
\text { Detects }\end{array}$ & $\begin{array}{c}\text { Average } \\
\text { Conc. } \\
\text { (mg/L) }\end{array}$ & $\begin{array}{l}\text { Median } \\
\text { Conc. } \\
\text { (mg/L) }\end{array}$ & $\begin{array}{c}\text { All } \\
\text { Samples } \\
\text { "ND" ? }\end{array}$ & $\begin{array}{c}\text { Mann- } \\
\text { Kendall } \\
\text { Trend }\end{array}$ & $\begin{array}{c}\text { Linear } \\
\text { Regression } \\
\text { Trend }\end{array}$ \\
\hline
\end{tabular}

NITRATE

\begin{tabular}{|c|c|}
\hline GW-014 & $\mathrm{S}$ \\
\hline GW-046 & $\mathrm{S}$ \\
\hline GW-052 & $T$ \\
\hline GW-053 & $T$ \\
\hline GW-058 & $T$ \\
\hline GW-068 & $T$ \\
\hline GW-071 & $\mathrm{S}$ \\
\hline GW-072 & $\mathrm{T}$ \\
\hline GW-079 & $\mathrm{T}$ \\
\hline GW-080 & $T$ \\
\hline GW-082 & $S$ \\
\hline GW-089 & $\mathrm{T}$ \\
\hline GW-242 & $\mathrm{T}$ \\
\hline GW-257 & $T$ \\
\hline GW-289 & $T$ \\
\hline GW-291 & $\mathrm{T}$ \\
\hline GW-624 & $S$ \\
\hline GW-626 & $S$ \\
\hline GW-627 & $\mathrm{T}$ \\
\hline GW-629 & $\mathrm{T}$ \\
\hline GW-653 & $\mathrm{T}$ \\
\hline GW-683 & $T$ \\
\hline GW-684 & $\mathrm{T}$ \\
\hline GW-694 & $T$ \\
\hline GW-703 & $\mathrm{T}$ \\
\hline GW-704 & $T$ \\
\hline GW-706 & $T$ \\
\hline GW-712 & $\mathrm{T}$ \\
\hline GW-713 & $\mathrm{T}$ \\
\hline GW-714 & $\mathrm{T}$ \\
\hline SS-4 & $\mathrm{T}$ \\
\hline SS-5 & $\mathrm{T}$ \\
\hline SS-6_6 & $\mathrm{T}$ \\
\hline SS-6E & $\mathrm{T}$ \\
\hline SS-7 & $\mathrm{T}$ \\
\hline SS-8 & $\mathrm{T}$ \\
\hline
\end{tabular}

\begin{tabular}{|c|c|c|}
\hline 2.0E-02 & $2.0 \mathrm{E}-02$ & Yes \\
\hline 7.2E-01 & 7.2E-01 & No \\
\hline $2.6 \mathrm{E}+00$ & $2.9 \mathrm{E}+00$ & No \\
\hline $2.2 \mathrm{E}-02$ & 2.0E-02 & No \\
\hline 3.7E+00 & $3.7 \mathrm{E}+00$ & No \\
\hline 2.0E-02 & 2.0E-02 & Yes \\
\hline 2.1E-02 & $2.0 \mathrm{E}-02$ & No \\
\hline $2.5 \mathrm{E}-02$ & 2.0E-02 & No \\
\hline $2.0 \mathrm{E}-02$ & $2.0 \mathrm{E}-02$ & Yes \\
\hline $2.0 \mathrm{E}-02$ & 2.0E-02 & Yes \\
\hline 3.0E-02 & $2.0 \mathrm{E}-02$ & No \\
\hline $5.0 \mathrm{E}-02$ & $5.0 \mathrm{E}-02$ & No \\
\hline $2.0 \mathrm{E}-02$ & 2.0E-02 & Yes \\
\hline $2.8 \mathrm{E}-01$ & $2.8 \mathrm{E}-01$ & No \\
\hline $2.9 \mathrm{E}-01$ & 3.0E-01 & No \\
\hline $2.5 \mathrm{E}-01$ & 2.4E-01 & No \\
\hline $2.0 \mathrm{E}-02$ & 2.0E-02 & Yes \\
\hline $3.6 \mathrm{E}-02$ & $2.5 \mathrm{E}-02$ & No \\
\hline $2.0 \mathrm{E}-02$ & $2.0 \mathrm{E}-02$ & Yes \\
\hline $2.0 \mathrm{E}-02$ & $2.0 \mathrm{E}-02$ & Yes \\
\hline $2.0 \mathrm{E}-02$ & 2.0E-02 & Yes \\
\hline $5.7 \mathrm{E}+00$ & 4.1E+00 & No \\
\hline $4.6 \mathrm{E}+00$ & $4.1 \mathrm{E}+00$ & No \\
\hline $6.4 \mathrm{E}+00$ & $5.8 \mathrm{E}+00$ & No \\
\hline 1.7E+01 & $1.8 \mathrm{E}+01$ & No \\
\hline $1.0 \mathrm{E}+01$ & $1.3 \mathrm{E}+01$ & No \\
\hline $2.9 \mathrm{E}+01$ & 2.4E+01 & No \\
\hline 7.6E-02 & 2.1E-02 & No \\
\hline 1.2E-01 & $2.0 \mathrm{E}-02$ & No \\
\hline $1.2 \mathrm{E}+00$ & 5.4E-01 & No \\
\hline $2.5 \mathrm{E}+01$ & $1.8 \mathrm{E}+01$ & No \\
\hline $1.0 \mathrm{E}+01$ & $6.4 \mathrm{E}+00$ & No \\
\hline $4.2 \mathrm{E}-01$ & 3.8E-01 & No \\
\hline $1.3 \mathrm{E}+00$ & $1.3 \mathrm{E}+00$ & No \\
\hline 4.6E-01 & 3.9E-01 & No \\
\hline $2.9 \mathrm{E}-01$ & $2.8 \mathrm{E}-01$ & No \\
\hline
\end{tabular}

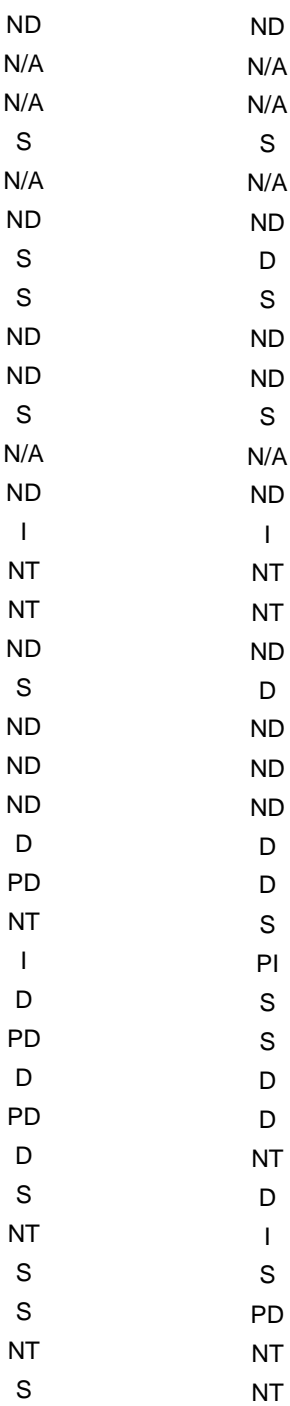

URANIUM

\begin{tabular}{|c|c|c|c|c|c|c|c|c|}
\hline GW-014 & $\mathrm{s}$ & 6 & 6 & $1.8 \mathrm{E}-03$ & $1.7 \mathrm{E}-03$ & No & $\mathrm{s}$ & s \\
\hline GW-046 & $\mathrm{s}$ & 6 & 2 & $2.2 \mathrm{E}-05$ & 4.6E-06 & No & NT & I \\
\hline GW-052 & $\mathrm{T}$ & 1 & 1 & 4.6E-02 & 4.6E-02 & No & N/A & N/A \\
\hline GW-053 & $\mathrm{T}$ & 3 & 3 & $2.8 \mathrm{E}-03$ & 3.0E-03 & No & N/A & $\mathrm{N} / \mathrm{A}$ \\
\hline GW-058 & $\mathrm{T}$ & 1 & 1 & 3.1E-02 & 3.1E-02 & No & N/A & N/A \\
\hline GW-068 & $\mathrm{T}$ & 3 & 0 & 4.6E-06 & 4.6E-06 & Yes & ND & $\mathrm{ND}$ \\
\hline GW-071 & $\mathrm{S}$ & 6 & 1 & $9.4 \mathrm{E}-05$ & 4.6E-06 & No & NT & NT \\
\hline GW-072 & $\mathrm{T}$ & 3 & 0 & 4.6E-06 & 4.6E-06 & Yes & ND & ND \\
\hline
\end{tabular}




\section{MAROS Statistical Trend Analysis Summary}

\begin{tabular}{|c|c|c|c|c|c|c|c|c|}
\hline Well & $\begin{array}{c}\text { Sourcel } \\
\text { Tail }\end{array}$ & $\begin{array}{c}\text { Number } \\
\text { of } \\
\text { Samples }\end{array}$ & $\begin{array}{c}\text { Number } \\
\text { of } \\
\text { Detects }\end{array}$ & $\begin{array}{c}\text { Average } \\
\text { Conc. } \\
\text { (mg/L) }\end{array}$ & $\begin{array}{l}\text { Median } \\
\text { Conc. } \\
\text { (mg/L) }\end{array}$ & $\begin{array}{c}\text { All } \\
\text { Samples } \\
\text { "ND" ? }\end{array}$ & $\begin{array}{l}\text { Mann- } \\
\text { Kendall } \\
\text { Trend }\end{array}$ & $\begin{array}{c}\text { Linear } \\
\text { Regression } \\
\text { Trend }\end{array}$ \\
\hline \multicolumn{9}{|l|}{ URANIUM } \\
\hline GW-077 & $\mathrm{T}$ & 6 & 1 & $1.9 \mathrm{E}-05$ & 4.6E-06 & No & NT & NT \\
\hline GW-078 & $\mathrm{T}$ & 6 & 1 & 4.1E-05 & 4.6E-06 & No & NT & NT \\
\hline GW-079 & $T$ & 6 & 1 & $1.5 \mathrm{E}-05$ & 4.6E-06 & No & NT & NT \\
\hline GW-080 & $T$ & 6 & 1 & $1.2 \mathrm{E}-05$ & 4.6E-06 & No & NT & NT \\
\hline GW-082 & S & 4 & 0 & 4.6E-06 & 4.6E-06 & Yes & ND & ND \\
\hline GW-089 & $\mathrm{T}$ & 1 & 0 & 4.6E-06 & 4.6E-06 & Yes & ND & ND \\
\hline GW-242 & $T$ & 2 & 0 & 4.6E-06 & 4.6E-06 & Yes & ND & ND \\
\hline GW-257 & $T$ & 2 & 0 & 4.6E-06 & 4.6E-06 & Yes & ND & ND \\
\hline GW-289 & $T$ & 4 & 0 & 4.6E-06 & 4.6E-06 & Yes & ND & ND \\
\hline GW-291 & $T$ & 2 & 1 & 7.2E-04 & 7.2E-04 & No & N/A & $\mathrm{N} / \mathrm{A}$ \\
\hline GW-624 & S & 2 & 2 & 1.6E-03 & 1.6E-03 & No & N/A & N/A \\
\hline GW-626 & S & 6 & 0 & 4.6E-06 & 4.6E-06 & Yes & ND & ND \\
\hline GW-627 & $\mathrm{T}$ & 6 & 0 & 4.6E-06 & 4.6E-06 & Yes & ND & ND \\
\hline GW-629 & $T$ & 3 & 0 & 4.6E-06 & 4.6E-06 & Yes & ND & ND \\
\hline GW-639 & $T$ & 6 & 0 & 4.6E-06 & 4.6E-06 & Yes & ND & ND \\
\hline GW-653 & $\mathrm{T}$ & 4 & 0 & 4.6E-06 & 4.6E-06 & Yes & ND & ND \\
\hline GW-683 & $T$ & 4 & 4 & $1.2 \mathrm{E}-02$ & $1.2 \mathrm{E}-02$ & No & NT & $\mathrm{PI}$ \\
\hline GW-684 & $\mathrm{T}$ & 4 & 4 & 1.6E-02 & $1.5 \mathrm{E}-02$ & No & NT & $\mathrm{PI}$ \\
\hline GW-694 & $T$ & 4 & 4 & 3.3E-02 & 3.2E-02 & No & NT & I \\
\hline GW-703 & $\mathrm{T}$ & 4 & 4 & 1.0E-02 & $9.7 \mathrm{E}-03$ & No & NT & NT \\
\hline GW-704 & $T$ & 6 & 3 & 7.5E-03 & $6.1 \mathrm{E}-03$ & No & NT & NT \\
\hline GW-706 & $\mathrm{T}$ & 6 & 6 & $6.4 \mathrm{E}-02$ & $6.3 \mathrm{E}-02$ & No & $\mathrm{s}$ & $\mathrm{s}$ \\
\hline GW-712 & $\mathrm{T}$ & 6 & 2 & $5.8 \mathrm{E}-05$ & 4.6E-06 & No & NT & I \\
\hline GW-713 & $\mathrm{T}$ & 6 & 2 & $1.9 \mathrm{E}-05$ & 4.6E-06 & No & NT & I \\
\hline GW-714 & $T$ & 6 & 2 & 5.4E-04 & 4.6E-06 & No & NT & I \\
\hline SS-4 & $\mathrm{T}$ & 5 & 5 & 7.3E-02 & 7.0E-02 & No & $\mathrm{s}$ & NT \\
\hline SS-5 & $\mathrm{T}$ & 7 & 7 & 2.4E-02 & $2.5 \mathrm{E}-02$ & No & I & I \\
\hline SS-6_6 & $T$ & 4 & 0 & 4.6E-06 & 4.6E-06 & Yes & ND & ND \\
\hline SS-6E & $T$ & 6 & 5 & $6.9 \mathrm{E}-03$ & $8.8 \mathrm{E}-03$ & No & NT & NT \\
\hline SS-7 & $\mathrm{T}$ & 3 & 2 & $9.0 \mathrm{E}-03$ & $1.2 \mathrm{E}-02$ & No & N/A & N/A \\
\hline SS-8 & $T$ & 4 & 0 & 4.6E-06 & 4.6E-06 & Yes & ND & ND \\
\hline
\end{tabular}

Note: Increasing (I); Probably Increasing (PI); Stable (S); Probably Decreasing (PD); Decreasing (D); No Trend (NT); Not Applicable (N/A); Not Applicable (N/A) - Due to insufficient Data (< 4 sampling events); No Detectable Concentration (NDC)

The Number of Samples and Number of Detects shown above are post-consolidation values. 


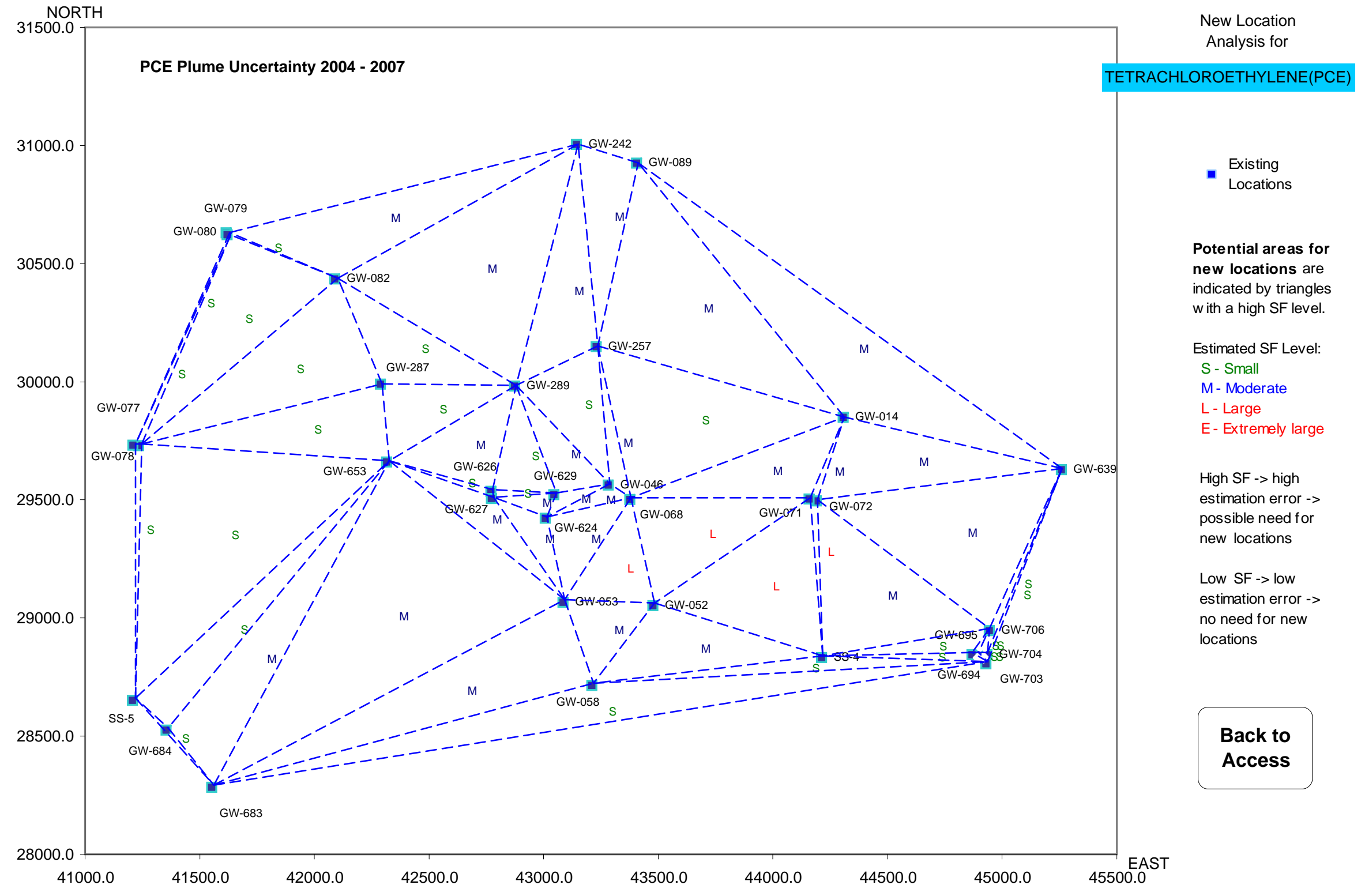


APPENDIX C.4

EAST S-3 AREA 


\section{MAROS COC Assessment}

Project: EF East S3 Beta 4

Location: East S-3

Toxicity:

\begin{tabular}{lccc}
\hline Coxicity: & $\begin{array}{c}\text { Representative } \\
\text { Concentration } \\
\text { (mg/L) }\end{array}$ & $\begin{array}{c}\text { PRG } \\
\text { (mg/L) }\end{array}$ & $\begin{array}{c}\text { Percent } \\
\text { Above } \\
\text { PRG }\end{array}$ \\
\hline NITRATE & $2.0 \mathrm{E}+03$ & $1.0 \mathrm{E}+01$ & $20143.7 \%$ \\
CADMIUM & $2.8 \mathrm{E}-01$ & $5.0 \mathrm{E}-03$ & $5523.0 \%$ \\
TETRACHLOROETHYLENE(PCE) & $2.2 \mathrm{E}-01$ & $5.0 \mathrm{E}-03$ & $4272.6 \%$ \\
TRICHLOROETHYLENE (TCE) & $1.3 \mathrm{E}-01$ & $5.0 \mathrm{E}-03$ & $2493.4 \%$ \\
GROSS BETA ACTIVITY & $1.0 \mathrm{E}+03$ & $5.0 \mathrm{E}+01$ & $1961.0 \%$ \\
VINYL CHLORIDE & $2.6 \mathrm{E}-02$ & $2.0 \mathrm{E}-03$ & $1219.5 \%$ \\
cis-1,2-DICHLOROETHYLENE & $6.7 \mathrm{E}-01$ & $7.0 \mathrm{E}-02$ & $853.0 \%$ \\
BENZENE & $3.5 \mathrm{E}-02$ & $5.0 \mathrm{E}-03$ & $590.9 \%$ \\
NICKEL & $3.8 \mathrm{E}-01$ & $7.3 \mathrm{E}-02$ & $424.2 \%$ \\
COPPER & $4.1 \mathrm{E}+00$ & $1.0 \mathrm{E}+00$ & $305.8 \%$ \\
MERCURY & $8.1 \mathrm{E}-03$ & $2.0 \mathrm{E}-03$ & $303.5 \%$ \\
1,1-DICHLOROETHENE & $2.8 \mathrm{E}-02$ & $7.0 \mathrm{E}-03$ & $301.3 \%$ \\
1,2-DICHLOROETHANE & $1.7 \mathrm{E}-02$ & $5.0 \mathrm{E}-03$ & $232.6 \%$ \\
GROSS ALPHA ACTIVITY & $4.6 \mathrm{E}+01$ & $1.5 \mathrm{E}+01$ & $206.4 \%$ \\
CARBON TETRACHLORIDE & $1.5 \mathrm{E}-02$ & $5.0 \mathrm{E}-03$ & $193.6 \%$ \\
LEAD & $2.6 \mathrm{E}-02$ & $1.5 \mathrm{E}-02$ & $74.6 \%$
\end{tabular}

Note: Top COCs by toxicity were determined by examining a representative concentration for each compound over the entire site. The compound representative concentrations are then compared with the chosen PRG for that compound, with the percentage exceedance from the PRG determining the compound's toxicity. All compounds above exceed the PRG.

\section{Prevalence:}

\begin{tabular}{|c|c|c|c|c|c|}
\hline Contaminant of Concern & Class & $\begin{array}{l}\text { Total } \\
\text { Wells }\end{array}$ & $\begin{array}{c}\text { Total } \\
\text { Exceedances }\end{array}$ & $\begin{array}{c}\text { Percent } \\
\text { Exceedances }\end{array}$ & $\begin{array}{l}\text { Total } \\
\text { detects }\end{array}$ \\
\hline TETRACHLOROETHYLENE(PCE) & ORG & 27 & 18 & $66.7 \%$ & 24 \\
\hline TRICHLOROETHYLENE (TCE) & ORG & 27 & 16 & $59.3 \%$ & 24 \\
\hline NITRATE & INO & 27 & 15 & $55.6 \%$ & 27 \\
\hline CADMIUM & MET & 27 & 15 & $55.6 \%$ & 21 \\
\hline GROSS ALPHA ACTIVITY & INO & 27 & 12 & $44.4 \%$ & 26 \\
\hline VINYL CHLORIDE & ORG & 27 & 12 & $44.4 \%$ & 12 \\
\hline 1,1-DICHLOROETHENE & ORG & 27 & 10 & $37.0 \%$ & 16 \\
\hline cis-1,2-DICHLOROETHYLENE & ORG & 27 & 9 & $33.3 \%$ & 19 \\
\hline GROSS BETA ACTIVITY & INO & 27 & 9 & $33.3 \%$ & 26 \\
\hline NICKEL & MET & 26 & 7 & $26.9 \%$ & 26 \\
\hline LEAD & MET & 23 & 6 & $26.1 \%$ & 23 \\
\hline BENZENE & ORG & 27 & 7 & $25.9 \%$ & 11 \\
\hline CARBON TETRACHLORIDE & ORG & 27 & 5 & $18.5 \%$ & 6 \\
\hline 1,2-DICHLOROETHANE & ORG & 26 & 4 & $15.4 \%$ & 7 \\
\hline MERCURY & MET & 14 & 2 & $14.3 \%$ & 14 \\
\hline COPPER & MET & 21 & 1 & $4.8 \%$ & 21 \\
\hline
\end{tabular}


Note: Top COCs by prevalence were determined by examining a representative concentration for each well location at the site. The total exceedances (values above the chosen PRGs) are compared to the total number of wells to determine the prevalence of the compound.

\section{Mobility:}

Contaminant of Concern

Kd

\begin{tabular}{lr}
\hline GROSS BETA ACTIVITY & \\
GROSS ALPHA ACTIVITY & \\
NITRATE & \\
VINYL CHLORIDE & 0.042 \\
1,2-DICHLOROETHANE & 0.0679 \\
CiS-1,2-DICHLOROETHYLENE & 0.0724 \\
BENZENE & 0.0984 \\
1,1-DICHLOROETHENE & 0.13 \\
CARBON TETRACHLORIDE & 0.277 \\
TRICHLOROETHYLENE (TCE) & 0.297 \\
TETRACHLOROETHYLENE(PCE) & 0.923 \\
LEAD & 10 \\
CADMIUM & 15 \\
NICKEL & 16 \\
COPPER & 40 \\
MERCURY & 52
\end{tabular}

Note: Top COCs by mobility were determined by examining each detected compound in the dataset and comparing their mobilities (Koc's for organics, assume foc $=0.001$, and Kd's for metals).

\section{Contaminants of Concern (COC's)}

NITRATE

TETRACHLOROETHYLENE(PCE)

TRICHLOROETHYLENE (TCE)

CADMIUM

GROSS ALPHA ACTIVITY 


\section{MAROS Spatial Moment Analysis Summary}

Project: EF S3 Beta 4

Location: East S-3
User Name: MV

State: Tennessee 2nd Moment (Spread)

Sigma XX Sigma $Y Y$

(sq ft)

(sq ft)
Number of Wells

CADMIUM

\begin{tabular}{|c|c|c|c|c|c|c|}
\hline 7/1/1996 & $0.0 \mathrm{E}+00$ & & & & & \\
\hline 7/1/1997 & $0.0 \mathrm{E}+00$ & & & & & \\
\hline 7/1/1998 & $0.0 \mathrm{E}+00$ & & & & & \\
\hline 7/1/1999 & $0.0 \mathrm{E}+00$ & & & & & \\
\hline $7 / 1 / 2000$ & $2.8 \mathrm{E}+00$ & 53,853 & 29,812 & 695 & 113,054 & 13,414 \\
\hline 7/1/2001 & $0.0 \mathrm{E}+00$ & & & & & \\
\hline $7 / 1 / 2002$ & $0.0 \mathrm{E}+00$ & & & & & \\
\hline $7 / 1 / 2003$ & $2.8 \mathrm{E}+00$ & 53,825 & 29,821 & 666 & 69,864 & 12,920 \\
\hline $7 / 1 / 2004$ & $0.0 \mathrm{E}+00$ & & & & & \\
\hline $7 / 1 / 2005$ & 9.6E-01 & 54,171 & 29,569 & 1,086 & 19,460 & 3,277 \\
\hline $7 / 1 / 2006$ & $6.9 \mathrm{E}-01$ & 53,615 & 29,731 & 530 & 111,453 & 13,881 \\
\hline $7 / 1 / 2007$ & $6.9 \mathrm{E}-01$ & 53,665 & 29,742 & 563 & 161,410 & 13,170 \\
\hline
\end{tabular}

GROSS BETA ACTIVITY

\begin{tabular}{|c|c|c|c|c|c|c|c|}
\hline $7 / 1 / 1996$ & $3.3 E+04$ & 53,570 & 30,024 & 366 & 96,883 & 49,852 & 14 \\
\hline 7/1/1997 & $9.6 \mathrm{E}+03$ & 53,664 & 29,965 & 469 & 94,925 & 36,947 & 7 \\
\hline $7 / 1 / 1998$ & $7.0 \mathrm{E}+03$ & 53,602 & 29,911 & 426 & 92,693 & 42,062 & 9 \\
\hline 7/1/1999 & $6.9 \mathrm{E}+03$ & 53,637 & 29,923 & 454 & 113,916 & 62,770 & 8 \\
\hline $7 / 1 / 2000$ & $5.4 \mathrm{E}+04$ & 53,453 & 30,030 & 249 & 50,250 & 31,748 & 12 \\
\hline 7/1/2001 & $5.2 \mathrm{E}+03$ & 53,664 & 29,968 & 469 & 67,537 & 36,011 & 6 \\
\hline $7 / 1 / 2002$ & $1.1 \mathrm{E}+04$ & 53,494 & 29,866 & 352 & 116,755 & 14,151 & 6 \\
\hline $7 / 1 / 2003$ & $8.6 \mathrm{E}+04$ & 53,474 & 29,976 & 283 & 42,075 & 28,916 & 15 \\
\hline $7 / 1 / 2004$ & $3.0 \mathrm{E}+04$ & 53,733 & 30,035 & 527 & 132,679 & 28,895 & 6 \\
\hline 7/1/2005 & $8.9 \mathrm{E}+03$ & 53,362 & 29,951 & 196 & 97,952 & 3,875 & 6 \\
\hline $7 / 1 / 2006$ & $1.1 \mathrm{E}+04$ & 53,260 & 30,042 & 60 & 29,322 & 21,990 & 23 \\
\hline $7 / 1 / 2007$ & $9.7 \mathrm{E}+02$ & 53,462 & 29,938 & 287 & 43,458 & 28,148 & 24 \\
\hline
\end{tabular}

NITRATE

$\begin{array}{rrrrrrrr}7 / 1 / 1996 & 1.5 \mathrm{E}+04 & 53,953 & 29,873 & 772 & 152,900 & 33,796 & 10 \\ 7 / 1 / 1997 & 1.5 \mathrm{E}+04 & 53,922 & 29,829 & 755 & 171,753 & 13,860 & 6 \\ 7 / 1 / 1998 & 8.2 \mathrm{E}+03 & 53,512 & 29,822 & 393 & 81,997 & 39,714 & 9 \\ 7 / 1 / 1999 & 0.0 \mathrm{E}+00 & & & & & & 4 \\ 7 / 1 / 2000 & 8.1 \mathrm{E}+04 & 53,749 & 29,956 & 554 & 153,260 & & \\ 7 / 1 / 2001 & 0.0 \mathrm{E}+00 & & & & & & 2 \\ 7 / 1 / 2002 & 0.0 \mathrm{E}+00 & & & & & & 35,283 \\ 7 / 1 / 2003 & 6.8 \mathrm{E}+04 & 53,542 & 30,037 & 337 & 72,532 & & 14 \\ 7 / 1 / 2004 & 0.0 \mathrm{E}+00 & & & & & & 5 \\ 7 / 1 / 2005 & 7.3 \mathrm{E}+03 & 53,518 & 29,787 & 421 & 115,383 & 13,297 & 6 \\ 7 / 1 / 2006 & 4.9 \mathrm{E}+04 & 53,519 & 29,972 & 327 & 65,339 & 32,432 & 23\end{array}$


Project: EF S3 Beta 4

Location: East S-3
User Name: MV

State: Tennessee

\begin{tabular}{|c|c|c|c|c|c|c|c|}
\hline \multirow[b]{2}{*}{ Effective Date } & \multirow{2}{*}{$\begin{array}{l}\text { Oth Moment } \\
\text { Estimated } \\
\text { Mass (kg) }\end{array}$} & \multicolumn{3}{|c|}{ 1st Moment (Center of Mass) } & \multicolumn{2}{|c|}{ 2nd Moment (Spread) } & \multirow[b]{2}{*}{$\begin{array}{c}\text { Number of } \\
\text { Wells }\end{array}$} \\
\hline & & $X c(f t)$ & Yc (ft) & $\begin{array}{c}\text { Source } \\
\text { Distance (ft) }\end{array}$ & $\begin{array}{c}\text { Sigma } X X \\
(s q \mathrm{ft})\end{array}$ & $\begin{array}{l}\text { Sigma } Y Y \\
(\mathrm{sq} \mathrm{ft})\end{array}$ & \\
\hline \multicolumn{8}{|l|}{ NITRATE } \\
\hline $7 / 1 / 2007$ & $5.2 E+04$ & 53,585 & 29,997 & 385 & 97,438 & 37,834 & 24 \\
\hline \multicolumn{8}{|c|}{ TETRACHLOROETHYLENE(PCE) } \\
\hline $7 / 1 / 1996$ & $1.6 \mathrm{E}+00$ & 53,980 & 29,600 & 905 & 119,244 & 26,882 & 11 \\
\hline 7/1/1997 & $1.3 \mathrm{E}+00$ & 54,101 & 29,983 & 898 & 161,503 & 18,739 & 7 \\
\hline 7/1/1998 & $1.7 \mathrm{E}+01$ & 54,319 & 29,900 & 1,125 & 150,350 & 66,157 & 11 \\
\hline 7/1/1999 & $1.6 \mathrm{E}+01$ & 54,004 & 29,741 & 862 & 143,724 & 23,758 & 8 \\
\hline $7 / 1 / 2000$ & $2.8 \mathrm{E}+01$ & 53,998 & 29,906 & 808 & 219,225 & 58,690 & 12 \\
\hline 7/1/2001 & $2.8 \mathrm{E}+00$ & 53,955 & 29,836 & 784 & 133,573 & 55,847 & 6 \\
\hline $7 / 1 / 2002$ & $2.3 \mathrm{E}+00$ & 53,781 & 29,663 & 703 & 139,760 & 17,787 & 6 \\
\hline $7 / 1 / 2003$ & $2.6 \mathrm{E}+01$ & 54,042 & 29,908 & 851 & 204,694 & 48,640 & 19 \\
\hline $7 / 1 / 2004$ & $1.2 \mathrm{E}+00$ & 53,897 & 29,875 & 717 & 417,763 & 23,701 & 6 \\
\hline $7 / 1 / 2005$ & $2.2 \mathrm{E}+00$ & 53,832 & 29,684 & 735 & 124,584 & 17,535 & 6 \\
\hline $7 / 1 / 2006$ & $2.6 \mathrm{E}+01$ & 53,771 & 29,885 & 594 & 139,283 & 65,243 & 23 \\
\hline $7 / 1 / 2007$ & $1.4 \mathrm{E}+01$ & 54,004 & 29,830 & 832 & 201,826 & 24,607 & 24 \\
\hline
\end{tabular}

TRICHLOROETHYLENE (TCE)

\begin{tabular}{|c|c|c|c|c|c|c|c|}
\hline $7 / 1 / 1996$ & $2.1 \mathrm{E}+00$ & 54,165 & 29,812 & 992 & 64,999 & 58,312 & 11 \\
\hline $7 / 1 / 1997$ & $2.6 \mathrm{E}+00$ & 53,867 & 29,953 & 670 & 181,222 & 28,484 & 7 \\
\hline 7/1/1998 & $1.5 \mathrm{E}+01$ & 54,234 & 29,919 & 1,038 & 139,237 & 65,103 & 11 \\
\hline 7/1/1999 & $1.4 \mathrm{E}+01$ & 54,108 & 29,798 & 941 & 75,602 & 38,735 & 8 \\
\hline $7 / 1 / 2000$ & $1.4 \mathrm{E}+01$ & 54,355 & 29,910 & 1,159 & 137,108 & 77,880 & 12 \\
\hline $7 / 1 / 2001$ & $2.8 \mathrm{E}+00$ & 53,990 & 29,883 & 805 & 116,822 & 51,491 & 6 \\
\hline $7 / 1 / 2002$ & $2.3 \mathrm{E}+00$ & 53,865 & 29,669 & 770 & 115,131 & 14,566 & 6 \\
\hline $7 / 1 / 2003$ & $1.2 \mathrm{E}+01$ & 54,338 & 29,869 & 1,148 & 95,673 & 57,997 & 19 \\
\hline $7 / 1 / 2004$ & 5.8E-01 & 53,941 & 29,879 & 759 & 384,654 & 23,315 & 6 \\
\hline $7 / 1 / 2005$ & $1.3 \mathrm{E}+00$ & 54,013 & 29,688 & 892 & 42,655 & 14,375 & 6 \\
\hline $7 / 1 / 2006$ & $8.6 \mathrm{E}+00$ & 54,135 & 29,752 & 981 & 123,183 & 38,891 & 23 \\
\hline $7 / 1 / 2007$ & $5.1 \mathrm{E}+00$ & 54,306 & 29,786 & 1,135 & 99,899 & 36,251 & 24 \\
\hline
\end{tabular}


Project: EF S3 Beta 4

Location: East S-3
User Name: $\mathrm{MV}$

State: Tennessee

\begin{tabular}{llllll} 
Moment Type & Constituent & $\begin{array}{l}\text { Coefficient } \\
\text { of Variation }\end{array}$ & $\begin{array}{c}\text { Mann-Kendall } \\
\text { S Statistic }\end{array}$ & $\begin{array}{c}\text { Confidence } \\
\text { in Trend }\end{array}$ & $\begin{array}{c}\text { Moment } \\
\text { Trend }\end{array}$ \\
\hline
\end{tabular}

Zeroth Moment: Mass

$\begin{array}{lcccc}\text { CADMIUM } & 1.60 & 17 & 86.0 \% & \text { NT } \\ \text { GROSS BETA ACTIVITY } & 1.16 & -10 & 72.7 \% & \text { NT } \\ \text { NITRATE } & 1.20 & 2 & 52.7 \% & \text { NT } \\ \text { TETRACHLOROETHYLENE(PCE) } & 0.95 & 4 & 58.0 \% & \text { NT } \\ \text { TRICHLOROETHYLENE (TCE) } & 0.85 & -12 & 77.0 \% & \mathrm{~S}\end{array}$

1st Moment: Distance to Source

CADMIUM
GROSS BETA ACTIVITY
NITRATE
TETRACHLOROETHYLENE(PCE)
TRICHLOROETHYLENE (TCE)

$\begin{array}{lccc}0.31 & -4 & 75.8 \% & \mathrm{~S} \\ 0.39 & -22 & 92.4 \% & \mathrm{PD} \\ 0.37 & -18 & 98.4 \% & \mathrm{D} \\ 0.16 & -38 & 99.6 \% & \mathrm{D} \\ 0.18 & 4 & 58.0 \% & \mathrm{NT}\end{array}$

2nd Moment: Sigma XX

CADMIUM
GROSS BETA ACTIVITY
NITRATE
TETRACHLOROETHYLENE(PCE)
TRICHLOROETHYLENE (TCE)

$\begin{array}{cccc}0.56 & 2 & 59.2 \% & \text { NT } \\ 0.42 & -14 & 81.0 \% & \mathrm{~S} \\ 0.36 & -12 & 91.1 \% & \text { PD } \\ 0.46 & 6 & 63.1 \% & \text { NT } \\ 0.67 & -8 & 68.1 \% & \mathrm{~S}\end{array}$

2nd Moment: Sigma YY

\begin{tabular}{ll} 
CADMIUM & 0.40 \\
GROSS BETA ACTIVITY & 0.48 \\
NITRATE & 0.40 \\
TETRACHLOROETHYLENE(PCE) & 0.53 \\
TRICHLOROETHYLENE (TCE) & 0.48 \\
\hline
\end{tabular}

\begin{tabular}{lccc}
0.40 & 0 & $40.8 \%$ & $\mathrm{~S}$ \\
0.48 & -42 & $99.8 \%$ & $\mathrm{D}$ \\
0.40 & 2 & $54.8 \%$ & $\mathrm{NT}$ \\
0.53 & -8 & $68.1 \%$ & $\mathrm{~S}$ \\
0.48 & -20 & $90.2 \%$ & PD \\
\hline
\end{tabular}

Note: The following assumptions were applied for the calculation of the Zeroth Moment:

Porosity: $0.10 \quad$ Saturated Thickness: Uniform: $50 \mathrm{ft}$

Mann-Kendall Trend test performed on all sample events for each constituent. Increasing (I); Probably Increasing (PI); Stable (S); Probably Decreasing (PD); Decreasing (D); No Trend (NT); Not Applicable (N/A)-Due to insufficient Data (< 4 sampling events).

Note: The Sigma $X X$ and Sigma $Y Y$ components are estimated using the given field coordinate system and then rotated to align with the estimated groundwater flow direction. Moments are not calculated for sample events with less than 6 wells. 


\section{MAROS Statistical Trend Analysis Summary}

Project: $\quad$ EF East S3 Beta 4

Location: East S-3

Time Period: $1 / 1 / 1996$ to $10 / 15 / 2007$

Consolidation Period: No Time Consolidation

Consolidation Type: Median

Duplicate Consolidation: Average

ND Values: Specified Detection Limit

J Flag Values : Actual Value
User Name: MV

State: Tennessee

\begin{tabular}{|c|c|c|c|c|c|c|c|c|}
\hline Well & $\begin{array}{c}\text { Sourcel } \\
\text { Tail }\end{array}$ & $\begin{array}{l}\text { Number } \\
\text { of } \\
\text { Samples }\end{array}$ & $\begin{array}{c}\text { Number } \\
\text { of } \\
\text { Detects }\end{array}$ & $\begin{array}{l}\text { Average } \\
\text { Conc. } \\
\text { (mg/L) }\end{array}$ & $\begin{array}{l}\text { Median } \\
\text { Conc. } \\
\text { (mg/L) }\end{array}$ & $\begin{array}{c}\text { All } \\
\text { Samples } \\
\text { "ND" ? }\end{array}$ & $\begin{array}{l}\text { Mann- } \\
\text { Kendall } \\
\text { Trend }\end{array}$ & $\begin{array}{l}\text { Linear } \\
\text { Regression } \\
\text { Trend }\end{array}$ \\
\hline \multicolumn{9}{|l|}{ CADMIUM } \\
\hline $55-1 A$ & $\mathrm{~T}$ & 2 & 0 & $5.1 \mathrm{E}-06$ & $5.1 \mathrm{E}-06$ & Yes & ND & ND \\
\hline $55-2 A$ & $\mathrm{~T}$ & 4 & 0 & $5.1 \mathrm{E}-06$ & $5.1 \mathrm{E}-06$ & Yes & ND & ND \\
\hline $55-2 B$ & $\mathrm{~T}$ & 4 & 0 & $5.1 \mathrm{E}-06$ & $5.1 \mathrm{E}-06$ & Yes & ND & ND \\
\hline $55-2 C$ & $\mathrm{~T}$ & 2 & 0 & $5.1 \mathrm{E}-06$ & $5.1 \mathrm{E}-06$ & Yes & ND & ND \\
\hline GW-105 & $\mathrm{T}$ & 4 & 2 & $1.1 \mathrm{E}-03$ & $1.1 \mathrm{E}-03$ & No & NT & $\mathrm{D}$ \\
\hline GW-106 & $\mathrm{T}$ & 4 & 2 & 3.8E-04 & $2.9 \mathrm{E}-04$ & No & NT & D \\
\hline GW-108 & s & 7 & 1 & 2.5E-04 & $5.1 \mathrm{E}-06$ & No & NT & D \\
\hline GW-109 & s & 7 & 7 & $1.6 \mathrm{E}+00$ & $1.7 \mathrm{E}+00$ & No & $D$ & D \\
\hline GW-192 & $\mathrm{T}$ & 2 & 0 & $5.1 \mathrm{E}-06$ & $5.1 \mathrm{E}-06$ & Yes & ND & ND \\
\hline GW-251 & $\mathrm{T}$ & 23 & 23 & $9.7 \mathrm{E}-02$ & $8.8 \mathrm{E}-02$ & No & PD & $\mathrm{s}$ \\
\hline GW-253 & $\mathrm{T}$ & 17 & 17 & $3.7 \mathrm{E}+00$ & $3.7 \mathrm{E}+00$ & No & NT & NT \\
\hline GW-265 & $\mathrm{T}$ & 4 & 0 & $5.1 \mathrm{E}-06$ & $5.1 \mathrm{E}-06$ & Yes & ND & ND \\
\hline GW-269 & $\mathrm{T}$ & 4 & 0 & 5.1E-06 & $5.1 \mathrm{E}-06$ & Yes & ND & ND \\
\hline GW-270 & $\mathrm{T}$ & 1 & 0 & $5.1 \mathrm{E}-06$ & $5.1 \mathrm{E}-06$ & Yes & ND & ND \\
\hline GW-272 & $\mathrm{T}$ & 2 & 1 & $1.0 \mathrm{E}-03$ & $1.0 \mathrm{E}-03$ & No & $N / A$ & $N / A$ \\
\hline GW-273 & $\mathrm{T}$ & 1 & 0 & $5.1 \mathrm{E}-06$ & $5.1 \mathrm{E}-06$ & Yes & ND & ND \\
\hline GW-274 & S & 5 & 3 & 7.2E-04 & $8.2 \mathrm{E}-04$ & No & $P D$ & $\mathrm{D}$ \\
\hline GW-275 & s & 2 & 0 & 5.1E-06 & 5.1E-06 & Yes & ND & ND \\
\hline GW-332 & $\mathrm{T}$ & 2 & 0 & 5.1E-06 & 5.1E-06 & Yes & ND & ND \\
\hline GW-336 & $\mathrm{T}$ & 2 & 0 & 5.1E-06 & 5.1E-06 & Yes & ND & ND \\
\hline GW-337 & $\mathrm{T}$ & 4 & 2 & $9.1 \mathrm{E}-04$ & 3.5E-04 & No & NT & $\mathrm{D}$ \\
\hline GW-505 & $\mathrm{T}$ & 1 & 0 & $5.1 \mathrm{E}-06$ & $5.1 \mathrm{E}-06$ & Yes & ND & ND \\
\hline GW-617 & $\mathrm{T}$ & 1 & 0 & 5.1E-06 & $5.1 \mathrm{E}-06$ & Yes & ND & ND \\
\hline GW-618 & $\mathrm{T}$ & 18 & 17 & $7.8 \mathrm{E}-03$ & $5.1 \mathrm{E}-03$ & No & $\mathrm{D}$ & $\mathrm{D}$ \\
\hline GW-619 & $\mathrm{T}$ & 1 & 0 & $5.1 \mathrm{E}-06$ & $5.1 \mathrm{E}-06$ & Yes & ND & ND \\
\hline GW-620 & $\mathrm{T}$ & 6 & 2 & 2.3E-04 & $5.1 \mathrm{E}-06$ & No & NT & $\mathrm{D}$ \\
\hline GW-633 & S & 9 & 6 & $6.5 \mathrm{E}-04$ & $6.9 \mathrm{E}-04$ & No & $\mathrm{D}$ & D \\
\hline
\end{tabular}

GROSS ALPHA ACTIVITY

$\begin{array}{lcc}55-1 A & \text { T } & 4 \\ 55-2 A & \text { T } & 4 \\ 55-2 B & \text { T } & 8 \\ 55-2 C & \text { T } & 10 \\ \text { GW-105 } & \text { T } & 3 \\ \text { GW-106 } & \text { T } & 4\end{array}$

$\begin{array}{llll}2 & 3.3 \mathrm{E}-01 & 1.1 \mathrm{E}-01 & \text { No } \\ 0 & 1.0 \mathrm{E}-02 & 1.0 \mathrm{E}-02 & \text { Yes } \\ 3 & 4.9 \mathrm{E}+00 & 1.0 \mathrm{E}-02 & \text { No } \\ 6 & 2.0 \mathrm{E}+00 & 1.4 \mathrm{E}+00 & \text { No } \\ 2 & 5.1 \mathrm{E}+01 & 3.2 \mathrm{E}+00 & \text { No } \\ 2 & 4.0 \mathrm{E}+01 & 6.6 \mathrm{E}-01 & \text { No }\end{array}$

$\begin{array}{ll}\text { NT } & \text { D } \\ \text { ND } & \text { ND } \\ \text { NT } & \text { NT } \\ \text { NT } & \text { NT } \\ \text { N/A } & \text { N/A } \\ \text { NT } & \text { PD }\end{array}$




\section{MAROS Statistical Trend Analysis Summary}

\begin{tabular}{ccccccccc} 
& & Number & Number & Average & Median & All & Mann- & Linear \\
Well & Sourcel & of & of & Conc. & Conc. & Samples & Kendall & Regression \\
Tail & Samples & Detects & (mg/L) & (mg/L) & "ND" ? & Trend & Trend \\
\hline
\end{tabular}

GROSS ALPHA ACTIVITY

\begin{tabular}{|c|c|}
\hline GW-108 & $\mathrm{s}$ \\
\hline GW-109 & $S$ \\
\hline GW-192 & $\mathrm{T}$ \\
\hline GW-251 & $\mathrm{T}$ \\
\hline GW-253 & $\mathrm{T}$ \\
\hline GW-265 & $\mathrm{T}$ \\
\hline GW-269 & $\mathrm{T}$ \\
\hline GW-270 & $\mathrm{T}$ \\
\hline GW-272 & $\mathrm{T}$ \\
\hline GW-273 & $\mathrm{T}$ \\
\hline GW-274 & $\mathrm{s}$ \\
\hline GW-275 & $\mathrm{s}$ \\
\hline GW-332 & $\mathrm{T}$ \\
\hline GW-336 & $\mathrm{T}$ \\
\hline GW-337 & $\mathrm{T}$ \\
\hline GW-505 & $\mathrm{T}$ \\
\hline GW-617 & $\mathrm{T}$ \\
\hline GW-618 & $\mathrm{T}$ \\
\hline GW-619 & $\mathrm{T}$ \\
\hline GW-620 & $\mathrm{T}$ \\
\hline GW-633 & $\mathrm{s}$ \\
\hline
\end{tabular}

23
7
9
22
18
3
5
2
3
1
5
6
3
2
6
4
4
18
4
17
9

$\begin{array}{cccc}18 & 2.3 \mathrm{E}+02 & 1.4 \mathrm{E}+02 & \text { No } \\ 5 & 1.2 \mathrm{E}+02 & 8.6 \mathrm{E}+01 & \text { No } \\ 8 & 1.2 \mathrm{E}+00 & 9.0 \mathrm{E}-01 & \text { No } \\ 21 & 6.9 \mathrm{E}+00 & 7.5 \mathrm{E}+00 & \text { No } \\ 18 & 4.5 \mathrm{E}+01 & 3.8 \mathrm{E}+01 & \text { No } \\ 0 & 1.0 \mathrm{E}-02 & 1.0 \mathrm{E}-02 & \text { Yes } \\ 2 & 6.7 \mathrm{E}-01 & 1.0 \mathrm{E}-02 & \text { No } \\ 2 & 4.3 \mathrm{E}+00 & 4.3 \mathrm{E}+00 & \text { No } \\ 3 & 2.2 \mathrm{E}+01 & 2.0 \mathrm{E}+01 & \text { No } \\ 1 & 1.1 \mathrm{E}+00 & 1.1 \mathrm{E}+00 & \text { No } \\ 3 & 4.0 \mathrm{E}+01 & 2.0 \mathrm{E}+00 & \text { No } \\ 4 & 7.5 \mathrm{E}+01 & 7.8 \mathrm{E}+01 & \text { No } \\ 1 & 3.4 \mathrm{E}-01 & 1.0 \mathrm{E}-02 & \text { No } \\ 2 & 3.1 \mathrm{E}+00 & 3.1 \mathrm{E}+00 & \text { No } \\ 4 & 6.8 \mathrm{E}-01 & 6.7 \mathrm{E}-01 & \text { No } \\ 4 & 3.0 \mathrm{E}+01 & 2.6 \mathrm{E}+01 & \text { No } \\ 3 & 6.7 \mathrm{E}-01 & 5.3 \mathrm{E}-01 & \text { No } \\ 12 & 1.4 \mathrm{E}+00 & 9.6 \mathrm{E}-01 & \text { No } \\ 3 & 2.1 \mathrm{E}+00 & 2.5 \mathrm{E}+00 & \text { No } \\ 15 & 1.6 \mathrm{E}+00 & 1.0 \mathrm{E}+00 & \text { No } \\ 8 & 3.2 \mathrm{E}+01 & 1.6 \mathrm{E}+01 & \text { No }\end{array}$

NITRATE

\begin{tabular}{|c|c|c|c|c|c|c|c|c|}
\hline $55-1 A$ & $\mathrm{~T}$ & 5 & 5 & $9.7 \mathrm{E}+00$ & $8.8 \mathrm{E}+00$ & No & $S$ & PD \\
\hline $55-2 A$ & $\mathrm{~T}$ & 4 & 4 & $1.8 \mathrm{E}+02$ & $1.8 \mathrm{E}+02$ & No & $\mathrm{s}$ & $\mathrm{s}$ \\
\hline $55-2 B$ & $\mathrm{~T}$ & 9 & 9 & $2.1 \mathrm{E}+02$ & $2.3 \mathrm{E}+02$ & No & I & 1 \\
\hline $55-2 C$ & $\mathrm{~T}$ & 10 & 10 & $1.6 \mathrm{E}+02$ & $1.5 \mathrm{E}+02$ & No & I & I \\
\hline GW-105 & $\mathrm{T}$ & 4 & 4 & $6.1 \mathrm{E}+02$ & $6.6 \mathrm{E}+02$ & No & NT & NT \\
\hline GW-106 & $\mathrm{T}$ & 4 & 4 & $7.1 \mathrm{E}+02$ & $7.1 \mathrm{E}+02$ & No & $\mathrm{s}$ & PD \\
\hline GW-108 & $\mathrm{S}$ & 11 & 11 & $1.8 \mathrm{E}+04$ & $1.1 \mathrm{E}+04$ & No & $\mathrm{D}$ & NT \\
\hline GW-109 & $\mathrm{S}$ & 6 & 6 & $9.4 \mathrm{E}+03$ & $9.2 \mathrm{E}+03$ & No & NT & I \\
\hline GW-192 & $\mathrm{T}$ & 6 & 4 & 3.0E-02 & 3.0E-02 & No & $\mathrm{D}$ & D \\
\hline GW-251 & $\mathrm{T}$ & 23 & 23 & $5.4 \mathrm{E}+01$ & $5.6 \mathrm{E}+01$ & No & PD & s \\
\hline GW-253 & $\mathrm{T}$ & 5 & 5 & $6.3 \mathrm{E}+02$ & $5.5 \mathrm{E}+02$ & No & $S$ & S \\
\hline GW-265 & $\mathrm{T}$ & 4 & 4 & 1.1E-01 & 1.1E-01 & No & NT & NT \\
\hline GW-269 & $\mathrm{T}$ & 6 & 6 & 5.9E-01 & 6.1E-01 & No & NT & 1 \\
\hline GW-270 & $\mathrm{T}$ & 3 & 3 & $7.1 \mathrm{E}+01$ & $6.5 \mathrm{E}+01$ & No & N/A & $\mathrm{N} / \mathrm{A}$ \\
\hline GW-272 & $\mathrm{T}$ & 3 & 3 & 4.7E+02 & $4.2 \mathrm{E}+02$ & No & N/A & $\mathrm{N} / \mathrm{A}$ \\
\hline GW-273 & $\mathrm{T}$ & 1 & 0 & $2.9 \mathrm{E}-04$ & $2.9 \mathrm{E}-04$ & Yes & ND & ND \\
\hline GW-274 & $\mathrm{s}$ & 6 & 6 & $3.3 \mathrm{E}+03$ & $3.0 \mathrm{E}+03$ & No & $\mathrm{D}$ & $\mathrm{D}$ \\
\hline GW-275 & $\mathrm{s}$ & 6 & 6 & $7.5 \mathrm{E}+03$ & $7.3 \mathrm{E}+03$ & No & $\mathrm{PI}$ & I \\
\hline GW-332 & $\mathrm{T}$ & 3 & 3 & $1.9 \mathrm{E}+00$ & $1.9 \mathrm{E}+00$ & No & $\mathrm{N} / \mathrm{A}$ & $\mathrm{N} / \mathrm{A}$ \\
\hline GW-336 & $\mathrm{T}$ & 4 & 4 & $1.7 \mathrm{E}+00$ & $1.7 \mathrm{E}+00$ & No & $\mathrm{s}$ & $\mathrm{s}$ \\
\hline GW-337 & $\mathrm{T}$ & 2 & 2 & $3.1 \mathrm{E}+00$ & $3.1 \mathrm{E}+00$ & No & $\mathrm{N} / \mathrm{A}$ & $\mathrm{N} / \mathrm{A}$ \\
\hline GW-505 & $\mathrm{T}$ & 3 & 3 & 2.7E-01 & 1.8E-01 & No & N/A & $\mathrm{N} / \mathrm{A}$ \\
\hline GW-617 & $\mathrm{T}$ & 6 & 6 & 1.7E+00 & $2.2 \mathrm{E}+00$ & No & $\mathrm{s}$ & NT \\
\hline
\end{tabular}

$\begin{array}{cc}\text { NT } & \text { NT } \\ \text { PD } & \text { D } \\ \text { D } & \text { D } \\ \text { D } & \text { D } \\ \text { S } & \text { S } \\ \text { ND } & \text { ND } \\ \text { NT } & \text { NT } \\ \text { N/A } & \text { N/A } \\ \text { N/A } & \text { N/A } \\ \text { N/A } & \text { N/A } \\ \text { D } & \text { D } \\ \text { S } & \text { D } \\ \text { N/A } & \text { N/A } \\ \text { N/A } & \text { N/A } \\ \text { D } & \text { D } \\ \text { S } & \text { D } \\ \text { D } & \text { D } \\ \text { D } & \text { D } \\ \text { D } & \text { D } \\ \text { D } & \text { D } \\ \text { NT } & \text { NT }\end{array}$




\section{MAROS Statistical Trend Analysis Summary}

\begin{tabular}{ccccccccc} 
& & Number & Number & Average & Median & All & Mann- & Linear \\
Well & Sourcel & of & of & Conc. & Conc. & Samples & Kendall & Regression \\
Tail & Samples & Detects & (mg/L) & (mg/L) & "ND" ? & Trend & Trend \\
\hline
\end{tabular}

NITRATE

$\begin{array}{cccccccccc}\text { GW-618 } & \text { T } & 11 & 10 & 8.3 \mathrm{E}-01 & 4.1 \mathrm{E}-01 & \text { No } & \text { D } & \text { D } \\ \text { GW-619 } & \mathrm{T} & 4 & 4 & 1.0 \mathrm{E}+00 & 1.0 \mathrm{E}+00 & \text { No } & \text { NT } & \text { NT } \\ \text { GW-620 } & \mathrm{T} & 23 & 23 & 1.6 \mathrm{E}+00 & 1.3 \mathrm{E}+00 & \text { No } & \text { D } & \text { D } \\ \text { GW-633 } & \mathrm{S} & 10 & 10 & 1.4 \mathrm{E}+03 & 1.4 \mathrm{E}+03 & \text { No } & \text { D } & \text { D }\end{array}$

TETRACHLOROETHYLENE(PCE)

\begin{tabular}{|c|c|c|c|c|c|c|c|c|}
\hline $55-1 A$ & $\mathrm{~T}$ & 5 & 0 & $6.0 \mathrm{E}-06$ & $6.0 \mathrm{E}-06$ & Yes & ND & ND \\
\hline $55-2 A$ & $\mathrm{~T}$ & 4 & 4 & 2.6E-01 & 2.6E-01 & No & D & D \\
\hline $55-2 B$ & $\mathrm{~T}$ & 9 & 9 & $5.8 \mathrm{E}-01$ & 5.7E-01 & No & NT & NT \\
\hline $55-2 C$ & $\mathrm{~T}$ & 10 & 10 & $4.9 \mathrm{E}-01$ & 5.0E-01 & No & $S$ & S \\
\hline GW-105 & $\mathrm{T}$ & 4 & 0 & $6.0 \mathrm{E}-06$ & 6.0E-06 & Yes & ND & ND \\
\hline GW-106 & $\mathrm{T}$ & 4 & 0 & $6.0 \mathrm{E}-06$ & 6.0E-06 & Yes & ND & ND \\
\hline GW-108 & $\mathrm{s}$ & 23 & 17 & $1.8 \mathrm{E}-03$ & 2.0E-03 & No & 1 & 1 \\
\hline GW-109 & $\mathrm{S}$ & 7 & 7 & 1.4E-01 & 1.5E-01 & No & D & D \\
\hline GW-192 & $\mathrm{T}$ & 15 & 10 & $2.1 \mathrm{E}-03$ & $2.0 \mathrm{E}-03$ & No & NT & NT \\
\hline GW-251 & $\mathrm{T}$ & 23 & 23 & 2.0E-01 & 1.6E-01 & No & S & S \\
\hline GW-253 & $\mathrm{T}$ & 17 & 16 & $5.6 \mathrm{E}-01$ & 5.7E-01 & No & $\mathrm{S}$ & NT \\
\hline GW-265 & $\mathrm{T}$ & 4 & 4 & $2.5 \mathrm{E}-02$ & $2.4 \mathrm{E}-02$ & No & $\mathrm{S}$ & S \\
\hline GW-269 & $\mathrm{T}$ & 6 & 6 & 2.4E-02 & $2.4 \mathrm{E}-02$ & No & D & D \\
\hline GW-270 & $\mathrm{T}$ & 3 & 0 & $6.0 \mathrm{E}-06$ & $6.0 \mathrm{E}-06$ & Yes & ND & ND \\
\hline GW-272 & $\mathrm{T}$ & 3 & 0 & $6.0 \mathrm{E}-06$ & $6.0 \mathrm{E}-06$ & Yes & ND & ND \\
\hline GW-273 & $\mathrm{T}$ & 3 & 0 & $6.0 \mathrm{E}-06$ & $6.0 \mathrm{E}-06$ & Yes & ND & ND \\
\hline GW-274 & $\mathrm{S}$ & 6 & 6 & $1.1 \mathrm{E}+00$ & $1.1 \mathrm{E}+00$ & No & 1 & 1 \\
\hline GW-275 & $\mathrm{S}$ & 6 & 3 & $2.2 \mathrm{E}-03$ & 1.0E-03 & No & NT & NT \\
\hline GW-332 & $\mathrm{T}$ & 5 & 5 & $1.0 \mathrm{E}+00$ & $9.4 \mathrm{E}-01$ & No & NT & NT \\
\hline GW-336 & $\mathrm{T}$ & 4 & 4 & 3.6E-01 & 2.7E-01 & No & $\mathrm{s}$ & $\mathrm{s}$ \\
\hline GW-337 & $\mathrm{T}$ & 8 & 8 & $6.8 \mathrm{E}-01$ & 6.1E-01 & No & $\mathrm{S}$ & PD \\
\hline GW-505 & $\mathrm{T}$ & 3 & 0 & $6.0 \mathrm{E}-06$ & $6.0 \mathrm{E}-06$ & Yes & ND & ND \\
\hline GW-617 & $\mathrm{T}$ & 6 & 1 & $1.2 \mathrm{E}-03$ & $6.0 \mathrm{E}-06$ & No & NT & NT \\
\hline GW-618 & $\mathrm{T}$ & 20 & 20 & 7.5E-03 & 6.3E-03 & No & D & D \\
\hline GW-619 & $\mathrm{T}$ & 6 & 6 & 3.3E-02 & 2.9E-02 & No & PD & D \\
\hline GW-620 & $\mathrm{T}$ & 24 & 23 & 4.3E-02 & 1.3E-02 & No & D & D \\
\hline GW-633 & $\mathrm{s}$ & 11 & 11 & 2.1E-01 & 2.0E-01 & No & PI & $\mathrm{PI}$ \\
\hline
\end{tabular}

TRICHLOROETHYLENE (TCE)

\begin{tabular}{|c|c|c|c|c|c|c|c|c|}
\hline $55-1 A$ & $T$ & 5 & 0 & $5.0 \mathrm{E}-06$ & $5.0 \mathrm{E}-06$ & Yes & ND & ND \\
\hline $55-2 A$ & $\mathrm{~T}$ & 4 & 4 & $1.2 \mathrm{E}-01$ & $1.1 \mathrm{E}-01$ & No & $\mathrm{S}$ & PD \\
\hline $55-2 B$ & $T$ & 9 & 9 & 2.4E-01 & 2.3E-01 & No & $\mathrm{D}$ & D \\
\hline $55-2 C$ & $T$ & 10 & 10 & 2.6E-01 & $2.3 \mathrm{E}-01$ & No & $\mathrm{D}$ & D \\
\hline GW-105 & $\mathrm{T}$ & 4 & 0 & $5.0 \mathrm{E}-06$ & $5.0 \mathrm{E}-06$ & Yes & ND & ND \\
\hline GW-106 & $\mathrm{T}$ & 4 & 0 & $5.0 \mathrm{E}-06$ & $5.0 \mathrm{E}-06$ & Yes & ND & ND \\
\hline GW-108 & $\mathrm{s}$ & 23 & 22 & $3.0 \mathrm{E}-03$ & $3.0 \mathrm{E}-03$ & No & 1 & 1 \\
\hline GW-109 & $\mathrm{s}$ & 7 & 7 & $3.9 \mathrm{E}-03$ & 4.0E-03 & No & $\mathrm{D}$ & $\mathrm{D}$ \\
\hline GW-192 & $\mathrm{T}$ & 15 & 13 & 3.7E-03 & $3.0 \mathrm{E}-03$ & No & NT & NT \\
\hline GW-251 & $\mathrm{T}$ & 23 & 23 & $8.8 \mathrm{E}-02$ & 7.6E-02 & No & $\mathrm{s}$ & s \\
\hline GW-253 & $T$ & 17 & 16 & $4.8 \mathrm{E}-01$ & 4.6E-01 & No & $S$ & NT \\
\hline
\end{tabular}




\section{MAROS Statistical Trend Analysis Summary}

\begin{tabular}{|c|c|c|c|c|c|c|c|c|}
\hline Well & $\begin{array}{c}\text { Sourcel } \\
\text { Tail }\end{array}$ & $\begin{array}{c}\text { Number } \\
\text { of } \\
\text { Samples }\end{array}$ & $\begin{array}{c}\text { Number } \\
\text { of } \\
\text { Detects }\end{array}$ & $\begin{array}{l}\text { Average } \\
\text { Conc. } \\
\text { (mg/L) }\end{array}$ & $\begin{array}{l}\text { Median } \\
\text { Conc. } \\
\text { (mg/L) }\end{array}$ & $\begin{array}{c}\text { All } \\
\text { Samples } \\
\text { "ND" ? }\end{array}$ & $\begin{array}{l}\text { Mann- } \\
\text { Kendall } \\
\text { Trend }\end{array}$ & $\begin{array}{l}\text { Linear } \\
\text { Regression } \\
\text { Trend }\end{array}$ \\
\hline \multicolumn{9}{|c|}{ TRICHLOROETHYLENE (TCE) } \\
\hline GW-265 & $\mathrm{T}$ & 4 & 4 & 4.0E-03 & $4.0 \mathrm{E}-03$ & No & $\mathrm{S}$ & $\mathrm{S}$ \\
\hline GW-269 & $\mathrm{T}$ & 6 & 6 & 3.7E-03 & 4.0E-03 & No & $\mathrm{S}$ & $\mathrm{S}$ \\
\hline GW-270 & $\mathrm{T}$ & 3 & 0 & $5.0 \mathrm{E}-06$ & 5.0E-06 & Yes & ND & ND \\
\hline GW-272 & $\mathrm{T}$ & 3 & 0 & 5.0E-06 & $5.0 \mathrm{E}-06$ & Yes & ND & ND \\
\hline GW-273 & $\mathrm{T}$ & 3 & 0 & $5.0 \mathrm{E}-06$ & 5.0E-06 & Yes & ND & ND \\
\hline GW-274 & $\mathrm{S}$ & 6 & 6 & 1.1E-02 & 1.1E-02 & No & $\mathrm{S}$ & 1 \\
\hline GW-275 & $\mathrm{S}$ & 6 & 0 & 5.0E-06 & 5.0E-06 & Yes & ND & ND \\
\hline GW-332 & $\mathrm{T}$ & 5 & 5 & $2.5 \mathrm{E}-01$ & 2.5E-01 & No & $\mathrm{S}$ & $\mathrm{S}$ \\
\hline GW-336 & $\mathrm{T}$ & 4 & 4 & 3.6E-01 & 3.2E-01 & No & $\mathrm{S}$ & PD \\
\hline GW-337 & $\mathrm{T}$ & 8 & 8 & 7.2E-01 & 7.3E-01 & No & PD & $\mathrm{D}$ \\
\hline GW-505 & $\mathrm{T}$ & 3 & 0 & 5.0E-06 & 5.0E-06 & Yes & ND & ND \\
\hline GW-617 & $\mathrm{T}$ & 6 & 1 & 1.0E-03 & 5.0E-06 & No & NT & NT \\
\hline GW-618 & $\mathrm{T}$ & 20 & 20 & 1.1E-02 & 1.1E-02 & No & D & $D$ \\
\hline GW-619 & $\mathrm{T}$ & 6 & 6 & $9.2 \mathrm{E}-03$ & 9.5E-03 & No & $\mathrm{S}$ & $D$ \\
\hline GW-620 & $\mathrm{T}$ & 24 & 18 & 1.3E-02 & $6.0 \mathrm{E}-03$ & No & D & $D$ \\
\hline GW-633 & $\mathrm{S}$ & 11 & 11 & 7.0E-03 & 8.0E-03 & No & D & PD \\
\hline
\end{tabular}

Note: Increasing (I); Probably Increasing (PI); Stable (S); Probably Decreasing (PD); Decreasing (D); No Trend (NT); Not Applicable (N/A); Not Applicable (N/A) - Due to insufficient Data (< 4 sampling events); No Detectable Concentration (NDC)

The Number of Samples and Number of Detects shown above are post-consolidation values. 


\section{MAROS Statistical Trend Analysis Summary}

Project: $\quad$ EF East S3 Beta 4

Location: East S-3

Time Period: $1 / 1 / 1996$ to $10 / 15 / 2007$

Consolidation Period: No Time Consolidation

Consolidation Type: Median

Duplicate Consolidation: Average

ND Values: Specified Detection Limit

J Flag Values : Actual Value
User Name: MV

State: Tennessee

\begin{tabular}{ccccccccc} 
& & Number & Number & Average & Median & All & Mann- & Linear \\
of & Sourcel & $\begin{array}{c}\text { of } \\
\text { Wall }\end{array}$ & $\begin{array}{c}\text { Samples } \\
\text { Tetects }\end{array}$ & $\begin{array}{c}\text { Conc. } \\
(\mathrm{mg} / \mathrm{L})\end{array}$ & $\begin{array}{c}\text { Samples } \\
(\mathrm{mg} / \mathrm{L})\end{array}$ & "ND" ? & $\begin{array}{c}\text { Kendall } \\
\text { Trend }\end{array}$ & $\begin{array}{c}\text { Regression } \\
\text { Trend }\end{array}$ \\
\hline
\end{tabular}

1,1-DICHLOROETHENE

$\begin{array}{ll}55-1 A & T \\ 55-2 A & T \\ 55-2 B & T \\ 55-2 C & T \\ \text { GW-105 } & \text { T } \\ \text { GW-106 } & \text { T } \\ \text { GW-108 } & \mathrm{S} \\ \text { GW-109 } & \mathrm{S} \\ \text { GW-192 } & \mathrm{T} \\ \text { GW-251 } & \mathrm{T} \\ \text { GW-253 } & \mathrm{T} \\ \text { GW-265 } & \mathrm{T} \\ \text { GW-269 } & \mathrm{T} \\ \text { GW-270 } & \mathrm{T} \\ \text { GW-272 } & \mathrm{T} \\ \text { GW-273 } & \mathrm{T} \\ \text { GW-274 } & \mathrm{S} \\ \text { GW-275 } & \mathrm{S} \\ \text { GW-332 } & \mathrm{T} \\ \text { GW-336 } & \mathrm{T} \\ \text { GW-337 } & \mathrm{T} \\ \text { GW-505 } & \mathrm{T} \\ \text { GW-617 } & \mathrm{T} \\ \text { GW-618 } & \mathrm{T} \\ \text { GW-619 } & \mathrm{T} \\ \text { GW-620 } & \mathrm{T} \\ \text { GW-633 } & \mathrm{S}\end{array}$

5
4
9
10
4
4
23
7
15
23
17
4
6
3
3
3
6
6
5
4
8
3
6
20
6
24
11

0
4
8
8
0
0
5
2
0
0
10
0
6
0
0
0
0
0
5
4
8
0
0
0
0
0
7

$\begin{array}{llc}8.0 \mathrm{E}-06 & 8.0 \mathrm{E}-06 & \text { Yes } \\ 8.3 \mathrm{E}-03 & 8.0 \mathrm{E}-03 & \text { No } \\ 2.0 \mathrm{E}-02 & 1.9 \mathrm{E}-02 & \text { No } \\ 2.6 \mathrm{E}-02 & 3.0 \mathrm{E}-02 & \text { No } \\ 8.0 \mathrm{E}-06 & 8.0 \mathrm{E}-06 & \text { Yes } \\ 8.0 \mathrm{E}-06 & 8.0 \mathrm{E}-06 & \text { Yes } \\ 2.7 \mathrm{E}-04 & 8.0 \mathrm{E}-06 & \text { No } \\ 4.3 \mathrm{E}-04 & 8.0 \mathrm{E}-06 & \text { No } \\ 8.0 \mathrm{E}-06 & 8.0 \mathrm{E}-06 & \text { Yes } \\ 8.0 \mathrm{E}-06 & 8.0 \mathrm{E}-06 & \text { Yes } \\ 2.0 \mathrm{E}-03 & 2.0 \mathrm{E}-03 & \text { No } \\ 8.0 \mathrm{E}-06 & 8.0 \mathrm{E}-06 & \text { Yes } \\ 1.7 \mathrm{E}-01 & 1.6 \mathrm{E}-01 & \text { No } \\ 8.0 \mathrm{E}-06 & 8.0 \mathrm{E}-06 & \text { Yes } \\ 8.0 \mathrm{E}-06 & 8.0 \mathrm{E}-06 & \text { Yes } \\ 8.0 \mathrm{E}-06 & 8.0 \mathrm{E}-06 & \text { Yes } \\ 8.0 \mathrm{E}-06 & 8.0 \mathrm{E}-06 & \text { Yes } \\ 8.0 \mathrm{E}-06 & 8.0 \mathrm{E}-06 & \text { Yes } \\ 4.6 \mathrm{E}-02 & 3.7 \mathrm{E}-02 & \text { No } \\ 8.2 \mathrm{E}-02 & 8.3 \mathrm{E}-02 & \text { No } \\ 1.1 \mathrm{E}-01 & 1.1 \mathrm{E}-01 & \text { No } \\ 8.0 \mathrm{E}-06 & 8.0 \mathrm{E}-06 & \text { Yes } \\ 8.0 \mathrm{E}-06 & 8.0 \mathrm{E}-06 & \text { Yes } \\ 8.0 \mathrm{E}-06 & 8.0 \mathrm{E}-06 & \text { Yes } \\ 8.0 \mathrm{E}-06 & 8.0 \mathrm{E}-06 & \text { Yes } \\ 8.0 \mathrm{E}-06 & 8.0 \mathrm{E}-06 & \text { Yes } \\ 1.9 \mathrm{E}-03 & 2.5 \mathrm{E}-03 & \text { No }\end{array}$

ND

$\mathrm{S}$

$\mathrm{S}$

$\mathrm{S}$

ND

ND

$\mathrm{PI}$

NT

ND

ND

I

ND

$D$

ND

$N D$

ND

ND

ND

$S$

$\mathrm{S}$

D

ND

ND

ND

ND

ND

NT

ND

$\mathrm{S}$

NT

PI

ND

ND

I

NT

ND

ND

I

ND

PD

$N D$

ND

ND

ND

ND

$\mathrm{S}$

D

D

ND

ND

ND

ND

ND

PI

BENZENE

\begin{tabular}{lcccccccc}
\hline $55-1 \mathrm{~A}$ & $\mathrm{~T}$ & 5 & 0 & $1.0 \mathrm{E}-06$ & $1.0 \mathrm{E}-06$ & Yes & ND & ND \\
$55-2 \mathrm{~A}$ & $\mathrm{~T}$ & 4 & 0 & $1.0 \mathrm{E}-06$ & $1.0 \mathrm{E}-06$ & Yes & ND & ND \\
$55-2 \mathrm{~B}$ & $\mathrm{~T}$ & 9 & 0 & $1.0 \mathrm{E}-06$ & $1.0 \mathrm{E}-06$ & Yes & ND & NT \\
$55-2 \mathrm{C}$ & $\mathrm{T}$ & 10 & 1 & $1.1 \mathrm{E}-05$ & $1.0 \mathrm{E}-06$ & No & ND & ND \\
GW-105 & $\mathrm{T}$ & 4 & 0 & $1.0 \mathrm{E}-06$ & $1.0 \mathrm{E}-06$ & Yes & ND & ND \\
GW-106 & $\mathrm{T}$ & 4 & 0 & $1.0 \mathrm{E}-06$ & $1.0 \mathrm{E}-06$ & Yes & & \\
\end{tabular}




\section{MAROS Statistical Trend Analysis Summary}

\begin{tabular}{ccccccccc} 
& & Number & Number & Average & Median & All & Mann- & Linear \\
Well & Sourcel & of & of & Conc. & Conc. & Samples & Kendall & Regression \\
Tail & Samples & Detects & (mg/L) & (mg/L) & "ND" ? & Trend & Trend \\
\hline
\end{tabular}

BENZENE

GW-108
GW-109
GW-192
GW-251
GW-253
GW-265
GW-269
GW-270
GW-272
GW-273
GW-274
GW-275
GW-332
GW-336
GW-337
GW-505
GW-617
GW-618
GW-619
GW-620
GW-633

cis-1,2-DICHLOROETHYLENE

\begin{tabular}{|c|c|c|c|c|c|c|c|c|}
\hline $55-1 A$ & $\mathrm{~T}$ & 5 & 0 & 9.0E-06 & $9.0 \mathrm{E}-06$ & Yes & ND & ND \\
\hline $55-2 A$ & $\mathrm{~T}$ & 4 & 4 & 4.1E-01 & 4.2E-01 & No & $S$ & s \\
\hline $55-2 B$ & $\mathrm{~T}$ & 9 & 9 & $1.1 \mathrm{E}+00$ & 8.5E-01 & No & $D$ & D \\
\hline $55-2 C$ & $\mathrm{~T}$ & 10 & 10 & $1.5 \mathrm{E}+00$ & $1.6 \mathrm{E}+00$ & No & $\mathrm{D}$ & D \\
\hline GW-105 & $\mathrm{T}$ & 4 & 0 & $9.0 \mathrm{E}-06$ & $9.0 \mathrm{E}-06$ & Yes & ND & ND \\
\hline GW-106 & $\mathrm{T}$ & 4 & 0 & $9.0 \mathrm{E}-06$ & $9.0 \mathrm{E}-06$ & Yes & ND & ND \\
\hline GW-108 & $\mathrm{S}$ & 23 & 0 & 9.0E-06 & $9.0 \mathrm{E}-06$ & Yes & ND & ND \\
\hline GW-109 & $\mathrm{S}$ & 7 & 1 & $2.9 \mathrm{E}-04$ & $9.0 \mathrm{E}-06$ & No & NT & NT \\
\hline GW-192 & $\mathrm{T}$ & 15 & 15 & $1.8 \mathrm{E}-02$ & 2.1E-02 & No & NT & NT \\
\hline GW-251 & $\mathrm{T}$ & 23 & 18 & 5.6E-03 & 5.0E-03 & No & NT & NT \\
\hline GW-253 & $\mathrm{T}$ & 17 & 17 & 2.2E-01 & 2.3E-01 & No & 1 & 1 \\
\hline GW-265 & $\mathrm{T}$ & 4 & 3 & 7.5E-04 & 1.0E-03 & No & $S$ & s \\
\hline GW-269 & $\mathrm{T}$ & 6 & 6 & $5.4 \mathrm{E}-02$ & 5.8E-02 & No & $S$ & s \\
\hline GW-270 & $\mathrm{T}$ & 3 & 0 & 9.0E-06 & $9.0 \mathrm{E}-06$ & Yes & ND & ND \\
\hline GW-272 & $\mathrm{T}$ & 3 & 0 & $9.0 \mathrm{E}-06$ & 9.0E-06 & Yes & ND & ND \\
\hline GW-273 & $\mathrm{T}$ & 3 & 0 & 9.0E-06 & $9.0 \mathrm{E}-06$ & Yes & ND & ND \\
\hline GW-274 & $\mathrm{s}$ & 6 & 6 & 1.4E-02 & 1.3E-02 & No & 1 & 1 \\
\hline GW-275 & $\mathrm{s}$ & 6 & 0 & $9.0 \mathrm{E}-06$ & $9.0 \mathrm{E}-06$ & Yes & ND & ND \\
\hline GW-332 & $\mathrm{T}$ & 5 & 5 & $1.9 \mathrm{E}+00$ & $1.2 \mathrm{E}+00$ & No & $\mathrm{s}$ & $\mathrm{s}$ \\
\hline GW-336 & $\mathrm{T}$ & 4 & 4 & $1.8 \mathrm{E}+00$ & $1.8 \mathrm{E}+00$ & No & $\mathrm{s}$ & D \\
\hline GW-337 & $\mathrm{T}$ & 8 & 8 & $3.8 \mathrm{E}+00$ & $4.1 \mathrm{E}+00$ & No & $\mathrm{D}$ & D \\
\hline GW-505 & $\mathrm{T}$ & 3 & 0 & 9.0E-06 & 9.0E-06 & Yes & ND & ND \\
\hline GW-617 & $\mathrm{T}$ & 6 & 2 & $1.8 \mathrm{E}-03$ & $9.0 \mathrm{E}-06$ & No & NT & NT \\
\hline
\end{tabular}




\section{MAROS Statistical Trend Analysis Summary}

\begin{tabular}{ccccccccc} 
& & Number & Number & Average & Median & All & Mann- & Linear \\
Well & Sourcel & of & of & Conc. & Conc. & Samples & Kendall & Regression \\
Tail & Samples & Detects & (mg/L) & (mg/L) & "ND" ? & Trend & Trend \\
\hline
\end{tabular}

cis-1,2-DICHLOROETHYLENE

$\begin{array}{ccccccccc}\text { GW-618 } & \text { T } & 20 & 20 & 2.3 \mathrm{E}-02 & 2.2 \mathrm{E}-02 & \text { No } & \text { D } & \text { D } \\ \text { GW-619 } & \text { T } & 6 & 6 & 1.1 \mathrm{E}-02 & 1.0 \mathrm{E}-02 & \text { No } & \text { D } & \text { D } \\ \text { GW-620 } & \mathrm{T} & 24 & 20 & 3.3 \mathrm{E}-02 & 1.4 \mathrm{E}-02 & \text { No } & \text { D } & \text { D } \\ \text { GW-633 } & \mathrm{S} & 11 & 11 & 1.1 \mathrm{E}-02 & 1.1 \mathrm{E}-02 & \text { No } & \text { NT } & \text { NT }\end{array}$

GROSS BETA ACTIVITY

\begin{tabular}{|c|c|c|c|c|c|c|c|c|}
\hline $55-1 \mathrm{~A}$ & $\mathrm{~T}$ & 4 & 2 & $4.9 \mathrm{E}+00$ & 3.7E-01 & No & NT & NT \\
\hline $55-2 A$ & $\mathrm{~T}$ & 4 & 0 & $9.9 \mathrm{E}-04$ & $9.9 \mathrm{E}-04$ & Yes & ND & ND \\
\hline $55-2 B$ & $\mathrm{~T}$ & 9 & 9 & $2.4 \mathrm{E}+01$ & $2.0 \mathrm{E}+01$ & No & 1 & 1 \\
\hline $55-2 C$ & $\mathrm{~T}$ & 10 & 9 & $1.5 \mathrm{E}+01$ & $1.1 \mathrm{E}+01$ & No & NT & $\mathrm{S}$ \\
\hline GW-105 & $\mathrm{T}$ & 2 & 0 & 9.9E-04 & 9.9E-04 & Yes & ND & ND \\
\hline GW-106 & $\mathrm{T}$ & 2 & 1 & 3.4E+01 & $3.4 \mathrm{E}+01$ & No & N/A & $\mathrm{N} / \mathrm{A}$ \\
\hline GW-108 & $s$ & 24 & 24 & $1.1 \mathrm{E}+04$ & $1.2 \mathrm{E}+04$ & No & I & 1 \\
\hline GW-109 & $\mathrm{s}$ & 7 & 7 & $1.0 \mathrm{E}+04$ & $7.4 \mathrm{E}+03$ & No & $\mathrm{s}$ & $\mathrm{s}$ \\
\hline GW-192 & $\mathrm{T}$ & 13 & 12 & $4.0 \mathrm{E}+00$ & $3.8 \mathrm{E}+00$ & No & $S$ & $D$ \\
\hline GW-251 & $\mathrm{T}$ & 22 & 18 & $5.6 \mathrm{E}+00$ & $5.0 \mathrm{E}+00$ & No & $\mathrm{D}$ & $D$ \\
\hline GW-253 & $\mathrm{T}$ & 18 & 18 & $2.9 \mathrm{E}+01$ & $2.7 \mathrm{E}+01$ & No & S & $\mathrm{S}$ \\
\hline GW-265 & $\mathrm{T}$ & 4 & 0 & $9.9 \mathrm{E}-04$ & 9.9E-04 & Yes & ND & ND \\
\hline GW-269 & $\mathrm{T}$ & 6 & 4 & $7.8 \mathrm{E}+00$ & $6.9 \mathrm{E}+00$ & No & S & $\mathrm{S}$ \\
\hline GW-270 & $\mathrm{T}$ & 3 & 3 & $1.4 \mathrm{E}+01$ & $1.5 \mathrm{E}+01$ & No & N/A & $\mathrm{N} / \mathrm{A}$ \\
\hline GW-272 & $\mathrm{T}$ & 1 & 0 & $9.9 \mathrm{E}-04$ & $9.9 \mathrm{E}-04$ & Yes & ND & ND \\
\hline GW-273 & $\mathrm{T}$ & 1 & 0 & $9.9 \mathrm{E}-04$ & $9.9 \mathrm{E}-04$ & Yes & ND & ND \\
\hline GW-274 & $\mathrm{s}$ & 7 & 7 & $6.6 \mathrm{E}+03$ & $6.0 \mathrm{E}+03$ & No & $\mathrm{S}$ & NT \\
\hline GW-275 & $\mathrm{s}$ & 6 & 4 & $2.3 \mathrm{E}+02$ & $2.2 \mathrm{E}+02$ & No & $\mathrm{D}$ & $D$ \\
\hline GW-332 & $\mathrm{T}$ & 3 & 1 & $1.3 \mathrm{E}+00$ & 9.9E-04 & No & $N / A$ & N/A \\
\hline GW-336 & $\mathrm{T}$ & 4 & 2 & $1.2 \mathrm{E}+00$ & 4.7E-01 & No & NT & D \\
\hline GW-337 & $\mathrm{T}$ & 7 & 5 & $3.4 \mathrm{E}+00$ & $3.4 \mathrm{E}+00$ & No & NT & D \\
\hline GW-505 & $\mathrm{T}$ & 4 & 3 & $1.1 \mathrm{E}+01$ & $9.9 \mathrm{E}+00$ & No & NT & NT \\
\hline GW-617 & $\mathrm{T}$ & 3 & 2 & $1.9 \mathrm{E}+00$ & 2.4E-01 & No & N/A & N/A \\
\hline GW-618 & $\mathrm{T}$ & 19 & 18 & $4.5 \mathrm{E}+00$ & $5.0 \mathrm{E}+00$ & No & PI & NT \\
\hline GW-619 & $\mathrm{T}$ & 4 & 4 & $1.3 \mathrm{E}+01$ & $1.2 \mathrm{E}+01$ & No & NT & NT \\
\hline GW-620 & $\mathrm{T}$ & 21 & 20 & $1.2 \mathrm{E}+01$ & $1.2 \mathrm{E}+01$ & No & NT & $\mathrm{S}$ \\
\hline GW-633 & $\mathrm{s}$ & 12 & 12 & $3.3 \mathrm{E}+03$ & $3.4 \mathrm{E}+03$ & No & $\mathrm{s}$ & 1 \\
\hline \multicolumn{9}{|l|}{ VINYL CHLORIDE } \\
\hline $55-1 \mathrm{~A}$ & $\mathrm{~T}$ & 5 & 0 & 1.0E-05 & 1.0E-05 & Yes & ND & ND \\
\hline $55-2 A$ & $\mathrm{~T}$ & 4 & 4 & $7.8 \mathrm{E}-03$ & 9.0E-03 & No & NT & $\mathrm{S}$ \\
\hline $55-2 B$ & $\mathrm{~T}$ & 9 & 9 & 2.5E-02 & 1.9E-02 & No & D & D \\
\hline $55-2 C$ & $\mathrm{~T}$ & 10 & 9 & $2.9 \mathrm{E}-02$ & $2.8 \mathrm{E}-02$ & No & D & NT \\
\hline GW-105 & $\mathrm{T}$ & 4 & 0 & 1.0E-05 & 1.0E-05 & Yes & ND & ND \\
\hline GW-106 & $\mathrm{T}$ & 4 & 0 & 1.0E-05 & 1.0E-05 & Yes & ND & ND \\
\hline GW-108 & $\mathrm{s}$ & 23 & 0 & 1.0E-05 & 1.0E-05 & Yes & ND & ND \\
\hline GW-109 & $\mathrm{s}$ & 7 & 0 & 1.0E-05 & 1.0E-05 & Yes & ND & ND \\
\hline GW-192 & $\mathrm{T}$ & 15 & 0 & 1.0E-05 & 1.0E-05 & Yes & ND & ND \\
\hline GW-251 & $\mathrm{T}$ & 23 & 1 & 5.3E-05 & 1.0E-05 & No & NT & PI \\
\hline GW-253 & $\mathrm{T}$ & 17 & 17 & 5.5E-02 & 5.3E-02 & No & 1 & 1 \\
\hline
\end{tabular}




\section{MAROS Statistical Trend Analysis Summary}

\begin{tabular}{|c|c|c|c|c|c|c|c|c|}
\hline Well & $\begin{array}{c}\text { Sourcel } \\
\text { Tail }\end{array}$ & $\begin{array}{c}\text { Number } \\
\text { of } \\
\text { Samples }\end{array}$ & $\begin{array}{c}\text { Number } \\
\text { of } \\
\text { Detects }\end{array}$ & $\begin{array}{l}\text { Average } \\
\text { Conc. } \\
\text { (mg/L) }\end{array}$ & $\begin{array}{l}\text { Median } \\
\text { Conc. } \\
\text { (mg/L) }\end{array}$ & $\begin{array}{c}\text { All } \\
\text { Samples } \\
\text { "ND" ? }\end{array}$ & $\begin{array}{l}\text { Mann- } \\
\text { Kendall } \\
\text { Trend }\end{array}$ & $\begin{array}{l}\text { Linear } \\
\text { Regression } \\
\text { Trend }\end{array}$ \\
\hline \multicolumn{9}{|c|}{ VINYL CHLORIDE } \\
\hline GW-265 & $\mathrm{T}$ & 4 & 0 & 1.0E-05 & $1.0 \mathrm{E}-05$ & Yes & ND & ND \\
\hline GW-269 & $\mathrm{T}$ & 6 & 2 & $3.4 \mathrm{E}-04$ & $1.0 \mathrm{E}-05$ & No & NT & NT \\
\hline GW-270 & $\mathrm{T}$ & 3 & 0 & 1.0E-05 & $1.0 \mathrm{E}-05$ & Yes & ND & ND \\
\hline GW-272 & $\mathrm{T}$ & 3 & 0 & 1.0E-05 & $1.0 \mathrm{E}-05$ & Yes & ND & ND \\
\hline GW-273 & $\mathrm{T}$ & 3 & 0 & 1.0E-05 & $1.0 \mathrm{E}-05$ & Yes & ND & ND \\
\hline GW-274 & $\mathrm{S}$ & 6 & 1 & $3.4 \mathrm{E}-04$ & $1.0 \mathrm{E}-05$ & No & NT & $\mathrm{PI}$ \\
\hline GW-275 & $\mathrm{S}$ & 6 & 0 & 1.0E-05 & $1.0 \mathrm{E}-05$ & Yes & ND & ND \\
\hline GW-332 & $\mathrm{T}$ & 5 & 4 & $2.2 \mathrm{E}-02$ & $2.0 \mathrm{E}-02$ & No & $\mathrm{S}$ & NT \\
\hline GW-336 & $\mathrm{T}$ & 4 & 4 & $2.8 \mathrm{E}-02$ & $2.7 \mathrm{E}-02$ & No & $\mathrm{S}$ & $D$ \\
\hline GW-337 & $\mathrm{T}$ & 8 & 8 & $4.2 \mathrm{E}-02$ & $4.6 \mathrm{E}-02$ & No & $\mathrm{D}$ & $D$ \\
\hline GW-505 & $\mathrm{T}$ & 3 & 0 & $1.0 \mathrm{E}-05$ & $1.0 \mathrm{E}-05$ & Yes & ND & ND \\
\hline GW-617 & $\mathrm{T}$ & 6 & 0 & 1.0E-05 & $1.0 \mathrm{E}-05$ & Yes & ND & ND \\
\hline GW-618 & $\mathrm{T}$ & 20 & 10 & $1.2 \mathrm{E}-03$ & 5.1E-04 & No & NT & NT \\
\hline GW-619 & $\mathrm{T}$ & 6 & 0 & $1.0 \mathrm{E}-05$ & $1.0 \mathrm{E}-05$ & Yes & ND & ND \\
\hline GW-620 & $\mathrm{T}$ & 24 & 0 & $1.0 \mathrm{E}-05$ & 1.0E-05 & Yes & ND & ND \\
\hline GW-633 & $\mathrm{S}$ & 11 & 0 & 1.0E-05 & $1.0 \mathrm{E}-05$ & Yes & ND & ND \\
\hline
\end{tabular}

Note: Increasing (I); Probably Increasing (PI); Stable (S); Probably Decreasing (PD); Decreasing (D); No Trend (NT); Not Applicable (N/A); Not Applicable (N/A) - Due to insufficient Data (< 4 sampling events); No Detectable Concentration (NDC)

The Number of Samples and Number of Detects shown above are post-consolidation values. 


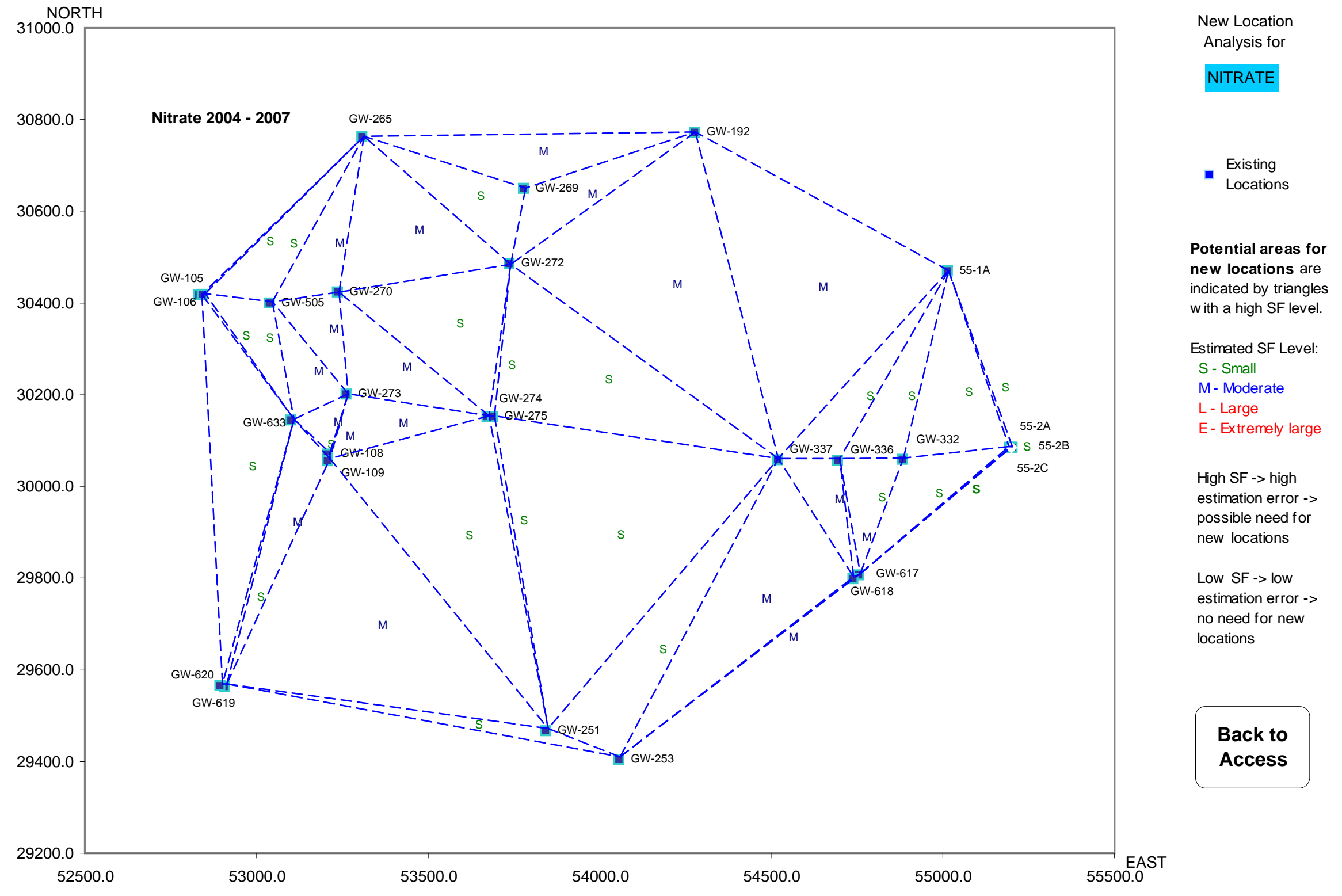




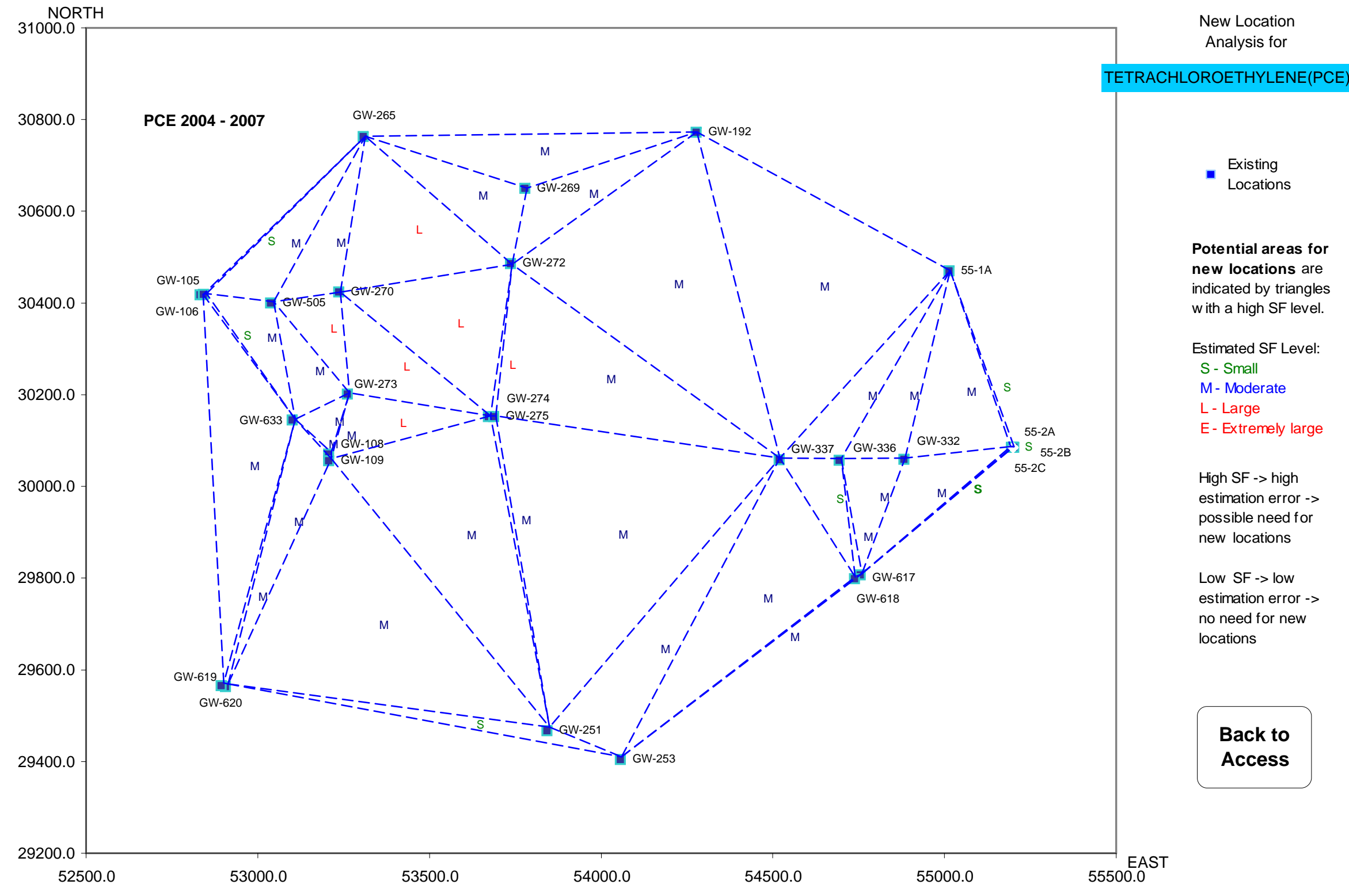


APPENDIX C.5

CENTRAL Y-12 AREA 


\section{MAROS COC Assessment}

Project: EF Central and Fuel

Location: Central Y-12

Toxicity:

\begin{tabular}{lccc}
\hline Toxicity: & $\begin{array}{c}\text { Representative } \\
\text { Concentration } \\
\text { (mg/L) }\end{array}$ & $\begin{array}{c}\text { PRG } \\
\text { (mg/L) }\end{array}$ & $\begin{array}{c}\text { Percent } \\
\text { Above } \\
\text { PRG }\end{array}$ \\
\hline TETRACHLOROETHYLENE(PCE) & $2.3 \mathrm{E}+00$ & $5.0 \mathrm{E}-03$ & $46130.1 \%$ \\
TRICHLOROETHYLENE (TCE) & $3.9 \mathrm{E}-01$ & $5.0 \mathrm{E}-03$ & $7765.8 \%$ \\
BENZENE & $1.9 \mathrm{E}-01$ & $5.0 \mathrm{E}-03$ & $3697.2 \%$ \\
MANGANESE & $1.2 \mathrm{E}+00$ & $5.0 \mathrm{E}-02$ & $2205.3 \%$ \\
VINYL CHLORIDE & $1.7 \mathrm{E}-02$ & $2.0 \mathrm{E}-03$ & $729.3 \%$ \\
CARBON TETRACHLORIDE & $3.0 \mathrm{E}-02$ & $5.0 \mathrm{E}-03$ & $492.1 \%$ \\
1,1-DICHLOROETHENE & $2.2 \mathrm{E}-02$ & $7.0 \mathrm{E}-03$ & $211.8 \%$ \\
URANIUM & $6.2 \mathrm{E}-02$ & $3.0 \mathrm{E}-02$ & $106.9 \%$ \\
CiS-1,2-DICHLOROETHYLENE & $1.4 \mathrm{E}-01$ & $7.0 \mathrm{E}-02$ & $100.2 \%$
\end{tabular}

Note: Top COCs by toxicity were determined by examining a representative concentration for each compound over the entire site. The compound representative concentrations are then compared with the chosen PRG for that compound, with the percentage exceedance from the PRG determining the compound's toxicity. All compounds above exceed the PRG.

\section{Prevalence:}

\begin{tabular}{|c|c|c|c|c|c|}
\hline Contaminant of Concern & Class & $\begin{array}{l}\text { Total } \\
\text { Wells }\end{array}$ & $\begin{array}{c}\text { Total } \\
\text { Exceedances }\end{array}$ & $\begin{array}{c}\text { Percent } \\
\text { Exceedances }\end{array}$ & $\begin{array}{c}\text { Total } \\
\text { detects }\end{array}$ \\
\hline MANGANESE & MET & 31 & 17 & $54.8 \%$ & 31 \\
\hline TETRACHLOROETHYLENE(PCE) & ORG & 53 & 28 & $52.8 \%$ & 37 \\
\hline TRICHLOROETHYLENE (TCE) & ORG & 53 & 22 & $41.5 \%$ & 33 \\
\hline URANIUM & MET & 50 & 14 & $28.0 \%$ & 26 \\
\hline VINYL CHLORIDE & ORG & 53 & 10 & $18.9 \%$ & 14 \\
\hline cis-1,2-DICHLOROETHYLENE & ORG & 53 & 10 & $18.9 \%$ & 27 \\
\hline 1,1-DICHLOROETHENE & ORG & 53 & 8 & $15.1 \%$ & 15 \\
\hline CARBON TETRACHLORIDE & ORG & 53 & 6 & $11.3 \%$ & 8 \\
\hline BENZENE & ORG & 53 & 5 & $9.4 \%$ & 7 \\
\hline
\end{tabular}

Note: Top COCs by prevalence were determined by examining a representative concentration for each well location at the site. The total exceedances (values above the chosen PRGs) are compared to the total number of wells to determine the prevalence of the compound.

\section{Mobility:}

Contaminant of Concern

\begin{tabular}{lr}
\hline VINYL CHLORIDE & 0.042 \\
CiS-1,2-DICHLOROETHYLENE & 0.0724 \\
BENZENE & 0.0984 \\
1,1-DICHLOROETHENE & 0.13 \\
CARBON TETRACHLORIDE & 0.277 \\
TRICHLOROETHYLENE (TCE) & 0.297 \\
TETRACHLOROETHYLENE(PCE) & 0.923 \\
MANGANESE & 50.1 \\
URANIUM & 2960
\end{tabular}


Project: EF Central and Fuel

User Name: MV

Location: Central Y-12

State: Tennessee

Note: Top COCs by mobility were determined by examining each detected compound in the dataset and comparing their mobilities (Koc's for organics, assume foc $=0.001$, and Kd's for metals).

Contaminants of Concern (COC's)

TETRACHLOROETHYLENE(PCE)

TRICHLOROETHYLENE (TCE)

CARBON TETRACHLORIDE

URANIUM

VINYL CHLORIDE 


\section{MAROS Spatial Moment Analysis Summary}

Project: EF Central and Fuel

Location: Central Y-12
User Name: MV

State: Tennessee

\begin{tabular}{|c|c|c|c|c|c|c|c|}
\hline \multirow[b]{2}{*}{ Effective Date } & \multirow{2}{*}{$\begin{array}{l}\text { 0th Moment } \\
\text { Estimated } \\
\text { Mass (Kg) }\end{array}$} & \multicolumn{3}{|c|}{ 1st Moment (Center of Mass) } & \multicolumn{2}{|c|}{ 2nd Moment (Spread) } & \multirow[b]{2}{*}{$\begin{array}{c}\text { Number o } \\
\text { Wells }\end{array}$} \\
\hline & & $X c(f t)$ & Yc (ft) & $\begin{array}{c}\text { Source } \\
\text { Distance (ft) }\end{array}$ & $\underset{(\mathrm{sq} \mathrm{ft})}{\operatorname{Sigma} x X}$ & $\underset{(s q \mathrm{ft})}{\operatorname{Sigma} Y Y}$ & \\
\hline
\end{tabular}

BENZENE

\begin{tabular}{|c|c|c|c|c|c|c|c|}
\hline 7/1/1996 & 4.6E-01 & 59,278 & 29,543 & 3,071 & $2,511,944$ & 123,157 & 18 \\
\hline 7/1/1997 & 2.6E-01 & 60,176 & 29,537 & 3,965 & $1,370,381$ & 102,992 & 13 \\
\hline 7/1/1998 & $1.1 \mathrm{E}+00$ & 60,083 & 29,605 & 3,867 & $1,979,819$ & 101,001 & 22 \\
\hline 7/1/1999 & 3.0E-01 & 60,007 & 29,480 & 3,803 & $1,632,404$ & 120,895 & 17 \\
\hline $7 / 1 / 2000$ & 3.9E-01 & 59,349 & 29,539 & 3,142 & $2,595,724$ & 114,498 & 16 \\
\hline $7 / 1 / 2001$ & 4.3E-01 & 59,599 & 29,442 & 3,402 & $2,103,179$ & 104,889 & 12 \\
\hline $7 / 1 / 2002$ & $4.5 \mathrm{E}+00$ & 60,824 & 29,546 & 4,611 & $1,532,822$ & 108,986 & 15 \\
\hline 7/1/2003 & $9.0 \mathrm{E}-01$ & 60,282 & 29,590 & 4,067 & $3,169,039$ & 151,354 & 19 \\
\hline $7 / 1 / 2004$ & $8.2 \mathrm{E}-01$ & 60,458 & 29,465 & 4,252 & $3,309,664$ & 87,354 & 18 \\
\hline 7/1/2005 & $8.4 \mathrm{E}-01$ & 60,419 & 29,475 & 4,213 & $3,495,616$ & 92,846 & 17 \\
\hline 7/1/2006 & $1.0 \mathrm{E}+00$ & 60,420 & 29,450 & 4,216 & $3,977,889$ & 114,102 & 40 \\
\hline $7 / 1 / 2007$ & $1.6 \mathrm{E}+00$ & 60,640 & 29,493 & 4,432 & $3,237,958$ & 168,932 & 44 \\
\hline
\end{tabular}

CARBON TETRACHLORIDE

\begin{tabular}{|c|c|c|c|c|c|c|c|}
\hline 7/1/1996 & $2.8 \mathrm{E}+00$ & 59,642 & 29,249 & 3,475 & 603,100 & 52,977 & 15 \\
\hline $7 / 1 / 1997$ & 3.1E-01 & 59,556 & 29,548 & 3,347 & $1,082,268$ & 134,800 & 10 \\
\hline 7/1/1998 & $9.1 \mathrm{E}-01$ & 58,507 & 29,574 & 2,302 & $1,417,373$ & 102,818 & 17 \\
\hline 7/1/1999 & 3.8E-01 & 59,076 & 29,611 & 2,863 & 634,064 & 103,544 & 13 \\
\hline $7 / 1 / 2000$ & $6.3 \mathrm{E}-01$ & 58,845 & 29,617 & 2,633 & 984,575 & 97,934 & 14 \\
\hline 7/1/2001 & $1.7 \mathrm{E}+00$ & 59,296 & 29,318 & 3,122 & 930,693 & 103,528 & 10 \\
\hline $7 / 1 / 2002$ & $1.9 \mathrm{E}+00$ & 59,444 & 29,324 & 3,267 & $1,169,044$ & 103,045 & 10 \\
\hline $7 / 1 / 2003$ & $1.8 \mathrm{E}+00$ & 59,778 & 29,475 & 3,575 & $1,121,131$ & 151,924 & 14 \\
\hline $7 / 1 / 2004$ & $1.9 \mathrm{E}+00$ & 59,515 & 29,327 & 3,336 & $1,231,300$ & 103,224 & 13 \\
\hline 7/1/2005 & $3.8 \mathrm{E}+00$ & 60,068 & 29,445 & 3,867 & $1,991,236$ & 100,193 & 17 \\
\hline $7 / 1 / 2006$ & $2.0 \mathrm{E}+00$ & 59,755 & 29,587 & 3,541 & $2,467,263$ & 119,613 & 40 \\
\hline $7 / 1 / 2007$ & $1.8 \mathrm{E}+00$ & 59,900 & 29,486 & 3,695 & $2,943,573$ & 181,406 & 44 \\
\hline
\end{tabular}

TETRACHLOROETHYLENE(PCE)

$\begin{array}{lrrrrrrr}7 / 1 / 1996 & 5.7 \mathrm{E}+01 & 56,956 & 29,875 & 730 & 474,146 & 19,873 & 18 \\ 7 / 1 / 1997 & 1.5 \mathrm{E}+00 & 59,688 & 29,662 & 3,469 & 1,985,709 & 131,274 & 13 \\ 7 / 1 / 1998 & 4.3 \mathrm{E}+01 & 57,332 & 29,807 & 1,108 & 832,182 & 32,875 & 22 \\ 7 / 1 / 1999 & 4.7 \mathrm{E}+00 & 60,341 & 29,375 & 4,146 & 2,595,086 & 151,150 & 17 \\ 7 / 1 / 2000 & 3.3 \mathrm{E}+01 & 58,112 & 29,767 & 1,890 & 1,937,735 & 66,326 & 16 \\ 7 / 1 / 2001 & 1.7 \mathrm{E}+01 & 58,623 & 29,632 & 2,410 & 1,848,698 & 84,881 & 12 \\ 7 / 1 / 2002 & 1.9 \mathrm{E}+01 & 59,288 & 29,734 & 3,066 & 2,272,875 & 91,630 & 15 \\ 7 / 1 / 2003 & 2.4 \mathrm{E}+01 & 58,718 & 29,742 & 2,496 & 3,602,533 & 187,752 & 19 \\ 7 / 1 / 2004 & 2.3 \mathrm{E}+01 & 58,743 & 29,762 & 2,520 & 2,604,222 & 88,439 & 18 \\ 7 / 1 / 2005 & 4.9 \mathrm{E}+01 & 57,670 & 29,848 & 1,444 & 2,458,324 & 77,231 & 17 \\ 7 / 1 / 2006 & 3.8 \mathrm{E}+01 & 58,214 & 29,548 & 2,016 & 3,741,866 & 117,369 & 40\end{array}$


Project: EF Central and Fuel

Location: Central Y-12
User Name: $\quad$ MV

State: Tennessee

\begin{tabular}{|c|c|c|c|c|c|c|c|}
\hline \multirow[b]{2}{*}{ Effective Date } & \multirow{2}{*}{$\begin{array}{l}\text { Oth Moment } \\
\text { Estimated } \\
\text { Mass (kg) }\end{array}$} & \multicolumn{3}{|c|}{ 1st Moment (Center of Mass) } & \multicolumn{2}{|c|}{ 2nd Moment (Spread) } & \multirow[b]{2}{*}{$\begin{array}{c}\text { Number of } \\
\text { Wells }\end{array}$} \\
\hline & & $\mathrm{Xc}(\mathrm{ft})$ & Yc (ft) & $\begin{array}{c}\text { Source } \\
\text { Distance (ft) }\end{array}$ & $\begin{array}{c}\text { Sigma } X X \\
(s q \mathrm{ft})\end{array}$ & $\begin{array}{l}\text { Sigma } Y Y \\
(\text { sq ft) }\end{array}$ & \\
\hline \multicolumn{8}{|c|}{ TETRACHLOROETHYLENE(PCE) } \\
\hline $7 / 1 / 2007$ & $2.4 \mathrm{E}+01$ & 57,677 & 29,708 & 1,461 & $5,590,097$ & 113,425 & 44 \\
\hline \multicolumn{8}{|c|}{ TRICHLOROETHYLENE (TCE) } \\
\hline 7/1/1996 & $2.9 \mathrm{E}+00$ & 58,601 & 29,592 & 2,393 & $3,603,171$ & 116,229 & 18 \\
\hline $7 / 1 / 1997$ & $5.5 \mathrm{E}-01$ & 60,542 & 29,416 & 4,341 & $1,757,296$ & 120,161 & 13 \\
\hline $7 / 1 / 1998$ & $8.9 E+00$ & 57,883 & 29,682 & 1,669 & $2,214,880$ & 87,778 & 22 \\
\hline 7/1/1999 & $2.7 \mathrm{E}+00$ & 61,119 & 29,183 & 4,943 & $1,606,134$ & 74,277 & 17 \\
\hline $7 / 1 / 2000$ & $8.9 \mathrm{E}+00$ & 57,925 & 29,640 & 1,716 & $1,913,560$ & 57,181 & 16 \\
\hline $7 / 1 / 2001$ & $2.3 \mathrm{E}+01$ & 57,669 & 29,595 & 1,471 & 499,658 & 18,338 & 12 \\
\hline $7 / 1 / 2002$ & $5.7 \mathrm{E}+00$ & 59,124 & 29,497 & 2,923 & $3,509,661$ & 80,878 & 15 \\
\hline $7 / 1 / 2003$ & $8.6 \mathrm{E}+00$ & 58,830 & 29,607 & 2,619 & $3,914,738$ & 125,885 & 19 \\
\hline $7 / 1 / 2004$ & $5.2 E+00$ & 58,867 & 29,502 & 2,668 & $3,731,954$ & 75,932 & 18 \\
\hline $7 / 1 / 2005$ & $1.6 \mathrm{E}+01$ & 57,523 & 29,676 & 1,314 & $2,622,524$ & 59,608 & 17 \\
\hline $7 / 1 / 2006$ & $2.9 \mathrm{E}+01$ & 58,100 & 29,504 & 1,912 & $1,966,337$ & 63,023 & 40 \\
\hline $7 / 1 / 2007$ & $2.4 \mathrm{E}+01$ & 57,695 & 29,619 & 1,492 & $2,057,661$ & 40,109 & 44 \\
\hline
\end{tabular}

URANIUM

\begin{tabular}{|c|c|c|c|c|c|c|c|}
\hline 7/1/1996 & 2.5E-01 & 59,629 & 29,354 & 3,444 & $2,359,312$ & 91,263 & 16 \\
\hline 7/1/1997 & 6.4E-02 & 61,111 & 29,056 & 4,955 & 557,744 & 57,768 & 10 \\
\hline 7/1/1998 & 4.2E-01 & 58,593 & 29,349 & 2,426 & $1,058,565$ & 54,595 & 15 \\
\hline 7/1/1999 & 8.1E-01 & 58,307 & 29,599 & 2,101 & 203,658 & 15,378 & 13 \\
\hline $7 / 1 / 2000$ & 9.9E-01 & 58,405 & 29,417 & 2,229 & 876,311 & 44,700 & 13 \\
\hline $7 / 1 / 2001$ & $1.2 \mathrm{E}+00$ & 58,452 & 29,362 & 2,286 & 801,530 & 29,649 & 10 \\
\hline $7 / 1 / 2002$ & $1.2 \mathrm{E}+00$ & 58,146 & 29,425 & 1,974 & $1,057,733$ & 39,944 & 10 \\
\hline $7 / 1 / 2003$ & 8.1E-01 & 58,662 & 29,409 & 2,482 & $1,071,761$ & 104,697 & 15 \\
\hline $7 / 1 / 2004$ & $2.2 \mathrm{E}+00$ & 59,413 & 29,250 & 3,250 & $1,169,270$ & 68,788 & 15 \\
\hline 7/1/2005 & $1.1 \mathrm{E}+00$ & 59,319 & 29,207 & 3,166 & $1,390,651$ & 68,121 & 14 \\
\hline $7 / 1 / 2006$ & 1.8E-01 & 58,093 & 29,643 & 1,883 & $5,572,059$ & 208,678 & 37 \\
\hline $7 / 1 / 2007$ & $1.5 \mathrm{E}+00$ & 59,280 & 29,164 & 3,138 & $1,348,628$ & 87,928 & 41 \\
\hline
\end{tabular}


Project: EF Central and Fuel

Location: Central Y-12
User Name: MV

State: Tennessee

\begin{tabular}{|c|c|c|c|c|c|}
\hline Moment Type & Constituent & $\begin{array}{l}\text { Coefficient } \\
\text { of Variation }\end{array}$ & $\begin{array}{l}\text { Mann-Kendall } \\
\text { S Statistic }\end{array}$ & $\begin{array}{l}\text { Confidence } \\
\text { in Trend }\end{array}$ & $\begin{array}{c}\text { Moment } \\
\text { Trend }\end{array}$ \\
\hline \multicolumn{6}{|c|}{ Zeroth Moment: Mass } \\
\hline & BENZENE & 1.10 & 30 & $97.8 \%$ & 1 \\
\hline & CARBON TETRACHLORIDE & 0.61 & 26 & $95.7 \%$ & 1 \\
\hline & TETRACHLOROETHYLENE(PCE) & 0.60 & 8 & $68.1 \%$ & NT \\
\hline & TRICHLOROETHYLENE (TCE) & 0.83 & 34 & $99.0 \%$ & $\mathrm{I}$ \\
\hline & URANIUM & 0.69 & 28 & $96.9 \%$ & 1 \\
\hline \multicolumn{6}{|c|}{ 1st Moment: Distance to Source } \\
\hline & BENZENE & 0.13 & 34 & $99.0 \%$ & 1 \\
\hline & CARBON TETRACHLORIDE & 0.14 & 30 & $97.8 \%$ & 1 \\
\hline & TETRACHLOROETHYLENE(PCE) & 0.45 & -2 & $52.7 \%$ & $\mathrm{~S}$ \\
\hline & TRICHLOROETHYLENE (TCE) & 0.47 & -18 & $87.5 \%$ & $\mathrm{~S}$ \\
\hline & URANIUM & 0.31 & -10 & $72.7 \%$ & $\mathrm{~S}$ \\
\hline \multicolumn{6}{|c|}{ 2nd Moment: Sigma XX } \\
\hline & BENZENE & 0.33 & 38 & $99.6 \%$ & 1 \\
\hline & CARBON TETRACHLORIDE & 0.52 & 44 & $99.9 \%$ & 1 \\
\hline & TETRACHLOROETHYLENE(PCE) & 0.54 & 44 & $99.9 \%$ & 1 \\
\hline & TRICHLOROETHYLENE (TCE) & 0.43 & 6 & $63.1 \%$ & NT \\
\hline & URANIUM & 0.96 & 30 & $97.8 \%$ & 1 \\
\hline \multicolumn{6}{|c|}{ 2nd Moment: Sigma YY } \\
\hline & BENZENE & 0.20 & 2 & $52.7 \%$ & NT \\
\hline & CARBON TETRACHLORIDE & 0.28 & 20 & $90.2 \%$ & $\mathrm{PI}$ \\
\hline & TETRACHLOROETHYLENE(PCE) & 0.49 & 18 & $87.5 \%$ & NT \\
\hline & TRICHLOROETHYLENE (TCE) & 0.42 & -26 & $95.7 \%$ & $\mathrm{D}$ \\
\hline & URANIUM & 0.69 & 16 & $84.5 \%$ & NT \\
\hline
\end{tabular}

Note: The following assumptions were applied for the calculation of the Zeroth Moment:

Porosity: $0.10 \quad$ Saturated Thickness: Uniform: $50 \mathrm{ft}$

Mann-Kendall Trend test performed on all sample events for each constituent. Increasing (I); Probably Increasing (PI); Stable (S);

Probably Decreasing (PD); Decreasing (D); No Trend (NT); Not Applicable (N/A)-Due to insufficient Data (< 4 sampling events).

Note: The Sigma $X X$ and Sigma $Y Y$ components are estimated using the given field coordinate system and then rotated to align with the estimated groundwater flow direction. Moments are not calculated for sample events with less than 6 wells. 


\section{MAROS Statistical Trend Analysis Summary}

Project: $\quad$ EF Central and Fuel

Location: Central Y-12

Time Period: 1/1/1996

to $10 / 15 / 2007$

Consolidation Period: No Time Consolidation

Consolidation Type: Median

Duplicate Consolidation: Average

ND Values: Specified Detection Limit

J Flag Values : Actual Value
User Name: $\quad$ MV

State: Tennessee

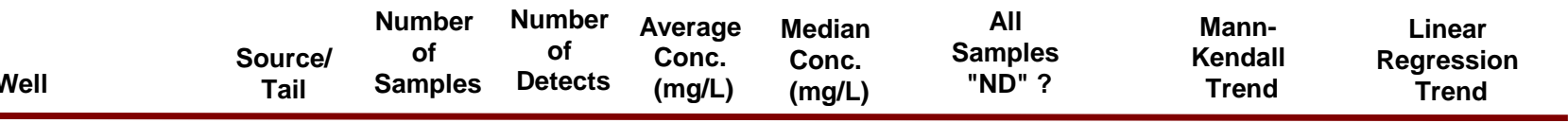

CARBON TETRACHLORIDE

\begin{tabular}{|c|c|}
\hline $55-2 A$ & $\mathrm{~S}$ \\
\hline $55-3 A$ & $\mathrm{~S}$ \\
\hline $55-3 B$ & $\mathrm{~S}$ \\
\hline $55-3 C$ & $\mathrm{~S}$ \\
\hline $56-1 A$ & $\mathrm{~T}$ \\
\hline $56-2 A$ & $\mathrm{~T}$ \\
\hline $56-2 B$ & $\mathrm{~T}$ \\
\hline $56-2 C$ & $\mathrm{~T}$ \\
\hline $56-3 A$ & $\mathrm{~T}$ \\
\hline $56-3 B$ & $\mathrm{~T}$ \\
\hline $56-3 C$ & $\mathrm{~T}$ \\
\hline $56-4 A$ & $\mathrm{~T}$ \\
\hline $56-6 A$ & $\mathrm{~T}$ \\
\hline $56-8 \mathrm{~A}$ & $\mathrm{~T}$ \\
\hline $60-1 A$ & $\mathrm{~T}$ \\
\hline GW-204 & $\mathrm{S}$ \\
\hline GW-219 & $\mathrm{S}$ \\
\hline GW-281 & $\mathrm{S}$ \\
\hline GW-605 & $\mathrm{S}$ \\
\hline GW-606 & $\mathrm{T}$ \\
\hline GW-656 & $\mathrm{S}$ \\
\hline GW-658 & $\mathrm{T}$ \\
\hline GW-686 & $\mathrm{T}$ \\
\hline GW-690 & $\mathrm{T}$ \\
\hline GW-691 & $\mathrm{S}$ \\
\hline GW-692 & $\mathrm{T}$ \\
\hline GW-698 & $\mathrm{S}$ \\
\hline GW-700 & $\mathrm{T}$ \\
\hline GW-762 & $\mathrm{T}$ \\
\hline GW-763 & $\mathrm{T}$ \\
\hline GW-765 & $\mathrm{T}$ \\
\hline GW-769 & $\mathrm{T}$ \\
\hline GW-770 & $\mathrm{T}$ \\
\hline GW-775 & $\mathrm{T}$ \\
\hline GW-776 & $\mathrm{T}$ \\
\hline
\end{tabular}

\begin{tabular}{|c|c|c|}
\hline 5.0E-04 & 5.0E-04 & Yes \\
\hline 5.0E-04 & 5.0E-04 & Yes \\
\hline 5.0E-04 & 5.0E-04 & Yes \\
\hline $5.0 \mathrm{E}-04$ & 5.0E-04 & Yes \\
\hline 5.0E-04 & 5.0E-04 & Yes \\
\hline 5.0E-04 & 5.0E-04 & Yes \\
\hline $5.0 \mathrm{E}-04$ & 5.0E-04 & Yes \\
\hline 5.0E-04 & 5.0E-04 & Yes \\
\hline 5.0E-04 & 5.0E-04 & Yes \\
\hline 5.0E-04 & 5.0E-04 & Yes \\
\hline 5.0E-04 & 5.0E-04 & Yes \\
\hline 5.0E-04 & 5.0E-04 & Yes \\
\hline 5.0E-04 & 5.0E-04 & Yes \\
\hline 5.0E-04 & 5.0E-04 & Yes \\
\hline 5.0E-04 & 5.0E-04 & Yes \\
\hline 5.0E-04 & 5.0E-04 & Yes \\
\hline 5.0E-04 & 5.0E-04 & Yes \\
\hline 5.0E-04 & 5.0E-04 & Yes \\
\hline 7.0E-02 & $3.9 \mathrm{E}-02$ & No \\
\hline 2.0E-01 & 8.7E-02 & No \\
\hline 5.0E-04 & 5.0E-04 & Yes \\
\hline 5.0E-04 & 5.0E-04 & Yes \\
\hline 5.0E-04 & 5.0E-04 & Yes \\
\hline 5.0E-04 & 5.0E-04 & Yes \\
\hline 5.0E-04 & 5.0E-04 & Yes \\
\hline $5.0 \mathrm{E}-04$ & 5.0E-04 & Yes \\
\hline 7.9E-03 & 9.0E-03 & No \\
\hline 5.0E-04 & 5.0E-04 & Yes \\
\hline 7.5E-04 & 5.0E-04 & No \\
\hline 5.0E-04 & 5.0E-04 & Yes \\
\hline 5.0E-04 & 5.0E-04 & Yes \\
\hline 5.9E-02 & 5.8E-02 & No \\
\hline 8.5E-03 & 5.0E-03 & No \\
\hline 5.0E-04 & 5.0E-04 & Ye \\
\hline 5.0E-04 & 5.0E-04 & Yes \\
\hline
\end{tabular}

ND

ND

ND

ND

ND

ND

ND

ND

ND

ND

ND

ND

ND

ND

ND

ND

ND

ND

NT

D

ND

ND

ND

ND

ND

ND

NT

ND

$S$

ND

ND

I

I

ND

ND

\section{ND}

ND

ND

ND

ND

ND

ND

ND

ND

ND

ND

ND

ND

ND

ND

ND

ND

ND

NT

D

ND

ND

ND

ND

ND

ND

NT

ND

$S$

ND

ND

I

ND

ND 
MAROS Statistical Trend Analysis Summary

\begin{tabular}{|c|c|c|c|c|c|c|c|c|}
\hline Well & $\begin{array}{c}\text { Sourcel } \\
\text { Tail }\end{array}$ & $\begin{array}{c}\text { Number } \\
\text { of } \\
\text { Samples }\end{array}$ & $\begin{array}{c}\text { Number } \\
\text { of } \\
\text { Detects }\end{array}$ & $\begin{array}{l}\text { Average } \\
\text { Conc. } \\
\text { (mg/L) }\end{array}$ & $\begin{array}{l}\text { Median } \\
\text { Conc. } \\
\text { (mg/L) }\end{array}$ & $\begin{array}{c}\text { All } \\
\text { Samples } \\
\text { "ND" ? }\end{array}$ & $\begin{array}{l}\text { Mann- } \\
\text { Kendall } \\
\text { Trend }\end{array}$ & $\begin{array}{l}\text { Linear } \\
\text { Regression } \\
\text { Trend }\end{array}$ \\
\hline \multicolumn{9}{|c|}{ CARBON TETRACHLORIDE } \\
\hline GW-779 & $\mathrm{T}$ & 5 & 0 & 5.0E-04 & $5.0 \mathrm{E}-04$ & Yes & ND & ND \\
\hline GW-781 & $\mathrm{T}$ & 11 & 0 & 5.0E-04 & 5.0E-04 & Yes & ND & ND \\
\hline GW-782 & $\mathrm{T}$ & 24 & 4 & 1.1E-03 & 5.0E-04 & No & NT & PD \\
\hline GW-783 & $\mathrm{T}$ & 11 & 4 & $9.5 \mathrm{E}-04$ & 5.0E-04 & No & NT & $\mathrm{S}$ \\
\hline GW-791 & $\mathrm{S}$ & 23 & 0 & $5.0 \mathrm{E}-04$ & 5.0E-04 & Yes & ND & ND \\
\hline GW-792 & $\mathrm{T}$ & 11 & 0 & 5.0E-04 & $5.0 \mathrm{E}-04$ & Yes & ND & ND \\
\hline GW-802 & $\mathrm{T}$ & 3 & 0 & 5.0E-04 & 5.0E-04 & Yes & ND & ND \\
\hline GW-820 & $\mathrm{S}$ & 10 & 0 & 5.0E-04 & 5.0E-04 & Yes & ND & ND \\
\hline GW-954-1 & $\mathrm{T}$ & 4 & 0 & 5.0E-04 & 5.0E-04 & Yes & ND & ND \\
\hline GW-954-2 & $\mathrm{T}$ & 4 & 0 & 5.0E-04 & $5.0 \mathrm{E}-04$ & Yes & ND & ND \\
\hline GW-954-3 & $\mathrm{T}$ & 4 & 0 & 5.0E-04 & 5.0E-04 & Yes & ND & ND \\
\hline GW-956-1 & $\mathrm{T}$ & 4 & 0 & 5.0E-04 & 5.0E-04 & Yes & ND & ND \\
\hline GW-956-2 & $\mathrm{T}$ & 4 & 0 & 5.0E-04 & 5.0E-04 & Yes & ND & ND \\
\hline GW-956-3 & $\mathrm{T}$ & 4 & 0 & 5.0E-04 & 5.0E-04 & Yes & ND & ND \\
\hline GW-956-4 & $\mathrm{T}$ & 4 & 0 & 5.0E-04 & 5.0E-04 & Yes & ND & ND \\
\hline GW-959 & $\mathrm{T}$ & 7 & 0 & 5.0E-04 & 5.0E-04 & Yes & ND & ND \\
\hline GW-960 & $\mathrm{T}$ & 2 & 0 & 5.0E-04 & 5.0E-04 & Yes & ND & ND \\
\hline UEFPC-SP17 & $\mathrm{T}$ & 9 & 0 & 5.0E-04 & 5.0E-04 & Yes & ND & ND \\
\hline
\end{tabular}

TETRACHLOROETHYLENE(PCE)

\begin{tabular}{|c|c|c|c|c|c|c|c|c|}
\hline $55-2 A$ & S & 4 & 4 & 2.6E-01 & 2.6E-01 & No & $D$ & D \\
\hline $55-3 A$ & S & 5 & 5 & $1.8 \mathrm{E}+01$ & $1.3 \mathrm{E}+01$ & No & NT & NT \\
\hline $55-3 B$ & S & 5 & 5 & $7.3 \mathrm{E}+01$ & $7.2 \mathrm{E}+01$ & No & NT & NT \\
\hline $55-3 C$ & S & 4 & 4 & $1.9 \mathrm{E}+01$ & $1.9 \mathrm{E}+01$ & No & NT & NT \\
\hline $56-1 \mathrm{~A}$ & $\mathrm{~T}$ & 4 & 0 & 5.0E-04 & $5.0 \mathrm{E}-04$ & Yes & ND & ND \\
\hline $56-2 A$ & $\mathrm{~T}$ & 5 & 5 & 2.0E-02 & 1.3E-02 & No & $\mathrm{s}$ & $\mathrm{PD}$ \\
\hline $56-2 B$ & $\mathrm{~T}$ & 5 & 5 & 9.4E-01 & 8.7E-01 & No & S & I \\
\hline $56-2 C$ & $\mathrm{~T}$ & 7 & 7 & $1.2 \mathrm{E}+00$ & 9.7E-01 & No & PD & D \\
\hline $56-3 A$ & $\mathrm{~T}$ & 4 & 4 & 4.7E-02 & 4.9E-02 & No & $\mathrm{s}$ & NT \\
\hline $56-3 B$ & $\mathrm{~T}$ & 4 & 4 & 2.1E-01 & 2.4E-01 & No & NT & NT \\
\hline $56-3 C$ & $\mathrm{~T}$ & 4 & 4 & 5.6E-01 & 5.5E-01 & No & $\mathrm{s}$ & $\mathrm{s}$ \\
\hline $56-4 A$ & $\mathrm{~T}$ & 4 & 3 & 3.4E-03 & 2.0E-03 & No & NT & NT \\
\hline $56-6 A$ & $\mathrm{~T}$ & 4 & 0 & 5.0E-04 & 5.0E-04 & Yes & ND & ND \\
\hline $56-8 A$ & $\mathrm{~T}$ & 2 & 0 & 5.0E-04 & 5.0E-04 & Yes & ND & ND \\
\hline $60-1 \mathrm{~A}$ & $\mathrm{~T}$ & 4 & 0 & 5.0E-04 & 5.0E-04 & Yes & ND & ND \\
\hline GW-204 & $\mathrm{s}$ & 16 & 1 & 1.5E-03 & 5.0E-04 & No & NT & PD \\
\hline GW-219 & $\mathrm{s}$ & 15 & 0 & 5.0E-04 & 5.0E-04 & Yes & ND & ND \\
\hline GW-281 & $\mathrm{S}$ & 5 & 0 & 5.0E-04 & 5.0E-04 & Yes & ND & ND \\
\hline GW-605 & $\mathrm{S}$ & 26 & 25 & 3.7E-02 & 3.0E-02 & No & 1 & I \\
\hline GW-606 & $\mathrm{T}$ & 26 & 23 & $5.6 \mathrm{E}-03$ & $6.0 \mathrm{E}-03$ & No & $\mathrm{s}$ & I \\
\hline GW-656 & $\mathrm{s}$ & 6 & 6 & 5.3E-02 & 5.2E-02 & No & $D$ & D \\
\hline GW-658 & $\mathrm{T}$ & 9 & 0 & 5.0E-04 & 5.0E-04 & Yes & ND & ND \\
\hline GW-686 & $\mathrm{T}$ & 6 & 0 & 5.0E-04 & 5.0E-04 & Yes & ND & ND \\
\hline GW-690 & $\mathrm{T}$ & 7 & 7 & 1.6E-01 & 4.8E-02 & No & PD & D \\
\hline GW-691 & $\mathrm{S}$ & 5 & 5 & $1.3 \mathrm{E}+00$ & $1.3 \mathrm{E}+00$ & No & $\mathrm{PI}$ & I \\
\hline GW-692 & $\mathrm{T}$ & 5 & 4 & 6.9E-03 & 4.0E-03 & No & NT & NT \\
\hline
\end{tabular}


MAROS Statistical Trend Analysis Summary

\begin{tabular}{ccccccccc} 
& & Number & Number & Average & Median & All & Mann- & Linear \\
Well & Sourcel & of & of & Conc. & Conc. \\
Tail & Samples & Detects & (mg/L) & (mg/L) & "ND" ? & $\begin{array}{c}\text { Kendall } \\
\text { Trend }\end{array}$ & $\begin{array}{c}\text { Regression } \\
\text { Trend }\end{array}$ \\
\hline
\end{tabular}

TETRACHLOROETHYLENE(PCE)

$\begin{array}{lll}\text { GW-698 } & \text { S } \\ \text { GW-700 } & \text { T } \\ \text { GW-762 } & \text { T } \\ \text { GW-763 } & \text { T } \\ \text { GW-765 } & \text { T } \\ \text { GW-769 } & \text { T } \\ \text { GW-770 } & \text { T } \\ \text { GW-775 } & \text { T } & 25 \\ \text { GW-776 } & \text { T } \\ \text { GW-779 } & \text { T } \\ \text { GW-781 } & \text { T } \\ \text { GW-782 } & \text { T } \\ \text { GW-783 } & \text { T } \\ \text { GW-791 } & \text { S } \\ \text { GW-792 } & \text { T } \\ \text { GW-802 } & \text { T } \\ \text { GW-820 } & \text { S } \\ \text { GW-954-1 } & \text { T } \\ \text { GW-954-2 } & \text { T } \\ \text { GW-954-3 } & \text { T } \\ \text { GW-956-1 } & \text { T } \\ \text { GW-956-2 } & \text { T } \\ \text { GW-956-3 } & \text { T } \\ \text { GW-956-4 } & \text { T } \\ \text { GW-959 } & \text { T } \\ \text { GW-960 } & \text { T } \\ \text { UEFPC-SP17 } & \text { T }\end{array}$

$\begin{array}{ccccc}17 & 16 & 1.2 \mathrm{E}-01 & 1.4 \mathrm{E}-01 & \text { No } \\ 7 & 7 & 1.5 \mathrm{E}-01 & 1.3 \mathrm{E}-01 & \text { No } \\ 18 & 18 & 2.3 \mathrm{E}+00 & 2.3 \mathrm{E}+00 & \text { No } \\ 24 & 15 & 1.6 \mathrm{E}-02 & 8.5 \mathrm{E}-03 & \text { No } \\ 3 & 0 & 5.0 \mathrm{E}-04 & 5.0 \mathrm{E}-04 & \text { Yes } \\ 25 & 24 & 1.1 \mathrm{E}-02 & 1.1 \mathrm{E}-02 & \text { No } \\ 25 & 1 & 5.2 \mathrm{E}-04 & 5.0 \mathrm{E}-04 & \text { No } \\ 12 & 0 & 5.0 \mathrm{E}-04 & 5.0 \mathrm{E}-04 & \text { Yes } \\ 12 & 4 & 9.2 \mathrm{E}-04 & 5.0 \mathrm{E}-04 & \text { No } \\ 5 & 0 & 5.0 \mathrm{E}-04 & 5.0 \mathrm{E}-04 & \text { Yes } \\ 11 & 8 & 1.0 \mathrm{E}-02 & 4.0 \mathrm{E}-03 & \text { No } \\ 24 & 24 & 1.7 \mathrm{E}-01 & 1.7 \mathrm{E}-01 & \text { No } \\ 11 & 11 & 2.1 \mathrm{E}-02 & 1.1 \mathrm{E}-02 & \text { No } \\ 23 & 23 & 5.6 \mathrm{E}-01 & 4.1 \mathrm{E}-01 & \text { No } \\ 11 & 11 & 6.3 \mathrm{E}-03 & 6.0 \mathrm{E}-03 & \text { No } \\ 7 & 0 & 5.0 \mathrm{E}-04 & 5.0 \mathrm{E}-04 & \text { Yes } \\ 10 & 10 & 3.7 \mathrm{E}+00 & 3.0 \mathrm{E}+00 & \text { No } \\ 4 & 0 & 5.0 \mathrm{E}-04 & 5.0 \mathrm{E}-04 & \text { Yes } \\ 4 & 0 & 5.0 \mathrm{E}-04 & 5.0 \mathrm{E}-04 & \text { Yes } \\ 4 & 0 & 5.0 \mathrm{E}-04 & 5.0 \mathrm{E}-04 & \text { Yes } \\ 4 & 1 & 1.6 \mathrm{E}-03 & 5.0 \mathrm{E}-04 & \text { No } \\ 4 & 4 & 4.0 \mathrm{E}-03 & 4.0 \mathrm{E}-03 & \text { No } \\ 4 & 1 & 6.3 \mathrm{E}-04 & 5.0 \mathrm{E}-04 & \text { No } \\ 4 & 1 & 6.3 \mathrm{E}-04 & 5.0 \mathrm{E}-04 & \text { No } \\ 7 & 0 & 5.0 \mathrm{E}-04 & 5.0 \mathrm{E}-04 & \text { Yes } \\ 2 & 0 & 5.0 \mathrm{E}-04 & 5.0 \mathrm{E}-04 & \text { Yes } \\ 9 & 0 & 5.0 \mathrm{E}-04 & 5.0 \mathrm{E}-04 & \text { Yes }\end{array}$

$\begin{array}{cc}\text { PI } & \text { NT } \\ \text { D } & \text { D } \\ \text { I } & \text { I } \\ \text { NT } & \text { NT } \\ \text { ND } & \text { ND } \\ \text { I } & \text { I } \\ \text { NT } & \text { NT } \\ \text { ND } & \text { ND } \\ \text { D } & \text { D } \\ \text { ND } & \text { ND } \\ \text { NT } & \text { NT } \\ \text { D } & \text { D } \\ \text { S } & \text { S } \\ \text { S } & \text { PD } \\ \text { D } & \text { D } \\ \text { ND } & \text { ND } \\ \text { S } & \text { S } \\ \text { ND } & \text { ND } \\ \text { ND } & \text { ND } \\ \text { ND } & \text { ND } \\ \text { NT } & \text { NT } \\ \text { S } & \text { D } \\ \text { NT } & \text { NT } \\ \text { S } & \text { S } \\ \text { ND } & \text { ND } \\ \text { ND } & \text { ND } \\ \text { ND } & \text { ND }\end{array}$

TRICHLOROETHYLENE (TCE)

\begin{tabular}{|c|c|c|c|c|c|c|c|c|}
\hline $55-2 A$ & S & 4 & 4 & $1.2 \mathrm{E}-01$ & 1.1E-01 & No & $S$ & PD \\
\hline $55-3 A$ & S & 5 & 5 & $9.9 \mathrm{E}-01$ & $1.1 \mathrm{E}+00$ & No & NT & NT \\
\hline $55-3 B$ & S & 5 & 5 & $6.2 \mathrm{E}+00$ & $5.8 \mathrm{E}+00$ & No & NT & NT \\
\hline $55-3 C$ & S & 4 & 4 & $1.6 \mathrm{E}+00$ & $1.6 \mathrm{E}+00$ & No & NT & NT \\
\hline $56-1 A$ & $\mathrm{~T}$ & 4 & 0 & $5.0 \mathrm{E}-04$ & 5.0E-04 & Yes & ND & ND \\
\hline $56-2 A$ & $\mathrm{~T}$ & 5 & 4 & 3.7E-03 & 3.0E-03 & No & $S$ & $\mathrm{~s}$ \\
\hline $56-2 B$ & $\mathrm{~T}$ & 5 & 5 & $6.0 \mathrm{E}-02$ & $5.8 \mathrm{E}-02$ & No & $S$ & NT \\
\hline $56-2 C$ & $\mathrm{~T}$ & 7 & 7 & $5.6 \mathrm{E}-01$ & 4.1E-01 & No & $S$ & $S$ \\
\hline $56-3 A$ & $\mathrm{~T}$ & 4 & 4 & $4.8 \mathrm{E}-03$ & $4.5 \mathrm{E}-03$ & No & NT & NT \\
\hline $56-3 B$ & $\mathrm{~T}$ & 4 & 4 & $1.6 \mathrm{E}-02$ & 1.7E-02 & No & NT & NT \\
\hline $56-3 C$ & $\mathrm{~T}$ & 4 & 4 & 3.7E-02 & 3.6E-02 & No & NT & NT \\
\hline $56-4 A$ & $\mathrm{~T}$ & 4 & 0 & $5.0 \mathrm{E}-04$ & $5.0 \mathrm{E}-04$ & Yes & ND & ND \\
\hline $56-6 A$ & $\mathrm{~T}$ & 4 & 0 & $5.0 \mathrm{E}-04$ & $5.0 \mathrm{E}-04$ & Yes & ND & ND \\
\hline $56-8 A$ & $\mathrm{~T}$ & 2 & 0 & $5.0 \mathrm{E}-04$ & 5.0E-04 & Yes & ND & ND \\
\hline $60-1 A$ & $\mathrm{~T}$ & 4 & 0 & $5.0 \mathrm{E}-04$ & $5.0 \mathrm{E}-04$ & Yes & ND & ND \\
\hline GW-204 & $\mathrm{S}$ & 16 & 1 & $5.9 \mathrm{E}-04$ & 5.0E-04 & No & $S$ & PD \\
\hline GW-219 & $\mathrm{s}$ & 15 & 1 & $6.0 \mathrm{E}-04$ & 5.0E-04 & No & $\mathrm{s}$ & $\mathrm{s}$ \\
\hline
\end{tabular}


MAROS Statistical Trend Analysis Summary

\begin{tabular}{|c|c|c|c|c|c|c|c|c|}
\hline Well & $\begin{array}{c}\text { Sourcel } \\
\text { Tail }\end{array}$ & $\begin{array}{l}\text { Number } \\
\text { of } \\
\text { Samples }\end{array}$ & $\begin{array}{c}\text { Number } \\
\text { of } \\
\text { Detects }\end{array}$ & $\begin{array}{l}\text { Average } \\
\text { Conc. } \\
\text { (mg/L) }\end{array}$ & $\begin{array}{l}\text { Median } \\
\text { Conc. } \\
\text { (mg/L) }\end{array}$ & $\begin{array}{c}\text { All } \\
\text { Samples } \\
\text { "ND" ? }\end{array}$ & $\begin{array}{l}\text { Mann- } \\
\text { Kendall } \\
\text { Trend }\end{array}$ & $\begin{array}{c}\text { Linear } \\
\text { Regression } \\
\text { Trend }\end{array}$ \\
\hline
\end{tabular}

TRICHLOROETHYLENE (TCE)

\begin{tabular}{|c|c|c|c|c|c|c|c|c|}
\hline GW-281 & $\mathrm{S}$ & 5 & 0 & $5.0 \mathrm{E}-04$ & $5.0 \mathrm{E}-04$ & Yes & ND & ND \\
\hline GW-605 & $\mathrm{S}$ & 26 & 25 & 3.8E-02 & $3.2 \mathrm{E}-02$ & No & I & I \\
\hline GW-606 & $\mathrm{T}$ & 26 & 1 & 1.1E-03 & $5.0 \mathrm{E}-04$ & No & NT & NT \\
\hline GW-656 & $\mathrm{S}$ & 6 & 6 & $3.8 \mathrm{E}+00$ & $4.0 \mathrm{E}+00$ & No & $\mathrm{D}$ & D \\
\hline GW-658 & $\mathrm{T}$ & 9 & 0 & 5.0E-04 & $5.0 \mathrm{E}-04$ & Yes & ND & ND \\
\hline GW-686 & $\mathrm{T}$ & 6 & 0 & 5.0E-04 & $5.0 \mathrm{E}-04$ & Yes & ND & ND \\
\hline GW-690 & $\mathrm{T}$ & 7 & 7 & 1.2E-02 & $6.0 \mathrm{E}-03$ & No & $\mathrm{s}$ & $\mathrm{D}$ \\
\hline GW-691 & $\mathrm{S}$ & 5 & 5 & 1.0E-02 & $8.0 \mathrm{E}-03$ & No & NT & NT \\
\hline GW-692 & $\mathrm{T}$ & 5 & 4 & 1.9E-03 & $2.0 \mathrm{E}-03$ & No & 1 & I \\
\hline GW-698 & $\mathrm{s}$ & 17 & 17 & 3.5E-01 & 3.4E-01 & No & 1 & I \\
\hline GW-700 & $\mathrm{T}$ & 7 & 7 & 1.2E-02 & $1.1 \mathrm{E}-02$ & No & $\mathrm{s}$ & $\mathrm{s}$ \\
\hline GW-762 & $\mathrm{T}$ & 18 & 18 & 1.4E-01 & $1.5 \mathrm{E}-01$ & No & NT & NT \\
\hline GW-763 & $\mathrm{T}$ & 24 & 15 & 4.8E-03 & $4.0 \mathrm{E}-03$ & No & $\mathrm{s}$ & $\mathrm{s}$ \\
\hline GW-765 & $\mathrm{T}$ & 3 & 0 & 5.0E-04 & $5.0 \mathrm{E}-04$ & Yes & ND & ND \\
\hline GW-769 & $\mathrm{T}$ & 25 & 20 & $2.6 \mathrm{E}-03$ & $3.0 \mathrm{E}-03$ & No & I & 1 \\
\hline GW-770 & $\mathrm{T}$ & 25 & 0 & 5.0E-04 & $5.0 \mathrm{E}-04$ & Yes & ND & ND \\
\hline GW-775 & $\mathrm{T}$ & 12 & 12 & 4.6E-03 & 4.5E-03 & No & $\mathrm{s}$ & PD \\
\hline GW-776 & $\mathrm{T}$ & 12 & 11 & $2.9 \mathrm{E}-03$ & $3.0 \mathrm{E}-03$ & No & PD & PD \\
\hline GW-779 & $\mathrm{T}$ & 5 & 0 & 5.0E-04 & $5.0 \mathrm{E}-04$ & Yes & ND & ND \\
\hline GW-781 & $\mathrm{T}$ & 11 & 3 & 1.7E-03 & $5.0 \mathrm{E}-04$ & No & NT & NT \\
\hline GW-782 & $\mathrm{T}$ & 24 & 24 & 5.3E-02 & $5.3 \mathrm{E}-02$ & No & $\mathrm{D}$ & $P D$ \\
\hline GW-783 & $\mathrm{T}$ & 11 & 10 & $9.0 \mathrm{E}-03$ & $6.0 \mathrm{E}-03$ & No & $\mathrm{s}$ & $\mathrm{s}$ \\
\hline GW-791 & $\mathrm{s}$ & 23 & 13 & $2.2 \mathrm{E}-03$ & $2.0 \mathrm{E}-03$ & No & NT & NT \\
\hline GW-792 & $\mathrm{T}$ & 11 & 0 & 5.0E-04 & $5.0 \mathrm{E}-04$ & Yes & ND & ND \\
\hline GW-802 & $\mathrm{T}$ & 7 & 0 & 5.0E-04 & $5.0 \mathrm{E}-04$ & Yes & ND & ND \\
\hline GW-820 & $\mathrm{s}$ & 10 & 10 & $6.4 \mathrm{E}-01$ & $5.7 \mathrm{E}-01$ & No & NT & NT \\
\hline GW-954-1 & $\mathrm{T}$ & 4 & 0 & 5.0E-04 & $5.0 \mathrm{E}-04$ & Yes & ND & ND \\
\hline GW-954-2 & $\mathrm{T}$ & 4 & 0 & 5.0E-04 & $5.0 \mathrm{E}-04$ & Yes & ND & ND \\
\hline GW-954-3 & $\mathrm{T}$ & 4 & 0 & 5.0E-04 & $5.0 \mathrm{E}-04$ & Yes & ND & ND \\
\hline GW-956-1 & $\mathrm{T}$ & 4 & 0 & 5.0E-04 & $5.0 \mathrm{E}-04$ & Yes & ND & ND \\
\hline GW-956-2 & $\mathrm{T}$ & 4 & 4 & $1.5 \mathrm{E}-03$ & $1.5 \mathrm{E}-03$ & No & $\mathrm{s}$ & PD \\
\hline GW-956-3 & $\mathrm{T}$ & 4 & 0 & 5.0E-04 & $5.0 \mathrm{E}-04$ & Yes & ND & ND \\
\hline GW-956-4 & $\mathrm{T}$ & 4 & 0 & 5.0E-04 & $5.0 \mathrm{E}-04$ & Yes & ND & ND \\
\hline GW-959 & $\mathrm{T}$ & 7 & 3 & 1.1E-03 & $5.0 \mathrm{E}-04$ & No & $\mathrm{s}$ & $\mathrm{s}$ \\
\hline GW-960 & $\mathrm{T}$ & 2 & 0 & 5.0E-04 & $5.0 \mathrm{E}-04$ & Yes & ND & ND \\
\hline UEFPC-SP17 & $\mathrm{T}$ & 9 & 0 & 5.0E-04 & $5.0 \mathrm{E}-04$ & Yes & ND & ND \\
\hline \multicolumn{9}{|l|}{ URANIUM } \\
\hline $55-2 A$ & $\mathrm{~S}$ & 4 & 4 & 4.4E-03 & 4.3E-03 & No & $\mathrm{s}$ & $\mathrm{s}$ \\
\hline $55-3 A$ & $\mathrm{~s}$ & 5 & 5 & $9.1 \mathrm{E}-04$ & $9.6 \mathrm{E}-04$ & No & $\mathrm{s}$ & $\mathrm{D}$ \\
\hline $55-3 B$ & $\mathrm{~S}$ & 5 & 0 & $5.9 \mathrm{E}-06$ & $5.9 \mathrm{E}-06$ & Yes & ND & ND \\
\hline $55-3 C$ & $\mathrm{~s}$ & 4 & 0 & $5.9 \mathrm{E}-06$ & $5.9 \mathrm{E}-06$ & Yes & ND & ND \\
\hline $56-1 A$ & $\mathrm{~T}$ & 4 & 4 & 2.3E-03 & $1.9 \mathrm{E}-03$ & No & $\mathrm{D}$ & PD \\
\hline $56-2 A$ & $\mathrm{~T}$ & 5 & 2 & $2.2 \mathrm{E}-04$ & $5.9 \mathrm{E}-06$ & No & NT & NT \\
\hline $56-2 B$ & $\mathrm{~T}$ & 5 & 0 & $5.9 \mathrm{E}-06$ & $5.9 \mathrm{E}-06$ & Yes & ND & ND \\
\hline $56-2 C$ & $\mathrm{~T}$ & 7 & 0 & 5.9E-06 & $5.9 \mathrm{E}-06$ & Yes & ND & ND \\
\hline
\end{tabular}




\section{MAROS Statistical Trend Analysis Summary}

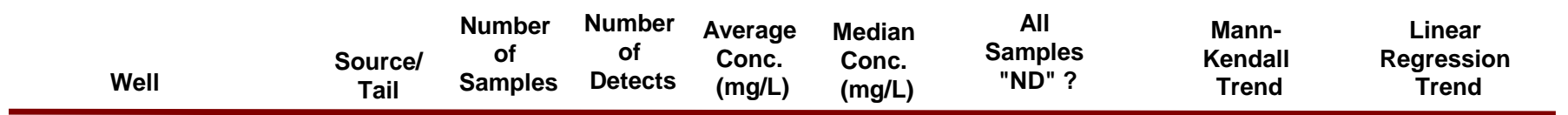

URANIUM

\section{6-3A}

\section{6-3B}

$56-3 \mathrm{C}$

56-4A

56-6A

$56-8 \mathrm{~A}$

60-1A

GW-204

GW-219

GW-605

GW-606

GW-656

GW-686

GW-690

GW-691

GW-692

GW-698

GW-700

GW-762

GW-763

GW-765

GW-769

GW-770

GW-775

GW-776

GW-779

GW-781

GW-782

GW-783

GW-791

GW-792

GW-820

GW-954-1

GW-954-2

GW-954-3

GW-956-1

GW-956-2

GW-956-3

GW-956-4

GW-959

GW-960

UEFPC-SP17

\begin{tabular}{lll}
$8.2 \mathrm{E}-04$ & $8.1 \mathrm{E}-04$ & No \\
$7.4 \mathrm{E}-04$ & $7.3 \mathrm{E}-04$ & No \\
$5.9 \mathrm{E}-06$ & $5.9 \mathrm{E}-06$ & Yes \\
$5.9 \mathrm{E}-06$ & $5.9 \mathrm{E}-06$ & Yes \\
$5.9 \mathrm{E}-06$ & $5.9 \mathrm{E}-06$ & Yes \\
$5.9 \mathrm{E}-06$ & $5.9 \mathrm{E}-06$ & Yes \\
$5.9 \mathrm{E}-06$ & $5.9 \mathrm{E}-06$ & Yes \\
$6.4 \mathrm{E}-02$ & $4.6 \mathrm{E}-02$ & No \\
$3.9 \mathrm{E}-01$ & $3.4 \mathrm{E}-01$ & No \\
$1.3 \mathrm{E}-01$ & $9.8 \mathrm{E}-02$ & No \\
$4.1 \mathrm{E}-03$ & $5.0 \mathrm{E}-03$ & No \\
$2.2 \mathrm{E}-04$ & $5.9 \mathrm{E}-06$ & No \\
$8.4 \mathrm{E}-04$ & $8.5 \mathrm{E}-04$ & No \\
$1.3 \mathrm{E}-04$ & $5.9 \mathrm{E}-06$ & No \\
$8.5 \mathrm{E}-04$ & $7.9 \mathrm{E}-04$ & No \\
$3.8 \mathrm{E}-04$ & $3.3 \mathrm{E}-04$ & No \\
$1.1 \mathrm{E}-03$ & $1.1 \mathrm{E}-03$ & No \\
$5.8 \mathrm{E}-04$ & $6.3 \mathrm{E}-04$ & No \\
$2.5 \mathrm{E}-05$ & $5.9 \mathrm{E}-06$ & No \\
$5.9 \mathrm{E}-06$ & $5.9 \mathrm{E}-06$ & Yes \\
$5.9 \mathrm{E}-06$ & $5.9 \mathrm{E}-06$ & Yes \\
$2.6 \mathrm{E}-05$ & $5.9 \mathrm{E}-06$ & No \\
$8.8 \mathrm{E}-04$ & $7.6 \mathrm{E}-04$ & No \\
$5.9 \mathrm{E}-06$ & $5.9 \mathrm{E}-06$ & Yes \\
$5.9 \mathrm{E}-06$ & $5.9 \mathrm{E}-06$ & Yes \\
$2.0 \mathrm{E}-03$ & $5.9 \mathrm{E}-06$ & No \\
$5.9 \mathrm{E}-06$ & $5.9 \mathrm{E}-06$ & Yes \\
$1.2 \mathrm{E}-03$ & $1.2 \mathrm{E}-03$ & No \\
$1.1 \mathrm{E}-03$ & $1.1 \mathrm{E}-03$ & No \\
$5.9 \mathrm{E}-06$ & $5.9 \mathrm{E}-06$ & Yes \\
$5.9 \mathrm{E}-06$ & $5.9 \mathrm{E}-06$ & Yes \\
$5.9 \mathrm{E}-06-03$ & $5.9 \mathrm{E}-06$ & Yes \\
$3.3 \mathrm{E}-04$ & $3.0 \mathrm{E}-04$ & No \\
$5.9 \mathrm{E}-06$ & $5.9 \mathrm{E}-06$ & Yes \\
$5.9 \mathrm{E}-06$ & $5.9 \mathrm{E}-06$ & Yes \\
$5.9 \mathrm{E}-06$ & $5.9 \mathrm{E}-06$ & Yes \\
$5.9 \mathrm{E}-06$ & $5.9 \mathrm{E}-06$ & Yes \\
$5.9 \mathrm{E}-06$ & $5.9 \mathrm{E}-06$ & Yes \\
$5.9 \mathrm{E}-06$ & $5.9 \mathrm{E}-06$ & Yes \\
$1.0 \mathrm{E}-03$ & No \\
\hline $.93-06$ & Yes \\
\hline & & No
\end{tabular}

\section{$\mathrm{s}$}

$S$

4

4

4

4

2

4

4
16

15

26

26
26

26
6

6
6

6
7

5

5
5

5
15

4

25

7
2

VINYL CHLORIDE

\begin{tabular}{|c|c|c|c|c|c|c|c|c|}
\hline $55-2 A$ & $\mathrm{~S}$ & 4 & 4 & $7.8 \mathrm{E}-03$ & $9.0 \mathrm{E}-03$ & No & NT & $\mathrm{s}$ \\
\hline $55-3 A$ & $\mathrm{~s}$ & 5 & 4 & $3.2 \mathrm{E}-02$ & 3.7E-02 & No & NT & $\mathrm{PI}$ \\
\hline
\end{tabular}


MAROS Statistical Trend Analysis Summary

\begin{tabular}{ccccccccc} 
& & Number & Number & Average & Median & All & Linear \\
Well & Sourcel & of & of & Conc. & Mann- & Conc. \\
(mgll & Samples & Detects & (mg/L) & "ND" ? & $\begin{array}{c}\text { Kendall } \\
\text { Trend }\end{array}$ & $\begin{array}{c}\text { Regression } \\
\text { Trend }\end{array}$ \\
\hline
\end{tabular}

VINYL CHLORIDE

\begin{tabular}{|c|c|}
\hline $55-3 B$ & $\mathrm{~s}$ \\
\hline $55-3 C$ & $S$ \\
\hline $56-1 A$ & $T$ \\
\hline $56-2 A$ & $\mathrm{~T}$ \\
\hline $56-2 B$ & $\mathrm{~T}$ \\
\hline $56-2 C$ & $\mathrm{~T}$ \\
\hline $56-3 A$ & $\mathrm{~T}$ \\
\hline $56-3 B$ & $\mathrm{~T}$ \\
\hline $56-3 C$ & $\mathrm{~T}$ \\
\hline $56-4 A$ & $\mathrm{~T}$ \\
\hline $56-6 \mathrm{~A}$ & $\mathrm{~T}$ \\
\hline $56-8 \mathrm{~A}$ & $\mathrm{~T}$ \\
\hline $60-1 \mathrm{~A}$ & $\mathrm{~T}$ \\
\hline GW-204 & $\mathrm{s}$ \\
\hline GW-219 & $\mathrm{s}$ \\
\hline GW-281 & $\mathrm{s}$ \\
\hline GW-605 & $\mathrm{S}$ \\
\hline GW-606 & $\mathrm{T}$ \\
\hline GW-656 & $\mathrm{s}$ \\
\hline GW-658 & $\mathrm{T}$ \\
\hline GW-686 & $\mathrm{T}$ \\
\hline GW-690 & $\mathrm{T}$ \\
\hline GW-691 & $\mathrm{s}$ \\
\hline GW-692 & $T$ \\
\hline GW-698 & $\mathrm{s}$ \\
\hline GW-700 & $\mathrm{T}$ \\
\hline GW-762 & $T$ \\
\hline GW-763 & $\mathrm{T}$ \\
\hline GW-765 & $\mathrm{T}$ \\
\hline GW-769 & $\mathrm{T}$ \\
\hline GW-770 & $\mathrm{T}$ \\
\hline GW-775 & $\mathrm{T}$ \\
\hline GW-776 & $\mathrm{T}$ \\
\hline GW-779 & $\mathrm{T}$ \\
\hline GW-781 & $\mathrm{T}$ \\
\hline GW-782 & $\mathrm{T}$ \\
\hline GW-783 & $\mathrm{T}$ \\
\hline GW-791 & $\mathrm{s}$ \\
\hline GW-792 & $\mathrm{T}$ \\
\hline GW-802 & $\mathrm{T}$ \\
\hline GW-820 & $\mathrm{S}$ \\
\hline GW-954-1 & $\mathrm{T}$ \\
\hline GW-954-2 & $\mathrm{T}$ \\
\hline GW-954-3 & $\mathrm{T}$ \\
\hline GW-956-1 & $\mathrm{T}$ \\
\hline GW-956-2 & $\mathrm{T}$ \\
\hline
\end{tabular}

\begin{tabular}{|c|c|c|}
\hline $2.5 \mathrm{E}-01$ & 3.2E-01 & No \\
\hline $1.8 \mathrm{E}-01$ & $1.7 \mathrm{E}-01$ & No \\
\hline $1.0 \mathrm{E}-03$ & $1.0 \mathrm{E}-03$ & Yes \\
\hline $1.0 \mathrm{E}-03$ & $1.0 \mathrm{E}-03$ & Yes \\
\hline $1.0 \mathrm{E}-03$ & $1.0 \mathrm{E}-03$ & Yes \\
\hline 2.6E-02 & 2.6E-02 & No \\
\hline $1.0 \mathrm{E}-03$ & $1.0 \mathrm{E}-03$ & Yes \\
\hline $1.0 \mathrm{E}-03$ & $1.0 \mathrm{E}-03$ & Yes \\
\hline $1.0 \mathrm{E}-03$ & $1.0 \mathrm{E}-03$ & Yes \\
\hline $1.0 \mathrm{E}-03$ & $1.0 \mathrm{E}-03$ & Yes \\
\hline 1.0E-03 & $1.0 \mathrm{E}-03$ & Yes \\
\hline $1.0 \mathrm{E}-03$ & $1.0 \mathrm{E}-03$ & Yes \\
\hline $1.0 \mathrm{E}-03$ & $1.0 \mathrm{E}-03$ & Yes \\
\hline $1.0 \mathrm{E}-03$ & $1.0 \mathrm{E}-03$ & Yes \\
\hline $1.0 \mathrm{E}-03$ & $1.0 \mathrm{E}-03$ & Yes \\
\hline $1.0 \mathrm{E}-03$ & $1.0 \mathrm{E}-03$ & Yes \\
\hline 1.3E-03 & $1.0 \mathrm{E}-03$ & No \\
\hline $1.0 \mathrm{E}-03$ & $1.0 \mathrm{E}-03$ & Yes \\
\hline 5.0E-03 & $5.0 \mathrm{E}-03$ & No \\
\hline $1.0 \mathrm{E}-03$ & $1.0 \mathrm{E}-03$ & Yes \\
\hline 2.3E-03 & $1.5 \mathrm{E}-03$ & No \\
\hline $1.0 \mathrm{E}-03$ & $1.0 \mathrm{E}-03$ & Yes \\
\hline $1.0 \mathrm{E}-03$ & $1.0 \mathrm{E}-03$ & Yes \\
\hline 1.0E-03 & $1.0 \mathrm{E}-03$ & Yes \\
\hline $1.0 \mathrm{E}-03$ & $1.0 \mathrm{E}-03$ & Yes \\
\hline 1.0E-03 & $1.0 \mathrm{E}-03$ & Yes \\
\hline 3.8E-03 & $5.0 \mathrm{E}-03$ & No \\
\hline 1.3E-03 & $1.0 \mathrm{E}-03$ & No \\
\hline $1.0 \mathrm{E}-03$ & $1.0 \mathrm{E}-03$ & Yes \\
\hline $1.0 \mathrm{E}-03$ & $1.0 \mathrm{E}-03$ & Yes \\
\hline $1.0 \mathrm{E}-03$ & $1.0 \mathrm{E}-03$ & Yes \\
\hline $1.0 \mathrm{E}-03$ & $1.0 \mathrm{E}-03$ & Yes \\
\hline $1.0 \mathrm{E}-03$ & $1.0 \mathrm{E}-03$ & Yes \\
\hline $1.0 \mathrm{E}-03$ & $1.0 \mathrm{E}-03$ & Yes \\
\hline 1.0E-03 & $1.0 \mathrm{E}-03$ & Yes \\
\hline 1.5E-03 & $1.0 \mathrm{E}-03$ & No \\
\hline 1.0E-03 & $1.0 \mathrm{E}-03$ & Yes \\
\hline 1.0E-03 & $1.0 \mathrm{E}-03$ & Yes \\
\hline $1.0 \mathrm{E}-03$ & $1.0 \mathrm{E}-03$ & Yes \\
\hline $1.0 \mathrm{E}-03$ & $1.0 \mathrm{E}-03$ & Yes \\
\hline 7.6E-02 & 7.6E-02 & No \\
\hline $1.0 \mathrm{E}-03$ & $1.0 \mathrm{E}-03$ & Yes \\
\hline 1.0E-03 & $1.0 \mathrm{E}-03$ & Yes \\
\hline $1.0 \mathrm{E}-03$ & $1.0 \mathrm{E}-03$ & No \\
\hline $1.0 \mathrm{E}-03$ & $1.0 \mathrm{E}-03$ & Yes \\
\hline $1.0 \mathrm{E}-03$ & $1.0 \mathrm{E}-03$ & Yes \\
\hline
\end{tabular}

I

NT NT

ND ND

ND ND

ND ND

N/A N/A

ND ND

ND ND

ND ND

ND ND

ND ND

ND ND

ND ND

ND ND

ND ND

ND ND

NT NT

ND ND

N/A N/A

ND ND

NT NT

ND ND

ND ND

ND ND

ND ND

ND ND

$S \quad S$

$S-S$

ND ND

ND ND

ND ND

ND ND

ND ND

ND ND

ND ND

$S \quad S$

ND ND

ND ND

ND ND

ND ND

NT NT

ND ND

ND ND

$S \quad S$

ND ND

ND ND 


\section{MAROS Statistical Trend Analysis Summary}

\begin{tabular}{|c|c|c|c|c|c|c|c|c|}
\hline Well & $\begin{array}{c}\text { Sourcel } \\
\text { Tail }\end{array}$ & $\begin{array}{l}\text { Number } \\
\text { of } \\
\text { Samples }\end{array}$ & $\begin{array}{l}\text { Number } \\
\text { of } \\
\text { Detects }\end{array}$ & $\begin{array}{l}\text { Average } \\
\text { Conc. } \\
\text { (mg/L) }\end{array}$ & $\begin{array}{l}\text { Median } \\
\text { Conc. } \\
\text { (mg/L) }\end{array}$ & $\begin{array}{c}\text { All } \\
\text { Samples } \\
\text { "ND" ? }\end{array}$ & $\begin{array}{l}\text { Mann- } \\
\text { Kendall } \\
\text { Trend }\end{array}$ & $\begin{array}{l}\text { Linear } \\
\text { Regression } \\
\text { Trend }\end{array}$ \\
\hline \multicolumn{9}{|l|}{ VINYL CHLORIDE } \\
\hline GW-956-3 & $\mathrm{T}$ & 4 & 0 & 1.0E-03 & $1.0 \mathrm{E}-03$ & Yes & ND & ND \\
\hline GW-956-4 & $\mathrm{T}$ & 4 & 0 & 1.0E-03 & 1.0E-03 & Yes & ND & ND \\
\hline GW-959 & $\mathrm{T}$ & 7 & 5 & 2.1E-02 & $1.0 \mathrm{E}-02$ & No & NT & PD \\
\hline GW-960 & $\mathrm{T}$ & 2 & 0 & 1.0E-03 & $1.0 \mathrm{E}-03$ & Yes & ND & ND \\
\hline UEFPC-SP17 & $\mathrm{T}$ & 1 & 0 & 1.0E-03 & 1.0E-03 & Yes & ND & ND \\
\hline
\end{tabular}

Note: Increasing (I); Probably Increasing (PI); Stable (S); Probably Decreasing (PD); Decreasing (D); No Trend (NT); Not Applicable (N/A); Not Applicable (N/A) - Due to insufficient Data (< 4 sampling events); No Detectable Concentration (NDC)

The Number of Samples and Number of Detects shown above are post-consolidation values. 


\section{MAROS Statistical Trend Analysis Summary}

Project: $\quad$ EF Central and Fuel

Location: Central Y-12

Time Period: 1/1/1996

to $10 / 15 / 2007$

Consolidation Period: No Time Consolidation

Consolidation Type: Median

Duplicate Consolidation: Average

ND Values: Specified Detection Limit

J Flag Values : Actual Value
User Name: $\quad$ MV

State: Tennessee

\begin{tabular}{ccccccccc} 
& & Number & Number & Average & Median & All & Mann- & Linear \\
of & Sourcel & $\begin{array}{c}\text { of } \\
\text { Wall }\end{array}$ & $\begin{array}{c}\text { Samples } \\
\text { Tetects }\end{array}$ & $\begin{array}{c}\text { Conc. } \\
(\mathrm{mg} / \mathrm{L})\end{array}$ & $\begin{array}{c}\text { Samples } \\
(\mathrm{mg} / \mathrm{L})\end{array}$ & "ND" ? & $\begin{array}{c}\text { Kendall } \\
\text { Trend }\end{array}$ & $\begin{array}{c}\text { Regression } \\
\text { Trend }\end{array}$ \\
\hline
\end{tabular}

1,1-DICHLOROETHENE

\begin{tabular}{|c|c|}
\hline $55-2 A$ & S \\
\hline $55-3 \mathrm{~A}$ & S \\
\hline $55-3 B$ & S \\
\hline $55-3 C$ & S \\
\hline $56-1 \mathrm{~A}$ & $\mathrm{~T}$ \\
\hline $56-2 A$ & $\mathrm{~T}$ \\
\hline $56-2 B$ & $\mathrm{~T}$ \\
\hline $56-2 C$ & $\mathrm{~T}$ \\
\hline $56-3 A$ & $T$ \\
\hline $56-3 B$ & $\mathrm{~T}$ \\
\hline $56-3 C$ & $\mathrm{~T}$ \\
\hline $56-4 A$ & $\mathrm{~T}$ \\
\hline $56-6 A$ & $\mathrm{~T}$ \\
\hline $56-8 A$ & $\mathrm{~T}$ \\
\hline $60-1 A$ & $\mathrm{~T}$ \\
\hline GW-204 & $\mathrm{s}$ \\
\hline GW-219 & $\mathrm{s}$ \\
\hline GW-281 & $\mathrm{s}$ \\
\hline GW-605 & $\mathrm{s}$ \\
\hline GW-606 & $\mathrm{T}$ \\
\hline GW-656 & $\mathrm{s}$ \\
\hline GW-658 & $\mathrm{T}$ \\
\hline GW-686 & $\mathrm{T}$ \\
\hline GW-690 & $\mathrm{T}$ \\
\hline GW-691 & $\mathrm{s}$ \\
\hline GW-692 & $\mathrm{T}$ \\
\hline GW-698 & $\mathrm{S}$ \\
\hline GW-700 & $\mathrm{T}$ \\
\hline GW-762 & $\mathrm{T}$ \\
\hline GW-763 & $\mathrm{T}$ \\
\hline GW-765 & $\mathrm{T}$ \\
\hline GW-769 & $\mathrm{T}$ \\
\hline GW-770 & $\mathrm{T}$ \\
\hline GW-775 & $\mathrm{T}$ \\
\hline GW-776 & $\mathrm{T}$ \\
\hline
\end{tabular}

$\begin{array}{lll}8.3 \mathrm{E}-03 & 8.0 \mathrm{E}-03 & \text { No } \\ 1.8 \mathrm{E}-02 & 2.3 \mathrm{E}-02 & \text { No } \\ 1.2 \mathrm{E}-01 & 1.6 \mathrm{E}-01 & \text { No } \\ 4.2 \mathrm{E}-02 & 3.9 \mathrm{E}-02 & \text { No } \\ 3.0 \mathrm{E}-03 & 3.0 \mathrm{E}-03 & \text { Yes } \\ 3.0 \mathrm{E}-03 & 3.0 \mathrm{E}-03 & \text { Yes } \\ 2.0 \mathrm{E}-03 & 2.0 \mathrm{E}-03 & \text { No } \\ 1.5 \mathrm{E}-02 & 1.5 \mathrm{E}-02 & \text { No } \\ 3.0 \mathrm{E}-03 & 3.0 \mathrm{E}-03 & \text { Yes } \\ 3.0 \mathrm{E}-03 & 3.0 \mathrm{E}-03 & \text { Yes } \\ 3.0 \mathrm{E}-03 & 3.0 \mathrm{E}-03 & \text { Yes } \\ 3.0 \mathrm{E}-03 & 3.0 \mathrm{E}-03 & \text { Yes } \\ 3.0 \mathrm{E}-03 & 3.0 \mathrm{E}-03 & \text { Yes } \\ 3.0 \mathrm{E}-03 & 3.0 \mathrm{E}-03 & \text { Yes } \\ 3.0 \mathrm{E}-03 & 3.0 \mathrm{E}-03 & \text { Yes } \\ 3.0 \mathrm{E}-03 & 3.0 \mathrm{E}-03 & \text { Yes } \\ 3.0 \mathrm{E}-03 & 3.0 \mathrm{E}-03 & \text { Yes } \\ 3.0 \mathrm{E}-03 & 3.0 \mathrm{E}-03 & \text { Yes } \\ 2.8 \mathrm{E}-03 & 3.0 \mathrm{E}-03 & \text { No } \\ 3.0 \mathrm{E}-03 & 3.0 \mathrm{E}-03 & \text { Yes } \\ 1.9 \mathrm{E}-01 & 1.9 \mathrm{E}-01 & \text { No } \\ 3.0 \mathrm{E}-03 & 3.0 \mathrm{E}-03 & \text { Yes } \\ 3.0 \mathrm{E}-03 & 3.0 \mathrm{E}-03 & \text { Yes } \\ 3.0 \mathrm{E}-03 & 3.0 \mathrm{E}-03 & \text { Yes } \\ 3.0 \mathrm{E}-03 & 3.0 \mathrm{E}-03 & \text { Yes } \\ 3.0 \mathrm{E}-03 & 3.0 \mathrm{E}-03 & \text { Yes } \\ 3.0 \mathrm{E}-03 & 3.0 \mathrm{E}-03 & \text { Yes } \\ 3.0 \mathrm{E}-03 & 3.0 \mathrm{E}-03 & \text { Yes } \\ 5.6 \mathrm{E}-02 & 6.2 \mathrm{E}-02 & \text { No } \\ 2.5 \mathrm{E}-03 & 3.0 \mathrm{E}-03 & \text { No } \\ 3.0 \mathrm{E}-03 & 3.0 \mathrm{E}-03 & \text { Yes } \\ 1.5 \mathrm{E}-03 & 1.5 \mathrm{E}-03 & \text { No } \\ 3.0 \mathrm{E}-03 & 3.0 \mathrm{E}-03 & \text { Yes } \\ 3.0 \mathrm{E}-03 & 3.0 \mathrm{E}-03 & \text { Yes } \\ 3.0 \mathrm{E}-03 & 3.0 \mathrm{E}-03 & \text { Yes } \\ & & \end{array}$

NT

ND

ND

N/A

N/A

ND

ND

ND

ND

ND

ND

ND

ND

ND

ND

$\mathrm{S}$

ND

N/A

ND

ND

ND

ND

ND

ND

ND

$\mathrm{S}$

NT

ND

NT

ND

ND

ND

\section{$\mathrm{S}$}

P

PI

NT

ND

ND

N/A

N/A

ND

ND

ND

ND

ND

ND

ND

ND

ND

ND

$S$

ND

N/A

ND

ND

ND

ND

ND

ND

ND

PD

NT

ND

NT

ND

ND

ND 
MAROS Statistical Trend Analysis Summary

\begin{tabular}{ccccccccc} 
& & Number & Number & Average & Median & All & Mann- & Linear \\
Well & Sourcel & of & of & Conc. & Conc. \\
Tail & Samples & Detects & (mg/L) & (mg/L) & "ND" ? & $\begin{array}{c}\text { Kendall } \\
\text { Trend }\end{array}$ & $\begin{array}{c}\text { Regression } \\
\text { Trend }\end{array}$ \\
\hline
\end{tabular}

1,1-DICHLOROETHENE

GW-779

GW-781

GW-782

GW-783

GW-791

GW-792

GW-802

GW-820

GW-954-1

GW-954-2

GW-954-3

GW-956-1

GW-956-2

GW-956-3

GW-956-4

GW-959

GW-960

UEFPC-SP17

$\begin{array}{ll}\mathrm{T} & 2 \\ \mathrm{~T} & 2 \\ \mathrm{~T} & 4 \\ \mathrm{~T} & 2 \\ \mathrm{~S} & 4 \\ \mathrm{~T} & 2 \\ \mathrm{~T} & 3 \\ \mathrm{~S} & 4 \\ \mathrm{~T} & 4 \\ \mathrm{~T} & 4 \\ \mathrm{~T} & 4 \\ \mathrm{~T} & 4 \\ \mathrm{~T} & 4 \\ \mathrm{~T} & 4 \\ \mathrm{~T} & 4 \\ \mathrm{~T} & 7 \\ \mathrm{~T} & 2 \\ \mathrm{~T} & 1\end{array}$

$\begin{array}{lll}3.0 \mathrm{E}-03 & 3.0 \mathrm{E}-03 & \text { Yes } \\ 3.0 \mathrm{E}-03 & 3.0 \mathrm{E}-03 & \text { Yes } \\ 4.8 \mathrm{E}-02 & 5.0 \mathrm{E}-02 & \text { No } \\ 4.0 \mathrm{E}-03 & 4.0 \mathrm{E}-03 & \text { No } \\ 3.0 \mathrm{E}-03 & 3.0 \mathrm{E}-03 & \text { Yes } \\ 3.0 \mathrm{E}-03 & 3.0 \mathrm{E}-03 & \text { Yes } \\ 3.0 \mathrm{E}-03 & 3.0 \mathrm{E}-03 & \text { Yes } \\ 5.0 \mathrm{E}-03 & 5.0 \mathrm{E}-03 & \text { No } \\ 3.0 \mathrm{E}-03 & 3.0 \mathrm{E}-03 & \text { Yes } \\ 3.0 \mathrm{E}-03 & 3.0 \mathrm{E}-03 & \text { Yes } \\ 2.8 \mathrm{E}-03 & 2.5 \mathrm{E}-03 & \text { No } \\ 3.0 \mathrm{E}-03 & 3.0 \mathrm{E}-03 & \text { Yes } \\ 3.0 \mathrm{E}-03 & 3.0 \mathrm{E}-03 & \text { Yes } \\ 3.0 \mathrm{E}-03 & 3.0 \mathrm{E}-03 & \text { Yes } \\ 3.0 \mathrm{E}-03 & 3.0 \mathrm{E}-03 & \text { Yes } \\ 3.0 \mathrm{E}-03 & 3.0 \mathrm{E}-03 & \text { Yes } \\ 3.0 \mathrm{E}-03 & 3.0 \mathrm{E}-03 & \text { Yes } \\ 3.0 \mathrm{E}-03 & 3.0 \mathrm{E}-03 & \text { Yes }\end{array}$

$\begin{array}{cc}N D & N D \\ N D & N D \\ S & S \\ N / A & N / A \\ N D & N D \\ N D & N D \\ N D & N D \\ S & S \\ N D & N D \\ N D & N D \\ S & S \\ N D & N D \\ N D & N D \\ N D & N D \\ N D & N D \\ N D & N D \\ N D & N D \\ N D & N D\end{array}$

BENZENE

\begin{tabular}{|c|c|c|c|c|c|c|c|c|}
\hline $55-2 A$ & S & 4 & 0 & $5.0 \mathrm{E}-04$ & $5.0 \mathrm{E}-04$ & Yes & ND & ND \\
\hline $55-3 A$ & S & 5 & 0 & $5.0 \mathrm{E}-04$ & $5.0 \mathrm{E}-04$ & Yes & ND & ND \\
\hline $55-3 B$ & S & 5 & 4 & $1.5 \mathrm{E}-03$ & 2.0E-03 & No & NT & NT \\
\hline $55-3 C$ & S & 4 & 0 & $5.0 \mathrm{E}-04$ & $5.0 \mathrm{E}-04$ & Yes & ND & ND \\
\hline $56-1 \mathrm{~A}$ & $\mathrm{~T}$ & 4 & 0 & 5.0E-04 & 5.0E-04 & Yes & ND & ND \\
\hline $56-2 A$ & T & 5 & 0 & $5.0 \mathrm{E}-04$ & $5.0 \mathrm{E}-04$ & Yes & ND & ND \\
\hline $56-2 B$ & $\mathrm{~T}$ & 5 & 0 & 5.0E-04 & 5.0E-04 & Yes & ND & ND \\
\hline $56-2 C$ & $\mathrm{~T}$ & 7 & 0 & 5.0E-04 & 5.0E-04 & Yes & ND & ND \\
\hline $56-3 A$ & $\mathrm{~T}$ & 4 & 0 & 5.0E-04 & 5.0E-04 & Yes & ND & ND \\
\hline $56-3 B$ & $\mathrm{~T}$ & 4 & 0 & $5.0 \mathrm{E}-04$ & 5.0E-04 & Yes & ND & ND \\
\hline $56-3 C$ & $\mathrm{~T}$ & 4 & 0 & $5.0 \mathrm{E}-04$ & 5.0E-04 & Yes & ND & ND \\
\hline $56-4 A$ & $\mathrm{~T}$ & 4 & 0 & $5.0 \mathrm{E}-04$ & 5.0E-04 & Yes & ND & ND \\
\hline $56-6 A$ & $\mathrm{~T}$ & 4 & 0 & $5.0 \mathrm{E}-04$ & 5.0E-04 & Yes & ND & ND \\
\hline $56-8 A$ & $\mathrm{~T}$ & 2 & 0 & $5.0 \mathrm{E}-04$ & 5.0E-04 & Yes & ND & ND \\
\hline $60-1 \mathrm{~A}$ & $\mathrm{~T}$ & 4 & 0 & $5.0 \mathrm{E}-04$ & 5.0E-04 & Yes & ND & ND \\
\hline GW-204 & $\mathrm{S}$ & 16 & 0 & $5.0 \mathrm{E}-04$ & 5.0E-04 & Yes & ND & ND \\
\hline GW-219 & $\mathrm{S}$ & 15 & 0 & $5.0 \mathrm{E}-04$ & 5.0E-04 & Yes & ND & ND \\
\hline GW-281 & $\mathrm{S}$ & 5 & 0 & $5.0 \mathrm{E}-04$ & 5.0E-04 & Yes & ND & ND \\
\hline GW-605 & $\mathrm{S}$ & 26 & 0 & $5.0 \mathrm{E}-04$ & 5.0E-04 & Yes & ND & ND \\
\hline GW-606 & $\mathrm{T}$ & 26 & 5 & $7.9 \mathrm{E}-04$ & 5.0E-04 & No & S & $\mathrm{S}$ \\
\hline GW-656 & $\mathrm{S}$ & 6 & 2 & $1.0 \mathrm{E}-03$ & 5.0E-04 & No & $S$ & $\mathrm{~S}$ \\
\hline GW-658 & $\mathrm{T}$ & 9 & 8 & $8.0 \mathrm{E}+00$ & $8.1 \mathrm{E}+00$ & No & NT & $\mathrm{PI}$ \\
\hline GW-686 & $\mathrm{T}$ & 6 & 0 & $5.0 \mathrm{E}-04$ & 5.0E-04 & Yes & ND & ND \\
\hline GW-690 & $\mathrm{T}$ & 7 & 0 & $5.0 \mathrm{E}-04$ & $5.0 \mathrm{E}-04$ & Yes & ND & ND \\
\hline GW-691 & $\mathrm{S}$ & 5 & 0 & $5.0 \mathrm{E}-04$ & 5.0E-04 & Yes & ND & ND \\
\hline GW-692 & $\mathrm{T}$ & 5 & 0 & 5.0E-04 & 5.0E-04 & Yes & ND & ND \\
\hline
\end{tabular}


MAROS Statistical Trend Analysis Summary

\begin{tabular}{ccccccccc} 
& & Number & Number & Average & Median & All & Mann- & Linear \\
Well & Sourcel & of & of & Conc. & Conc. \\
Tail & Samples & Detects & (mg/L) & (mg/L) & "ND" ? & $\begin{array}{c}\text { Kendall } \\
\text { Trend }\end{array}$ & $\begin{array}{c}\text { Regression } \\
\text { Trend }\end{array}$ \\
\hline
\end{tabular}

BENZENE

\section{GW-698}

GW-700

GW-762

GW-763

GW-765

GW-769

GW-770

GW-775

GW-776

GW-779

GW-781

GW-782

GW-783

GW-791

GW-792

GW-802

GW-820

GW-954-1

GW-954-2

GW-954-3

GW-956-1

GW-956-2

GW-956-3

GW-956-4

GW-959

GW-960

UEFPC-SP17

$\begin{array}{lll}5.0 \mathrm{E}-04 & 5.0 \mathrm{E}-04 & \text { Yes } \\ 5.0 \mathrm{E}-04 & 5.0 \mathrm{E}-04 & \text { Yes } \\ 5.0 \mathrm{E}-04 & 5.0 \mathrm{E}-04 & \text { Yes } \\ 5.0 \mathrm{E}-04 & 5.0 \mathrm{E}-04 & \text { Yes } \\ 5.0 \mathrm{E}-04 & 5.0 \mathrm{E}-04 & \text { Yes } \\ 5.0 \mathrm{E}-04 & 5.0 \mathrm{E}-04 & \text { Yes } \\ 5.0 \mathrm{E}-04 & 5.0 \mathrm{E}-04 & \text { Yes } \\ 5.0 \mathrm{E}-04 & 5.0 \mathrm{E}-04 & \text { Yes } \\ 5.0 \mathrm{E}-04 & 5.0 \mathrm{E}-04 & \text { Yes } \\ 5.0 \mathrm{E}-04 & 5.0 \mathrm{E}-04 & \text { Yes } \\ 5.0 \mathrm{E}-04 & 5.0 \mathrm{E}-04 & \text { Yes } \\ 6.3 \mathrm{E}-04 & 5.0 \mathrm{E}-04 & \text { No } \\ 5.0 \mathrm{E}-04 & 5.0 \mathrm{E}-04 & \text { Yes } \\ 5.0 \mathrm{E}-04 & 5.0 \mathrm{E}-04 & \text { Yes } \\ 6.4 \mathrm{E}-04 & 5.0 \mathrm{E}-04 & \text { No } \\ 5.0 \mathrm{E}-04 & 5.0 \mathrm{E}-04 & \text { Yes } \\ 5.0 \mathrm{E}-04 & 5.0 \mathrm{E}-04 & \text { Yes } \\ 5.0 \mathrm{E}-04 & 5.0 \mathrm{E}-04 & \text { Yes } \\ 5.0 \mathrm{E}-04 & 5.0 \mathrm{E}-04 & \text { Yes } \\ 5.0 \mathrm{E}-04 & 5.0 \mathrm{E}-04 & \text { Yes } \\ 5.0 \mathrm{E}-04 & 5.0 \mathrm{E}-04 & \text { Yes } \\ 5.0 \mathrm{E}-04 & 5.0 \mathrm{E}-04 & \text { Yes } \\ 5.0 \mathrm{E}-04 & 5.0 \mathrm{E}-04 & \text { Yes } \\ 5.0 \mathrm{E}-04 & 5.0 \mathrm{E}-04 & \text { Yes } \\ 5.0 \mathrm{E}-04 & 5.0 \mathrm{E}-04 & \text { Yes } \\ 5.0 \mathrm{E}-04 & 5.0 \mathrm{E}-04 & \text { Yes } \\ 5.0 \mathrm{E}-04 & 5.0 \mathrm{E}-04 & \text { Yes }\end{array}$

\begin{tabular}{|c|c|}
\hline ND & ND \\
\hline ND & ND \\
\hline ND & ND \\
\hline ND & ND \\
\hline ND & ND \\
\hline ND & ND \\
\hline ND & ND \\
\hline ND & ND \\
\hline ND & ND \\
\hline ND & ND \\
\hline ND & ND \\
\hline S & $S$ \\
\hline ND & $\mathrm{ND}$ \\
\hline ND & ND \\
\hline $\mathrm{S}$ & $\mathrm{S}$ \\
\hline ND & ND \\
\hline ND & $\mathrm{NL}$ \\
\hline ND & $\mathrm{NL}$ \\
\hline ND & $\mathrm{NL}$ \\
\hline ND & $\mathrm{NL}$ \\
\hline ND & $\mathrm{NL}$ \\
\hline ND & $\mathrm{NL}$ \\
\hline ND & ND \\
\hline ND & $\mathrm{NL}$ \\
\hline ND & ND \\
\hline ND & $\mathrm{N}$ \\
\hline ND & $\mathrm{N}$ \\
\hline
\end{tabular}

cis-1,2-DICHLOROETHYLENE

\begin{tabular}{|c|c|c|c|c|c|c|c|c|}
\hline $55-2 A$ & $S$ & 4 & 4 & $4.1 \mathrm{E}-01$ & 4.2E-01 & No & $\mathrm{S}$ & $S$ \\
\hline $55-3 A$ & $S$ & 5 & 5 & $9.9 \mathrm{E}-01$ & $1.0 \mathrm{E}+00$ & No & NT & NT \\
\hline 55-3B & $S$ & 5 & 5 & $1.4 \mathrm{E}+00$ & $1.3 \mathrm{E}+00$ & No & $\mathrm{PI}$ & $\mathrm{PI}$ \\
\hline $55-3 C$ & $S$ & 4 & 4 & $1.9 \mathrm{E}+00$ & $2.0 \mathrm{E}+00$ & No & $\mathrm{S}$ & $\mathrm{S}$ \\
\hline $56-1 A$ & $\mathrm{~T}$ & 4 & 0 & $5.0 \mathrm{E}-03$ & $5.0 \mathrm{E}-03$ & Yes & ND & ND \\
\hline $56-2 A$ & $\mathrm{~T}$ & 2 & 1 & $6.0 \mathrm{E}-03$ & $6.0 \mathrm{E}-03$ & No & $\mathrm{N} / \mathrm{A}$ & N/A \\
\hline $56-2 B$ & $\mathrm{~T}$ & 2 & 2 & 7.0E-02 & 7.0E-02 & No & N/A & $\mathrm{N} / \mathrm{A}$ \\
\hline $56-2 C$ & $\mathrm{~T}$ & 2 & 2 & 8.3E-01 & 8.3E-01 & No & N/A & N/A \\
\hline $56-3 A$ & $\mathrm{~T}$ & 4 & 4 & 8.3E-03 & $8.0 \mathrm{E}-03$ & No & $\mathrm{S}$ & $S$ \\
\hline 56-3B & $T$ & 4 & 4 & 2.7E-02 & 2.7E-02 & No & NT & NT \\
\hline $56-3 C$ & $\mathrm{~T}$ & 4 & 4 & 6.7E-02 & $6.8 \mathrm{E}-02$ & No & NT & I \\
\hline $56-4 A$ & $\mathrm{~T}$ & 4 & 0 & $5.0 \mathrm{E}-03$ & $5.0 \mathrm{E}-03$ & Yes & ND & ND \\
\hline $56-6 A$ & $\mathrm{~T}$ & 4 & 0 & $5.0 \mathrm{E}-03$ & $5.0 \mathrm{E}-03$ & Yes & ND & ND \\
\hline $56-8 A$ & $\mathrm{~T}$ & 2 & 2 & 2.2E-02 & $2.2 \mathrm{E}-02$ & No & $\mathrm{N} / \mathrm{A}$ & N/A \\
\hline $60-1 \mathrm{~A}$ & $\mathrm{~T}$ & 4 & 0 & $5.0 \mathrm{E}-03$ & $5.0 \mathrm{E}-03$ & Yes & ND & ND \\
\hline GW-204 & $S$ & 4 & 0 & $5.0 \mathrm{E}-03$ & $5.0 \mathrm{E}-03$ & Yes & ND & ND \\
\hline GW-219 & $S$ & 3 & 0 & $5.0 \mathrm{E}-03$ & $5.0 \mathrm{E}-03$ & Yes & ND & ND \\
\hline
\end{tabular}


MAROS Statistical Trend Analysis Summary

\begin{tabular}{ccccccccc} 
& & Number & Number & Average & Median & All & Mann- & Linear \\
Well & Sourcel & of & of & Conc. & Conc. & Samples & Kendall & Regression \\
Tail & Samples & Detects & (mg/L) & (mg/L) & "ND" ? & Trend & Trend \\
\hline
\end{tabular}

cis-1,2-DICHLOROETHYLENE

\begin{tabular}{|c|c|c|c|c|c|c|c|c|}
\hline GW-281 & $\mathrm{s}$ & 3 & 0 & $5.0 \mathrm{E}-03$ & $5.0 \mathrm{E}-03$ & Yes & ND & ND \\
\hline GW-605 & $S$ & 6 & 6 & $1.2 \mathrm{E}-01$ & $9.7 \mathrm{E}-02$ & No & I & I \\
\hline GW-606 & $T$ & 6 & 0 & $5.0 \mathrm{E}-03$ & $5.0 \mathrm{E}-03$ & Yes & ND & ND \\
\hline GW-656 & $\mathrm{S}$ & 2 & 2 & $1.5 \mathrm{E}-01$ & $1.5 \mathrm{E}-01$ & No & N/A & $N / A$ \\
\hline GW-658 & $\mathrm{T}$ & 3 & 0 & $5.0 \mathrm{E}-03$ & $5.0 \mathrm{E}-03$ & Yes & ND & $N D$ \\
\hline GW-686 & $\mathrm{T}$ & 4 & 4 & 2.2E-02 & 2.2E-02 & No & $\mathrm{s}$ & NT \\
\hline GW-690 & $\mathrm{T}$ & 2 & 2 & $6.5 \mathrm{E}-03$ & $6.5 \mathrm{E}-03$ & No & N/A & $N / A$ \\
\hline GW-691 & $\mathrm{s}$ & 2 & 2 & 1.4E-02 & $1.5 \mathrm{E}-02$ & No & $\mathrm{N} / \mathrm{A}$ & $\mathrm{N} / \mathrm{A}$ \\
\hline GW-692 & $\mathrm{T}$ & 2 & 2 & $2.9 \mathrm{E}-02$ & $2.9 \mathrm{E}-02$ & No & $\mathrm{N} / \mathrm{A}$ & $\mathrm{N} / \mathrm{A}$ \\
\hline GW-698 & $\mathrm{S}$ & 6 & 6 & 3.1E-02 & $3.4 \mathrm{E}-02$ & No & NT & NT \\
\hline GW-700 & $\mathrm{T}$ & 2 & 2 & 4.3E-02 & 4.3E-02 & No & $\mathrm{N} / \mathrm{A}$ & $\mathrm{N} / \mathrm{A}$ \\
\hline GW-762 & $\mathrm{T}$ & 6 & 6 & 5.7E-02 & 6.6E-02 & No & $S$ & PD \\
\hline GW-763 & $\mathrm{T}$ & 4 & 3 & $1.4 \mathrm{E}-02$ & $1.1 \mathrm{E}-02$ & No & $\mathrm{S}$ & PD \\
\hline GW-765 & $\mathrm{T}$ & 1 & 0 & $5.0 \mathrm{E}-03$ & $5.0 \mathrm{E}-03$ & Yes & ND & ND \\
\hline GW-769 & $\mathrm{T}$ & 6 & 6 & $3.2 \mathrm{E}-03$ & $3.0 \mathrm{E}-03$ & No & NT & PI \\
\hline GW-770 & $\mathrm{T}$ & 6 & 0 & $5.0 \mathrm{E}-03$ & $5.0 \mathrm{E}-03$ & Yes & ND & ND \\
\hline GW-775 & $\mathrm{T}$ & 1 & 0 & $5.0 \mathrm{E}-03$ & $5.0 \mathrm{E}-03$ & Yes & ND & ND \\
\hline GW-776 & $\mathrm{T}$ & 1 & 0 & $5.0 \mathrm{E}-03$ & $5.0 \mathrm{E}-03$ & Yes & ND & ND \\
\hline GW-779 & $\mathrm{T}$ & 2 & 0 & $5.0 \mathrm{E}-03$ & $5.0 \mathrm{E}-03$ & Yes & ND & ND \\
\hline GW-781 & $\mathrm{T}$ & 2 & 0 & $5.0 \mathrm{E}-03$ & $5.0 \mathrm{E}-03$ & Yes & ND & ND \\
\hline GW-782 & $\mathrm{T}$ & 4 & 4 & $1.8 \mathrm{E}-02$ & $1.7 \mathrm{E}-02$ & No & NT & NT \\
\hline GW-783 & $\mathrm{T}$ & 2 & 2 & $5.0 \mathrm{E}-03$ & $5.0 \mathrm{E}-03$ & No & N/A & $\mathrm{N} / \mathrm{A}$ \\
\hline GW-791 & $\mathrm{s}$ & 4 & 1 & 4.0E-03 & $5.0 \mathrm{E}-03$ & No & NT & NT \\
\hline GW-792 & $\mathrm{T}$ & 2 & 0 & $5.0 \mathrm{E}-03$ & $5.0 \mathrm{E}-03$ & Yes & ND & ND \\
\hline GW-802 & $\mathrm{T}$ & 3 & 0 & $5.0 \mathrm{E}-03$ & $5.0 \mathrm{E}-03$ & Yes & ND & ND \\
\hline GW-820 & $\mathrm{S}$ & 4 & 4 & $1.0 \mathrm{E}+00$ & $1.0 \mathrm{E}+00$ & No & $S$ & NT \\
\hline GW-954-1 & $\mathrm{T}$ & 4 & 0 & $5.0 \mathrm{E}-03$ & $5.0 \mathrm{E}-03$ & Yes & ND & ND \\
\hline GW-954-2 & $\mathrm{T}$ & 4 & 0 & $5.0 \mathrm{E}-03$ & $5.0 \mathrm{E}-03$ & Yes & ND & ND \\
\hline GW-954-3 & $\mathrm{T}$ & 4 & 0 & $5.0 \mathrm{E}-03$ & $5.0 \mathrm{E}-03$ & Yes & ND & ND \\
\hline GW-956-1 & $\mathrm{T}$ & 4 & 0 & $5.0 \mathrm{E}-03$ & $5.0 \mathrm{E}-03$ & Yes & ND & ND \\
\hline GW-956-2 & $\mathrm{T}$ & 4 & 0 & $5.0 \mathrm{E}-03$ & $5.0 \mathrm{E}-03$ & Yes & ND & ND \\
\hline GW-956-3 & $\mathrm{T}$ & 4 & 0 & $5.0 \mathrm{E}-03$ & $5.0 \mathrm{E}-03$ & Yes & ND & ND \\
\hline GW-956-4 & $\mathrm{T}$ & 4 & 0 & $5.0 \mathrm{E}-03$ & $5.0 \mathrm{E}-03$ & Yes & ND & ND \\
\hline GW-959 & $\mathrm{T}$ & 7 & 7 & $9.7 \mathrm{E}-02$ & $6.1 \mathrm{E}-02$ & No & PD & D \\
\hline GW-960 & $\mathrm{T}$ & 2 & 0 & $5.0 \mathrm{E}-03$ & $5.0 \mathrm{E}-03$ & Yes & ND & ND \\
\hline UEFPC-SP17 & $\mathrm{T}$ & 1 & 0 & $5.0 \mathrm{E}-03$ & $5.0 \mathrm{E}-03$ & Yes & ND & ND \\
\hline
\end{tabular}

GROSS ALPHA ACTIVITY

\begin{tabular}{|c|c|c|c|c|c|c|c|c|}
\hline $55-2 A$ & $\mathrm{~S}$ & 4 & 0 & $1.0 \mathrm{E}-02$ & $1.0 \mathrm{E}-02$ & Yes & ND & ND \\
\hline $55-3 A$ & $S$ & 5 & 0 & $1.0 \mathrm{E}-02$ & $1.0 \mathrm{E}-02$ & Yes & ND & ND \\
\hline $55-3 B$ & $S$ & 5 & 0 & $1.0 \mathrm{E}-02$ & $1.0 \mathrm{E}-02$ & Yes & ND & ND \\
\hline $55-3 C$ & $\mathrm{~S}$ & 4 & 1 & $1.5 \mathrm{E}+00$ & $1.0 \mathrm{E}-02$ & No & NT & NT \\
\hline $56-1 A$ & $\mathrm{~T}$ & 4 & 0 & $1.0 \mathrm{E}-02$ & $1.0 \mathrm{E}-02$ & Yes & ND & ND \\
\hline $56-2 A$ & $\mathrm{~T}$ & 4 & 2 & $6.0 \mathrm{E}-01$ & $5.1 \mathrm{E}-01$ & No & NT & D \\
\hline $56-2 B$ & $\mathrm{~T}$ & 4 & 3 & $9.0 \mathrm{E}-01$ & $1.1 \mathrm{E}+00$ & No & S & $\mathrm{S}$ \\
\hline $56-2 C$ & $\mathrm{~T}$ & 6 & 5 & $1.9 \mathrm{E}+00$ & $1.6 \mathrm{E}+00$ & No & NT & $\mathrm{S}$ \\
\hline
\end{tabular}


MAROS Statistical Trend Analysis Summary

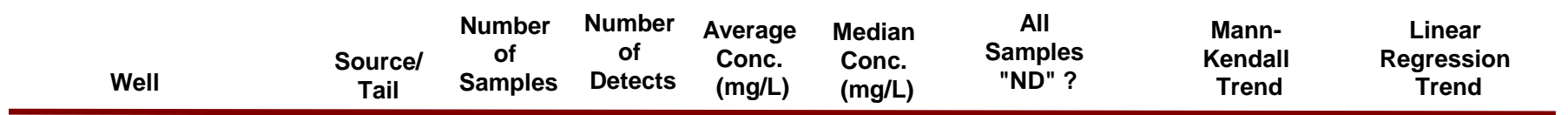

GROSS ALPHA ACTIVITY

\begin{tabular}{|c|c|}
\hline $56-3 A$ & $\mathrm{~T}$ \\
\hline $56-3 B$ & $\mathrm{~T}$ \\
\hline $56-3 C$ & $\mathrm{~T}$ \\
\hline $56-4 A$ & $\mathrm{~T}$ \\
\hline $56-6 A$ & $\mathrm{~T}$ \\
\hline $56-8 A$ & $\mathrm{~T}$ \\
\hline $60-1 \mathrm{~A}$ & $\mathrm{~T}$ \\
\hline GW-204 & $\mathrm{s}$ \\
\hline GW-219 & $\mathrm{s}$ \\
\hline GW-605 & $\mathrm{S}$ \\
\hline GW-606 & $\mathrm{T}$ \\
\hline GW-656 & $\mathrm{S}$ \\
\hline GW-658 & $\mathrm{T}$ \\
\hline GW-686 & $\mathrm{T}$ \\
\hline GW-690 & $\mathrm{T}$ \\
\hline GW-691 & $\mathrm{S}$ \\
\hline GW-692 & $\mathrm{T}$ \\
\hline GW-698 & $\mathrm{S}$ \\
\hline GW-700 & $\mathrm{T}$ \\
\hline GW-762 & $\mathrm{T}$ \\
\hline GW-763 & $\mathrm{T}$ \\
\hline GW-765 & $\mathrm{T}$ \\
\hline GW-769 & $\mathrm{T}$ \\
\hline GW-770 & $\mathrm{T}$ \\
\hline GW-775 & $\mathrm{T}$ \\
\hline GW-776 & $\mathrm{T}$ \\
\hline GW-779 & $\mathrm{T}$ \\
\hline GW-781 & $\mathrm{T}$ \\
\hline GW-782 & $\mathrm{T}$ \\
\hline GW-783 & $\mathrm{T}$ \\
\hline GW-791 & $\mathrm{S}$ \\
\hline GW-792 & $\mathrm{T}$ \\
\hline GW-820 & $\mathrm{s}$ \\
\hline GW-954-1 & $\mathrm{T}$ \\
\hline GW-954-2 & $\mathrm{T}$ \\
\hline GW-954-3 & $\mathrm{T}$ \\
\hline GW-956-1 & $\mathrm{T}$ \\
\hline GW-956-2 & $\mathrm{T}$ \\
\hline GW-956-3 & $\mathrm{T}$ \\
\hline GW-956-4 & $\mathrm{T}$ \\
\hline GW-959 & $\mathrm{T}$ \\
\hline GW-960 & $\mathrm{T}$ \\
\hline UEFPC-SP17 & $\mathrm{T}$ \\
\hline
\end{tabular}

$\begin{array}{lll}1.0 \mathrm{E}-02 & 1.0 \mathrm{E}-02 & \text { Yes } \\ 1.0 \mathrm{E}-02 & 1.0 \mathrm{E}-02 & \text { Yes } \\ 1.0 \mathrm{E}-02 & 1.0 \mathrm{E}-02 & \text { Yes } \\ 1.0 \mathrm{E}-02 & 1.0 \mathrm{E}-02 & \text { Yes } \\ 1.0 \mathrm{E}-02 & 1.0 \mathrm{E}-02 & \text { Yes } \\ 3.1 \mathrm{E}+00 & 3.1 \mathrm{E}+00 & \text { No } \\ 1.0 \mathrm{E}-02 & 1.0 \mathrm{E}-02 & \text { Yes } \\ 4.8 \mathrm{E}+01 & 3.7 \mathrm{E}+01 & \text { No } \\ 1.2 \mathrm{E}+02 & 1.1 \mathrm{E}+02 & \text { No } \\ 6.9 \mathrm{E}+01 & 5.2 \mathrm{E}+01 & \text { No } \\ 7.0 \mathrm{E}+00 & 7.0 \mathrm{E}+00 & \text { No } \\ 1.9 \mathrm{E}+00 & 1.9 \mathrm{E}+00 & \text { No } \\ 1.1 \mathrm{E}+00 & 1.1 \mathrm{E}+00 & \text { No } \\ 3.2 \mathrm{E}+00 & 1.0 \mathrm{E}-02 & \text { No } \\ 5.4 \mathrm{E}+00 & 3.1 \mathrm{E}+00 & \text { No } \\ 1.4 \mathrm{E}+00 & 1.4 \mathrm{E}+00 & \text { No } \\ 4.8 \mathrm{E}+00 & 3.2 \mathrm{E}+00 & \text { No } \\ 4.6 \mathrm{E}+00 & 2.6 \mathrm{E}+00 & \text { No } \\ 1.5 \mathrm{E}+00 & 1.7 \mathrm{E}+00 & \text { No } \\ 6.9 \mathrm{E}-01 & 1.0 \mathrm{E}-02 & \text { No } \\ 2.6 \mathrm{E}+00 & 8.2 \mathrm{E}-01 & \text { No } \\ 1.0 \mathrm{E}+00 & 1.3 \mathrm{E}+00 & \text { No } \\ 2.5 \mathrm{E}+00 & 1.4 \mathrm{E}+00 & \text { No } \\ 1.2 \mathrm{E}+00 & 6.5 \mathrm{E}-01 & \text { No } \\ 1.7 \mathrm{E}+00 & 1.2 \mathrm{E}+00 & \text { No } \\ 2.6 \mathrm{E}+00 & 1.2 \mathrm{E}+00 & \text { No } \\ 3.2 \mathrm{E}+00 & 2.1 \mathrm{E}+00 & \text { No } \\ 1.5 \mathrm{E}+00 & 4.2 \mathrm{E}-01 & \text { No } \\ 3.9 \mathrm{E}+01 & 4.0 \mathrm{E}+01 & \text { No } \\ 4.0 \mathrm{E}+00 & 3.0 \mathrm{E}+00 & \text { No } \\ 1.5 \mathrm{E}+00 & 1.5 \mathrm{E}+00 & \text { No } \\ 2.0 \mathrm{E}+00 & 1.6 \mathrm{E}+00 & \text { No } \\ 9.3 \mathrm{E}-01 & 3.8 \mathrm{E}-01 & \text { No } \\ 7.3 \mathrm{E}+00 & 6.9 \mathrm{E}+00 & \text { No } \\ 2.9 \mathrm{E}+00 & 2.4 \mathrm{E}+00 & \text { No } \\ 2.9 \mathrm{E}+00 & 3.8 \mathrm{E}+00 & \text { No } \\ 1.0 \mathrm{E}-02 & 1.0 \mathrm{E}-02 & \text { Yes } \\ 5.5 \mathrm{E}+00 & 2.5 \mathrm{E}+00 & \text { No } \\ 5.1 \mathrm{E}+00 & 3.7 \mathrm{E}+00 & \text { No } \\ 2.1 \mathrm{E}+00 & 1.0 \mathrm{E}-02 & \text { No } \\ 3.2 \mathrm{E}-01 & 1.0 \mathrm{E}-02 & \text { No } \\ 1.0 \mathrm{E}-02 & 1.0 \mathrm{E}-02 & \text { Yes } \\ 2.4 \mathrm{E}+00 & 1.9 \mathrm{E}+00 & \text { No }\end{array}$

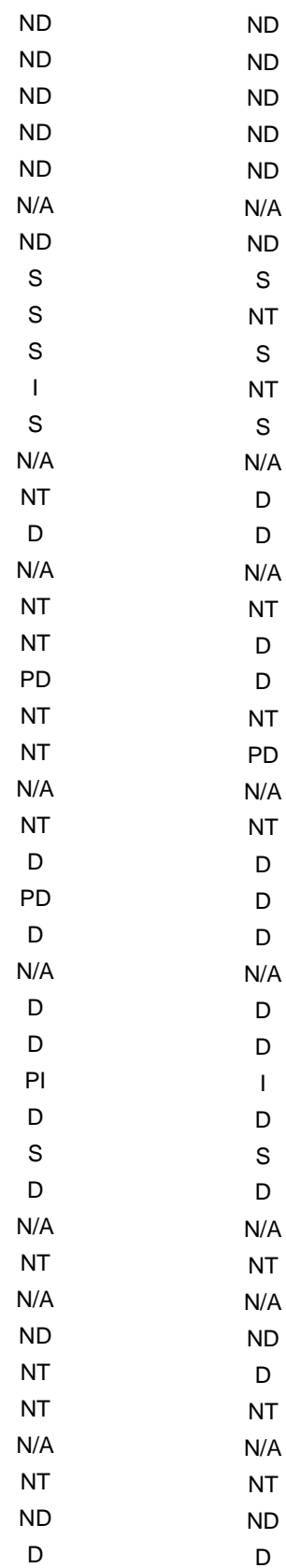

GROSS BETA ACTIVITY

\begin{tabular}{cccccccc}
\hline $55-2 \mathrm{~A}$ & $\mathrm{~S}$ & 4 & 0 & $2.1 \mathrm{E}+00$ & $2.1 \mathrm{E}+00$ & Yes & ND \\
\hline MAROS Version 2.2, 2006, AFCEE & \multicolumn{9}{c}{ Tuesday, December 09, 2008 } & & & Page 5 of 7
\end{tabular}


MAROS Statistical Trend Analysis Summary

\begin{tabular}{|c|c|c|c|c|c|c|c|c|}
\hline Well & $\begin{array}{c}\text { Sourcel } \\
\text { Tail }\end{array}$ & $\begin{array}{l}\text { Number } \\
\text { of } \\
\text { Samples }\end{array}$ & $\begin{array}{c}\text { Number } \\
\text { of } \\
\text { Detects }\end{array}$ & $\begin{array}{l}\text { Average } \\
\text { Conc. } \\
\text { (mg/L) }\end{array}$ & $\begin{array}{l}\text { Median } \\
\text { Conc. } \\
\text { (mg/L) }\end{array}$ & $\begin{array}{c}\text { All } \\
\text { Samples } \\
\text { "ND" ? }\end{array}$ & $\begin{array}{l}\text { Mann- } \\
\text { Kendall } \\
\text { Trend }\end{array}$ & $\begin{array}{c}\text { Linear } \\
\text { Regression } \\
\text { Trend }\end{array}$ \\
\hline
\end{tabular}

GROSS BETA ACTIVITY

\begin{tabular}{|c|c|}
\hline $55-3 A$ & $\mathrm{~S}$ \\
\hline $55-3 B$ & $\mathrm{~S}$ \\
\hline $55-3 C$ & $\mathrm{~S}$ \\
\hline $56-1 A$ & $\mathrm{~T}$ \\
\hline $56-2 A$ & $\mathrm{~T}$ \\
\hline $56-2 B$ & $\mathrm{~T}$ \\
\hline $56-2 C$ & $\mathrm{~T}$ \\
\hline $56-3 A$ & $\mathrm{~T}$ \\
\hline $56-3 B$ & $\mathrm{~T}$ \\
\hline $56-3 C$ & $\mathrm{~T}$ \\
\hline $56-4 A$ & $\mathrm{~T}$ \\
\hline $56-6 A$ & $\mathrm{~T}$ \\
\hline $56-8 A$ & $\mathrm{~T}$ \\
\hline $60-1 \mathrm{~A}$ & $\mathrm{~T}$ \\
\hline GW-204 & $\mathrm{S}$ \\
\hline GW-219 & $\mathrm{s}$ \\
\hline GW-605 & $\mathrm{s}$ \\
\hline GW-606 & $\mathrm{T}$ \\
\hline GW-656 & $\mathrm{S}$ \\
\hline GW-686 & $\mathrm{T}$ \\
\hline GW-690 & $\mathrm{T}$ \\
\hline GW-691 & $\mathrm{s}$ \\
\hline GW-692 & $\mathrm{T}$ \\
\hline GW-698 & $\mathrm{S}$ \\
\hline GW-700 & $\mathrm{T}$ \\
\hline GW-762 & $\mathrm{T}$ \\
\hline GW-763 & $\mathrm{T}$ \\
\hline GW-765 & $\mathrm{T}$ \\
\hline GW-769 & $\mathrm{T}$ \\
\hline GW-770 & $\mathrm{T}$ \\
\hline GW-775 & $\mathrm{T}$ \\
\hline GW-776 & $\mathrm{T}$ \\
\hline GW-779 & $\mathrm{T}$ \\
\hline GW-781 & $\mathrm{T}$ \\
\hline GW-782 & $\mathrm{T}$ \\
\hline GW-783 & $\mathrm{T}$ \\
\hline GW-791 & $\mathrm{S}$ \\
\hline GW-792 & $\mathrm{T}$ \\
\hline GW-820 & $\mathrm{s}$ \\
\hline GW-954-1 & $\mathrm{T}$ \\
\hline GW-954-2 & $\mathrm{T}$ \\
\hline GW-954-3 & $\mathrm{T}$ \\
\hline GW-956-1 & $\mathrm{T}$ \\
\hline GW-956-2 & $\mathrm{T}$ \\
\hline GW-956-3 & $\mathrm{T}$ \\
\hline GW-956-4 & $\mathrm{T}$ \\
\hline
\end{tabular}

\begin{tabular}{lll}
$4.3 \mathrm{E}+00$ & $2.1 \mathrm{E}+00$ & No \\
$3.6 \mathrm{E}+00$ & $2.1 \mathrm{E}+00$ & No \\
$1.0 \mathrm{E}+01$ & $1.1 \mathrm{E}+01$ & No \\
$4.3 \mathrm{E}+00$ & $2.1 \mathrm{E}+00$ & No \\
$2.1 \mathrm{E}+00$ & $2.1 \mathrm{E}+00$ & Yes \\
$2.1 \mathrm{E}+00$ & $2.1 \mathrm{E}+00$ & Yes \\
$2.1 \mathrm{E}+00$ & $2.1 \mathrm{E}+00$ & Yes \\
$3.3 \mathrm{E}+00$ & $2.1 \mathrm{E}+00$ & No \\
$2.1 \mathrm{E}+00$ & $2.1 \mathrm{E}+00$ & Yes \\
$2.1 \mathrm{E}+00$ & $2.1 \mathrm{E}+00$ & Yes \\
$3.2 \mathrm{E}+00$ & $2.1 \mathrm{E}+00$ & No \\
$4.6 \mathrm{E}+00$ & $3.8 \mathrm{E}+00$ & No \\
$2.1 \mathrm{E}+00$ & $2.1 \mathrm{E}+00$ & Yes \\
$3.8 \mathrm{E}+00$ & $2.1 \mathrm{E}+00$ & No \\
$1.3 \mathrm{E}+01$ & $1.2 \mathrm{E}+01$ & No \\
$5.2 \mathrm{E}+01$ & $5.6 \mathrm{E}+01$ & No \\
$2.7 \mathrm{E}+01$ & $2.5 \mathrm{E}+01$ & No \\
$5.4 \mathrm{E}+00$ & $5.6 \mathrm{E}+00$ & No \\
$5.9 \mathrm{E}+00$ & $5.9 \mathrm{E}+00$ & No \\
$2.1 \mathrm{E}+00$ & $2.1 \mathrm{E}+00$ & Yes \\
$4.6 \mathrm{E}+00$ & $4.6 \mathrm{E}+00$ & No \\
$2.1 \mathrm{E}+00$ & $2.1 \mathrm{E}+00$ & Yes \\
$9.0 \mathrm{E}+00$ & $9.0 \mathrm{E}+00$ & No \\
$5.2 \mathrm{E}+00$ & $2.1 \mathrm{E}+00$ & No \\
$2.1 \mathrm{E}+00$ & $2.1 \mathrm{E}+00$ & Yes \\
$5.1 \mathrm{E}+00$ & $5.7 \mathrm{E}+00$ & No \\
$4.0 \mathrm{E}+00$ & $3.9 \mathrm{E}+00$ & No \\
$2.1 \mathrm{E}+00$ & $2.1 \mathrm{E}+00$ & Yes \\
$6.1 \mathrm{E}+00$ & $3.9 \mathrm{E}+00$ & No \\
$2.1 \mathrm{E}+00$ & $2.1 \mathrm{E}+00$ & Yes \\
$2.1 \mathrm{E}+00$ & $2.1 \mathrm{E}+00$ & Yes \\
$8.3 \mathrm{E}+00$ & $8.3 \mathrm{E}+00$ & No \\
$2.1 \mathrm{E}+00$ & $2.1 \mathrm{E}+00$ & Yes \\
$7.8 \mathrm{E}+00$ & $7.8 \mathrm{E}+00$ & No \\
$5.6 \mathrm{E}+00$ & $2.1 \mathrm{E}+00$ & No \\
$5.1 \mathrm{E}+00$ & $5.1 \mathrm{E}+00$ & No \\
$8.6 \mathrm{E}+00$ & $2.1 \mathrm{E}+00$ & No \\
$2.1 \mathrm{E}+00$ & $2.1 \mathrm{E}+00$ & Yes \\
$2.8 \mathrm{E}+00$ & $2.1 \mathrm{E}+00$ & No \\
$4.0 \mathrm{E}+00$ & $2.1 \mathrm{E}+00$ & No \\
$2.6 \mathrm{E}+00$ & $2.1 \mathrm{E}+00$ & No \\
$5.6 \mathrm{E}+00$ & $2.5 \mathrm{E}+00$ & No \\
$6.7 \mathrm{E}+00$ & $7.1 \mathrm{E}+00$ & Yes \\
& $7.6 \mathrm{E}+00$ & No \\
\hline $\mathrm{E}+00$ & No \\
\hline .000 & & \\
2.00 & \\
2.00 &
\end{tabular}

\section{PI}

$S$

$5 \quad 1$

$4 \quad 4$

4

2

2

2

4

4

4

4

4

2

4

4

3

3
6

6

6
2

4

4

2
2

2

2

6

6
2

2
6
4

4

1

6

6

6
1

1
1

1

2
2

2
4
2

2
4

4

2
4

4

4
4
4

4
4

4
4

4

$\begin{array}{ll}4 & 2 \\ 4 & 3\end{array}$

$\begin{array}{ll}S & S \\ S & S\end{array}$

$\begin{array}{cc}S & S \\ N T & N T\end{array}$

ND ND

ND ND

ND ND

$\mathrm{S}$

ND $\quad$ ND

NT ND

NT I

ND ND

NT NT

$\mathrm{S} \quad \mathrm{NT}$

N/A N/A

$\mathrm{S} \quad \mathrm{PD}$

NT NT

N/A N/A

$\begin{array}{ll}N / A & N / A \\ N D & N D\end{array}$

N/A N/A

ND ND

N/A N/A

NT PI

ND ND

$S \quad P D$

$S \quad S$

ND ND

NT ।

ND ND

$\begin{array}{ll}N D & N D \\ N D & N D\end{array}$

N/A N/A

ND N/A

N/A N/A

NT PI

N/A N/A

NT $\quad \mathrm{P} / \mathrm{A}$

$\begin{array}{cc}N D & \text { ND } \\ S & S\end{array}$

$\begin{array}{ll}S & S \\ S & S \\ S & S\end{array}$

$\begin{array}{cc}\text { NT } & S \\ N T\end{array}$

ND ND

$\begin{array}{lc}\mathrm{S} & \mathrm{ND} \\ \mathrm{NT}\end{array}$

$\begin{array}{cc}\text { NT } & \text { S } \\ \text { NT } & \text { NT }\end{array}$

\begin{tabular}{lr} 
NT & NT \\
\hline
\end{tabular}




\section{MAROS Statistical Trend Analysis Summary}

\begin{tabular}{|c|c|c|c|c|c|c|c|c|}
\hline Well & $\begin{array}{c}\text { Sourcel } \\
\text { Tail }\end{array}$ & $\begin{array}{c}\text { Number } \\
\text { of } \\
\text { Samples }\end{array}$ & $\begin{array}{c}\text { Number } \\
\text { of } \\
\text { Detects }\end{array}$ & $\begin{array}{c}\text { Average } \\
\text { Conc. } \\
\text { (mg/L) }\end{array}$ & $\begin{array}{l}\text { Median } \\
\text { Conc. } \\
\text { (mg/L) }\end{array}$ & $\begin{array}{c}\text { All } \\
\text { Samples } \\
\text { "ND" ? }\end{array}$ & $\begin{array}{l}\text { Mann- } \\
\text { Kendall } \\
\text { Trend }\end{array}$ & $\begin{array}{l}\text { Linear } \\
\text { Regression } \\
\text { Trend }\end{array}$ \\
\hline \multicolumn{9}{|c|}{ GROSS BETA ACTIVITY } \\
\hline GW-959 & $\mathrm{T}$ & 7 & 3 & $5.4 \mathrm{E}+00$ & $2.1 \mathrm{E}+00$ & No & NT & NT \\
\hline GW-960 & $\mathrm{T}$ & 2 & 1 & $5.3 E+00$ & $5.3 E+00$ & No & N/A & N/A \\
\hline UEFPC-SP17 & $\mathrm{T}$ & 1 & 0 & $2.1 \mathrm{E}+00$ & $2.1 \mathrm{E}+00$ & Yes & ND & ND \\
\hline
\end{tabular}

Note: Increasing (I); Probably Increasing (PI); Stable (S); Probably Decreasing (PD); Decreasing (D); No Trend (NT); Not Applicable (N/A); Not Applicable (N/A) - Due to insufficient Data (< 4 sampling events); No Detectable Concentration (NDC)

The Number of Samples and Number of Detects shown above are post-consolidation values. 


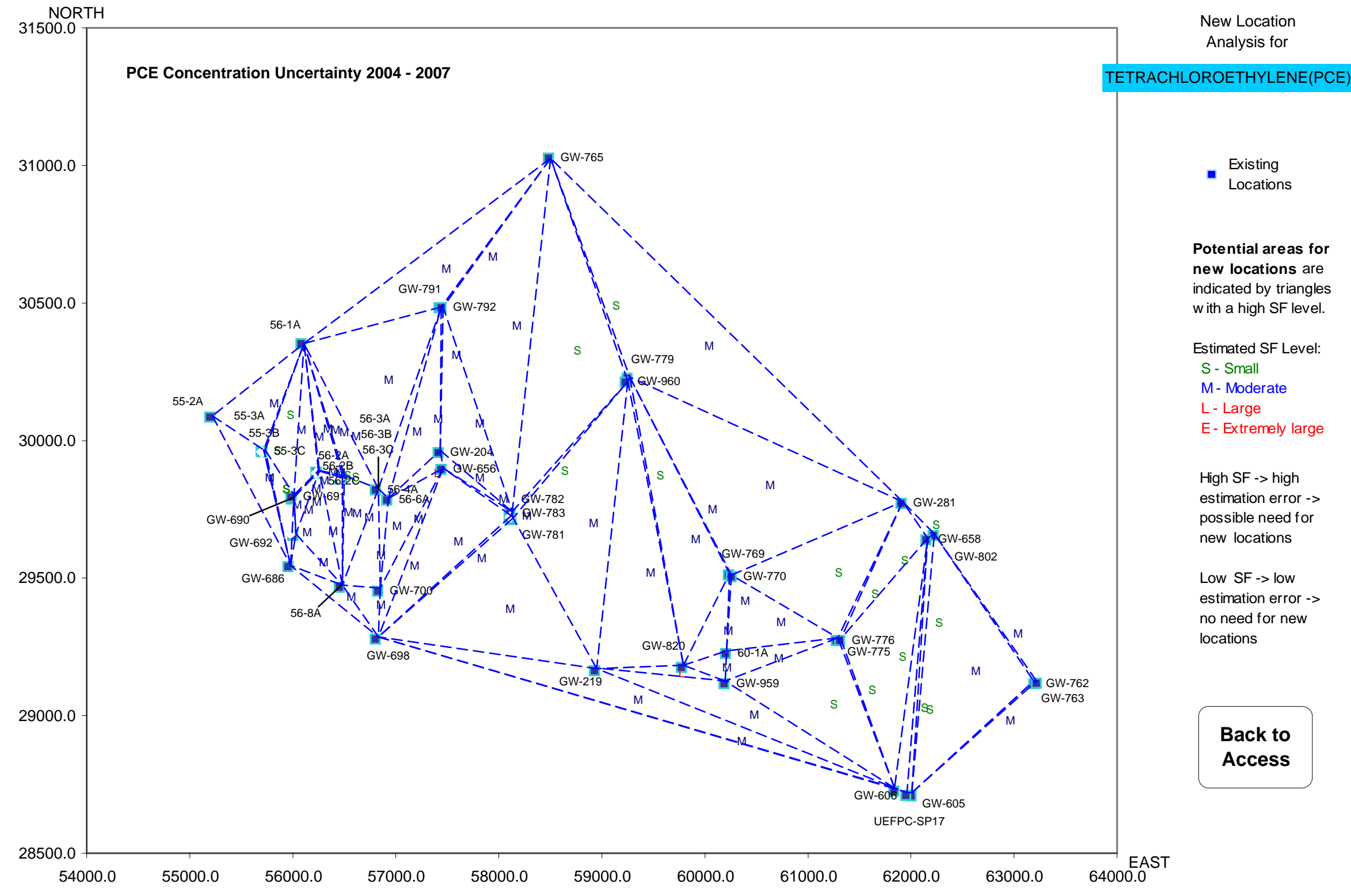


APPENDIX C.6

EAST Y-12 AREA 


\section{MAROS COC Assessment}

Project: $\quad$ EF New Hope Pond

Location: East Y-12

Toxicity:

\begin{tabular}{lccc}
\hline Contaminant of Concern & $\begin{array}{c}\text { Representative } \\
\text { Concentration } \\
\text { (mg/L) }\end{array}$ & $\begin{array}{c}\text { PRG } \\
\text { (mg/L) }\end{array}$ & $\begin{array}{c}\text { Percent } \\
\text { Above } \\
\text { PRG }\end{array}$ \\
\hline CARBON TETRACHLORIDE & $3.4 \mathrm{E}-01$ & $5.0 \mathrm{E}-03$ & $6768.9 \%$ \\
MANGANESE & $7.2 \mathrm{E}-01$ & $5.0 \mathrm{E}-02$ & $1342.5 \%$ \\
TETRACHLOROETHYLENE(PCE) & $4.1 \mathrm{E}-02$ & $5.0 \mathrm{E}-03$ & $717.3 \%$ \\
TRICHLOROETHYLENE (TCE) & $1.8 \mathrm{E}-02$ & $5.0 \mathrm{E}-03$ & $255.7 \%$ \\
GROSS ALPHA ACTIVITY & $1.8 \mathrm{E}+01$ & $1.5 \mathrm{E}+01$ & $17.2 \%$
\end{tabular}

Note: Top COCs by toxicity were determined by examining a representative concentration for each compound over the entire site. The compound representative concentrations are then compared with the chosen PRG for that compound, with the percentage exceedance from the PRG determining the compound's toxicity. All compounds above exceed the PRG.

\section{Prevalence:}

\begin{tabular}{lccccc} 
Contaminant of Concern & Class & $\begin{array}{c}\text { Total } \\
\text { Wells }\end{array}$ & $\begin{array}{c}\text { Total } \\
\text { Exceedances }\end{array}$ & $\begin{array}{c}\text { Percent } \\
\text { Exceedances } \\
\text { detects }\end{array}$ \\
\hline MANGANESE & MET & 25 & 16 & $64.0 \%$ & 25 \\
CARBON TETRACHLORIDE & ORG & 26 & 11 & $42.3 \%$ & 15 \\
TETRACHLOROETHYLENE(PCE) & ORG & 26 & 7 & $26.9 \%$ & 17 \\
TRICHLOROETHYLENE (TCE) & ORG & 26 & 5 & $19.2 \%$ & 9 \\
GROSS ALPHA ACTIVITY & INO & 26 & 1 & $3.8 \%$ & 26
\end{tabular}

Note: Top COCs by prevalence were determined by examining a representative concentration for each well location at the site. The total exceedances (values above the chosen PRGs) are compared to the total number of wells to determine the prevalence of the compound.

\section{Mobility:}

Contaminant of Concern

GROSS ALPHA ACTIVITY

CARBON TETRACHLORIDE

TRICHLOROETHYLENE (TCE)

TETRACHLOROETHYLENE(PCE)

MANGANESE
User Name: MV

State: Tennessee 


\section{MAROS Spatial Moment Analysis Summary}

Project: EF New Hope Pond

Location: East Y-12
User Name: MV

State: Tennessee

\begin{tabular}{|c|c|c|c|c|c|c|c|}
\hline \multirow[b]{2}{*}{ Effective Date } & \multirow{2}{*}{$\begin{array}{l}\text { Dth Moment } \\
\text { Estimated } \\
\text { Mass (Kg) }\end{array}$} & \multicolumn{3}{|c|}{$\underline{1 s t}$ Moment (Center of Mass) } & \multicolumn{2}{|c|}{$\underline{\text { 2nd Moment (Spread) }}$} & \multirow[b]{2}{*}{$\begin{array}{c}\text { Number of } \\
\text { Wells }\end{array}$} \\
\hline & & $\mathrm{Xc}(\mathrm{ft})$ & Yc (ft) & $\begin{array}{c}\text { Source } \\
\text { Distance (ft) }\end{array}$ & $\begin{array}{c}\underset{(s q}{\text { Sigma }} x X \\
\end{array}$ & $\begin{array}{l}\text { Sigma } Y Y \\
(s q \mathrm{ft})\end{array}$ & \\
\hline \multicolumn{8}{|l|}{ CARBON TETRACHLORIDE } \\
\hline 7/1/1996 & $5.7 \mathrm{E}+00$ & 64,976 & 28,886 & 2,035 & $1,098,923$ & 149,058 & 23 \\
\hline $7 / 1 / 1997$ & $8.3 \mathrm{E}+00$ & 64,892 & 28,774 & 1,945 & $1,040,280$ & 137,282 & 22 \\
\hline $7 / 1 / 1998$ & $7.0 \mathrm{E}+00$ & 64,887 & 28,824 & 1,942 & $1,104,444$ & 147,718 & 21 \\
\hline 7/1/1999 & $8.4 \mathrm{E}+00$ & 64,639 & 28,822 & 1,694 & $1,123,379$ & 122,194 & 23 \\
\hline $7 / 1 / 2000$ & $5.7 \mathrm{E}+00$ & 64,480 & 28,977 & 1,554 & $1,016,341$ & 177,112 & 23 \\
\hline $7 / 1 / 2001$ & $5.5 \mathrm{E}+00$ & 64,535 & 28,922 & 1,600 & 928,672 & 166,424 & 23 \\
\hline $7 / 1 / 2002$ & $7.2 \mathrm{E}+00$ & 64,493 & 28,809 & 1,547 & 693,164 & 106,799 & 23 \\
\hline $7 / 1 / 2003$ & $6.5 \mathrm{E}+00$ & 64,445 & 28,830 & 1,502 & 803,924 & 117,074 & 23 \\
\hline $7 / 1 / 2004$ & $6.2 \mathrm{E}+00$ & 64,543 & 28,826 & 1,599 & 816,360 & 122,392 & 23 \\
\hline $7 / 1 / 2005$ & $6.0 \mathrm{E}+00$ & 64,529 & 28,830 & 1,585 & 837,558 & 123,967 & 23 \\
\hline $7 / 1 / 2006$ & $4.5 \mathrm{E}+00$ & 64,765 & 28,952 & 1,832 & 984,994 & 146,035 & 24 \\
\hline $7 / 1 / 2007$ & $3.6 \mathrm{E}+00$ & 64,711 & 28,926 & 1,776 & $1,121,547$ & 176,189 & 24 \\
\hline
\end{tabular}

GROSS ALPHA ACTIVITY

\begin{tabular}{|c|c|c|c|c|c|c|c|}
\hline 7/1/1996 & $2.6 \mathrm{E}+03$ & 65,329 & 29,444 & 2,490 & $1,904,362$ & 255,843 & 22 \\
\hline $7 / 1 / 1997$ & $1.1 \mathrm{E}+03$ & 64,414 & 29,316 & 1,584 & $1,048,423$ & 323,976 & 21 \\
\hline 7/1/1998 & $3.4 \mathrm{E}+03$ & 65,573 & 29,409 & 2,715 & $1,923,427$ & 243,560 & 20 \\
\hline 7/1/1999 & $2.3 E+03$ & 65,323 & 29,426 & 2,479 & $2,028,566$ & 253,872 & 23 \\
\hline $7 / 1 / 2000$ & $1.4 \mathrm{E}+03$ & 64,771 & 29,567 & 2,012 & $1,340,797$ & 274,422 & 23 \\
\hline $7 / 1 / 2001$ & $5.4 \mathrm{E}+03$ & 64,702 & 29,678 & 2,001 & $1,794,920$ & 268,317 & 20 \\
\hline $7 / 1 / 2002$ & $2.4 \mathrm{E}+03$ & 65,349 & 29,474 & 2,518 & $2,197,078$ & 275,619 & 23 \\
\hline $7 / 1 / 2003$ & $1.2 \mathrm{E}+03$ & 64,753 & 29,246 & 1,881 & $1,191,702$ & 201,769 & 22 \\
\hline $7 / 1 / 2004$ & $1.9 \mathrm{E}+03$ & 64,244 & 29,501 & 1,515 & 548,650 & 325,558 & 17 \\
\hline $7 / 1 / 2005$ & $5.0 \mathrm{E}+02$ & 63,584 & 29,043 & 716 & 120,505 & 121,484 & 18 \\
\hline $7 / 1 / 2006$ & $1.4 \mathrm{E}+02$ & 63,913 & 29,152 & 1,060 & 383,463 & 185,349 & 18 \\
\hline $7 / 1 / 2007$ & $1.4 \mathrm{E}+03$ & 63,893 & 29,382 & 1,157 & 147,430 & 36,484 & 17 \\
\hline
\end{tabular}

GROSS BETA ACTIVITY

\begin{tabular}{|c|c|c|c|c|c|c|c|}
\hline 7/1/1996 & $4.9 \mathrm{E}+03$ & 65,912 & 29,525 & 3,072 & $1,839,592$ & 150,839 & 22 \\
\hline 7/1/1997 & $6.6 \mathrm{E}+03$ & 65,784 & 29,568 & 2,962 & $1,756,068$ & 207,653 & 20 \\
\hline 7/1/1998 & 7.7E+03 & 65,686 & 29,441 & 2,833 & $1,674,243$ & 224,095 & 20 \\
\hline 7/1/1999 & $6.4 \mathrm{E}+03$ & 65,530 & 29,546 & 2,713 & $1,964,216$ & 254,547 & 23 \\
\hline $7 / 1 / 2000$ & $4.5 \mathrm{E}+03$ & 65,181 & 29,382 & 2,331 & $1,163,289$ & 405,153 & 23 \\
\hline $7 / 1 / 2001$ & $8.5 E+03$ & 65,816 & 29,455 & 2,962 & $1,903,932$ & 306,592 & 23 \\
\hline $7 / 1 / 2002$ & $1.2 \mathrm{E}+04$ & 65,173 & 29,585 & 2,389 & $2,269,092$ & 306,857 & 22 \\
\hline $7 / 1 / 2003$ & $1.0 \mathrm{E}+04$ & 65,375 & 29,457 & 2,538 & $2,067,346$ & 277,152 & 21 \\
\hline $7 / 1 / 2004$ & $3.2 \mathrm{E}+03$ & 64,560 & 29,399 & 1,752 & 589,407 & 286,635 & 16 \\
\hline 7/1/2005 & $2.4 \mathrm{E}+02$ & 64,677 & 29,085 & 1,768 & 730,958 & 142,810 & 18 \\
\hline 7/1/2006 & $1.2 \mathrm{E}+03$ & 64,354 & 29,277 & 1,514 & 567,505 & 268,672 & 19 \\
\hline
\end{tabular}


Project: EF New Hope Pond

Location: East Y-12

\begin{tabular}{|c|c|c|c|c|c|c|c|}
\hline \multirow[b]{2}{*}{ Effective Date } & \multirow{2}{*}{$\begin{array}{l}\text { th Moment } \\
\text { Estimated } \\
\text { Mass (kg) }\end{array}$} & \multicolumn{3}{|c|}{ 1st Moment (Center of Mass) } & \multicolumn{2}{|c|}{ 2nd Moment (Spread) } & \multirow[b]{2}{*}{$\begin{array}{c}\text { Number of } \\
\text { Wells }\end{array}$} \\
\hline & & $\mathrm{Xc}$ (ft) & Yc (ft) & $\begin{array}{c}\text { Source } \\
\text { Distance (ft) }\end{array}$ & $\underset{(s q \mathrm{ft})}{\text { Sigma } X X}$ & $\begin{array}{c}\text { Sigma } Y Y \\
(s q \mathrm{ft})\end{array}$ & \\
\hline \multicolumn{8}{|l|}{ GROSS BETA ACTIVITY } \\
\hline $7 / 1 / 2007$ & $2.3 E+02$ & 64,148 & 28,937 & 1,220 & 893,784 & 181,736 & 19 \\
\hline \multicolumn{8}{|c|}{ TETRACHLOROETHYLENE(PCE) } \\
\hline 7/1/1996 & $2.5 E+00$ & 64,618 & 29,524 & 1,856 & $1,454,404$ & 326,501 & 23 \\
\hline $7 / 1 / 1997$ & $2.2 \mathrm{E}+00$ & 64,667 & 29,456 & 1,872 & $1,758,329$ & 414,384 & 22 \\
\hline $7 / 1 / 1998$ & $2.7 \mathrm{E}+00$ & 64,828 & 29,404 & 2,002 & $1,892,006$ & 430,954 & 21 \\
\hline 7/1/1999 & $3.3 \mathrm{E}+00$ & 64,402 & 29,300 & 1,567 & $1,576,627$ & 396,519 & 23 \\
\hline $7 / 1 / 2000$ & $4.1 \mathrm{E}+00$ & 64,301 & 29,459 & 1,544 & $1,230,832$ & 300,113 & 23 \\
\hline $7 / 1 / 2001$ & $3.8 \mathrm{E}+00$ & 64,338 & 29,442 & 1,569 & $1,228,333$ & 312,281 & 23 \\
\hline $7 / 1 / 2002$ & $3.5 \mathrm{E}+00$ & 64,491 & 29,363 & 1,673 & $1,310,582$ & 342,025 & 23 \\
\hline $7 / 1 / 2003$ & $3.8 \mathrm{E}+00$ & 64,441 & 29,360 & 1,627 & $1,234,050$ & 335,899 & 23 \\
\hline $7 / 1 / 2004$ & $3.6 \mathrm{E}+00$ & 64,458 & 29,359 & 1,642 & $1,233,876$ & 332,128 & 23 \\
\hline $7 / 1 / 2005$ & $3.6 \mathrm{E}+00$ & 64,466 & 29,366 & 1,652 & $1,258,123$ & 336,867 & 23 \\
\hline 7/1/2006 & $2.8 \mathrm{E}+00$ & 64,636 & 29,422 & 1,831 & $1,431,165$ & 352,801 & 24 \\
\hline $7 / 1 / 2007$ & $3.3 E+00$ & 64,489 & 29,393 & 1,684 & $1,305,355$ & 332,473 & 24 \\
\hline
\end{tabular}

TRICHLOROETHYLENE (TCE)

$\begin{array}{llllllll}7 / 1 / 2005 & 5.3 \mathrm{E}+00 & 64,982 & 29,475 & 2,171 & 1,973,304 & 377,375 \\ 7 / 1 / 2006 & 5.6 \mathrm{E}+00 & 65,034 & 29,455 & 2,213 & 1,981,410 & 363,684 & 23 \\ 7 / 1 / 2007 & 5.2 \mathrm{E}+00 & 65,041 & 29,496 & 2,233 & 2,097,488 & 379,949\end{array}$


Project: EF New Hope Pond

Location: East Y-12
User Name: $\mathrm{MV}$

State: Tennessee

\begin{tabular}{|c|c|c|c|c|c|}
\hline Moment Type & Constituent & $\begin{array}{l}\text { Coefficient } \\
\text { of Variation }\end{array}$ & $\begin{array}{l}\text { Mann-Kendall } \\
\text { S Statistic }\end{array}$ & $\begin{array}{l}\text { Confidence } \\
\text { in Trend }\end{array}$ & $\begin{array}{c}\text { Moment } \\
\text { Trend }\end{array}$ \\
\hline \multicolumn{6}{|c|}{ Zeroth Moment: Mass } \\
\hline & CARBON TETRACHLORIDE & 0.22 & -30 & $97.8 \%$ & $\mathrm{D}$ \\
\hline & GROSS ALPHA ACTIVITY & 0.72 & -26 & $95.7 \%$ & $\mathrm{D}$ \\
\hline & GROSS BETA ACTIVITY & 0.70 & -22 & $92.4 \%$ & PD \\
\hline & TETRACHLOROETHYLENE(PCE) & 0.18 & 14 & $81.0 \%$ & NT \\
\hline & TRICHLOROETHYLENE (TCE) & 0.00 & 0 & $0.0 \%$ & $\mathrm{~N} / \mathrm{A}$ \\
\hline \multicolumn{6}{|c|}{ 1st Moment: Distance to Source } \\
\hline & CARBON TETRACHLORIDE & 0.11 & -24 & $94.2 \%$ & PD \\
\hline & GROSS ALPHA ACTIVITY & 0.35 & -38 & $99.6 \%$ & $\mathrm{D}$ \\
\hline & GROSS BETA ACTIVITY & 0.27 & -52 & $100.0 \%$ & $\mathrm{D}$ \\
\hline & TETRACHLOROETHYLENE(PCE) & 0.08 & 2 & $52.7 \%$ & NT \\
\hline & TRICHLOROETHYLENE (TCE) & 0.00 & 0 & $0.0 \%$ & N/A \\
\hline \multicolumn{6}{|c|}{ 2nd Moment: Sigma XX } \\
\hline & CARBON TETRACHLORIDE & 0.15 & -14 & $81.0 \%$ & $\mathrm{~S}$ \\
\hline & GROSS ALPHA ACTIVITY & 0.63 & -32 & $98.4 \%$ & $\mathrm{D}$ \\
\hline & GROSS BETA ACTIVITY & 0.43 & -20 & $90.2 \%$ & PD \\
\hline & TETRACHLOROETHYLENE(PCE) & 0.16 & -16 & $84.5 \%$ & $\mathrm{~S}$ \\
\hline & TRICHLOROETHYLENE (TCE) & 0.00 & 0 & $0.0 \%$ & $\mathrm{~N} / \mathrm{A}$ \\
\hline \multicolumn{6}{|c|}{ 2nd Moment: Sigma YY } \\
\hline & CARBON TETRACHLORIDE & 0.17 & 0 & $47.3 \%$ & $\mathrm{~S}$ \\
\hline & GROSS ALPHA ACTIVITY & 0.36 & -22 & $92.4 \%$ & PD \\
\hline & GROSS BETA ACTIVITY & 0.30 & 4 & $58.0 \%$ & NT \\
\hline & TETRACHLOROETHYLENE(PCE) & 0.12 & -6 & $63.1 \%$ & $\mathrm{~S}$ \\
\hline & TRICHLOROETHYLENE (TCE) & 0.00 & 0 & $0.0 \%$ & $\mathrm{~N} / \mathrm{A}$ \\
\hline
\end{tabular}

Note: The following assumptions were applied for the calculation of the Zeroth Moment:

Porosity: $0.10 \quad$ Saturated Thickness: Uniform: $50 \mathrm{ft}$

Mann-Kendall Trend test performed on all sample events for each constituent. Increasing (I); Probably Increasing (PI); Stable (S); Probably Decreasing (PD); Decreasing (D); No Trend (NT); Not Applicable (N/A)-Due to insufficient Data (< 4 sampling events).

Note: The Sigma $X X$ and Sigma $Y Y$ components are estimated using the given field coordinate system and then rotated to align with the estimated groundwater flow direction. Moments are not calculated for sample events with less than 6 wells. 


\section{MAROS Statistical Trend Analysis Summary}

Project: $\quad$ EF New Hope Pond

Location: East Y-12

Time Period: $1 / 1 / 1996$ to $10 / 15 / 2007$

Consolidation Period: No Time Consolidation

Consolidation Type: Median

Duplicate Consolidation: Average

ND Values: Specified Detection Limit

J Flag Values : Actual Value
User Name: MV

State: Tennessee

\begin{tabular}{|c|c|c|c|c|c|c|c|c|}
\hline Well & $\begin{array}{c}\text { Sourcel } \\
\text { Tail }\end{array}$ & $\begin{array}{c}\text { Number } \\
\text { of } \\
\text { Samples }\end{array}$ & $\begin{array}{c}\text { Number } \\
\text { of } \\
\text { Detects }\end{array}$ & $\begin{array}{c}\text { Average } \\
\text { Conc. } \\
\text { (mg/L) }\end{array}$ & $\begin{array}{l}\text { Median } \\
\text { Conc. } \\
\text { (mg/L) }\end{array}$ & $\begin{array}{c}\text { All } \\
\text { Samples } \\
\text { "ND" ? }\end{array}$ & $\begin{array}{l}\text { Mann- } \\
\text { Kendall } \\
\text { Trend }\end{array}$ & $\begin{array}{l}\text { Linear } \\
\text { Regression } \\
\text { Trend }\end{array}$ \\
\hline \multicolumn{9}{|c|}{ CARBON TETRACHLORIDE } \\
\hline GW-148 & $\mathrm{T}$ & 11 & 0 & $5.0 \mathrm{E}-04$ & $5.0 \mathrm{E}-04$ & Yes & ND & ND \\
\hline GW-151 & S & 25 & 24 & $1.1 \mathrm{E}+00$ & $1.1 \mathrm{E}+00$ & No & I & I \\
\hline GW-153 & $\mathrm{T}$ & 24 & 23 & $1.4 \mathrm{E}-01$ & $1.3 \mathrm{E}-01$ & No & $\mathrm{D}$ & s \\
\hline GW-154 & $\mathrm{s}$ & 26 & 0 & $5.0 \mathrm{E}-04$ & $5.0 \mathrm{E}-04$ & Yes & ND & ND \\
\hline GW-169 & $\mathrm{T}$ & 30 & 0 & $5.0 \mathrm{E}-04$ & $5.0 \mathrm{E}-04$ & Yes & ND & ND \\
\hline GW-170 & $\mathrm{T}$ & 36 & 36 & $1.5 \mathrm{E}-02$ & $4.0 \mathrm{E}-03$ & No & NT & $\mathrm{D}$ \\
\hline GW-171 & $\mathrm{T}$ & 22 & 0 & $5.0 \mathrm{E}-04$ & $5.0 \mathrm{E}-04$ & Yes & ND & ND \\
\hline GW-172 & $\mathrm{T}$ & 21 & 0 & $5.0 \mathrm{E}-04$ & $5.0 \mathrm{E}-04$ & Yes & ND & ND \\
\hline GW-220 & $\mathrm{T}$ & 28 & 28 & 7.9E-01 & $8.0 \mathrm{E}-01$ & No & I & I \\
\hline GW-223 & S & 21 & 6 & 4. $8 \mathrm{E}-03$ & $5.0 \mathrm{E}-04$ & No & D & D \\
\hline GW-230 & $\mathrm{T}$ & 21 & 0 & $5.0 \mathrm{E}-04$ & $5.0 \mathrm{E}-04$ & Yes & ND & ND \\
\hline GW-240 & $\mathrm{T}$ & 10 & 10 & 7.1E-03 & $5.5 \mathrm{E}-03$ & No & $\mathrm{s}$ & D \\
\hline GW-380 & $\mathrm{T}$ & 22 & 1 & $6.1 \mathrm{E}-04$ & $5.0 \mathrm{E}-04$ & No & NT & NT \\
\hline GW-381 & S & 14 & 14 & 2.2E-01 & $9.4 \mathrm{E}-02$ & No & $\mathrm{D}$ & $\mathrm{D}$ \\
\hline GW-382 & S & 17 & 17 & $7.9 \mathrm{E}-01$ & 7.2E-01 & No & D & D \\
\hline GW-383 & $\mathrm{s}$ & 27 & 2 & $6.1 \mathrm{E}-04$ & $5.0 \mathrm{E}-04$ & No & $\mathrm{s}$ & D \\
\hline GW-733 & $\mathrm{T}$ & 27 & 27 & $1.3 \mathrm{E}-02$ & $8.0 \mathrm{E}-03$ & No & D & $\mathrm{D}$ \\
\hline GW-735 & $\mathrm{T}$ & 24 & 0 & $5.0 \mathrm{E}-04$ & $5.0 \mathrm{E}-04$ & Yes & ND & ND \\
\hline GW-744 & $\mathrm{T}$ & 24 & 0 & $5.0 \mathrm{E}-04$ & $5.0 \mathrm{E}-04$ & Yes & ND & ND \\
\hline GW-747 & $\mathrm{T}$ & 24 & 0 & $5.0 \mathrm{E}-04$ & $5.0 \mathrm{E}-04$ & Yes & ND & ND \\
\hline GW-748 & $\mathrm{T}$ & 7 & 0 & $5.0 \mathrm{E}-04$ & $5.0 \mathrm{E}-04$ & Yes & ND & ND \\
\hline GW-750 & $\mathrm{T}$ & 23 & 0 & $5.0 \mathrm{E}-04$ & $5.0 \mathrm{E}-04$ & Yes & ND & ND \\
\hline GW-816 & $\mathrm{T}$ & 24 & 0 & $5.0 \mathrm{E}-04$ & $5.0 \mathrm{E}-04$ & Yes & ND & ND \\
\hline GW-832 & $\mathrm{T}$ & 23 & 23 & $1.5 \mathrm{E}-02$ & $1.0 \mathrm{E}-02$ & No & $\mathrm{D}$ & $\mathrm{D}$ \\
\hline SCR7_1SP & $\mathrm{T}$ & 23 & 9 & $1.4 \mathrm{E}-03$ & $5.0 \mathrm{E}-04$ & No & D & D \\
\hline SCR7_8SP & $\mathrm{T}$ & 21 & 0 & $5.0 \mathrm{E}-04$ & $5.0 \mathrm{E}-04$ & Yes & ND & ND \\
\hline
\end{tabular}

GROSS ALPHA ACTIVITY

\begin{tabular}{|c|c|c|c|c|c|c|c|c|}
\hline GW-148 & $\mathrm{T}$ & 10 & 9 & $1.6 \mathrm{E}+00$ & $1.4 \mathrm{E}+00$ & No & $\mathrm{D}$ & $\mathrm{D}$ \\
\hline GW-151 & $\mathrm{S}$ & 23 & 8 & 6.6E-01 & 1.0E-02 & No & NT & NT \\
\hline GW-153 & $\mathrm{T}$ & 23 & 20 & $1.9 \mathrm{E}+00$ & $1.7 \mathrm{E}+00$ & No & $\mathrm{D}$ & D \\
\hline GW-154 & $\mathrm{S}$ & 26 & 26 & $5.4 \mathrm{E}+02$ & $5.1 \mathrm{E}+02$ & No & 1 & $\mathrm{Pl}$ \\
\hline GW-169 & $\mathrm{T}$ & 30 & 21 & $1.2 \mathrm{E}+00$ & $1.4 \mathrm{E}+00$ & No & $S$ & PD \\
\hline GW-170 & $\mathrm{T}$ & 35 & 21 & $1.0 \mathrm{E}+00$ & $1.0 \mathrm{E}+00$ & No & PD & PD \\
\hline GW-171 & $\mathrm{T}$ & 16 & 15 & $3.0 \mathrm{E}+00$ & $2.3 \mathrm{E}+00$ & No & NT & NT \\
\hline
\end{tabular}




\section{MAROS Statistical Trend Analysis Summary}

\begin{tabular}{ccccccccc} 
& & Number & Number & Average & Median & All & Mann- & Linear \\
Well & Sourcel & of & of & Conc. & Conc. & Samples & Kendall & Regression \\
Tail & Samples & Detects & (mg/L) & (mg/L) & "ND" ? & Trend & Trend \\
\hline
\end{tabular}

GROSS ALPHA ACTIVITY

GW-172

GW-220

GW-223

GW-230

GW-240

GW-380

GW-381

GW-382

GW-383

GW-733

GW-735

GW-744

GW-747

GW-748

GW-750

GW-816

GW-832

SCR7 1SP

SCR7_8SP

\section{5}

21

21

14

7

21

10

17

18

25

18

19

17

4

19

21

23

15

11

\section{6}

14

14

8.7E-01

$1.3 \mathrm{E}+00$

1.4E+01

3. $5 \mathrm{E}+00$

2. $0 \mathrm{E}+00$

$2.5 \mathrm{E}+00$

1. $3 \mathrm{E}+00$

$1.9 \mathrm{E}+00$

2.2 $\mathrm{E}+00$

$1.2 \mathrm{E}+00$

2. $4 \mathrm{E}+00$

$1.7 \mathrm{E}+00$

1. $0 \mathrm{E}+00$

2.7E-01

$1.1 \mathrm{E}+00$

1.3 $\mathrm{E}+00$

4.7E+00

9.2E-01

3.5E-01

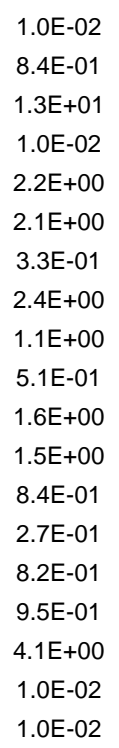

1.0E-02

No
No
No
No
No
No
No
No
No
No
No
No
No
No
No
No
No
No
No

NT

D NT

GROSS BETA ACTIVITY

\begin{tabular}{|c|c|c|c|c|c|c|c|c|}
\hline GW-148 & $T$ & 8 & 7 & $3.3 \mathrm{E}+00$ & $3.6 \mathrm{E}+00$ & No & S & D \\
\hline GW-151 & S & 21 & 16 & $2.7 \mathrm{E}+00$ & $3.1 \mathrm{E}+00$ & No & NT & NT \\
\hline GW-153 & $T$ & 19 & 15 & $3.0 \mathrm{E}+00$ & $2.9 \mathrm{E}+00$ & No & $P D$ & $\mathrm{D}$ \\
\hline GW-154 & S & 26 & 26 & $1.3 \mathrm{E}+02$ & $1.0 \mathrm{E}+02$ & No & 1 & I \\
\hline GW-169 & $T$ & 30 & 26 & $3.4 \mathrm{E}+00$ & $3.3 \mathrm{E}+00$ & No & $\mathrm{s}$ & S \\
\hline GW-170 & $\mathrm{T}$ & 35 & 35 & $9.5 \mathrm{E}+00$ & $7.8 \mathrm{E}+00$ & No & 1 & I \\
\hline GW-171 & $\mathrm{T}$ & 16 & 16 & $1.0 \mathrm{E}+01$ & 8.7E+00 & No & NT & NT \\
\hline GW-172 & $\mathrm{T}$ & 15 & 10 & $4.0 \mathrm{E}+00$ & $2.5 \mathrm{E}+00$ & No & NT & NT \\
\hline GW-220 & $\mathrm{T}$ & 24 & 18 & $3.0 \mathrm{E}+00$ & $3.1 \mathrm{E}+00$ & No & $\mathrm{D}$ & D \\
\hline GW-223 & $\mathrm{S}$ & 20 & 20 & $1.1 \mathrm{E}+01$ & $9.3 \mathrm{E}+00$ & No & 1 & I \\
\hline GW-230 & $\mathrm{T}$ & 14 & 12 & $1.9 \mathrm{E}+02$ & $3.8 \mathrm{E}+00$ & No & 1 & NT \\
\hline GW-240 & $\mathrm{T}$ & 9 & 9 & $3.8 \mathrm{E}+00$ & $3.3 \mathrm{E}+00$ & No & 1 & 1 \\
\hline GW-380 & $\mathrm{T}$ & 18 & 16 & $4.6 \mathrm{E}+00$ & 4.4E+00 & No & NT & $\mathrm{s}$ \\
\hline GW-381 & $\mathrm{S}$ & 10 & 6 & $1.6 \mathrm{E}+00$ & $6.7 \mathrm{E}-01$ & No & NT & D \\
\hline GW-382 & $\mathrm{S}$ & 17 & 15 & $5.1 \mathrm{E}+00$ & $5.4 \mathrm{E}+00$ & No & NT & $\mathrm{s}$ \\
\hline GW-383 & $\mathrm{S}$ & 19 & 13 & $3.1 \mathrm{E}+00$ & $2.0 \mathrm{E}+00$ & No & NT & D \\
\hline GW-733 & $\mathrm{T}$ & 25 & 21 & $3.0 \mathrm{E}+00$ & $2.4 \mathrm{E}+00$ & No & NT & PD \\
\hline GW-735 & $\mathrm{T}$ & 18 & 15 & $2.6 \mathrm{E}+00$ & 2.7E+00 & No & S & D \\
\hline GW-744 & $\mathrm{T}$ & 18 & 15 & $3.6 \mathrm{E}+00$ & $3.0 \mathrm{E}+00$ & No & $\mathrm{s}$ & D \\
\hline GW-747 & $\mathrm{T}$ & 19 & 16 & $3.0 \mathrm{E}+00$ & $3.1 \mathrm{E}+00$ & No & NT & D \\
\hline GW-748 & $\mathrm{T}$ & 4 & 3 & 4.6E-01 & $3.2 \mathrm{E}-01$ & No & D & D \\
\hline GW-750 & $\mathrm{T}$ & 21 & 19 & $3.9 \mathrm{E}+00$ & $4.4 \mathrm{E}+00$ & No & S & $\mathrm{s}$ \\
\hline GW-816 & $\mathrm{T}$ & 24 & 20 & $5.2 \mathrm{E}+00$ & $5.0 \mathrm{E}+00$ & No & $\mathrm{D}$ & D \\
\hline GW-832 & $\mathrm{T}$ & 23 & 20 & $5.8 \mathrm{E}+00$ & $5.8 \mathrm{E}+00$ & No & $\mathrm{PI}$ & NT \\
\hline SCR7_1SP & $\mathrm{T}$ & 16 & 12 & $2.9 \mathrm{E}+00$ & $3.3 \mathrm{E}+00$ & No & NT & NT \\
\hline
\end{tabular}




\section{MAROS Statistical Trend Analysis Summary}

\begin{tabular}{ccccccccc} 
& & Number & Number & Average & Median & All & Mann- & Linear \\
Well & Sourcel & of & of & Conc. & Conc. & Samples & Kendall & Regression \\
Tail & Samples & Detects & (mg/L) & (mg/L) & "ND" ? & Trend & Trend \\
\hline
\end{tabular}

GROSS BETA ACTIVITY

SCR7_8SP

11

$9 \quad 1.9 \mathrm{E}+00 \quad 2.0 \mathrm{E}+00$

No

NT

NT

TETRACHLOROETHYLENE(PCE)

\begin{tabular}{|c|c|c|c|c|c|c|c|c|}
\hline GW-148 & $\mathrm{T}$ & 11 & 0 & $5.0 \mathrm{E}-04$ & 5.0E-04 & Yes & ND & ND \\
\hline GW-151 & $\mathrm{S}$ & 25 & 25 & 3.5E-01 & 2.4E-01 & No & 1 & 1 \\
\hline GW-153 & $\mathrm{T}$ & 24 & 23 & $4.1 \mathrm{E}-03$ & 4.0E-03 & No & $D$ & D \\
\hline GW-154 & $S$ & 26 & 1 & $5.6 \mathrm{E}-04$ & 5.0E-04 & No & $S$ & PD \\
\hline GW-169 & $\mathrm{T}$ & 30 & 28 & $1.6 \mathrm{E}-03$ & 2.0E-03 & No & NT & 1 \\
\hline GW-170 & $\mathrm{T}$ & 36 & 35 & 2.3E-03 & 2.0E-03 & No & D & D \\
\hline GW-171 & $\mathrm{T}$ & 22 & 0 & $5.0 \mathrm{E}-04$ & 5.0E-04 & Yes & ND & ND \\
\hline GW-172 & $\mathrm{T}$ & 21 & 0 & $5.0 \mathrm{E}-04$ & 5.0E-04 & Yes & ND & ND \\
\hline GW-220 & $\mathrm{T}$ & 28 & 28 & 2.2E-01 & 1.1E-01 & No & 1 & 1 \\
\hline GW-223 & $\mathrm{S}$ & 21 & 21 & 8.6E-02 & 4.2E-02 & No & $\mathrm{D}$ & D \\
\hline GW-230 & $\mathrm{T}$ & 21 & 0 & 5.0E-04 & 5.0E-04 & Yes & ND & ND \\
\hline GW-240 & $\mathrm{T}$ & 10 & 0 & 5.0E-04 & 5.0E-04 & Yes & ND & ND \\
\hline GW-380 & $\mathrm{T}$ & 22 & 1 & $5.2 \mathrm{E}-04$ & $5.0 \mathrm{E}-04$ & No & $\mathrm{S}$ & S \\
\hline GW-381 & $\mathrm{S}$ & 14 & 12 & $3.9 \mathrm{E}-03$ & 4.0E-03 & No & PD & PD \\
\hline GW-382 & $\mathrm{s}$ & 17 & 17 & 2.2E-02 & 1.3E-02 & No & NT & NT \\
\hline GW-383 & $\mathrm{S}$ & 27 & 27 & 4.3E-01 & 4.0E-01 & No & $\mathrm{S}$ & D \\
\hline GW-733 & $\mathrm{T}$ & 27 & 5 & $6.2 \mathrm{E}-04$ & $5.0 \mathrm{E}-04$ & No & $\mathrm{D}$ & $\mathrm{D}$ \\
\hline GW-735 & $\mathrm{T}$ & 24 & 0 & 5.0E-04 & 5.0E-04 & Yes & ND & ND \\
\hline GW-744 & $\mathrm{T}$ & 24 & 0 & $5.0 \mathrm{E}-04$ & 5.0E-04 & Yes & ND & ND \\
\hline GW-747 & $\mathrm{T}$ & 24 & 0 & $5.0 \mathrm{E}-04$ & 5.0E-04 & Yes & ND & ND \\
\hline GW-748 & $\mathrm{T}$ & 7 & 0 & $5.0 \mathrm{E}-04$ & 5.0E-04 & Yes & ND & ND \\
\hline GW-750 & $\mathrm{T}$ & 23 & 0 & $5.0 \mathrm{E}-04$ & 5.0E-04 & Yes & ND & ND \\
\hline GW-816 & $\mathrm{T}$ & 24 & 0 & $5.0 \mathrm{E}-04$ & 5.0E-04 & Yes & ND & ND \\
\hline GW-832 & $\mathrm{T}$ & 23 & 23 & $5.1 \mathrm{E}-03$ & $5.0 \mathrm{E}-03$ & No & $\mathrm{s}$ & $\mathrm{s}$ \\
\hline SCR7_1SP & $\mathrm{T}$ & 23 & 2 & $5.4 \mathrm{E}-04$ & 5.0E-04 & No & $\mathrm{s}$ & D \\
\hline SCR7_8SP & $\mathrm{T}$ & 21 & 4 & 7.4E-04 & $5.0 \mathrm{E}-04$ & No & D & D \\
\hline
\end{tabular}

TRICHLOROETHYLENE (TCE)

\begin{tabular}{|c|c|c|c|c|c|c|c|c|}
\hline GW-148 & $\mathrm{T}$ & 1 & 0 & $2.0 \mathrm{E}-03$ & 2.0E-03 & Yes & ND & ND \\
\hline GW-151 & $\mathrm{s}$ & 6 & 6 & $1.3 \mathrm{E}-01$ & 1.3E-01 & No & D & D \\
\hline GW-153 & $\mathrm{T}$ & 4 & 0 & $2.0 \mathrm{E}-03$ & 2.0E-03 & Yes & ND & ND \\
\hline GW-154 & $\mathrm{S}$ & 6 & 0 & $2.0 \mathrm{E}-03$ & 2.0E-03 & Yes & ND & ND \\
\hline GW-169 & $\mathrm{T}$ & 8 & 1 & $2.1 \mathrm{E}-03$ & $2.0 \mathrm{E}-03$ & No & $\mathrm{s}$ & S \\
\hline GW-170 & $\mathrm{T}$ & 9 & 9 & $1.8 \mathrm{E}-03$ & 2.0E-03 & No & PD & D \\
\hline GW-171 & $\mathrm{T}$ & 4 & 0 & 2.0E-03 & 2.0E-03 & Yes & ND & ND \\
\hline GW-172 & $\mathrm{T}$ & 4 & 0 & $2.0 \mathrm{E}-03$ & 2.0E-03 & Yes & ND & ND \\
\hline GW-220 & $\mathrm{T}$ & 6 & 6 & $1.0 \mathrm{E}-01$ & 1.0E-01 & No & NT & NT \\
\hline GW-223 & $S$ & 6 & 6 & $1.4 \mathrm{E}-02$ & 1.4E-02 & No & S & NT \\
\hline GW-230 & $\mathrm{T}$ & 4 & 0 & $2.0 \mathrm{E}-03$ & 2.0E-03 & Yes & ND & ND \\
\hline GW-240 & $\mathrm{T}$ & 2 & 0 & $2.0 \mathrm{E}-03$ & 2.0E-03 & Yes & ND & ND \\
\hline GW-380 & $\mathrm{T}$ & 6 & 0 & $2.0 \mathrm{E}-03$ & 2.0E-03 & Yes & ND & ND \\
\hline GW-381 & $\mathrm{S}$ & 4 & 0 & 2.0E-03 & 2.0E-03 & Yes & ND & ND \\
\hline GW-382 & $\mathrm{S}$ & 6 & 3 & 2.7E-03 & 2.0E-03 & No & $\mathrm{s}$ & D \\
\hline
\end{tabular}




\section{MAROS Statistical Trend Analysis Summary}

\begin{tabular}{|c|c|c|c|c|c|c|c|c|}
\hline Well & $\begin{array}{c}\text { Sourcel } \\
\text { Tail }\end{array}$ & $\begin{array}{c}\text { Number } \\
\text { of } \\
\text { Samples }\end{array}$ & $\begin{array}{l}\text { Number } \\
\text { of } \\
\text { Detects }\end{array}$ & $\begin{array}{l}\text { Average } \\
\text { Conc. } \\
\text { (mg/L) }\end{array}$ & $\begin{array}{l}\text { Median } \\
\text { Conc. } \\
\text { (mg/L) }\end{array}$ & $\begin{array}{c}\text { All } \\
\text { Samples } \\
\text { "ND" ? }\end{array}$ & $\begin{array}{l}\text { Mann- } \\
\text { Kendall } \\
\text { Trend }\end{array}$ & $\begin{array}{l}\text { Linear } \\
\text { Regression } \\
\text { Trend }\end{array}$ \\
\hline \multicolumn{9}{|c|}{ TRICHLOROETHYLENE (TCE) } \\
\hline GW-383 & $\mathrm{S}$ & 6 & 6 & 1.6E-01 & 1.6E-01 & No & NT & NT \\
\hline GW-733 & $\mathrm{T}$ & 6 & 0 & 2.0E-03 & 2.0E-03 & Yes & ND & ND \\
\hline GW-735 & $\mathrm{T}$ & 3 & 0 & 2.0E-03 & 2.0E-03 & Yes & ND & ND \\
\hline GW-744 & $\mathrm{T}$ & 4 & 0 & 2.0E-03 & $2.0 \mathrm{E}-03$ & Yes & ND & ND \\
\hline GW-747 & $\mathrm{T}$ & 4 & 0 & 2.0E-03 & 2.0E-03 & Yes & ND & ND \\
\hline GW-748 & $\mathrm{T}$ & 1 & 0 & 2.0E-03 & 2.0E-03 & Yes & ND & ND \\
\hline GW-750 & $\mathrm{T}$ & 3 & 0 & $2.0 \mathrm{E}-03$ & $2.0 \mathrm{E}-03$ & Yes & ND & ND \\
\hline GW-816 & $\mathrm{T}$ & 4 & 0 & 2.0E-03 & 2.0E-03 & Yes & ND & ND \\
\hline GW-832 & $\mathrm{T}$ & 6 & 4 & 1.2E-03 & 1.0E-03 & No & $\mathrm{S}$ & $\mathrm{S}$ \\
\hline SCR7_1SP & $\mathrm{T}$ & 4 & 1 & $1.8 \mathrm{E}-03$ & $2.0 \mathrm{E}-03$ & No & NT & NT \\
\hline SCR7_8SP & $\mathrm{T}$ & 4 & 0 & 2.0E-03 & 2.0E-03 & Yes & ND & ND \\
\hline
\end{tabular}

Note: Increasing (I); Probably Increasing (PI); Stable (S); Probably Decreasing (PD); Decreasing (D); No Trend (NT); Not Applicable (N/A); Not Applicable (N/A) - Due to insufficient Data (< 4 sampling events); No Detectable Concentration (NDC)

The Number of Samples and Number of Detects shown above are post-consolidation values. 


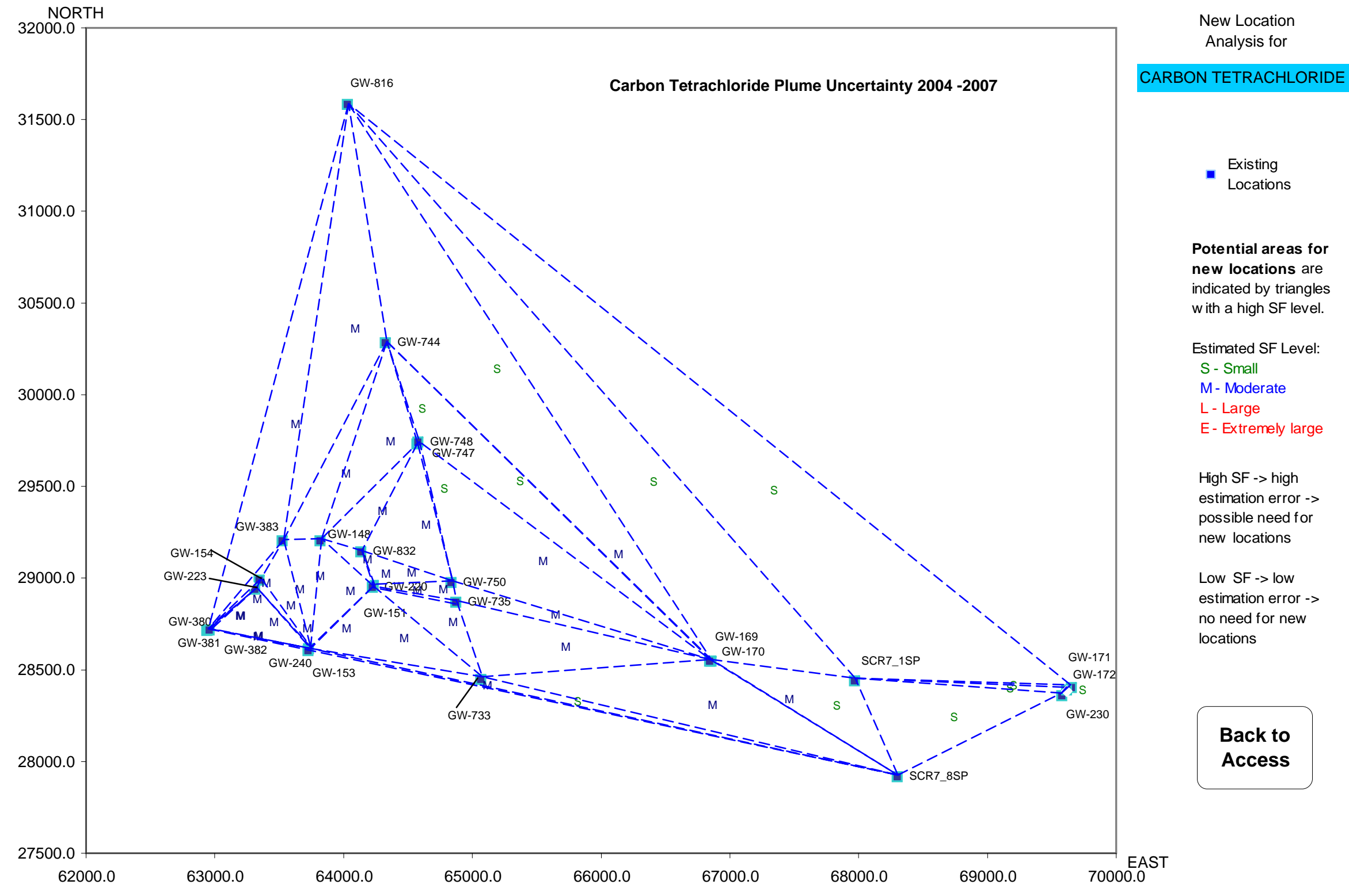


APPENDIX C.7

WEST CHESTNUT RIDGE 


\section{MAROS COC Assessment}

Project: CR Landfill VI

Location: West Chestnut Ridge
User Name: $\quad$ MV

State: Tennessee

\section{Toxicity:}

No priority, plume-wide COCs were found for the West Chestnut Ridge Landfill VI

\section{Prevalence:}

Mobility: 


\section{MAROS Spatial Moment Analysis Summary}

Project: CR Landfill VI

Location: West Chestnut Ridge
User Name: $\mathrm{MV}$

State: Tennessee 2nd Moment (Spread)

0th Moment

1st Moment (Center of Mass)
Estimated

Mass (Kg)

Source

Effective Date

\section{1,1-DICHLOROETHENE}

$\begin{array}{llllllll}7 / 1 / 2005 & 1.2 \mathrm{E}+00 & 52,861 & 26,571 & 2,128 & 234,819 & 1,112,038 & 15 \\ 7 / 1 / 2006 & 1.2 \mathrm{E}+00 & 52,853 & 26,591 & 2,115 & 238,252 & 1,131,334 & 15 \\ 7 / 1 / 2007 & 1.2 \mathrm{E}+00 & 52,859 & 26,604 & 2,101 & 234,750 & 1,115,457 & 15\end{array}$

CADMIUM

\begin{tabular}{|c|c|c|c|c|c|c|c|}
\hline 7/1/1996 & 2.4E-02 & 52,914 & 27,443 & 1,430 & 295,972 & 678,598 & 16 \\
\hline 7/1/1997 & 3.3E-02 & 53,167 & 26,387 & 2,148 & 337,856 & $1,490,541$ & 17 \\
\hline 7/1/1998 & 2.7E-02 & 53,095 & 26,697 & 1,900 & 393,663 & $1,237,287$ & 18 \\
\hline $7 / 1 / 1999$ & 2.7E-02 & 53,095 & 26,697 & 1,900 & 392,921 & $1,246,287$ & 19 \\
\hline $7 / 1 / 2000$ & 2.7E-02 & 53,095 & 26,697 & 1,900 & 392,921 & $1,246,287$ & 19 \\
\hline $7 / 1 / 2001$ & 2.7E-02 & 53,095 & 26,697 & 1,900 & 392,921 & $1,246,287$ & 19 \\
\hline $7 / 1 / 2002$ & $1.9 \mathrm{E}-02$ & 52,870 & 27,202 & 1,625 & 332,554 & 614,408 & 17 \\
\hline $7 / 1 / 2003$ & 2.7E-02 & 53,095 & 26,697 & 1,900 & 392,921 & $1,246,287$ & 19 \\
\hline $7 / 1 / 2004$ & 2.7E-02 & 53,095 & 26,697 & 1,900 & 392,921 & $1,246,287$ & 19 \\
\hline 7/1/2005 & 3.4E-02 & 53,091 & 26,562 & 2,021 & 359,753 & $1,669,734$ & 19 \\
\hline $7 / 1 / 2006$ & 3.3E-02 & 53,040 & 26,810 & 1,830 & 382,760 & $1,264,773$ & 19 \\
\hline $7 / 1 / 2007$ & 2.8E-02 & 53,079 & 26,733 & 1,877 & 395,993 & $1,272,707$ & 19 \\
\hline
\end{tabular}

GROSS ALPHA ACTIVITY

\begin{tabular}{|c|c|c|c|c|c|c|c|}
\hline 7/1/1996 & $2.6 \mathrm{E}+03$ & 52,817 & 27,225 & 1,647 & 219,206 & 746,585 & 15 \\
\hline 7/1/1997 & $2.3 \mathrm{E}+03$ & 53,087 & 26,342 & 2,221 & 311,236 & $1,257,658$ & 17 \\
\hline 7/1/1998 & $1.8 \mathrm{E}+03$ & 53,206 & 26,661 & 1,881 & 274,727 & $1,029,322$ & 16 \\
\hline 7/1/1999 & $1.9 \mathrm{E}+03$ & 52,823 & 26,486 & 2,219 & 285,837 & $1,331,894$ & 19 \\
\hline $7 / 1 / 2000$ & $3.3 E+02$ & 53,489 & 25,892 & 2,525 & 309,155 & 889,617 & 19 \\
\hline $7 / 1 / 2001$ & $5.2 \mathrm{E}+02$ & 53,523 & 25,086 & 3,313 & 72,091 & 775,688 & 19 \\
\hline $7 / 1 / 2002$ & $3.9 \mathrm{E}+02$ & 53,585 & 27,260 & 1,181 & 173,998 & 177,472 & 17 \\
\hline $7 / 1 / 2003$ & $8.6 \mathrm{E}+02$ & 53,528 & 25,435 & 2,967 & 75,275 & 971,937 & 19 \\
\hline $7 / 1 / 2004$ & $7.0 \mathrm{E}+02$ & 53,596 & 25,842 & 2,554 & 154,008 & $1,392,220$ & 19 \\
\hline 7/1/2005 & $7.5 \mathrm{E}+01$ & 53,383 & 26,741 & 1,738 & 223,548 & $1,604,847$ & 19 \\
\hline $7 / 1 / 2006$ & $2.4 \mathrm{E}+02$ & 53,238 & 26,386 & 2,122 & 303,616 & 898,738 & 18 \\
\hline $7 / 1 / 2007$ & $9.3 \mathrm{E}+01$ & 53,242 & 26,248 & 2,249 & 315,565 & 874,664 & 19 \\
\hline \multicolumn{8}{|l|}{ GROSS BETA ACTIVITY } \\
\hline $7 / 1 / 1996$ & $3.2 \mathrm{E}+03$ & 52,910 & 27,083 & 1,687 & 163,213 & 379,810 & 13 \\
\hline 7/1/1997 & $5.7 \mathrm{E}+03$ & 52,996 & 26,696 & 1,951 & 213,104 & $1,156,940$ & 17 \\
\hline $7 / 1 / 1998$ & $6.6 \mathrm{E}+03$ & 53,106 & 26,941 & 1,684 & 217,234 & 782,535 & 17 \\
\hline $7 / 1 / 1999$ & $3.9 \mathrm{E}+03$ & 52,891 & 26,718 & 1,988 & 396,578 & 730,782 & 19 \\
\hline $7 / 1 / 2000$ & $4.9 \mathrm{E}+02$ & 53,324 & 26,774 & 1,730 & 58,871 & 535,391 & 18 \\
\hline
\end{tabular}


Project: CR Landfill VI

Location: West Chestnut Ridge

\begin{tabular}{|c|c|c|c|c|c|c|c|}
\hline \multirow[b]{2}{*}{ Effective Date } & \multirow{2}{*}{$\begin{array}{r}\text { 0th Moment } \\
\text { Estimated } \\
\text { Mass }(\mathrm{kg})\end{array}$} & \multicolumn{3}{|c|}{ 1st Moment (Center of Mass) } & \multicolumn{2}{|c|}{$\underline{\text { 2nd Moment (Spread) }}$} & \multirow[b]{2}{*}{$\begin{array}{c}\text { Number of } \\
\text { Wells }\end{array}$} \\
\hline & & $\mathrm{Xc}(\mathrm{ft})$ & Yc (ft) & $\begin{array}{c}\text { Source } \\
\text { Distance (ft) }\end{array}$ & $\underset{(s q \mathrm{ft})}{\operatorname{Sigma} X X}$ & $\begin{array}{c}\text { Sigma } Y Y \\
(\mathrm{sq} \mathrm{ft})\end{array}$ & \\
\hline \multicolumn{8}{|l|}{ GROSS BETA ACTIVITY } \\
\hline $7 / 1 / 2001$ & $6.2 \mathrm{E}+03$ & 53,335 & 26,979 & 1,539 & 90,005 & 200,392 & 19 \\
\hline $7 / 1 / 2002$ & $2.7 \mathrm{E}+03$ & 53,383 & 27,145 & 1,369 & 248,537 & 220,328 & 17 \\
\hline $7 / 1 / 2003$ & $2.3 E+03$ & 53,509 & 26,492 & 1,936 & 232,949 & 914,658 & 19 \\
\hline $7 / 1 / 2004$ & $3.5 \mathrm{E}+03$ & 53,512 & 26,470 & 1,957 & 171,051 & 861,333 & 19 \\
\hline $7 / 1 / 2005$ & $1.2 \mathrm{E}+03$ & 53,650 & 27,124 & 1,289 & 92,788 & 342,211 & 19 \\
\hline $7 / 1 / 2006$ & $2.4 \mathrm{E}+03$ & 53,460 & 27,235 & 1,254 & 143,342 & 224,166 & 19 \\
\hline $7 / 1 / 2007$ & $1.1 \mathrm{E}+03$ & 53,235 & 27,090 & 1,490 & 25,360 & 40,895 & 19 \\
\hline \multicolumn{8}{|l|}{ NITRATE } \\
\hline $7 / 1 / 2005$ & $2.9 \mathrm{E}+02$ & 53,263 & 26,696 & 1,826 & 472,836 & $1,322,473$ & 19 \\
\hline $7 / 1 / 2006$ & $2.7 \mathrm{E}+02$ & 53,295 & 26,522 & 1,974 & 464,840 & $1,508,834$ & 19 \\
\hline $7 / 1 / 2007$ & $1.9 \mathrm{E}+02$ & 53,401 & 26,234 & 2,214 & 391,004 & $1,452,500$ & 19 \\
\hline
\end{tabular}


Project: CR Landfill VI

Location: West Chestnut Ridge
User Name: $\mathrm{MV}$

State: Tennessee

\begin{tabular}{|c|c|c|c|c|c|}
\hline Moment Type & Constituent & $\begin{array}{l}\text { Coefficient } \\
\text { of Variation }\end{array}$ & $\begin{array}{l}\text { Mann-Kendall } \\
\text { S Statistic }\end{array}$ & $\begin{array}{l}\text { Confidence } \\
\text { in Trend }\end{array}$ & $\begin{array}{c}\text { Moment } \\
\text { Trend }\end{array}$ \\
\hline \multicolumn{6}{|c|}{ Zeroth Moment: Mass } \\
\hline & 1,1-DICHLOROETHENE & 0.00 & 0 & $0.0 \%$ & $\mathrm{~N} / \mathrm{A}$ \\
\hline & CADMIUM & 0.14 & 12 & $77.0 \%$ & NT \\
\hline & GROSS ALPHA ACTIVITY & 0.92 & -44 & $99.9 \%$ & $\mathrm{D}$ \\
\hline & GROSS BETA ACTIVITY & 0.62 & -28 & $96.9 \%$ & $\mathrm{D}$ \\
\hline & NITRATE & 0.00 & 0 & $0.0 \%$ & $\mathrm{~N} / \mathrm{A}$ \\
\hline \multicolumn{6}{|c|}{ 1st Moment: Distance to Source } \\
\hline & 1,1-DICHLOROETHENE & 0.00 & 0 & $0.0 \%$ & $\mathrm{~N} / \mathrm{A}$ \\
\hline & CADMIUM & 0.10 & -2 & $52.7 \%$ & $\mathrm{~S}$ \\
\hline & GROSS ALPHA ACTIVITY & 0.26 & 10 & $72.7 \%$ & NT \\
\hline & GROSS BETA ACTIVITY & 0.16 & -24 & $94.2 \%$ & PD \\
\hline & NITRATE & 0.00 & 0 & $0.0 \%$ & $\mathrm{~N} / \mathrm{A}$ \\
\hline \multicolumn{6}{|c|}{ 2nd Moment: Sigma XX } \\
\hline & 1,1-DICHLOROETHENE & 0.00 & 0 & $0.0 \%$ & $\mathrm{~N} / \mathrm{A}$ \\
\hline & CADMIUM & 0.09 & 6 & $63.1 \%$ & NT \\
\hline & GROSS ALPHA ACTIVITY & 0.40 & 4 & $58.0 \%$ & NT \\
\hline & GROSS BETA ACTIVITY & 0.59 & -16 & $84.5 \%$ & $\mathrm{~S}$ \\
\hline & NITRATE & 0.00 & 0 & $0.0 \%$ & $\mathrm{~N} / \mathrm{A}$ \\
\hline \multicolumn{6}{|c|}{ 2nd Moment: Sigma YY } \\
\hline & 1,1-DICHLOROETHENE & 0.00 & 0 & $0.0 \%$ & N/A \\
\hline & CADMIUM & 0.24 & 30 & $97.8 \%$ & 1 \\
\hline & GROSS ALPHA ACTIVITY & 0.37 & 4 & $58.0 \%$ & NT \\
\hline & GROSS BETA ACTIVITY & 0.66 & -24 & $94.2 \%$ & PD \\
\hline & NITRATE & 0.00 & 0 & $0.0 \%$ & N/A \\
\hline
\end{tabular}

Note: The following assumptions were applied for the calculation of the Zeroth Moment:

Porosity: $0.10 \quad$ Saturated Thickness: Uniform: $50 \mathrm{ft}$

Mann-Kendall Trend test performed on all sample events for each constituent. Increasing (I); Probably Increasing (PI); Stable (S); Probably Decreasing (PD); Decreasing (D); No Trend (NT); Not Applicable (N/A)-Due to insufficient Data (< 4 sampling events).

Note: The Sigma XX and Sigma YY components are estimated using the given field coordinate system and then rotated to align with the estimated groundwater flow direction. Moments are not calculated for sample events with less than 6 wells. 


\section{MAROS Statistical Trend Analysis Summary}

Project: $\quad$ CR Landfill VI

Location: West Chestnut Ridge

Time Period: $1 / 1 / 1996$ to $10 / 15 / 2007$

Consolidation Period: No Time Consolidation

Consolidation Type: Median

Duplicate Consolidation: Average

ND Values: Specified Detection Limit

J Flag Values : Actual Value
User Name: $\quad$ MV

State: Tennessee

\begin{tabular}{|c|c|c|c|c|c|c|c|c|}
\hline Well & $\begin{array}{c}\text { Sourcel } \\
\text { Tail }\end{array}$ & $\begin{array}{l}\text { Number } \\
\text { of } \\
\text { Samples }\end{array}$ & $\begin{array}{c}\text { Number } \\
\text { of } \\
\text { Detects }\end{array}$ & $\begin{array}{l}\text { Average } \\
\text { Conc. } \\
\text { (mg/L) }\end{array}$ & $\begin{array}{l}\text { Median } \\
\text { Conc. } \\
\text { (mg/L) }\end{array}$ & $\begin{array}{c}\text { All } \\
\text { Samples } \\
\text { "ND" ? }\end{array}$ & $\begin{array}{l}\text { Mann- } \\
\text { Kendall } \\
\text { Trend }\end{array}$ & $\begin{array}{l}\text { Linear } \\
\text { Regression } \\
\text { Trend }\end{array}$ \\
\hline \multicolumn{9}{|c|}{ 1,1-DICHLOROETHENE } \\
\hline GW-141 & s & 6 & 0 & $1.0 \mathrm{E}-03$ & $1.0 \mathrm{E}-03$ & Yes & ND & ND \\
\hline GW-217 & s & 6 & 0 & $1.0 \mathrm{E}-03$ & $1.0 \mathrm{E}-03$ & Yes & ND & ND \\
\hline GW-305 & $\mathrm{T}$ & 12 & 12 & $5.7 \mathrm{E}-03$ & $5.8 \mathrm{E}-03$ & No & NT & NT \\
\hline GW-540 & $\mathrm{T}$ & 6 & 0 & $1.0 \mathrm{E}-03$ & $1.0 \mathrm{E}-03$ & Yes & ND & ND \\
\hline GW-542 & $\mathrm{T}$ & 6 & 0 & $1.0 \mathrm{E}-03$ & $1.0 \mathrm{E}-03$ & Yes & ND & ND \\
\hline GW-543 & $\mathrm{T}$ & 6 & 0 & $1.0 \mathrm{E}-03$ & $1.0 \mathrm{E}-03$ & Yes & ND & ND \\
\hline GW-544 & $\mathrm{T}$ & 6 & 0 & $1.0 \mathrm{E}-03$ & $1.0 \mathrm{E}-03$ & Yes & ND & ND \\
\hline GW-709 & $\mathrm{T}$ & 6 & 0 & $1.0 \mathrm{E}-03$ & $1.0 \mathrm{E}-03$ & Yes & ND & ND \\
\hline SCR2_2SP & $\mathrm{T}$ & 4 & 0 & $1.0 \mathrm{E}-03$ & $1.0 \mathrm{E}-03$ & Yes & ND & ND \\
\hline
\end{tabular}

GROSS ALPHA ACTIVITY

\begin{tabular}{|c|c|c|c|c|c|c|c|c|}
\hline 1090 & $\mathrm{~s}$ & 22 & 14 & $1.3 \mathrm{E}+00$ & $1.3 \mathrm{E}+00$ & No & $\mathrm{s}$ & $\mathrm{s}$ \\
\hline GW-141 & $\mathrm{s}$ & 23 & 7 & $1.3 \mathrm{E}+00$ & 1.0E-02 & No & $\mathrm{D}$ & D \\
\hline GW-203 & $\mathrm{s}$ & 21 & 14 & $2.2 \mathrm{E}+00$ & $1.1 \mathrm{E}+00$ & No & NT & NT \\
\hline GW-205 & $\mathrm{s}$ & 23 & 14 & $1.6 \mathrm{E}+00$ & $1.0 \mathrm{E}+00$ & No & NT & NT \\
\hline GW-217 & $\mathrm{s}$ & 21 & 5 & 2.9E-01 & 1.0E-02 & No & $\mathrm{D}$ & D \\
\hline GW-221 & $\mathrm{T}$ & 22 & 9 & $1.2 \mathrm{E}+00$ & 1.0E-02 & No & NT & NT \\
\hline GW-305 & $\mathrm{T}$ & 38 & 11 & 7.4E-01 & 1.0E-02 & No & PD & $\mathrm{D}$ \\
\hline GW-521 & $\mathrm{T}$ & 21 & 5 & 2.7E-01 & 1.0E-02 & No & $\mathrm{D}$ & D \\
\hline GW-522 & $\mathrm{T}$ & 21 & 5 & $6.2 \mathrm{E}-01$ & 1.0E-02 & No & $\mathrm{D}$ & D \\
\hline GW-540 & $\mathrm{T}$ & 21 & 5 & 4.8E-01 & 1.0E-02 & No & $\mathrm{D}$ & $\mathrm{D}$ \\
\hline GW-542 & $\mathrm{T}$ & 21 & 5 & $6.8 \mathrm{E}-01$ & 1.0E-02 & No & $\mathrm{D}$ & D \\
\hline GW-543 & $\mathrm{T}$ & 22 & 6 & 2.4E-01 & 1.0E-02 & No & $\mathrm{D}$ & $\mathrm{D}$ \\
\hline GW-544 & $\mathrm{T}$ & 24 & 11 & $1.1 \mathrm{E}+00$ & 1.0E-02 & No & $\mathrm{D}$ & D \\
\hline GW-709 & $\mathrm{T}$ & 21 & 10 & $1.2 \mathrm{E}+00$ & 1.0E-02 & No & NT & PD \\
\hline GW-757 & $\mathrm{T}$ & 24 & 20 & $2.5 E+00$ & $2.4 \mathrm{E}+00$ & No & $\mathrm{D}$ & $\mathrm{s}$ \\
\hline GW-827 & $\mathrm{T}$ & 21 & 6 & 2.3E-01 & 1.0E-02 & No & $\mathrm{D}$ & D \\
\hline SCR1_25SP & $\mathrm{T}$ & 18 & 14 & $4.2 \mathrm{E}+00$ & $2.5 E+00$ & No & NT & $\mathrm{PD}$ \\
\hline SCR2_1SP & $\mathrm{T}$ & 17 & 14 & $2.7 \mathrm{E}+00$ & $2.3 E+00$ & No & $\mathrm{D}$ & $\mathrm{D}$ \\
\hline
\end{tabular}




\section{MAROS Statistical Trend Analysis Summary}

\begin{tabular}{ccccccccc} 
& & Number & Number & Average & Median & All & Mann- & Linear \\
Well & Sourcel & of & of & Conc. & Conc. & Samples & Kendall & Regression \\
Tail & Samples & Detects & (mg/L) & (mg/L) & "ND" ? & Trend & Trend \\
\hline
\end{tabular}

GROSS ALPHA ACTIVITY

SCR2_2SP

15

$12 \quad 1.4 \mathrm{E}+00 \quad 1.2 \mathrm{E}+00$

No

S

D

GROSS BETA ACTIVITY

\begin{tabular}{|c|c|c|c|c|c|c|c|c|}
\hline 1090 & S & 22 & 15 & $3.0 \mathrm{E}+00$ & $2.6 \mathrm{E}+00$ & No & NT & NT \\
\hline GW-141 & $\mathrm{S}$ & 22 & 10 & $2.1 \mathrm{E}+00$ & 1.0E-03 & No & D & $\mathrm{D}$ \\
\hline GW-203 & S & 20 & 16 & $5.7 \mathrm{E}+00$ & $3.5 \mathrm{E}+00$ & No & NT & NT \\
\hline GW-205 & $S$ & 22 & 21 & $5.8 \mathrm{E}+01$ & $6.5 \mathrm{E}+01$ & No & I & 1 \\
\hline GW-217 & $\mathrm{s}$ & 23 & 14 & $1.4 \mathrm{E}+00$ & $1.5 E+00$ & No & NT & $P D$ \\
\hline GW-221 & $\mathrm{T}$ & 22 & 12 & $2.3 \mathrm{E}+00$ & 7.3E-01 & No & NT & NT \\
\hline GW-305 & $\mathrm{T}$ & 36 & 8 & $1.4 \mathrm{E}+00$ & 1.0E-03 & No & PD & D \\
\hline GW-521 & $\mathrm{T}$ & 21 & 6 & 8.3E-01 & 1.0E-03 & No & $\mathrm{D}$ & D \\
\hline GW-522 & $\mathrm{T}$ & 20 & 5 & 6.7E-01 & 1.0E-03 & No & D & D \\
\hline GW-540 & $\mathrm{T}$ & 20 & 10 & $1.4 \mathrm{E}+00$ & 8.0E-01 & No & NT & $P D$ \\
\hline GW-542 & T & 22 & 17 & $2.4 \mathrm{E}+00$ & $2.5 \mathrm{E}+00$ & No & D & $\mathrm{s}$ \\
\hline GW-543 & $\mathrm{T}$ & 23 & 10 & $1.1 \mathrm{E}+00$ & 1.0E-03 & No & D & D \\
\hline GW-544 & $\mathrm{T}$ & 22 & 12 & $2.3 \mathrm{E}+00$ & $2.6 \mathrm{E}+00$ & No & D & D \\
\hline GW-709 & $\mathrm{T}$ & 20 & 13 & $2.2 \mathrm{E}+00$ & $2.1 \mathrm{E}+00$ & No & NT & NT \\
\hline GW-757 & $\mathrm{T}$ & 24 & 24 & $1.3 \mathrm{E}+01$ & $1.5 \mathrm{E}+01$ & No & 1 & I \\
\hline GW-827 & $\mathrm{T}$ & 22 & 11 & $1.7 \mathrm{E}+00$ & $6.2 \mathrm{E}-01$ & No & PD & D \\
\hline SCR1_25SP & $\mathrm{T}$ & 18 & 12 & $2.0 \mathrm{E}+00$ & $2.0 \mathrm{E}+00$ & No & NT & $\mathrm{S}$ \\
\hline SCR2_1SP & $\mathrm{T}$ & 15 & 11 & $2.1 \mathrm{E}+00$ & $1.6 \mathrm{E}+00$ & No & NT & NT \\
\hline SCR2_2SP & $\mathrm{T}$ & 13 & 9 & $2.0 \mathrm{E}+00$ & $1.9 \mathrm{E}+00$ & No & D & D \\
\hline \multicolumn{9}{|l|}{ LEAD } \\
\hline 1090 & $\mathrm{~s}$ & 17 & 2 & 1.2E-04 & 5.2E-05 & No & NT & D \\
\hline GW-141 & $\mathrm{S}$ & 18 & 4 & 1.6E-03 & 5.2E-05 & No & D & D \\
\hline GW-203 & $S$ & 16 & 2 & 1.3E-04 & 5.2E-05 & No & NT & D \\
\hline GW-205 & $\mathrm{S}$ & 17 & 2 & 2.4E-04 & 5.2E-05 & No & NT & D \\
\hline GW-217 & $\mathrm{S}$ & 18 & 5 & 1.6E-04 & 5.2E-05 & No & $\mathrm{D}$ & D \\
\hline GW-221 & $\mathrm{T}$ & 17 & 1 & 1.2E-04 & 5.2E-05 & No & NT & NT \\
\hline GW-305 & $\mathrm{T}$ & 28 & 1 & 9.7E-05 & 5.2E-05 & No & NT & PD \\
\hline GW-521 & $\mathrm{T}$ & 20 & 4 & 2.1E-04 & 5.2E-05 & No & PD & D \\
\hline GW-522 & $\mathrm{T}$ & 18 & 5 & $4.8 \mathrm{E}-04$ & 5.2E-05 & No & D & D \\
\hline GW-540 & $\mathrm{T}$ & 16 & 2 & $1.8 \mathrm{E}-04$ & 5.2E-05 & No & NT & NT \\
\hline GW-542 & $\mathrm{T}$ & 17 & 6 & 2.4E-04 & 5.2E-05 & No & D & D \\
\hline GW-543 & $\mathrm{T}$ & 18 & 0 & $5.2 \mathrm{E}-05$ & 5.2E-05 & Yes & ND & ND \\
\hline GW-544 & T & 18 & 1 & 6.5E-05 & 5.2E-05 & No & $\mathrm{s}$ & $\mathrm{S}$ \\
\hline GW-709 & $\mathrm{T}$ & 18 & 1 & $9.4 \mathrm{E}-05$ & 5.2E-05 & No & NT & PD \\
\hline GW-757 & $\mathrm{T}$ & 18 & 1 & $9.2 \mathrm{E}-05$ & $5.2 \mathrm{E}-05$ & No & NT & PD \\
\hline GW-827 & $\mathrm{T}$ & 18 & 0 & $5.2 \mathrm{E}-05$ & 5.2E-05 & Yes & ND & ND \\
\hline SCR1_25SP & $\mathrm{T}$ & 12 & 0 & $5.2 \mathrm{E}-05$ & 5.2E-05 & Yes & ND & ND \\
\hline SCR2_1SP & T & 14 & 4 & 2.0E-04 & 5.2E-05 & No & NT & NT \\
\hline SCR2_2SP & $\mathrm{T}$ & 14 & 2 & 4.7E-03 & 5.2E-05 & No & NT & D \\
\hline
\end{tabular}

NITRATE

\begin{tabular}{lllllllllll}
\hline 1090 & $S$ & 6 & 6 & $6.8 \mathrm{E}-01$ & $6.7 \mathrm{E}-01$ & No & & \\
\hline
\end{tabular}




\section{MAROS Statistical Trend Analysis Summary}

\begin{tabular}{|c|c|c|c|c|c|c|c|c|}
\hline Well & $\begin{array}{c}\text { Sourcel } \\
\text { Tail }\end{array}$ & $\begin{array}{l}\text { Number } \\
\text { of } \\
\text { Samples }\end{array}$ & $\begin{array}{c}\text { Number } \\
\text { of } \\
\text { Detects }\end{array}$ & $\begin{array}{l}\text { Average } \\
\text { Conc. } \\
\text { (mg/L) }\end{array}$ & $\begin{array}{l}\text { Median } \\
\text { Conc. } \\
\text { (mg/L) }\end{array}$ & $\begin{array}{c}\text { All } \\
\text { Samples } \\
\text { "ND" ? }\end{array}$ & $\begin{array}{l}\text { Mann- } \\
\text { Kendall } \\
\text { Trend }\end{array}$ & $\begin{array}{l}\text { Linear } \\
\text { Regression } \\
\text { Trend }\end{array}$ \\
\hline \multicolumn{9}{|l|}{ NITRATE } \\
\hline GW-141 & $\mathrm{S}$ & 6 & 5 & 3.3E-01 & $3.4 \mathrm{E}-01$ & No & $\mathrm{D}$ & PD \\
\hline GW-203 & $\mathrm{S}$ & 6 & 6 & 7.8E-01 & 5.7E-01 & No & $\mathrm{D}$ & $\mathrm{D}$ \\
\hline GW-205 & $\mathrm{S}$ & 6 & 6 & $9.0 \mathrm{E}-02$ & 5.2E-02 & No & NT & NT \\
\hline GW-217 & $\mathrm{S}$ & 6 & 5 & 2.9E-01 & $3.2 \mathrm{E}-01$ & No & $\mathrm{S}$ & PD \\
\hline GW-221 & $\mathrm{T}$ & 6 & 6 & 4.0E-01 & 4.1E-01 & No & $\mathrm{D}$ & $\mathrm{S}$ \\
\hline GW-305 & $\mathrm{T}$ & 12 & 9 & 3.7E-01 & 3.8E-01 & No & $\mathrm{D}$ & D \\
\hline GW-521 & $\mathrm{T}$ & 6 & 5 & 2.1E-01 & $2.2 \mathrm{E}-01$ & No & $\mathrm{S}$ & $\mathrm{S}$ \\
\hline GW-522 & $\mathrm{T}$ & 6 & 5 & 3.6E-01 & 4.0E-01 & No & $\mathrm{S}$ & PD \\
\hline GW-540 & $\mathrm{T}$ & 6 & 2 & 4.1E-02 & 2.0E-02 & No & PD & $\mathrm{D}$ \\
\hline GW-542 & $\mathrm{T}$ & 6 & 5 & $2.5 \mathrm{E}-01$ & $2.9 \mathrm{E}-01$ & No & NT & $\mathrm{S}$ \\
\hline GW-543 & $\mathrm{T}$ & 6 & 5 & 2.0E-01 & 2.3E-01 & No & NT & $\mathrm{S}$ \\
\hline GW-544 & $\mathrm{T}$ & 6 & 6 & 4.8E-01 & 4.6E-01 & No & NT & NT \\
\hline GW-709 & $\mathrm{T}$ & 6 & 3 & 2.6E-02 & $2.2 \mathrm{E}-02$ & No & $\mathrm{S}$ & $\mathrm{S}$ \\
\hline GW-757 & $\mathrm{T}$ & 6 & 5 & 2.3E-01 & 2.6E-01 & No & NT & $\mathrm{S}$ \\
\hline GW-827 & $\mathrm{T}$ & 6 & 5 & 7.1E-02 & 7.9E-02 & No & $\mathrm{S}$ & $\mathrm{S}$ \\
\hline SCR1_25SP & $\mathrm{T}$ & 6 & 6 & $1.1 \mathrm{E}-01$ & 1.1E-01 & No & $\mathrm{PI}$ & I \\
\hline SCR2_1SP & $\mathrm{T}$ & 4 & 4 & $2.2 \mathrm{E}-01$ & 2.0E-01 & No & NT & NT \\
\hline SCR2_2SP & $\mathrm{T}$ & 4 & 4 & $1.0 \mathrm{E}+00$ & 8.9E-01 & No & $\mathrm{S}$ & $\mathrm{S}$ \\
\hline
\end{tabular}

Note: Increasing (I); Probably Increasing (PI); Stable (S); Probably Decreasing (PD); Decreasing (D); No Trend (NT); Not Applicable (N/A); Not Applicable (N/A) - Due to insufficient Data (< 4 sampling events); No Detectable Concentration (NDC)

The Number of Samples and Number of Detects shown above are post-consolidation values. 


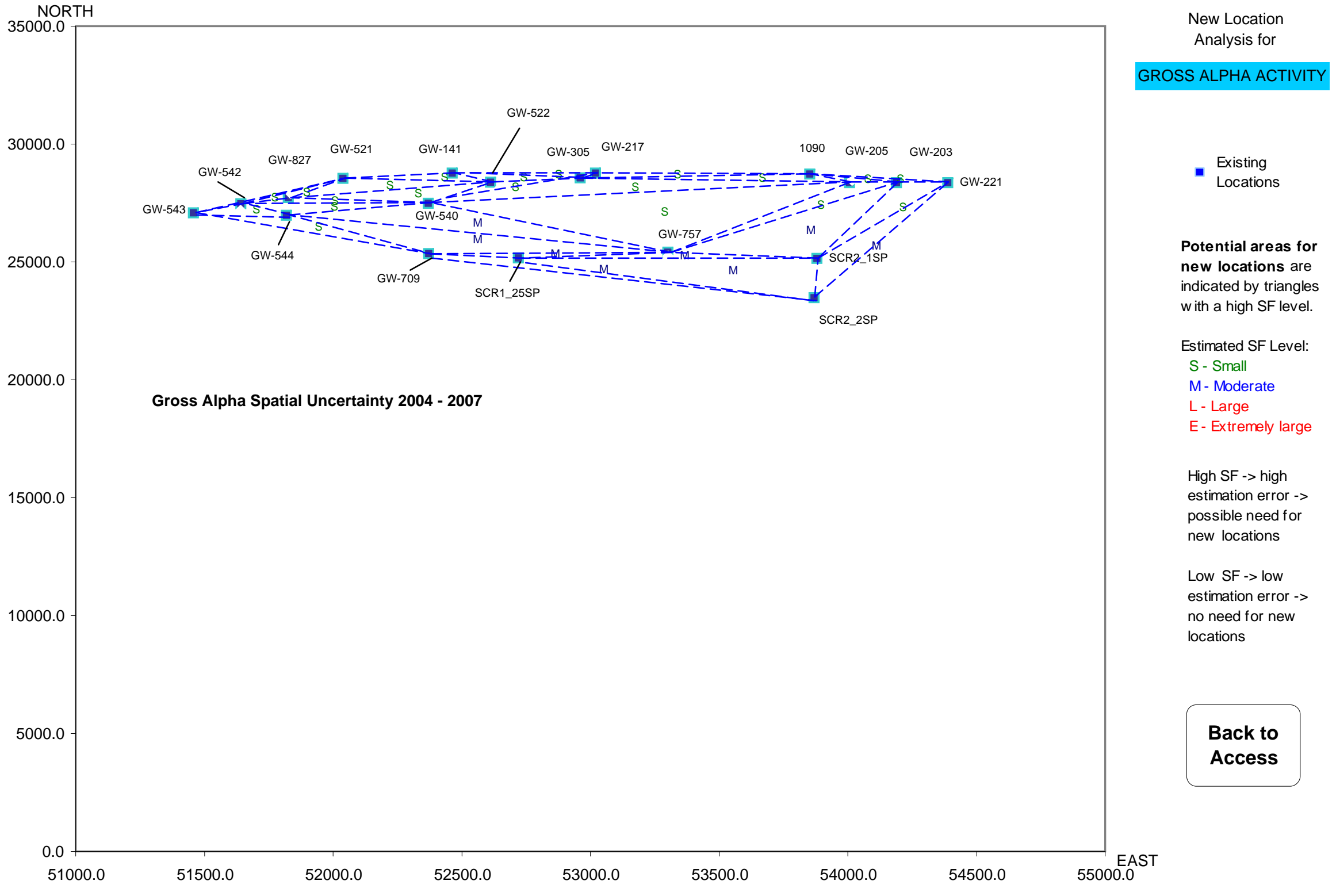


APPENDIX C.8

\section{CHESTNUT RIDGE SECURITY PITS}




\section{MAROS COC Assessment}

Project: $\quad$ CR Security Pits

Location: CRSP

Toxicity:

Contaminant of Concern

TETRACHLOROETHYLENE(PCE)

1,1-DICHLOROETHENE
User Name: $\quad$ MV

State: Tennessee

Note: Top COCs by toxicity were determined by examining a representative concentration for each compound over the entire site. The compound representative concentrations are then compared with the chosen PRG for that compound, with the percentage exceedance from the PRG determining the compound's toxicity. All compounds above exceed the PRG.

\section{Prevalence:}

\begin{tabular}{|c|c|c|c|c|c|}
\hline Contaminant of Concern & Class & $\begin{array}{l}\text { Total } \\
\text { Wells }\end{array}$ & $\begin{array}{c}\text { Total } \\
\text { Exceedances }\end{array}$ & $\begin{array}{c}\text { Percent } \\
\text { Exceedances }\end{array}$ & $\begin{array}{c}\text { Total } \\
\text { detects }\end{array}$ \\
\hline TETRACHLOROETHYLENE(PCE) & ORG & 13 & 8 & $61.5 \%$ & 10 \\
\hline 1,1-DICHLOROETHENE & ORG & 13 & 4 & $30.8 \%$ & 10 \\
\hline
\end{tabular}

Note: Top COCs by prevalence were determined by examining a representative concentration for each well location at the site. The total exceedances (values above the chosen PRGs) are compared to the total number of wells to determine the prevalence of the compound.

\section{Mobility:}

Contaminant of Concern

1,1-DICHLOROETHENE

TETRACHLOROETHYLENE(PCE)
Kd

\subsection{3}

0.923

Note: Top COCs by mobility were determined by examining each detected compound in the dataset and comparing their mobilities (Koc's for organics, assume foc $=0.001$, and Kd's for metals).

\section{Contaminants of Concern (COC's)}

TETRACHLOROETHYLENE(PCE)

\section{1,1-DICHLOROETHENE}




\section{MAROS Spatial Moment Analysis Summary}

Project: CR Security Pits

Location: CRSP
User Name: MV

State: Tennessee

\begin{tabular}{|c|c|c|c|}
\hline $\mathrm{h}$ Moment & 1st & ent (Cer & of Mass) \\
\hline $\begin{array}{l}\text { ted } \\
\text { Ka }\end{array}$ & $c(\mathrm{ft})$ & $Y c(f t)$ & \\
\hline
\end{tabular}

2nd Moment (Spread)

Sigma $X X \quad$ Sigma $Y Y$

(sq ft)

(sq ft)

Number of

Wells

1,1-DICHLOROETHENE

$\begin{array}{rrrrrrrr}1 / 1 / 1997 & 9.3 \mathrm{E}-01 & 57,976 & 27,560 & 1,158 & 234,839 & 616,057 & 8 \\ 1 / 1 / 1999 & 0.0 \mathrm{E}+00 & & & & & & 4 \\ 1 / 1 / 2001 & 9.0 \mathrm{E}-01 & 57,959 & 27,555 & 1,174 & 253,151 & 626,393 & 9 \\ 1 / 1 / 2003 & 0.0 \mathrm{E}+00 & & & & & & 3 \\ 1 / 1 / 2005 & 2.6 \mathrm{E}+00 & 57,770 & 27,374 & 1,434 & 229,973 & 668,040 & 9 \\ 1 / 1 / 2007 & 1.4 \mathrm{E}+00 & 58,185 & 27,817 & 841 & 243,169 & 525,230 & 13\end{array}$

GROSS ALPHA ACTIVITY

$\begin{array}{lllllcrl}1 / 1 / 1997 & 1.4 \mathrm{E}+02 & 57,875 & 27,059 & 1,572 & 259,802 & 370,064 & 6 \\ 1 / 1 / 1999 & 0.0 \mathrm{E}+00 & & & & & & 3 \\ 1 / 1 / 2001 & 6.1 \mathrm{E}+02 & 57,763 & 27,254 & 1,514 & 203,896 & 647,577 & 8 \\ 1 / 1 / 2003 & 0.0 \mathrm{E}+00 & & & & & & 3 \\ 1 / 1 / 2005 & 4.6 \mathrm{E}+02 & 57,469 & 26,950 & 1,936 & 64,195 & 523,062 & 7 \\ 1 / 1 / 2007 & 1.1 \mathrm{E}+02 & 57,507 & 26,714 & 2,075 & 117,167 & 308,597 & 13\end{array}$

GROSS BETA ACTIVITY

\begin{tabular}{|c|c|c|c|c|}
\hline 2005 & $0.0 \mathrm{E}+00$ & & & \\
\hline $1 / 1 / 2007$ & $4.9 \mathrm{E}+03$ & 57,662 & 26,819 & 1,893 \\
\hline
\end{tabular}

NITRATE

\begin{tabular}{|c|c|c|c|c|c|c|}
\hline 1/1/2005 & $0.0 \mathrm{E}+00$ & & & & & \\
\hline $1 / 1 / 2007$ & $2.4 \mathrm{E}+02$ & 58,374 & 27,101 & 1,260 & 213,385 & 327,238 \\
\hline
\end{tabular}

TETRACHLOROETHYLENE(PCE)

$\begin{array}{lrrrrcrc}1 / 1 / 1997 & 2.3 \mathrm{E}+00 & 58,244 & 27,243 & 1,201 & 382,186 & 588,949 & 8 \\ 1 / 1 / 1999 & 0.0 \mathrm{E}+00 & & & & & & 4 \\ 1 / 1 / 2001 & 1.3 \mathrm{E}+00 & 58,137 & 27,122 & 1,361 & 451,458 & 567,035 & 9 \\ 1 / 1 / 2003 & 0.0 \mathrm{E}+00 & & & & & & 3 \\ 1 / 1 / 2005 & 7.2 \mathrm{E}-01 & 57,588 & 27,058 & 1,775 & 165,225 & 622,686 & 9 \\ 1 / 1 / 2007 & 1.3 \mathrm{E}+00 & 58,169 & 27,341 & 1,167 & 445,159 & 703,495 & 13\end{array}$


Project: CR Security Pits

Location: CRSP
User Name: $\mathrm{MV}$

State: Tennessee

\begin{tabular}{|c|c|c|c|c|c|}
\hline Moment Type & Constituent & $\begin{array}{l}\text { Coefficient } \\
\text { of Variation }\end{array}$ & $\begin{array}{l}\text { Mann-Kendall } \\
\text { S Statistic }\end{array}$ & $\begin{array}{l}\text { Confidence } \\
\text { in Trend }\end{array}$ & $\begin{array}{c}\text { Moment } \\
\text { Trend }\end{array}$ \\
\hline \multicolumn{6}{|c|}{ Zeroth Moment: Mass } \\
\hline & 1,1-DICHLOROETHENE & 0.99 & 4 & $70.3 \%$ & NT \\
\hline & GROSS ALPHA ACTIVITY & 1.16 & 0 & $42.3 \%$ & NT \\
\hline & GROSS BETA ACTIVITY & 0.00 & 0 & $0.0 \%$ & $\mathrm{~N} / \mathrm{A}$ \\
\hline & NITRATE & 0.00 & 0 & $0.0 \%$ & $\mathrm{~N} / \mathrm{A}$ \\
\hline & TETRACHLOROETHYLENE(PCE) & 0.95 & -2 & $57.0 \%$ & $\mathrm{~S}$ \\
\hline \multicolumn{6}{|c|}{ 1st Moment: Distance to Source } \\
\hline & 1,1-DICHLOROETHENE & 0.21 & 0 & $37.5 \%$ & $\mathrm{~s}$ \\
\hline & GROSS ALPHA ACTIVITY & 0.15 & 4 & $83.3 \%$ & NT \\
\hline & GROSS BETA ACTIVITY & 0.00 & 0 & $0.0 \%$ & $\mathrm{~N} / \mathrm{A}$ \\
\hline & NITRATE & 0.00 & 0 & $0.0 \%$ & $\mathrm{~N} / \mathrm{A}$ \\
\hline & TETRACHLOROETHYLENE(PCE) & 0.20 & 0 & $37.5 \%$ & $\mathrm{~S}$ \\
\hline \multicolumn{6}{|c|}{ 2nd Moment: Sigma XX } \\
\hline & 1,1-DICHLOROETHENE & 0.04 & 0 & $37.5 \%$ & $\mathrm{~S}$ \\
\hline & GROSS ALPHA ACTIVITY & 0.54 & -4 & $83.3 \%$ & $\mathrm{~S}$ \\
\hline & GROSS BETA ACTIVITY & 0.00 & 0 & $0.0 \%$ & $\mathrm{~N} / \mathrm{A}$ \\
\hline & NITRATE & 0.00 & 0 & $0.0 \%$ & $\mathrm{~N} / \mathrm{A}$ \\
\hline & TETRACHLOROETHYLENE(PCE) & 0.37 & 0 & $37.5 \%$ & $\mathrm{~S}$ \\
\hline \multicolumn{6}{|c|}{ 2nd Moment: Sigma YY } \\
\hline & 1,1-DICHLOROETHENE & 0.10 & 0 & $37.5 \%$ & $\mathrm{~S}$ \\
\hline & GROSS ALPHA ACTIVITY & 0.33 & -2 & $62.5 \%$ & $\mathrm{~S}$ \\
\hline & GROSS BETA ACTIVITY & 0.00 & 0 & $0.0 \%$ & $\mathrm{~N} / \mathrm{A}$ \\
\hline & NITRATE & 0.00 & 0 & $0.0 \%$ & $\mathrm{~N} / \mathrm{A}$ \\
\hline & TETRACHLOROETHYLENE(PCE) & 0.10 & 4 & $83.3 \%$ & NT \\
\hline
\end{tabular}

Note: The following assumptions were applied for the calculation of the Zeroth Moment:

Porosity: $0.10 \quad$ Saturated Thickness: Uniform: $50 \mathrm{ft}$

Mann-Kendall Trend test performed on all sample events for each constituent. Increasing (I); Probably Increasing (PI); Stable (S); Probably Decreasing (PD); Decreasing (D); No Trend (NT); Not Applicable (N/A)-Due to insufficient Data (< 4 sampling events).

Note: The Sigma $X X$ and Sigma $Y Y$ components are estimated using the given field coordinate system and then rotated to align with the estimated groundwater flow direction. Moments are not calculated for sample events with less than 6 wells. 


\section{MAROS Statistical Trend Analysis Summary}

Project: $\quad$ CR Security Pits

Location: CRSP

Time Period: $1 / 1 / 1986$ to $10 / 15 / 2007$

Consolidation Period: No Time Consolidation

Consolidation Type: Median

Duplicate Consolidation: Average

ND Values: Specified Detection Limit

J Flag Values : Actual Value
User Name: $\quad$ MV

State: Tennessee

\begin{tabular}{|c|c|c|c|c|c|c|c|c|}
\hline Well & $\begin{array}{c}\text { Sourcel } \\
\text { Tail }\end{array}$ & $\begin{array}{l}\text { Number } \\
\text { of } \\
\text { Samples }\end{array}$ & $\begin{array}{c}\text { Number } \\
\text { of } \\
\text { Detects }\end{array}$ & $\begin{array}{l}\text { Average } \\
\text { Conc. } \\
\text { (mg/L) }\end{array}$ & $\begin{array}{l}\text { Median } \\
\text { Conc. } \\
\text { (mg/L) }\end{array}$ & $\begin{array}{c}\text { All } \\
\text { Samples } \\
\text { "ND" ? }\end{array}$ & $\begin{array}{l}\text { Mann- } \\
\text { Kendall } \\
\text { Trend }\end{array}$ & $\begin{array}{l}\text { Linear } \\
\text { Regression } \\
\text { Trend }\end{array}$ \\
\hline \multicolumn{9}{|c|}{ 1,1-DICHLOROETHENE } \\
\hline GW-174 & s & 28 & 6 & $6.0 \mathrm{E}-04$ & $5.0 \mathrm{E}-04$ & No & PD & PD \\
\hline GW-175 & $\mathrm{T}$ & 36 & 5 & $6.1 \mathrm{E}-04$ & $5.0 \mathrm{E}-04$ & No & $\mathrm{s}$ & $\mathrm{s}$ \\
\hline GW-176 & $\mathrm{T}$ & 30 & 29 & $1.6 \mathrm{E}-02$ & $1.4 \mathrm{E}-02$ & No & 1 & I \\
\hline GW-179 & $\mathrm{T}$ & 26 & 26 & 4.4E-02 & 4.3E-02 & No & $\mathrm{D}$ & $\mathrm{D}$ \\
\hline GW-180 & s & 22 & 3 & $6.6 \mathrm{E}-04$ & $5.0 \mathrm{E}-04$ & No & $\mathrm{s}$ & $\mathrm{s}$ \\
\hline GW-322 & s & 23 & 23 & $9.9 \mathrm{E}-02$ & $9.5 \mathrm{E}-02$ & No & NT & $\mathrm{s}$ \\
\hline GW-514 & $\mathrm{T}$ & 28 & 0 & $5.0 \mathrm{E}-04$ & $5.0 \mathrm{E}-04$ & Yes & ND & ND \\
\hline GW-609 & s & 39 & 1 & $5.4 \mathrm{E}-04$ & $5.0 \mathrm{E}-04$ & No & NT & NT \\
\hline
\end{tabular}

GROSS ALPHA ACTIVITY

\begin{tabular}{|c|c|c|c|c|c|c|c|c|}
\hline GW-174 & $\mathrm{s}$ & 27 & 23 & $4.0 \mathrm{E}+00$ & $1.8 \mathrm{E}+00$ & No & PD & PD \\
\hline GW-175 & $\mathrm{T}$ & 25 & 24 & $1.6 \mathrm{E}+00$ & $1.2 \mathrm{E}+00$ & No & $\mathrm{D}$ & $\mathrm{D}$ \\
\hline GW-176 & $\mathrm{T}$ & 27 & 21 & $4.6 \mathrm{E}+00$ & $1.3 \mathrm{E}+00$ & No & NT & NT \\
\hline GW-177 & $\mathrm{T}$ & 55 & 50 & $2.3 \mathrm{E}+00$ & $2.2 \mathrm{E}+00$ & No & 1 & NT \\
\hline GW-178 & $\mathrm{T}$ & 16 & 15 & $2.8 \mathrm{E}+00$ & $2.3 \mathrm{E}+00$ & No & PD & D \\
\hline GW-179 & $\mathrm{T}$ & 24 & 19 & $3.2 \mathrm{E}+00$ & $2.8 \mathrm{E}+00$ & No & NT & 1 \\
\hline GW-180 & $\mathrm{s}$ & 16 & 15 & 1.7E+00 & $1.1 \mathrm{E}+00$ & No & NT & D \\
\hline GW-322 & $\mathrm{s}$ & 19 & 18 & $2.9 \mathrm{E}+00$ & $1.6 \mathrm{E}+00$ & No & NT & D \\
\hline GW-514 & $\mathrm{T}$ & 19 & 18 & $1.2 \mathrm{E}+00$ & 6.9E-01 & No & s & D \\
\hline GW-609 & $\mathrm{S}$ & 30 & 25 & $1.4 \mathrm{E}+00$ & 9.0E-01 & No & $\mathrm{D}$ & D \\
\hline GW-612 & $\mathrm{T}$ & 16 & 14 & $1.8 \mathrm{E}+00$ & $1.7 \mathrm{E}+00$ & No & PD & D \\
\hline GW-831 & $\mathrm{T}$ & 21 & 16 & $1.8 \mathrm{E}+00$ & $1.3 \mathrm{E}+00$ & No & NT & NT \\
\hline SCR3_5SP & $\mathrm{T}$ & 21 & 11 & $1.4 \mathrm{E}+00$ & $1.1 \mathrm{E}+00$ & No & NT & NT \\
\hline \multicolumn{9}{|c|}{ GROSS BETA ACTIVITY } \\
\hline GW-174 & $\mathrm{s}$ & 1 & 0 & $2.0 \mathrm{E}+00$ & $2.0 \mathrm{E}+00$ & Yes & ND & ND \\
\hline GW-175 & $\mathrm{T}$ & 1 & 0 & $2.0 \mathrm{E}+00$ & $2.0 \mathrm{E}+00$ & Yes & ND & ND \\
\hline GW-176 & $\mathrm{T}$ & 1 & 0 & $2.0 \mathrm{E}+00$ & $2.0 \mathrm{E}+00$ & Yes & ND & ND \\
\hline GW-177 & $\mathrm{T}$ & 6 & 3 & $3.2 \mathrm{E}+00$ & $2.6 \mathrm{E}+00$ & No & $\mathrm{s}$ & $\mathrm{s}$ \\
\hline GW-178 & $\mathrm{T}$ & 1 & 0 & $2.0 \mathrm{E}+00$ & $2.0 \mathrm{E}+00$ & Yes & ND & ND \\
\hline
\end{tabular}




\section{MAROS Statistical Trend Analysis Summary}

\begin{tabular}{ccccccccc} 
& & Number & Number & Average & Median & All & Mann- & Linear \\
Well & Sourcel & of & of & Conc. & Conc. & Samples & Kendall & Regression \\
Tail & Samples & Detects & (mg/L) & (mg/L) & "ND" ? & Trend & Trend \\
\hline
\end{tabular}

GROSS BETA ACTIVITY

GW-179

GW-180

GW-322

GW-514

GW-609

GW-612

GW-831

SCR3_5SP

(mg/L)

(mg/L)

$\begin{array}{lll}2.0 \mathrm{E}+00 & 2.0 \mathrm{E}+00 & \text { Yes } \\ 2.0 \mathrm{E}+00 & 2.0 \mathrm{E}+00 & \text { Yes } \\ 7.9 \mathrm{E}+00 & 7.9 \mathrm{E}+00 & \text { No } \\ 2.0 \mathrm{E}+00 & 2.0 \mathrm{E}+00 & \text { Yes } \\ 2.0 \mathrm{E}+00 & 2.0 \mathrm{E}+00 & \text { Yes } \\ 2.0 \mathrm{E}+00 & 2.0 \mathrm{E}+00 & \text { Yes } \\ 5.8 \mathrm{E}+02 & 2.7 \mathrm{E}+00 & \text { No } \\ 3.6 \mathrm{E}+00 & 4.2 \mathrm{E}+00 & \text { No }\end{array}$

$\begin{array}{lc}\text { ND } & \text { ND } \\ \text { ND } & \text { ND } \\ \text { N/A } & \text { N/A } \\ \text { ND } & \text { ND } \\ \text { ND } & \text { ND } \\ \text { ND } & \text { ND } \\ \text { NT } & \text { NT } \\ \text { PD } & \text { D }\end{array}$

NITRATE

\begin{tabular}{|c|c|c|c|c|c|c|c|c|}
\hline GW-174 & $\mathrm{s}$ & 1 & 1 & $6.0 \mathrm{E}-01$ & 6.0E-01 & No & N/A & N/A \\
\hline GW-175 & $T$ & 1 & 1 & 2.6E-01 & 2.6E-01 & No & N/A & $\mathrm{N} / \mathrm{A}$ \\
\hline GW-176 & $\mathrm{T}$ & 1 & 1 & 5.4E-01 & 5.4E-01 & No & N/A & $\mathrm{N} / \mathrm{A}$ \\
\hline GW-178 & $\mathrm{T}$ & 1 & 1 & 3.4E-01 & 3.4E-01 & No & $\mathrm{N} / \mathrm{A}$ & $\mathrm{N} / \mathrm{A}$ \\
\hline GW-179 & $\mathrm{T}$ & 1 & 1 & 5.8E-01 & 5.8E-01 & No & $\mathrm{N} / \mathrm{A}$ & $\mathrm{N} / \mathrm{A}$ \\
\hline GW-180 & $\mathrm{S}$ & 1 & 1 & 2.7E-01 & 2.7E-01 & No & $\mathrm{N} / \mathrm{A}$ & $\mathrm{N} / \mathrm{A}$ \\
\hline GW-322 & $\mathrm{S}$ & 1 & 1 & 4.2E-01 & 4.2E-01 & No & $\mathrm{N} / \mathrm{A}$ & $\mathrm{N} / \mathrm{A}$ \\
\hline GW-514 & $T$ & 1 & 0 & 2.0E-02 & 2.0E-02 & Yes & ND & ND \\
\hline GW-609 & $\mathrm{s}$ & 1 & 1 & $1.2 \mathrm{E}+00$ & $1.2 \mathrm{E}+00$ & No & $\mathrm{N} / \mathrm{A}$ & $\mathrm{N} / \mathrm{A}$ \\
\hline GW-612 & $\mathrm{T}$ & 2 & 2 & 1.3E-01 & 1.3E-01 & No & $\mathrm{N} / \mathrm{A}$ & $\mathrm{N} / \mathrm{A}$ \\
\hline SCR3_5SP & $\mathrm{T}$ & 5 & 5 & $2.9 \mathrm{E}-01$ & $2.5 \mathrm{E}-01$ & No & $S$ & $\mathrm{~s}$ \\
\hline \multicolumn{9}{|c|}{ TETRACHLOROETHYLENE(PCE) } \\
\hline GW-174 & $\mathrm{s}$ & 28 & 27 & $3.1 \mathrm{E}-02$ & 3.5E-02 & No & $\mathrm{D}$ & $D$ \\
\hline GW-175 & $\mathrm{T}$ & 36 & 36 & $1.9 \mathrm{E}-02$ & 1.6E-02 & No & $D$ & $D$ \\
\hline GW-176 & $\mathrm{T}$ & 30 & 28 & 7.3E-03 & 7.0E-03 & No & $D$ & PD \\
\hline GW-177 & $T$ & 55 & 11 & 8.5E-04 & $5.0 \mathrm{E}-04$ & No & $\mathrm{D}$ & $D$ \\
\hline GW-178 & $\mathrm{T}$ & 20 & 5 & 8.0E-04 & 5.0E-04 & No & NT & NT \\
\hline GW-179 & $\mathrm{T}$ & 26 & 23 & $7.2 \mathrm{E}-03$ & 5.0E-03 & No & $\mathrm{D}$ & $\mathrm{D}$ \\
\hline GW-180 & $\mathrm{s}$ & 22 & 22 & 8.6E-02 & 7.3E-02 & No & $\mathrm{D}$ & D \\
\hline GW-322 & $\mathrm{s}$ & 23 & 20 & 5.6E-03 & 6.0E-03 & No & 1 & NT \\
\hline GW-514 & $\mathrm{T}$ & 28 & 0 & 5.0E-04 & 5.0E-04 & Yes & ND & ND \\
\hline GW-609 & $\mathrm{s}$ & 39 & 37 & 1.7E-02 & 1.3E-02 & No & $\mathrm{D}$ & $\mathrm{D}$ \\
\hline GW-612 & $T$ & 18 & 16 & $6.7 \mathrm{E}-03$ & 7.0E-03 & No & $\mathrm{D}$ & D \\
\hline GW-831 & $T$ & 24 & 0 & $5.0 \mathrm{E}-04$ & $5.0 \mathrm{E}-04$ & Yes & ND & ND \\
\hline SCR3_5SP & $T$ & 21 & 0 & $5.0 \mathrm{E}-04$ & $5.0 \mathrm{E}-04$ & Yes & ND & ND \\
\hline
\end{tabular}

Note: Increasing (I); Probably Increasing (PI); Stable (S); Probably Decreasing (PD); Decreasing (D); No Trend (NT); Not Applicable (N/A); Not Applicable (N/A) - Due to insufficient Data (< 4 sampling events); No Detectable Concentration (NDC)

The Number of Samples and Number of Detects shown above are post-consolidation values. 


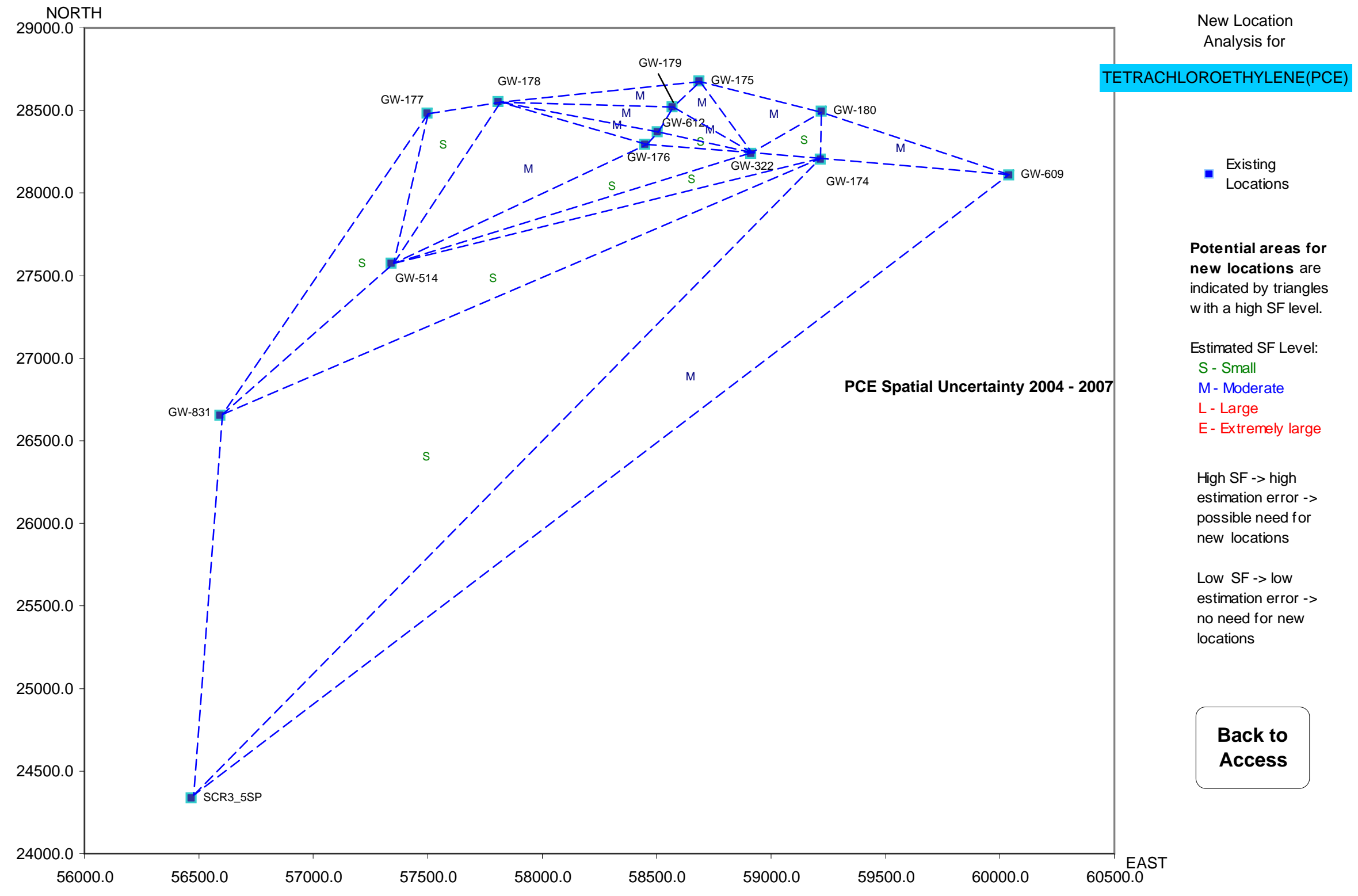


APPENDIX C.9

\section{LANDFILLS V AND VII AREA}




\section{MAROS COC Assessment}

Project: Landfill V and VII

Location: LandfillsV and VII
User Name: $\quad$ MV

State: Tennessee

Toxicity:

$$
\begin{aligned}
& \text { No priority, plume-wide COCs were found } \\
& \text { for the Landfill V and VII Area. }
\end{aligned}
$$

\section{Prevalence:}

Mobility: 


\section{MAROS Spatial Moment Analysis Summary}

Project: CR Landfill V and VII

Location: LandfillsV and VII
User Name: MV

State: Tennessee

\begin{tabular}{|c|c|c|c|c|c|c|c|}
\hline \multirow[b]{2}{*}{ Effective Date } & \multirow{2}{*}{$\begin{array}{l}\text { Oth Moment } \\
\text { Estimated } \\
\text { Mass (Kg) }\end{array}$} & \multicolumn{3}{|c|}{$\underline{1 s t}$ Moment (Center of Mass) } & \multicolumn{2}{|c|}{ 2nd Moment (Spread) } & \multirow[b]{2}{*}{$\begin{array}{c}\text { Number of } \\
\text { Wells }\end{array}$} \\
\hline & & $X c(f t)$ & Yc (ft) & $\begin{array}{c}\text { Source } \\
\text { Distance (ft) }\end{array}$ & $\underset{(\mathrm{sq} \mathrm{ft})}{\operatorname{Sigma} X X}$ & $\begin{array}{c}\text { Sigma } Y Y \\
(\mathrm{sq} \mathrm{ft})\end{array}$ & \\
\hline
\end{tabular}

GROSS ALPHA ACTIVITY

$\begin{array}{llllllll}7 / 1 / 2005 & 7.9 \mathrm{E}+02 & 60,134 & 26,484 & 2,406 & 589,180 & 386,340 & 10 \\ 7 / 1 / 2006 & 7.6 \mathrm{E}+02 & 60,150 & 26,463 & 2,432 & 598,624 & 395,169 & 10 \\ 7 / 1 / 2007 & 7.8 \mathrm{E}+02 & 60,194 & 26,444 & 2,479 & 608,367 & 389,993 & 10\end{array}$

GROSS BETA ACTIVITY

$\begin{array}{llllllll}7 / 1 / 2005 & 6.4 \mathrm{E}+02 & 60,100 & 26,505 & 2,367 & 620,886 & 413,034 & 10 \\ 7 / 1 / 2006 & 8.4 \mathrm{E}+02 & 60,102 & 26,514 & 2,363 & 589,817 & 403,814 & 10 \\ 7 / 1 / 2007 & 7.1 \mathrm{E}+02 & 60,225 & 26,383 & 2,539 & 597,525 & 413,289 & 10\end{array}$

NITRATE

$\begin{array}{llllllll}7 / 1 / 2005 & 3.0 \mathrm{E}+02 & 59,939 & 26,647 & 2,153 & 514,250 & 375,668 & 10 \\ 7 / 1 / 2006 & 3.0 \mathrm{E}+02 & 59,944 & 26,636 & 2,163 & 477,912 & 379,263 & 10 \\ 7 / 1 / 2007 & 2.2 \mathrm{E}+02 & 59,792 & 26,828 & 1,928 & 417,537 & 312,322 & 10\end{array}$

TETRACHLOROETHYLENE(PCE)

$\begin{array}{llllllll}7 / 1 / 1996 & 3.1 \mathrm{E}-01 & 60,145 & 26,465 & 2,427 & 599,576 & 394,724 & 10 \\ 7 / 1 / 1997 & 3.1 \mathrm{E}-01 & 60,145 & 26,465 & 2,427 & 599,576 & 394,724 & 10 \\ 7 / 1 / 1998 & 2.2 \mathrm{E}-01 & 59,795 & 26,615 & 2,059 & 330,386 & 324,786 & 7 \\ 7 / 1 / 1999 & 2.2 \mathrm{E}-01 & 59,795 & 26,615 & 2,059 & 330,386 & 324,786 & 7 \\ 7 / 1 / 2000 & 3.9 \mathrm{E}-01 & 60,145 & 26,540 & 2,383 & 585,716 & 375,581 & 10 \\ 7 / 1 / 2001 & 4.4 \mathrm{E}-01 & 60,145 & 26,573 & 2,364 & 579,535 & 363,472 & 10 \\ 7 / 1 / 2002 & 5.0 \mathrm{E}-01 & 60,145 & 26,602 & 2,347 & 574,109 & 351,025 & 10 \\ 7 / 1 / 2003 & 5.4 \mathrm{E}-01 & 60,145 & 26,620 & 2,337 & 570,835 & 342,692 & 10 \\ 7 / 1 / 2004 & 4.6 \mathrm{E}-01 & 60,145 & 26,585 & 2,357 & 577,346 & 358,654 & 10 \\ 7 / 1 / 2005 & 4.5 \mathrm{E}-01 & 60,145 & 26,580 & 2,359 & 578,123 & 360,394 & 10 \\ 7 / 1 / 2006 & 4.5 \mathrm{E}-01 & 60,145 & 26,578 & 2,361 & 578,548 & 361,334 & 10 \\ 7 / 1 / 2007 & 4.6 \mathrm{E}-01 & 60,145 & 26,586 & 2,356 & 577,167 & 358,248 & 10\end{array}$

TRICHLOROETHYLENE (TCE)

\begin{tabular}{llllllll}
\hline $7 / 1 / 2005$ & $5.3 \mathrm{E}-01$ & 60,145 & 26,410 & 2,460 & 609,896 & 401,782 & 10 \\
$7 / 1 / 2006$ & $5.2 \mathrm{E}-01$ & 60,145 & 26,400 & 2,466 & 611,736 & 402,395 & 10 \\
$7 / 1 / 2007$ & $5.4 \mathrm{E}-01$ & 60,145 & 26,415 & 2,457 & 608,883 & 401,361 & 10
\end{tabular}


Project: CR Landfill V and VII

Location: LandfillsV and VII
User Name: $\mathrm{MV}$

State: Tennessee

\begin{tabular}{|c|c|c|c|c|c|}
\hline Moment Type & Constituent & $\begin{array}{l}\text { Coefficient } \\
\text { of Variation }\end{array}$ & $\begin{array}{l}\text { Mann-Kendall } \\
\text { S Statistic }\end{array}$ & $\begin{array}{l}\text { Confidence } \\
\text { in Trend }\end{array}$ & $\begin{array}{c}\text { Moment } \\
\text { Trend }\end{array}$ \\
\hline \multicolumn{6}{|c|}{ Zeroth Moment: Mass } \\
\hline & GROSS ALPHA ACTIVITY & 0.00 & 0 & $0.0 \%$ & $\mathrm{~N} / \mathrm{A}$ \\
\hline & GROSS BETA ACTIVITY & 0.00 & 0 & $0.0 \%$ & $\mathrm{~N} / \mathrm{A}$ \\
\hline & NITRATE & 0.00 & 0 & $0.0 \%$ & $\mathrm{~N} / \mathrm{A}$ \\
\hline & TETRACHLOROETHYLENE(PCE) & 0.27 & 32 & $98.4 \%$ & 1 \\
\hline & TRICHLOROETHYLENE (TCE) & 0.00 & 0 & $0.0 \%$ & $\mathrm{~N} / \mathrm{A}$ \\
\hline \multicolumn{6}{|c|}{ 1st Moment: Distance to Source } \\
\hline & GROSS ALPHA ACTIVITY & 0.00 & 0 & $0.0 \%$ & $\mathrm{~N} / \mathrm{A}$ \\
\hline & GROSS BETA ACTIVITY & 0.00 & 0 & $0.0 \%$ & $N / A$ \\
\hline & NITRATE & 0.00 & 0 & $0.0 \%$ & $\mathrm{~N} / \mathrm{A}$ \\
\hline & TETRACHLOROETHYLENE(PCE) & 0.05 & -10 & $72.7 \%$ & $\mathrm{~S}$ \\
\hline & TRICHLOROETHYLENE (TCE) & 0.00 & 0 & $0.0 \%$ & $\mathrm{~N} / \mathrm{A}$ \\
\hline \multicolumn{6}{|c|}{ 2nd Moment: Sigma XX } \\
\hline & GROSS ALPHA ACTIVITY & 0.00 & 0 & $0.0 \%$ & $\mathrm{~N} / \mathrm{A}$ \\
\hline & GROSS BETA ACTIVITY & 0.00 & 0 & $0.0 \%$ & $\mathrm{~N} / \mathrm{A}$ \\
\hline & NITRATE & 0.00 & 0 & $0.0 \%$ & $\mathrm{~N} / \mathrm{A}$ \\
\hline & TETRACHLOROETHYLENE(PCE) & 0.18 & -10 & $72.7 \%$ & $\mathrm{~S}$ \\
\hline & TRICHLOROETHYLENE (TCE) & 0.00 & 0 & $0.0 \%$ & N/A \\
\hline \multicolumn{6}{|c|}{ 2nd Moment: Sigma YY } \\
\hline & GROSS ALPHA ACTIVITY & 0.00 & 0 & $0.0 \%$ & $\mathrm{~N} / \mathrm{A}$ \\
\hline & GROSS BETA ACTIVITY & 0.00 & 0 & $0.0 \%$ & $N / A$ \\
\hline & NITRATE & 0.00 & 0 & $0.0 \%$ & $\mathrm{~N} / \mathrm{A}$ \\
\hline & TETRACHLOROETHYLENE(PCE) & 0.06 & -10 & $72.7 \%$ & $\mathrm{~S}$ \\
\hline & TRICHLOROETHYLENE (TCE) & 0.00 & 0 & $0.0 \%$ & N/A \\
\hline
\end{tabular}

Note: The following assumptions were applied for the calculation of the Zeroth Moment:

Porosity: $0.10 \quad$ Saturated Thickness: Uniform: $50 \mathrm{ft}$

Mann-Kendall Trend test performed on all sample events for each constituent. Increasing (I); Probably Increasing (PI); Stable (S); Probably Decreasing (PD); Decreasing (D); No Trend (NT); Not Applicable (N/A)-Due to insufficient Data (< 4 sampling events).

Note: The Sigma XX and Sigma YY components are estimated using the given field coordinate system and then rotated to align with the estimated groundwater flow direction. Moments are not calculated for sample events with less than 6 wells. 


\section{MAROS Statistical Trend Analysis Summary}

Project: $\quad$ CR Landfill V and VII

Location: LandfillsV and VII

Time Period: $4 / 1 / 1993$ to $7 / 15 / 2007$

Consolidation Period: No Time Consolidation

Consolidation Type: Median

Duplicate Consolidation: Average

ND Values: Specified Detection Limit

J Flag Values : Actual Value
User Name: MV

State: Tennessee

\begin{tabular}{|c|c|c|c|c|c|c|c|c|}
\hline Well & $\begin{array}{c}\text { Sourcel } \\
\text { Tail }\end{array}$ & $\begin{array}{c}\text { Number } \\
\text { of } \\
\text { Samples }\end{array}$ & $\begin{array}{c}\text { Number } \\
\text { of } \\
\text { Detects }\end{array}$ & $\begin{array}{c}\text { Average } \\
\text { Conc. } \\
\text { (mg/L) }\end{array}$ & $\begin{array}{l}\text { Median } \\
\text { Conc. } \\
\text { (mg/L) }\end{array}$ & $\begin{array}{c}\text { All } \\
\text { Samples } \\
\text { "ND" ? }\end{array}$ & $\begin{array}{l}\text { Mann- } \\
\text { Kendall } \\
\text { Trend }\end{array}$ & $\begin{array}{l}\text { Linear } \\
\text { Regression } \\
\text { Trend }\end{array}$ \\
\hline \multicolumn{9}{|c|}{ GROSS ALPHA ACTIVITY } \\
\hline GW-557 & $\mathrm{T}$ & 6 & 0 & $1.2 \mathrm{E}+00$ & $1.2 \mathrm{E}+00$ & Yes & ND & ND \\
\hline GW-560 & $\mathrm{T}$ & 6 & 1 & $1.2 \mathrm{E}+00$ & $1.2 \mathrm{E}+00$ & No & NT & NT \\
\hline GW-562 & $\mathrm{T}$ & 6 & 1 & $1.6 \mathrm{E}+00$ & $1.2 \mathrm{E}+00$ & No & NT & $\mathrm{PI}$ \\
\hline GW-564 & $\mathrm{T}$ & 6 & 0 & $1.2 \mathrm{E}+00$ & $1.2 \mathrm{E}+00$ & Yes & ND & ND \\
\hline GW-796 & S & 6 & 1 & $1.3 \mathrm{E}+00$ & $1.2 \mathrm{E}+00$ & No & NT & NT \\
\hline GW-797 & s & 6 & 0 & $1.2 \mathrm{E}+00$ & $1.2 \mathrm{E}+00$ & Yes & ND & ND \\
\hline GW-798 & $\mathrm{T}$ & 6 & 1 & $1.3 \mathrm{E}+00$ & $1.2 \mathrm{E}+00$ & No & $\mathrm{s}$ & PD \\
\hline GW-799 & $\mathrm{T}$ & 6 & 2 & $1.3 \mathrm{E}+00$ & $1.2 \mathrm{E}+00$ & No & S & S \\
\hline GW-801 & S & 6 & 0 & $1.2 \mathrm{E}+00$ & $1.2 \mathrm{E}+00$ & Yes & ND & ND \\
\hline SCR4_3SP & $\mathrm{T}$ & 6 & 0 & $1.2 \mathrm{E}+00$ & $1.2 \mathrm{E}+00$ & Yes & ND & ND \\
\hline
\end{tabular}

GROSS BETA ACTIVITY

\begin{tabular}{|c|c|c|c|c|c|c|c|c|}
\hline GW-557 & $\mathrm{T}$ & 6 & 2 & $1.4 \mathrm{E}+00$ & $1.0 \mathrm{E}+00$ & No & NT & NT \\
\hline GW-560 & $\mathrm{T}$ & 6 & 1 & $1.1 \mathrm{E}+00$ & $1.0 \mathrm{E}+00$ & No & NT & NT \\
\hline GW-562 & $\mathrm{T}$ & 6 & 0 & $1.0 \mathrm{E}+00$ & $1.0 \mathrm{E}+00$ & Yes & ND & ND \\
\hline GW-564 & $\mathrm{T}$ & 6 & 1 & $1.2 \mathrm{E}+00$ & $1.0 \mathrm{E}+00$ & No & NT & NT \\
\hline GW-796 & $\mathrm{S}$ & 6 & 2 & $2.0 \mathrm{E}+00$ & $1.0 \mathrm{E}+00$ & No & $\mathrm{S}$ & $\mathrm{s}$ \\
\hline GW-797 & $\mathrm{s}$ & 6 & 1 & $1.1 \mathrm{E}+00$ & $1.0 \mathrm{E}+00$ & No & $\mathrm{s}$ & $\mathrm{s}$ \\
\hline GW-798 & $\mathrm{T}$ & 6 & 1 & 1.7E+00 & $1.0 \mathrm{E}+00$ & No & NT & NT \\
\hline GW-799 & $\mathrm{T}$ & 6 & 1 & $1.2 \mathrm{E}+00$ & $1.0 \mathrm{E}+00$ & No & NT & NT \\
\hline GW-801 & $\mathrm{s}$ & 6 & 0 & $1.0 \mathrm{E}+00$ & $1.0 \mathrm{E}+00$ & Yes & ND & ND \\
\hline SCR4_3SP & $\mathrm{T}$ & 6 & 3 & 1.7E+00 & $1.5 \mathrm{E}+00$ & No & I & 1 \\
\hline
\end{tabular}

NITRATE

\begin{tabular}{|c|c|c|c|c|c|c|c|c|}
\hline GW-557 & $\mathrm{T}$ & 6 & 5 & 4.2E-01 & 4.8E-01 & No & PD & PD \\
\hline GW-560 & $\mathrm{T}$ & 6 & 5 & $1.5 \mathrm{E}-01$ & $1.7 \mathrm{E}-01$ & No & NT & $\mathrm{s}$ \\
\hline GW-562 & $\mathrm{T}$ & 6 & 5 & $1.8 \mathrm{E}-01$ & $1.7 \mathrm{E}-01$ & No & S & PD \\
\hline GW-564 & $\mathrm{T}$ & 6 & 5 & $3.8 \mathrm{E}-01$ & 4.6E-01 & No & $\mathrm{s}$ & $\mathrm{s}$ \\
\hline GW-796 & $\mathrm{s}$ & 6 & 5 & $9.7 \mathrm{E}-02$ & $8.1 \mathrm{E}-02$ & No & $S$ & $P D$ \\
\hline GW-797 & $\mathrm{S}$ & 6 & 6 & $2.1 \mathrm{E}+00$ & $2.1 \mathrm{E}+00$ & No & 1 & 1 \\
\hline GW-798 & $\mathrm{T}$ & 6 & 6 & $6.9 \mathrm{E}-01$ & $6.8 \mathrm{E}-01$ & No & S & S \\
\hline GW-799 & $\mathrm{T}$ & 6 & 6 & $1.1 \mathrm{E}+00$ & $1.1 \mathrm{E}+00$ & No & NT & $\mathrm{PI}$ \\
\hline GW-801 & $\mathrm{S}$ & 6 & 5 & $1.1 \mathrm{E}-01$ & $9.5 \mathrm{E}-02$ & No & S & S \\
\hline SCR4_3SP & $\mathrm{T}$ & 6 & 6 & 7.7E-01 & $7.9 \mathrm{E}-01$ & No & NT & NT \\
\hline
\end{tabular}




\section{MAROS Statistical Trend Analysis Summary}

\begin{tabular}{|c|c|c|c|c|c|c|c|c|}
\hline Well & $\begin{array}{c}\text { Sourcel } \\
\text { Tail }\end{array}$ & $\begin{array}{c}\text { Number } \\
\text { of } \\
\text { Samples }\end{array}$ & $\begin{array}{c}\text { Number } \\
\text { of } \\
\text { Detects }\end{array}$ & $\begin{array}{c}\text { Average } \\
\text { Conc. } \\
\text { (mg/L) }\end{array}$ & $\begin{array}{c}\text { Median } \\
\text { Conc. } \\
\text { (mg/L) }\end{array}$ & $\begin{array}{c}\text { All } \\
\text { Samples } \\
\text { "ND" ? }\end{array}$ & $\begin{array}{c}\text { Mann- } \\
\text { Kendall } \\
\text { Trend }\end{array}$ & $\begin{array}{c}\text { Linear } \\
\text { Regression } \\
\text { Trend }\end{array}$ \\
\hline LC & CE) & & & & & & & \\
\hline
\end{tabular}

\section{TETRACHLOROETHYLENE(PCE)}

\begin{tabular}{|c|c|c|c|c|c|c|c|c|}
\hline GW-557 & $\mathrm{T}$ & 33 & 0 & $5.0 \mathrm{E}-04$ & $5.0 \mathrm{E}-04$ & Yes & ND & ND \\
\hline GW-560 & $\mathrm{T}$ & 30 & 0 & $5.0 \mathrm{E}-04$ & 5.0E-04 & Yes & ND & ND \\
\hline GW-562 & $\mathrm{T}$ & 30 & 0 & $5.0 \mathrm{E}-04$ & 5.0E-04 & Yes & ND & ND \\
\hline GW-564 & $\mathrm{T}$ & 30 & 0 & $5.0 \mathrm{E}-04$ & 5.0E-04 & Yes & ND & ND \\
\hline GW-796 & $\mathrm{s}$ & 33 & 0 & $5.0 \mathrm{E}-04$ & 5.0E-04 & Yes & ND & ND \\
\hline GW-797 & $\mathrm{S}$ & 33 & 0 & 5.0E-04 & 5.0E-04 & Yes & ND & ND \\
\hline GW-798 & $\mathrm{T}$ & 36 & 19 & $2.6 \mathrm{E}-03$ & 1.5E-03 & No & 1 & 1 \\
\hline GW-799 & $\mathrm{T}$ & 33 & 0 & 5.0E-04 & 5.0E-04 & Yes & ND & ND \\
\hline GW-801 & $\mathrm{S}$ & 32 & 0 & $5.0 \mathrm{E}-04$ & $5.0 \mathrm{E}-04$ & Yes & ND & ND \\
\hline SCR4_3SP & $\mathrm{T}$ & 28 & 0 & $5.0 \mathrm{E}-04$ & 5.0E-04 & Yes & ND & ND \\
\hline \multicolumn{9}{|c|}{ TRICHLOROETHYLENE (TCE) } \\
\hline GW-557 & $\mathrm{T}$ & 6 & 0 & $1.0 \mathrm{E}-03$ & 1.0E-03 & Yes & ND & ND \\
\hline GW-560 & $\mathrm{T}$ & 6 & 0 & $1.0 \mathrm{E}-03$ & $1.0 \mathrm{E}-03$ & Yes & ND & ND \\
\hline GW-562 & $\mathrm{T}$ & 6 & 0 & $1.0 \mathrm{E}-03$ & 1.0E-03 & Yes & ND & ND \\
\hline GW-564 & $\mathrm{T}$ & 6 & 0 & $1.0 \mathrm{E}-03$ & $1.0 \mathrm{E}-03$ & Yes & ND & ND \\
\hline GW-796 & $\mathrm{s}$ & 6 & 0 & $1.0 \mathrm{E}-03$ & $1.0 \mathrm{E}-03$ & Yes & ND & ND \\
\hline GW-797 & $\mathrm{s}$ & 6 & 0 & $1.0 \mathrm{E}-03$ & 1.0E-03 & Yes & ND & ND \\
\hline GW-798 & $\mathrm{T}$ & 6 & 6 & $3.8 \mathrm{E}-04$ & $4.0 \mathrm{E}-04$ & No & S & NT \\
\hline GW-799 & $\mathrm{T}$ & 6 & 0 & $1.0 \mathrm{E}-03$ & $1.0 \mathrm{E}-03$ & Yes & ND & ND \\
\hline GW-801 & $\mathrm{s}$ & 6 & 0 & $1.0 \mathrm{E}-03$ & 1.0E-03 & Yes & ND & ND \\
\hline SCR4_3SP & $\mathrm{T}$ & 6 & 0 & $1.0 \mathrm{E}-03$ & 1.0E-03 & Yes & ND & ND \\
\hline
\end{tabular}

Note: Increasing (I); Probably Increasing (PI); Stable (S); Probably Decreasing (PD); Decreasing (D); No Trend (NT); Not Applicable (N/A); Not Applicable (N/A) - Due to insufficient Data (< 4 sampling events); No Detectable Concentration (NDC)

The Number of Samples and Number of Detects shown above are post-consolidation values. 
APPENDIX C.10

EAST CHESTNUT RIDGE 


\section{MAROS COC Assessment}

Project: $\quad$ CR Kerr Hollow

Location: East Chestnut Ridge

\section{Toxicity:}

Contaminant of Concern

TRICHLOROETHYLENE (TCE)
User Name: $\quad$ MV

State: Tennessee

Note: Top COCs by toxicity were determined by examining a representative concentration for each compound over the entire site. The compound representative concentrations are then compared with the chosen PRG for that compound, with the percentage exceedance from the PRG determining the compound's toxicity. All compounds above exceed the PRG.

\section{Prevalence:}

\begin{tabular}{|c|c|c|c|c|c|}
\hline Contaminant of Concern & Class & $\begin{array}{l}\text { Total } \\
\text { Wells }\end{array}$ & $\begin{array}{c}\text { Total } \\
\text { Exceedances }\end{array}$ & $\begin{array}{c}\text { Percent } \\
\text { Exceedances }\end{array}$ & $\begin{array}{c}\text { Total } \\
\text { detects }\end{array}$ \\
\hline TRICHLOROETHYLENE (TCE) & ORG & 17 & 1 & $5.9 \%$ & 10 \\
\hline
\end{tabular}

Note: Top COCs by prevalence were determined by examining a representative concentration for each well location at the site. The total exceedances (values above the chosen PRGs) are compared to the total number of wells to determine the prevalence of the compound.

\section{Mobility:}

Contaminant of Concern

$\mathrm{Kd}$

TRICHLOROETHYLENE (TCE) 0.297

Note: Top COCs by mobility were determined by examining each detected compound in the dataset and comparing their mobilities (Koc's for organics, assume foc $=0.001$, and Kd's for metals).

Contaminants of Concern (COC's)

GROSS ALPHA ACTIVITY

GROSS BETA ACTIVITY

NITRATE

TRICHLOROETHYLENE (TCE)

URANIUM 


\section{MAROS Spatial Moment Analysis Summary}

Project: CR East Kerr Hollow

Location: East Chestnut Ridge
User Name: MV

State: Tennessee

\begin{tabular}{|c|c|c|c|c|c|c|c|}
\hline \multirow[b]{2}{*}{ Effective Date } & \multirow{2}{*}{$\begin{array}{l}\text { 0th Moment } \\
\begin{array}{l}\text { Estimated } \\
\text { Mass (Kg) }\end{array}\end{array}$} & \multicolumn{3}{|c|}{ 1st Moment (Center of Mass) } & \multicolumn{2}{|c|}{ 2nd Moment (Spread) } & \multirow[b]{2}{*}{$\begin{array}{c}\text { Number of } \\
\text { Wells }\end{array}$} \\
\hline & & Xc (ft) & Yc (ft) & $\begin{array}{c}\text { Source } \\
\text { Distance (ft) }\end{array}$ & $\underset{(s q \mathrm{ft})}{\operatorname{Sigma} x X}$ & $\underset{(\mathrm{sq} \mathrm{ft})}{\operatorname{Sigma} Y Y}$ & \\
\hline
\end{tabular}

GROSS ALPHA ACTIVITY

$\begin{array}{ll}7 / 1 / 1996 & 1.6 \mathrm{E}+03 \\ 7 / 1 / 1997 & 0.0 \mathrm{E}+00 \\ 7 / 1 / 1998 & 0.0 \mathrm{E}+00 \\ 7 / 1 / 1999 & 0.0 \mathrm{E}+00 \\ 7 / 1 / 2000 & 0.0 \mathrm{E}+00 \\ 7 / 1 / 2001 & 0.0 \mathrm{E}+00 \\ 7 / 1 / 2002 & 0.0 \mathrm{E}+00 \\ 7 / 1 / 2003 & 0.0 \mathrm{E}+00 \\ 7 / 1 / 2004 & 0.0 \mathrm{E}+00 \\ 7 / 1 / 2005 & 0.0 \mathrm{E}+00 \\ 7 / 1 / 2006 & 0.0 \mathrm{E}+00 \\ 7 / 1 / 2007 & 3.5 \mathrm{E}+01\end{array}$

63,157

26,787

1,690

157,162

665,443

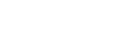

GROSS BETA ACTIVITY

\begin{tabular}{llllllll}
\hline & $7 / 1 / 2005$ & $0.0 \mathrm{E}+00$ & & & & & 5 \\
& $7 / 1 / 2006$ & $0.0 \mathrm{E}+00$ & & & & & 5 \\
& $7 / 1 / 2007$ & $6.4 \mathrm{E}+02$ & 62,825 & 26,167 & 2,087 & 90,568 & $748,336 \quad$ \\
NITRATE & & & & & & & \\
\hline
\end{tabular}

62,923

25,807

97,631

$\begin{array}{cc} & 5 \\ 4 \\ 5 \\ \\ & 5 \\ & 5 \\ & 5 \\ & 5 \\ & 5 \\ 714,871 & 5 \\ & 5 \\ & 10\end{array}$

$\begin{array}{llllllll}7 / 1 / 2007 & 5.0 \mathrm{E}+00 & 62,303 & 27,854 & 327 & 7,245 & 12,890 & 6\end{array}$

TRICHLOROETHYLENE (TCE)

\begin{tabular}{|c|c|c|c|c|c|c|c|c|}
\hline & 7/1/1996 & $9.6 \mathrm{E}-02$ & 62,679 & 26,406 & 1,814 & 63,149 & 634,906 & 8 \\
\hline & $7 / 1 / 1997$ & $1.8 \mathrm{E}+00$ & 64,047 & 24,873 & 3,781 & 215,855 & 357,895 & 7 \\
\hline & 7/1/1998 & $0.0 \mathrm{E}+00$ & & & & & & 5 \\
\hline & 7/1/1999 & $2.4 \mathrm{E}+00$ & 64,040 & 24,885 & 3,767 & 191,741 & 337,697 & 7 \\
\hline & $7 / 1 / 2000$ & $0.0 \mathrm{E}+00$ & & & & & & 5 \\
\hline & $7 / 1 / 2001$ & $1.0 \mathrm{E}+00$ & 64,044 & 24,868 & 3,783 & 239,713 & 372,967 & 7 \\
\hline & $7 / 1 / 2002$ & $1.1 \mathrm{E}+00$ & 64,069 & 24,843 & 3,817 & 260,138 & 398,691 & 7 \\
\hline & $7 / 1 / 2003$ & 7.0E-01 & 64,010 & 24,896 & 3,742 & 234,671 & 353,097 & 7 \\
\hline & $7 / 1 / 2004$ & 4.6E-01 & 63,966 & 24,929 & 3,692 & 235,290 & 332,569 & 7 \\
\hline & $7 / 1 / 2005$ & 8.4E-01 & 64,007 & 24,904 & 3,733 & 212,527 & 336,002 & 7 \\
\hline & $7 / 1 / 2006$ & $1.4 \mathrm{E}+00$ & 64,069 & 24,846 & 3,815 & 246,302 & 389,548 & 7 \\
\hline & $7 / 1 / 2007$ & $1.3 \mathrm{E}+00$ & 64,031 & 25,139 & 3,544 & 282,342 & 534,562 & 13 \\
\hline \multicolumn{9}{|l|}{ URANIUM } \\
\hline & $7 / 1 / 1996$ & $4.9 \mathrm{E}-02$ & 63,175 & 26,817 & 1,676 & 162,408 & $1,142,763$ & 12 \\
\hline & $7 / 1 / 1997$ & 3.5E-02 & 63,574 & 26,356 & 2,286 & 133,642 & $1,265,790$ & 9 \\
\hline
\end{tabular}


Project: CR East Kerr Hollow

Location: East Chestnut Ridge
User Name: $\quad$ MV

State: Tennessee

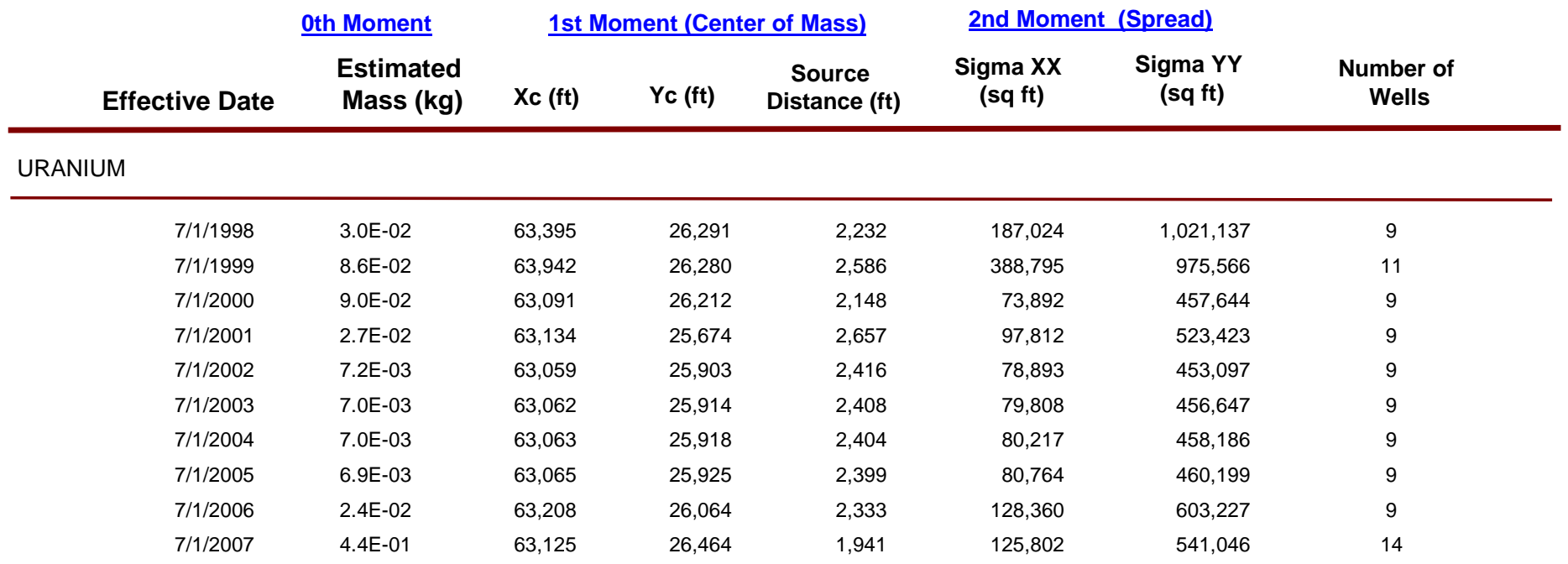


Project: CR East Kerr Hollow

Location: East Chestnut Ridge
User Name: $\mathrm{MV}$

State: Tennessee

\begin{tabular}{|c|c|c|c|c|c|}
\hline Moment Type & Constituent & $\begin{array}{l}\text { Coefficient } \\
\text { of Variation }\end{array}$ & $\begin{array}{l}\text { Mann-Kendall } \\
\text { S Statistic }\end{array}$ & $\begin{array}{l}\text { Confidence } \\
\text { in Trend }\end{array}$ & $\begin{array}{c}\text { Moment } \\
\text { Trend }\end{array}$ \\
\hline \multicolumn{6}{|c|}{ Zeroth Moment: Mass } \\
\hline & GROSS ALPHA ACTIVITY & 3.39 & -1 & $50.0 \%$ & NT \\
\hline & GROSS BETA ACTIVITY & 0.00 & 0 & $0.0 \%$ & $\mathrm{~N} / \mathrm{A}$ \\
\hline & NITRATE & 0.00 & 0 & $0.0 \%$ & $\mathrm{~N} / \mathrm{A}$ \\
\hline & TRICHLOROETHYLENE (TCE) & 0.80 & 11 & $74.9 \%$ & NT \\
\hline & URANIUM & 1.80 & -22 & $92.4 \%$ & PD \\
\hline \multicolumn{6}{|c|}{ 1st Moment: Distance to Source } \\
\hline & GROSS ALPHA ACTIVITY & 0.00 & 0 & $0.0 \%$ & $\mathrm{~N} / \mathrm{A}$ \\
\hline & GROSS BETA ACTIVITY & 0.00 & 0 & $0.0 \%$ & $N / A$ \\
\hline & NITRATE & 0.00 & 0 & $0.0 \%$ & $\mathrm{~N} / \mathrm{A}$ \\
\hline & TRICHLOROETHYLENE (TCE) & 0.17 & -3 & $56.9 \%$ & $\mathrm{~S}$ \\
\hline & URANIUM & 0.12 & -2 & $52.7 \%$ & $\mathrm{~S}$ \\
\hline \multicolumn{6}{|c|}{ 2nd Moment: Sigma XX } \\
\hline & GROSS ALPHA ACTIVITY & 0.00 & 0 & $0.0 \%$ & $\mathrm{~N} / \mathrm{A}$ \\
\hline & GROSS BETA ACTIVITY & 0.00 & 0 & $0.0 \%$ & $\mathrm{~N} / \mathrm{A}$ \\
\hline & NITRATE & 0.00 & 0 & $0.0 \%$ & $\mathrm{~N} / \mathrm{A}$ \\
\hline & TRICHLOROETHYLENE (TCE) & 0.28 & 23 & $97.7 \%$ & 1 \\
\hline & URANIUM & 0.65 & -10 & $72.7 \%$ & $\mathrm{~S}$ \\
\hline \multicolumn{6}{|c|}{ 2nd Moment: Sigma YY } \\
\hline & GROSS ALPHA ACTIVITY & 0.00 & 0 & $0.0 \%$ & $\mathrm{~N} / \mathrm{A}$ \\
\hline & GROSS BETA ACTIVITY & 0.00 & 0 & $0.0 \%$ & $\mathrm{~N} / \mathrm{A}$ \\
\hline & NITRATE & 0.00 & 0 & $0.0 \%$ & $\mathrm{~N} / \mathrm{A}$ \\
\hline & TRICHLOROETHYLENE (TCE) & 0.25 & -3 & $56.9 \%$ & $\mathrm{~S}$ \\
\hline & URANIUM & 0.44 & -22 & $92.4 \%$ & PD \\
\hline
\end{tabular}

Note: The following assumptions were applied for the calculation of the Zeroth Moment:

Porosity: $0.10 \quad$ Saturated Thickness: Uniform: $50 \mathrm{ft}$

Mann-Kendall Trend test performed on all sample events for each constituent. Increasing (I); Probably Increasing (PI); Stable (S); Probably Decreasing (PD); Decreasing (D); No Trend (NT); Not Applicable (N/A)-Due to insufficient Data (< 4 sampling events).

Note: The Sigma XX and Sigma YY components are estimated using the given field coordinate system and then rotated to align with the estimated groundwater flow direction. Moments are not calculated for sample events with less than 6 wells. 


\section{MAROS Statistical Trend Analysis Summary}

Project: CR Kerr Hollow

Location: East Chestnut Ridge

Time Period: $1 / 1 / 1986$ to $10 / 15 / 2007$

Consolidation Period: No Time Consolidation

Consolidation Type: Median

Duplicate Consolidation: Average

ND Values: Specified Detection Limit

J Flag Values : Actual Value
User Name: MV

State: Tennessee

\begin{tabular}{|c|c|c|c|c|c|c|c|c|}
\hline Well & $\begin{array}{c}\text { Sourcel } \\
\text { Tail }\end{array}$ & $\begin{array}{c}\text { Number } \\
\text { of } \\
\text { Samples }\end{array}$ & $\begin{array}{c}\text { Number } \\
\text { of } \\
\text { Detects }\end{array}$ & $\begin{array}{c}\text { Average } \\
\text { Conc. } \\
\text { (mg/L) }\end{array}$ & $\begin{array}{l}\text { Median } \\
\text { Conc. } \\
\text { (mg/L) }\end{array}$ & $\begin{array}{c}\text { All } \\
\text { Samples } \\
\text { "ND" ? }\end{array}$ & $\begin{array}{l}\text { Mann- } \\
\text { Kendall } \\
\text { Trend }\end{array}$ & $\begin{array}{l}\text { Linear } \\
\text { Regression } \\
\text { Trend }\end{array}$ \\
\hline \multicolumn{9}{|c|}{ GROSS ALPHA ACTIVITY } \\
\hline GW-143 & $\mathrm{T}$ & 51 & 45 & $3.8 \mathrm{E}+00$ & $3.5 \mathrm{E}+00$ & No & $\mathrm{D}$ & $\mathrm{s}$ \\
\hline GW-144 & $\mathrm{T}$ & 51 & 49 & $3.0 \mathrm{E}+00$ & $2.3 \mathrm{E}+00$ & No & D & $\mathrm{s}$ \\
\hline GW-145 & $\mathrm{T}$ & 51 & 51 & $1.1 \mathrm{E}+01$ & $1.1 \mathrm{E}+01$ & No & D & I \\
\hline GW-156 & $\mathrm{T}$ & 35 & 34 & $1.2 \mathrm{E}+01$ & $3.2 \mathrm{E}+00$ & No & PD & NT \\
\hline GW-159 & $\mathrm{T}$ & 38 & 37 & $6.2 \mathrm{E}+00$ & $2.8 \mathrm{E}+00$ & No & PD & NT \\
\hline GW-161 & $\mathrm{T}$ & 2 & 0 & $1.0 \mathrm{E}-02$ & $1.0 \mathrm{E}-02$ & Yes & ND & ND \\
\hline GW-231 & $\mathrm{T}$ & 43 & 32 & $1.3 \mathrm{E}+00$ & $8.1 \mathrm{E}-01$ & No & D & PD \\
\hline GW-292 & S & 21 & 18 & $1.1 \mathrm{E}+00$ & $9.0 \mathrm{E}-01$ & No & NT & $\mathrm{D}$ \\
\hline GW-293 & S & 22 & 19 & $1.7 \mathrm{E}+00$ & $1.7 \mathrm{E}+00$ & No & $\mathrm{s}$ & D \\
\hline GW-294 & S & 2 & 0 & $1.0 \mathrm{E}-02$ & $1.0 \mathrm{E}-02$ & Yes & ND & ND \\
\hline GW-296 & S & 2 & 0 & $1.0 \mathrm{E}-02$ & $1.0 \mathrm{E}-02$ & Yes & ND & ND \\
\hline GW-298 & $\mathrm{T}$ & 26 & 25 & $2.7 \mathrm{E}+00$ & $1.7 \mathrm{E}+00$ & No & NT & $\mathrm{D}$ \\
\hline GW-301 & $\mathrm{T}$ & 43 & 35 & $1.5 \mathrm{E}+00$ & $1.3 E+00$ & No & $\mathrm{D}$ & $\mathrm{D}$ \\
\hline GW-731 & $\mathrm{T}$ & 20 & 20 & $3.7 \mathrm{E}+00$ & $1.3 E+00$ & No & D & D \\
\hline GW-732 & $\mathrm{T}$ & 20 & 20 & $1.1 \mathrm{E}+01$ & $2.6 \mathrm{E}+00$ & No & NT & NT \\
\hline
\end{tabular}

GROSS BETA ACTIVITY

\begin{tabular}{|c|c|c|c|c|c|c|c|c|}
\hline GW-143 & $\mathrm{T}$ & 5 & 5 & $1.8 \mathrm{E}+01$ & $1.9 \mathrm{E}+01$ & No & $S$ & D \\
\hline GW-144 & $\mathrm{T}$ & 5 & 3 & $2.9 \mathrm{E}+00$ & $3.3 E+00$ & No & s & $P D$ \\
\hline GW-145 & $\mathrm{T}$ & 5 & 5 & 1.7E+01 & $1.5 \mathrm{E}+01$ & No & D & D \\
\hline GW-161 & T & 1 & 0 & $2.1 \mathrm{E}+00$ & $2.1 \mathrm{E}+00$ & Yes & ND & ND \\
\hline GW-231 & $\mathrm{T}$ & 5 & 2 & $4.1 \mathrm{E}+00$ & $2.1 \mathrm{E}+00$ & No & S & $\mathrm{S}$ \\
\hline GW-292 & S & 1 & 0 & $2.1 \mathrm{E}+00$ & $2.1 \mathrm{E}+00$ & Yes & ND & ND \\
\hline GW-293 & S & 1 & 0 & $2.1 \mathrm{E}+00$ & $2.1 \mathrm{E}+00$ & Yes & ND & ND \\
\hline GW-294 & S & 1 & 0 & $2.1 \mathrm{E}+00$ & $2.1 \mathrm{E}+00$ & Yes & ND & ND \\
\hline GW-296 & S & 1 & 0 & $2.1 \mathrm{E}+00$ & $2.1 \mathrm{E}+00$ & Yes & ND & ND \\
\hline GW-298 & $\mathrm{T}$ & 1 & 0 & $2.1 \mathrm{E}+00$ & $2.1 \mathrm{E}+00$ & Yes & ND & ND \\
\hline GW-301 & $\mathrm{T}$ & 4 & 2 & 3.7E+02 & $2.5 E+00$ & No & NT & NT \\
\hline \multicolumn{9}{|l|}{ NITRATE } \\
\hline GW-161 & $\mathrm{T}$ & 2 & 1 & 1.2E-01 & 1.2E-01 & No & $N / A$ & $\mathrm{~N} / \mathrm{A}$ \\
\hline GW-292 & s & 2 & 2 & 3.7E-01 & 3.7E-01 & No & $\mathrm{N} / \mathrm{A}$ & N/A \\
\hline GW-293 & S & 2 & 2 & 1.5E-01 & 1.5E-01 & No & $N / A$ & N/A \\
\hline GW-294 & $\mathrm{s}$ & 2 & 2 & $1.5 \mathrm{E}+00$ & $1.5 E+00$ & No & $\mathrm{N} / \mathrm{A}$ & N/A \\
\hline GW-296 & $\mathrm{s}$ & 2 & 2 & 3.8E-01 & 3.8E-01 & No & N/A & $N / A$ \\
\hline
\end{tabular}




\section{MAROS Statistical Trend Analysis Summary}

\begin{tabular}{ccccccccc} 
& & Number & Number & Average & Median & All & Mann- & Linear \\
Well & Sourcel & of & of & Conc. & Conc. & Samples & Kendall & Regression \\
Tail & Samples & Detects & (mg/L) & (mg/L) & "ND" ? & Trend & Trend \\
\hline
\end{tabular}

NITRATE

$\begin{array}{lllllllll}\text { GW-298 } & T & 2 & 2 & 2.4 \mathrm{E}-01 & 2.4 \mathrm{E}-01 & \text { No } & \text { N/A } & \text { N/A }\end{array}$

TRICHLOROETHYLENE (TCE)

\begin{tabular}{|c|c|c|c|c|c|c|c|c|}
\hline GW-143 & $\mathrm{T}$ & 47 & 1 & $5.1 \mathrm{E}-04$ & 5.0E-04 & No & $D$ & $D$ \\
\hline GW-144 & $\mathrm{T}$ & 48 & 1 & $5.3 \mathrm{E}-04$ & $5.0 \mathrm{E}-04$ & No & $D$ & $D$ \\
\hline GW-145 & $\mathrm{T}$ & 47 & 2 & 5.3E-04 & 5.0E-04 & No & 1 & 1 \\
\hline GW-156 & $\mathrm{T}$ & 35 & 1 & $6.0 \mathrm{E}-04$ & 5.0E-04 & No & $\mathrm{s}$ & $\mathrm{S}$ \\
\hline GW-159 & $\mathrm{T}$ & 38 & 2 & $5.5 \mathrm{E}-04$ & $5.0 \mathrm{E}-04$ & No & S & $P D$ \\
\hline GW-161 & $\mathrm{T}$ & 2 & 0 & $5.0 \mathrm{E}-04$ & $5.0 \mathrm{E}-04$ & Yes & ND & ND \\
\hline GW-231 & $\mathrm{T}$ & 46 & 1 & 5.1E-04 & 5.0E-04 & No & 1 & 1 \\
\hline GW-292 & $\mathrm{S}$ & 23 & 0 & 5.0E-04 & 5.0E-04 & Yes & ND & ND \\
\hline GW-293 & $\mathrm{s}$ & 23 & 1 & $5.2 \mathrm{E}-04$ & $5.0 \mathrm{E}-04$ & No & $\mathrm{S}$ & $\mathrm{S}$ \\
\hline GW-294 & $\mathrm{s}$ & 2 & 0 & $5.0 \mathrm{E}-04$ & 5.0E-04 & Yes & ND & ND \\
\hline GW-296 & $\mathrm{s}$ & 2 & 0 & $5.0 \mathrm{E}-04$ & $5.0 \mathrm{E}-04$ & Yes & ND & ND \\
\hline GW-298 & $\mathrm{T}$ & 26 & 0 & $5.0 \mathrm{E}-04$ & 5.0E-04 & Yes & ND & ND \\
\hline GW-301 & $\mathrm{T}$ & 47 & 1 & $5.3 \mathrm{E}-04$ & $5.0 \mathrm{E}-04$ & No & D & $\mathrm{S}$ \\
\hline GW-731 & $\mathrm{T}$ & 17 & 0 & 5.0E-04 & 5.0E-04 & Yes & ND & ND \\
\hline GW-732 & $\mathrm{T}$ & 17 & 0 & $5.0 \mathrm{E}-04$ & 5.0E-04 & Yes & ND & ND \\
\hline GW-841 & $\mathrm{T}$ & 13 & 13 & $8.8 \mathrm{E}-02$ & 2.4E-02 & No & PD & PD \\
\hline GW-842 & $\mathrm{T}$ & 16 & 15 & $4.2 \mathrm{E}-03$ & 3.0E-03 & No & NT & NT \\
\hline \multicolumn{9}{|l|}{ URANIUM } \\
\hline GW-143 & $\mathrm{T}$ & 28 & 15 & $8.2 \mathrm{E}-04$ & 3.6E-04 & No & D & $D$ \\
\hline GW-144 & $\mathrm{T}$ & 28 & 16 & $1.0 \mathrm{E}-03$ & 1.1E-03 & No & $\mathrm{D}$ & D \\
\hline GW-145 & $\mathrm{T}$ & 28 & 27 & $1.1 \mathrm{E}-02$ & $1.2 \mathrm{E}-02$ & No & $\mathrm{S}$ & NT \\
\hline GW-156 & $\mathrm{T}$ & 26 & 14 & $1.1 \mathrm{E}-03$ & 9.7E-04 & No & $\mathrm{D}$ & D \\
\hline GW-159 & $\mathrm{T}$ & 26 & 14 & $7.9 \mathrm{E}-04$ & 1.1E-03 & No & $\mathrm{D}$ & $D$ \\
\hline GW-161 & $\mathrm{T}$ & 2 & 2 & 2.2E-04 & 2.2E-04 & No & N/A & $\mathrm{N} / \mathrm{A}$ \\
\hline GW-231 & $\mathrm{T}$ & 29 & 6 & $2.3 \mathrm{E}-04$ & $4.9 \mathrm{E}-06$ & No & NT & NT \\
\hline GW-292 & $\mathrm{s}$ & 5 & 2 & $1.2 \mathrm{E}-04$ & 4.9E-06 & No & PI & 1 \\
\hline GW-293 & $\mathrm{S}$ & 5 & 2 & $1.3 \mathrm{E}-04$ & 4.9E-06 & No & NT & 1 \\
\hline GW-294 & $\mathrm{S}$ & 2 & 2 & $1.5 \mathrm{E}-04$ & 1.5E-04 & No & N/A & $\mathrm{N} / \mathrm{A}$ \\
\hline GW-296 & $\mathrm{S}$ & 2 & 2 & $1.2 \mathrm{E}-04$ & 1.2E-04 & No & $\mathrm{N} / \mathrm{A}$ & $\mathrm{N} / \mathrm{A}$ \\
\hline GW-298 & $\mathrm{T}$ & 5 & 5 & $1.5 \mathrm{E}-03$ & 1.6E-03 & No & $\mathrm{S}$ & PD \\
\hline GW-301 & $\mathrm{T}$ & 24 & 2 & $3.1 \mathrm{E}-05$ & 4.9E-06 & No & NT & NT \\
\hline GW-731 & $\mathrm{T}$ & 26 & 5 & 9.6E-05 & 4.9E-06 & No & NT & NT \\
\hline GW-732 & $\mathrm{T}$ & 26 & 12 & 7.5E-04 & 4.9E-06 & No & D & D \\
\hline GW-841 & $\mathrm{T}$ & 1 & 0 & 4.9E-06 & 4.9E-06 & Yes & ND & ND \\
\hline GW-842 & $\mathrm{T}$ & 1 & 0 & 4.9E-06 & 4.9E-06 & Yes & ND & ND \\
\hline
\end{tabular}

Note: Increasing (I); Probably Increasing (PI); Stable (S); Probably Decreasing (PD); Decreasing (D); No Trend (NT); Not Applicable (N/A); Not Applicable (N/A) - Due to insufficient Data (< 4 sampling events); No Detectable Concentration (NDC)

The Number of Samples and Number of Detects shown above are post-consolidation values. 


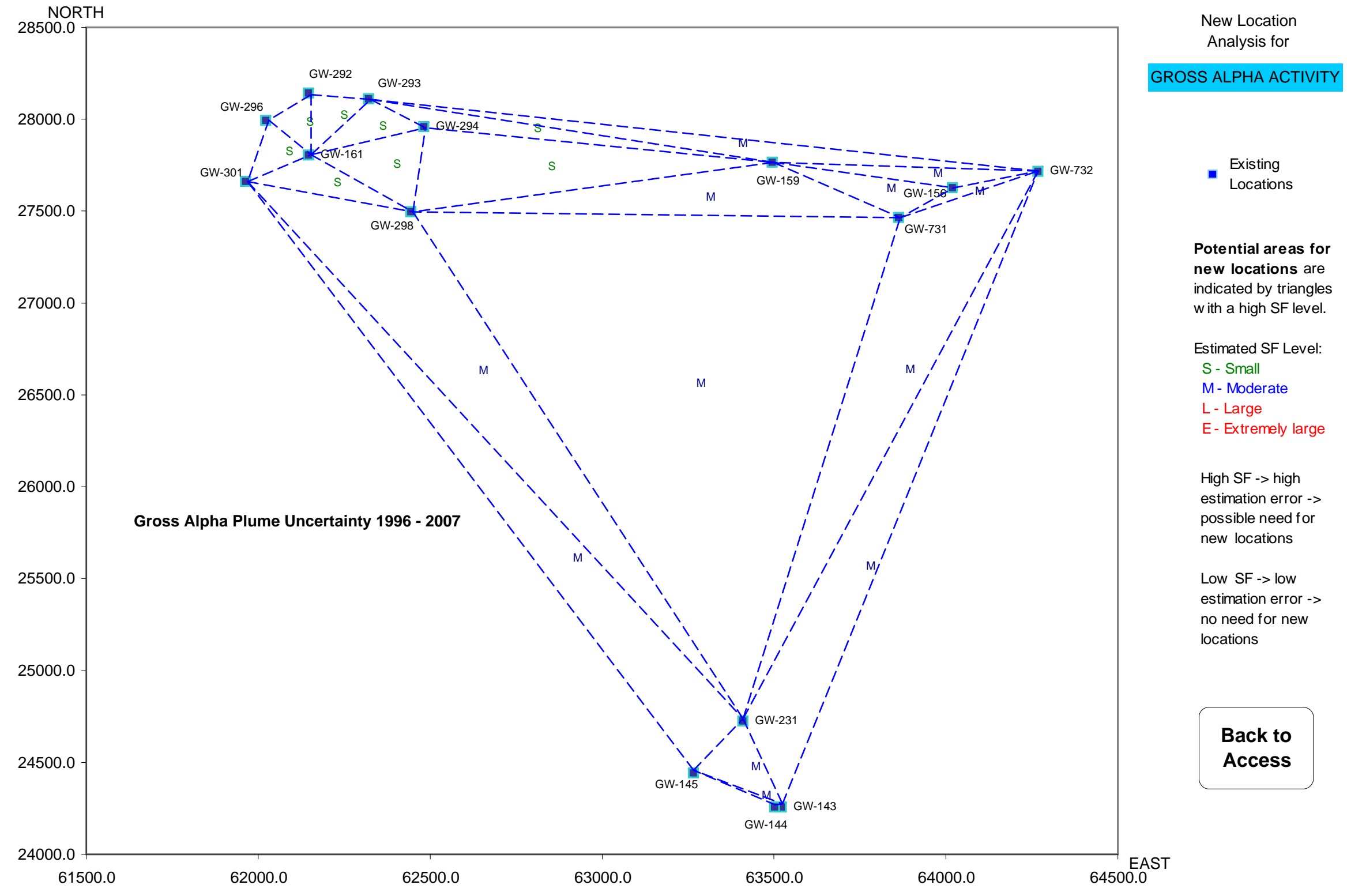


APPENDIX D

SUPPLEMENTAL MAROS ASSESSMENT SAMPLING RECOMMENDATIONS 
APPENDIX D.1

BEAR CREEK HYDROGEOLOGIC REGIME 
APPENDIX D.1

SUMMARY OF SAMPLING RECOMMENDATIONS FOR THE BEAR CREEK REGIME

\begin{tabular}{|c|c|c|c|c|c|c|c|}
\hline Regime & Functional Area & $\begin{array}{c}\text { Location } \\
\text { Name }\end{array}$ & $\begin{array}{l}\text { Recent } \\
\text { Trend }\end{array}$ & $\begin{array}{l}\text { Overall } \\
\text { Trend }\end{array}$ & $\begin{array}{l}\text { Preliminary } \\
\text { Frequency }\end{array}$ & Final Frequency & Recommendation \\
\hline \multicolumn{8}{|c|}{ Regulated Locations } \\
\hline $\mathrm{BC}$ & Oil Landfarm WMA & GW-008 & I & I & Quarterly & Regulated & {$\left[\begin{array}{l}\text { Monitor for PCE and TCE center of Oil } \\
\text { Landfarm area. Sample as per permit. }\end{array}\right.$} \\
\hline $\mathrm{BC}$ & $\begin{array}{l}\text { Bear Creek Burial } \\
\text { Grounds WMA }\end{array}$ & GW-046 & NT & I & Annual & Regulated & $\begin{array}{l}\text { Sample as per permit, sample all COCs } \\
\text { annually. }\end{array}$ \\
\hline $\mathrm{BC}$ & $\begin{array}{l}\text { Bear Creek Burial } \\
\text { Grounds WMA }\end{array}$ & GW-077 & NT & NT & Biennial & Regulated & $\begin{array}{l}\text { Sample as per ROD, largely ND well, } \\
\text { consider reduced effort. }\end{array}$ \\
\hline $\mathrm{BC}$ & $\begin{array}{l}\text { Bear Creek Burial } \\
\text { Grounds WMA }\end{array}$ & GW-078 & NT & NT & Biennial & Regulated & $\begin{array}{l}\text { Sample as per ROD, largely ND well, } \\
\text { consider reduced effort. }\end{array}$ \\
\hline $\mathrm{BC}$ & $\begin{array}{l}\text { Bear Creek Burial } \\
\text { Grounds WMA }\end{array}$ & GW-079 & NT & NT & Biennial & Regulated & $\begin{array}{l}\text { Sample as per ROD, concentrations } \\
\text { consistent w/Biennial sampling. }\end{array}$ \\
\hline $\mathrm{BC}$ & $\begin{array}{l}\text { Bear Creek Burial } \\
\text { Grounds WMA }\end{array}$ & GW-080 & NT & NT & Biennial & Regulated & $\begin{array}{l}\text { Sample as per ROD, largely ND well, } \\
\text { consider reduced effort. }\end{array}$ \\
\hline $\mathrm{BC}$ & EMWMF & GW-363 & ND & ND & Biennial & Regulated & $\begin{array}{l}\text { Alternate RCRA well. Located in largely } \\
\text { unaffected area. Eliminate from program if } \\
\text { possible or reduce frequency to biennial. }\end{array}$ \\
\hline $\mathrm{BC}$ & EMWMF & GW-639 & S & $\mathrm{S}$ & Biennial & Regulated & $\begin{array}{l}\text { Sample as per permit/ROD, consider } \\
\text { reduced effort. }\end{array}$ \\
\hline $\mathrm{BC}$ & $\begin{array}{l}\text { Exit Pathway - } \\
\text { Traverse A }\end{array}$ & GW-683 & $\mathrm{D}$ & $\mathrm{D}$ & Annual & Regulated & $\begin{array}{l}\text { Sample as per ROD, consider annual } \\
\text { sampling. }\end{array}$ \\
\hline $\mathrm{BC}$ & $\begin{array}{l}\text { Exit Pathway - } \\
\text { Traverse A }\end{array}$ & GW-684 & $\mathrm{D}$ & PD & Annual & Regulated & $\begin{array}{l}\text { Sample as per ROD, consider annual } \\
\text { sampling. }\end{array}$ \\
\hline $\mathrm{BC}$ & $\begin{array}{l}\text { Exit Pathway - } \\
\text { Traverse B }\end{array}$ & GW-704 & $\mathrm{D}$ & $\mathrm{D}$ & Annual & Regulated & Sample as per ROD \\
\hline $\mathrm{BC}$ & $\begin{array}{l}\text { Exit Pathway - } \\
\text { Traverse B }\end{array}$ & GW-706 & NT & I & Annual & Regulated & Sample as per ROD \\
\hline $\mathrm{BC}$ & $\begin{array}{l}\text { Exit Pathway - } \\
\text { Traverse W }\end{array}$ & GW-712 & NT & NT & Biennial & Regulated & $\begin{array}{l}\text { Sample as per permit; possible outlier, } \\
\text { largely non-detect, consider reduced effort }\end{array}$ \\
\hline$B C$ & $\begin{array}{l}\text { Exit Pathway - } \\
\text { Traverse W }\end{array}$ & GW-713 & NT & NT & Annual & Regulated & $\begin{array}{l}\text { Sample as per permit, consider annual } \\
\text { sampling. }\end{array}$ \\
\hline$B C$ & $\begin{array}{l}\text { Exit Pathway - } \\
\text { Traverse } \mathrm{W}\end{array}$ & GW-714 & $S$ & PD & Biennial & Regulated & $\begin{array}{l}\text { Sample as per permit, consider annual } \\
\text { sampling. }\end{array}$ \\
\hline $\mathrm{BC}$ & EMWMF & GW-916 & NT & NT & Biennial & Regulated & $\begin{array}{l}\text { Sample as per permit (largely non-detect; } \\
\text { consider reducing sampling frequency in } \\
\text { future) }\end{array}$ \\
\hline
\end{tabular}


APPENDIX D.1

SUMMARY OF SAMPLING RECOMMENDATIONS FOR THE BEAR CREEK REGIME

\begin{tabular}{|c|c|c|c|c|c|c|c|}
\hline Regime & Functional Area & $\begin{array}{l}\text { Location } \\
\text { Name }\end{array}$ & $\begin{array}{l}\text { Recent } \\
\text { Trend }\end{array}$ & $\begin{array}{l}\text { Overall } \\
\text { Trend }\end{array}$ & $\begin{array}{l}\text { Preliminary } \\
\text { Frequency }\end{array}$ & Final Frequency & Recommendation \\
\hline $\mathrm{BC}$ & EMWMF & GW-917 & NT & NT & Biennial & Regulated & $\begin{array}{l}\text { Sample as per permit (largely non-detect; } \\
\text { consider reducing sampling frequency in } \\
\text { future) }\end{array}$ \\
\hline $\mathrm{BC}$ & EMWMF & GW-918 & NT & NT & Biennial & Regulated & $\begin{array}{l}\text { Sample as per permit (largely non-detect; } \\
\text { consider reducing sampling frequency in } \\
\text { future) }\end{array}$ \\
\hline $\mathrm{BC}$ & EMWMF & GW-920 & NT & NT & Biennial & Regulated & $\begin{array}{l}\text { Sample as per permit (largely non-detect; } \\
\text { consider reducing sampling frequency in } \\
\text { future) }\end{array}$ \\
\hline $\mathrm{BC}$ & EMWMF & GW-921 & NT & NT & Biennial & Regulated & $\begin{array}{l}\text { Sample as per permit (largely non-detect; } \\
\text { consider reducing sampling frequency in } \\
\text { future) }\end{array}$ \\
\hline $\mathrm{BC}$ & EMWMF & GW-922 & NT & NT & Biennial & Regulated & $\begin{array}{l}\text { Sample as per permit (largely non-detect; } \\
\text { consider reducing sampling frequency in } \\
\text { future) }\end{array}$ \\
\hline $\mathrm{BC}$ & EMWMF & GW-923 & NT & NT & Biennial & Regulated & $\begin{array}{l}\text { Sample as per permit (largely non-detect; } \\
\text { consider reducing sampling frequency in } \\
\text { future) }\end{array}$ \\
\hline $\mathrm{BC}$ & EMWMF & GW-924 & ND & ND & Annual & Regulated & $\begin{array}{l}\text { Sample as per permit (non-detect for } \\
\text { COPCs; consider reducing sampling } \\
\text { frequency in future) }\end{array}$ \\
\hline$B C$ & EMWMF & GW-925 & NT & NT & Biennial & Regulated & $\begin{array}{l}\text { Sample as per permit (largely non-detect; } \\
\text { consider reducing sampling frequency in } \\
\text { future) }\end{array}$ \\
\hline $\mathrm{BC}$ & EMWMF & GW-926 & NT & NT & Annual & Regulated & $\begin{array}{l}\text { Sample as per permit (largely non-detect; } \\
\text { consider reducing sampling frequency in } \\
\text { future) }\end{array}$ \\
\hline $\mathrm{BC}$ & EMWMF & GW-927 & NT & $\mathrm{N} / \mathrm{A}$ & Annual & Regulated & $\begin{array}{l}\text { Sample as per permit (largely non-detect; } \\
\text { consider reducing sampling frequency in } \\
\text { future) }\end{array}$ \\
\hline $\mathrm{BC}$ & $\begin{array}{c}\text { Exit Pathway } \\
\text { Spring/Surface Water }\end{array}$ & SS-6_6 & ND & ND & Biennial & Regulated & Sampled as spring location SS-6. \\
\hline$B C$ & $\begin{array}{l}\text { Exit Pathway } \\
\text { Spring/Surface Water }\end{array}$ & SS-6E & NT & 1 & Biennial & Regulated & Sampled as spring SS-6. \\
\hline
\end{tabular}


APPENDIX D.1

SUMMARY OF SAMPLING RECOMMENDATIONS FOR THE BEAR CREEK REGIME

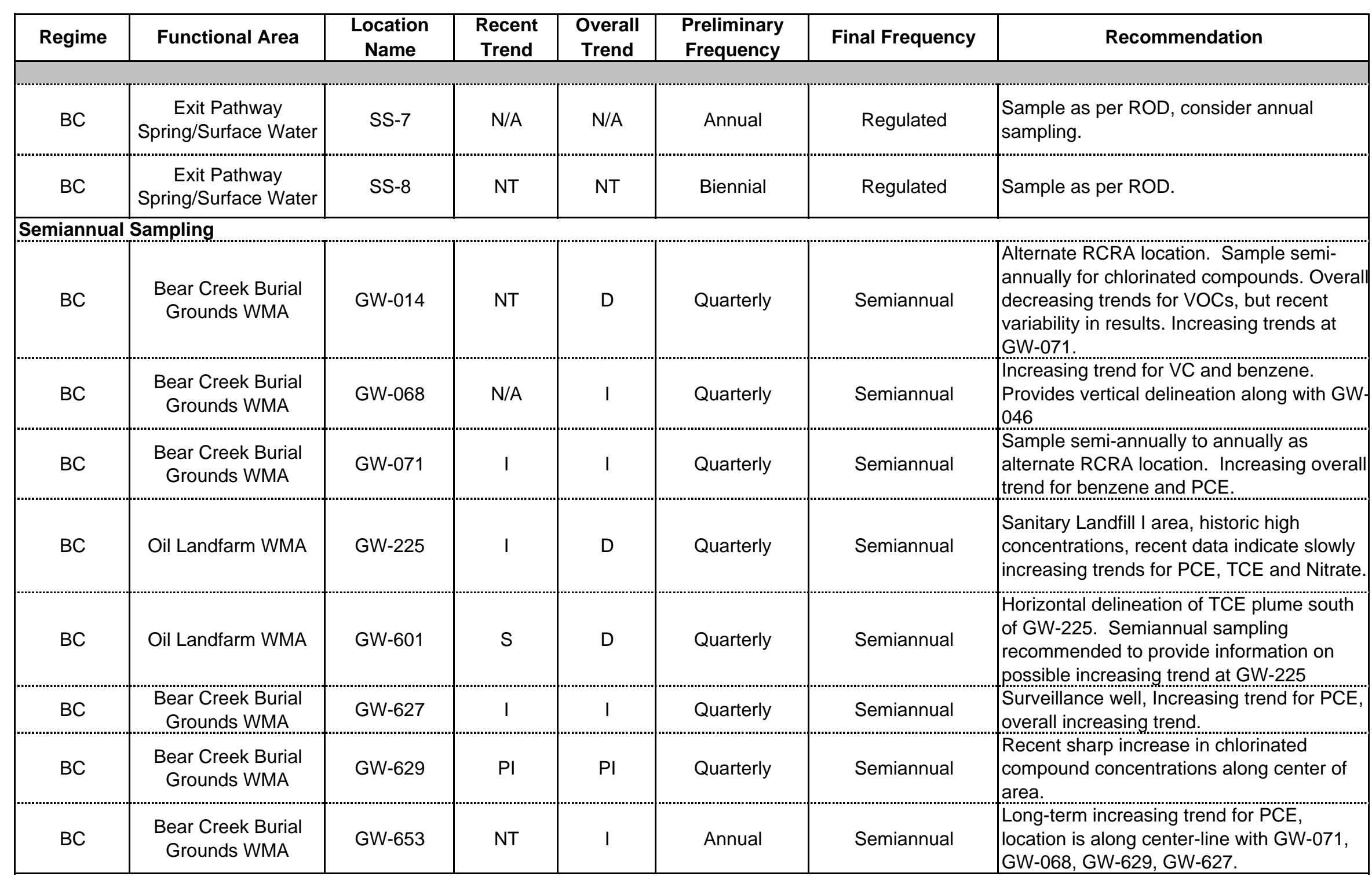


APPENDIX D.1

SUMMARY OF SAMPLING RECOMMENDATIONS FOR THE BEAR CREEK REGIME

\begin{tabular}{|c|c|c|c|c|c|c|c|}
\hline Regime & Functional Area & $\begin{array}{l}\text { Location } \\
\text { Name }\end{array}$ & $\begin{array}{l}\text { Recent } \\
\text { Trend }\end{array}$ & $\begin{array}{l}\text { Overall } \\
\text { Trend }\end{array}$ & $\begin{array}{l}\text { Preliminary } \\
\text { Frequency }\end{array}$ & Final Frequency & Recommendation \\
\hline \multicolumn{8}{|c|}{ Annual Sampling } \\
\hline $\mathrm{BC}$ & $\begin{array}{l}\text { Bear Creek Burial } \\
\text { Grounds WMA }\end{array}$ & GW-082 & NT & I & Quarterly & Annual & $\begin{array}{l}\text { Variable concentrations, sample as an } \\
\text { alternate RCRA location for vinyl chloride. }\end{array}$ \\
\hline $\mathrm{BC}$ & Oil Landfarm WMA & GW-085 & $\mathrm{S}$ & $\mathrm{D}$ & Annual & Annual & $\begin{array}{l}\text { Nitrate is the only major COC, sample } \\
\text { annually. On-strike with GW-587 and GW- } \\
829 \text {, also recommended for annual } \\
\text { sampling. }\end{array}$ \\
\hline $\mathrm{BC}$ & Oil Landfarm WMA & GW-098 & NT & NT & Annual & Annual & $\begin{array}{l}\text { Deeper location next to GS-097. } \\
\text { Surveillance well, vertical delineation. }\end{array}$ \\
\hline $\mathrm{BC}$ & S-3 Site & GW-100 & $\mathrm{N} / \mathrm{A}$ & PD & Quarterly & Annual & Probably decreasing trend, centerline well \\
\hline $\mathrm{BC}$ & S-3 Site & GW-101 & N/A & $\mathrm{D}$ & Quarterly & Annual & $\begin{array}{l}\text { Limited recent samples; sample annually to } \\
\text { delineate northern edge }\end{array}$ \\
\hline $\mathrm{BC}$ & Oil Landfarm WMA & GW-229 & NT & I & Annual & Annual & $\begin{array}{l}\text { Monitors VC degradation product. } \\
\text { Concentrations may show seasonal effects. } \\
\text { Sample in the same season each year to } \\
\text { monitor degradation of chlorinated parent } \\
\text { compounds. }\end{array}$ \\
\hline $\mathrm{BC}$ & $\begin{array}{l}\text { Bear Creek Burial } \\
\text { Grounds WMA }\end{array}$ & GW-242 & NT & NT & Quarterly & Annual & $\begin{array}{l}\text { Variable concentrations, low spatial } \\
\text { uncertainty, horizontal delineation. }\end{array}$ \\
\hline $\mathrm{BC}$ & S-3 Site & GW-246 & S & $\mathrm{PI}$ & Annual & Annual & $\begin{array}{l}\text { Monitors Gross Beta in source area, retain } \\
\text { at annual frequency. }\end{array}$ \\
\hline $\mathrm{BC}$ & S-3 Site & GW-276 & $\mathrm{D}$ & $\mathrm{D}$ & Annual & Annual & $\begin{array}{l}\text { Sample as per permit, consider annual } \\
\text { sampling as trend is decreasing. }\end{array}$ \\
\hline $\mathrm{BC}$ & $\begin{array}{l}\text { Bear Creek Burial } \\
\text { Grounds WMA }\end{array}$ & GW-289 & I & $\mathrm{S}$ & Quarterly & Annual & $\begin{array}{l}\text { Recent increasing trends for PCE and TCE, } \\
\text { long-term stable trend. }\end{array}$ \\
\hline $\mathrm{BC}$ & Rust Spoil Area & GW-307 & $\mathrm{N} / \mathrm{A}$ & $\mathrm{D}$ & Quarterly & Annual & $\begin{array}{l}\text { Defines downgradient boundary of plume } \\
\text { before Exit Pathway Traverse C, limited } \\
\text { recent samples, sample annually. }\end{array}$ \\
\hline $\mathrm{BC}$ & Rust Spoil Area & GW-310 & $N / A$ & $\mathrm{D}$ & Quarterly & Annual & $\begin{array}{l}\text { Long-term decreasing trend, history of } \\
\text { sampling at nearby GW-311 }\end{array}$ \\
\hline $\mathrm{BC}$ & Rust Spoil Area & GW-312 & N/A & $\mathrm{S}$ & Quarterly & Annual & $\begin{array}{l}\text { Well GW-311 is inactive, GW-312 provides } \\
\text { downgradient delineation of TCE affected } \\
\text { groundwater. }\end{array}$ \\
\hline BC & Spoil Area I" & GW-315 & $\mathrm{D}$ & $\mathrm{D}$ & Annual & Annual & Monitor annually, Decreasing trend \\
\hline
\end{tabular}


APPENDIX D.1

SUMMARY OF SAMPLING RECOMMENDATIONS FOR THE BEAR CREEK REGIME

\begin{tabular}{|c|c|c|c|c|c|c|c|}
\hline Regime & Functional Area & $\begin{array}{c}\text { Location } \\
\text { Name }\end{array}$ & $\begin{array}{l}\text { Recent } \\
\text { Trend }\end{array}$ & $\begin{array}{l}\text { Overall } \\
\text { Trend }\end{array}$ & $\begin{array}{l}\text { Preliminary } \\
\text { Frequency }\end{array}$ & Final Frequency & Recommendation \\
\hline $\mathrm{BC}$ & Industrial Landfill I & GW-365 & NT & $\mathrm{PI}$ & Quarterly & Annual & $\begin{array}{l}\text { Monitors VC degradation product } \\
\text { downgradient from GW-229. Decreasing } \\
\text { TCE concentrations may result in } \\
\text { increasing VC. Samples provide } \\
\text { information on horizontal extent of TCE } \\
\text { degradation. }\end{array}$ \\
\hline $\mathrm{BC}$ & S-3 Site & GW-526 & S & I & Annual & Annual & $\begin{array}{l}\text { Sample as per ROD, consider annual } \\
\text { sampling as trend has stabilized. }\end{array}$ \\
\hline $\mathrm{BC}$ & Oil Landfarm WMA & GW-537 & $\mathrm{D}$ & $\mathrm{D}$ & Annual & Annual & $\begin{array}{l}\text { On strike with GW-085 and GW-829. Long } \\
\text { and short-term decreasing trend for Nitrate. }\end{array}$ \\
\hline $\mathrm{BC}$ & S-3 Site & GW-616 & S & NT & Semiannual & Annual & $\begin{array}{l}\text { Monitors nitrate downgradient from source, } \\
\text { along centerline }\end{array}$ \\
\hline $\mathrm{BC}$ & $\begin{array}{l}\text { Exit Pathway - } \\
\text { Traverse B }\end{array}$ & GW-703 & $\mathrm{D}$ & $\mathrm{D}$ & Annual & Annual & $\begin{array}{l}\text { Decreasing trends, but monitored as part of } \\
\text { exit pathway, reduced frequency } \\
\text { appropriate. }\end{array}$ \\
\hline $\mathrm{BC}$ & $\begin{array}{l}\text { Exit Pathway - } \\
\text { Traverse C }\end{array}$ & GW-724 & $\mathrm{D}$ & NT & Annual & Annual & Exit pathway surveillance well. \\
\hline $\mathrm{BC}$ & $\begin{array}{l}\text { Exit Pathway - } \\
\text { Traverse C }\end{array}$ & GW-725 & NT & $\mathrm{D}$ & Semiannual & Annual & $\begin{array}{l}\text { Intermittent concentration spikes, overall } \\
\text { decreasing trend, monitor as part of Exit } \\
\text { Pathway Traverse C. }\end{array}$ \\
\hline $\mathrm{BC}$ & $\begin{array}{l}\text { Exit Pathway - } \\
\text { Traverse C }\end{array}$ & GW-738 & $\mathrm{D}$ & $\mathrm{D}$ & Annual & Annual & $\begin{array}{l}\text { Surveillance well, overall decreasing trend, } \\
\text { monitor as part of Exit Pathway Traverse C. }\end{array}$ \\
\hline $\mathrm{BC}$ & $\begin{array}{l}\text { Exit Pathway - } \\
\text { Traverse C }\end{array}$ & GW-740 & $\mathrm{D}$ & $\mathrm{D}$ & Annual & Annual & $\begin{array}{l}\text { Surveillance well, long-term decreasing } \\
\text { trend; sample annually to monitor Exit } \\
\text { Pathway Traverse C picket. }\end{array}$ \\
\hline $\mathrm{BC}$ & S-3 Site & GW-829 & $\mathrm{D}$ & $\mathrm{D}$ & Quarterly & Annual & $\begin{array}{l}\text { Limited samples between } 2001 \text { and } 2006 \text {, } \\
\text { decreasing trend, sample annually. }\end{array}$ \\
\hline $\mathrm{BC}$ & $\begin{array}{c}\text { Exit Pathway } \\
\text { Spring/Surface Water }\end{array}$ & SS-1 & $\mathrm{D}$ & D & Annual & Annual & $\begin{array}{l}\text { Spring shows Decreasing trend, sample } \\
\text { annually. }\end{array}$ \\
\hline $\mathrm{BC}$ & $\begin{array}{c}\text { Exit Pathway } \\
\text { Spring/Surface Water }\end{array}$ & SS-4 & $S$ & D & Annual & Annual & $\begin{array}{l}\text { Spring, potential exposure point, } \\
\text { decreasing long-term trend. }\end{array}$ \\
\hline $\mathrm{BC}$ & $\begin{array}{c}\text { Exit Pathway } \\
\text { Spring/Surface Water }\end{array}$ & SS-5 & NT & $\mathrm{D}$ & Annual & Annual & $\begin{array}{l}\text { Spring, potential exposure point, } \\
\text { decreasing long-term trend. }\end{array}$ \\
\hline
\end{tabular}


APPENDIX D.1

SUMMARY OF SAMPLING RECOMMENDATIONS FOR THE BEAR CREEK REGIME

\begin{tabular}{|c|c|c|c|c|c|c|c|}
\hline Regime & Functional Area & $\begin{array}{l}\text { Location } \\
\text { Name }\end{array}$ & $\begin{array}{l}\text { Recent } \\
\text { Trend }\end{array}$ & $\begin{array}{l}\text { Overall } \\
\text { Trend }\end{array}$ & $\begin{array}{l}\text { Preliminary } \\
\text { Frequency }\end{array}$ & Final Frequency & Recommendation \\
\hline \multicolumn{8}{|c|}{ Biennial Sampling } \\
\hline $\mathrm{BC}$ & $\begin{array}{l}\text { Bear Creek Burial } \\
\text { Grounds WMA }\end{array}$ & GW-053 & NT & NT & Semiannual & Biennial & $\begin{array}{l}\text { Decreasing trend for VC, low levels of GA, } \\
\text { extensive long-term data record. Sample to } \\
\text { monitor decreasing trends over time. }\end{array}$ \\
\hline $\mathrm{BC}$ & $\begin{array}{l}\text { Bear Creek Burial } \\
\text { Grounds WMA }\end{array}$ & GW-058 & N/A & $\mathrm{S}$ & Quarterly & Biennial & $\begin{array}{l}\text { Horizontal delineation. Stable GA trend. } \\
\text { Intermittent detections of PCE }\end{array}$ \\
\hline \multicolumn{8}{|c|}{ Remove from the Active Network } \\
\hline $\mathrm{BC}$ & $\begin{array}{l}\text { Bear Creek Burial } \\
\text { Grounds WMA }\end{array}$ & GW-052 & $N / A$ & PD & Quarterly & Eliminate & $\begin{array}{l}\text { Non-detect for PCE/TCE, overall } \\
\text { decreasing trend for Gross Alpha. }\end{array}$ \\
\hline $\mathrm{BC}$ & $\begin{array}{l}\text { Bear Creek Burial } \\
\text { Grounds WMA }\end{array}$ & GW-072 & NT & I & Quarterly & Eliminate & $\begin{array}{l}\text { Very few detections, shallower than GW- } \\
071 \text {. }\end{array}$ \\
\hline $\mathrm{BC}$ & $\begin{array}{l}\text { Bear Creek Burial } \\
\text { Grounds WMA }\end{array}$ & GW-089 & N/A & NT & Annual & Eliminate & Redundant with GW-242 \\
\hline $\mathrm{BC}$ & S-3 Site & GW-122 & S & $\mathrm{D}$ & Annual & Eliminate & $\begin{array}{l}\text { Overall decreasing trend, redundant with } \\
\text { GW-616. }\end{array}$ \\
\hline $\mathrm{BC}$ & S-3 Site & GW-127 & $N / A$ & $\mathrm{D}$ & Quarterly & Eliminate & $\begin{array}{l}\text { Redundant with GW-616, low } \\
\text { concentrations of Gross Alpha, not as } \\
\text { heavily affected as surrounding wells. }\end{array}$ \\
\hline $\mathrm{BC}$ & Oil Landfarm WMA & GW-226 & $S$ & 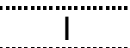 & Annual' & Eliminate & Redundant with GW-225 \\
\hline $\mathrm{BC}$ & Oil Landfarm WMA & GW-227 & $N / A$ & $\mathrm{D}$ & Quarterly & Eliminate & $\begin{array}{l}\text { Long-term decreasing trend confirmed, } \\
\text { recent reduction in sampling frequency. } \\
\text { Eliminate from routine monitoring. }\end{array}$ \\
\hline BC' & S-3 Site & GW-236 & N/A & D" & Quarterly & Eliminate & Decreasing trend, redundant with SS-1. \\
\hline $\mathrm{BC}$ & S-3 Site & GW-244 & N/A & $\mathrm{D}$ & Quarterly & Eliminate & $\begin{array}{l}\text { Stable to decreasing trend, redundant with } \\
\text { other locations. }\end{array}$ \\
\hline $\mathrm{BC}$ & S-3 Site & GW-245 & $\mathrm{N} / \mathrm{A}$ & $\mathrm{S}$ & Quarterly & Eliminate & $\begin{array}{l}\text { Stable to decreasing trend, redundant with } \\
\text { other locations. }\end{array}$ \\
\hline $\mathrm{BC}$ & S-3 Site & $\mathrm{GW}-247$ & N/A & S & Quarterly & Eliminate & Redundant with GW-246. \\
\hline $\mathrm{BC}$ & $\begin{array}{l}\text { Bear Creek Burial } \\
\text { Grounds WMA }\end{array}$ & GW-257 & NT & NT & Semiannual & Eliminate & $\begin{array}{l}\text { Alternate RCRA location. Largely non- } \\
\text { detect for most COPCs, concentrations of } \\
\text { PCE show no trend. Redundant with GW- } \\
289 \text {. }\end{array}$ \\
\hline $\mathrm{BC}$ & S-3 Site & GW-277 & $N / A$ & $\mathrm{~S}$ & Quarterly & Eliminate & $\begin{array}{l}\text { Stable to decreasing trend, redundant with } \\
\text { other source locations. }\end{array}$ \\
\hline $\mathrm{BC}$ & $\begin{array}{l}\text { Bear Creek Burial } \\
\text { Grounds WMA }\end{array}$ & GW-291 & $\mathrm{S}$ & $\mathrm{S}$ & Quarterly & Eliminate & $\begin{array}{l}\text { Alternate RCRA location, historic high } \\
\text { concentrations, but stable trends. }\end{array}$ \\
\hline BC' & Spoil Area I' & GW-313 & N/A & NT" & Quarterly & Eliminate & Redundant with GW-315 \\
\hline
\end{tabular}


APPENDIX D.1

SUMMARY OF SAMPLING RECOMMENDATIONS FOR THE BEAR CREEK REGIME

\begin{tabular}{|c|c|c|c|c|c|c|c|}
\hline Regime & Functional Area & $\begin{array}{c}\text { Location } \\
\text { Name }\end{array}$ & $\begin{array}{l}\text { Recent } \\
\text { Trend }\end{array}$ & $\begin{array}{l}\text { Overall } \\
\text { Trend }\end{array}$ & $\begin{array}{l}\text { Preliminary } \\
\text { Frequency }\end{array}$ & Final Frequency & Recommendation \\
\hline $\mathrm{BC}$ & Industrial Landfill I & GW-368 & $\mathrm{N} / \mathrm{A}$ & $\mathrm{D}$ & Quarterly & Eliminate & $\begin{array}{l}\text { Redundant with GW-601; long-term } \\
\text { decreasing trend for TCE. }\end{array}$ \\
\hline $\mathrm{BC}$ & Industrial Landfill I & GW-369 & $\mathrm{N} / \mathrm{A}$ & $\mathrm{D}$ & Quarterly & Eliminate & $\begin{array}{l}\text { Redundant with GW-601; long-term } \\
\text { decreasing trend for TCE. }\end{array}$ \\
\hline $\mathrm{BC}$ & S-3 Site & GW-615 & NT & I & Quarterly & Eliminate & $\begin{array}{l}\text { High concentrations are largely stable, no } \\
\text { rapid changes, redundant with other source } \\
\text { area wells. }\end{array}$ \\
\hline $\mathrm{BC}$ & $\begin{array}{l}\text { Bear Creek Burial } \\
\text { Grounds WMA }\end{array}$ & GW-624 & $\mathrm{S}$ & $\mathrm{S}$ & Quarterly & Eliminate & Redundant with GW-629 \\
\hline $\mathrm{BC}$ & $\begin{array}{l}\text { Bear Creek Burial } \\
\text { Grounds WMA }\end{array}$ & GW-626 & I & I & Quarterly & Eliminate & Redundant with GW-627 \\
\hline $\mathrm{BC}$ & $\begin{array}{l}\text { Exit Pathway - } \\
\text { Traverse B }\end{array}$ & GW-694 & NT & $\mathrm{D}$ & Annual & Eliminate & $\begin{array}{l}\text { Decreasing trends, low concentrations, low } \\
\text { slope factor indicate low spatial uncertainty } \\
\text { in area. Redundant with GW-704 and GW- } \\
695\end{array}$ \\
\hline $\mathrm{BC}$ & $\begin{array}{l}\text { Exit Pathway - } \\
\text { Traverse C }\end{array}$ & GW-736 & $\mathrm{S}$ & $\mathrm{D}$ & Quarterly & Eliminate & $\begin{array}{l}\text { Redundant with GW-725; decreasing } \\
\text { concentrations of Nitrate and TCE }\end{array}$ \\
\hline $\mathrm{BC}$ & $\begin{array}{l}\text { Exit Pathway - } \\
\text { Traverse C }\end{array}$ & GW-739 & S & $\mathrm{S}$ & Quarterly & Eliminate & $\begin{array}{l}\text { Redundant with GW-740; very stable to } \\
\text { decreasing trend; low rate of change } \\
\text { supports elimination from routine } \\
\text { monitoring. }\end{array}$ \\
\hline \multicolumn{8}{|c|}{ Locations with no data 2005 - 2007 (to be sampled in 2008) } \\
\hline $\mathrm{BC}$ & Oil Landfarm WMA & GW-006 & S & $\mathrm{S}$ & Annual & Biennial & $\begin{array}{l}\text { If results still show area of low } \\
\text { concentrations, low rate of change, low } \\
\text { uncertainty. Monitor biennially to every } 5 \\
\text { years to confirm low concentrations. }\end{array}$ \\
\hline $\mathrm{BC}$ & $\begin{array}{l}\text { Bear Creek Burial } \\
\text { Grounds WMA }\end{array}$ & GW-259 & N/A & NT & Annual & Every 5 years & $\begin{array}{l}\text { Location has historically high } \\
\text { concentrations of PCE. May be redundant } \\
\text { with GW-257. If concentration of PCE has } \\
\text { not changed significantly since } 1990 \text { (PCE } \\
2 \text { mg/L)) or if concentration has decreased } \\
\text { significantly, consider inactive }\end{array}$ \\
\hline $\mathrm{BC}$ & Rust Spoil Area & GW-306 & $\mathrm{N} / \mathrm{A}$ & NT & Biennial & Every 5 years & $\begin{array}{l}\text { Confirm concentrations within expected } \\
\text { range (70 ug/L TCE; } 10 \mathrm{mg} / \mathrm{L} \mathrm{NO})\end{array}$ \\
\hline $\mathrm{BC}$ & Rust Spoil Area & GW-309 & $\mathrm{N} / \mathrm{A}$ & $\mathrm{D}$ & Biennial & Every 5 years & $\begin{array}{l}\text { Confirm concentrations of NO3 and GB in } \\
\text { center of Rust Spoils Area. }\end{array}$ \\
\hline
\end{tabular}


APPENDIX D.1

SUMMARY OF SAMPLING RECOMMENDATIONS FOR THE BEAR CREEK REGIME

\begin{tabular}{|c|c|c|c|c|c|c|c|}
\hline Regime & Functional Area & $\begin{array}{c}\text { Location } \\
\text { Name }\end{array}$ & $\begin{array}{c}\text { Recent } \\
\text { Trend } \\
\end{array}$ & $\begin{array}{l}\text { Overall } \\
\text { Trend }\end{array}$ & $\begin{array}{c}\text { Preliminary } \\
\text { Frequency }\end{array}$ & Final Frequency & Recommendation \\
\hline $\mathrm{BC}$ & Spoil Area I & GW-314 & N/A & $S$ & Annual & Eliminate & $\begin{array}{l}\text { If PCE and NO3 concentrations still low, } \\
\text { consider inactive status. Redundant with } \\
\text { GW-315. }\end{array}$ \\
\hline $\mathrm{BC}$ & Industrial Landfill I & GW-367 & N/A & D & Biennial & Every 5 years & $\begin{array}{l}\text { Only well in area to show detections of } \\
\text { TCE, unknown screen depth. Monitor } \\
\text { every } 5 \text { years to confirm decreasing trends } \\
\text { of TCE; provides horizontal delineation of } \\
\text { affected groundwater. }\end{array}$ \\
\hline $\mathrm{BC}$ & Lysimeter Demo & GW-531 & $\mathrm{N} / \mathrm{A}$ & $\mathrm{S}$ & Biennial & Eliminate & $\begin{array}{l}\text { If results still below screening levels, } \\
\text { consider inactive status. If concentrations } \\
\text { have increased, sample annually until trend } \\
\text { confirmed. }\end{array}$ \\
\hline $\mathrm{BC}$ & $\begin{array}{l}\text { Bear Creek Burial } \\
\text { Grounds WMA }\end{array}$ & GW-623 & N/A & I & Annual & Semiannual & $\begin{array}{l}\text { Location has historically high } \\
\text { concentrations of PCE in deep } \\
\text { groundwater. The general area shows high } \\
\text { spatial uncertainty (see Plume Uncertainty } \\
\text { Figure). Sample along with GW-071 to } \\
\text { characterize TCE concentrations over time }\end{array}$ \\
\hline
\end{tabular}


APPENDIX D.2

UPPER EAST FORK POPLAR CREEK HYDROGEOLOGIC REGIME 
APPENDIX D.2

SUMMARY OF SAMPLING RECOMMENDATIONS FOR THE EAST FORK REGIME

\begin{tabular}{|c|c|c|c|c|c|c|c|}
\hline Regime & Functional Area & $\begin{array}{l}\text { Location } \\
\text { Name }\end{array}$ & $\begin{array}{l}\text { Recent } \\
\text { Trend }\end{array}$ & $\begin{array}{l}\text { Overall } \\
\text { Trend }\end{array}$ & $\begin{array}{l}\text { Preliminary } \\
\text { Frequency }\end{array}$ & Final Frequency & Recommendation \\
\hline \multicolumn{8}{|c|}{ Regulated Locations } \\
\hline EF & S-3 Site & GW-108 & $\mathrm{D}$ & $\mathrm{D}$ & Semiannual & Regulated & $\begin{array}{l}\text { Limited recent (2004 -2007) sample events } \\
\text { trigger quarterly monitoring recommendation, } \\
\text { Historically high concentrations but stable to } \\
\text { decreasing trends. Monitors nitrate source } \\
\text { area. Continue limited sampling frequency. }\end{array}$ \\
\hline EF & New Hope Pond & GW-151 & 1 & 1 & Quarterly & Regulated & Sample as per ROD \\
\hline EF & New Hope Pond & GW-153 & $\mathrm{D}$ & $\mathrm{D}$ & Annual & Regulated & $\begin{array}{l}\text { Sample as per permit. Decreasing short and } \\
\text { long-term trends, horizontal delineation. }\end{array}$ \\
\hline EF & New Hope Pond & GW-154 & "ai" & "ai" & Quarterly & Regulated & Sample as per ROD \\
\hline EF & $\begin{array}{c}\text { Union Valley - Exit } \\
\text { Pathway }\end{array}$ & GW-169 & $\mathrm{S}$ & $\mathrm{S}$ & Annual & Regulated & Sample as per ROD \\
\hline EF & $\begin{array}{c}\text { Union Valley - Exit } \\
\text { Pathway }\end{array}$ & GW-170 & NT & $\mathrm{D}$ & Annual & Regulated & Sample as per ROD \\
\hline EF & $\begin{array}{c}\text { Union Valley - Exit } \\
\text { Pathway }\end{array}$ & GW-171 & NT & NT & -- & Regulated & Sample as per ROD \\
\hline EF & $\begin{array}{c}\text { Union Valley - Exit } \\
\text { Pathway }\end{array}$ & GW-172 & NT & NT & -- & Regulated & Sample as per ROD \\
\hline EF & Uranium Oxide Vault & GW-219 & $\mathrm{S}$ & $\mathrm{S}$ & Quarterly & Regulated & $\begin{array}{l}\text { Sample as per ROD. Stable long-term trend, } \\
\text { but recent (2004 - 2007) results indicate a } \\
\text { possible increase -- triggers Quarterly } \\
\text { sampling recommendation. }\end{array}$ \\
\hline$E F$ & New Hope Pond & $\mathrm{GW}-220$ & 1 & ! & Quarterly & Regulated & Sample as per ROD. \\
\hline EF & New Hope Pond & GW-223 & D...... & $\mathrm{D}$ & Annual & Regulated & Sample as per ROD \\
\hline EF & $\begin{array}{c}\text { Union Valley - Exit } \\
\text { Pathway }\end{array}$ & GW-230 & $\mathrm{S}$ & $\mathrm{S}$ & Biennial & Regulated & Sample as per ROD \\
\hline EF & S-2 Site & GW-253 & NT & $\mathrm{S}$ & Quarterly & Regulated & $\begin{array}{l}\text { Sample as per ROD, High historic cadmium } \\
\text { concentrations, }\end{array}$ \\
\hline EF & Y-12 Fuel Station & GW-281 & ND & NT & Biennial & Regulated & Sample as per ROD \\
\hline EF & New Hope Pond & GW-380 & $\mathrm{S}$ & $\mathrm{D}$ & Annual & Regulated & Sample as per ROD \\
\hline EF & New Hope Pond & GW-381 & $\mathrm{D}$ & $\mathrm{D}$ & Annual & Regulated & Sample as per ROD \\
\hline EF & New Hope Pond & $\mathrm{GW}-382$ & $\mathrm{D}$ & $\mathrm{D}$ & Annual & Regulated & Sample as perROD \\
\hline EF & New Hope Pond & GW-383 & $\mathrm{S}$ & 1 & Annual & Regulated & Sample as per ROD \\
\hline EF & $\begin{array}{c}\text { Exit Pathway - } \\
\text { Traverse I }\end{array}$ & GW-605 & I & 1 & Quarterly & Regulated & Sample as per ROD or permit \\
\hline EF & $\begin{array}{l}\text { Exit Pathway - } \\
\text { Traverse I }\end{array}$ & GW-606 & $\mathrm{D}$ & $\mathrm{D}$ & Annual & Regulated & Sample as per ROD or permit \\
\hline EF & $\begin{array}{l}\text { Exit Pathway - } \\
\text { Traverse E }\end{array}$ & GW-618 & $\mathrm{D}$ & $\mathrm{D}$ & Quarterly & Regulated & $\begin{array}{l}\text { Sample as per ROD, historic high } \\
\text { concentrations of VOC and cadmium with } \\
\text { largely decreasing trends. }\end{array}$ \\
\hline
\end{tabular}


APPENDIX D.2

SUMMARY OF SAMPLING RECOMMENDATIONS FOR THE EAST FORK REGIME

\begin{tabular}{|c|c|c|c|c|c|c|c|}
\hline Regime & Functional Area & $\begin{array}{l}\text { Location } \\
\text { Name }\end{array}$ & $\begin{array}{l}\text { Recent } \\
\text { Trend }\end{array}$ & $\begin{array}{l}\text { Overall } \\
\text { Trend }\end{array}$ & $\begin{array}{l}\text { Preliminary } \\
\text { Frequency }\end{array}$ & Final Frequency & Recommendation \\
\hline EF & Y-12 Fuel Station & GW-658 & NT & NT & Quarterly & Regulated & Sample as per ROD \\
\hline EF & $\begin{array}{l}\text { Exit Pathway - } \\
\text { Traverse J }\end{array}$ & GW-733 & $\mathrm{D}$ & $\mathrm{D}$ & Annual & Regulated & Sample as per ROD \\
\hline EF & Y-12 Grid Well K1 & $\mathrm{GW}-744$ & PD & S & Annual & Regulated & Sample as per ROD \\
\hline EF' & Y-12 Grid Well K2 & GW-747 & $S$ & NT" & Biennial & Regulated & Sample as perROD \\
\hline EF & $\begin{array}{l}\text { Y-12 Grid Well J- } \\
\text { Primary }\end{array}$ & GW-762 & I & I & Quarterly & Regulated & Sample as per ROD \\
\hline EF & Y-12 Fuel Station & GW-802 & ND & ND & Biennial & Regulated & Sample as per ROD \\
\hline EF & $\begin{array}{c}\text { Exit Pathway Scarboro } \\
\text { Road/Pine Ridge }\end{array}$ & GW-816 & $\mathrm{D}$ & $\mathrm{D}$ & Annual & Regulated & Sample as per ROD \\
\hline$E F$ & New Hope Pond & GW-832 & D...... & D..... & Annual & Regulated & Sample as per ROD \\
\hline EF & $\begin{array}{l}\text { Union Valley - Exit } \\
\text { Pathway }\end{array}$ & SCR7_1SP & $\mathrm{D}$ & $\mathrm{D}$ & Annual & Regulated & Sample as per ROD \\
\hline EF & $\begin{array}{c}\text { Exit Pathway } \\
\text { Spring/Surface Water }\end{array}$ & SCR7_8SP & D & $\mathrm{D}$ & Biennial & Regulated & Sample as per ROD \\
\hline \multicolumn{8}{|c|}{ Semiannual Sampling } \\
\hline EF & -- & $55-3 A$ & NT & NT & Quarterly & Semiannual & $\begin{array}{l}\text { New well monitoring source area. Monitor } \\
\text { semiannually until trend stabilizes. }\end{array}$ \\
\hline EF & -- & $55-3 B$ & NT & NT & Quarterly & Semiannual & $\begin{array}{l}\text { New well monitoring source area. Monitor } \\
\text { semiannually until trend stabilizes. }\end{array}$ \\
\hline EF & -- & $55-3 C$ & NT & NT & Quarterly & Semiannual & $\begin{array}{l}\text { New well monitoring source area. Monitor } \\
\text { semiannually until trend stabilizes. }\end{array}$ \\
\hline EF & Coal Pile Trench & GW-691 & $\mathrm{PI}$ & $\mathrm{PI}$ & Quarterly & Semiannual & $\begin{array}{l}\text { Shallow well near GW-690, higher } \\
\text { concentrations with possible increasing trends. }\end{array}$ \\
\hline EF & Building 8110 & GW-698 & I & I & Quarterly & Semiannual & $\begin{array}{l}\text { Increasing to probably increasing trends, well } \\
\text { monitors centerline of Maynardville Limestone, } \\
\text { monitor until plume stabilizes. }\end{array}$ \\
\hline EF & Y-12 Grid Well G3 & GW-769 & 1 & I & Quarterly & Semiannual & $\begin{array}{l}\text { Surveillance well, steadily increasing trend for } \\
\text { Carbon Tetrachloride. Monitor semiannually }\end{array}$ \\
\hline EF & Y-12 Grid Well G3 & GW-770 & I & I & Annual & Semiannual & $\begin{array}{l}\text { Surveillance well, steadily increasing trend for } \\
\text { Carbon Tetrachloride. Monitor semiannually }\end{array}$ \\
\hline
\end{tabular}


APPENDIX D.2

SUMMARY OF SAMPLING RECOMMENDATIONS FOR THE EAST FORK REGIME

\begin{tabular}{|c|c|c|c|c|c|c|c|}
\hline Regime & Functional Area & $\begin{array}{l}\text { Location } \\
\text { Name }\end{array}$ & $\begin{array}{l}\text { Recent } \\
\text { Trend }\end{array}$ & $\begin{array}{l}\text { Overall } \\
\text { Trend }\end{array}$ & $\begin{array}{l}\text { Preliminary } \\
\text { Frequency }\end{array}$ & Final Frequency & Recommendation \\
\hline \multicolumn{8}{|c|}{ Annual Sampling } \\
\hline EF & Y-12 Grid Well B3 & $55-2 B$ & NT & NT & Semiannual & Annual & $\begin{array}{l}\text { Increasing concentrations of nitrate, no trend } \\
\text { for PCE. Monitor for horizontal and vertical } \\
\text { delineation of nitrate and PCE. }\end{array}$ \\
\hline EF & Y-12 Grid Well B3 & $55-2 \mathrm{C}$ & $\mathrm{S}$ & $\mathrm{S}$ & Quarterly & Annual & $\begin{array}{l}\text { Surveillance well, Increasing concentrations of } \\
\text { nitrate, stable PCE concentrations, monitor } \\
\text { annually along with 55-2B to delineate } \\
\text { horizontal and vertical concentrations of nitrate } \\
\text { and PCE. }\end{array}$ \\
\hline EF" & $--\cdot$ & $56-1 A$ & NT" & NT & Biennial & Annual' & Delineates northern area of plume. \\
\hline EF & Y-12 Grid Well C3 & $56-2 A$ & $\mathrm{~S}$ & $\mathrm{~S}$ & Annual & Annual & $\begin{array}{l}\text { Low concentrations stable trends. Consider } \\
\text { reducing frequency if trend continues to be } \\
\text { stable. }\end{array}$ \\
\hline EF & Y-12 Grid Well C3 & $56-2 B$ & $\mathrm{~S}$ & S & Annual & Annual & $\begin{array}{l}\text { Stable recent trend, monitors vertical } \\
\text { distribution of } P C E \text { in center of plume. }\end{array}$ \\
\hline EF & Y-12 Grid Well C3 & $56-2 C$ & PD & PD & Quarterly & Annual & $\begin{array}{l}\text { Stable recent trend, monitors vertical } \\
\text { distribution of } P C E \text { in center of plume. }\end{array}$ \\
\hline EF & -- & $56-3 A$ & $\mathrm{~S}$ & S & Annual & Annual & reducing frequency if trend continues to be \\
\hline EF & -.:- & $56-3 B$ & NT & NT & Quarterly & Annual & Variable recent concentrations, monitors \\
\hline EF & -- & $56-3 C$ & $\mathrm{~S}$ & S & Annual & Annual & $\begin{array}{l}\text { Stable recent trend, monitors vertical } \\
\text { distribution of PCE in center of plume. }\end{array}$ \\
\hline EF & -- & $56-4 A$ & NT & NT & Annual & Annual & $\begin{array}{l}\text { Monitors center of PCE plume, monitor } \\
\text { annually along with } 56-2 \text { wells. }\end{array}$ \\
\hline EF & -- & $56-8 \mathrm{~A}$ & $\mathrm{~N} / \mathrm{A}$ & N/A & Annual & Annual & $\begin{array}{l}\text { Monitor annually until sufficient samples to } \\
\text { determine a trend. }\end{array}$ \\
\hline EF & New Hope Pond & GW-240 & $\mathrm{S}$ & $\mathrm{D}$ & Annual & Annual & $\begin{array}{l}\text { Decreasing long-term trend, low levels of } \\
\text { COPCs. }\end{array}$ \\
\hline EF & S-2 Site & GW-251 & $\mathrm{S}$ & $\mathrm{D}$ & Annual & Annual & $\begin{array}{l}\text { Surveillance well, horizontal delineation to the } \\
\text { south, Centerline of Maynardville Limestone, } \\
\text { supports data from GW-253 on VOC plume }\end{array}$ \\
\hline EF & Y-12 Salvage Yard & GW-272 & N/A & I & Quarterly & Annual & $\begin{array}{l}\text { No recent trend information, long-term } \\
\text { increasing trend. Monitor for nitrate along } \\
\text { center-line of plume, along with GW-505 for } \\
\text { possible movement of nitrate. }\end{array}$ \\
\hline EF & Y-12 Salvage Yard & GW-274 & I & I & Quarterly & Annual & $\begin{array}{l}\text { Alternate RCRA regulatory location. } \\
\text { Decreasing trend for nitrate, long and short- } \\
\text { term increasing trends for PCE. }\end{array}$ \\
\hline
\end{tabular}


APPENDIX D.2

SUMMARY OF SAMPLING RECOMMENDATIONS FOR THE EAST FORK REGIME

\begin{tabular}{|c|c|c|c|c|c|c|c|}
\hline Regime & Functional Area & $\begin{array}{l}\text { Location } \\
\text { Name }\end{array}$ & $\begin{array}{l}\text { Recent } \\
\text { Trend }\end{array}$ & $\begin{array}{l}\text { Overall } \\
\text { Trend }\end{array}$ & $\begin{array}{l}\text { Preliminary } \\
\text { Frequency }\end{array}$ & Final Frequency & Recommendation \\
\hline EF & Y-12 Salvage Yard & GW-275 & $\mathrm{PI}$ & $\mathrm{S}$ & Quarterly & Annual & $\begin{array}{l}\text { Alternate RCRA regulatory location. Recent } \\
\text { increasing trend in nitrate, long-term stable } \\
\text { trend. }\end{array}$ \\
\hline EF & $\begin{array}{l}\text { Waste Coolant } \\
\text { Processing Facility }\end{array}$ & GW-332 & NT & NT & Quarterly & Annual & $\begin{array}{l}\text { Historic high concentrations with limited rate of } \\
\text { change for PCE. Monitor center-line of PCE } \\
\text { with 55-2B and GW-337. }\end{array}$ \\
\hline EF & $\begin{array}{l}\text { Waste Coolant } \\
\text { Processing Facility }\end{array}$ & GW-337 & $\mathrm{S}$ & $\mathrm{D}$ & Quarterly & Annual & $\begin{array}{l}\text { Historic high concentrations with long-term } \\
\text { decreasing trend for PCE. Monitor center-line } \\
\text { of PCE with 55-2B and GW-332. }\end{array}$ \\
\hline EF & Fire Training Facility & GW-620 & $\mathrm{D}$ & $\mathrm{D}$ & Annual & Annual & $\begin{array}{l}\text { Surveillance well in deeper groundwater near } \\
\text { GW-619. Monitor annually to document } \\
\text { decreasing concentrations of PCE, delineates } \\
\text { both horizontally and vertically. }\end{array}$ \\
\hline EF & Rust Garage Area & GW-633 & I & I & Quarterly & Annual & $\begin{array}{l}\text { Surveillance well, Increasing trend for } \\
\text { benzene, monitor for benzene trend. }\end{array}$ \\
\hline EF & Coal Pile Trench & GW-686 & NT & NT & Annual & Annual & $\begin{array}{l}\text { Delineates plume south of source area, } \\
\text { monitor annually for chlorinated hydrocarbons. }\end{array}$ \\
\hline EF & Coal Pile Trench & GW-690 & PD & PD & Quarterly & Annual & $\begin{array}{l}\text { Surveillance well with probably decreasing } \\
\text { trends. }\end{array}$ \\
\hline EF & Coal Pile Trench & GW-692 & NT & NT & Annual & Annual & $\begin{array}{l}\text { Low concentrations, monitor along with other } \\
\text { wells in Coal Pile Trench area. }\end{array}$ \\
\hline EF & Building 8110 & GW-700 & $\mathrm{D}$ & D & Quarterly & Annual & $\begin{array}{l}\text { Strongly decreasing trend for PCE in shallow } \\
\text { groundwater. }\end{array}$ \\
\hline EF & $\begin{array}{l}\text { Exit Pathway - } \\
\text { Traverse J }\end{array}$ & GW-735 & NT & NT & Annual & Annual & $\begin{array}{l}\text { Exit pathway well, low detections of COPCs, } \\
\text { monitor annually as early warning for possible } \\
\text { migration. }\end{array}$ \\
\hline EF & $\begin{array}{l}\text { Y-12 Grid Well J- } \\
\text { Primary }\end{array}$ & GW-763 & NT & NT & Annual & Annual & Delineates shallow groundwater near GW-762. \\
\hline EF & Y-12 Grid Well E3 & GW-781 & NT & NT & Annual & Annual & $\begin{array}{l}\text { Concentration spiked in 1998, decreasing } \\
\text { since, annual monitoring to confirm decreasing } \\
\text { recent trend. }\end{array}$ \\
\hline EF & Y-12 Grid Well E3 & GW-782 & $\mathrm{D}$ & D & Annual & Annual & $\begin{array}{l}\text { Surveillance well, Concentration of VOC } \\
\text { decreasing. Monitors shallow groundwater }\end{array}$ \\
\hline EF & Y-12 Grid Well E3 & GW-783 & $\mathrm{S}$ & $\mathrm{D}$ & Quarterly & Annual & $\begin{array}{l}\text { Stable to decreasing trend with fairly low } \\
\text { concentrations. Monitor annually with GW-781 } \\
\text { and } 782 \text {. }\end{array}$ \\
\hline
\end{tabular}


APPENDIX D.2

SUMMARY OF SAMPLING RECOMMENDATIONS FOR THE EAST FORK REGIME

\begin{tabular}{|c|c|c|c|c|c|c|c|}
\hline Regime & Functional Area & $\begin{array}{l}\text { Location } \\
\text { Name }\end{array}$ & $\begin{array}{l}\text { Recent } \\
\text { Trend }\end{array}$ & $\begin{array}{l}\text { Overall } \\
\text { Trend }\end{array}$ & $\begin{array}{l}\text { Preliminary } \\
\text { Frequency }\end{array}$ & Final Frequency & Recommendation \\
\hline EF & Y-12 Grid Well D2 & GW-791 & $\mathrm{S}$ & $\mathrm{D}$ & Annual & Annual & $\begin{array}{l}\text { Stable to decreasing trend in area of high } \\
\text { concentrations of VOCs, long-term monitoring } \\
\text { record, monitors affected deeper groundwater } \\
\text { in northern area of plume. }\end{array}$ \\
\hline EF & Building 9201-2 & GW-820 & $\mathrm{S}$ & $\mathrm{S}$ & Annual & Annual & $\begin{array}{l}\text { Along line of } G W-219 \text { and } G W-959 \text {. Higher } \\
\text { concentrations of } V O C \text {, but stable trend. }\end{array}$ \\
\hline EF & -- & GW-954-1 & N/A & N/A & -- & Annual & $\begin{array}{l}\text { Low level detections, monitor annually to } \\
\text { delineate horizontal distribution of COPCs. }\end{array}$ \\
\hline EF & -- & GW-954-2 & NT & NT & -- & Annual & $\begin{array}{l}\text { Low level detections, monitor annually to } \\
\text { delineate horizontal distribution of COPCs. }\end{array}$ \\
\hline EF & -- & GW-954-3 & $\mathrm{S}$ & $\mathrm{S}$ & -- & Annual & $\begin{array}{l}\text { Low level detections, monitor annually to } \\
\text { delineate horizontal distribution of COPCs. }\end{array}$ \\
\hline EF & -- & GW-956-1 & NT & NT & -- & Annual & $\begin{array}{l}\text { Low level detections, monitor annually to } \\
\text { delineate horizontal distribution of COPCs. }\end{array}$ \\
\hline EF & -- & GW-956-2 & NT & NT & -- & Annual & $\begin{array}{l}\text { Low level detections, monitor annually to } \\
\text { delineate horizontal distribution of COPCs. }\end{array}$ \\
\hline EF & -- & GW-956-3 & NT & NT & -- & Annual & $\begin{array}{l}\text { Low level detections, monitor annually to } \\
\text { delineate horizontal distribution of COPCs. }\end{array}$ \\
\hline EF & -- & GW-956-4 & N/A & N/A & -- & Annual & $\begin{array}{l}\text { Low level detections, monitor annually to } \\
\text { delineate horizontal distribution of COPCS. }\end{array}$ \\
\hline EF & -- & GW-959 & NT & NT & Annual & Annual & $\begin{array}{l}\text { Monitors degradation of VOC in plume, } \\
\text { downgradient from GW-820, monitor annually. }\end{array}$ \\
\hline EF & -- & GW-960 & N/A & $N / A$ & Annual & Annual & $\begin{array}{l}\text { New well near GW-779, outside of VOC } \\
\text { plume. Delineates groundwater to the north. } \\
\text { Sample annually and evaluate trend. }\end{array}$ \\
\hline EF & $\begin{array}{c}\text { Exit Pathway } \\
\text { Spring/Surface Water }\end{array}$ & UEFPC-SP17 & NT & NT & Semiannual & Annual & Sample as per permit. \\
\hline \multicolumn{8}{|c|}{ Biennial Sampling } \\
\hline EF & Y-12 Grid Well B3 & $55-2 A$ & $\mathrm{D}$ & $\mathrm{D}$ & Annual & Biennial & $\begin{array}{l}\text { Shallow well in nested group, decreasing } \\
\text { recent trends for PCE. }\end{array}$ \\
\hline EF & Y-12 Grid Well C2 & $56-6 A$ & NT & NT & Biennial & Biennial & $\begin{array}{l}\text { Low concentrations of Gross Beta and nitrate, } \\
\text { non-detect for other site COPCs. }\end{array}$ \\
\hline EF & Y-12 Plant Site & $60-1 A$ & NT & NT & Biennial & Biennial & $\begin{array}{l}\text { Very limited detections, delineation of plumes } \\
\text { horizontally. }\end{array}$ \\
\hline EF & S-3 Site & GW-106 & $\mathrm{S}$ & $\mathrm{D}$ & Quarterly & Biennial & $\begin{array}{l}\text { Surveillance well, Limited recent (2004-2007) } \\
\text { sample events trigger quarterly monitoring } \\
\text { recommendation, stable to decreasing trends. } \\
\text { Monitors nitrate source area. }\end{array}$ \\
\hline
\end{tabular}


APPENDIX D.2

SUMMARY OF SAMPLING RECOMMENDATIONS FOR THE EAST FORK REGIME

\begin{tabular}{|c|c|c|c|c|c|c|c|}
\hline Regime & Functional Area & $\begin{array}{l}\text { Location } \\
\text { Name }\end{array}$ & $\begin{array}{l}\text { Recent } \\
\text { Trend }\end{array}$ & $\begin{array}{l}\text { Overall } \\
\text { Trend }\end{array}$ & $\begin{array}{l}\text { Preliminary } \\
\text { Frequency }\end{array}$ & Final Frequency & Recommendation \\
\hline & & & & & & & \\
\hline EF & S-3 Site & GW-109 & NT & NT & Quarterly & Biennial & $\begin{array}{l}\text { Limited recent (2004 -2007) sample events } \\
\text { trigger quarterly monitoring recommendation, } \\
\text { Historically high concentrations but stable to } \\
\text { decreasing trends. Monitors nitrate source } \\
\text { area. Alternate regulatory location. Continue } \\
\text { limited sampling frequency. }\end{array}$ \\
\hline EF & New Hope Pond & GW-148 & $\mathrm{N} / \mathrm{A}$ & N/A & -- & Biennial & $\begin{array}{l}\text { Monitors shallow groundwater between GW- } \\
383 \text { and GW-832, delineates plume, largely } \\
\text { non-detect results }\end{array}$ \\
\hline EF & Y-12 Plant Site & GW-204 & $\mathrm{S}$ & I & Semiannual & Biennial & $\begin{array}{l}\text { Surveillance well, Sample biennially, monitor } \\
\text { trend for Gross Alpha }\end{array}$ \\
\hline EF & Rust Garage Area & GW-505 & $\mathrm{S}$ & NT & Semiannual & Biennial & $\begin{array}{l}\text { Surveillance well. Low levels of gross alpha } \\
\text { and nitrate, stable to decreasing trends. } \\
\text { Limited VOC detections. Stable to no trend. }\end{array}$ \\
\hline EF & Fire Training Facility & GW-619 & PD & $\mathrm{D}$ & Quarterly & Biennial & $\begin{array}{l}\text { Decreasing trends for VOCS, in shallow } \\
\text { groundwater. }\end{array}$ \\
\hline EF & Y-12 Plant Site & GW-656 & $\mathrm{D}$ & $\mathrm{D}$ & Quarterly & Biennial & $\begin{array}{l}\text { Strong decreasing trend, relatively high } \\
\text { concentrations. Monitor along with GW-204 to } \\
\text { delineate VOC plume. }\end{array}$ \\
\hline $\mathrm{EF}$ & Y-12 Grid Well K2 & GW-748 & $\mathrm{S}$ & $\mathrm{D}$ & Biennial & Biennial & $\begin{array}{l}\text { Monitors property boundary, decreasing long- } \\
\text { term trend, monitor biennially as early warning } \\
\text { for possible migration. }\end{array}$ \\
\hline $\mathrm{EF}$ & $\begin{array}{l}\text { Exit Pathway - } \\
\text { Traverse J }\end{array}$ & GW-750 & $\mathrm{S}$ & S & Biennial & Biennial & $\begin{array}{l}\text { Monitors property boundary, stable long-term } \\
\text { trend, monitor biennially as early warning for } \\
\text { possible migration. }\end{array}$ \\
\hline EF & Y-12 Grid Well E1 & GW-765 & $N / A$ & $\mathrm{D}$ & Annual & Biennial & $\begin{array}{l}\text { Occasional sampling to delineate northern } \\
\text { extent of affected groundwater. }\end{array}$ \\
\hline $\mathrm{EF}$ & Y-12 Grid Well H3 & GW-775 & $\mathrm{S}$ & NT & Semiannual & Biennial & $\begin{array}{l}\text { Monitors deeper groundwater at the eastern } \\
\text { end of the Central Y-12/Fuel Station. Stable } \\
\text { trend, reduce sample frequency. }\end{array}$ \\
\hline EF & Y-12 Grid Well H3 & GW-776 & PD & $\mathrm{D}$ & Annual & Biennial & $\begin{array}{l}\text { Monitors shallow groundwater at the eastern } \\
\text { end of the Central Y-12/Fuel Station. Stable } \\
\text { trend, reduce sample frequency. }\end{array}$ \\
\hline $\mathrm{EF}$ & Y-12 Grid Well F2 & GW-779 & $N / A$ & NT & Annual & Biennial & $\begin{array}{l}\text { Low detections, provides unique information, } \\
\text { recommended limited sampling. }\end{array}$ \\
\hline EF & Y-12 Grid Well D2 & GW-792 & $\mathrm{D}$ & D & Semiannual & Biennial & $\begin{array}{l}\text { Located near GW-791, but in shallow zone, } \\
\text { much lower concentrations, stable trends, } \\
\text { reduce monitoring frequency. }\end{array}$ \\
\hline
\end{tabular}


APPENDIX D.2

SUMMARY OF SAMPLING RECOMMENDATIONS FOR THE EAST FORK REGIME

\begin{tabular}{|c|c|c|c|c|c|c|c|}
\hline Regime & Functional Area & $\begin{array}{l}\text { Location } \\
\text { Name }\end{array}$ & $\begin{array}{l}\text { Recent } \\
\text { Trend }\end{array}$ & $\begin{array}{l}\text { Overall } \\
\text { Trend }\end{array}$ & $\begin{array}{l}\text { Preliminary } \\
\text { Frequency }\end{array}$ & Final Frequency & Recommendation \\
\hline EF & Y-12 Grid Well B2 & $55-1 A$ & $\mathrm{~S}$ & $\mathrm{~S}$ & Annual & Every 5 years & $\begin{array}{l}\text { Delineates northern edge of affected } \\
\text { groundwater, limited detections of COPCs, } \\
\text { reduce sampling }\end{array}$ \\
\hline EF & Beta-4 Security Pits & GW-192 & NT & 1 & Semiannual & Every 5 years & $\begin{array}{l}\text { Monitor to delineate northern VOC plume, } \\
\text { monitor infrequently, along with 55-1A, to } \\
\text { delineate plume. }\end{array}$ \\
\hline $\mathrm{EF}$ & Y-12 Salvage Yard & GW-265 & $\mathrm{S}$ & $\mathrm{D}$ & Annual & Every 5 years & $\begin{array}{l}\text { Stable to decreasing trends, monitor } \\
\text { occasionally to delineate southern edge of } \\
\text { plume. }\end{array}$ \\
\hline $\mathrm{EF}$ & Y-12 Salvage Yard & GW-269 & $\mathrm{D}$ & I & Annual & Every 5 years & $\begin{array}{l}\text { Recent decreasing trends, monitor } \\
\text { occasionally to delineate southern edge of } \\
\text { plume. }\end{array}$ \\
\hline \multicolumn{8}{|c|}{ Remove from the Active Network } \\
\hline EF & S-3 Site & GW-105 & NT & $\mathrm{D}$ & Quarterly & Eliminate & $\begin{array}{l}\text { Surveillance well, Limited recent (2004 -2007) } \\
\text { sample events trigger quarterly monitoring } \\
\text { recommendation, stable to decreasing trends. } \\
\text { Monitors nitrate source area. Does not provide } \\
\text { unique information. }\end{array}$ \\
\hline EF & Y-12 Salvage Yard & $G W-270$ & N/A & $\mathrm{D}$ & Quarterly & Eliminate & Redundant with GW-505. \\
\hline EF & Y-12 Salvage Yard & $\mathrm{GW}-273$ & ND & $\mathrm{D}$ & Annual & Eliminate & Redundant with GW-633. \\
\hline EF & $\begin{array}{c}\text { Waste Coolant } \\
\text { Processing Facility }\end{array}$ & GW-336 & $\mathrm{S}$ & NT & Quarterly & Eliminate & Redundant with GW-332 and GW-337. \\
\hline EF & $\begin{array}{l}\text { Exit Pathway - } \\
\text { Traverse E }\end{array}$ & GW-617 & $\mathrm{S}$ & NT & Annual & Eliminate & $\begin{array}{l}\text { Low-level and non-detect results for COPCS. } \\
\text { Monitors very shallow groundwater, redundant } \\
\text { with GW-618. }\end{array}$ \\
\hline
\end{tabular}


APPENDIX D.2

SUMMARY OF SAMPLING RECOMMENDATIONS FOR THE EAST FORK REGIME

\begin{tabular}{|c|c|c|c|c|c|c|c|}
\hline Regime & Functional Area & $\begin{array}{l}\text { Location } \\
\text { Name }\end{array}$ & $\begin{array}{l}\text { Recent } \\
\text { Trend }\end{array}$ & $\begin{array}{c}\text { Overall } \\
\text { Trend }\end{array}$ & $\begin{array}{l}\text { Preliminary } \\
\text { Frequency }\end{array}$ & Final Frequency & Recommendation \\
\hline \multicolumn{8}{|c|}{ Locations with no data 2005 - 2007} \\
\hline $\mathrm{EF}$ & New Hope Pond & GW-222 & $\mathrm{s}$ & NT & Annual & Annual & $\begin{array}{l}\text { Not sampled since } 2004 \text {, recommendation as } \\
\text { per } 2005 \text { analysis. }\end{array}$ \\
\hline $\mathrm{EF}$ & Y-12 Plant Site & GW-190 & ND & NT & Biennial & Every 5 years & $\begin{array}{l}\text { To be Sampled in 2008. Confirm PCE } \\
\text { concentrations below MCLs (or ND), monitor } \\
\text { metal concentrations, consider reduced } \\
\text { sampling frequency. }\end{array}$ \\
\hline $\mathrm{EF}$ & Rust Garage Area & GW-508 & N/A & D & Remove & Every 5 years & $\begin{array}{l}\text { To be Sampled 2008. Area of high benzene } \\
\text { concentrations, Nearby GW- } 633 \text { has } \\
\text { increasing trend for benzene. If } 2008 \text { results } \\
\text { confirm decreasing benzene trend, sample } \\
\text { every five years to confirm trend. }\end{array}$ \\
\hline
\end{tabular}


APPENDIX D.3

\section{CHESTNUT RIDGE HYDROGEOLOGIC REGIME}


APPENDIX D.3

SUMMARY SAMPLING RECOMMENDATIONS FOR THE CHESTNUT RIDGE REGIME

\begin{tabular}{|c|c|c|c|c|c|c|c|}
\hline Regime & Functional Area & $\begin{array}{c}\text { Location } \\
\text { Name }\end{array}$ & $\begin{array}{l}\text { Recent } \\
\text { Trend }\end{array}$ & $\begin{array}{l}\text { Overall } \\
\text { Trend }\end{array}$ & $\begin{array}{l}\text { Preliminary } \\
\text { Frequency }\end{array}$ & $\begin{array}{c}\text { Final } \\
\text { Frequency }\end{array}$ & Recommendation \\
\hline \multicolumn{8}{|c|}{ Regulated Locations } \\
\hline $\mathrm{CR}$ & $\begin{array}{l}\text { United Nuclear Corporation } \\
\text { Site }\end{array}$ & 1090 & $\mathrm{~S}$ & $\mathrm{D}$ & Biennial & Regulated & Sample as per permit \\
\hline CR & Industrial Landfill IV & $\mathrm{GW}-141$ & 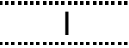 & 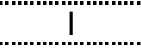 & Biennial & Regulated & Sample as per permit \\
\hline CR & Kerr Hollow Quarry & GW-143 & $\mathrm{S}$ & $\mathrm{S}$ & Biennial & Regulated & Sample as per permit or as per ROD \\
\hline CR & Kerr Hollow Quarry & $\mathrm{GW}-144$ & NT & $\mathrm{D}$ & Biennial & Regulated & Sample as per permit or as per ROD \\
\hline CR & Kerr Hollow Quarry & $\mathrm{GW}-145$ & $\mathrm{~S}$ & $\mathrm{D}$ & Annual & Regulated & Sample as per permit or as per ROD \\
\hline $\mathrm{CR}$ & $\begin{array}{l}\text { Chestnut Ridge Sediment } \\
\text { Disposal Basin }\end{array}$ & GW-156 & $\mathrm{D}$ & $\mathrm{D}$ & Biennial & Regulated & Sample as per permit \\
\hline CR & $\begin{array}{l}\text { Chestnut Ridge Sediment } \\
\text { Disposal Basin }\end{array}$ & GW-159 & $\mathrm{D}$ & $\mathrm{D}$ & Biennial & Regulated & Sample as per permit \\
\hline CR & $\begin{array}{l}\text { East Chestnut Ridge Waste } \\
\text { Pile }\end{array}$ & GW-161 & $\mathrm{N} / \mathrm{A}$ & N/A & Quarterly & Regulated & $\begin{array}{l}\text { New well, very limited detections of COPCs, } \\
\text { sample annually as sentinel well. }\end{array}$ \\
\hline $\mathrm{CR}$ & Chestnut Ridge Security Pits & GW-177 & $\mathrm{S}$ & I & Annual & Regulated & Sample as per permit. \\
\hline CR & $\begin{array}{l}\text { United Nuclear Corporation } \\
\text { Site }\end{array}$ & GW-203 & NT & I & Biennial & Regulated & Sample as per permit \\
\hline $\mathrm{CR}$ & $\begin{array}{l}\text { United Nuclear Corporation } \\
\text { Site }\end{array}$ & GW-205 & I & I & Annual & Regulated & Sample as per permit \\
\hline CR & Industrial Landfill IV & $\mathrm{GW}-217$ & NTT & $\mathrm{D}$ & Biennial & Regulated & Sample as per permit \\
\hline $\mathrm{CR}$ & $\begin{array}{l}\text { United Nuclear Corporation } \\
\text { Site }\end{array}$ & GW-221 & NT & $\mathrm{D}$ & Annual & Regulated & Sample as per permit \\
\hline CR & Kerr Hollow Quarry & $\mathrm{GW}-231$ & S & S & Biennial & Regulated & Sample as per permit or as per ROD \\
\hline $\mathrm{CR}$ & $\begin{array}{l}\text { East Chestnut Ridge Waste } \\
\text { Pile }\end{array}$ & GW-292 & N/A & N/A & Annual & Regulated & $\begin{array}{l}\text { Non-detect for TCE, limited samples for nitrate } \\
\text { and other COPCs. Detection monitoring for } \\
\text { any COPCS. }\end{array}$ \\
\hline $\mathrm{CR}$ & $\begin{array}{l}\text { East Chestnut Ridge Waste } \\
\text { Pile }\end{array}$ & GW-293 & N/A & N/A & Annual & Regulated & $\begin{array}{l}\text { Only one detection of TCE, low levels of gross } \\
\text { alpha. }\end{array}$ \\
\hline CR & $\begin{array}{l}\text { East Chestnut Ridge Waste } \\
\text { Pile }\end{array}$ & GW-294 & N/A & N/A & Annual & Regulated & $\begin{array}{l}\text { New well, very limited detections of COPCS, } \\
\text { sample annually until sufficient samples to } \\
\text { detect a trend. }\end{array}$ \\
\hline CR & $\begin{array}{l}\text { East Chestnut Ridge Waste } \\
\text { Pile }\end{array}$ & GW-296 & N/A & N/A & Annual & Regulated & $\begin{array}{l}\text { New well, very limited detections of COPCs, } \\
\text { sample annually until sufficient samples to } \\
\text { detect a trend. }\end{array}$ \\
\hline $\mathrm{CR}$ & $\begin{array}{l}\text { Chestnut Ridge Borrow Area } \\
\text { Waste Pile }\end{array}$ & GW-298 & N/A & NT & Annual & Regulated & $\begin{array}{l}\text { No-detect for TCE, gross alpha and uranium } \\
\text { found at low levels. }\end{array}$ \\
\hline $\mathrm{CR}$ & $\begin{array}{l}\text { Chestnut Ridge Borrow Area } \\
\text { Waste Pile }\end{array}$ & GW-301 & NT & NT & Quarterly & Regulated & Sample as per permit \\
\hline
\end{tabular}


APPENDIX D.3

SUMMARY SAMPLING RECOMMENDATIONS FOR THE CHESTNUT RIDGE REGIME

\begin{tabular}{|c|c|c|c|c|c|c|c|}
\hline Regime & Functional Area & $\begin{array}{l}\text { Location } \\
\text { Name }\end{array}$ & $\begin{array}{l}\text { Recent } \\
\text { Trend }\end{array}$ & $\begin{array}{l}\text { Overall } \\
\text { Trend }\end{array}$ & $\begin{array}{l}\text { Preliminary } \\
\text { Frequency }\end{array}$ & $\begin{array}{c}\text { Final } \\
\text { Frequency }\end{array}$ & Recommendation \\
\hline CR & Industrial Landfill IV & GW-305 & NT & NT & Annual & Regulated & Sample as per permit \\
\hline CR & Industrial Landfill IV & GW-521 & $\mathrm{D}$ & $\mathrm{D}$ & Biennial & Regulated & Sample as per permit \\
\hline CR & Industrial Landfill IV & GW-522 & $\mathrm{S}$ & $\mathrm{S}$ & Biennial & Regulated & Sample as per permit \\
\hline CR & Industrial Landfill II & $\mathrm{GW}-540$ & NT & PD & Biennial & Regulated & Sample as per permit \\
\hline CR & $\begin{array}{l}\text { Construction/Demolition } \\
\text { Landfill VI }\end{array}$ & GW-542 & $\mathrm{D}$ & D & Biennial & Regulated & Sample as per permit \\
\hline $\mathrm{CR}$ & $\begin{array}{l}\text { Construction/Demolition } \\
\text { Landfill VI }\end{array}$ & GW-543 & NT & NT & Biennial & Regulated & Sample as per permit \\
\hline $\mathrm{CR}$ & $\begin{array}{l}\text { Construction/Demolition } \\
\text { Landfill VI }\end{array}$ & GW-544 & NT & NT & Biennial & Regulated & Sample as per permit \\
\hline CR & Industrial Landfill V & $G W-557$ & PD & PD & Annual & Regulated & Sample as per permit. \\
\hline CR & $\begin{array}{l}\text { Construction/Demolition } \\
\text { Landfill VII }\end{array}$ & GW-560 & NT & NT & Annual & Regulated & Sample as per permit. \\
\hline CR & $\begin{array}{l}\text { Construction/Demolition } \\
\text { Landfill VII }\end{array}$ & GW-562 & NT & NT & Annual & Regulated & Sample as per permit. \\
\hline CR & $\begin{array}{l}\text { Construction/Demolition } \\
\text { Landfill VII }\end{array}$ & GW-564 & $\mathrm{S}$ & $\mathrm{S}$ & Annual & Regulated & Sample as per permit. \\
\hline CR & Industrial Landfill II & GW-709 & NT & $\mathrm{D}$ & Biennial & Regulated & Sample as per permit \\
\hline $\mathrm{CR}$ & $\begin{array}{l}\text { Chestnut Ridge Sediment } \\
\text { Disposal Basin }\end{array}$ & GW-731 & NT & NT & Biennial & Regulated & Sample as per permit \\
\hline CR & $\begin{array}{l}\text { Chestnut Ridge Sediment } \\
\text { Disposal Basin }\end{array}$ & GW-732 & PD & D & Biennial & Regulated & Sample as per permit \\
\hline CR & Industrial Landfill II & $\mathrm{GW}-757$ & 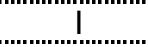 & (1)... & Biennial & Regulated & Sample as per permit \\
\hline CRR & Industrial Landfill V & GW-796 & $\mathrm{S}$ & $\mathrm{S}$ & Annual & Regulated & Sample as per permit. \\
\hline CR & Industrial Landfili V & $\mathrm{GW}-797$ & alu. & (1) & Annual & Regulated & Sample as per permit. \\
\hline CR & $\begin{array}{l}\text { Construction/Demolition } \\
\text { Landfill VII }\end{array}$ & GW-798 & I & 1 & Annual & Regulated & Sample as per permit. \\
\hline CR & Industrial Landfill V & GW-799 & S & S & Annual & Regulated & Sample as per permit. \\
\hline CR & Industrial Landfill V & GW-801 & S & S & Annual & Regulated & Sample as per permit. \\
\hline CR & $\begin{array}{l}\text { Construction/Demolition } \\
\text { Landfill VI }\end{array}$ & GW-827 & PD & PD & Biennial & Regulated & Sample as per permit \\
\hline $\mathrm{CR}$ & Chestnut Ridge Security Pits & GW-831 & NT & NT & Quarterly & Regulated & Sample as per permit. \\
\hline $\mathrm{CR}$ & Chestnut Ridge Security Pits & GW-831 & NT & NT & Quarterly & Regulated & Sample as per permit. \\
\hline CR & $\begin{array}{l}\text { South Campus Facility, Bethel } \\
\text { Valley }\end{array}$ & GW-841 & PD & PD & Quarterly & Regulated & Sample as per ROD \\
\hline
\end{tabular}


APPENDIX D.3

SUMMARY SAMPLING RECOMMENDATIONS FOR THE CHESTNUT RIDGE REGIME

\begin{tabular}{|c|c|c|c|c|c|c|c|}
\hline Regime & Functional Area & $\begin{array}{l}\text { Location } \\
\text { Name }\end{array}$ & $\begin{array}{l}\text { Recent } \\
\text { Trend }\end{array}$ & $\begin{array}{l}\text { Overall } \\
\text { Trend }\end{array}$ & $\begin{array}{l}\text { Preliminary } \\
\text { Frequency }\end{array}$ & $\begin{array}{c}\text { Final } \\
\text { Frequency }\end{array}$ & Recommendation \\
\hline $\mathrm{CR}$ & $\begin{array}{l}\text { South Campus Facility, Bethel } \\
\text { Valley }\end{array}$ & GW-842 & NT & NT & Annual & Regulated & Sample as per ROD \\
\hline CR & $\begin{array}{l}\text { Exit Pathway Spring/Surface } \\
\text { Water }\end{array}$ & SCR1_25SP & NT & NT & Biennial & Regulated & Sample as per permit or ROD \\
\hline CR & $\begin{array}{l}\text { Exit Pathway Spring/Surface } \\
\text { Water }\end{array}$ & SCR2_1SP & $\mathrm{D}$ & $\mathrm{D}$ & Annual & Regulated & Sample as per ROD \\
\hline $\mathrm{CR}$ & $\begin{array}{l}\text { Exit Pathway Spring/Surface } \\
\text { Water }\end{array}$ & SCR2_2SP & NT & NT & Annual & Regulated & Sample as per ROD \\
\hline $\mathrm{CR}$ & $\begin{array}{l}\text { Exit Pathway Spring/Surface } \\
\text { Water }\end{array}$ & SCR3_5SP & $\mathrm{PI}$ & $\mathrm{PI}$ & Annual & Regulated & Sample as per permit or ROD \\
\hline $\mathrm{CR}$ & Industrial Landfill V & SCR4_3SP & ND & ND & Biennial & Regulated & Sample as per permit. \\
\hline \multicolumn{8}{|c|}{ Annual Sampling } \\
\hline $\mathrm{CR}$ & Chestnut Ridge Security Pits & GW-322 & $\mathrm{S}$ & NT & Annual & Annual & $\begin{array}{l}\text { Stable to variable trend in 11DCE, } \\
\text { concentrations above screening level. Monitor } \\
\text { extent of 11DCE. }\end{array}$ \\
\hline \multicolumn{8}{|c|}{ Biennial Sampling } \\
\hline CR & Chestnut Ridge Security Pits & GW-174 & N/A & D & Quarterly & Biennial & $\begin{array}{l}\text { Decreasing long-term trend, recent } \\
\text { concentration below screening level. Monitor to } \\
\text { confirm decreasing trend. }\end{array}$ \\
\hline $\mathrm{CR}$ & Chestnut Ridge Security Pits & GW-175 & S & $\mathrm{D}$ & Annual & Biennial & $\begin{array}{l}\text { Decreasing long-term trend, recent } \\
\text { concentration below screening level. Alternate } \\
\text { regulatory point. Monitor to confirm decreasing } \\
\text { trend. }\end{array}$ \\
\hline $\mathrm{CR}$ & Chestnut Ridge Security Pits & GW-176 & $N / A$ & I & Quarterly & Biennial & $\begin{array}{l}\text { Decreasing trend for PCE, but increasing trend } \\
\text { for 11DCE. Sample along with GW-174 and } \\
\text { GW-175. }\end{array}$ \\
\hline CR & Chestnut Ridge Security Pits & GW-180 & N/A & D & Quarterly & Biennial & $\begin{array}{l}\text { Decreasing long-term trend for PCE, retain to } \\
\text { delineate plume. }\end{array}$ \\
\hline $\mathrm{CR}$ & Filled Coal Ash Pond & GW-514 & $N / A$ & S & Annual & Biennial & $\begin{array}{l}\text { Concentrations below screening levels, monitor } \\
\text { occasionally to detect any change in } \\
\text { concentrations. Alternate regulatory point. }\end{array}$ \\
\hline CR & Chestnut Ridge Security Pits & GW-609 & $\mathrm{D}$ & D & Annual & Biennial & $\begin{array}{l}\text { Decreasing short and long-term trends for } \\
\text { PCE, recent concentrations below screening } \\
\text { levels. Alternate regulatory point. Well } \\
\text { provides unique information -- spatially isolated. }\end{array}$ \\
\hline
\end{tabular}


APPENDIX D.3

SUMMARY SAMPLING RECOMMENDATIONS FOR THE CHESTNUT RIDGE REGIME

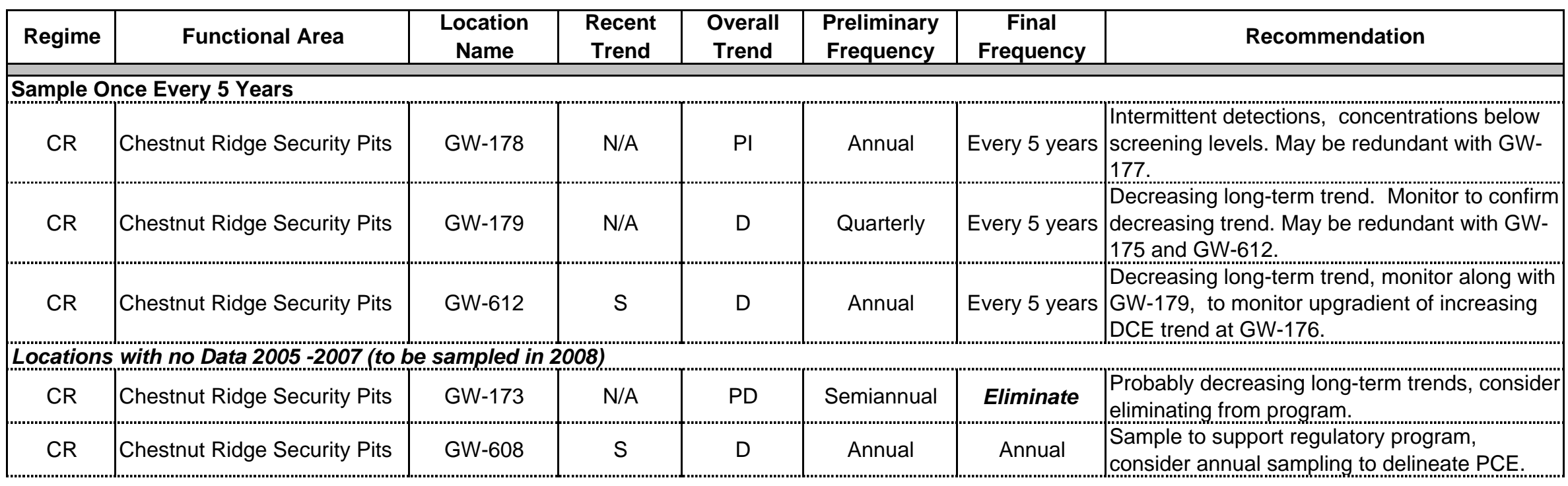




\section{DISTRIBUTION}

\section{U.S. DEPARTMENT OF ENERGY}

J. D. Darby, DOE-EM

J. P. Donnelly, DOE-NNSA *

TENNESSEE DEPARTMENT OF

ENVIRONMENT AND CONSERVATION

DOE-ORR OVERSIGHT DIVISION

J. E. Sebastian *

B\&W Y-12, L.L.C.

ENVIRONMENTAL COMPLIANCE

DEPARTMENT

S. M. Field

C. C. Hill

S. B. Jones *

E. R. Schultz *

O. D. Stevens

L. O. Vaughan

Y-12 Central Files *

9114DMC-01971865.6551-RC

Y-12 Records Services (Electronic copy- OSTI)

YDCC - RC *
BECHTEL JACOBS COMPANY LLC

E. L. Berglund

R. H. Ketelle

L. M. Sims *

File - EMEF-DMC *

File - Y-12 Project PDCC *

UT-BATTELLE, LLC

D. B. Watson

GSI ENVIRONMENTAL, INC.

M. Vanderford

ELVADO ENVIRONMENTAL LLC

T. R. Harrison *

J. R. Walker *

Note: $*=$ receives hard copy version 